TRAVAUX ET DOCUMENTS DU CIRAC

\title{
FRANCE-ALLEMAGNE : CULTURES MONÉTAIRES ET BUDGÉTAIRES
}

Vers une nouvelle gouvernance européenne?

Sous la direction de

Solène HAZOUARD

René LASSERRE

Henrik UTERWEDDE

CIRAC 


\section{France-Allemagne : Cultures monétaires et budgétaires}

Vers une nouvelle gouvernance européenne?

\section{Solène Hazouard, René Lasserre et Henrik Uterwedde (dir.)}

DOI : 10.4000/books.cirac. 100

Éditeur : IFAEE

Année d'édition : 2015

Date de mise en ligne : 13 décembre 2017

Collection : Travaux et documents du CIRAC

ISBN électronique : 9782905518712

\section{Qbooks}

http://books.openedition.org

\section{Édition imprimée}

ISBN : 9782905518484

Nombre de pages : 282

\section{Référence électronique}

HAZOUARD, Solène (dir.) ; LASSERRE, René (dir.) ; et UTERWEDDE, Henrik (dir.). France-Allemagne Cultures monétaires et budgétaires : Vers une nouvelle gouvernance européenne ? Nouvelle édition [en ligne]. Cergy-Pontoise : IFAEE, 2015 (généré le 02 octobre 2020). Disponible sur Internet : <http:// books.openedition.org/cirac/100>. ISBN : 9782905518712. DOI : https://doi.org/10.4000/books.cirac. 100 .

(c) IFAEE, 2015

Conditions d'utilisation:

http://www.openedition.org/6540 
TRAVAUX ET DOCUMENTS DU CIRAC

\section{FRANCE-ALLEMAGNE : CULTURES MONÉTAIRES ET BUDGÉTAIRES}

Vers une nouvelle gouvernance européenne?

Sous la direction de

Solène HAZOUARD René LASSERRE Henrik UTERWEDDE 


\section{FRANCE-ALLEMAGNE : CULTURES MONÉTAIRES ET BUDGÉTAIRES}

Vers une nouvelle gouvernance européenne?

Sous la direction de Solène HAZOUARD, René LASSERRE, Henrik UTERWEDDE

\section{CIRAC}

TRAVAUX ET DOCUMENTS DU CIRAC 


\section{Travaux et documents du CIRAC}

Collection dirigée par René Lasserre

Ouvrage publié avec le concours du Centre interdisciplinaire d'études et de recherches sur l'Allemagne (CIERA), ainsi que de l'Institut d'études avancées et du centre de recherche Civilisations et identités culturelles comparées (CICC) de l'université de Cergy-Pontoise

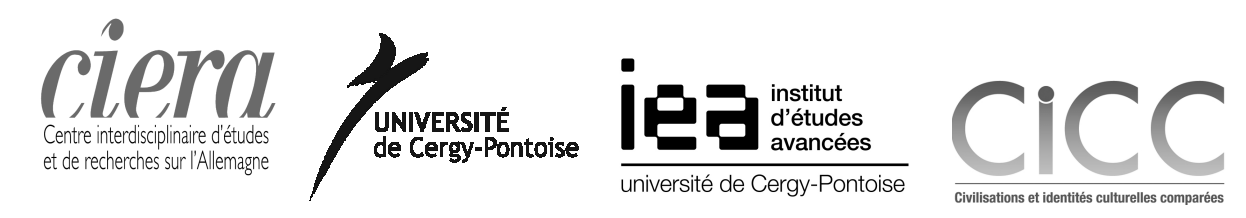

Les contributions publiées dans cet ouvrage engagent uniquement la responsabilité de leurs auteurs.

(C) CIRAC, 2015

http://www.cirac.u-cergy.fr

CIRAC, c/o Université de Cergy-Pontoise

33, boulevard du Port - 95011 CERGY-PONTOISE CEDEX

ISBN : 978-2-905518-48-4 


\section{France-Allemagne : CULTURES MONÉTAIRES ET BUDGÉTAIRES}

\section{VERS UNE NOUVELLE GOUVERNANCE EUROPÉENNE ?}

\section{TABLE DES MATIÈRES}

REMERCIEMENTS

\section{INTRODUCTION}

Avancer vers plus de fédéralisme en Europe, malgré les divergences nationales

Solène Hazouard, René Lasserre, Henrik Uterwedde.

I - Cultures monétaires en France et en Allemagne

Principes fondateurs et constantes en matière de politique monétaire en République fédérale d'Allemagne depuis 1945

Manfred J.M. Neumann.

Les voies et moyens d'une longue mutation de la culture et des pratiques monétaires en France depuis 1945

Michel Margairaz

Le couple franco-allemand : (més-)entente cordiale ?

Hans-Helmut Kotz

L'UEM, Maastricht et la BCE : controverses franco-allemandes

Pascal Kauffmann

Table ronde : Convergences et divergences franco-allemandes

sur la politique monétaire européenne. État des lieux et perspectives

Exposé introductif : Paul J.J. Welfens.

Ouverture de la table ronde : Rainer Klump.

Exposés des intervenants

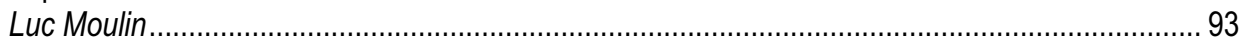

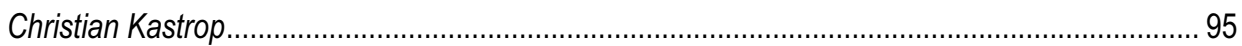

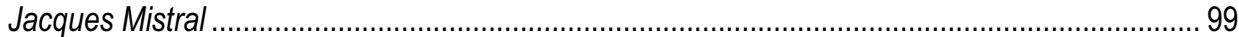

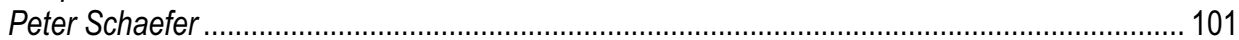

II - Cultures budgétaires en France et en AlLemagne............................. 107

Principes fondateurs et constantes de la politique budgétaire de la République fédérale d'Allemagne

Heinz Grossekettler.

Principes fondateurs et constantes en matière de politique budgétaire

en France depuis 1945

Frédéric Tristram... 
La politique budgétaire allemande après Maastricht :

l'expression d'une culture budgétaire ?

Michael Thöne

La politique budgétaire depuis le Pacte de stabilité et de croissance :

point de vue français

Jérôme Creel.

Table ronde : Les contraintes budgétaires liées au Pacte de stabilité et de croissance et au pacte budgétaire européen (TSCG), dont le MES, sont-elles praticables et adaptées pour sortir l'économie européenne de la crise ?

Ouverture de la table ronde : Rainer Klump.

Exposés des intervenants

Christian Kastrop

Sandrine Gaudin

Conclusion : Rainer Klump

III - GOUVERNANCE ÉCONOMIQUE, FINANCIÈRE ET MONÉTAIRE

DE L'UNION EUROPÉENNE

Table ronde : Quelle(s) politique(s) économique(s) pour la zone euro ?

Ouverture de la table ronde : René Lasserre.

Exposés des intervenants

Christian Kastrop

Jean-Marc Daniel

Henrik Uterwedde

Natacha Valla

Table ronde : Quel rôle doit jouer la BCE aux yeux des responsables politiques français et allemands et quelle est sa politique actuelle ?

Ouverture de la table ronde : Christian de Boissieu.

Exposés des intervenants

Jacques Mistral

Philippe Moutot

Friedrich Heinemann

Gerald Braunberger

Conclusion : Christian de Boissieu

Table ronde : Regards communautaires sur les enjeux de la politique économique et financière européenne

Ouverture de la table ronde : Guntram B. Wolff.

Exposés des intervenants

Miguel Moltó Calvo.

Gustavo Piga.

Antoin E. Murphy 


\section{REMERCIEMENTS}

Cet ouvrage collectif constitue la synthèse des travaux de deux journées d'étude et d'un colloque, organisés en 2013 et 2014 dans le cadre du projet: "Culture monétaire, culture budgétaire en Allemagne et en France. Divergences et convergences francoallemandes. Sur la voie d'une nouvelle gouvernance européenne? 》 Mené par le Centre d'information et de recherche sur l'Allemagne contemporaine (CIRAC), en partenariat avec le Deutsch-Französisches Institut de Ludwigsburg (DFI), ce projet s'inscrit dans le cadre des programmes de formation-recherche soutenus par le Centre interdisciplinaire d'études et de recherches sur l'Allemagne (CIERA). Il avait pour objet d'étudier les origines historiques, politiques et culturelles et la dimension institutionnelle des différends monétaires et budgétaires entre la France et l'Allemagne, ainsi que leur évolution. $\mathrm{Si}$ ces différends furent en partie surmontés dans le grand moment de la création de l'euro et si de nouveaux rapprochements se dessinent actuellement sous l'impulsion conjointe des chefs d'État et de gouvernement, ils continuent malgré tout de peser sur la relation franco-allemande dans la gestion de la crise que traverse l'Europe aujourd'hui. Il apparaissait donc essentiel d'en mettre à jour les racines, d'en analyser les continuités et les manifestations actuelles dans le contexte de l'Union économique et monétaire (UEM), ceci afin de mieux discerner les risques qu'ils font courir au couple francoallemand dans sa capacité à promouvoir une gouvernance économique durable de l'Europe.

Pour mener à bien ce projet, les organisateurs ont pu bénéficier, en majeure partie, du concours financier apporté par le CIERA, auquel s'est associé le centre de recherche Civilisations et identités culturelles comparées (CICC) et l'Institut d'études avancées (IEA) de l'université de Cergy-Pontoise. Nous remercions vivement les auteurs, issus de divers pays et de différents horizons scientifiques ou professionnels, pour leur contribution à cet ouvrage, ainsi que Marie-Céline Georg et Marie Gravey, qui ont assuré la traduction de leurs travaux.

Nous tenons également à remercier l'équipe du CIRAC pour son concours, en particulier Meryem Richter, stagiaire, pour sa coopération aux travaux d'édition, Werner Zettelmeier, chargé de recherches, pour ses relectures attentives, ainsi qu'Héloïse Cuillier, documentaliste, pour l'aide précieuse apportée à la finalisation de l'ouvrage.

$\begin{array}{ccc}\text { Solène Hazouard } & \text { René Lasserre } & \text { Henrik Uterwedde } \\ \text { Ingénieur d'études au CIRAC } & \text { Directeur du CIRAC } & \text { Chercheur associé au DFI }\end{array}$





\section{Avancer vers plus de fédéralisme en Europe, malgré les divergences nationales}

\section{Solène HazouARD, René LASSERRE, Henrik UTERWEDDE}

Depuis ses débuts, malgré la volonté commune de réconciliation et d'intégration européenne de l'après-guerre, la coopération économique entre la France et l'Allemagne a toujours été difficile. Si les controverses et les conflits franco-allemands dans ce domaine ont été nombreux (politique commerciale, politique agricole commune dans les années 1960, politique industrielle, politique monétaire dans les années 1970 et 1980), ils ont finalement été surmontés pour déboucher sur l'avancée décisive qu'a constitué l'Union économique et monétaire (UEM) et la création de l'euro en 2002. Mais depuis le déclenchement de la crise financière de 2008, puis de celle de la zone euro en 2010, les désaccords et les affrontements franco-allemands se sont multipliés. Les deux gouvernements n'ont cessé de s'opposer quand il s'est agi de trouver des réponses politiques à la crise de la dette souveraine, à la crédibilité de l'euro et à la reprise de la croissance en Europe. Les points d'achoppement n'ont pas manqué : le soutien financier à la Grèce, le rôle de la Banque centrale européenne (BCE) et le débat sur la mutualisation de la dette, l'instauration d'un gouvernement économique, le pacte fiscal et l'instauration d'une règle d'or, l'Union bancaire, la critique du modèle d'exportation et de compétitivité allemand, sans oublier le sempiternel débat sur les sanctions à l'égard des pays réfractaires aux règles communes de discipline budgétaire... Ces différends, souvent amplifiés par les médias et le débat public, ont même donné lieu, au gré des circonstances et des échéances politiques européennes, à des tensions palpables au sein du "couple franco-allemand ", au point que la fonction motrice qu'avait antérieurement jouée l'entente franco-allemande dans le processus d'intégration européenne s'est trouvée ouvertement mise en doute. Certains observateurs allant même jusqu'à voir dans cette mésentente persistante un élément d'incertitude majeur pour la capacité de l'Union européenne à surmonter sa crise de crédibilité.

Certes, les deux gouvernements ont su pour l'essentiel surmonter leurs désaccords et trouver les compromis nécessaires pour consolider l'UEM. Ainsi la dialectique qui est au cœur de la coopération franco-allemande a-t-elle continué à jouer son rôle qui consiste, à partir du constat des divergences initiales des intérêts et approches, à générer la volonté politique commune permettant de les transformer en compromis constructifs au service de l'Europe. Ce fut toutefois dans des conditions plus difficiles, à la fois en raison d'un contexte global plus contraignant, mais sans doute aussi du fait que les enjeux à traiter touchaient désormais non seulement à des intérêts vitaux, mais plus encore à des traditions politiques et conceptuelles constitutives de la culture, pour ne pas dire de l'identité économique des deux pays.

C'est à l'exploration de ces rémanences culturelles respectives qui, dans cette phase cruciale de la construction européenne, sous-tendent et inspirent les conceptions différentes que la France et l'Allemagne ont de la gouvernance 
économique de l'Europe qu'a été consacré notre programme de formationrecherche, lequel s'est délibérément focalisé sur les deux champs cruciaux des cultures monétaires et budgétaires.

Le projet, mené sur deux ans, s'est articulé autour de deux journées d'étude et d'un colloque final rassemblant un ensemble de chercheurs et experts français, allemands et européens de différentes disciplines, reconnus pour leur compétence et leur expérience dans les champs considérés. Leurs contributions ont été ensuite mises en forme pour donner lieu au présent ouvrage qui répond à un triple objectif :

- identifier les principaux éléments constitutifs des cultures monétaires et budgétaires contrastées qui ont été au cœur des modèles économiques français et allemand d'après-guerre, dégager leurs racines, ainsi que les choix politiques fondamentaux qui en ont initialement découlé dans la période de reconstruction, mais aussi en analyser le développement ultérieur dans le contexte de l'internationalisation et de l'intégration croissante de l'économie européenne. L'analyse s'efforçant de faire la part, dans chacun de ces deux champs, des trajectoires nationales et de leurs évolutions spécifiques, en même temps que des rapprochements et convergences relatives entre les deux pays ;

- analyser l'interaction entre ces cultures nationales, dans la conception et la mise en œuvre de l'UEM. D'une part, en tentant d'apprécier l'impact des cultures monétaires et budgétaires sur les approches allemande et française de l'Europe économique et monétaire, ainsi que sur l'architecture de l'UEM proprement dite. D'autre part aussi pour voir dans quelle mesure la dynamique de l'intégration européenne, ses limites et les ajustements qu'elle a imposés sous l'effet de la crise, ont eu un impact sur les conceptions nationales, induisant des modifications, voire poussant à des changements de paradigme ;

- s'interroger, enfin, sur les implications de ces changements au niveau européen : quelles sont, entre les cultures différentes et leurs évolutions respectives, les chances d'un rapprochement? L'existence de visions et de choix différents est-elle un frein, sinon un handicap pour l'émergence d'une gouvernance économique, financière et monétaire européenne ? Ou bien peut-elle au contraire enrichir la réflexion et ouvrir ainsi la voie à une gouvernance appropriée ?

La notion de «culture » économique, monétaire ou budgétaire ne s'impose pas d'emblée dans les éléments qu'elle recouvre. Nous avons opté pour une approche pragmatique, qui définit ce terme comme l'ensemble des valeurs, principes doctrinaux et préférences collectives qui guident les choix dans le domaine concerné, et permettent d'expliquer les grandes orientations de la politique dans une perspective longue, en l'occurrence depuis la fin de la Seconde Guerre mondiale. Cette dernière a en effet marqué une rupture par le fait 
que tous les pays européens ont dû définir un nouveau modèle économique et social qui s'est ensuite inséré dans le nouveau cadre international défini par les accords de Bretton Woods et l'accord général sur les tarifs douaniers et le commerce (General Agreement on Tariffs and Trade, GATT). À l'intérieur de ce nouveau cadre, ainsi que dans celui qu'elles contribuent conjointement à promouvoir pour l'intégration européenne, les choix de l'Allemagne et de la France portent chacun le sceau de leurs trajectoires et expériences collectives antérieures assez fortement contrastées.

Ainsi, l'Allemagne, sortant de deux hyperinflations, de l'échec de la République de Weimar et de la dictature sanglante du nazisme, a eu un souci particulier pour la stabilité, un terme qui, bien au-delà de l'économie, s'est aussi appliqué au régime politique et au système social. En revanche, elle pouvait compter sur une structure productive solide développée depuis la fin du XIX ${ }^{\mathrm{e}}$ siècle et sur une compétitivité internationale incontestée. La France, pour sa part, avait accumulé tout au long de la III $^{\mathrm{e}}$ République un retard de développement économique et social séculaire ; après 1944, elle s'est trouvée confrontée à un défi énorme de modernisation accélérée afin de réaliser son rattrapage vis-à-vis de ses voisins. Cet objectif ambitieux ne pouvait être réalisé qu'à la condition d'une croissance soutenue placée au premier rang des priorités de l'État. D'où une différence fondamentale entre les mythes fondateurs de l'après-guerre : la modernisation pilotée par l'État en France, l'ordolibéralisme et l'économie sociale de marché (soziale Marktwirtschaft) en Allemagne, rendue populaire par Ludwig Erhard, le «père » du miracle économique allemand, dont l'objectif prioritaire était de délivrer les Allemands de près d'un siècle de dirigisme et de leur rendre la liberté et la capacité d'entreprendre. Alors qu'en France, l'État se vit érigé en promoteur de la croissance et en pilote du développement, s'installait enfin de l'autre côté du Rhin un «État modeste », dont la principale préoccupation était de créer les conditions-cadre optimales d'un marché dans lequel les acteurs pourraient donner libre cours au développement de leur capacité créatrice et de leur savoir-faire.

\section{Approches française et allemande en matière de politique monétaire...}

Les deux premières parties de l'ouvrage explicitent de manière détaillée ce tableau franco-allemand contrasté. Elles s'emploient également à cerner les évolutions, en faisant la part des constantes et des changements, voire des ruptures dans certains cas, et s'interrogent également sur l'impact européen des différences : divergences, convergences, capacités de compromis à l'échelle européenne.

Dans la première partie consacrée aux cultures monétaires, il apparaît clairement à quel point les grandes orientations de la politique monétaire ont été tributaires des grands choix fondateurs de l'après-1945 : en Allemagne, l'ordolibéralisme et le référentiel de l'économie sociale de marché, qui ont commandé 
la grande valeur attachée à la stabilité monétaire, à l'indépendance de la Bundesbank et à la prévention du risque de change, ces trois priorités qui constituent la constante de la politique monétaire allemande jusqu'à nos jours. La tendance à l'appréciation de la monnaie, qui s'est exprimée tant dans le régime de Bretton Woods jusqu'en 1972 que par la suite dans le Système monétaire européen (SME) jusqu'à la création de l'euro, a pu être compensée par - et a renforcé - la spécialisation industrielle dégageant des avantages qualitatifs en termes d'innovation et de gamme de produits (M.J.M. NEUMANN). En France, la politique monétaire a été mise au service de l'effort de modernisation de l'appareil productif et subordonnée à la politique du crédit, à la fois quantitative et sélective. Son caractère inflationniste a été dénoncé à plusieurs reprises, sans toutefois se voir durablement infléchi dans les années 1960 et 1970, jusqu'au changement de paradigme introduit par la politique de rigueur et de libéralisation des marchés financiers dans les années 1983-1986 (M. MARGAIRAZ).

Ces approches nationales différentes ont trouvé leur prolongement dans les postures respectives des deux pays face à l'Union monétaire. Celle-ci a été largement calquée sur l'approche allemande, qui a insisté sur l'indépendance de la $\mathrm{BCE}$ et son attachement à l'objectif de stabilité monétaire, ainsi que sur des règles contraignant les politiques budgétaires nationales à combattre tout déficit excessif. Si, à la suite de la crise, l'Allemagne a accepté l'instauration d'un Mécanisme européen de stabilité (MES) doté de ressources, conçu comme un mécanisme de résolution de crise, elle a en revanche revendiqué - et obtenu - le renforcement des règles de stabilité, notamment à travers le traité sur la stabilité, la coordination et la gouvernance (TSCG) imposant des règles strictes aux États membres en matière d'équilibre budgétaire et d'endettement. L'Allemagne a consenti à l'Union bancaire mais reste hostile à l'idée d'émission d'eurobonds et à toute forme de mutualisation de la dette (H.-H. KOTZ).

D'autres désaccords ont concerné la politique dite non conventionnelle de la BCE (rachat de dettes souveraines, assouplissement des conditions de crédit etc.), qui suscite des inquiétudes en Allemagne, ainsi que la politique de change, revendiquée par la France pour mettre fin à l'euro trop fort (P. KAUFFMANN). Dans les deux cas, la BCE a agi de manière autonome sans qu'il y ait eu de compromis négociés au niveau gouvernemental. Ce faisant, elle est allée jusqu'aux limites de son mandat. La balle est de nouveau dans le camp des gouvernements, qui devront répondre à la nécessité de définir un concept de gouvernance économique de l'Union européenne, et de renforcer le caractère politique, voire fédéral, de l'UEM.

\section{... et de politique budgétaire}

La deuxième partie de l'ouvrage, portant sur les cultures budgétaires française et allemande, fait également ressortir des constantes nationales mais aussi des évolutions significatives. L'Allemagne reste marquée par le fédéralisme budgé- 
taire, ancré dans la Constitution, qui combine la gestion séparée et autonome par les Länder et des éléments coopératifs, exprimés dans des lois fédérales qui contraignent Bund et Länder à faire concorder les règles budgétaires et à mener des politiques concertées. Autres constantes : l'aversion de la population allemande pour tout endettement excessif et un droit budgétaire conçu de telle sorte qu'il préserve la viabilité des finances publiques (H. GROSSEKETTLER).

L'évolution de la politique budgétaire a été plus mouvementée en France, oscillant entre le volontarisme dépensier dans la période de reconstruction des années 1950, la recherche de l'équilibre budgétaire introduit en 1959 à la suite d'une rupture réglementaire et doctrinale (plan Pinay-Rueff), et la période postérieure au premier choc pétrolier en 1973, donnant lieu à une nouvelle doctrine marquée par une nette augmentation des recettes et des dépenses et l'apparition d'un niveau élevé de déficit et d'endettement. Cette doctrine et cette pratique se trouvent de nouveau remises en question avec le renforcement des règles budgétaires européennes. Les constantes depuis 1945 résident dans un niveau élevé de la dépense publique comme des prélèvements obligatoires, et surtout dans la volonté constamment réaffirmée d'utiliser le budget comme instrument de réglage conjoncturel, ce qui revient à considérer le déficit comme l'un des principaux leviers pour augmenter la croissance (F. TRISTRAM). Les différences francoallemandes en termes de culture budgétaire se font sentir dans les pratiques politiques : ainsi en France, la part des dépenses publiques dans le produit intérieur brut (PIB) est nettement supérieure (57\%) à celle observée en Allemagne (45\%), une différence considérable qui ne se retrouve pas forcément dans la qualité des services publics offerts en France. On peut en conclure qu'il existe des marges pour réduire la dépense publique en améliorant l'efficacité des politiques publiques et sans forcément tomber dans une politique d'austérité et du moins-disant social (M. THÖNE). À cette interprétation, qui met l'accent sur le caractère structurel des déficits budgétaires français, s'oppose une autre vue, très présente dans le débat français, selon laquelle la responsabilité majeure de l'aggravation de la situation budgétaire française est à chercher dans la crise conjoncturelle depuis 2008. Dans cette perspective, une politique de désendettement pourrait mener à l'austérité, avec un risque déflationniste pesant en retour sur la capacité de remboursement de l'État (J. CREEL).

Face à ces différends importants, comment peut-on trouver un terrain commun pour stabiliser l'Union monétaire et sortir l'Europe de la crise économique ? Les contributions de deux hauts fonctionnaires des deux ministères des Finances font apparaître comment les deux gouvernements ont su dépasser certains clivages et trouver des pistes d'action communes, qui se manifestent dans les progrès atteints en matière de stabilité budgétaire, économique et financière et de gestion de crise. Ce faisant, les responsables des deux pays ont démontré qu'ils sont d'accord sur l'essentiel. Certes, les nouveaux accents à promouvoir peuvent varier, l'Allemagne insistant davantage sur les réformes structurelles nationales, qu'il conviendrait de mieux concerter, tandis que la France pointe 
l'absence d'un outil européen de stabilisation macroéconomique en cas de choc, absence qu'il faudrait combler en renforçant l'outil de coordination budgétaire et en créant une capacité budgétaire de la zone euro. Restent les questions controversées de l'opportunité ou non de créer des instruments de mutualisation de la dette (eurobonds) ou de nouveaux transferts financiers, qui demanderaient en tout cas de nouveaux dispositifs de contrôle et de prise de décision parlementaires à l'échelle européenne (C. KASTROP, S. GAUDIN).

\section{Vers une nouvelle gouvernance européenne?}

Cinq ans après le déclenchement de la crise de la zone euro, le débat sur les perspectives de la gouvernance économique, financière et monétaire de l'UEM s'est dépassionné et des convergences se dégagent. C'est ce que font ressortir l'ensemble des contributions aux trois tables rondes, rassemblées dans la troisième partie de l'ouvrage.

Ainsi, il semble d'ores et déjà admis qu'il est vain d'opposer réformes structurelles et stimulation macroéconomique, consolidation budgétaire et croissance, en somme : stabilité et croissance. La situation européenne commande d'agir sur plusieurs fronts à la fois. La consolidation budgétaire reste essentielle, mais elle gagnerait à être programmée et échelonnée dans le temps pour éviter des effets procycliques. Afin d'augmenter le potentiel de croissance, il faudra y ajouter des programmes de réformes structurelles appropriés aux situations nationales respectives, portant sur le fonctionnement des marchés du travail, des biens et des services, sur l'efficacité des administrations et des politiques publiques ou sur la formation et l'innovation. Là encore, le souci d'éviter des effets contracycliques est légitime, à condition de ne pas rejeter ces réformes $a d$ vitam aeternam au prétexte que «ce n'est pas le moment». Enfin, un effort d'investissement, tant au niveau national qu'à l'échelle européenne, pourra à la fois compléter les efforts structurels et stimuler la croissance. Dans ce contexte, la Banque européenne d'investissement (BEI) et les banques d'inves-tissement nationales (qui pourront davantage se mettre en réseau) pourraient potentiellement et dans un avenir prévisible jouer un rôle important.

Quant au rôle de la BCE, si la politique non conventionnelle, très active, est saluée par la plupart des observateurs, elle semble également avoir atteint ses limites. La politique monétaire ne peut pas tout; elle ne pourra en aucun cas combler la carence des gouvernements nationaux. Resurgit alors le caractère hybride de l'UEM, ayant créé une monnaie unique sans pouvoir politique commun, ce qui l'expose à des fragmentations et menace de la déstabiliser. Or une vision plus politique, voire fédérale de l'UEM s'impose si l'on veut rendre l'Union monétaire plus robuste, qu'il s'agisse des problèmes d'une gouvernance plus efficace ou des problèmes croissants de légitimité démocratique des décisions prises. 
Cette perspective soulignant le caractère politique de l'UEM et la nécessité d'une vision plus fédérale est également présente dans les regards communautaires sur les enjeux européens venant d'Espagne, d'Italie et d'Irlande. Par ailleurs, les trajectoires de ces pays rappellent que la crise de la zone euro a eu des origines nationales diverses, comme l'éclatement de «bulles » dans l'immobilier en Espagne et en Irlande et la crise bancaire qui s'en est ensuivie.

Les exemples de ces trois pays illustrent parfaitement la permanence des différences culturelles en Europe concernant les grands choix économiques, les approches politiques et les valeurs, qui sont l'objet central de ce livre. Si les contributions ont montré que malgré ces différences, la gestion de crise, notamment franco-allemande, a malgré tout permis d'avancer et de poser les bases d'une UEM plus solide, il conviendra d'aller plus loin. Il faudra dépasser une vision trop parcellaire des problèmes par une perspective plus ouverte, et mener un débat sur une stratégie d'ensemble. De même devra-t-on enfin assumer le caractère politique du projet de monnaie commune et s'orienter vers une perspective plus fédérale, condition sine qua non du renforcement, voire de la pérennisation de l'UEM.

Enfin, les cultures économiques mettront du temps à évoluer et à converger. Seul un climat de confiance mutuelle et l'émergence progressive de valeurs partagées permettront d'ouvrir de nouvelles perspectives à l'UEM, dans laquelle on pourra passer d'une logique normative, dominée par des contraintes et des sanctions, à une logique plus contractuelle. En tout état de cause, cependant, il sera difficile de continuer à vouloir faire progresser et approfondir l'UEM en l'absence de toute perspective d'union politique. 

$-I-$

CULTURES MONÉTAIRES en France et en AlLEmagne 



\title{
Principes fondateurs et constantes en matière de politique monétaire en République fédérale d'Allemagne depuis $1945^{1}$
}

\author{
Manfred J.M. NEUMANN
}

" En tant que chancelier, il m'arrive d'avoir des problèmes avec la Bundesbank. En tant que citoyen, je suis heureux qu'elle existe. "

Helmut Kohl, chancelier fédéral, 1997

La réforme monétaire de juin 1948 introduit le deutsche mark dans les zones d'occupation occidentales, en remplacement du reichsmark jusqu'alors en vigueur. Le reichsmark avait été totalement ébranlé par l'inflation destinée à soutenir l'effort de guerre et, à l'issue du conflit, il avait progressivement perdu sa fonction de moyen de paiement universellement accepté. Avant même la fondation de la République fédérale d'Allemagne (RFA) en mai 1949, des banques centrales régionales avaient été instituées dans les trois zones d'occupation occidentales, auxquelles la compétence d'émission de la nouvelle monnaie avait été transférée. En mars 1948 fut créée la Banque des Länder allemands afin de permettre la coordination d'une politique monétaire commune. Son organe de direction se composait des présidents des banques centrales régionales². Jusqu'en 1956, la Banque des Länder allemands eut pour mission d'élaborer la politique monétaire ouest-allemande et d'en assumer la responsabilité. La Bundesbank allemande prit ensuite le relais et conduisit la politique monétaire et financière de la RFA jusqu'en 1998, soit pendant quarante-deux ans.

Si l'on observe les cinquante années de politique monétaire et financière, avec leurs aléas, de 1948 à 1998, à la recherche de principes fondateurs ou de constantes, on relève les traits suivants : premièrement, la poursuite résolue de l'objectif de stabilité monétaire interne ; deuxièmement, à partir du début des années 1970, une acceptation croissante du principe de cours de change flexibles ou ajustables ; troisièmement, l'attachement constant à l'indépendance de la banque centrale vis-à-vis de la politique et des groupes d'intérêts. Cependant, en forçant un peu le trait, on pourrait dire que la politique monétaire allemande n'obéissait qu'à un seul principe directeur, auquel tout le reste était subordonné : la garantie d'une stabilité monétaire durable ${ }^{3}$. En effet, on ne saurait préserver la stabilité de la valeur d'une monnaie au sens de la stabilité du niveau des prix dans le pays si l'on devait dans le même temps maintenir la stabilité du cours des changes par rapport à une monnaie dominante, à moins que celle-ci n'obéisse également à l'objectif de stabilité monétaire interne. II n'est pas davantage possible d'assurer la stabilité monétaire interne si la direction de la banque centrale

\footnotetext{
${ }^{1}$ Article rédigé en novembre 2013, NdE.

${ }^{2}$ Sur l'organisation détaillée de la banque centrale à l'époque, voir Buchheim (1998), p. 105-117.

${ }^{3}$ La stabilité monétaire interne doit se comprendre comme la stabilité du niveau des prix. Aujourd'hui toutefois, suivant l'usage anglo-saxon, on parle plus souvent de « stabilité des prix ». Or il est clair qu'il s'agit non pas de la stabilité des différents prix, mais seulement d'une moyenne pondérée.
} 
n'est pas libre de mener la politique monétaire et financière qu'elle considère appropriée, mais qu'elle est au contraire assujettie aux préoccupations des politiques face à la conjoncture et aux prévisions budgétaires.

L'expérience engrangée pendant des décennies par la Bundesbank, mais également par la Banque nationale suisse, avec ce statut de banque indépendante, ont conduit à prendre conscience, au plan international, qu'une banque centrale doit être indépendante, faute de quoi l'on ne peut s'attendre à une politique monétaire suffisamment soucieuse d'assurer la stabilité des prix. C'est pourquoi le statut de la Banque centrale européenne, créée dans les années 1990, suit à plusieurs égards le modèle de la Bundesbank. La première partie analysera, en s'appuyant sur l'histoire monétaire allemande, différents aspects de l'indépendance de cet établissement. Les deuxième et troisième parties examineront dans quelle mesure la Bundesbank a rempli sa mission légale de préservation de la monnaie. À cette fin, nous commencerons par une présentation assez précise de la stabilité interne réelle du mark, en nous appuyant sur l'indice du coût de la vie et en comparant la situation allemande aux évolutions en France et aux États-Unis (deuxième partie). II s'agira également de mettre en évidence les fluctuations dues aux différents régimes de change. Dans la troisième partie, nous analyserons plus en détail la manière dont la Bundesbank a fait face aux différentes situations de change, ce que nous illustrerons à l'aide de quelques exemples.

\section{L'indépendance, une condition fondamentale}

L'État peut financer ses dépenses grâce à des recettes ordinaires et, quand celles-ci ne suffisent plus, recourir en outre à l'émission de dettes d'État ou à la création monétaire. Le monopole de l'État en matière de création monétaire met ainsi à la disposition des responsables politiques une réponse séduisante aux difficultés financières. Alors que les hausses d'impôts doivent être largement communiquées et s'imposer dans la procédure parlementaire, l'accroissement de la masse monétaire peut se faire en toute discrétion ou presque. Lorsqu'est imprimé un billet de banque supplémentaire, le bénéfice de la création monétaire se calcule en soustrayant les coûts d'émission à la valeur nominale du billet, le bénéfice total issu de la création monétaire apparaissant dans les bénéfices annuels de la banque centrale. D'un point de vue strictement technique, une banque d'émission peut ainsi augmenter ses bénéfices et les verser au budget de l'État en augmentant progressivement l'achat d'actifs portant intérêt qu'elle paie à l'aide de la base monétaire nouvellement créée ${ }^{4}$. Si une telle politique est conduite sur la durée, elle entraîne un processus d'expansion monétaire qui fait augmenter plus rapidement le crédit et les placements bancaires, stimule au bout d'un certain temps l'activité économique mais qui, finalement, se répercute sur le niveau général des prix car le potentiel de croissance du PIB réel est limité. Cette dynamique peut en-

\footnotetext{
${ }^{4}$ La base monétaire, dite également masse monétaire centrale, correspond à la somme des liquidités en circulation et des comptes à vue détenus par les banques auprès de la banque centrale.
} 
traîner une inflation, laquelle a l'effet d'une imposition de l'épargne des citoyens, la valeur réelle de la trésorerie baissant à mesure que le niveau des prix augmente. Les citoyens ne peuvent échapper à cet impôt inflationniste car ils sont contraints d'utiliser de l'argent public. Cet impôt touche en particulier ceux qui perçoivent des revenus modestes car ils n'ont en général ni les connaissances ni les moyens nécessaires pour se prémunir contre l'inflation.

Deux solutions radicalement différentes sont envisageables pour empêcher les gouvernements de recourir à cette manne financière que représente la création monétaire. Soit des dispositions constitutionnelles lient la banque centrale à des règles limitant sa marge de manœuvre discrétionnaire en matière de politique monétaire, soit la banque centrale bénéficie d'un statut indépendant vis-à-vis du gouvernement.

Les tenants de la première solution, notamment les économistes américains libéraux de l'École de Chicago depuis Simons (1936), considéraient qu'il valait mieux soumettre la création monétaire à une règle stricte. La règle la plus connue, prônée par Milton Friedman, prévoyait un accroissement de la masse monétaire de $k$ pour cent chaque année, le taux $k$ se calculant sur la base des taux de croissance prévisibles du revenu national et de la vitesse de circulation de la monnaie ${ }^{5}$. L'idée d'une règle fixe a longtemps eu une place importante dans le courant monétariste, mais elle n'est finalement pas parvenue à s'imposer. De fait, l'idée qu'il vaudrait mieux confier la politique monétaire à un système automatique plutôt qu'à des gouverneurs faillibles est un peu naïve. L'évolution aurait dû nous apprendre que les automates sont condamnés à disparaître car ils n'ont pas la capacité d'innover ${ }^{6}$. Les tenants de la règle fixe n'ont pas suffisamment pris en compte la nécessité d'établir également une méta-règle définissant les conditions dans lesquelles il faudrait modifier la règle.

L'alternative à une règle fixe consiste à accorder un statut indépendant à la banque centrale. L'indépendance s'applique à l'institution, mais elle ne peut jouer son rôle protecteur que si les gouverneurs de la banque centrale sont eux-mêmes indépendants vis-à-vis du pouvoir politique. L'interdiction de financer l'État et la liberté d'action sont les indispensables piliers de l'indépendance de l'institution. Les modalités de nomination, la durée des mandats et le renouvellement éventuel de ceux-ci sont des éléments constitutifs de l'indépendance des gouverneurs. Il faut analyser ces caractéristiques plus en détail pour comprendre pourquoi la Bundesbank allemande et la Banque des Länder allemands qui l'a précédée ont réussi à faire du deutsche mark, créé en 1948, l'une des monnaies les plus stables de leur époque.

La liberté d'action était déjà garantie à la Banque des Länder allemands par le gouvernement militaire en vertu de la loi $\mathrm{n}^{\circ} 60 \mathrm{du} 1^{\mathrm{er}}$ mars 1948 . Il est dit à l'art. I, § 3 que «...la banque n'est subordonnée aux instructions

${ }^{5}$ Cf. Friedman (1959).

${ }^{6}$ Cf. Neumann (1992). 
d'aucune espèce d'entité politique ou organisme public à l'exception des autorités judiciaires ». Cette liberté d'action explicite a été reprise par le législateur allemand à l'alinéa 12 , phrase 2 , de la loi portant création de la banque centrale (Bundesbankgesetz) de 1957. Le gouvernement fédéral se voit certes concéder le droit (art. 13 (2)) de participer aux réunions du conseil des gouverneurs de la banque centrale, mais il n'a pas le droit de vote. Si une décision de cet organe ne lui convenait pas, le gouvernement était autorisé à opposer sous huit jours un veto suspensif. Le conseil pouvait alors rejeter ce veto à la majorité des voix lors de sa réunion suivante. Dans les faits, aucun gouvernement fédéral n'a jamais fait usage de cette clause de veto. En effet, dans les rares cas où un gouvernement a souhaité invoquer ce droit, la Bundesbank en a rejeté la validité au motif que le gouvernement avait déjà fait usage de son veto en émettant préalablement des objections contre un projet de décision ${ }^{7}$. L'indépendance de la banque se trouva également renforcée du fait qu'elle interprétait de facto son obligation de conseiller le gouvernement dans les affaires importantes en matière de politique monétaire (art. 13 (1) Bundesbankgesetz) comme un droit de conseil ; elle justifiait cette interprétation en arguant que, sans cela, un gouvernement pourrait éventuellement esquiver un conseil jugé inopportun en ne sollicitant pas la banque centrale ${ }^{8}$.

Il fut d'emblée interdit à la Bundesbank de financer l'État, c'est-à-dire la Fédération et les Länder. Comparativement à ce que lui permet son statut actuel, celui de l'époque du deutsche mark l'autorisait à accorder à la Fédération et aux Länder des «crédits de caisse » à court terme, très limités en volume. Ces crédits servaient à garantir la fluidité des paiements pour les budgets publics, eux-mêmes dans l'obligation de maintenir leurs liquidités sur les comptes courants de la Bundesbank.

Pour ce qui est de l'indépendance individuelle des membres du conseil des gouverneurs de la banque centrale, il convient de préciser qu'en vertu de l'organisation fédérale du pays, les gouvernements régionaux pouvaient également, en plus du gouvernement fédéral, proposer au président fédéral des candidats à la nomination. L'âge des différents membres du conseil des gouverneurs permettait d'étaler les nominations dans le temps. En général, pour une année donnée, une seule personne était nommée. Les mandats étaient d'une durée de huit ans, avec la possibilité d'être ensuite nommé de nouveau pour un deuxième mandat de même durée. Neumann (1998) a examiné la durée de mandat dans les faits pour 38 personnes membres du conseil des gouverneurs de la banque centrale dans la période $1969-1995^{9}$. Il en est ressorti une durée de 14 ans en moyenne. Cela signifie que les personnes nommées une première fois ont vu leur mandat renouvelé de manière quasi automatique. C'est un phénomène tout à fait remarquable dans la mesure où la cou-

\footnotetext{
${ }^{7}$ Cf. Neumann (1998a), note de bas de page $n^{\circ} 33$, p. 331.

${ }^{8}$ Cf. Deutsche Bundesbank (1972), p. 16-17.

${ }^{9}$ Cf. Neumann (1998a), p. 319.
} 
leur politique du gouvernement fédéral comme des gouvernements régionaux a connu des changements réels au cours des 25 années étudiées. En dépit des alternances, les gouvernements n'ont donc pas nécessairement tenté de nommer leurs partisans à des postes de gouverneurs devenus vacants. Dans ses travaux, Neumann (1998) s'est également demandé si l'appartenance à un parti avait une incidence sur les comportements de vote des membres du conseil des gouverneurs de la banque centrale lorsqu'il s'agissait de modifier le taux d'escompte ou le taux des avances sur titre («taux Lombard ») ${ }^{10}$. Il n'a pas été possible d'établir la preuve systématique d'une telle hypothèse.

En résumé, on peut retenir que la Bundesbank allemande (et, avant elle, la Banque des Länder allemands), disposait d'une grande indépendance vis-à-vis des gouvernements fédéraux et de la vie politique. Une condition fondamentale et nécessaire de la stabilité monétaire interne fut ainsi présente dès 1948 en Allemagne. L'indépendance est une condition nécessaire pour la raison suivante : en garantissant un tel statut, la sphère politique se lie les mains (Neumann 1991a, 1991b). Elle ne peut plus prendre les commandes. L'indépendance n'est toutefois pas une condition suffisante car elle donne aux gouverneurs une marge de manœuvre que ceux-ci pourraient tout à fait utiliser pour mener une politique monétaire inflationniste, pour quelque raison que ce soit.

Il faut toutefois avoir à l'esprit que l'indépendance octroyée à la Bundesbank n'était alors pas inscrite dans la constitution de l'État mais dans une simple loi. Il aurait suffi d'une majorité simple au Bundestag pour pouvoir amender cette loi sur la Bundesbank. Les gouverneurs étaient conscients que cette protection légale ne deviendrait pérenne que s'ils parvenaient à se forger une excellente réputation auprès de la population. Cela supposait d'une part de rendre visible l'objectif de stabilité comme ligne directrice de la politique monétaire, d'autre part de ne pas éviter les conflits avec le gouvernement fédéral, mais de les affronter pleinement, y compris publiquement ${ }^{11}$.

De fait, dès le milieu des années 1950, la Banque des Länder allemands fut en capacité de refuser publiquement une baisse du taux d'escompte souhaitée par Konrad Adenauer, alors chancelier fédéral, car l'opinion publique faisait confiance à l'établissement pour mener une politique garantissant la stabilité interne de la monnaie. La Bundesbank a toujours veillé à ne pas décevoir cette confiance. C'est pourquoi aucun gouvernement fédéral n'a jamais osé proposer de modifier la loi sur la Bundesbank dans un sens qui n'eût pas l'appui de l'établissement lui-même.

\section{Stabilité réelle : une brève comparaison}

Pour apprécier le niveau de stabilité intérieure d'une monnaie, on se base sur les taux d'évolution de l'indice des prix à la consommation. Nous comparerons la

\footnotetext{
${ }^{10}$ Cf. Neumann (1998a), p. 320-329.
}

${ }^{11}$ Cf. également Spahn (1995), p. 9. 
RFA à trois pays : les États-Unis, en leur qualité de pays de la monnaie de référence du système de changes fixes de Bretton Woods, la France, en tant que principal partenaire de l'Allemagne dans le cadre de l'Union européenne, et la Suisse, en tant que petit pays frontalier à la monnaie traditionnellement stable.

Pour toute cette période de près de cinquante ans, il apparaît que la stabilité interne a été relativement bien assurée en Allemagne et en Suisse, avec une hausse générale des prix de respectivement 2,8 \% et $3 \%$ par an en moyenne (cf. tableau 1). C'est en tout cas ce qui ressort de la comparaison avec les Etats-Unis (4\%) ou la France (5,7\%). En valeur absolue, c'est-à-dire sans tenir compte des conditions historiques, ce ne sont en revanche pas de bons résultats. En effet, une hausse générale des prix de $2,8 \%$ par an signifie qu'en cinquante ans, la monnaie a perdu les trois quarts de sa valeur de pouvoir d'achat (cf. dernière ligne du tableau 1).

Tableau 1 : Taux d'inflation moyens (indices des prix à la consommation), en \%

\begin{tabular}{|l|c|c|c|c|c|}
\cline { 3 - 6 } \multicolumn{2}{c}{} & \multirow{2}{*}{} & Allemagne & \multicolumn{3}{c|}{ Pour comparaison : } \\
\cline { 4 - 6 } \multicolumn{2}{c|}{} & $\begin{array}{c}\text { États- } \\
\text { Unis }\end{array}$ & France & Suisse \\
\hline $\begin{array}{l}\text { Union européenne } \\
\text { des paiements }\end{array}$ & $1950-1958$ & 1,16 & 1,92 & 6,21 & 1,34 \\
\hline Indexation sur le dollar & $1959-1972$ & 2,77 & 2,69 & 4,41 & 3,39 \\
\hline Serpent monétaire européen & $1973-1978$ & 5,11 & 7,71 & 10,18 & 4,88 \\
\hline Système monétaire européen & $1979-1998$ & 2,76 & 4,73 & 5,14 & 2,95 \\
\hline Total & $\mathbf{1 9 5 0 - 1 9 9 8}$ & $\mathbf{2 , 7 6}$ & $\mathbf{4 , 0 0}$ & $\mathbf{5 , 7 5}$ & $\mathbf{3 , 0 1}$ \\
\hline $\begin{array}{l}\text { Valeur réelle de la monnaie } \\
\text { (en \%) }\end{array}$ & 1949 & 100 & 100 & 100 & 100 \\
\hline
\end{tabular}

Sources : Destatis, Réserve fédérale américaine, Insee, Banque nationale suisse.

L'érosion de la valeur de la monnaie sur le marché domestique s'est faite de manière irrégulière. Dans les années 1950, période de reconstruction forcée de l'économie après la Seconde Guerre mondiale, la hausse moyenne des prix fut particulièrement basse : à peine $1,2 \%$. Des facteurs propices contribuèrent à cette situation: les États-Unis menaient globalement une politique monétaire modérée qui maintenait la stabilité du dollar, et les syndicats, faisant le pari d'un développement rapide de l'emploi, avaient accepté une politique de modération salariale. Le ministre allemand de l'Économie Ludwig Erhard poursuivait en outre la libéralisation des marchés et notamment celle du commerce extérieur. On arriva ainsi dès 1956 à une convertibilité de facto du deutsche mark qui privait prématurément de sa raison d'être l'Union européenne des paiements (1950-1958), institution de compensation multilatérale.

C'est dans les années 1970 que la monnaie connaît sa plus forte perte de valeur, et cela vaut pour tous les pays comparés ici. Si l'on fait la moyenne 
des années 1973-1978, le taux d'inflation fut de 5,1\% en Allemagne; en France, il fut même deux fois plus élevé. Cela tient d'une part au fait que les États-Unis avaient mené pendant des années une politique monétaire trop expansionniste et exporté en s'appuyant sur un déficit de la balance des paiements. D'autre part, l'OPEP, le cartel fixant les prix du pétrole, multiplia les prix du pétrole par quatre à l'automne 1973, créant ainsi un « choc pétrolier » mondial auquel de nombreuses économies réagirent par une politique monétaire trop accommodante.

\section{Ancrage dans des régimes de change fixes : sources de perturbation de la stabilité}

En juillet 1957, au moment d'adopter la première loi sur la Bundesbank (Bundesbankgesetz), dont l'alinéa 3 formulait l'objectif «de préserver la monnaie $»^{12}$, certains députés allemands au Bundestag se seraient demandé si cette formulation visait la valeur du deutsche mark sur le marché domestique ou bien sa valeur nominale dans les échanges extérieurs, représentée par le taux de change par rapport à la monnaie de référence de l'époque, le dollar américain. En effet, une banque centrale ne peut garantir à la fois la stabilité interne et externe d'une monnaie ${ }^{13}$. La stabilité interne d'une monnaie est tout à fait compatible avec des fluctuations relativement importantes des cours des changes : en effet le cours correspond au taux de change entre deux devises et ne dépend donc pas uniquement de la conjoncture domestique, mais également de la situation économique à l'étranger.

Ce conflit fondamental entre l'orientation vers la stabilité interne ou vers la stabilité externe a immanquablement marqué la politique monétaire de la Bundesbank. Jusqu'au milieu des années 1970, l'ancrage dans le système monétaire mondial de Bretton Woods fut une constante essentielle de la politique monétaire allemande; elle fut ensuite remplacée par l'inscription dans le Système monétaire européen (SME), entré en vigueur en mars 1979. Par conséquent, pendant les trois premières décennies d'existence du deutsche mark, la politique monétaire allemande se focalisa surtout sur le cours du mark par rapport au dollar ; puis sur le cours du mark par rapport à l'ECU ${ }^{14}$, la nouvelle unité monétaire européenne. Les deux systèmes monétaires étaient des régimes à taux de change fixe, mais les parités étaient ajustables. Pour l'Allemagne, dont la banque centrale s'était donné la stabilité interne de la monnaie pour impératif suprême, cela

\footnotetext{
${ }^{12} \S 3$ de la Bundesbankgesetz : "La Bundesbank allemande règle, au moyen des compétences de politique monétaire qui lui sont conférées en vertu de la présente loi, la circulation monétaire et l'octroi de crédits à l'économie, l'objectif étant de préserver la monnaie. Elle veille au règlement bancaire des paiements dans le pays et à l'étranger. » Cf. Bundesbank (1993), p. 120.

${ }^{13}$ Emminger (1975, p. 486) affirme que « les grands représentants de la banque centrale » auraient cru initialement que l'on pouvait du même coup atteindre les deux objectifs.

${ }^{14}$ L'acronyme ECU se réfère à l'anglais European currency unit [en français : Unité de compte européenne].
} 
signifiait nécessairement qu'il fallait négocier régulièrement avec les partenaires les modifications des parités.

\section{Ancrage dans le système de Bretton Woods}

En 1952, l'Allemagne rejoint le système monétaire de Bretton Woods, à la parité qui était en vigueur avant 1933 d'un dollar pour 4,20 marks ${ }^{15}$. Afin de favoriser une reconstruction plus rapide du pays, les syndicats acceptent, pour toute la période des années 1950, une politique de modération salariale. Il s'ensuivit une croissance économique remarquablement forte, de $7 \%$ par an en moyenne et, dès le début pratiquement, des excédents de la balance des transactions courantes. Dès le milieu des années 1950, la Banque des Länder allemands fut confrontée à un premier dilemme quant à l'orientation à privilégier entre stabilité interne et stabilité externe. Afin d'atténuer la pression à la hausse constatée pour la première fois sur le mark, il aurait fallu baisser les taux d'intérêt ; mais pour freiner le boom économique de l'époque et ainsi parer au risque d'aggravation de la flambée des prix, il fallait au contraire une hausse des taux d'intérêt. C'est cette dernière option que la Banque des Länder allemands considéra comme nécessaire. Contrairement à ce que souhaitait expressément le chancelier fédéral Konrad Adenauer, elle releva donc à plusieurs reprises le taux d'escompte et accepta l'afflux de devises qui en résulta comme une conséquence inévitable du régime de change fixe. Ainsi, les réserves de devises passèrent de 1,1 milliard de deutsche mark en 1950 à 13,3 milliards de deutsche mark en 1955, et même à 33,2 milliards de deutsche mark en $1960^{16}$.

Dans la seconde moitié des années 1950, il eût été possible de demander une réévaluation du mark dans le cadre du Fonds monétaire international plutôt que de le maintenir servilement arrimé au dollar. Cette solution aurait vraisemblablement reçu le soutien du ministre fédéral de l'Économie si la banque centrale l'avait sollicitée. Mais le conseil des gouverneurs, considérant le maintien de la parité mark/dollar comme un indispensable gage de stabilité, n'était dans sa large majorité pas prêt à franchir ce pas. L'afflux de devises, qui se poursuivit pendant des années, obligea finalement la Bundesbank à réévaluer le mark pour la première fois en mars 1961. Cette réévaluation fut décidée à l'instigation de Ludwig Erhard, membre du gouvernement, et ce, contre la volonté de la Bundesbank. Konrad Adenauer ordonna une revalorisation de $5 \%$, soit un taux sensiblement plus faible que celui qu'avait proposé le ministre de l'Économie Ludwig Erhard $(7,7 \%)^{17}$. La sous-évaluation du mark, apparue en une décennie, ne fut pas complètement corrigée.

La revalorisation de 1961 constitua cependant une césure dans l'histoire monétaire allemande. Elle est cruciale car elle marque un tournant dans le rai-

\footnotetext{
${ }^{15}$ Pour ce qui suit, cf. Neumann (1998b).

${ }^{16}$ Deutsche Bundesbank (1976), p. 48.

${ }^{17}$ Pour le détail de la discussion menée à l'époque sur la réévaluation, cf. Emminger (1986), p. 98-134.
} 
sonnement des gouverneurs de la banque centrale allemande. La glace des taux de change intangibles était brisée. L'on commençait à raisonner autrement. Pour la première fois, l'on avait en outre osé imposer une dégradation de la compétitivité en termes de prix. En Allemagne, mais également dans les pays partenaires, on apprivoisait l'idée qu'il ne fallait pas considérer simplement la modification des parités comme une mesure d'urgence à éviter par principe. On commençait à l'envisager comme une voie praticable et même élégante s'agissant de réduire durablement les déséquilibres en matière de commerce extérieur. L'industrie allemande, et en particulier les secteurs d'exportation, commença à apprendre qu'à long terme une revalorisation de la monnaie est inévitable lorsque l'on a acquis une avance compétitive réelle et durable.

$\mathrm{Au}$ cours des années 1960, les tensions s'intensifièrent à nouveau entre le dollar et le mark, mais aussi et surtout entre le mark et les monnaies des partenaires européens comme le franc et la livre sterling. En 1968, les tensions culminèrent dans une vaste crise franc/deutsche mark. Il eût été facile de la surmonter en combinant dévaluation du franc et appréciation du mark. Mais en Allemagne, les avis divergeaient: la Bundesbank était favorable à un ajustement des cours, mais le gouvernement fédéral, compétent en matière de modification des parités, rejetait cette solution. Il refusait de modifier durablement la parité mark/dollar, lui préférant une mesure provisoire qui, en outre, n'affecterait pas les prix agricoles. C'est ainsi qu'en novembre 1968, le gouvernement opta pour un "ersatz de réévaluation ", à savoir une solution fiscale consistant à combiner une taxe exceptionnelle sur les exportations de $4 \%$ et une réduction fiscale sur les importations, également de $4 \%$. Cette mesure s'avéra bientôt inadaptée, en particulier car elle ne contribuait pas à atténuer le boom économique de 1969 provoqué par la politique fiscale. C'est pourquoi il fallut, en octobre 1969, substituer à la solution fiscale une revalorisation du mark de $9,3 \%$. Mais en réalité, cette réévaluation intervenait plus d'un an trop tard, et le taux d'appréciation était bien trop faible, de sorte qu'une vague de hausse des prix et des salaires se produisit en 1969-1970. Si la RFA avait opté dès 1968 pour une réévaluation de $10 \%$ voire un peu plus, elle aurait sans doute pu empêcher l'explosion des salaires qui eut lieu à l'automne 1969.

Au début de la décennie 1970, le dollar montrait des signes de faiblesse, et le système à taux de change fixe de Bretton Woods touchait à sa fin. Les ÉtatsUnis suspendirent de manière unilatérale l'obligation de détenir la contrepartie en or de la masse monétaire. Le dollar fut soutenu à l'international à l'aide d'interventions considérables et l'on tenta même de déréguler provisoirement la parité mark/dollar. Vint alors une succession de crises. Il ne s'agit pas ici d'en retracer le détail, mais ce qui est crucial, c'est que dans de nombreux pays, la conviction s'imposa peu à peu qu'un « flottement» durable des monnaies serait inévitable. Or, en Allemagne, les industries d'exportation s'y opposaient. Des responsables politiques de premier plan partageaient leurs doutes, notamment Helmut Schmidt, alors ministre fédéral des Finances, et Karl Klasen, alors pré- 
sident de la Bundesbank. À l'inverse, Otmar Emminger, vice-président du même établissement et gouverneur en charge des questions monétaires internationales, défendait depuis longtemps déjà un point de vue moins rigide. Depuis le début des années 1960, il s'était à plusieurs reprises prononcé pour une réévaluation du mark.

Face à la crise du marché des changes, qui entraîna en février 1973 un déferlement de dollars sur le franc suisse et le deutsche mark, Emminger acquit la conviction - et, comme lui, une majorité au sein du conseil des gouverneurs de la banque centrale, mais non son président - qu'un flottement du mark serait la seule issue praticable si l'on souhaitait stopper le gonflement de la masse monétaire allemande et la hausse du niveau des prix qui l'accompagnait. La seule question était de savoir si le flottement indépendant du mark était inévitable ou si l'on pourrait parvenir à la solution, plus attrayante, d'un flottement concerté avec le franc et d'autres devises européennes. Dans la première hypothèse, les décideurs politiques auraient vraisemblablement renoncé assez rapidement au flottement pour revenir à un régime de change fixe. En revanche, avec un flottement concerté, les nombreux intérêts impliqués rendaient peu probables un revirement et une nouvelle indexation des monnaies sur le dollar.

Le $1^{\text {er }}$ mars 1973, un hasard de l'histoire permit à Emminger de préparer la sortie définitive du système de Bretton Woods ${ }^{18}$. Ce jour-là, la Bundesbank dut intervenir en faveur du dollar à hauteur de près de 8 milliards de marks. Cette somme représentait quatre fois l'augmentation totale des liquidités en circulation pour l'ensemble de l'année $1973^{19}$. Il n'était pas possible de continuer ainsi. Les adversaires d'un flottement immédiat n'étaient pas en situation de lutter : le président de la Bundesbank, Karl Klasen, se trouvait hospitalisé, tout comme le ministre fédéral des Finances Helmut Schmidt; quant à son secrétaire d'État Karl-Otto Pöhl, en charge des questions monétaires, il était en vacances de ski en Suisse, et n'était pas joignable par téléphone. En sa qualité de vice-président, c'est Emminger qui, en l'absence de Klasen, gérait les affaires de la Bundesbank et dirigeait le conseil des gouverneurs. Il décida d'agir. Il se fit donner mandat par le conseil des gouverneurs et se rendit à Bonn pour y consulter le chancelier fédéral Willy Brandt, qui n'était guère versé dans les questions de politique monétaire. Emminger parvint, avec le soutien du libéral Hans Friderichs, ministre fédéral de l'Économie, à convaincre le chancelier Brandt de la nécessité de fermer immédiatement les marchés des changes pour mettre fin au soutien au dollar, source d'inflation. À compter du 2 mars, les marchés des changes restèrent fermés en Allemagne et dans les pays partenaires, et ce pour deux semaines. Au cours de cette période, les pays se mirent d'accord lors d'une réunion du Conseil des ministres de la Communauté européenne, le

\footnotetext{
${ }^{18} \mathrm{Cf}$. Emminger (1986, p. 240-251) pour une présentation passionnante, quoique narcissique, des événements.

${ }^{19}$ Deutsche Bundesbank (1976), tab. B III. 1.03, p. 32. Emminger (1986, p. 240) établit quant à lui une comparaison avec un accroissement «normal » indéterminé de l'argent de la banque centrale.
} 
11 mars, pour un flottement généralisé au niveau européen auquel participèrent, outre l'Allemagne et la France, six autres pays, mais non l'Angleterre.

\section{Flottement et pilotage du Système monétaire européen (SME)}

Le passage au flottement libéra les États de leur pesante obligation d'intervention et, surtout, du déferlement impitoyable de dollars et des risques d'inflation qui y étaient associés. La Bundesbank avait désormais les mains libres pour gérer la masse monétaire. Cependant, cela signifiait aussi qu'il fallait un nouvel ancrage indiquant de façon crédible au grand public la volonté de préserver la stabilité interne de la monnaie. Cette donne radicalement nouvelle ne prenait pas la Bundesbank tout à fait au dépourvu. Dès 1970, le conseil de la banque centrale avait en effet mené en toute discrétion un vaste débat sur le paradigme des théories monétaristes et sur les principes défendus par Milton Friedman en matière de politique monétaire.

La Bundesbank refusait l'idée de fonder la politique monétaire sur une loi mécanique applicable à la masse monétaire ; mais elle reprit à son compte l'idée de communiquer à l'opinion des objectifs chiffrés et de déclarer, en s'appuyant sur la théorie quantitative classique, quelles étaient les prévisions en matière de croissance économique et de vitesse de circulation de la monnaie utilisées pour établir les objectifs annuels concrets de croissance de la masse monétaire ${ }^{20}$. Fin 1974, la Bundesbank publia pour la première fois un objectif chiffré pour le taux d'augmentation de la masse monétaire de l'année 1975. Par la suite, elle mit en place la publication de fourchettes cibles, puis changea même d'agrégat cible. Jusqu'en 1987, l'agrégat cible qualifié de masse monétaire centrale correspondait à la somme des liquidités détenues hors du système bancaire et des réserves obligatoires, dépôts à vue, livrets d'épargne et dépôts à court terme des établissements non-bancaires dans le pays, calculés à coefficient constant de réserves obligatoires ${ }^{21}$. À compter de 1987, ce fut la masse monétaire M3 définie au sens large (liquidités détenues hors du système bancaire plus dépôts à vue, livrets d'épargne et dépôts à court terme) qui servit d'agrégat cible. Des expériences diverses furent donc menées, et il n'aurait pu en aller autrement puisque cette communication autour de la politique monétaire était sans précédent dans l'histoire.

Rétrospectivement, il est intéressant de noter que le système des objectifs de masse monétaire a toujours bien fonctionné, et ce jusqu'à ce que la Banque centrale européenne prenne le relais de la Bundesbank en 1998. Ce système a fait ses preuves, cet instrument permettant d'influencer les attentes de l'opinion publique en matière de stabilité des prix. Avec ses objectifs annuels, la Bundesbank traça, dans la pratique, le cadre dans lequel les attentes annuelles des ménages et des entreprises en termes de salaires et de prix pouvaient selon toute

\footnotetext{
${ }^{20}$ À propos de l'influence du monétarisme sur la nouvelle politique monétaire de la Bundesbank, voir Richter (1998), notamment p. 578-586.

${ }^{21}$ Neumann (1997), p. 181-182.
} 
vraisemblance évoluer sans heurts. Ce procédé transparent, qui permettait d'anticiper, valut à la Bundesbank une forte crédibilité ${ }^{22}$. Bien sûr, l'objectif visé fut manqué à plusieurs reprises, mais le procédé établi permettait alors à la banque de déclarer de manière transparente pourquoi l'objectif de masse monétaire défini n'avait pas été atteint. Cette ouverture renforça la confiance de l'opinion dans la politique de la banque centrale.

Après 1973, la Bundesbank ne réussit pas dans un premier temps à empêcher une hausse sensible du niveau général des prix. Le taux d'inflation passa de 1,8\% en 1968 à 7,1\%, et la multiplication par quatre des prix du pétrole par l'Organisation des pays exportateurs de pétrole à l'automne 1973 menaçait de faire grimper le taux d'inflation bien au-delà de $10 \%$, comme cela se produisit en 1974 dans de nombreux pays. Mais, contrairement à certaines de ses homologues, la banque centrale allemande refusa de prendre des mesures d'accommodation monétaire. Le taux d'escompte fut laissé à un niveau élevé afin de couper court à la crainte indésirable d'une aggravation de la hausse des prix. Ce tour de force réussit, mais contribua certainement à la récession mondiale de 1974-1975. De même, lors du second choc pétrolier, en 1979, la Bundesbank refusa toute mesure d'accommodation monétaire. Début 1979, elle avait renoncé à la politique monétaire très expansionniste menée en 1978 afin de soutenir le dollar américain. Elle prit un cap plus restrictif et introduisit une hausse des taux d'intérêt. Malgré les critiques virulentes émanant du gouvernement fédéral, la banque centrale ne laissa pas la nouvelle hausse considérable des prix du pétrole la détourner d'une politique restrictive. Au contraire, en concertation avec la Réserve fédérale américaine, dirigée depuis 1979 par le faucon Paul Volcker, elle durcit ses orientations anti-inflationnistes afin de parer à une nouvelle vague de hausse des prix. En un an, le taux d'escompte fut porté de $3 \%$ à $7,5 \%$; il fut maintenu à ce niveau extrêmement élevé pendant deux ans, soit jusqu'au milieu de l'année 1982.

Le graphique 1 présente l'évolution des taux d'inflation en Allemagne (RFA) sur l'ensemble de la période 1950-1998 et, pour comparaison, les taux d'inflation en France. Si l'évolution des courbes en RFA fait apparaitre l'impact sensible des chocs pétroliers de 1973 et 1979, le succès de la Bundesbank se traduit par le fait que la pression à la hausse fut à chaque fois moins forte en Allemagne qu'en France. Ce graphique apporte également un éclairage sur les effets de la politique monétaire allemande sur le taux de change bilatéral. Pendant près de cinquante ans et sans exception ou presque, le taux d'inflation a été plus élevé en France qu'en RFA, ce qui à long terme ne pouvait que faire perdre de sa valeur au franc. Le cours bilatéral moyen au comptant du franc est passé de 1,19 mark en 1950 à 0,30 mark en 1998, soit une valeur relative divisée par quatre (cf. partie basse du graphique 1). D'un point de vue économique, on note avec un intérêt particulier que l'évolution du taux de change bilatéral mark/franc coïncide de manière impressionnante avec la célèbre théorie de la

\footnotetext{
${ }^{22}$ Pour une analyse empirique des objectifs de masse monétaire, voir Neumann (1997), section 4, The Bundesbank's Performance.
} 
parité de pouvoir d'achat, une théorie des changes élaborée par l'économiste suédois Gustav Cassel (1866-1945). La courbe en pointillés décrit l'évolution de la parité de pouvoir d'achat du franc par rapport au mark. Abstraction faite du début des années 1950, elle ne s'écarte du taux de change effectivement observé que de façon non significative ${ }^{23}$.

\section{Graphique 1: Inflation en Allemagne et en France (prix à la consommation, en \%)}

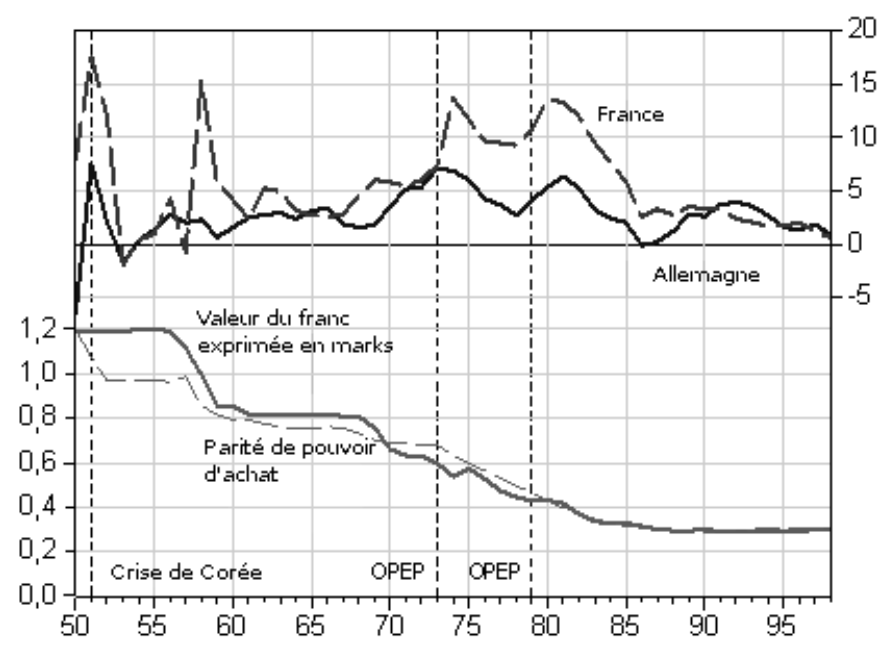

Sources : Destatis, Deutsche Bundesbank, Insee ; présentation de l'auteur.

S'il n'avait tenu qu'à la Bundesbank, il n'y aurait pas nécessairement eu, après l'effondrement du système de Bretton Woods, de nouveau système monétaire dans un cadre européen restreint. En effet, le flottement avait permis à la banque centrale allemande de recouvrer une liberté d'action illimitée en matière de politique monétaire. L'expérience des objectifs de masse monétaire publiés était en outre encourageante, et cette expérimentation fut pour quelques années accueillie favorablement par d'autres banques. Se lier à un nouveau système de change ne pouvait qu'être synonyme de nouvelles menaces pour la force du mark sur le marché intérieur et l'indépendance de la Bundesbank ${ }^{24}$. En outre, le serpent monétaire européen n'avait pas été une expérience convaincante. Créé en 1972 par

\footnotetext{
${ }^{23}$ Pour calculer la courbe de la parité de pouvoir d'achat, nous avons pris pour point de départ le taux de change réel de l'année 1950 . Si $\mathrm{k}_{\mathrm{t}}$ représente la parité pour la période $\mathrm{t}, \mathrm{k}_{\mathrm{t}-1}$ celle de la période précédente, $\pi_{\mathrm{t}}^{\mathrm{D}}$ le taux d'inflation de l'Allemagne et $\pi_{\mathrm{t}}^{\mathrm{F}}$ celui de la France pour la période $\mathrm{t}$, la parité peut être décrite comme suit : $\mathrm{k}_{\mathrm{t}}=\mathrm{k}_{\mathrm{t}-1} *\left(1+\pi_{\mathrm{t}}^{\mathrm{D}}\right) /\left(1+\pi_{\mathrm{t}}^{\mathrm{F}}\right)$.

${ }^{24}$ La Bundesbank ne se conforma donc pas au préjugé de Milton Friedman sur les banques centrales. Selon Emminger (1986, p. 292), Friedman aurait déclaré en mai 1972 lors d'une conférence bancaire internationale à Montréal qu'il n'y aurait jamais de flottement car les banques centrales perdraient alors leur raison d'être. «Les cours de change fixes génèrent régulièrement de nouvelles crises et conférences monétaires ; cela permet aux banques centrales de se donner de l'importance et de se démarquer. "
} 
l'Allemagne, la France, l'Italie et les États du Benelux, ce dispositif multilatéral d'intervention avait pour vocation de réduire les marges de fluctuation des cours entre eux. Mais cet instrument se solda pour ainsi dire par un échec, une fois disparue l'obligation d'intervenir au profit du dollar américain. Et surtout, la France, qui avait joué le jeu au début, préféra, après une deuxième tentative en ce sens, le flottement indépendant. Le serpent perdait ainsi tout sens pour l'Allemagne également.

C'est pourquoi, lorsque Helmut Schmidt et Valéry Giscard d'Estaing proposèrent en 1978 une construction technocratique alambiquée en vue du Système monétaire européen (SME), la Bundesbank commença par faire obstacle à cette proposition. Ce projet, élaboré par Horst Schulmann, alors chef de service à la Chancellerie fédérale, prévoyait de mettre en place des taux de change fixes mais ajustables entre les monnaies nationales d'une part et un panier européen de monnaies, l'unité de compte ECU, d'autre part. Un indicateur de divergence devait établir laquelle des devises nationales s'écartait le plus fortement de l'ECU et donc de l'ensemble des autres monnaies. Cette devise devait donc, à l'aide d'interventions en ce sens, être alignée sur le cours des autres devises.

La Bundesbank vit tout de suite le défaut de cette construction. Si les banques centrales des autres pays décidaient de mener sur la durée une politique monétaire plutôt expansionniste, l'indicateur de divergence montrerait que la Bundesbank s'écartait trop du cours pivot et devrait donc s'aligner sur les autres. Un tel scénario impliquait pour la Bundesbank l'obligation de mener une politique plus inflationniste. Il fallait donc s'attendre à ce que le SME empêche la Bundesbank d'honorer sa mission légale, à savoir assurer la stabilité interne de la monnaie. La banque centrale allemande s'y refusait unanimement. Diverses modifications furent apportées au projet initial lors de négociations avec le gouvernement fédéral et les partenaires étrangers. La plus importante consistait à retirer à l'indicateur de divergence sa fonction de déclencheur automatique d'une obligation d'intervention, l'ECU étant relégué de facto à un rôle purement symbolique. Dans la nouvelle version du projet, les obligations d'intervention étaient associées à des évolutions critiques des taux de change bilatéraux entre deux monnaies à l'intérieur de marges de fluctuations fixes de $+/-2,25 \%$. Les obligations bilatérales d'intervention devaient s'appliquer de manière symétrique. Cela signifie que les deux parties devaient intervenir, et ce de manière «automatique » et «illimitée ». Prise au mot, cette disposition n'était pas sans danger. Mais elle restait théorique. La Bundesbank pouvait donc supposer qu'en sa qualité de banque centrale de la plus grande zone monétaire, elle aurait disposé d'une marge de négociation suffisante en cas de situation concrète d'intervention.

La Bundesbank n'en resta pas là. Elle formula par écrit ce qui devait être un prérequis pour donner son accord au SME : le principe selon lequel, dans le cas particulier où «des obligations démesurées d'intervention » menacent la stabilité interne d'une devise, le gouvernement fédéral "préserve la Bundesbank 
d'un tel embarras, soit en corrigeant les parités au sein du SME, soit, si nécessaire, en la déliant au moins provisoirement de l'obligation d'intervention ${ }^{25}$. En novembre 1978, au cours d'une réunion extraordinaire du conseil des gouverneurs de la banque centrale, le chancelier Helmut Schmidt tenta de gagner l'accord définitif de la Bundesbank en faveur du SME sans avoir eu l'engagement du gouvernement fédéral sur cette suspension de l'obligation d'intervention. Mais le conseil des gouverneurs ne démordit pas de ce principe. Le chancelier finit par céder car il avait conscience que c'était la Bundesbank qui disposerait des meilleures cartes en cas de conflit ouvert. Mais le procès-verbal de la réunion rapporte ainsi ses propos : "Un tel accord ne se donne pas par écrit, Messieurs. $\|^{26}$

Le SME a duré officiellement vingt ans. Au cours de cette période, il fallut redéfinir 23 fois le cours pivot du mark. Une crise des taux de change survint pratiquement tous les ans. À chaque crise ou presque, il fallut négocier âprement pour décider quelles parités il s'agissait de modifier et surtout dans quelle proportion. Outre les seuls faits économiques, des aspects purement politiques jouèrent souvent un rôle. Les trois réévaluations les plus fortes du mark se produisirent en 1981, 1983 et 1986, avec des taux d'appréciation de 4,7 \% à 5,6\% ${ }^{27}$. Le freinage d'urgence concédé à la Bundesbank par le gouvernement fédéral, consistant à suspendre les interventions obligatoires en faveur de la devise d'un pays partenaire si l'ampleur de cette intervention menaçait la stabilité interne de la monnaie en Allemagne, se produisit pour la première fois en mars 1983, alors que le franc faisait une nouvelle fois face à une forte pression à la baisse. Lorsque la Bundesbank déclara ne plus être obligée d'intervenir encore en faveur du franc, la Banque de France tomba des nues, tout comme le gouvernement français. Il fallut alors combiner dévaluation du franc et réévaluation du mark. Jusque-là, l'arrangement interne allemand, en contradiction flagrante avec l'accord officiel sur le SME, était resté inconnu des partenaires européens. L'action déterminée de la Bundesbank fut un choc qui poussa l'administration française dirigée par le président François Mitterrand à se détourner en 1983 de sa politique socialiste et keynésienne expansionniste et protectionniste ${ }^{28}$. Par la suite, la Bundesbank invoqua une seconde fois son droit de suspendre ses interventions, à savoir en septembre 1992, face à la Banca d'Italia. Cette attitude contraignit l'Italie à quitter le mécanisme de change du SME.

D'un point de vue économique, on peut arguer que le SME n'a fonctionné que jusqu'au milieu de l'année 1993 comme un système à taux de change fixe. À cette époque également, la situation monétaire était volatile depuis un certain temps, ce qui se traduisit fin juillet par une crise du franc. La Banque de France

\footnotetext{
${ }^{25}$ Emminger (1986), p. 361 ; cf. également Neumann (1998a), p. 338.

${ }^{26}$ Le procès-verbal de la réunion, approuvé par les deux parties, fit office d'accord écrit.

${ }^{27}$ Deutsche Bundesbank, Statistische Beihefte Reihe 5 - Devisenkursstatistik, janvier 2000, tableau III.2, p. 44.

${ }^{28}$ Hetzel $(2002$, p. 53) souligne à juste titre que ce retournement était une condition nécessaire pour permettre l'union monétaire avec l'Allemagne.
} 
fut alors la proie d'une attaque spéculative si forte qu'elle perdit en quelques heures seulement la moitié de ses réserves internationales. Le 2 août, les ministres des Finances et gouverneurs des banques centrales des États membres du SME organisèrent une réunion à Paris, laquelle se déroula de manière dramatique. Il n'y était plus question de réévaluation ou de dévaluation. Au lieu de cela, le gouvernement français d'Édouard Balladur créa la surprise en déclarant, par la voix de son ministre des Finances Edmond Alphandéry que, pour résoudre les problèmes, l'Allemagne devait quitter le SME. Cela n'aura pas lieu, les représentants des Pays-Bas et d'autres petits pays ayant laissé entendre au cours des négociations que, si jamais l'Allemagne quittait le SME, leurs gouvernements devraient étudier l'éventualité de faire de même. Le ministre français des Finances retira finalement sa proposition, et l'on s'entendit sur une autre solution radicale : les marges bilatérales des taux de change furent élargies de $+/-2,25 \%$ à $+/-15 \%$. Cela revenait de facto à supprimer le régime de change fixe, la marge de fluctuation autorisée étant tellement accrue qu'aucune banque centrale n'avait plus à craindre de devoir soutenir d'autres monnaies par des interventions conséquentes portant préjudice à la stabilité interne de sa propre monnaie.

\section{Conclusion : une culture de la stabilité}

Dans cette courte étude de la politique monétaire allemande depuis la fin de la Seconde Guerre mondiale, nous avons tenté de montrer que la préservation de la stabilité interne du mark fut le principe qui régissait tout. Pendant le demi-siècle au cours duquel le deutsche mark fut la monnaie allemande, la politique monétaire fut menée par sept présidents ; une centaine d'autres personnes - responsables politiques, banquiers, universitaires et journalistes - firent partie du conseil de la banque centrale, en qualité de membres du directoire de la Bundesbank ou en tant que présidents des banques centrales régionales, contribuant ainsi à forger l'histoire de la politique monétaire. Au-delà de la diversité des personnalités, l'axe fondamental de la Bundesbank - la préservation de la stabilité interne de la monnaie - ne fut jamais sérieusement remis en question. Il fallut certes de temps à autre tenir compte de l'évolution conjoncturelle du moment. Mais on considérait toujours que l'on s'écartait, à titre temporaire uniquement, de la voie nécessaire à moyen terme en matière de monnaie et de crédit, à laquelle il fallait toujours revenir. L'idée que l'objectif de stabilité n'eût été qu'un but parmi d'autres et qu'il pût être nécessaire d'y renoncer si et aussi longtemps que la conjoncture n'était pas favorable, ou même l'idée qu'il eût été possible d'utiliser une politique monétaire habile pour générer de la croissance économique et des emplois durables n'avaient aucune place dans l'esprit des responsables de la politique monétaire et financière allemande aux commandes. Et il en va de même aujourd'hui.

La Bundesbank a développé une «culture de la stabilité ». C'est Helmut Schlesinger, chef économiste de l'établissement pendant de longues années et président de la Bundesbank de 1991 à 1993, qui a forgé cette notion, mais sans 
jamais la définir précisément par écrit ${ }^{29}$. L'on peut comprendre cette notion comme l'ensemble des mesures visibles prises par la Bundesbank avec trois objectifs à destination des citoyens du pays et des représentants des institutions politiques : premièrement, leur rappeler régulièrement que la préservation de la valeur interne de la monnaie est essentielle pour le bien du pays et de ses citoyens; deuxièmement, les informer sur les interactions monétaires; et troisièmement, leur montrer, en faisant référence à l'histoire de l'Allemagne, quelles conséquences dévastatrices l'inflation peut avoir sur la prospérité. Aucun citoyen de l'Union européenne actuelle n'a jamais vécu d'hyperinflation. Cependant, nombreux sont les Allemands qui ont connaissance de la grande inflation qui a démarré dans leur pays en 1914 avec la Première Guerre mondiale et a culminé en novembre 1923 sous la forme d'une hyperinflation, avec une explosion de l'indice du coût de la vie (placé à $100 \%$ en janvier 1922) à 17,9 milliards de $\%$. Cette inflation plongea une grande partie de la population dans la pauvreté et entraîna une radicalisation politique. La Bundesbank se fait fort de transmettre ce savoir aux jeunes.

Le refus de l'inflation, largement répandu dans la population allemande, n'est donc pas, comme le pensent certains observateurs étrangers, une préférence typiquement allemande ${ }^{30}$. Un tel postulat signifierait que ce phénomène ne s'explique pas. Or ce rejet de l'inflation est le fruit d'une éducation. Il est en revanche intéressant de se demander pourquoi une banque centrale perpétue une telle tradition.

Nous suggérerons brièvement deux hypothèses. La première pourrait être qualifiée d'hypothèse du sens prussien du devoir. Dans ce schéma, une personne qui endosse des responsabilités publiques se sent des obligations vis-à-vis du bien commun et s'efforce par conséquent de réaliser au mieux de l'intérêt public la mission qui lui a été confiée. Or, pour la direction d'une banque centrale, entretenir une culture de la stabilité facilite l'accomplissement du devoir. L'autre hypothèse, radicalement différente, est celle du plaisir d'exercer le pouvoir. Elle suppose que ce qui intéresse les membres du conseil de la banque centrale n'est pas tant l'objectif de stabilité en tant que tel, mais bien plus le fait d'accroitre et de conserver le pouvoir de l'institution et, ainsi, leur propre pouvoir personnel. Or perpétuer une culture de la stabilité peut également servir cet objectif de pérennisation du pouvoir.

Laquelle de ces deux hypothèses est la plus pertinente ? Il est impossible de trancher. L'une et l'autre sont plus ou moins compatibles avec l'histoire de la politique monétaire allemande.

\section{Traduction de Marie GRAVEY}

\footnotetext{
${ }^{29}$ Voir toutefois Neumann (1998), p. 343-344.

${ }^{30}$ L'économiste suisse Thomas Straubhaar croit par exemple savoir que «la peur de l'inflation est inscrite dans l'ADN allemand». Thomas Straubhaar, «Inflation gibt es nur noch in Geschichtsbüchern » [en ligne], Welt.de, 12/11/2013. Disponible sur: http://www.welt.de/wirtschaft/article121805628/Inflation-gibt-es-nurnoch-in-Geschichtsbuechern.html [consulté le 30/01/2015].
} 


\section{Indications bibliographiques}

BrunNer K., «The Art of Central Banking », in H. GöPPL, R. HenN (eds), Geld, Banken und Versicherungen, vol. 1, Athenäum Verlag, Königstein, 1981

Buchнеiм C., « Die Errichtung der Bank deutscher Länder und die Währungsreform in Westdeutschland », in Deutsche Bundesbank, Fünfzig Jahre Deutsche Mark - Notenbank und Währung in Deutschland seit 1948, C.H. Beck Verlag, Munich, 1998, p. 91-138

Cagan P., "The Monetary Dynamics of Hyperinflation », in Friedman M. (ed), Studies in the Quantity Theory of Money, University of Chicago Press, Chicago, 1956, p. 25-117

Deutsche BundeSBANK, « Bundesregierung und Bundesbank », Monatsbericht, vol. 20, n 8, 1972, p. 15-17

Deutsche BundeSBank, Deutsches Geld- und Bankwesen in Zahlen 1876-1975, Fritz Knapp, Francfort-surle-Main, 1976

Deutsche Bundesbank, Die Deutsche Bundesbank - Geldpolitische Aufgaben und Instrumente, $6^{\mathrm{e}}$ édition, Francfort-sur-le-Main, 1993 (Sonderdrucke der Deutschen Bundesbank ; 7)

Deutsche BundeSBank, Statistische Beihefte Reihe 5 - Devisenkursstatistik, janvier 2000, p. [n.c.]

EMminger O., «Deutsche Geld- und Währungspolitik im Spannungsfeld zwischen innerem und äußerem Gleichgewicht 1948-1952 », in DeuTSChE BundeSBANK, Währung und Wirtschaft in Deutschland 18761975, Fritz Knapp, Francfort-sur-le-Main, 1975, p. 447-554

Emminger O., D-Mark, Dollar, Währungskrisen, Deutsche Verlags-Anstalt, Stuttgart, 1986

Friedman M., A Program for Monetary Stability, Fordham University Press, New York, 1959

Friedman M., «Should There Be an Independent Monetary Authority? », in YeAGER L.B. (ed), In Search of a Monetary Constitution, Harvard University Press, Cambridge, 1962, p. 219-243

Hetzel R.L., " German Monetary History in the Second Half of the Twentieth Century: From the Deutsche Mark to the Euro », Economic Quarterly, vol. 88, n² 2, 2002, p. 32-64

Neumann M.J.M., « Central Bank Independence as a Prerequisite of Price Stability », European Economy, special edition 1, 1991a, p. 76-106 (The Economics of EMU)

NeumanN M.J.M., « Die deutschen Währungen im internationalen Währungsgefüge seit dem Zweiten Weltkrieg », Historisch-Politische Mitteilungen, n5, 1998b, p. 219-226

NeUManN M.J.M., « Geldwertstabilität : Bedrohung und Bewährung », in DeUTSCHE BundESBANK, Fünfzig Jahre Deutsche Mark - Notenbank und Währung in Deutschland seit 1948, C.H. Beck Verlag, Munich, 1998a, p. 309-346

NeUmann M.J.M., « Monetary Reform », The New Palgrave Dictionary of Money and Finance 2, Macmillan Press, Londres, 1992, p. 751-756

Neumann M.J.M., « Monetary Targeting in Germany », in KuROda I. (ed), Towards More Effective Monetary Policy, MacMillan, Londres, 1997, p. 176-198

Neumann M.J.M., «Precommitment by Central Bank Independence », Open Economies Review, n², 1991b, p. 95-112

RiCHTER R., « Die Geldpolitik im Spiegel der wissenschaftlichen Diskussion », in DEUTSCHE BUNDESBANK, Fünfzig Jahre Deutsche Mark - Notenbank und Währung in Deutschland seit 1948, C.H. Beck Verlag, Munich, 1998, p. 561-606

Simons H.C., « Rules versus Authorities in Monetary Policy », Journal of Political Economy, vol. 44, n¹, février 1936, p. 1-30

SPAHN H.-P., « Die Krise des EWS und die brüchigen Grundlagen der Leitwährungsordnung », in THOMASBERGER C. (ed), Europäische Geldpolitik zwischen Marktzwängen und neuen institutionellen Regelungen Zur politischen Ökonomie der europäischen Währungsintegration, Metropolis-Verlag, Marbourg, 1995, p. 171-196. 


\section{Les voies et moyens d'une longue mutation de la culture et des pratiques monétaires en France depuis $1945^{1}$}

\section{Michel MARGAIRAZ}

Cette contribution vise à éclairer les origines historiques, politiques et culturelles des différends monétaires entre la France et l'Allemagne, qui auraient été partiellement surmontés dans le second XX siècle. Une des hypothèses les plus couramment répandues oppose des conceptions et des normes différentes de politique monétaire forgées depuis 1945. Pour la République fédérale allemande (RFA) et l'Allemagne après 1990, une véritable culture de stabilité et d'indépendance monétaire reposerait sur deux piliers fondamentaux, toujours actifs en 2014. Le premier s'identifie à la stabilité à long terme de la monnaie, le deutsche mark, dont on sait que sa naissance (1948) précède celle de l'État lui-même, et depuis 1999, l'euro. Le second s'incarne dans l'indépendance de la banque centrale : la Bundesbank, puis la Banque centrale européenne (BCE). À la différence de la France, qui se distinguerait par une culture de soutien monétaire à la croissance, mais qui, depuis les années 1980, serait conduite à se convertir à la culture de stabilité et d'indépendance monétaire, avant même l'institution de l'euro et de la BCE. Pour rendre compte de cette différenciation et de cette dynamique de convergence des années 1945 à nos jours, il convient de replacer la question dans un temps plus long et de mettre à jour les dynamiques d'une "culture monétaire " dans chacun des deux pays à partir des structures monétaires et des politiques monétaires effectivement conduites.

Trois questions préalables méritent d'être précisées.

Les cultures monétaires s'inscrivent dans des institutions et des politiques monétaires.

L'approche culturelle en matière de monnaie ne saurait être dissociée de questions structurelles relatives aux systèmes monétaires, bancaires et du crédit et des politiques monétaires elles-mêmes. Peut-on réellement parler d'une culture monétaire nationale dans les deux cas ? La culture monétaire est constituée de représentations, de valeurs et de croyances partagées quant au rôle et au statut de la monnaie. Mais il ne s'agit pas uniquement d'analyser des représentations et des mentalités. La question n'est pas seulement culturelle, mais également structurelle. Une telle culture monétaire s'enracine dans des institutions, des normes, des instruments, qui eux-mêmes encadrent les pratiques des autorités monétaires et constituent pour elles un véritable socle de références pour l'action. Mais ces institutions monétaires s'insèrent elles-mêmes dans d'autres institutions plus larges, dont la chronologie peut fortement différer en France et en Allemagne. Distinguons trois domaines qui s'imbriquent les uns dans les autres.

\footnotetext{
${ }^{1}$ Article rédigé en octobre $2014, \mathrm{NdE}$.
} 
D'abord, l'État lui-même en France et en Allemagne, on le sait, ne présente ni la même chronologie, ni la même trajectoire historique, ni le même contenu institutionnel, ni encore les mêmes interrelations avec la société. Et il ne suffit pas d'opposer, comme souvent, structures fédérales de la RFA vs structures centralisées de la France, dont les premières seraient plus favorables à ce que certains auteurs nomment « les incitations de marché ».

Second aspect, la monnaie n'est pas dissociable du système bancaire et des formes de contrôle qui le supervisent. En France, l'expérience longue a été marquée par des formes fluctuantes de partage de la supervision bancaire entre la Banque de France et le Trésor. En outre, les relations entre la banque centrale et les banques commerciales pèsent également sur les représentations. De même, les relations entre la monnaie et l'économie, particulièrement les interactions banques/industries ne manquent pas d'influer sur les conceptions et pratiques à l'œuvre de la monnaie. On sait que sous cet angle, les structures bancaires en France et en RFA diffèrent, selon notamment que les banques se trouvent plus (plutôt en Allemagne) ou moins (en France) organiquement liées aux entreprises industrielles et commerciales.

Ajoutons que la culture de stabilité monétaire en Allemagne ne peut être considérée comme la seule source de la trajectoire de croissance depuis 1945. Les structures industrielles de la RFA et les atouts de leur spécialisation technologique importent tout autant si ce n'est davantage, puisque seuls des produits de haute valeur ajoutée peuvent supporter une monnaie périodiquement revalorisée par rapport à ses concurrentes immédiates (des années 1960 aux années 1980), sans pour autant perdre leurs avantages compétitifs. Ainsi, la position internationale des monnaies qui en découle, et notamment l'évolution des taux de change, diffèrent fortement dans les deux pays. Les excédents ou les déficits extérieurs qui y sont liés impriment également leur marque sur la culture monétaire.

\section{Les cultures monétaires s'inscrivent dans une histoire longue.}

Une deuxième série de réflexions préalables mérite des précisions quant à la chronologie proposée. Peut-on souscrire à l'idée, avancée par le CIRAC pour cette contribution, de l'année 1945 comme «point zéro » pour la France comme pour la future RFA?

Les conditions de relèvement des deux pays en 1945 ne s'éclairent ellesmêmes qu'à travers une plongée dans une histoire plus vaste, tant il est vrai que les cultures monétaires s'élaborent et se consolident sur le temps long. Si on prend en compte un espace de temps plus étendu, l'expérience allemande apparaît marquée davantage que la France par des chocs majeurs réitérés : deux catastrophes monétaires (en 1923 et 1948) et une catastrophe politique (de 1933 à 1945), perçues comme telles après 1945. L'Allemagne a connu une histoire dramatique de l'État, à travers les douze années d'existence du $\mathrm{III}^{\mathrm{e}}$ Reich ainsi que le traumatisme de son effondrement total lors de la capitulation sans condi- 
tion de 1945, au point de forger pour une longue période après cette date - jusqu'à nos jours - une image fortement répulsive de l'État central.

La France n'a pas vécu cette expérience traumatique, même si la défaite de 1940, les années d'occupation et le régime de Vichy constituent un triple choc pour l'expérience démocratique. Mais en 1944-1945, les Français - et sans doute d'abord le général de Gaulle en leur nom - ont rétabli la continuité républicaine en relégitimant l'État comme acteur majeur et démocratique de la Reconstruction, en particulier à travers le programme du Conseil national de la Résistance et ses « réformes de structures ».

Parallèlement, l'histoire monétaire de l'Allemagne est marquée par deux catastrophes majeures - distinctes chronologiquement, rappelons-le, de la précédente $^{2}$ - où, par deux fois en 25 ans dans les deux après-guerres (en 1923 et en 1948), la monnaie disparaît dans une hyperinflation spectaculaire. Ce double effondrement nécessite la création d'une nouvelle monnaie, prélude à l'affirmation d'un nouvel État allemand surmontant la défaite militaire et rompant de manière nette avec le passé - impérial d'avant 1919 ; nazi d'avant 1945. Historiquement, l'Allemagne resurgit sous les traits de la République de Weimar (1920), puis trente ans plus tard de la République fédérale allemande, dont les deux fondements, constitués en 1949 et maintenus par-delà l'unification allemande (1990) sont précisément une monnaie stable, le deutsche mark, et la démocratie politique sous sa forme fédérale.

La France connaît, elle, après plus de 110 ans de stabilité du franc germinal jusqu'en 1914, une longue dépréciation monétaire, plus ou moins vive selon les fluctuations du $\mathrm{XX}^{\mathrm{e}}$ siècle, mais sans effondrement. Et les structures anciennes, telle la Banque de France, née en 1800, perdurent, même si sa nationalisation de facto (en 1936), puis de jure (en 1945) en modifie la place relative et les marges de manœuvre. De manière (apparemment) paradoxale, l'histoire monétaire française procède davantage par ajustements successifs, faits de dévaluations périodiques, alors que l'histoire allemande est totalement reconfigurée en 1949, afin de surmonter trois catastrophes nationales, à la fois politiques et monétaires (1923, 1933 et 1945-48). Il en résulte la combinaison née de deux hantises conjuguées et imbriquées l'une dans l'autre dans les mentalités allemandes d'après 1945: une double condamnation, à l'égard de l'État centralisé et de l'inflation, mêlés dans une même répulsion ${ }^{3}$. Les deux piliers fondamentaux de la RFA résultent de cette double hantise. En 1948, le deutsche mark, fondé avant même la nouvelle République, devient ainsi une composante essentielle de la future RFA, née une année

\footnotetext{
${ }^{2}$ À ce propos, rappelons que l'expérience hitlérienne (1933-1945) n'a pas surgi de l'hyperinflation - comme on peut le lire parfois dans des approches mêlant l'effondrement monétaire de 1923 et le nazisme - mais de l'une des plus graves expériences de déflation connue en Allemagne de 1930 à 1933 lors de la crise de 1929 et de ses suites.

${ }^{3}$ Ce télescopage chronologique s'explique fréquemment par le fait que, dans les mentalités des dirigeants comme de la population de la RFA après 1949, la double condamnation de l'État nazi et de l'hyperinflation conduit à les mêler dans une même réprobation et représentation répulsive alors que, on l'a dit, le nazisme s'installe en réalité au pouvoir à l'occasion d'une grave situation déflationniste de chômage massif.
} 
plus tard, conjointement avec la structure démocratique et fédérale. Et la création de la Banque centrale des Länder, puis d'une Bundesbank indépendante en 1957, conforte la culture de stabilité et d'indépendance monétaire.

Pour ce qui est de la période récente, peut-on accepter l'idée d'une convergence mondiale en matière monétaire à partir des années 1980 ? Ou à tout le moins d'une convergence monétaire européenne affectant la France et la RFA, amorcée en 1979 avec la création du Système monétaire européen, puis spectaculairement accélérée et mise en scène lors de la dévaluation française de mars 1983 ? Assiste-t-on à une libéralisation des marchés financiers et à une ouverture des marchés monétaires, préludant à une politique monétaire "neutre » «de marché », reposant sur les taux d'intérêt et n'influant pas sur les modes d'allocation du crédit, ni sur la concurrence entre banques et établissements financiers ? Il semble que les enchaînements chronologiques, si on veut s'y attarder quelque peu, soient plus complexes que ces évolutions (trop) générales.

On sait que les structures monétaires en France ont été, à travers la loi du 2 décembre 1945 et les institutions et réglementations qui s'en suivent, imbriquées, mêlées, encastrées (embedded) à la "politique du crédit », qui présente le double aspect pour les autorités monétaires de fixer des limites quantitatives aux crédits accordés quasi continûment de 1945 à 1984 et de procéder à des mesures sélectives de différenciation (taux) de crédits selon les activités privilégiées, d'où un système monétaire et financier administré, segmenté, fragmenté, cloisonné selon les destinataires du crédit. Cet ensemble d'institutions et de pratiques apparaît comme une partie intégrante et constitutive de la culture monétaire dominante en France des années 1945 aux années 1970, voire 1980.

Dans ce cas, est-ce la mondialisation des années 1980-1990, puis la construction de l'Union européenne (1993-1999) de manière exogène qui, en France, transforment la politique monétaire et la culture qui s'y rapporte ? Ne trouve-t-on pas plutôt des traces dès avant cela, au cours des années 1960-1970, au sein des autorités monétaires françaises (à la Banque de France, au Trésor) et aussi parmi les économistes et experts monétaires, de critiques plus ou moins radicales des structures et des politiques monétaires, faites d'avancées plus ou moins inabouties et de reculs, montrant une voie française marquée par une véritable révolution culturelle en matière monétaire ? Ne peut-on déceler le passage complexe dans sa chronologie comme dans ses modalités d'une culture monétaire imbriquée dans la politique administrée ou dirigiste de crédit vers une culture monétaire de marché conduisant elle-même à une culture de la stabilité monétaire de l'euro après 1999 ?

\section{Une hypothétique culture monétaire nationale?}

Enfin, une troisième série de questions porte sur le fait de savoir si cette culture monétaire évolutive des élites financières et monétaires est relayée dans la $\mathrm{Na}$ tion. Quelles implications socio-politiques ou culturelles plus larges peuvent en- 
traîner ces déphasages éventuels ? Si en France les élites administratives, politiques et financières se rallient progressivement, on l'a dit, à une culture de stabilité monétaire dans les années 1980-1990, est-ce pour autant également le cas pour l'ensemble de la Nation? Peut-on parler de culture monétaire nationale en relation avec une culture politique nationale ? Peut-on réduire cela au rôle de l'État dans l'économie, en opposant l'économie sociale de marché et l'ordolibéralisme en RFA au dirigisme en France ? Et à cet égard, quel rôle jouent les éventuels modèles ou transferts d'un pays à l'autre?

Nous envisagerons cette traversée du second $\mathrm{XX}^{\mathrm{e}}$ siècle de mutation de la culture monétaire en France en trois moments successifs, scandés par les deux ruptures de 1958 et de 1974-75 et en insistant davantage sur les périodes les plus lointaines, moins abordées dans l'ouvrage.

\section{La culture et les pratiques monétaires imbriquées dans la politique quantitative et sélective du crédit (1945-1958)}

Le système monétaire est marqué dans la France de la Libération par la loi du 2 décembre 1945, adoptée sous la présidence à la tête du gouvernement provisoire - encore pour quelques semaines - du général de Gaulle, dont la haute figure symbolique va en légitimer le contenu pour plusieurs décennies. Le contenu résulte largement de l'application du programme du Conseil national de la Résistance (CNR), adopté dans la clandestinité le 15 mars 1944 de manière consensuelle par toutes les composantes (partis politiques, confédérations syndicales, mouvements de Résistance) de la Résistance intérieure. Tout en reprenant certaines dispositions des «lois» des 13 et 14 juin 1941 adoptées sous Vichy, la loi de décembre 1945, outre la nationalisation de la Banque de France et des quatre grandes banques de dépôts, crée des institutions nouvelles, telles que le Conseil national du crédit (CNC) composé de 38 membres, ou la commission de contrôle des banques. Mais surtout la loi institutionnalise une politique nationale du crédit, subordonnée à la reconstruction et à la croissance recouvrée. La Banque de France perd son indépendance en droit, mais dans les pratiques, elle assure des fonctions nouvelles de supervision bancaire sur un système bancaire réglementé et cartellisé et assure la présidence de fait du CNC, qui fixe sous l'autorité du ministre des Finances la politique du crédit. La Banque d'émission, et plus largement la monnaie sont désormais soumises au double impératif de favoriser la croissance et de freiner l'inflation, deux objectifs annoncés de la politique du crédit, qui surplombe de fait la politique monétaire. Car il s'agit, on va le constater, d'une fausse symétrie, l'objectif de croissance surpassant celui relatif à l'inflation.

La monnaie est subordonnée à partir de 1946 et, de manière durable, aux priorités définies par le Plan de modernisation et d'équipement (ou Plan Monnet), lui-même issu de la consultation des «commissions de modernisation », emblématique de l'«économie concertée ». La politique du crédit est largement 
contrôlée par la Banque de France, à travers une politique sélective des crédits conforme aux priorités du Plan et une politique sélective de réescompte sur ressources monétaires de crédits à moyen terme, qui mobilise l'ensemble du système financier semi-public (Caisse des dépôts, Crédit national, Crédit foncier). En 1947-1948, la politique demeure sélective en faveur des « secteurs clés » du Plan. Il s'y ajoute, dans un souci anti-inflationniste, une politique quantitative appuyée sur des plafonds de réescompte et des planchers de bons du Trésor pour toutes les banques. Par ailleurs, une large politique de financement public des investissements se poursuit, alimentée pour une grande part par la contrepartie en francs de l'aide Marshall. Mais, afin d'éviter un effet déflationniste, cette politique est assortie de crédits « hors plafonds » pour des crédits destinés à des activités jugées prioritaires pour «l'expansion et le plein emploi » ainsi que pour les crédits à moyen terme réescomptables à la Banque de France. La politique monétaire stricto sensu - au sens d'une politique menée à travers les taux d'intérêt - ne joue quasiment aucun rôle, du fait du maintien de taux d'intérêt très bas, alors que l'inflation dans ces années 1946-1951 atteint ou dépasse fréquemment $30 \%$ à $40 \%$ l'an ! De surcroît, il n'existe pas à proprement parler de marché monétaire, la plupart des banques connaissant un endettement quasi permanent auprès de la Banque de France, qui veille de ce fait à maintenir un taux d'escompte bas, d'autant plus que le Trésor pousse dans ce sens afin de limiter les charges d'endettement.

Cette politique administrée, volontariste et sélective du crédit est largement partagée par les autorités monétaires, administratives et politiques, de manière transpartisane, et même par une large partie des milieux professionnels. En effet, le très grand écart entre la faiblesse des ressources financières et des capitaux disponibles par rapport aux objectifs de reconstruction et de croissance écarte les modes de financement par les marchés, financier et monétaire, très affaiblis par l'inflation, et incite à définir des priorités dans les secteurs de base de l'économie (énergie, transports, acier, ciment, matériaux de construction...), quasi incontestables et dont le bien-fondé est reconnu par la plupart des acteurs administratifs, politiques et sociaux. En ce sens, la politique du crédit nourrit une culture monétaire de soutien administré à la croissance, qui dispose, outre de l'appui des acteurs économiques et socio-politiques, des instruments institutionnels, administratifs et réglementaires, en particulier de la co-direction du système assuré conjointement par la Banque de France et le Trésor. Cette politique se maintient au moins pendant les deux décennies qui suivent la fin de la guerre et se trouve d'autant moins mise en question qu'elle accompagne une forte croissance, même supérieure dans les années 1960 à la croissance du « miracle allemand » en RFA.

Dans une importante note rétrospective destinée au ministre et rédigée en 1950 par le directeur général de la nouvelle direction du Crédit à la Banque de France, E. de Sèze, celui-ci justifie le maintien de taux d'intérêt très bas et, par suite, l'inexistence d'une politique monétaire stricto sensu: " [...] dans le même 
esprit qui avait présidé à l'institution du crédit moyen terme mobilisable à l'Institut d'émission, il s'agissait d'encourager par tous les moyens sans exception, fussent-ils de nature inflationniste, les premiers efforts de rééquipement et de production, de manière à réamorcer la vie économique ${ }^{4} »$.

Les pratiques et instruments de la politique du crédit se poursuivent pendant les années 1950, mis en œuvre par un système monétaire et financier inchangé et codirigé par la Banque et le Trésor. Il s'y ajoute toutefois à partir de février 1958 des mesures dites de «plafonnement du crédit», levées entre 1959 et 1963 et périodiquement rétablies sous le nom d' " encadrement du crédit », afin de limiter l'inflation. La stabilisation monétaire de fait opérée par-delà le changement de République et le retour de Charles de Gaulle, la création d'un «nouveau franc » en 1960 incitent désormais à miser sur la double stabilité - intérieure et internationale - de la monnaie. Mais la culture monétaire demeure suspendue à la politique administrée et sélective du crédit, confortée par la résurgence du Plan « ardente obligation » aux yeux du général de Gaulle - placé sous la responsabilité du commissaire général Pierre Massé, qui y voit un « réducteur d'incertitude ».

\section{Les critiques à bas bruits de la culture monétaire dominante (1958-1975)}

L'ouverture commerciale de l'économie française à travers la signature des traités de Rome en 1957 et la marche au Marché commun de l'Europe des Six même si le contrôle des changes demeure sur l'essentiel de la période - incite certains responsables de la politique monétaire à desserrer l'appareil de la politique de crédit dans un sens plus libéral. Cela apparaît souvent comme un débat strictement limité aux échanges feutrés entre autorités monétaires et financières sans prise sur l'opinion, dont les effets demeurent limités et réversibles. De surcroît, les phénomènes d'instabilité monétaire et économique en fin de période freinent les évolutions à peine engagées et conduisent plutôt à conforter la culture monétaire de soutien administré à la croissance. De manière schématique, distinguons trois moments : l'épisode de Jacques Rueff, les réformes DebréHaberer et le rapport Wormser.

\section{Les critiques isolées de Jacques Rueff}

Jacques Rueff, bien qu'assez marginal dans le milieu des décideurs financiers et monétaires en France, demeure toutefois un conseiller écouté par le général de Gaulle. Celui-ci lui a confié l'élaboration du premier plan de stabilisation en 1958 - auquel a été adjoint le nom du ministre Antoine Pinay - avant de le placer, avec Louis Armand, à la tête du Comité destiné à surmonter les obstacles à la croissance en 1960. Très hostile au système du Gold Exchange Standard et notamment à la suprématie de l'étalon dollar, Jacques Rueff, dans la lignée de

\footnotetext{
${ }^{4}$ Archives de la Banque de France, 1331 2003-01/16, Note de M. de Sèze au ministre, 26 mai 1950, p. 2 (souligné par l'auteur).
} 
ses idées déjà exprimées dans les années 1920, se réfère à une culture monétaire libérale où, à l'instar de la place de Londres et du système monétaire britannique, il préconise pour la place de Paris un vaste marché monétaire unifié et régulé de manière libérale par des taux d'intérêt variables, à la hausse comme à la baisse. Cela le conduit à critiquer le système de crédit mis en place depuis 1945 , tout particulièrement les crédits à moyen terme réescomptables, considérés comme inflationnistes, le taux d'escompte de la Banque de France maintenu délibérément très bas, les phénomènes d'éviction dus aux interventions du Trésor sur le marché, la politique segmentée et sélective du crédit, jugée trop dirigiste, la politique de limitation du réseau bancaire par le CNC....

Mais l'auteur du Discours sur le crédit (1961) se heurte à de multiples obstacles. Structurels, du fait de l'endettement permanent des banques commerciales auprès de la Banque de France, du captage des capitaux extérieurs par le Trésor et ses satellites ou encore de la place encore élevée de la monnaie fiduciaire en France aux dépens de la monnaie de banque et qui absorbe une grande part de l'entrée des devises. Mais, plus fondamentalement, cette culture libérale très anglophile remet en cause tout le système de crédit administré mis en place par la Banque de France, par le Trésor et les établissements publics parallèlement au financement public direct ou indirect des investissements et à la planification indicative, jugée alors efficace pour maintenir des taux de croissance élevés - de l'ordre de $4 \%$ à $5 \%$ l'an de 1959 à 1972 - et un chômage très limité. Vingt ans de politique du crédit administrée et sélective ont accoutumé les responsables monétaires à manier les outils visant d'abord à maintenir l'expansion ${ }^{5}$. L'expérience cumulée des années 1930 et de l'Occupation ont acclimaté chez eux l'idée que l'inflation était un mal moindre que la dépression des années 1932-1945, au point d'en faire l'ingrédient majeur d'une véritable culture monétaire. Comme l'indique un ancien directeur des Finances et sous-gouverneur de la Banque de France : "toute manipulation en hausse du taux d'intérêt suscitait l'effroi ${ }^{6} »$, par crainte de refroidir par trop l'activité économique.

À la hantise allemande de l'inflation débridée répond chez les responsables français la hantise de la déflation des années 1930 et du chômage massif.

\section{Les réformes Debré-Haberer (1966-1967) et leurs limites}

Lors du second septennat du général de Gaulle et de l'arrivée de Michel Debré rue de Rivoli, une tentative de desserrement du système de crédit s'accompagne de critiques et de réformes. Tout en maintenant la sélectivité de la politique du crédit selon les secteurs, le conseiller du ministre Jean-Yves Haberer propose de

\footnotetext{
${ }^{5} \mathrm{Cf}$. à ce sujet les témoignages des principaux responsables de cette génération de hauts fonctionnaires des Finances, du Plan ou de l'Insee d'après-guerre : François Bloch-Lainé, Claude Gruson, Roger Goetze, Paul Delouvrier..., ainsi que le volume de mémoires et témoignages réunis in François Fourquet, Les comptes de la puissance. Histoire de la comptabilité nationale et du Plan, Éditions Recherche, Paris, 1980 (Encres).

${ }_{6}^{6}$ André de Lattre, témoignage in De Gaulle en son siècle, tome III, Moderniser la France, Plon, Paris, 1990, p. 94 .
} 
libérer les ouvertures de guichets du système bancaire, d'assouplir le contrôle des changes, de remplacer dans les banques les planchers de bons du Trésor par le système plus «neutre » des réserves obligatoires et de stimuler le marché financier afin de favoriser le financement des investissements par des mécanismes de marché, et de pousser la Banque de France à conduire une politique plus conforme aux orientations des marchés en menant «une vraie politique d'open market, au lieu de s'enfermer dans son obsession du réescompte ${ }^{7} »$.

Au même moment, le directeur général des Études et du Crédit à la Banque de France Henri Fournier indique à propos du rétablissement depuis 1963 de « l'encadrement du crédit» qu'il " n'est pas possible de pétrifier, de manière durable, la structure du système bancaire ${ }^{8} »$.

Mais la crise de mai 1968 et l'affaiblissement consécutif du franc conduisent à rétablir le contrôle des changes et à maintenir l'encadrement du crédit, et à ne pas modifier dans ses fondements le système monétaire.

\section{Le rapport Marjolin-Sadrin-Wormser (1969)}

Le nouveau gouverneur de la Banque de France, Olivier Wormser, nommé par son ami Couve de Murville peu avant le départ du général de Gaulle en avril 1969 , est le principal rédacteur d'un rapport - pour lequel il fait également appel à Robert Marjolin et Jean Sadrin - publié en juin 1969 sur le système de crédit ${ }^{9}$. Bien que très technique et limité à un cercle étroit de décideurs, le rapport vise à une transformation sur un mode libéral du système de crédit et à la création d'un véritable marché monétaire libéral à l'anglaise. Pour cela, les rapporteurs préconisent de supprimer l'encadrement du crédit, de libéraliser les taux bancaires, de faire fonctionner un marché monétaire par des taux variables et unifiés et, pour ce faire, de limiter l'action de la Banque de France à une politique d'open market et de faire passer le taux d'escompte de la Banque à un niveau pénalisant, au-dessus du taux du marché interbancaire. Une partie des mesures du rapport sont appliquées en 1971, avec la mise en œuvre de la politique d'open market, et en janvier 1972, avec la suppression des plafonds de réescompte et de l'encadrement du crédit. Mais l'éclatement du système monétaire international en août 1971 et l'entrée dans un système de changes flottants, la remontée de l'inflation poussent à rétablir l'encadrement du crédit - pour quinze années - en décembre 1972, au point qu'une économiste reconnue parle de retour au « dirigisme monétaire ${ }^{10} »$.

\footnotetext{
${ }^{7}$ Centre d'Histoire de Sciences Po, papiers Michel Debré, 4 DE 35, Note pour le ministre, de Jean-Yves Haberer, MEF-Cabinet, ${ }^{\circ} 421 / \mathrm{CAB} 8,1^{\mathrm{er}}$ avril 1967.

${ }^{8}$ Archives de la Banque de France, 1330 2011-02/1, DGEC, «Le problème du réglage de la liquidité en France ", mars 1967, signée Henri Fournier.

${ }^{9}$ Robert Marjolin, Jean Sadrin, Olivier Wormser, Rapport sur le marché monétaire et les conditions du crédit, demandé par décision en date du 6 décembre 1968, La Documentation française, Paris, 1969.

${ }^{10}$ Cf. Guillaumont-Jeanneney (1991), p. 519.
} 


\section{Vers une lente mutation culturelle (1975-années 1980)}

Même si le rapport Wormser n'est pas suivi d'effets durables, les critiques se multiplient chez les décideurs, publics et privés, à l'encontre d'un système administré et rigide, désormais mal adapté à une économie plus ouverte, à une inflation plus élevée et à une croissance ralentie. L'encadrement du crédit, bien que rétabli, est contourné par des « soupapes ", destinées à l'alléger et est considéré comme un "mal nécessaire » par Clappier, le gouverneur de la Banque de France en 1979. C'est le moment où plusieurs économistes et responsables de la Banque critiquent l' "économie d'endettement administrée », en appellent à « une économie de marché libéralisée » et en viennent, suivant en cela les outils de politique monétaire d'outre-Atlantique, à définir un ciblage «neutre » de masse monétaire. Toutefois, il demeure encore un certain scepticisme sur la capacité de la seule politique monétaire à limiter l'inflation et, par conséquent, le refus d'une politique monétaire ouvertement restrictive en ces débuts de désindustrialisation : la cible est délibérément fixée au-dessus de l'inflation. Avec la création du Système monétaire européen en 1979 et la nécessaire convergence qu'il implique avec le deutsche mark, l'inflation est désormais vue davantage comme un effet, non de la politique monétaire et de crédit, mais de la politique salariale et de l'indexation des salaires instituée par la politique de la « Nouvelle société » dans les années 1969-1972. Dès cette fin des années 1980, d'ailleurs, plusieurs des conseillers économiques du futur gouvernement Mauroy, tels Jean Peyrelevade ou Henri Guillaume, considèrent que, à la différence de la RFA en 1974-1975, la France a fait supporter les charges de la facture pétrolière aux entreprises et comporte de ce fait, un excès de demande intérieure qui se traduit en termes négatifs de compétitivité et de différence d'inflation aux dépens de la France.

Mais ces débats ont lieu à bas bruit, sans écho véritable dans le débat public.

On sait que c'est en juin 1982, lors du premier plan de rigueur, qu'est décidée la désindexation des salaires sur l'inflation et lors de la troisième dévaluation du gouvernement Mauroy-Delors, en mars 1983, que l'ancrage monétaire européen et la politique ouverte de désinflation sont désormais légitimés comme objectifs majeurs, substitués à la relance et aux « réformes de structures » achevées en 1981-1982. La loi bancaire de 1984 et la libéralisation des marchés monétaires et financiers de 1986-1987 conduisent à la double décision du marché unique et de la marche à la monnaie unique et à la banque centrale indépendante.

AINSI, EN QUATRE DÉCENNIES, ON A PU MESURER, même de manière rapide et nécessairement raccourcie, la mutation culturelle qui s'opère chez les décideurs publics en matière monétaire et qui les conduit à substituer à une culture de soutien monétaire à la croissance une culture de stabilité et d'indépendance monétaire. En cela, il s'agit bien d'une révolution culturelle, incluant une convergence explicite vers la culture monétaire allemande. Mais autant la culture mo- 
nétaire forgée dans les années qui suivent la Libération entrait en résonance avec la culture politique nationale de la Reconstruction, largement répandue dans l'ensemble de la population française parce qu'assise sur les « réformes de structures » issues du programme de la Résistance (nationalisations, Sécurité sociale, Plan...) et identifiée à la Grande Croissance des années 1950-début des années 1970, autant la culture de stabilité monétaire tarde à trouver ses assises dans l'ensemble de la Nation.

\section{Indications bibliographiques}

Conti G., Feiertag O., Scatamacchia R. (eds), Credito e nazione in Francia e in Italia (XIX-XX secolo), Pisa University Press, Pise, 2009

Feiertag O., Margairaz M. (eds), Politiques et pratiques des banques d'émission en Europe (XVII -XXe siècle). Le bicentenaire de la Banque de France dans la perspective de l'identité monétaire européenne, Albin Michel, Paris, 2003 (Mission historique de la Banque de France) (Bibliothèque Albin Michel Histoire)

Guillaumont-JEANNENEY S., "L'alternance entre dirigisme et libéralisme monétaires (1945-1990)», in LÉvy-Leboyer M., CaSAnova J.-C. (eds), Entre l'État et le marché. L'économie française des années 1880 à nos jours, Gallimard, Paris, 1991, p. $507-543$ (Bibliothèque des sciences humaines)

Plessis A., Histoires de la Banque de France, Albin Michel, Paris, 1998 (Mission historique de la Banque de France) (Bibliothèque Albin Michel Histoire)

Plessis A., «La Banque de France », in Lévy-Leboyer M. (ed), Les banques en Europe de l'Ouest de 1920 à nos jours - Colloque tenu à Bercy les 7 et 8 octobre 1993, Comité pour l'histoire économique et financière de la France, Paris, 1995, p. 273-282

SAINT-PÉRIER A. DE, La France, l'Allemagne et l'Europe monétaire de 1974 à 1981 - La persévérance récompensée, Les Presses de Sciences Po, Paris, 2013 (Mission historique de la Banque de France). 



\section{Le couple franco-allemand : (més-)entente cordiale ? ${ }^{1}$}

\section{Hans-Helmut KoTZ}

II existe un besoin bien réel - et persistant - d'échange réflexif. En effet, des malentendus surgissent de temps à autre, et il arrive qu'ils ne soient plus même cordiaux. Dans de tels cas, c'est la caricature qui prédomine, de part et d'autre. Or ce programme de formationrecherche vise sans aucun doute à tenter d'inscrire les discussions et les débats dans une meilleure compréhension réciproque.

Le CIRAC m'a chargé de me risquer à présenter une perspective allemande sur l'Union monétaire, le modèle de Maastricht - en particulier le Pacte de stabilité et de croissance (PSC) - ainsi que sur la politique monétaire de la Banque centrale européenne (BCE). Répondre à cette demande est une tâche ardue, et pas uniquement parce que le sujet est vaste. II m'est surtout difficile d'adopter une perspective que l'on pourrait qualifier d'allemande. Le métier que j'exerce n'est à vrai dire pas national, bien que nous disposions en Allemagne du terme particulier de Nationalökonomie (tout comme de celui de Volkswirt pour désigner l'économiste). La profession travaille plutôt avec un outil appliqué de manière transversale à cette catégorie. Cependant, la "dimension nationale " peut jouer un rôle, moins pour ce qui est des instruments d'analyse utilisés que s'agissant de la perspective de perception à partir de laquelle les questions économiques sont étudiées. En la matière, des variantes nationales peuvent certainement intervenir.

Cela vaut par exemple pour la politique monétaire, un sujet sur lequel on prête à "l'Allemand moyen " une plus forte aversion contre l'inflation qu'au " Français représentatif ". Est considéré comme cause de cette aversion le spectre d'un effondrement, survenu à deux reprises, du système monétaire (Toni Pierenkemper, 1996 ; Kurt Andreas, 1996). L'hyperinflation des années 1920, dont Stefan Zweig a décrit les conséquences de manière impressionnante, est considérée comme ayant largement ouvert la voie au national-socialisme. En revanche, en Allemagne, le taux de chômage, alors extrêmement élevé, est bien plus rarement mis en relation avec l'arrivée au pouvoir du NSDAP. Toujours est-il que le nazisme mena tout droit, inéluctablement, à la Seconde Guerre mondiale et au deuxième effondrement monétaire.

\section{Points de vue franco-allemands}

Avant d'aborder les différences nationales, encore faut-il définir préalablement les positions respectives. De tels agrégats existent-t-ils? Quelle est 1'opinion moyenne et quel degré de dispersion convient-il de prendre en compte, en France ou en Allemagne? L'on pourrait regarder les journaux dominants dans chacun des pays. L'on pourrait également envisager d'examiner les institutions qui conseillent le gouvernement sur les questions économiques - le Cercle des

\footnotetext{
${ }^{1}$ Article rédigé à l'été 2013, NdE.
} 
économistes ou le Conseil d'experts allemand pour l'évaluation de la situation économique (Sachverständigenrat). Il y a un peu plus d'une décennie, Wim Kösters et moi-même nous sommes essayés à une caricature traçant les contours de l'Allemagne par opposition à la France. Au cours de ce travail, nous nous sommes demandé si le point de vue allemand était celui du gouvernement en place ou encore celui du Verein für Socialpolitik. De même, le point de vue français est-il celui de l'Association française de science économique ou celui du gouvernement? L'opinion publique, est-ce avant tout ce que nous trouvons dans les journaux comme le FAZ, le Handelsblatt, Le Monde, Les Échos ou Le Figaro ? Le 22 avril 2013, Le Figaro publiait un article traitant du fait que l'Allemagne n'avait plus goût à l'Europe ${ }^{2}$. Enfin, on pourrait aussi tout simplement prendre pour référence le gouvernement en place, dans la mesure où il représente potentiellement "l'électeur médian », notion chère aux économistes politiques. Mais sa position évolue avec le temps, et c'est là un simple constat. Cette évolution peut être due à un comportement tactique (certainement légitime), mais aussi à un changement de contexte. Prenons l'exemple de la position du gouvernement allemand sur les plans européens de sauvetage. Elle est passée du rejet total (en 2010 encore) à l'acceptation, à titre provisoire, du Fonds européen de stabilité financière (FESF) pour aboutir finalement à l'accord sur l'instauration d'un mécanisme permanent : le Mécanisme européen de stabilité (MES), à partir de 2013.

Nous tracerons les grandes lignes d'une position allemande (et française) telle qu'on peut la déduire des discours et des actes des acteurs. Il est intéressant de constater que l'écart entre les experts, ceux qui conçoivent les politiques et ceux qui les mettent en œuvre est le plus souvent moins grand que le débat public n'en donne l'impression. Il est vrai que les médias ont besoin de forcer le trait. Mais il se pourrait aussi que les opinions publiques respectives, qui comptent pour la sphère politique, soient plus nettement divergentes. Cette idée d'une tendance centrifuge entre des groupes relativement homogènes est développée par Cass R. Sunstein (Going to Extremes, Oxford University Press USA, 2011), qui s'appuie sur de nombreux exemples. Je ne la développerai toutefois pas plus avant.

$\mathrm{Au}$ bout du compte, tous ces facteurs sont probablement soumis à des phénomènes d'interaction. La question de la position du gouvernement allemand est très importante dans ce contexte. À l'automne 2009 encore, il était inconcevable de mettre en place, en matière de coordination de la politique financière, une sorte de plan de sauvetage, une telle mesure étant, d'un point de vue allemand, incompatible avec la clause de non-renflouement. En mai 2010, il n'était pas encore envisageable de voir le FESF, créé à cette période, devenir à terme le MES. Ce qui s'est produit dans le domaine budgétaire n'était pas imaginable. Il me serait difficile de trouver en la matière un axe cohérent.

\footnotetext{
${ }^{2}$ Patrick Saint-Paul, « L'Allemagne peut-elle se passer de l'Europe ? », Le Figaro, 22/04/2013, p. 15.
} 


\section{France-Allemagne : des attentes divergentes}

Quel est actuellement, au début de l'été 2013, le point de vue dominant lorsque l'on considère les médias ? En Allemagne, ils sont majoritairement devenus très sceptiques au cours de la crise. (Et l'on peut dresser le même constat s'agissant des journaux néerlandais, finlandais ou autrichiens.) Ainsi, le 7 avril 2013, l'éditorial publié en une du Welt am Sonntag était intitulé : «Zurück zur DMark» [Retour au deutsche mark]. Les frustrations que nous percevons aujourd'hui dans la sphère médiatique - qui apparaissent de manière particulièrement flagrante dans les émissions de débat télévisé - trouvent leurs racines dans le fait que les règles préalablement établies solennellement dans les traités n'ont pas été respectées. En d'autres termes, les promesses (formulées de manière inconditionnelle) du début de l'Union monétaire, n'ont pas été tenues - et cela peut aujourd'hui alimenter des stéréotypes.

En Allemagne, on avait considéré que les traités seraient tout bonnement appliqués et que serait instaurée une répartition des compétences claire entre les différents domaines politiques, soit une démarche assez proche de ce à quoi on était habitué dans ce pays. Cette répartition des compétences était fondée sur une logique analytique. À l'époque, on parlait souvent d' "Ordnungspolitik». L'idée ne venait toutefois pas directement de l'École de Fribourg, mais consistait à dire que la politique monétaire n'est pas en mesure de produire de la croissance, que l'on ne peut pas jouer avec la courbe de Phillips, laquelle ne permet pas de choisir à la carte. Il n'y a pas de rapport d'interchangeabilité exploitable entre chômage et inflation. La politique monétaire est responsable de la stabilité du niveau des prix et la politique budgétaire, de la soutenabilité des finances publiques. L'emploi et les salaires font l'objet de négociations - en toute autonomie - entre les partenaires sociaux.

Cette idée macroéconomique moderne, telle qu'elle fut conçue au début des années 1970, se concrétisa dans les traités sur lesquels repose l'Union économique et monétaire (UEM). La BCE est plus indépendante que la Bundesbank, dans la mesure où son indépendance ne saurait être modifiée qu'en changeant les traités de l'Union européenne (UE). Or, en la matière, le principe d'unanimité s'applique. De surcroît, des référendums sont nécessaires dans certains pays. L'on a également inscrit dans les traités l'interdiction de financer les États, laquelle découle des expériences du début des années 1920 évoquées ci-dessus. Ont été inscrites dans la constitution des procédures de sanction et de retour au sérieux budgétaire pour les pays qui étaient sortis des rails. Et l'on s'en tint à cela : il n'y avait pas besoin de plus de coordination en matière de politique économique. Charge à chacun de maintenir l'ordre chez soi. C'était une Europe qui promettait de fonctionner.

Wim Kösters et moi-même avons établi il y a une bonne dizaine d'années une représentation schématique (et un peu caricaturale, à vrai dire) portant sur des thèmes macroéconomiques. L'idée était de faire apparaître ce que pouvait 
être la vision de l'économiste français moyen et celle de son homologue allemand sur la situation macroéconomique (cf. tableau 1).

Tableau 1 : Visions du monde au plan macroéconomique

\begin{tabular}{|l|l|l|l|}
\cline { 2 - 4 } \multicolumn{1}{c|}{} & \multicolumn{1}{c|}{$\begin{array}{c}\text { Stabilité } \\
\text { du secteur privé }\end{array}$} & \multicolumn{1}{c|}{$\begin{array}{c}\text { Efficacité de la } \\
\text { politique de stabilité }\end{array}$} & $\begin{array}{c}\text { Besoin } \\
\text { de concertation }\end{array}$ \\
\hline Vision allemande & $\begin{array}{l}\text { Auto-correction, } \\
\text { pas de nécessité } \\
\text { d'intervention }\end{array}$ & $\begin{array}{l}\text { Inefficacité des prévisions } \\
\text { politiques, préférence } \\
\text { pour les règles }\end{array}$ & $\begin{array}{l}\text { Claire répartition } \\
\text { des compétences }\end{array}$ \\
\hline Vision française & $\begin{array}{l}\text { Rigidités nominales, } \\
\text { échec de coordination, } \\
\text { besoin de macro- } \\
\text { intervention }\end{array}$ & $\begin{array}{l}\text { Efficacité, préférence } \\
\text { pour des interventions } \\
\text { au cas par cas }\end{array}$ & $\begin{array}{l}\text { Concertation, } \\
\text { harmonisation } \\
\text { minimale }\end{array}$ \\
\hline
\end{tabular}

Source : Wim Kösters, Hans-Helmut Kotz, 2001.

Des débats de ce type existaient de toute évidence déjà avant l'Union monétaire. $\mathrm{Au}$ cours de la première année d'existence de cette union, le Commissariat général du plan publiait un rapport très instructif de Robert Boyer ${ }^{3}$ qui définissait quatre scénarios de développement de l'Union monétaire :

- Maastricht à la lettre (le statu quo institutionnel, perpétuel) ;

- une UEM conduite par des règles et des procédures, sans possibilité d'intervention au cas par cas ;

- un fédéralisme européen ;

- le pragmatisme : apprendre et adapter.

Lorsque ces scénarios furent examinés, celui dit du «statu quo », qui correspondait à la vision allemande, fut classé dans la rubrique "guère plausible ». Dès le début de l'Union monétaire, l'idée que celle-ci resterait telle qu'elle avait été créée à l'origine était donc considérée comme plutôt improbable en France. En effet, des questions furent soulevées qui allaient faire l'objet de débats intenses dix ans plus tard - c'est-à-dire après la plus grande crise depuis les années 1930. Ainsi, dans un rapport du Conseil d'analyse économique (CAE), Pierre-Alain Muet invitait à imaginer ce qui se passerait aux États-Unis s'il n'y avait pas de mécanisme de coordination régissant les 50 États fédérés ${ }^{4}$. Mais le Conseil d'experts allemand pour l'évaluation de la situation économique ne voulait pas faire sienne une telle idée de politique macroéconomique concertée. Dans un autre rapport du CAE, Olivier Blanchard argumentait qu'une règle d'équilibre budgétaire pourrait peut-être être utile pour régler le problème du

\footnotetext{
${ }^{3}$ Robert Boyer (ed), Le gouvernement économique de la zone euro, Commissariat général du Plan, Paris, 1999.

${ }^{4}$ Conseil d'analyse économique, Coordination européenne des politiques économiques, Rapports : Michel Aglietta, Christian de Boissieu, Dominique Bureau (et al.), La Documentation française, décembre 1998, p. 26 (Les Rapports du Conseil d'analyse économique).
} 
déficit. Mais il estimait d'un autre côté que l'on perdrait un instrument macroéconomique majeur. Selon ce rapport, c'était un prix bien trop lourd à payer.

L'on peut interpréter la réforme des institutions de politique budgétaire comme une réponse à certaines de ces questions. À l'été 2010, le semestre européen fut instauré afin de renforcer la coordination et la cohérence des politiques nationales. Suivit au printemps 2011 le Pacte pour l'euro plus, qui souligne également le besoin de concertation, mais qui prend surtout la compétitivité en considération. En décembre de la même année, il fut complété par le « six-pack », un instrument que l'on peut considérer comme renforçant le Pacte de stabilité et de croissance (marge de manœuvre réduite, majorité qualifiée inversée). En mars 2012 enfin, le pacte budgétaire inclus dans le traité sur la stabilité, la coordination et la gouvernance (TSCG) fut signé par 25 États membres de l'UE : il comprenait une règle d'équilibre budgétaire à moyen terme plafonnant le déficit structurel autorisé à $0,5 \%$ du produit intérieur brut (PIB).

C'est sans doute en matière de politique monétaire que les divergences sont les plus faibles. Dans ce domaine en effet, les recoupements entre les positions représentatives allemande et française semblent importants et sont en tout cas devenus nettement plus nombreux ces deux dernières décennies.

\section{Comment cela est-il (fut-il) possible?}

$\mathrm{Au}$ fil des ans, le modèle a connu des évolutions. Il est apparu nécessaire d'instaurer davantage de coopération entre des économies fortement intégrées. On s'est rendu compte que l'on pouvait faire mieux que de considérer le comportement du partenaire comme étant simplement donné et de s'y adapter. Telles furent les leçons tirées de la grande crise financière. Elles ont conduit à repenser les institutions de la zone euro, à mettre en place les différents plans de sauvetage et à tenter de coordonner la politique financière, comme il a été dit plus haut.

Je vais maintenant me focaliser surtout sur la place actuelle de la politique monétaire et sur ce qu'elle sera à l'avenir. J'aimerais d'emblée souligner que la BCE (ou l'Eurosystème) n'a pas planifié son changement de rôle. Cette institution opère du reste dans un cadre autorisant plusieurs interprétations. En Allemagne, on interprète ce cadre d'une manière bien spécifique.

C'est surtout la crise survenue sur les marchés financiers qui a contraint la $\mathrm{BCE}$ à endosser un nouveau rôle. Et c'est seulement à la lumière de cette crise que l'on peut comprendre pourquoi la BCE, considérée par de nombreux acteurs (non sans raison) comme un clone de la Deutsche Bundesbank, adopte en apparence une autre attitude que celle à laquelle on était habitué s'agissant de la Bundesbank. Mais est-ce vraiment exact? Une question contrefactuelle s'impose : s'il avait été confronté aux problèmes de l'été 2007 (ou, plus encore, 2008-2009) et ce, sur un périmètre géographique semblable à celui de l'Union monétaire, le conseil des gouverneurs de la Bundesbank aurait-il réellement agi autrement ? Ou, en d'autres termes : la BCE se démarque-t-elle clairement de la Bundesbank de jadis? 


\section{Graphiques 1 et 2 : Évolution des spreads européens et tensions sur le marché monétaire}
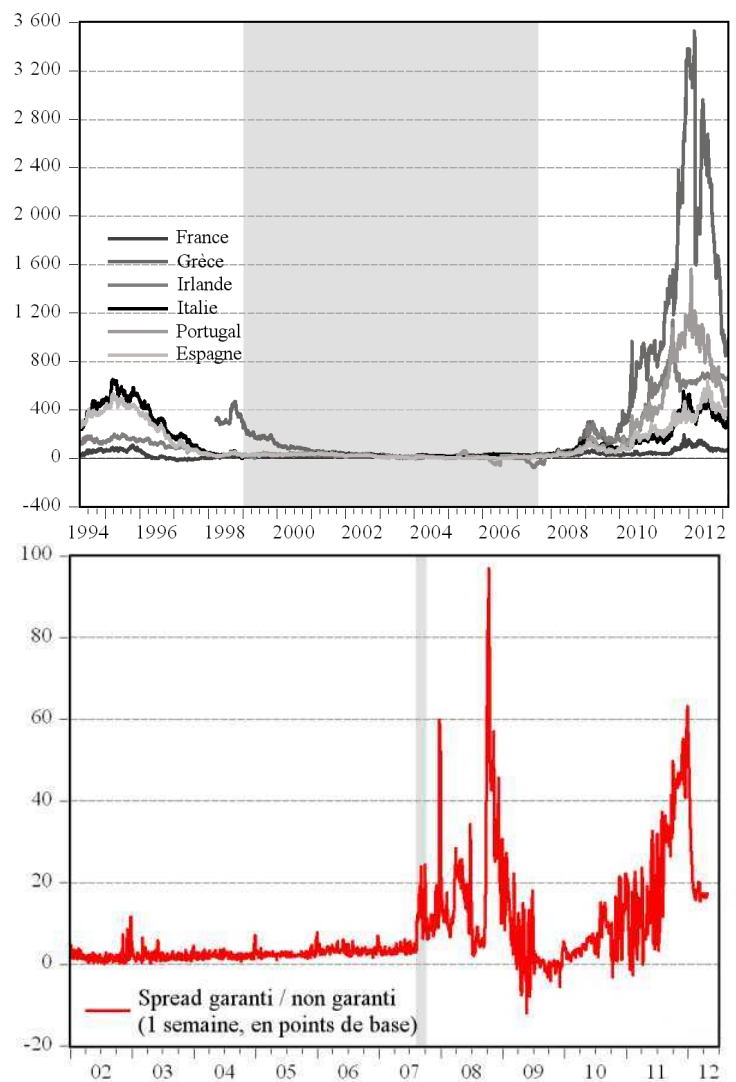

Source : Bundesbank. Le graphique du dessus (graphique 1) montre la hausse des spreads souverains européens par rapport au taux allemand. La hausse la plus forte a commencé seulement un peu plus d'un an après la faillite de Lehman Brothers. Les spreads se sont resserrés après l'annonce par la BCE que l'établissement allait faire tout ce qui serait nécessaire pour garantir l'euro. Le graphique du dessous (graphique 2) retrace l'évolution des écarts de taux d'intérêts entre les prêts interbancaires non garantis et ceux adossés à des garanties sur le marché monétaire. Nous ne traitons que de la phase marquée par la colonne gris clair, autrement dit, la flambée des spreads à partir du 9 août 2007. La hausse beaucoup plus nette ensuite n'était à l'évidence pas connue des acteurs.

Un argument notamment invite à répondre de manière négative : le fait que la Bundesbank n'a cessé de poursuivre une politique adaptée à la situation. Même le monétarisme, qui fit office de modèle depuis 1975, fut interprété de manière pragmatique - selon les mots de Helmut Schlesinger, ancien président de la Bundesbank - et surtout mis en œuvre de manière pragmatique, ce qui signifie que dans un cas sur deux, l'objectif intermédiaire (d'abord la croissance de la masse monétaire de la banque centrale, puis de l'agrégat M3) ne fut pas atteint. Cela a d'ailleurs conduit quelques monétaristes universitaires à critiquer régulièrement la politique menée pour son manque de discipline et son caractère 
discrétionnaire. La Bundesbank parvint en revanche à atteindre l'objectif proprement dit - la lutte contre l'inflation - avec un succès remarquable. De surcroît, pour ce qui est de leurs préférences en matière d'objectifs, les responsables de la BCE ne se distinguent guère de ceux qui siégeaient au conseil des gouverneurs de la Bundesbank de l'époque. Robert Raymond, ancien directeur général de l'Institut monétaire européen (l'ancêtre de la BCE), a par exemple constaté que "la Banque de France serait peu ou prou monétariste».

Jusqu'à l'apparition de turbulences sur les marchés financiers - c'est ainsi en effet que l'on appela la crise survenue entre l'été 2007 et septembre 2008 -, le comportement de la BCE ne se distingua pas de celui de la Bundesbank. Ce fut la période interprétée (rétrospectivement!) comme problématique du point de vue des marchés des capitaux. Les risques de défaillance furent sous-estimés, de manière flagrante et sans différenciation entre les pays. Les écarts de taux d'intérêts par rapport aux emprunts d'État allemands étaient insignifiants. Les taux à 10 ans de la Grèce n'excédaient les taux allemands que d'à peine 20 points de base.

S'agissant de ces faibles spreads, on évoque souvent l'idée que les financiers des banques d'investissement, pourtant généreusement rémunérés, n'auraient manifestement pas été véritablement vigilants, et ce pendant des années. Mais une autre interprétation est plus plausible : la promesse de ne pas intervenir était considérée comme impossible à réaliser et, par conséquent, dépourvue de crédibilité. Le marché européen des banques est (était ?) fortement intégré, caractérisé par des établissements bancaires perçus comme trop grands pour faire faillite. Dans le contexte d'une crise des marchés financiers, on ne pouvait se permettre de ne pas sauver ces banques, y renoncer eût porté préjudice à l'ensemble du système. C'est là un argument - l'incohérence temporelle de la clause de non-renflouement - déjà avancé dans la littérature universitaire avant même l'Union monétaire.

Même à un stade avancé de la crise, l'Union monétaire faisait l'objet d'appréciations largement positives. L'on ne voyait pas de raison de réaménager les institutions. Les petits pays en particulier se considéraient à l'abri derrière un rempart protecteur. (La perception ne changea qu'à l'automne 2009, lorsque les problèmes budgétaires grecs furent rendus publics.) On eut tendance à ignorer l'évolution distincte des coûts salariaux unitaires, avec l'appréciation différenciée, en termes réels, de la monnaie qui en résultait et ses conséquences en termes de compétitivité et de balance des transactions courantes à l'échelle nationale. Ces phénomènes s'accompagnèrent immanquablement d'une accumulation de dettes externes intenables, mais, avant la crise, ce problème n'attira pas davantage l'attention. De même, les anomalies furent balayées d'un revers de main. Par exemple celle sur laquelle Thomas Mayer, alors économiste en chef de la Deutsche Bank, attira l'attention en 2006 : près du tiers de la croissance de l'Union monétaire européenne provenait alors du secteur du BTP en Espagne un secteur d'activité dont il est apparu rétrospectivement qu'il était surdimen- 
sionné. Les déficits de la balance des transactions courantes, élevés pendant près d'une décennie, et leur corollaire nécessaire, les afflux de capitaux, étaient ex ante considérés comme normaux.

\section{L'obligation d'apporter des réponses non conventionnelles}

La perception changea après l'éclatement de la crise, même si la prise de conscience mit du temps à mûrir. Les dysfonctionnements du marché monétaire interbancaire jouèrent là un rôle crucial. Le graphique 2 montre l'écart entre les prêts interbancaires non garantis (Euribor) et garantis. Sur une longue période, ces derniers valaient, selon l'échéance, entre 5 et 7 points de base. Mais le 9 août 2007, les taux bondirent à des niveaux extraordinaires.

À l'époque, au moment où la crise a éclaté, deux explications avaient cours. Selon la première - qui correspondait alors à la position majoritaire -, les gens avaient fait des erreurs et allaient devoir assumer les conséquences de leurs actes. Toujours selon cette lecture, intervenir conduirait les investisseurs à agir de manière encore plus impitoyable à la prochaine occasion : l'aléa moral, donc. C'était la théorie défendue notamment par la Banque d'Angleterre. La seconde approche, soutenue par l'Eurosystème qui la mit en œuvre, était autre : les liquidités du marché interbancaire avaient disparu. Le système bancaire implosa. On voyait se concrétiser un modèle qui rendait probable la défiance des dépositaires ( $r u n »)$.

Les 8 et 9 août 2007, BNP Paribas avait annoncé : "Nous ne sommes plus en mesure d'estimer certains de nos fonds investis dans des titres de créance structurés. » Le vrai problème était qu'il y avait de nombreux établissements comme BNP Paribas. Dans cette situation, la BCE est intervenue en fournissant plus de 90 milliards d'euros bruts - soit plus de 60 milliards nets - de liquidités supplémentaires à taux fixe. Elle tentait ainsi de soutenir le marché monétaire interbancaire, lequel a une fonction d'infrastructure irremplaçable et de référence pour le marché des capitaux. Cela donna lieu à des critiques virulentes. On put lire dans un journal britannique : " $L a B C E$ se comporte de manière hyperactive, sous le coup de la panique. " Il s'agissait d'éviter que le système n'implose. En temps normal, la BCE met à la disposition du secteur financier uniquement la quantité de liquidités dont le système a besoin du fait des exigences en termes de réserves obligatoires et de monnaie en espèces. Or l'Eurosystème sait très bien estimer les volumes requis. La BCE injecta donc beaucoup plus, non pas en raison de difficultés d'ordre technique, mais parce que l'implosion aurait été inéluctable en l'absence d'une telle mesure.

Les failles les plus graves survinrent à l'automne 2008, juste après Lehman/AIG. Au fil du temps, la crise des marchés financiers devint une crise des banques puis, finalement, une crise des dettes publiques dans quelques pays de la périphérie de la zone euro. Le graphique 4 montre la hausse radicale des primes d'assurance contre la défaillance des obligations d'État. Ces primes constituant la base de tarification des emprunts bancaires et obligations d'entre- 
prises, la hausse conduisit immédiatement à un net renchérissement des coûts de financement pour les entreprises, en particulier pour les petites structures tributaires de crédits bancaires.

\section{Graphiques 3 et 4 : Bilans des banques d'émission et primes de CDS (credit default swap) sur les banques européennes}
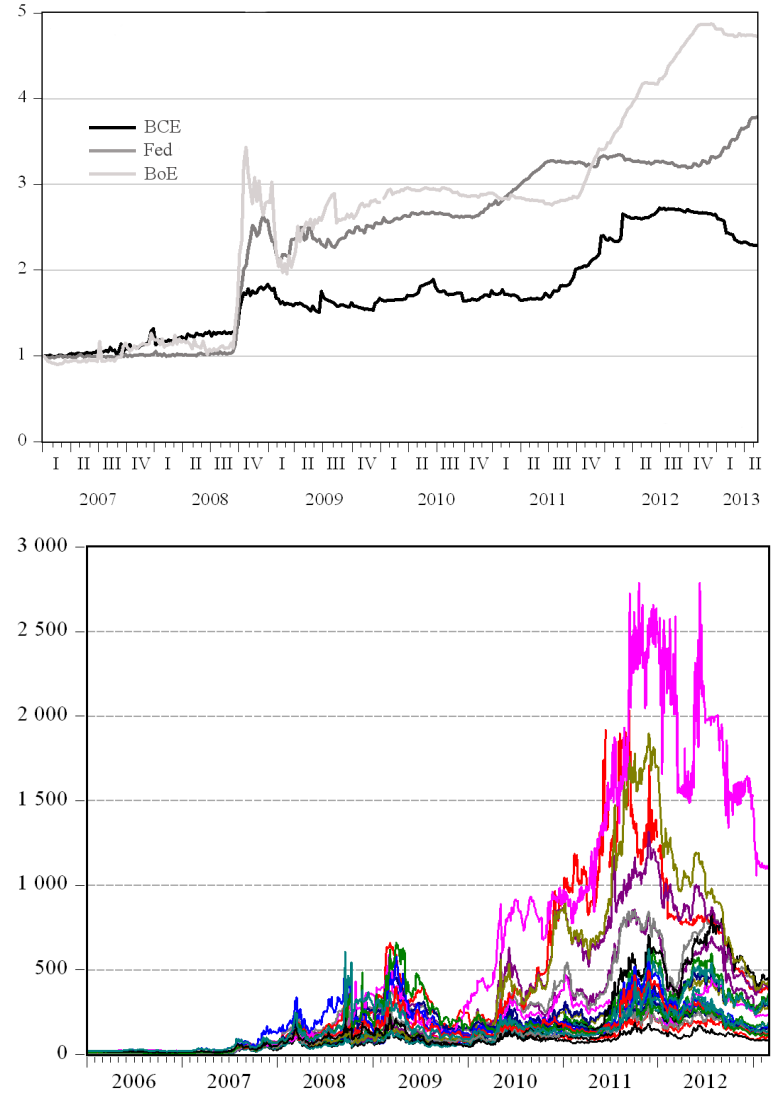

Sources : graphique 3 : BCE, Réserve fédérale américaine (Fed), Banque d'Angleterre (BoE) ; graphique 4 : Bundesbank. Le graphique 3 montre l'évolution des bilans des banques d'émission. Au fil de la crise, ils ont connu une croissance nette, variable selon les établissements. La Fed et la BoE ont mené une politique d'assouplissement quantitatif, stabilisant surtout quelques marchés ciblés. La BCE développa le principe de soutien renforcé au crédit. Elle a surtout mis des liquidités à la disposition des banques. Le graphique 4 fait apparaître l'écart considérable entre les coûts d'assurance contre les défaillances bancaires. On observe en la matière une forte dispersion en fonction du pays dans lequel la banque est domiciliée, avec des écarts en conséquence pour les coûts de financement pour les entreprises.

Le principal problème de la politique monétaire européenne réside dans l'existence de conditions monétaires extrêmement différentes à l'échelle nationale. La même politique monétaire a des effets très différents d'un pays à l'autre. De ce 
point de vue, l'Union monétaire ressemble plutôt à un système de changes fixes qui fonctionnerait mal. En conséquence, les marchés financiers européens présentent maintenant un niveau d'intégration comparable à celui que nous connaissions au milieu des années 1990. Les activités interbancaires transfrontalières se trouvent fortement réduites. Face à de telles perspectives, les banques sont très prudentes. L'Eurosystème intervient et fait office d'intermédiaire.

Fin 2011, il y eut de nouveau des tensions très fortes. Elles étaient liées aux craintes de voir survenir des problèmes résultant de l'arrivée à échéance de crédits au seuil de la nouvelle année. La BCE répondit en portant à trois ans l'échéance de ses opérations de refinancement à long terme. Les bilans des banques d'émission connurent alors une extension énorme dans le monde (cf. graphique 3).

Le solde TARGET2, auquel Hans-Werner Sinn, de l'institut de conjoncture ifo de Munich, fait régulièrement référence, n'est que le reflet du problème sous-jacent. Un épiphénomène, pas un problème en soi. Les marchés privés ont pris conscience que les déficits de la balance des transactions courantes qu'ils avaient financés auparavant n'étaient pas tenables. S'y ajouta à partir de 2010 une fuite des capitaux pour tenter d'échapper au risque de redénonimation. L'arrêt brutal, par la force des choses, du flux de capitaux dans les pays émergents est alors atténué par l'Eurosystème. Le bilan de la banque d'émission fait l'équilibre entre le Nord et le Sud, ce qui n'est pas tenable sur la durée.

La BCE n'a pas endossé ce rôle parce qu'elle planifiait de prendre les commandes afin de devenir l'institution européenne la plus puissante. Elle se trouve au contraire dans une situation dont elle veut sortir le plus vite possible. La BCE est en effet très proche d'une situation que les économistes qualifient de « dominance budgétaire ». Cette configuration progresse dans la mesure où la politique budgétaire ne résout pas les problèmes d'endettement, obligeant ou forçant au bout du compte la politique monétaire à utiliser la planche à billets. En l'occurrence, la $\mathrm{BCE}$ se trouve régulièrement confrontée au dilemme suivant: soit laisser le système imploser, soit gagner du temps. Cette seconde option peut se traduire par un programme pour les marchés de titres (securities markets program, SMP), par des opérations de refinancement à long terme (longer-term refinancing operations, LTROs) ou par des opérations monétaires sur titres (outright monetary transactions, OMT).

\section{Encore une fois : comment en sommes-nous arrivés là ?}

Quel est le diagnostic allemand - caricatural à n'en point douter - des problèmes ? En Allemagne, selon la vision prédominante, les dysfonctionnements proviennent surtout du fait que les pays membres de la zone euro n'ont pas respecté leurs engagements, à commencer par la refonte du Pacte de stabilité et de croissance en coopération entre la France et l'Allemagne. C'est aussi le constat sur lequel se basent en Allemagne les critiques, portées surtout par les juristes 
constitutionnalistes, mais aussi par des sociologues, à l'encontre de la politique menée par le gouvernement: des lois, des engagements ne sont pas respectés. Les droits des parlements sont en outre sapés. Il y a donc un manque de légitimité démocratique. On souligne en particulier le fait que les prérogatives parlementaires en matière budgétaire devraient être inconditionnelles (no taxation without representation). Le problème de toute règle est cependant, pour considérer les choses de manière dépassionnée, qu'elle tourne à vide si elle n'est pas assortie de mécanismes de mise en œuvre et de sanction.

Outre la question de l'efficacité (et du caractère adéquat) des règles de politique budgétaire, il existe bien sûr le problème des déséquilibres intenables des balances des transactions courantes d'un pays à l'autre de la zone euro. L'évolution différenciée des taux de change réels a eu un fort impact négatif sur la compétitivité de certaines régions en termes de prix. L'Union monétaire européenne est divisée en une Europe du Nord forte (avec des balances des transactions courantes excédentaires) et une Europe du Sud fragile (avec un niveau élevé de déficits commerciaux extérieurs et d'engagements nets). Cette situation n'est pas tenable sur la durée. Le taux nominal de change étant par définition inexistant dans la zone euro, il reste trois mécanismes d'ajustement : la migration de main-d'œuvre, les transferts budgétaires et, surtout, la flexibilité des prix relatifs. Les deux premiers mécanismes étant soit faibles, soit dépourvus de plausibilité d'un point de vue politique, les ajustements nécessaires interviennent ainsi majoritairement via les salaires, c'est-à-dire à travers une dévaluation interne. Et, de plus, il est inéluctable de traiter des questions qui résultent de l'intégration financière approfondie : l'Union bancaire...

\section{En guise de conclusion : quelques interrogations}

En 2013, les positions des gouvernements ont changé à bien des égards. Le point de vue dominant aujourd'hui consiste à dire qu'il nous faut un Pacte de stabilité et de croissance plus fort. Dans l'intervalle, le gouvernement fédéral allemand a accepté la mise en place à long terme du mécanisme de crise (le mécanisme de sauvetage permanent). L'Allemagne a aussi donné son aval aux premières étapes en direction d'une union bancaire. Mais elle ne veut à aucun prix des eurobonds, c'est-à-dire d'une mutualisation des dettes anciennes.

Si l'on avait laissé construire l'Union monétaire par les économistes, ils auraient porté leur attention d'abord sur ce qu'ils estiment être les critères d'une union monétaire optimale. Ils auraient donc en particulier réfléchi au degré de flexibilité que requiert pareil système lorsqu'il ne peut plus agir sur la valeur nominale des taux de change. On se serait demandé quel était le volume des migrations transfrontalières et quelles mesures de coopération budgétaire les États étaient prêts à accepter pour atténuer les effets de l'Union monétaire. On se serait en outre demandé quel était le degré de flexibilité des prix relatifs. 
Un tel modèle relève toutefois du monde des années 1960-1970. Les marchés financiers et les banques n'y ont aucune place. Bien peu de gens ont à l'époque réfléchi en profondeur au fait qu'une politique monétaire commune (donc une union monétaire) requiert d'être complétée par une politique bancaire commune (appelée aujourd'hui Union bancaire). Il faut par conséquent non seulement des règles communes, mais aussi une autorité de surveillance commune. Un mécanisme commun est nécessaire pour liquider les banques qui ne fonctionnent pas et il faut également quelque chose comme une garantie commune des dépôts.

Ce sont les enseignements tirés par exemple des États-Unis. Dans les années 1990, au moment des prémices de l'Union monétaire, Charles Goodhart, Garry Schinasi et quelques autres avaient abordé cette question. Actuellement, le gouvernement fédéral allemand estime toutefois que l'on peut s'en tenir à un Mécanisme de surveillance unique (MSU), avec des garanties exclusivement nationales qui plus est. Cela ne résout bien évidemment pas le problème de la dépendance fatale des entreprises vis-à-vis des États.

D'autres questions demeurent, notamment celle de savoir comment gérer la problématique du surendettement du secteur privé. C'est un point que le Pacte de stabilité et de croissance ne traite pas. Ainsi, l'Espagne présentait des déficits autorisés au titre du PSC. Elle a maintenant, tout comme l'Irlande, une dette nettement supérieure au taux de PIB admis. Autre question : que signifie le fait que les marchés traitent de facto les pays de la zone euro, d'un point de vue monétaire, comme les Länder allemands, c'est-à-dire comme des entités infranationales ? Lorsque la Bavière se trouve en difficulté, elle ne peut recourir à l'émission monétaire, pas plus que la France ne le pourrait.

Une coordination européenne de la politique budgétaire, ou encore un budget de l'Union monétaire européenne sont-ils nécessaires ? Dès 1966, Alfred Müller-Armack, l'un des grands noms de l'Ordnungspolitik allemande, écrivait: "Nous avons la mission de développer pour l'Europe des stabilisateurs conjoncturels qui assurent la cohérence des objectifs des différents pays avec les besoins de coopération internationale. »

Les défis auxquels l'UEM - et, au premier chef, le couple franco-allemand doit faire face consistent à gérer les bilans fragiles de toute une série de pays et de secteurs. L'UE a déjà opéré la mutualisation et la monétisation dans de nombreux domaines. Cela permet de gagner du temps, mais non de résoudre les problèmes. Il se pourrait même que le fait de gagner du temps encore et encore devienne un jour le problème en soi. De plus, cette attitude ne correspond pas aux représentations sacrées d'une politique monétaire, par principe censée être neutre. Mais à vrai dire, de quelle neutralité peut-on parler lorsque des technocrates non élus prennent des décisions sur des questions de redistribution ? Cette situation est devenue très claire, conséquence obligée de la dilution des responsabilités dans la politique monétaire et budgétaire, inéluctable du fait de la crise. 
L'on peut imaginer ce que seraient les réponses types allemande et française à quelques questions brûlantes. Revenir à Maastricht? La réponse allemande serait probablement : «Ja! » et la réponse française : «Non!» Davantage de fédéralisme ? Côté allemand, du moins aujourd'hui encore : «Oui, certainement! » Côté français, sans doute plutôt : «En aucun cas! »

CElA FAIT TROP DE MÉSENTENTES, ce qui ne convient toujours pas. L'issue de telles divergences irait toutefois de pair avec des coûts prohibitifs. Mais nous savons ce qu'il y a à faire, tout comme nous savons où se trouvent les recoupements possibles. Depuis 2007, bien des choses ont changé. L'Union monétaire sera irréversible dès lors que l'écart entre les visions allemande et française ne pèsera guère plus que celui entre les Länder de Bavière et de Rhénanie du NordWestphalie. Cela suppose toutefois de pouvoir débattre de manière équilibrée et nuancée. Cela suppose que les pays soient en mesure d'apprendre bien plus les uns des autres.

Traduction de Marie GRAVEY

\section{Indications bibliographiques}

ANDREAS K., « Les Allemands et le mark », in ASSOCIATION D’ÉCONOMIE FINANCIÈRE, Rapport moral sur l'argent dans le monde, Paris, 1996, p. [n.c.]

Pierenkemper T., «La peur des Allemands devant l'inflation », in Association D'ÉCONOMiE FinANCIÈRE, Rapport moral sur l'argent dans le monde, Paris, 1996, p. [n.c.]

Sunstein C. R., Going to Extremes - How Like Minds Unite and Divide, Oxford University Press USA, Cary/New York, 2011. 



\section{L'UEM, Maastricht et la BCE : controverses franco-allemandes ${ }^{1}$}

\section{Pascal KaUfFMANN}

La France n'a jamais été parfaitement à l'aise avec la construction monétaire européenne issue du traité de Maastricht. En première analyse, il s'agit là d'un paradoxe. À l'époque, en effet, la France a obtenu que soient entérinés les principaux objectifs qu'elle s'était fixés dans la négociation communautaire. En l'occurrence, le traité prévoyait la mise en circulation d'une monnaie unique, gérée par une banque centrale " unique » (la BCE), conduisant une politique monétaire unique. Précisément, la France ne voulait plus de multiples monnaies nationales liées par les règles d'un Système monétaire européen (SME) asymétrique - en pratique, une zone mark -, et de surcroît très exposé aux crises de change. Elle ne voulait pas davantage la perpétuation de l'hégémonie de la Bundesbank sur les taux d'intérêt dans l'Union.

Si l'édifice constitué du Système européen de banques centrales (SEBC), de l'euro et de la politique monétaire unique répond a priori très bien à ce cahier des charges, il présente toutefois deux particularités s'inscrivant profondément en faux par rapport à la conception française de l'Union économique et monétaire (UEM). La première est le caractère clairement ordolibéral du dispositif né du traité de Maastricht. La chose est flagrante s'agissant des statuts de la BCE, calqués sur ceux de l'ancienne Bundesbank. L'institution de Francfort est ainsi totalement indépendante de tout pouvoir politique, qu'il soit européen ou national. II est en outre interdit à la BCE de financer directement les États, ce qu'a longtemps fait la Banque de France. Le Pacte de stabilité et de croissance (PSC), qui encadre les politiques budgétaires nationales, a lui aussi une saveur typiquement ordolibérale.

Le PSC illustre en outre, quoiqu'en creux, une seconde caractéristique de l'UEM contraire aux conceptions françaises. II s'agit de l'absence de véritables leviers de politique macroéconomique dans la zone euro (de politique budgétaire et de change en particulier). C'est la raison des appels répétés de la France à la création d'un " gouvernement économique » en Europe.

Ce " modèle " d'union monétaire ordolibérale se trouve aujourd'hui remis en cause pour au moins deux raisons. La première réside en un besoin accru d'intervention publique européenne (en matière monétaire, budgétaire, prudentielle, etc.), qui est apparu dès la grande récession de 2008-2009, et fut exacerbé par la crise de l'endettement souverain et ses métastases. La seconde raison est que cette même crise a montré que l'architecture actuelle de l'UEM était vulnérable, au point de risquer l'éclatement. La pérennisation de la construction monétaire européenne suppose alors d'aller au-delà de Maastricht, dans un sens globalement plus fédéral, notamment aux plans bancaire et budgétaire.

II en résulte un certain nombre de controverses qui divisent le tandem franco-allemand - et par-delà, l'Europe entière. La ligne schématique de partage des positions en présence dessine un clivage Nord-Sud, avec d'un côté l'Allemagne et les pays de culture

\footnotetext{
${ }^{1}$ Article rédigé en juillet 2013, NdE.
} 
germanique (Autriche, Finlande, Pays-Bas, etc.), de l'autre un vaste groupe méditerranéen, dont la France. Dans les développements qui suivent, nous identifions trois grands foyers de controverse autour de l'architecture monétaire européenne. Nous commençons par examiner celui qui a trait à la politique non conventionnelle de la BCE. Nous analysons ensuite les oppositions, beaucoup plus vives, autour des programmes de rachat de dette souveraine par l'Institut d'émission. Enfin, nous présentons l'avancement de l'Union bancaire européenne, et les difficultés que soulève son approfondissement.

\section{La politique monétaire non conventionnelle de la BCE}

Les politiques monétaires non conventionnelles, mises en œuvre par toutes les grandes banques centrales depuis la crise des subprimes, s'articulent autour de deux principaux concepts : 1'assouplissement qualitatif (qualitative easing) et l'assouplissement quantitatif (quantitative easing). Ces politiques ont donné lieu à débat dans de nombreux pays. Au sein de la zone euro, certaines pratiques développées par la BCE sont contestées en Europe du Nord, et plus particulièrement en Allemagne en raison de son attachement à l'objectif traditionnel de stabilité des prix.

\section{L'assouplissement qualitatif}

Parallèlement à sa politique de taux d'intérêt historiquement bas, la BCE a mis en œuvre, dès le commencement de la crise mondiale, un ensemble de mesures destinées à assouplir les conditions de liquidité du système bancaire européen. Au rang de celles-ci figure l'allongement de la maturité des opérations de refinancement. Cette démarche a culminé avec les opérations de refinancement à long terme (longer-term refinancing operations, LTROs), dont l'horizon est de trois ans - là où, en temps normal, la $\mathrm{BCE}$ prêtait aux établissements de crédit tout au plus à trois mois. Dans le même ordre d'idées, les appels d'offres de la banque centrale se font désormais à taux fixe, tandis que le coefficient de réserves obligatoires a été abaissé de $2 \%$ à $1 \%$.

La principale mesure de qualitative easing qui ait réellement fait débat concerne les garanties liées au refinancement (collateral). D'une façon générale, les critères d'éligibilité ont été assouplis, permettant d'élargir la gamme des actifs que les banques sont susceptibles de mettre en pension. En particulier, la note de crédit «plancher» requise pour qu'une créance soit éligible au refinancement a été supprimée par la $\mathrm{BCE}$ depuis la crise grecque. Cette dernière porte ainsi dans son bilan des créances de moins bonne qualité que par le passé. À la limite, il n'est pas exclu que certaines de ces garanties puissent occasionnellement faire problème. La banque centrale se trouverait alors directement exposée à un risque de contrepartie, donc à des pertes éventuelles qui, si elles étaient avérées, devraient être supportées par les actionnaires de la BCE (soit, en ultime recours, par les États concernés). 
Les réticences allemandes relatives au qualitative easing portent autant sur le risque de contrepartie auquel s'expose ainsi la banque centrale que sur le fait qu'elle vienne de facto soulager les banques commerciales d'actifs risqués dont ces dernières souhaitent se défaire. En d'autres termes, ce qui est dénoncé, c'est que la $\mathrm{BCE}$ sorte de son rôle de fournisseur de liquidité pour venir en aide à des établissements qui sont en proie à des problèmes d'insolvabilité. L'apurement des bilans bancaires, qui semble nécessaire dans la plupart des pays membres de la zone euro, devrait, dans cette logique, relever de véritables structures de défaisance (bad banks). Ce sont les autorités de tutelle compétentes, et non la $\mathrm{BCE}$, qui devraient les mettre en place, à l'instar de ce qu'entreprend par exemple le gouvernement espagnol.

D'un point de vue français, l'assouplissement qualitatif participe des efforts qui doivent être entrepris, de façon générale, pour réactiver le canal du crédit bancaire, sévèrement endommagé depuis plusieurs années. Que la banque centrale soutienne à cette fin les établissements, par des taux de refinancement très bas ou par le portage de créances de moindre qualité, n'est pas jugé hétérodoxe.

\section{L'assouplissement quantitatif}

Plus que l'assouplissement qualitatif, c'est toutefois l'assouplissement quantitatif qui a fait débat en Europe du Nord. La controverse se nourrit du fait que la $\mathrm{BCE}$, à l'instar de la plupart de ses consœurs du monde développé, a créé de grandes quantités de monnaie centrale dans le cadre de sa politique non conventionnelle. Depuis le début de la crise mondiale, le bilan de la banque centrale a ainsi plus que doublé, ce qui ne peut manquer d'impressionner.

Pour certains, cette expansion considérable de la base monétaire est problématique dans la perspective du maintien de la stabilité des prix. L'explosion de l'offre de monnaie centrale serait un facteur d'inflation future, qui serait par conséquent à redouter. On retrouve ici une approche classiquement quantitativiste des phénomènes monétaires, ainsi que l'expression de l'aversion traditionnelle de l'Allemagne pour l'inflation. Cette lecture de l'assouplissement quantitatif est en outre parfaitement « conventionnelle», au sens de ce qui était devenu, dans les années de Grande modération, la norme en matière de politique monétaire - à savoir fixer et viser une cible d'inflation, quasiment à l'exclusion de toute autre préoccupation.

C'est bien à cette aune que la politique de la $\mathrm{BCE}$ dans les années récentes peut être qualifiée de non conventionnelle. En effet, l'objectif devenu prioritaire en pratique, depuis 2007, consiste à assurer la stabilité du système bancaire et financier. C'est à ce titre que de grandes quantités de monnaie centrale ont été créées, afin d'éviter toute pénurie de liquidité.

La critique quantitativiste de cette politique peut être rejetée en s'appuyant sur plusieurs lignes d'argumentation. La première consiste à faire observer que, depuis 2007, il n'est nulle trace de tensions inflationnistes dans la zone euro. En 
particulier, les anticipations d'inflation sont restées proches de l'objectif usuel de $2 \%$. À l'inverse, on peut arguer que le risque de déflation n'est pas totalement conjuré, en vertu d'un scénario qui pourrait ressembler à celui de l'économie japonaise des années 1990. En deuxième lieu, si la base monétaire en euro a bel et bien augmenté, ni le crédit aux agents non financiers (ANF) ni l'agrégat monétaire M3 n'ont fait de même. Or M3 est censé être pertinent pour mesurer les déséquilibres de nature monétaire, et il est à ce titre explicitement surveillé par la BCE. Sa grande stabilité n'annonce - pour l'instant du moins aucune dérive.

Quant au crédit aux ANF, son manque de dynamisme est devenu une source majeure de préoccupation pour la BCE. L'un des objets de l'assouplissement quantitatif et de la baisse historique des taux directeurs était, on le sait, de faire redémarrer le crédit bancaire, après son effondrement à la suite de la crise des subprimes. Or, malgré l'activisme de la BCE, ce redémarrage n'a pas eu lieu. D'une certaine manière, la monnaie centrale récemment créée est restée cantonnée au sein du système bancaire européen, sans se traduire par une création significative de monnaie de second rang (via l'octroi de crédits) au bénéfice des ménages et des entreprises.

Une controverse connexe à celle relative au quantitative easing a eu lieu à propos de la cible d'inflation de la BCE - à la différence que cette fois, c'est le point de vue allemand qui a prévalu. Lorsque le Fonds monétaire international a préconisé que cette cible soit rehaussée d'une valeur d'environ $2 \%$ à une valeur de $4 \%$, il a trouvé un appui dans les pays à dette publique élevée ainsi que dans les pays qui, comme la France, se sont longtemps accommodés d'une inflation modérée. À l'inverse, l'Allemagne et les pays de culture germanique s'y sont vigoureusement opposés, montrant à quel point leur crainte - légitime - de l'hyperinflation s'est muée en une peur - phobique - de la moindre hausse des prix.

\section{Les programmes de rachat de titres souverains par la BCE}

Au paroxysme de la crise grecque, au printemps 2010, s'est posée la question de savoir si la BCE devait intervenir sur les marchés de dette publique au sein de la zone euro. Les raisons de cette interrogation sont claires. D'une part la crise, par effet de contagion, se propageait rapidement à d'autres pays participant à l'UEM, non nécessairement frappés par des problèmes de mauvaise gestion des finances publiques - à l'instar de l'Irlande. D'autre part, aucun autre acteur que la banque centrale n'avait la capacité d'acheter en grande quantité les titres souverains mis en difficulté.

Il en est résulté un premier programme d'acquisition de tels titres par la BCE, le SMP (pour securities markets program). Interrompu dès l'été 2011, il fut suivi en 2012 de l'annonce d'un nouveau dispositif, aujourd'hui prêt à entrer en fonction si nécessaire : le programme d'opérations monétaires sur titres (ou- 
tright monetary transactions, $\mathrm{OMT}^{2}$ ). Nous examinons les caractéristiques de ce programme, puis les controverses qu'il a suscitées.

\section{Les caractéristiques du programme OMT}

Les opérations monétaires sur titres (OMT) ont été imaginées à un moment où la crise de l'endettement souverain dans la zone euro ne parvenait pas à être endiguée, menaçant de se propager à de «grands pays» tels que l'Italie ou l'Espagne. Plus encore, la zone elle-même paraissait menacée de la possible sortie (exit) de tel ou tel État membre en difficulté, voire d'un éclatement pur et simple $^{3}$. À cette aune, il est alors remarquable que la simple annonce du programme OMT (qui, à ce jour, n'a pas encore été utilisé) ait pu avoir un effet significatif de stabilisation des marchés de titres publics.

Dans le cadre de l'OMT comme antérieurement avec le SMP, la BCE pourra acquérir de la dette souveraine européenne, et ce exclusivement sur les marchés secondaires. Que les opérations ne puissent s'effectuer sur les marchés primaires est fondamental pour ne pas contrevenir aux textes en vigueur. L'interdiction faite à la BCE par les traités (article 123 du traité sur le fonctionnement de l'Union européenne, TFUE) de financer directement les États membres devait impérativement être respectée. Tel est bien le cas lorsque la banque centrale acquiert des titres seulement sur le marché secondaire, puisqu'alors ceux-ci préexistent, tandis que l'opération ne modifie pas l'endettement du pays émetteur.

L'OMT a également pour caractéristique clé que les interventions éventuelles de la BCE seraient potentiellement illimitées. La banque centrale ne s'impose ainsi aucun plafond ex ante sur ses achats de titres souverains, ce qui a pour vertu d'impressionner la spéculation. C'est là une importante raison expliquant les effets dissuasifs avérés de l'OMT.

En troisième lieu, le nouveau programme tient compte des imperfections de son prédécesseur, le SMP. D'une part, la BCE ne revendique plus le statut de créancier senior ${ }^{4}$. D'autre part, une intervention au titre de l'OMT ne pourra se faire qu'au bénéfice d'un État par ailleurs engagé dans un programme d'ajustement assorti d'une conditionnalité stricte, sous l'égide du Fonds européen de stabilité financière (FESF) ou du Mécanisme européen de stabilité (MES). Il s'agit ici de limiter l'aléa de moralité, que l'on a vu à l'œuvre avec le $\mathrm{SMP}^{5}$. La BCE se réserve la possibilité d'interrompre son programme de rachat

\footnotetext{
${ }^{2}$ Voir Banque centrale européenne (2012a).

${ }^{3}$ Voir par exemple Clerc et Kauffmann (2012).

${ }^{4}$ Par ce biais, la banque centrale cherchait à minimiser le risque de pertes, en cas de défaut partiel d'un émetteur. Ce faisant, la BCE augmentait de facto le risque porté par les autres détenteurs de dette publique. Ces derniers savaient qu'ils seraient les premiers mis à contribution en cas d'incident. L'action de la banque centrale avait donc ceci de paradoxal qu'en soulageant temporairement un pays en difficulté, elle aggravait la perception du risque souverain correspondant pour les investisseurs privés.

${ }^{5}$ Un pays confronté à des problèmes structurels de finances publiques, et se sachant soutenu par la BCE, peut être tenté de relâcher ses efforts d'ajustement. C'est précisément ce qui s'est produit avec le gouvernement ita-
} 
si l'État concerné ne respectait pas scrupuleusement ses engagements vis-à-vis des institutions de l'Union. Ces rachats concerneraient des titres souverains de maturités courtes, comprises entre un et trois ans. Ce choix limite en pratique le risque de pertes auquel serait exposée la banque centrale, même s'il ne le fait pas disparaître.

Enfin, il est à noter que, pour l'OMT comme pour son prédécesseur, la BCE prévoit de stériliser la création de monnaie centrale qui en résulterait. Cette disposition n'est nullement anodine. En effet, alors que la base monétaire a augmenté de centaines de milliards d'euros dans le cadre de sa politique monétaire non conventionnelle, comment comprendre que la banque centrale tienne à stériliser des opérations qui, s'agissant du SMP, ont à peine dépassé 200 milliards ? La réponse tient à l'évidence au caractère très sensible des achats de titres souverains aux yeux de certains États membres. On entre ici au cœur des controverses autour de ces programmes.

\section{Controverses autour du programme OMT}

Comme on l'a vu, il y eut en Europe du Nord quelques voix, minoritaires, pour s'élever contre certaines modalités de la politique monétaire non conventionnelle de la BCE. En particulier, l'assouplissement quantitatif a fait l'objet de critiques en Allemagne, en raison de la création de monnaie centrale qu'il induit, et donc de son potentiel inflationniste. Toutefois, le consensus au sein des instances dirigeantes du SEBC n'a jamais été rompu sur l'orientation et les moyens de la politique monétaire non conventionnelle. Il en va très différemment s'agissant des programmes de rachats de titres souverains.

En effet, le SMP et l'OMT ont fait vivement débat au sein même du Conseil des gouverneurs ${ }^{6}$. Or on ne peut manquer d'observer que leur propre potentiel inflationniste est limité. D'une part, au moins à ce jour, les montants en cause sont modérés. D'autre part, la BCE les a soigneusement stérilisés et prévoit de faire de même à l'avenir. Force est donc d'admettre que ce ne sont pas de simples considérations quantitativistes, ni la crainte de l'inflation stricto sen$s u$, qui motivent l'hostilité allemande au SMP et à l'OMT.

Ce qui est en cause est bien davantage le lien entre banque centrale et pouvoir politique. C'est l'ombre de la République de Weimar qui est ici perceptible, moins dans sa dimension strictement macroéconomique - les excès de la création monétaire - que dans sa dimension politico-institutionnelle - la soumission de la Reichsbank au gouvernement allemand. Si le SMP et l'OMT ont tant contrarié la Bundesbank, c'est en raison de la connexion qu'ils sont suscep-

\footnotetext{
lien en 2011. C'est cet effet pervers qui a conduit la banque centrale à mettre un terme prématurément à son programme SMP.

${ }^{6}$ La Bundesbank était très hostile au SMP - tout comme elle l'est à l'égard de l'OMT. La vivacité des tensions et des désaccords s'est traduite, à l'époque, par deux démissions remarquées de hauts dirigeants allemands : celle du président de la Bundesbank A. Weber, et celle de l'économiste en chef de la BCE et membre du directoire J. Stark.
} 
tibles de (re)créer entre dépenses publiques et déficits publics d'une part, création de monnaie d'autre part. L'indépendance même de la BCE, attribut traditionnel et emblématique de la Bundesbank, serait ici écornée. La contestation de l'OMT est, il faut le souligner, quasi exclusivement allemande, ce qui renforce la conviction que c'est bien le poids d'un épisode historique singulier propre à l'Allemagne qui est à l'œuvre. On mesure incidemment la vivacité de l'opposition à l'OMT outre-Rhin lorsque l'on sait que ce programme a fait l'objet de multiples plaintes devant la Cour constitutionnelle de Karlsruhe.

Plusieurs types de considérations permettent toutefois de relativiser les critiques à l'égard des rachats de titres souverains par la BCE.

En premier lieu, cette pratique n'est nullement spécifique à la Banque centrale européenne. Tout au contraire, ses grands homologues américain, britannique ou japonais ont procédé, parfois de longue date, à des opérations similaires, et pour des montants généralement bien plus importants ${ }^{7}$. Or on n'observe, dans les pays concernés, nulle trace de tensions inflationnistes. Parallèlement, ni la Réserve fédérale ni la Banque d'Angleterre ne sont considérées comme ayant perdu leur indépendance à l'égard du pouvoir politique ${ }^{8}$.

Précisément, et en deuxième lieu, aucun des grands éléments constitutifs de l'indépendance d'une banque centrale à l'égard du pouvoir politique n'est remis en cause par l'OMT. Ni les caractéristiques des mandats des dirigeants du SEBC, ni l'indépendance financière de l'institution, ni son autonomie dans la conduite de sa politique monétaire ne sont affectées. De surcroît, le Conseil des gouverneurs gardera toute latitude pour s'engager ou se désengager d'un programme d'achat de titres souverains; il n'aura pas les mains liées à l'égard de tel ou tel État membre de la zone euro.

En conséquence, et en troisième lieu, le parallèle entre la situation contemporaine de la BCE et celle, datée, de la Reichsbank est largement fallacieux. En particulier, le rachat d'obligations publiques sur le marché secondaire, prévu par l'OMT, n'est nullement équivalent à la monétisation des déficits du budget de l'État' qu'a connue la République de Weimar. Les achats d'obligations sur le marché primaire, qui s'analyseraient comme un financement monétaire direct des États, sont totalement exclus. Enfin et surtout, l'OMT a été conçu de manière à n'induire aucun laxisme dans la conduite des politiques budgétaires nationales. Les éventuels programmes de rachats seront destinés à des États engagés dans une démarche d'ajustement sous conditions vis-à-vis du FESF ou du MES. La BCE peut donc, à juste titre, se prévaloir du fait que l'outil dont elle s'est dotée n'est pas de nature à inciter au relâchement des comportements des gouvernements en matière de finances publiques.

\footnotetext{
${ }^{7}$ Là où la BCE a racheté, via le SMP, environ 220 milliards d'euros de titres publics, la Réserve fédérale américaine (Fed) en a acquis pour plus de 1600 milliards de dollars dans le cadre de son propre quantitative easing.

${ }^{8}$ Le cas de la Banque du Japon est un peu différent, compte tenu des pressions qui s'exercent sur elle depuis l'avènement du gouvernement de $\mathrm{S}$. Abe.

${ }^{9}$ Voir Banque centrale européenne (2012b).
} 


\section{Vers l'Union bancaire européenne ?}

C'est dans le courant de l'année 2012 que la problématique de l'Union bancaire est devenue prégnante en Europe. Alors que cette préoccupation aurait pu s'imposer antérieurement, ne serait-ce qu'en raison de l'existence du marché unique, c'est la crise de l'endettement souverain qui mit cette question en exergue. S'est notamment fait jour un redoutable cercle vicieux, liant au sein d'un État membre de la zone euro crise de la dette publique et crise bancaire.

Les enchaînements en cause sont schématiquement les suivants. Considérons un pays participant à l'UEM, au sein duquel se produit une importante crise bancaire. En vertu des règles en vigueur à ce jour, si certains établissements sont menacés de défaillance alors qu'ils ont un caractère systémique, ce sont les finances publiques de l'État concerné qui seront mises à contribution pour venir en aide aux banques en difficulté. Le gouvernement procède$\mathrm{ra}$, sur ses ressources, à d'éventuelles recapitalisations ou nationalisations, comme récemment en Espagne. Si les montants en jeu sont significatifs, tandis que le pays est en situation de déficit budgétaire, il faudra recourir à l'emprunt, ce qui alourdira la dette publique. Si, par ailleurs et préalablement, la situation générale des finances publiques est déjà dégradée, les emprunts additionnels viendront détériorer la qualité de l'ensemble des titres souverains en circulation.

Dans la mesure où les banques locales sont elles-mêmes, de façon générale, d'importantes détentrices de titres publics, la détérioration que subissent ces derniers, qui peut se traduire par une forte baisse de leur cours, affectera à son tour les bilans bancaires. Certains établissements, non nécessairement concernés par la crise initiale, enregistreront alors des pertes sur leur portefeuille de valeurs mobilières. La boucle est bouclée : la crise bancaire originelle s'est aggravée, moyennant une crise de l'endettement souverain.

C'est pour briser cette spirale que le Conseil européen a fixé pour objectif, en juin 2012, la création d'une union bancaire dans la zone euro. Nous commençons par en présenter les éléments constitutifs. Nous en précisons ensuite les premiers acquis, avant de montrer pourquoi certaines avancées vers une union bancaire pleine et entière sont aujourd'hui controversées.

\section{Les éléments constitutifs d'une union bancaire}

Schématiquement, une union bancaire au sein d'une entité telle que la zone euro est réalisée lorsque sont satisfaites les quatre conditions suivantes :

- la supervision micro-prudentielle des banques est unifiée, sous la responsabilité d'une autorité commune ;

- le droit bancaire est pleinement harmonisé, afin de n'engendrer aucune distorsion de concurrence d'un État à l'autre, ni de permettre un quelconque arbitrage réglementaire de la part des banques ; 
- les procédures de traitement des crises bancaires sont unifiées, et placées idéalement sous la responsabilité d'un organisme unique de résolution ;

- il existe un dispositif commun (paneuropéen) d'assurance des dépôts, adossé à des ressources budgétaires clairement identifiées, idéalement communautaires.

La deuxième condition d'harmonisation complète du droit bancaire dans la zone euro tombe sous le sens. En toute logique, elle devrait être satisfaite même dans le cadre d'un simple marché intérieur - sans parler d'une union monétaire. C'est dès ce stade d'intégration, en effet, que les banques ont besoin de règles d'activité unifiées (le level playing field des anglo-saxons). Il est alors paradoxal que le marché unique européen existe depuis plus de vingt ans sans que la problématique de l'unification du droit bancaire ne se soit jamais pleinement imposée - $\mathrm{y}$ compris après l'éclatement de la crise des subprimes ${ }^{10}$.

Parallèlement, le caractère fondamental et logiquement premier de la supervision unique semble avéré. C'est seulement lorsque les banques sont strictement soumises aux mêmes règles du jeu et à une même autorité de surveillance que l'on peut envisager de " communautariser » l'assurance des dépôts et la résolution des crises - donc les modalités et les moyens de sauvetage des banques.

Les deux dernières conditions sont en quelque sorte les points d'aboutissement d'une union bancaire. C'est leur pleine réalisation qui permet de rompre le cercle vicieux, analysé précédemment, liant crise bancaire et crise de la dette souveraine. En effet, lorsque l'assurance-dépôts et la résolution des crises sont unifiées, les États concernés ne sont plus placés dans un périlleux tête-à-tête budgétaire avec leur système bancaire. En cas de difficulté, ce sont des mécanismes et des ressources communautaires (en l'occurrence, à l'échelle de la zone euro) qui sont mobilisés. De surcroît, la troisième condition garantit un traitement plus rapide et plus efficace des défaillances d'établissements plurinationaux. En l'état actuel en effet, les banques européennes ayant une activité transfrontière sont soumises, en cas de faillite, aux procédures parfois divergentes des différents États dans lesquels elles sont implantées. Il peut en résulter de longs imbroglios politico-juridiques, peu propices à une bonne résolution des crises ${ }^{11}$.

\section{Les acquis : supervision unifiée et single rulebook}

Placés face au cercle vicieux liant crise bancaire et crise de la dette souveraine, certains États de la zone euro ont, dans le courant de l'année 2012, émis l'idée qu'il soit fait appel à des fonds européens pour recapitaliser leurs établissements en difficulté. La possibilité d'une intervention en ce sens du MES fut ainsi étudiée. Toutefois, pour qu'une telle action soit utile au regard du problème posé, il faudrait qu'elle ne se traduise pas par une aide conditionnelle classique à l'État demandeur. Dans ce cas en effet, la dette publique de ce dernier s'alourdirait à

\footnotetext{
${ }^{10}$ Voir par exemple Kauffmann (2011).

${ }^{11}$ Les exemples de Fortis ou de Dexia illustrent clairement ce phénomène.
} 
hauteur des prêts du MES, si bien que la « spirale infernale » à briser serait toujours à l'œuvre.

Toutefois, pour que le MES intervienne directement dans la résolution d'une crise bancaire nationale, une condition fut formulée par les pays d'Europe du Nord, dont l'Allemagne. Ces derniers demandèrent, fort logiquement, que les banques concernées soient préalablement soumises à une autorité de supervision paneuropéenne. Il s'agissait en particulier de prévenir tout phénomène d'aléa moral, de la part des banques elles-mêmes ou de leurs autorités de tutelle nationales. C'est sous cette impulsion que la mise en place d'un Mécanisme de surveillance unique (MSU) en Europe devint une priorité. Le processus connut un aboutissement rapide puisque dès l'automne 2012, un consensus au sein de la zone euro conduisit à faire de la $\mathrm{BCE}$ l'autorité supérieure de supervision bancaire. La banque centrale se vit donc confier de nouvelles prérogatives, destinées à entrer progressivement en application.

La BCE supervisera ainsi directement un certain nombre de grands établissements, considérés comme systémiques. Par ailleurs, les autorités nationales existantes continueront d'exercer leur surveillance sur les banques de plus petite taille. Ce partage des tâches dessine une architecture de type fédéral pour la supervision bancaire. Notons toutefois que la BCE pourra, chaque fois qu'elle le jugera utile, avoir un accès direct aux données relatives à tel ou tel établissement qu'elle ne surveille pas directement. Il s'agit de la sorte d'éviter, comme on l'a trop souvent vu par le passé, que les superviseurs nationaux ne "protègent » leurs banques en tendant à minimiser leurs difficultés réelles.

La BCE aura ainsi bien plus de pouvoirs effectifs que l'Agence bancaire européenne $(\mathrm{ABE})$ n'en avait jusque-là. Cette dernière était, certes, censée coiffer l'action des superviseurs nationaux. Elle manquait toutefois de capacités coercitives à leur égard et d'un accès en temps utile à certaines informations sur les systèmes bancaires nationaux. Au demeurant, le nouveau MSU suppose que le rôle de l'ABE soit redéfini. Laissant à la $\mathrm{BCE}$ les fonctions exécutives en matière de supervision, l'agence se concentrera sur le contenu de la réglementation et du droit bancaire en Europe. C'est à elle qu'il reviendra de réfléchir à faire évoluer ces derniers, et à garantir l'existence de règles du jeu parfaitement harmonisées dans l'Union (le single rulebook).

\section{Les controverses : résolution des crises bancaires et assurance des dépôts}

L'avancement de l'Union bancaire européenne procède désormais des deux chantiers que sont la mise en place d'un Mécanisme de résolution unique des crises (MRU), et d'un dispositif paneuropéen d'assurance-dépôts. Or ces deux dossiers viennent buter sur une même contrainte, qui n'est pas spécifiquement inhérente à l'Europe bancaire, et concerne la possibilité de transferts budgétaires entre États. Ce sont ces derniers qui soulèvent l'opposition de l'Allemagne, tandis que la perspective d'un MRU et d'une assurance-dépôts unifiée dans la zone euro est perçue très favorablement en France. 
Ainsi de l'assurance-dépôts. Actuellement, la législation européenne prévoit simplement que ce dispositif est obligatoire dans tous les États membres, et harmonise le degré de couverture des déposants. Ce sont cependant des ressources purement nationales qui viennent abonder les fonds existants ${ }^{12}$, généralement au moyen d'une cotisation versée par les banques au prorata de leurs dépôts. Si ces fonds s'avèrent insuffisants, ce sont les ressources budgétaires de l'État concerné qui sont mobilisées. À ce titre, les mécanismes existants d'assurance-dépôts participent donc au fameux cercle vicieux à circonscrire.

Pour le rompre, la solution la plus simple consiste à concevoir un dispositif unique (paneuropéen) d'assurance des dépôts. Celui-ci pourrait être basé sur un fond communautaire, abondé par l'ensemble des banques de la zone euro. Ce fond serait lui-même adossé, en cas de besoin, à des ressources budgétaires européennes, dont la nature exacte reste à préciser (recettes de la taxe sur les transactions financières, contributions directes des États, etc.). Sous pareilles hypothèses, une crise bancaire nécessitant l'indemnisation des déposants dans un État membre ne mobiliserait plus uniquement les ressources propres à cet État. Ce sont des fonds issus de l'ensemble de la zone euro qui, par construction, seraient engagés. Il est alors clair que des transferts entre États pourraient intervenir. L'indemnisation de déposants espagnols pourrait se faire, au moins partiellement, à partir de fonds allemands - et vice versa. Cette perspective suscite les plus vives réserves outre-Rhin.

La conception d'un mécanisme européen " unique » de résolution des crises bancaires soulève in fine des problèmes similaires. En effet, de nombreux protagonistes (dont la Commission) pensent qu'un bon traitement des crises bancaires dans la zone euro, au vu de l'expérience des dernières années, suppose une autorité communautaire spécifique. Celle-ci, qui pourrait être une agence rattachée à la Commission, prendrait en charge les établissements en difficulté, auxquels elle appliquerait des règles prédéfinies et, par construction, identiques pour toute la zone euro. Ce MRU pourrait décider de la mise en liquidation d'une banque, de sa recapitalisation, de son adossement à un établissement sain, etc.

Là où le bât blesse, en Europe du Nord et particulièrement en Allemagne, c'est que - à tout le moins dans les prochaines années ${ }^{13}$ - ce sont des ressources issues des États membres qui serviront à la résolution des crises. Celles-ci devraient être complétées par des financements issus du MES, selon des modalités qui restent à préciser. Par conséquent, la possibilité qu'une crise bancaire dans un pays membre soit pour partie résolue avec des fonds issus d'autres pays membres est avérée.

Cette perspective de transferts entre États fait naître, en Allemagne, une double opposition. L'une est d'ordre juridique : la République fédérale consi-

\footnotetext{
${ }^{12}$ Encore aura-t-on garde de distinguer les fonds pré-financés, en vigueur dans certains États, et les fonds constitués ex post, en cas de sinistre avéré.

${ }^{13}$ À terme (à l'horizon d'une dizaine d'années), la Commission envisage la création d'un véritable fonds européen de résolution des crises, qui serait doté de 60 milliards d'euros environ.
} 
dère que les traités en vigueur ne permettent pas d'engager de la sorte " l'argent du contribuable allemand ». L'autre est d'ordre politique : ni le gouvernement ni l'opinion publique ne veulent voir étendue à l'Europe ou à la zone euro l'union de transferts (Transferunion) qui prévaut entre les Länder, et qui a coûté si cher à certains d'entre eux depuis la réunification. C'est peut-être parce que la France n'a pas l'expérience de ce fédéralisme budgétaire que l'éventualité d'une union de transferts n'y est pas négativement connotée.

L'EXAMEN DES GRANDS FOYERS DE CONTROVERSES FRANCO-ALLEMANDES, et plus généralement européennes, autour de l'architecture de l'Union monétaire révèle que leurs points focaux ne doivent rien au hasard. Dans l'ordre où nous les avons examinés, ils ont trait : à l'aversion pour l'inflation, s'agissant de la politique monétaire non conventionnelle de la $\mathrm{BCE}$, à la relation entre banque centrale et pouvoir politique, s'agissant des programmes de rachat de dette publique et au fédéralisme budgétaire, s'agissant de la formation d'une union bancaire. Ces points d'achoppement ont tous des racines profondes, ancrées dans les histoires respectives des protagonistes.

Le traité de Maastricht a dessiné une zone euro empreinte d'ordolibéralisme, au sein de laquelle, conformément aux préférences allemandes, la lutte contre l'inflation était prioritaire, la banque centrale était totalement indépendante du politique et les transferts budgétaires entre États étaient proscrits. L'expérience ayant montré que cette architecture n'était pas viable, il appartient aux pays participants à l'UEM de remettre l'ouvrage sur le métier. Un nouveau traité sera sans doute indispensable, non seulement pour dépasser les controverses monétaires analysées précédemment, mais pour en appréhender d'autres (gouvernement économique, eurobonds, politique de compétitivité, etc.), non moins importantes pour l'avenir de l'intégration européenne.

\section{Indications bibliographiques}

BANQUE CENTRALE EUROPÉENNE, «Éditorial », Bulletin mensuel, septembre 2012, p. [n.c.] (2012a)

BANQUE CENTRALE EUROPÉENNE, «Éditorial », Bulletin mensuel, octobre 2012, p. [n.c.] (2012b)

Clerc O., Kauffmann P., "Vers la désunion monétaire européenne », Revue de l'Union européenne, $\mathrm{n}^{\circ}$ 563, décembre 2012, p. [n.c.]

KaUfFMann P., «Les banques européennes et la crise : quelles mutations? », in GraRd L., KaUfFManN P. (eds), L'Europe des banques - Approches juridique et économique, Pedone, Paris, 2011, p. [n.c.] (Droits Européens)

LAROsIÈRE J. DE, Rapport sur la surveillance financière dans l'Union européenne [en ligne], [s.e.], Bruxelles, 2009. Disponible sur : http://ec.europa.eu/internal_market/finances/docs/de_larosiere_report_fr.pdf [consulté le 31/07/2014]

Pisani-Ferry J., WolfF G., "The Fiscal Implications of a Banking Union », Bruegel Policy Brief, n $^{\circ}$, septembre 2012, p. [n.c.]

VÉRON N., «Europe's Single Supervisory Mechanism and the Long Journey Towards Banking Union », Bruegel Policy Contribution, $\mathrm{n}^{\circ}$ 16, octobre 2012, p. [n.c.]. 


\section{Table ronde : Convergences et divergences franco-allemandes sur la politique monétaire européenne. État des lieux et perspectives ${ }^{1}$}

\section{EXPOSÉ INTRODUCTIF}

Paul J.J. Welfens : La première décennie de l'euro s'est avérée un succès, au vu des faibles taux d'inflation, de la croissance de l'emploi, et d'une certaine convergence économique dans la zone euro. Cependant, le monde politique, lors de la phase de démarrage à onze pays - y compris l'Italie -, était certainement conscient que ce grand groupe initial impliquait aussi des risques en 1999. L'intégration de la Grèce en 2001, qui fut une surprise, s'explique par la pression des États-Unis pour faire accéder la Turquie au statut de candidat à l'Union européenne (UE). Les États leaders de l'euro ont accordé leur soutien à la Grèce pour sa demande d'adhésion à l'euro uniquement à condition qu'Athènes lève ses réserves vis-à-vis de l'ouverture des négociations d'adhésion de la Turquie à l'UE, et que la Grèce remplisse les critères de convergence. La zone euro à $12 \mathrm{a}$ encore grandi dans les années qui ont suivi et lorsque la crise a éclaté en 2010, 17 pays étaient membres de l'Union monétaire.

Le Pacte de stabilité et de croissance, avec ses plafonds de déficit et d'endettement, était déjà dépourvu de sens à l'époque : les pays membres (l'Allemagne et la France en 2003) l'avaient trop souvent enfreint. Ce qui manquait depuis le début, c'était une institution indépendante qui établisse et surtout publie chaque année un rapport sur le respect des règles de l'euro. Ce qui manquait aussi, c'étaient des évidences techniques, à savoir un logiciel unique pour les ministères nationaux des Finances, avec un droit de regard de la Commission sur tous les processus budgétaires nationaux en cours. Enfin, il était évident que le taux de dépenses publiques supranationales de $1 \%$ appliqué à Bruxelles était bien trop faible pour assurer un équilibre optimal entre politique monétaire et politique budgétaire, et on ne savait pas vraiment comment réaliser une coordination pertinente des politiques budgétaires nationales en cas de récession. Il devait sembler improbable que l'on parvienne à une coordination rapide et efficace des politiques budgétaires nationales en cas de récession grave. On pensait probablement, en Allemagne et en France, que les deux principaux pays de la zone euro pourraient exercer suffisamment de pression sur leurs partenaires afin d'accroître la coopération en matière de politique budgétaire en cas de crise. On ne s'attendait pas vraiment à ce que ce soient de petits pays, comme la Grèce et l'Irlande, qui puissent déstabiliser toute la zone euro avec leur politique économique singulière en matière de déficit ou de contrôle bancaire (cas de l'Irlande).

${ }^{1}$ Cette table ronde s'est tenue dans le cadre de la journée d'étude Cultures monétaires en France et en Allemagne, coorganisée par le CIRAC le 22 avril 2013, NdE. 


\section{Les problèmes persistants de la crise de l'euro}

Même après le séisme déclenché par la faillite de Lehman Brothers en 2008, les responsables politiques de la zone euro n'ont toujours pas cherché à concrétiser une coopération politique renforcée au vu du changement dramatique de situation. En Allemagne surtout, on a encouragé une politique de réformes et consolidations à petits pas ; pourtant, en 2013, l'Irlande est tombée dans sa deuxième récession en peu de temps, la Grèce n'a toujours pas renoué avec la croissance et le Portugal pourrait avoir besoin d'un second plan d'aide en 2014. Pendant ce temps, les États-Unis ont surmonté leur crise. Les chiffres positifs de la croissance aux USA laissent penser que certains pays de la zone euro présentant des symptômes de crise pourraient bénéficier d'une croissance économique accrue grâce au renforcement des exportations vers les États-Unis. Malte, la GrandeBretagne, la Lituanie, l'Irlande, l'Estonie et le Danemark réalisent des exportations à valeur ajoutée qui représentent $3 \%$ ou plus du produit intérieur brut (PIB), Malte atteignant même 6,4 \% du PIB en 2009 (calculs effectués à partir de la base de données ÉVA de l'OCDE-OMC). Les exportations à valeur ajoutée de l'Allemagne ont atteint $2 \%$ du PIB, celles de la France seulement 1,2\%, de sorte que l'expansion de l'Allemagne devrait être supérieure à celle de la France en cas de reprise économique américaine. Les taux se situaient à 1,8\% et 1,5\% respectivement pour la Grèce et l'Italie en 2009 , à $1 \%$ pour le Portugal et l'Espagne. Dans tous les cas, l'expansion économique des États-Unis contribuera à stabiliser la zone euro ainsi que l'économie britannique.

En 2013, la zone euro était coincée dans sa quatrième année de crise, et les progrès vers la stabilisation étaient maigres. Avec l'Irlande, le Portugal, l'Espagne, l'Italie et la Grèce, plus Chypre, au tournant 2012-2013, 31 \% de la zone euro - en pourcentage du PIB - étaient en récession. Cela pèse aussi sur les perspectives de croissance de l'Allemagne et encore plus de la France, l'industrie allemande profitant plus que l'industrie française de la mondialisation et donc de l'expansion des marchés asiatiques en forte croissance. De plus, l'Allemagne est plus engagée que la France dans la création de valeur ajoutée et dans l'exportation (nette) de biens d'équipement. En 2007, la part du secteur des biens d'équipement dans la création brute de valeur ajoutée de toute l'économie allemande s'élevait à $14,8 \%$, tandis qu'elle ne représentait que $5,9 \%$ en France - un taux aussi faible qu'aux États-Unis et en GrandeBretagne.

Le solde de la balance commerciale des biens d'équipement, en pourcentage du PIB, s'élevait à $9,5 \%$ en Allemagne, contre $-0,3 \%$ pour la France. Cela montre bien qu'il existe de profondes différences de spécialisation entre l'Allemagne et la France, et que la faible importance du secteur des biens d'équipement fait partie du sous-dimensionnement relatif de l'industrie en France. Il en va de même pour d'autres pays de la zone euro; de plus, l'Allemagne dispose d'une avance dans les dépenses consacrées aux technolo- 
gies de l'information et de la communication (TIC), un domaine important pour la compétitivité internationale de tous les secteurs ainsi que pour l'évolution des coûts salariaux unitaires.

Tableau 1: Balance des transactions courantes et structure de l'économie (2007)

\begin{tabular}{|l|l|c|c|c|}
\cline { 2 - 5 } \multicolumn{1}{c|}{} & $\begin{array}{l}\text { Part du secteur des biens d'équipement } \\
\text { dans la création brute de valeur ajoutée } \\
\text { de l'économie allemande (en \%) }\end{array}$ & $\begin{array}{l}\text { Solde de la balance commerciale } \\
\text { des biens d'équipement } \\
\text { (en \% du PIB) }\end{array}$ & $\begin{array}{l}\text { Solde global de la } \\
\text { balance commerciale } \\
\text { (en \% du PIB) }\end{array}$ & $\begin{array}{l}\text { Solde de la balance des } \\
\text { transactions courantes } \\
\text { (en \% du PIB) }\end{array}$ \\
\hline KR & 18,1 & 10,8 & 11,1 & 2,1 \\
\hline DE & 14,8 & 9,5 & 12,0 & 7,5 \\
\hline JP & 11,6 & 7,5 & 5,9 & 4,9 \\
\hline SE & 11,0 & 2,8 & 4,7 & 9,1 \\
\hline AT & 10,6 & 3,0 & 2,7 & 3,5 \\
\hline CH & 10,1 & 2,6 & 3,7 & 8,6 \\
\hline IT & 9,2 & 1,9 & 3,3 & $-1,2$ \\
\hline ES & 6,3 & $-4,1$ & $-6,5$ & $-10,0$ \\
\hline BE & 6,2 & 1,2 & 8,9 & 1,6 \\
\hline DK & 6,2 & $-3,1$ & $-0,8$ & 1,4 \\
\hline FR & 5,9 & $-0,3$ & $-1,3$ & $-1,0$ \\
\hline IE & 5,9 & $-1,3$ & 15,6 & $-5,4$ \\
\hline US & 5,4 & $-2,0$ & $-4,2$ & $-5,1$ \\
\hline LU & 5,1 & $-0,5$ & $-7,5$ & 10,1 \\
\hline GB & 5,0 & $-2,9$ & $-5,9$ & $-2,3$ \\
\hline NL & 4,7 & 1,7 & 8,9 & 6,7 \\
\hline PT & 4,0 & $-5,1$ & $-7,0$ & $-10,7$ \\
\hline GR & 2,5 & $-7,9$ & $-14,0$ & $-14,6$ \\
\hline
\end{tabular}

Source : IWD, « Investitionsgüter führen zu Überschüssen » (Les biens d'équipement génèrent des excédents), iw-dienst, vol. 39, n 15, 2013, p 3. Portugal : données 2006 ; KR : République de Corée du Sud.

Malgré quelques progrès dans la réduction des dépenses publiques et l'augmentation des recettes fiscales par rapport au PIB, la Grèce reste plongée dans une crise sérieuse. Le déficit persistant de la balance des transactions courantes en 2012 montre que le pays souffre d'une dette extérieure qui continue à augmenter. Les responsables du mensonge politique sur le déficit en 2009 n'ont pas dû rendre de comptes lorsque le gouvernement a annoncé $4 \%$ de déficit pendant l'année électorale, alors qu'il atteignait en réalité 15,6\%. Comme, d'expérience, on sait qu'il est impossible de réduire le déficit de plus de $3 \%$ par an, le taux avec lequel le gouvernement conservateur d'Athènes a tenté de s'acheter presque illégalement la victoire dans les urnes annonçait que dans les cinq années suivantes, le déficit allait augmenter d'au moins 45 points de pourcentage - un désastre, sachant que la dette de départ était supérieure à $110 \%$. Toutefois, la Grèce disposait d'après le Fonds monétaire international (FMI, 2010) d'un patrimoine public largement supérieur à sa dette à la fin de l'année 2010 : une grande campagne de privatisation, alliée à des réformes structurelles et des mesures de consolidation, aurait pu lui permettre d'échapper à la faillite partielle. Pourtant, ni l'Allemagne, ni la France, ni les autres pays de la zone euro n'ont insisté, s'appuyant sur l'expérience des membres de l'UE d'Europe de l'Est, pour que la Grèce engage un vaste programme de privatisations : il aurait fallu 
pour cela mobiliser l'aide de la Banque européenne pour la reconstruction et le développement (BERD) à Londres, qui a expérimenté cette politique dans 29 pays d'Europe de l'Est et d'Asie centrale. Entre 2010 et 2013, la Grèce a privatisé à peine $2 \%$ de son patrimoine. C'est une inertie tacitement tolérée par Paris, Berlin et Bruxelles, voire une solidarité négative avec les pays partenaires de l'euro, d'autant plus que les privatisations constituent la base d'une augmentation de la productivité et de l'innovation. Si la Grèce est devenue membre de la zone euro en 2001, c'est indirectement dû à la pression des États-Unis en matière de relations entre la Turquie et l'UE, qui a de fait conféré à la Turquie le statut de candidat après la guerre du Kosovo (déclarations lors du sommet du G8 à Cologne), Athènes ayant abandonné sa position jusqu'alors négative pour obtenir en contrepartie le soutien - notamment de l'Allemagne - à sa candidature à l'euro.

La crise de l'euro, qui touche non seulement la Grèce, mais aussi le Portugal, l'Irlande, l'Espagne, Chypre et, de fait, l'Italie, a mis en évidence les faiblesses du cadre réglementaire de l'UE et de la zone euro. Le manque de discipline budgétaire de ces pays - parfois lié aux charges imposées par la crise bancaire transatlantique - est largement responsable des problèmes actuels. Dans les faits, la clause de non-renflouement du traité de Maastricht n'a pas pu être appliquée. À la place, après l'étape intermédiaire du programme bilatéral de crédit pour la Grèce, on a créé le fonds de crédit du Fonds européen de stabilité financière (FESF) qui s'appuie sur les garanties des pays de la zone euro (sans les membres du programme qui étaient en mode de crise); depuis la fin de l'année 2012, le fonds de sauvetage du Mécanisme européen de stabilité (MES) basé sur la levée de capitaux peut être mobilisé pour un volume d'environ 500 milliards d'euros. Ce fonds n'est pas vraiment élevé, lorsqu'on pense à l'éventualité d'une double crise Espagne/Italie, ou que l'on prend comme point de comparaison le fonds de sauvetage bancaire allemand SoFFin qui disposait d'un volume de 480 milliards d'euros, dont environ la moitié a dû être utilisée entre-temps. La dette publique de l'Italie atteignait largement 2000 milliards d'euros, celle de l'Espagne environ 800 milliards d'euros. La capacité de grands pays tels que l'Allemagne et la France à apporter leur aide contre la crise de l'euro est limitée politiquement; en Allemagne, l'émergence du nouveau parti Alternative für Deutschland (AfD) devrait restreindre la capacité du système politique à élaborer des solutions généreuses pour les pays en crise. En outre, la publication des chiffres de la Banque centrale européenne (BCE, 2013 : cf. tableaux 2 et 3 ) sur le patrimoine des ménages dans les pays de la zone euro pour la période 2009-2010 - pour certains pays, les chiffres concernent 2008 - devrait, au vu du rang obtenu par l'Allemagne sur la base des chiffres médians, se traduire plutôt par une limitation renforcée de la capacité d'action pour le financement de mesures de sauvetage étendues.

Comme les banques détiennent en général d'importants stocks de titres d'État, un effacement des dettes auprès des créanciers privés, comme cela a été 
le cas en Grèce en 2012, provoque une crise bancaire nationale ou exige une recapitalisation des établissements bancaires. Dans certains pays, la crise de la dette publique est liée à la crise bancaire (Artus/Gravet, 2013), une crise bancaire impliquant elle-même une réduction des octrois de prêts, ce qui accentue les problèmes de récession ou empêche le redémarrage de l'économie, et donc a tendance à creuser le déficit public.

La restructuration de la dette de la Grèce, lors de laquelle les créanciers privés ont perdu 107 milliards d'euros, aurait pu être moins importante si une privatisation d'envergure avait été réalisée. Au début de l'année 2013, la Grèce avait accompli des progrès certains. Toutefois, sa balance des transactions courantes restait déficitaire et on ne pouvait guère espérer un retour à la croissance économique avant 2014. En Irlande, on notait des progrès relativement satisfaisants jusqu'au début de l'année 2013, mais ce pays illustrait lui aussi un sérieux problème structurel européen, puisque les directives de contrôle bancaire de l'UE étaient restées lettre morte pendant des années. En raison de ce manquement grave aux règles de l'UE contre lequel la Commission européenne n'a pas levé le petit doigt, les banques irlandaises ont pu se disperser exagérément dans un nombre excessif de projets et de produits financiers à haut risque. En 2010, le déficit de l'Irlande s'élevait à $31 \%$, dont les deux tiers étaient dus à des recapitalisations bancaires par les fonds publics.

Le président de la Commission, José Manuel Barroso, n'a publiquement réprimandé ni la Grèce pour ses mensonges sur son déficit, ni l'Irlande pour son laxisme réglementaire. Voilà qui ternit l'image de la Commission, voire de l'UE et de la zone euro. Le Portugal a fait quelques progrès pour améliorer sa situation, mais les contraintes et les restrictions imposées par la Cour constitutionnelle compliquent le processus de stabilisation. Après la restructuration du déficit grec, l'Espagne et l'Italie ont subi de fortes hausses des taux d'intérêt, parfois de 2 à 3 points, tandis que le taux des emprunts à long terme pour l'Allemagne est, d'après les indications de la Bundesbank (2010), de 2 points inférieur à la normale : en avril 2013, elle a pu placer un emprunt à $1,3 \%$ sur le marché des capitaux. La France aussi profite de cet effet de refuge sur ses taux d'intérêt. Ainsi, dans la zone euro, les taux d'intérêt sont extrêmement différents, ce qui ne peut pas être considéré comme une situation normale dans une union monétaire. En Espagne, les mesures de réduction du déficit se prolongeront encore jusqu'en 2015 environ afin de passer sous la barre des $3 \%$, le pays souffrant d'une crise bancaire conjuguée à la crise nationale de l'immobilier. Le pays a reçu 40 milliards d'euros de crédits pour aider son secteur bancaire. Avec la crise chypriote, c'est un autre pays de la zone euro qui s'enfonce dans de sérieux problèmes, là encore dans un contexte de défaillances largement prévisibles dans le secteur bancaire, renforcées par la restructuration de la dette grecque. Certes, des éléments d'adaptation du marché et de la politique économique agissent dans les pays en crise, mais ceux-ci ne semblent pas près de la 
surmonter durablement. Du reste, la Grande-Bretagne est elle aussi dans une situation difficile.

On ne sait toujours pas clairement comment appliquer les règles sur les déficits ni parvenir à une coordination raisonnable des politiques économiques. Les nouvelles règles de déficit du pacte budgétaire (maximum $0,5 \%$ de déficit structurel) sont contradictoires avec les objectifs du Pacte de stabilité et de croissance (déficit limité à $3 \%$ ), ce qui ne facilite pas le rétablissement de la confiance. Jusqu'à présent, l'Allemagne et la France n'ont proposé aucune initiative commune de réforme. De la part des grands acteurs de la politique économique, seule la $\mathrm{BCE}$ a apporté une contribution pertinente à la stabilisation : en annonçant le 6 septembre 2012 que les pays en crise qui adopteraient un programme d'adaptation recevraient en contrepartie une aide éventuellement illimitée sous la forme d'achat d'emprunts d'État à 1 à 3 ans d'échéance, elle a permis à l'Espagne et à l'Italie d'obtenir, à court terme et au moins provisoirement, une baisse des taux. Le fait que l'Allemagne, la France et d'autres pays présents aux sommets de l'euro aient pris l'initiative dans la gestion de la crise de la zone euro est préoccupant dans la mesure où ce n'est plus la Commission européenne, en charge de l'intérêt général de l'UE, qui est au premier plan, mais que ce sont les chefs de gouvernement des grands pays influents qui sont sous les feux de la rampe - dans cette situation, Angela Merkel a parfois elle-même contribué à se voir attribuer le rôle de bouc émissaire. Il est évident que la méthode précédente de gestion de la crise ne peut être maintenue pendant de nombreuses années sans nuire gravement à l'image de l'UE et encourager un renouveau préoccupant du nationalisme.

\section{Les avantages de l'euro}

L'Union monétaire a été élaborée à partir de nombreuses années de réflexion théorique. L'euro et la BCE sont nés le $1^{\text {er }}$ janvier 1999 avec onze pays membres. Pour une capitalisation à très long terme, sur l'espérance de vie du citoyen européen moyen, les bénéfices de l'euro s'élèvent à environ $10000 €$ (Welfens, 2012 ; 2013b). Ces avantages découlent non seulement des économies sur les frais de transaction, mais surtout de l'avantage que présente la zone euro en tant que réserve monétaire : on peut y réaliser quasiment sans frais des importations nettes à hauteur de $0,5 \%$ du produit intérieur brut, parce que le taux d'intérêt payé sur les emprunts européens en réserves de devises étrangères est faible, et devrait se situer à environ 2 points en dessous du rendement global du capital. L'hypothèse en question est prudente, et suppose une participation à hauteur de $20 \%$ dans les réserves monétaires mondiales. Si l'on effectue le calcul sur la base de 3 points de différence en termes de rendement et de $30 \%$ des réserves monétaires, l'effet bénéfique de l'euro est encore plus évident.

Par ailleurs, l'intégration monétaire accentue la concurrence sur le marché intérieur (dans la zone euro). 
Après une certaine période de stabilisation, la zone euro pourrait à nouveau être considérée comme un espace d'intégration attractif auquel d'autres pays d'Europe de l'Est voudraient rapidement adhérer. La question de 1'extension de la zone euro peut être considérée comme critique : d'un point de vue économique, il faudrait se préoccuper plus qu'avant des critères de zones monétaires optimales. Ces critères prévoient que les pays sont les plus susceptibles de renoncer à la flexibilité des changes lorsqu'ils disposent d'une forte mobilité de la main-d'œuvre (critère de Mundell), d'une forte différenciation des produits (c'est la tendance dans les grands pays ; critère de Kenen), et d'une forte proportion de biens échangeables dans leur production (en pratique, on pourrait ici s'appuyer sur le taux d'exportation ; critère de McKinnon). Dans l'Union monétaire, ou dans l'UE et ses pays membres, la mobilité de la main-d'œuvre peut être encore renforcée, mais il faudrait pour cela rendre les systèmes nationaux de sécurité sociale plus perméables. Avec la hausse des taux de chômage dans les pays touchés, la crise de l'euro a accentué la pression migratoire : l'Allemagne à elle seule devrait absorber un flux d'immigration de 2 millions de personnes environ dans la décennie qui suit 2009. Ainsi, la population de l'Allemagne pourrait augmenter à moyen terme.

Envisager le scénario de l'effondrement d'une union monétaire européenne préparée pendant des décennies reviendrait à accepter la possibilité que certains États des États-Unis puissent quitter l'union monétaire autour du dollar. En fait, on ne peut pas imaginer un tel échec politique historique dans la zone euro. Hormis le cas particulier de la Grèce, aucun pays ne semble candidat à l'hypothèse d'une sortie de l'euro. En cas de faillite des pays touchés par la crise de l'euro, 1'Allemagne, la France et le Benelux seraient les plus fortement affectés, ce qui laisse à penser que dans le doute, une union politique de la zone euro serait mise en place. Le coût d'une sortie de la zone euro serait en tout cas très élevé, comme la société Prognos AG et la Bertelsmann Stiftung (2012) l'ont constaté : le PIB allemand serait pénalisé pendant huit ans de 1700 milliards d'euros, ce qui représente environ 20000 euros par habitant, tandis que la France perdrait 2900 milliards d'euros et que l'économie mondiale devrait affronter un recul de ses revenus de 17000 milliards d'euros.

Aucun chef de gouvernement en Allemagne, en France ou au Benelux ne pourrait survivre politiquement à un processus de démantèlement de l'Union monétaire ; en même temps, on peut supposer que les pays en crise susmentionnés veulent très sérieusement éviter toute forme de faillite publique - à l'exception peut-être de la Grèce, marquée par l'instabilité politique et économique. Il est quelque peu étonnant de constater avec quelle indifférence la Grande-Bretagne, ancienne puissance protectrice politique de la Grèce, assiste au processus de déstabilisation d'Athènes. La France, quant à elle, peut faire preuve d'une certaine sympathie pour quelques positions des pays d'Europe du Sud en crise ; tout du moins, les milieux politiques français sont probablement d'avis que l'Allemagne, avec ses excédents d'exportations nets structurelle- 
ment élevés, représente quasiment le reflet inversé du problème des importants déficits de la balance des transactions courantes dans les pays en crise. Si, à l'avenir, les salaires n'augmentent fortement qu'en Allemagne, alors, de ce point de vue, la situation de la balance des transactions courantes devrait s'améliorer dans les pays en crise et leur dette extérieure devrait augmenter moins vite qu'auparavant. Cependant, cette opinion n'est guère adaptée au cœur du problème. Le principal facteur d'amortissement salarial en Allemagne est la forte présence des entreprises allemandes à l'étranger, c'est-à-dire la forte proportion de multinationales (principalement dans le secteur manufacturier) qui produisent à faible coût à l'étranger et peuvent donc, dans certaines limites, faire peser la menace crédible d'une délocalisation des étapes créatrices de valeur ajoutée vers les sites étrangers. En France, par rapport à l'Allemagne, d'une part le secteur manufacturier est relativement faible en pourcentage du produit intérieur brut, d'autre part les gouvernements successifs n'ont pas soutenu la mondialisation de l'économie française de la même manière que le gouvernement fédéral.

\section{Différences traditionnelles entre l'Allemagne et la France}

La République fédérale d'Allemagne est une économie sociale de marché, faiblement centralisée, avec une multitude d'institutions indépendantes - par exemple le Bundeskartellamt (Office fédéral des cartels) et la Bundesnetzagentur (Agence fédérale des réseaux) en matière de politique de la concurrence et de la régulation, ou encore la Deutsche Bundesbank, la banque centrale allemande. Depuis des décennies, l'économie allemande a acquis un avantage comparatif dans le secteur de l'industrie et exploité depuis les années 1990 l'importation de prestations intermédiaires en provenance de pays à bas salaires en Asie et en Europe de l'Est pour renforcer sa position face à la concurrence (Sinn développe une idée contradictoire, mais erronée, avec sa thèse de l'économie de bazar selon laquelle l'Allemagne aurait un problème de compétitivité internationale).

La France, elle, est un État plutôt centralisé qui s'est un temps distingué par ses succès dus aux interventions publiques dans la vie économique. Cependant, on peut se demander, par exemple dans le domaine de l'énergie nucléaire, à quels risques (cachés) intertemporels elle s'expose avec l'extension de la production nucléaire d'électricité : du point de vue de l'industrie française, c'est de l'électricité bon marché, mais seulement tant que l'on n'intègre pas le véritable coût des risques liés à la production nucléaire se traduisant par des primes d'assurances colossales (Hennicke/Welfens, 2012). La natalité naturelle plus élevée par rapport à l'Allemagne et le vieillissement plus lent de la société peuvent également être considérés comme un avantage par rapport à l'Allemagne. Depuis le traité de Maastricht, qui n'a certes amené aucune union politique, la France a milité à maintes reprises pour une coordination plus forte de la poli- 
tique économique à l'échelle de l'UE ou de la zone euro; de fait, depuis la crise grecque, les sommets des pays de la zone euro ont abouti à une coordination renforcée, mais sans création d'institutions indépendantes. Kauffmann/Uterwedde (2010, p. 18) écrivent : "l'Allemagne insiste sur un renforcement de la surveillance budgétaire ex ante et sur des sanctions claires et efficaces. Ces propositions n'auraient rien de contradictoire avec celles avancées par la France si Berlin ne semblait camper sur la ligne historique en vertu de laquelle la " bonne gouvernance » se limite à des règles, et non à des choix politiques. La coordination n'a de sens que si les partenaires se rencontrent, échangent, débattent, et enfin décident. Les règles seules ne peuvent produire ce résultatet des sommets réguliers de l'Eurogroupe en sont une condition nécessaire, quoique non suffisante. 》

En France, la politique de crise contre les instabilités de l'euro va renforcer le débat sur les réformes. Toutefois, au vu des difficultés de certains de ses voisins du Sud, il n'est absolument pas évident que la France puisse mettre en œuvre les réformes nécessaires dans la mesure requise afin d'augmenter la croissance et l'emploi. La commission Gallois mise en place par le président Hollande a du reste déjà attiré l'attention, fin 2012, sur un problème de la France, à savoir la relative faiblesse de la production industrielle et le manque d'entreprises de taille intermédiaire (ETI) innovantes. La France est également handicapée, du point de vue économique, par un ratio des dépenses publiques par rapport au PIB inhabituellement élevé, dépassant les $50 \%$. On peut être sceptique sur l'efficacité des rapports de cette commission. Certes, le président Sarkozy avait demandé à la commission Attali de compiler une sorte d'atlas de la croissance, mais sur les plus de 300 mesures, très peu ont été véritablement appliquées.

En Allemagne, dans le contexte des réformes Hartz IV, la grande coalition a esquissé quelques pistes raisonnables pour plus de croissance et d'emploi, et certains problèmes importants en matière de réforme des retraites ont été abordés assez rapidement, par exemple avec l'augmentation de l'âge de départ à la retraite. Sous la coalition conservatrice-libérale, l'Allemagne a poursuivi certaines réformes, et la chancelière a en particulier donné quelques impulsions pour une dynamique d'innovation et de croissance en maintenant le Sommet national sur les technologies de l'information et de la communication (TIC), qui a efficacement promu l'expansion de l'économie numérique pendant presque dix ans. Le taux de dépenses pour la recherche et le développement devrait approcher $3 \%$ du PIB en Allemagne en 2013, ce qui lui permettrait d'atteindre l'un des jalons du programme européen de Lisbonne 2010.

L'Allemagne et la France doivent se demander si l'on peut raisonnablement croire qu'il est possible de rétablir une stabilité durable en durcissant les règles sur les déficits, tout en conservant l'ancienne architecture des institutions de la zone euro. C'est plus que douteux. D'après les réflexions présentées ci-après, il est nécessaire de former une union politique de la zone euro pour surmonter la 
crise (Welfens, 2012 ; 2013). Cependant, ni l'Allemagne ni la France n'ont mené de débat politique à ce sujet jusqu'à présent.

\section{Calcul par la BCE du patrimoine dans les pays de la zone euro, en données corrigées}

La première publication par la $\mathrm{BCE}$ des chiffres du patrimoine des ménages dans les pays de la zone euro n'a soulevé qu'une discussion minime : c'est en avril 2013 que la Banque centrale européenne a présenté pour la première fois les chiffres du patrimoine net des ménages établis sur la base de sondages. Pour l'essentiel, des ménages représentatifs ont été priés d'indiquer le montant total de leur patrimoine, déduction faite des dettes. Certains résultats se rapportant à 2009-2010 sont remarquables : d'après eux, l'Allemagne dispose du plus faible patrimoine par ménage, lorsqu'on considère la médiane (valeur partageant les ménages en deux ensembles, $50 \%$ d'entre eux se situant au-dessus, $50 \%$ en dessous). Les grands écarts relevés entre les pays ont été principalement attribués, dans le débat public, aux différences en termes de patrimoine immobilier. En vérité, il ne s'agit pas là d'un point capital.

L'Espagne, l'Italie, l'Autriche, la France, l'Allemagne et les Pays-Bas occupent les places 5 à 10 en ordre décroissant sur la base du patrimoine moyen par ménage en 2009-2010. Le patrimoine par ménage en Grèce se situait 47000 euros en dessous de la valeur moyenne de l'Allemagne. En revanche, l'Italie se situait 80000 euros au-dessus de cette moyenne. Si l'on examine les chiffres de plus près, avec un œil critique, on obtient des valeurs parfois très différentes pour le patrimoine par ménage, ou par habitant (les différences de taille des ménages d'un pays à l'autre signifient naturellement que les résultats du calcul par ménage changent de perspective dès que l'on étudie la valeur du patrimoine par personne) : du point de vue économique, une étude critique doit commencer par l'affectation de la dette publique et des obligations d'État, qui ont été représentées dans le questionnaire comme un élément du patrimoine privé - or, sur l'ensemble des ménages, les obligations d'État ne sont, du point de vue économique, pas un patrimoine ; au mieux, on peut les considérer comme tel avec un abattement. Dans les pays où la sécurité sociale est peu développée, comme en Italie ou en Espagne, une majorité des ménages mise pendant la vie active sur l'achat de fonds contenant des obligations d'État dont le rendement ou la vente progressive constitueront la base du paiement de quasi-retraites une fois l'âge atteint. Ainsi, la faiblesse du système d'assurance sociale ou de l'assurance retraite publique favorise une dette publique élevée. À l'inverse, une sécurité sociale solidement financée, comme en Allemagne ou en Autriche, assure une demande plutôt limitée des ménages en obligations d'État. On ignore ici volontairement la crise de l'euro, car elle nécessite la prise en compte de facteurs spécifiques, en particulier le renforcement de la demande internationale en titres d'emprunts d'État venant de quelques pays considérés comme moins que 
sûrs. Outre ces réflexions sur le niveau de la dette publique, on peut se demander par principe, au sens du théorème d'équivalence de Ricardo, si les titres de dette publique peuvent être considérés comme un patrimoine. Si l'État, aujourd'hui, émet un emprunt pour 10 milliards d'euros mis sur le marché à un taux de $10 \%$, cela signifie que les générations actuelles et futures, à compter de ce jour, devront tabler sur une hausse des impôts (escomptée au présent) de 10 milliards d'euros. Si l'on compense la hausse de la dette fiscale future par celle du solde des emprunts d'État, l'effet net sur le patrimoine est nul (ou alors on peut modifier l'hypothèse de départ).

\section{Tableau 2 : Données de patrimoine de la BCE, corrigées sur la base de l'hypothèse de l'ElIW}

\begin{tabular}{|c|c|c|c|c|}
\hline 2009-2010 & \multicolumn{2}{|c|}{$\begin{array}{c}\text { BCE - patrimoine moyen } \\
\text { (moyenne arithmétique) }\end{array}$} & $\begin{array}{c}\text { ElIW - patrimoine moyen } \\
\text { (hors dette publique } \\
\text { ou emprunts d'État) }\end{array}$ & $\begin{array}{c}\text { Surestimation } \\
\text { (en \%) }\end{array}$ \\
\hline $\begin{array}{c}\text { en milliers } \\
\text { d'euros }\end{array}$ & $\begin{array}{c}\text { a) } \\
\text { par ménage }\end{array}$ & $\begin{array}{c}\text { b) } \\
\text { par habitant }\end{array}$ & $\begin{array}{c}\text { c) } \\
\text { par habitant }\end{array}$ & b) par rapport à c) \\
\hline BE & 338,60 & 141,38 & 110,58 & 21,79 \\
\hline DE & 195,20 & 95,64 & 70,50 & 26,28 \\
\hline GR & 147,80 & 47,93 & 18,77 & 60,84 \\
\hline ES & 291,40 & 105,89 & 91,90 & 13,22 \\
\hline FR & 233,40 & 93,19 & 66,04 & 29,13 \\
\hline IT & 275,20 & 110,88 & 79,46 & 28,33 \\
\hline CY & 670,90 & 16,96 & 4,87 & 71,27 \\
\hline LU & 710,10 & 240,86 & 225,67 & 6,31 \\
\hline MT & 366,00 & 122,33 & 112,04 & 8,42 \\
\hline NL & 170,20 & 73,84 & 51,57 & 30,16 \\
\hline AT & 265,00 & 114,51 & 88,53 & 22,69 \\
\hline PT & 152,90 & 52,87 & 37,48 & 29,11 \\
\hline SI & 148,70 & 49,62 & 42,94 & 13,45 \\
\hline SK & 79,70 & 30,59 & 25,59 & 16,35 \\
\hline FI & 161,50 & 76,05 & 59,90 & 21,23 \\
\hline TOTAL & $\mathbf{2 3 0 , 8 0}$ & $\mathbf{9 6 , 0 0}$ & $\mathbf{7 1 , 6 6}$ & $\mathbf{2 5 , 3 5}$ \\
\hline
\end{tabular}

Source des chiffres de base : BCE, « The Eurosystem Household Finance and Consumption Survey », Statistic Paper Series, $\mathrm{n}^{\circ}$ 2, avril 2013, p. [n.c.] ; calculs EIIW. Données de taille du patrimoine d'après CEEONU 2010 ou derniers chiffres disponibles, données sur la dette publique par l'Ameco (UE).

Si l'on suit la théorie classique de David Ricardo en supposant que les ménages interrogés ne sont pas conscients que les charges fiscales futures constituent un endettement implicite, et que les ménages sans patrimoine paieront les « impôts sur les intérêts » futurs sous la forme de la taxe sur la valeur ajoutée et de l'impôt sur le revenu, alors l'encours de titres nationaux de dette publique doit être déduit, dans tous les pays, du patrimoine présenté par la BCE. Le résultat 
est présenté ci-après à partir d'une moyenne arithmétique et montre que le patrimoine par habitant est surévalué dans plusieurs pays : en Grèce et à Chypre, il est respectivement de $61 \%$ et $71 \%$ trop haut, tandis que pour l'Italie, la surestimation est de $28 \%$. Beaucoup de pays sont surévalués de $20 \%$, seuls le Luxembourg et Malte montrent une surcote inférieure à $10 \%$. D'après les calculs de l'Institut européen des relations économiques internationales (EEIW), le patrimoine réel corrigé par habitant est très proche en Allemagne et en Italie : des 80000 euros de différence d'après la BCE pour le patrimoine médian, il ne reste que 9000 euros d'avance pour l'Italie selon la méthode de l'EEIW (la différence par rapport aux chiffres de la BCE se montant à 15000 euros si l'on considère la moyenne arithmétique). Pour l'Espagne, la correction sur la base des chiffres par habitant est, à $13 \%$, aussi faible qu'en Slovénie. Pour l'Allemagne, la surestimation est tout de même de $26 \%$. Dans la mesure où les emprunts d'État ont été émis pour financer des investissements publics, on peut toujours arguer que la hausse des capitaux publics entraîne une augmentation de la productivité des facteurs (travail et capital privé), de sorte que les rentrées fiscales devraient également être supérieures. Toutefois, la zone euro ne dispose pas d'indications claires sur l'affectation de la dette publique : il manque une prescription légale stipulant que les nouvelles dettes ne peuvent être utilisées que pour le financement d'investissements publics. Reste à vérifier dans quelle mesure on pourrait créer une clause d'exception pour les cas de déséquilibre de l'économie entière. Les chiffres de patrimoine de la BCE corrigés montrent par ailleurs que, du reste, les possibilités d'imposition sur la fortune sont plus réduites qu'on le suppose souvent dans le débat public.

\section{L'union politique de la zone euro}

En Allemagne et dans quelques autres pays, une partie de la classe politique ainsi que de nombreux économistes sont d'avis que pour surmonter la crise de l'euro, il faut avant tout des règles plus strictes sur les déficits pour les pays membres ; au demeurant, l'ancienne architecture de la zone euro pourrait etre conservée telle quelle. C'est une idée en partie erronée, car même le pacte budgétaire ne précise absolument pas comment le nouveau plafond à $0,5 \%$ du PIB doit être appliqué en cas de déficit structurel (indépendant de la conjoncture). La juxtaposition de la nouvelle limite de déficit avec le plafond de $3 \%$ fixé dans le traité de Maastricht représente une réglementation confuse, comme le FMI l'a fait remarquer dans son rapport Article IV sur la zone euro (FMI, 2012). De fait, le pacte budgétaire ne contient aucune possibilité de plainte, puisque la présidence du Conseil en exercice, la précédente et la suivante devraient toutes être en faveur d'un recours.

Il existe deux possibilités pour faire réellement appliquer une limitation des déficits - au-delà de l'ancrage souhaitable d'un frein à l'endettement dans la constitution. La première réside dans une condition jusqu'à présent inexistante : 
que les pays de la zone euro en situation de boom économique soient soumis à une obligation d'excédent budgétaire associée à des sanctions ou des pénalités automatiques. Les pays qui souscrivent à une telle réglementation en plus des précédentes devraient alors être autorisés à procéder à l'émission conjointe d'obligations communautaires en euros, ce qui entraînerait pour presque tous les pays de la zone euro (sauf l'Allemagne et la France) d'importantes économies sur les intérêts. Par ailleurs, on pourrait suivre la proposition du Conseil d'experts allemands pour l'évaluation de la situation économique (SVR, 2011) et convenir d'un fonds d'amortissement des charges héritées pour la partie de la dette publique qui dépasse les $60 \%$ de l'endettement. Toutefois, une mise en œuvre en trois étapes de cinq années chacune est pertinente. L'étape I s'appliquerait pour le premier tiers des dettes à court terme dépassant le seuil de $60 \%$ des dettes ; le pays qui rembourserait ces dettes conformément à l'accord accéderait au niveau II, qui concerne un deuxième tiers, celui des titres à moyen terme; celui qui réglerait le niveau II serait admis au niveau III, son taux d'endettement devant diminuer d'une étape à l'autre.

Une seconde possibilité, complémentaire, de mise en œuvre des limites de déficit réside dans l'intégration politique des partis, dans le cadre d'une union politique de la zone euro dotée d'une constitution pertinente. Seuls des partis couvrant l'ensemble de la zone euro, dans lesquels un poste attractif de décision au niveau supranational constituerait le clou d'une carrière politique, permettront de réaliser une véritable union politique de la zone euro. Sans union politique ni intégration des partis de la zone euro, il n'existe aucune incitation systématique pour les grandes pointures politiques nationales à garder toujours à l'esprit l'intérêt général de la zone euro.

Enfin, la coordination sera renforcée par l'Union bancaire dans le cadre du contrôle bancaire qui sera aux mains de la BCE à partir du printemps 2014 pour les grandes banques. Cette affectation institutionnelle des tâches n'est pas vraiment convaincante, puisque la BCE peut se trouver en conflit d'intérêts latent entre la politique monétaire et la politique de contrôle bancaire, ce qui risquerait de saper à long terme sa réputation de stabilité. Si l'euro était marqué par un taux d'inflation élevé, la part de l'euro dans les réserves monétaires mondiales qui est passée d'environ $17 \%$ en 1998 à $25 \%$ en 2010 - baisserait et porterait atteinte, par là même, à un facteur important de l'utilité économique particulière de la monnaie commune.

Une véritable coordination des politiques économiques nationales dans la zone euro est de fait exclue à moins d'une union politique. Si la zone euro veut exprimer son potentiel d'avantages économiques, il lui faut une union politique incluant les éléments suivants :

- un Europarlement (reste à savoir si le Parlement européen pourra endosser cette fonction) ;

- un eurogouvernement élu par l'Europarlement ; 
- des missions et des dépenses au niveau supranational qui devraient représenter environ $5 \%$ du PIB sur la base des dépenses d'infrastructures et de défense ainsi que des dépenses de soutien à l'innovation et à la formation (Welfens, 2013a) ;

- une eurotaxe à part entière, constituée non seulement d'une taxe modérée sur les transactions financières, mais également d'une imposition des revenus. Il faut également penser aux recettes des taxes environnementales ou des certificats d'émission ;

- il serait possible d'émettre des emprunts communautaires sous forme d'eurobonds, en plus de quoi les gouvernements nationaux pourraient transférer au niveau supranational jusqu'à $20 \%$ de leur dette publique nationale en l'accompagnant du patrimoine correspondant - par exemple autoroutes, ports et voies de chemin de fer.

Une union politique de la zone euro implique des partis organisés au niveau de cette zone. Jamais on n'aurait vécu les tricheries historiques de la Grèce sur son déficit s'il existait - un peu comme aux États-Unis - un parti européen conservateur et un parti européen social-démocrate ainsi qu'un parti libéral, etc., et si le sommet des perspectives de carrière politique s'appelait Bruxelles; le premier ministre conservateur de la Grèce, membre de la zone euro, qui aurait mis en place une fraude au gigantesque déficit, aurait vu sa carrière au sein de son parti prendre fin immédiatement, et n'aurait par conséquent même pas tenté de mettre en place une telle tromperie. C'est pour cela qu'il est au moins aussi important d'avoir des structures européennes de partis que des règles sur les déficits si l'on souhaite que la zone euro reste un modèle de réussite. Une analyse purement économique de la zone euro, en mettant naïvement l'accent sur de nouvelles règles de déficit - ou sur le pacte budgétaire - ne peut qu'être trompeuse.

L'introduction des eurobonds fait baisser la part des dépenses d'intérêt d'environ $0,5 \%$ du PIB dans la zone euro, ce qui devrait constituer une incitation bienvenue à la baisse des impôts à moyen terme. Si, à l'avenir, les États de la zone euro ne se comportent pas conformément aux règles ou se lancent dans le surendettement comme la Grèce, ils feront faillite au sein de l'Union monétaire. Avec un pourcentage raisonnable de dépenses et d'imposition supranationales - on pourrait le réaliser si besoin -, l'Etat en tant qu'institution ne serait pas incapable de fonctionner - comme c'est le cas dans le système actuel. Cela permettrait à la zone euro de tirer d'immenses avantages économiques de l'Union monétaire. L'Europe devrait se trouver des règles simples et claires et des responsabilités identifiables. Du point de vue institutionnel, ce sont les États-Unis, le Canada, la Suisse et l'Australie qui constituent les modèles de l'union politique. Aucun de ces États fédéraux n'aurait l'idée absurde de réaliser un taux de dépenses publiques fédérales de $1 \%$, comme c'est le cas dans l'UE. Les $5 \%$ proposés représenteraient à peine la moitié du taux appliqué en Suisse. De bons points de départ pour augmenter la croissance seraient le déve- 
loppement des technologies de l'information et de la communication ainsi que l'amélioration des perspectives pour les créateurs d'entreprise.

Quelques points de réforme énergiques suffiraient à stabiliser la zone euro, afin de consolider le modèle de l'Union économique et monétaire au sein de l'UE et le modèle de l'économie sociale de marché de la zone euro. S'il existait une union politique de la zone euro, incluant une eurotaxe et une gestion fiscale autonome, la zone euro resterait un acteur stable et de poids, même en période de difficultés internationales. Si le projet d'union politique de la zone euro est retardé et sans cesse renvoyé à plus tard, celle-ci et toute l'UE risquent être marginalisées dans la politique mondiale. Cela sapera en outre la volonté d'autres régions du monde de parvenir à une intégration économique et politique. Voilà qui ne serait dans l'intérêt ni des Européens, ni de l'économie mondiale.

Pour finir, on peut se demander ce qui pourrait maintenant pousser les députés à transférer vers Bruxelles des compétences et des moyens budgétaires. À cela, il n'existe que trois raisons :

- au cœur de la crise de l'euro, les marchés financiers mènent les parlements nationaux par le bout du nez, et le primat de la politique ne pourra être rétabli qu'en donnant plus de pouvoir au niveau supranational ;

- l'ensemble des mesures de fondation d'une union politique de la zone euro peut être organisé conjointement avec l'émission d'eurobonds de manière à ce que la sphère politique bénéficie, au plan national et international, de marges de manœuvre grâce à l'effet de la baisse des taux d'intérêt dans la zone euro ;

- seule une union politique de la zone euro donnera aux États membres une capacité réelle d'action à long terme dans les organisations internationales. La stratégie jusqu'ici mise en œuvre, qui consiste à simplement essayer de prolonger les sièges nationaux au FMI et à la Banque mondiale, a peu de chances de succès.

Il revient à l'Allemagne et à la France, et aux membres fondateurs dans leur ensemble, d'assumer toute leur responsabilité économique dans la crise, et de lancer à temps de nouvelles initiatives bien réfléchies.

Les perspectives économiques à moyen terme pour les pays en crise de la zone euro sont relativement positives, car outre leurs propres efforts de réforme, la reprise économique aux États-Unis les soulage. C'est surtout l'Irlande qui profitera de cet effet, ainsi que la Grande-Bretagne. À son tour, la relance économique dans ces deux pays facilitera la stabilisation en France. Néanmoins, d'importantes réformes restent nécessaires en France comme en Allemagne. Il ne faut pas s'imaginer qu'une amélioration de la conjoncture rendra moins nécessaire la création d'une meilleure architecture institutionnelle dans l'UE et dans la zone euro. 


\section{Annexe 1 - Tableau 3 : répartition du patrimoine dans la zone euro (patrimoines moyen et médian en milliers d'euros par ménage)}

\begin{tabular}{|c|c|c|c|c|c|}
\hline & \begin{tabular}{|l} 
Patrimoines moyen \\
et médian en milliers \\
d'euros par ménage - \\
valeur médiane*
\end{tabular} & $\begin{array}{l}\text { Patrimoines moyen } \\
\text { et médian en milliers } \\
\text { d'euros par ménage - } \\
\text { valeur moyenne }\end{array}$ & \begin{tabular}{|l|} 
Pourcentage des \\
ménages propriétaires \\
immobiliers
\end{tabular} & $\begin{array}{l}\text { Revenu annuel brut } \\
\text { médian, en milliers } \\
\text { d'euros par ménage }\end{array}$ & $\begin{array}{l}\text { Revenu annuel brut } \\
\text { moyen, en milliers } \\
\text { d'euros par ménage }\end{array}$ \\
\hline Luxembourg (2010) & 398 & 710 & 67 & 65 & 84 \\
\hline Belgique (2010) & 206 & 339 & 70 & 34 & 50 \\
\hline Espagne (2008) & 183 & 291 & 83 & 25 & 31 \\
\hline Italie (2010) & 174 & 275 & 69 & 26 & 34 \\
\hline France (2010) & 116 & 233 & 55 & 29 & 37 \\
\hline Pays-Bas (2009) & 104 & 170 & 57 & 41 & 46 \\
\hline Portugal (2010) & 75 & 153 & 72 & 15 & 20 \\
\hline Slovaquie (2010) & 61 & 80 & 90 & 11 & 14 \\
\hline Allemagne (2010) & 51 & 195 & 44 & 33 & 44 \\
\hline Zone euro & 109 & 231 & 60 & 29 & 38 \\
\hline
\end{tabular}

Source : BCE, « The Eurosystem Household Finance and Consumption Survey », Statistic Paper Series, $\mathrm{n}^{\circ}$ 2, avril 2013, p. [n.c.]. * Données les plus récentes disponibles : 2008 à 2010. Hors Estonie et Irlande. Patrimoine après déduction des dettes. La valeur médiane sépare l'ensemble des ménages en deux groupes : $50 \%$ des ménages se situent au-dessus, $50 \%$ en dessous. Remarque : le rapport entre le patrimoine moyen et le patrimoine médian est d'environ 4:1 en Allemagne, de 2:1 dans la zone euro ; le rapport $\beta$ entre le revenu moyen et le revenu médian dépend, dans une distribution lognormale (qui devrait être pertinente), exclusivement de la dispersion $\mathrm{S}$ des patrimoines : $\mathrm{d} \ln \beta / \mathrm{dS}=1$. Si l'on suppose par ailleurs qu'une répartition très inégale des patrimoines entraîne également un pourcentage élevé de fraude fiscale internationale et de dissimulation de patrimoine (dans un sondage, les fraudeurs n'indiqueront pas le patrimoine non déclaré qu'ils possèdent à l'étranger), il faut alors considérer avec prudence les chiffres venant notamment d'Allemagne et d'Autriche.

\section{Annexe 2 - Fragmentation des partis en Allemagne}

La formation du parti Alternative für Deutschland (AfD), dont le congrès fondateur a eu lieu en avril 2013 à Berlin, a fait entrer en scène un parti eurosceptique. Cela ne dénote pas seulement une fragmentation du paysage politique sur l'aile droite ou conservatrice, mais illustre probablement aussi le goût parfois problématique de l'Allemagne pour la voie particulière (Sonderweg). On n'a jamais vu un pays jouer un rôle déterminant dans la fondation d'une union monétaire puis amorcer un mouvement de retrait. Ce nouveau parti est encore petit, mais il témoigne depuis le début d'une perte totale du sens des réalités, ce qui n'est pas forcément le terreau naturel du succès politique, mais on ne peut pas l'exclure : sur le site Internet du parti, on peut lire dès les premières phrases (en date du 14/04/2013) que "la République fédérale d'Allemagne traverse la plus grave crise de son histoire »; cette affirmation est absurde et bien éloignée de la réalité, probablement aussi représentative de la situation réelle que la légende du coup de poignard dans le dos répandue par les partis de droite pendant la République de Weimar. On ne peut que souhaiter « $\operatorname{Bad} \operatorname{Luck}(\mathrm{e})$ » à ce nouveau parti, mais naturellement, le verdict viendra des urnes. 
La crise de l'euro, tout comme la crise bancaire transatlantique, est un problème déstabilisant non seulement du point de vue économique, mais aussi au plan politique. En effet, pour surmonter la crise bancaire comme la crise de l'euro, il faut prendre des mesures non conventionnelles et trouver des solutions pragmatiques bien éloignées des principes politiques et économiques couramment appliqués et acceptés par le plus grand nombre, ce qui provoque une vive incertitude parmi la population. C'est là l'un des principaux problèmes de l'organisation institutionnelle insuffisante de la zone euro, comme lors de la crise bancaire. Si l'État, pour sauver les banques ou stabiliser l'économie de marché, doit enfreindre des principes éprouvés tels que la souveraineté et des notions acceptées de responsabilité, il sape d'une manière générale l'acceptation des règles et peut-être l'économie de marché elle-même. En Allemagne, avec ses écoles de politique réglementaire et ordolibérales influentes parmi les économistes, cela conduit, qui plus est dans le contexte des foyers de crise dans la zone euro, à la recherche de nouvelles assurances en matière de politique réglementaire. Le parti AfD répond apparemment à ce besoin et l'on retrouve de nombreux économistes engagés dans la politique réglementaire parmi ses soutiens les plus proches; il semble par ailleurs compter également des membres au sens national particulièrement développé, qui jugent approprié de porter une écharpe aux couleurs allemandes en bandoulière ou de hisser le drapeau le jour du congrès du parti. Comme on trouve aussi certainement à l'AfD de riches électeurs conservateurs déçus, la situation financière du parti doit être plutôt satisfaisante.

\section{Annexe 3}

Le tableau qui suit est la première évaluation par l'EIIW de la nouvelle base de données ÉVA de l'OCDE et de ses analyses d'entrées-sorties, qui permettent pour la première fois de représenter, outre le traditionnel ratio exportations/PIB, le rapport entre les « exportations à valeur ajoutée » (exportations hors importations de prestations préalables) et le produit intérieur brut. Dans une économie mondialisée, les exportations de produits de grande valeur incluent typiquement des produits semi-finis sophistiqués importés, de sorte qu'en principe, les exportations tout comme les exportations à valeur ajoutée seront plus importantes dans les petits pays que dans les grands. La même réflexion s'applique aux importations et aux importations à valeur ajoutée. La différence entre les deux indicateurs d'exportations est plus forte en Allemagne qu'en France et dans de nombreux autres pays. Pour les importations, on note également une différence légèrement plus grande en Allemagne qu'en France. De plus, selon les derniers chiffres des tableaux d'entrées-sorties (non indiqués ici), sur la base des exportations à valeur ajoutée, la France n'est plus le premier destinataire des exportations allemandes, elle est détrônée par les États-Unis : apparemment, il y a moins de produits semi-finis américains dans les exportations allemandes à destination des USA que de produits semi-finis français dans les exportations allemandes à destination de l'Hexagone. 


\section{Tableau 4 : Exportations brutes et exportations en valeur ajoutée (en pourcentage du PIB)}

\begin{tabular}{|c|c|c|c|c|c|c|c|c|c|}
\hline \multirow{2}{*}{ Pays } & \multicolumn{3}{|c|}{ X_GDP } & \multicolumn{3}{|c|}{ XVA_GDP } & \multicolumn{3}{|c|}{ DIFFÉRENCE } \\
\hline & 2005 & 2008 & 2009 & 2005 & 2008 & 2009 & 2005 & 2008 & 2009 \\
\hline Brésil & 14,1 & 12,6 & 10,3 & 12,4 & 11,2 & 9.4 & 1.7 & 1.4 & 0.9 \\
\hline États-Unis & 8.8 & 11,3 & 10,0 & 7.8 & 9.6 & 8,9 & 1.0 & 1.7 & 1,1 \\
\hline Japon & 13,7 & 17.0 & 12,3 & 11.9 & 13.7 & 10.5 & 1.8 & 3,3 & 1.8 \\
\hline Fédération de Russie & 33,3 & 29.7 & 26.6 & 30,1 & 27.0 & 24,7 & 3,2 & 2,7 & 1.9 \\
\hline Australie & 18.4 & 21.4 & 18.7 & 16,2 & 18.6 & 16.4 & 2,3 & 2,8 & 2,3 \\
\hline Reste du monde & 45,7 & 45,0 & 38,3 & 39.5 & 40.6 & 35,3 & 6.2 & 4,4 & 3,0 \\
\hline Indonésie & 33,2 & 29.5 & 24,0 & 27,2 & 24,1 & 20.5 & 6,0 & 5.4 & 3.5 \\
\hline Espagne & 21.0 & 21,1 & 19.2 & 15.6 & 16.3 & 15.5 & 5.5 & 4.8 & 3.6 \\
\hline Italie & 22.6 & 24,4 & 20,3 & 17,2 & 19.1 & 16.7 & 5.5 & 5.2 & 3.6 \\
\hline Grèce & 21.8 & 22,3 & 17,7 & 16.5 & 16.5 & 13,7 & 5,3 & 5.7 & 4,0 \\
\hline Royaume-Uni & 22,4 & 25,2 & 24,4 & 18.7 & 20.6 & 20,3 & 3.7 & 4,6 & 4,1 \\
\hline Afrique du Sud & 25.7 & 33.5 & 25.8 & 21,2 & 25,7 & 21.5 & 4,5 & 7.8 & 4.4 \\
\hline Inde & 18,3 & 22,6 & 18.8 & 14,6 & 17,0 & 14,4 & 3.7 & 5,6 & 4,4 \\
\hline Turquie & 20.1 & 22,2 & 21.7 & 16.1 & 16.4 & 17,1 & 4,0 & 5.8 & 4,6 \\
\hline Nouvelle-Zélande & 26,6 & 29.9 & 27,1 & 21.0 & 23,0 & 21.9 & 5,5 & 6.9 & 5.2 \\
\hline France & 24,4 & 25,0 & 21,6 & 18,2 & 18.0 & 16,1 & 6.2 & 7,0 & 5,6 \\
\hline Canada & 36,1 & 32,6 & 26,7 & 26.7 & 25,5 & 21,1 & 9.5 & 7,1 & 5.6 \\
\hline Norvège & 42,8 & 46.6 & 39,9 & 37.0 & 40.0 & 34,2 & 5.8 & 6.6 & 5.7 \\
\hline Chili & 41,3 & 44,7 & 39.0 & 34,0 & 35,2 & 31.7 & 7.3 & 9.5 & 7.2 \\
\hline Chine & 36,5 & 34,8 & 27.5 & 23,3 & 23,7 & 19.6 & 13,2 & 11,1 & 7.8 \\
\hline Portugal & 256 & 31.0 & 26.7 & 18.7 & 19.9 & 18,3 & 6.9 & 11,1 & 8.4 \\
\hline Mexique & 26,5 & 27.6 & 27,0 & 18.0 & 18,8 & 18,6 & 8.5 & 8.8 & 8.5 \\
\hline Allemagne & 33,5 & 38.6 & 33.6 & 25,2 & 28,4 & 25,1 & 8,3 & 10,2 & 8,5 \\
\hline Autriche & 43,9 & 48,1 & 39.4 & 29.9 & 32,2 & 29.8 & 14,0 & 159 & 9.6 \\
\hline Pologne & 35,6 & 36.9 & 36.8 & 24.5 & 25.7 & 26.6 & 11,1 & 11.2 & 10.2 \\
\hline Israël & 40,5 & 38.1 & 32,8 & 24,8 & 23.6 & 22,3 & 15.7 & 14,5 & 10.5 \\
\hline Finlande & 39.1 & 43,3 & 35.0 & 26,3 & 27,9 & 23,7 & 12,7 & 15.4 & 11.3 \\
\hline Danemark & 40,2 & 44,5 & 39.1 & 27.9 & 30.2 & 27,1 & 12,2 & 14,3 & 11,9 \\
\hline Suède & 43,3 & 47.5 & 43,0 & 29.9 & 31.4 & 29.1 & 13,4 & 16.1 & 13,9 \\
\hline Slovénie & 51,4 & 53,4 & 47,1 & 32,2 & 34,7 & 32,7 & 19.2 & 18.7 & 14,4 \\
\hline Pays-Bas & 48,7 & 47.7 & 42,7 & 34,1 & 30,9 & 28,3 & 14,5 & 16.8 & 14,5 \\
\hline Suisse & 47.6 & 54.8 & 50,3 & 32.7 & 36.8 & 34.9 & 15.0 & 18.0 & 15,3 \\
\hline Belgique & 54,8 & 56,1 & 48,7 & 32,2 & 34,9 & 33,1 & 22,7 & 21,2 & 15,6 \\
\hline Islande & 30.6 & 42,8 & 50,8 & 18.0 & 26.9 & 33,7 & 12,6 & 15.8 & 17,1 \\
\hline Corée & 38.6 & 50.6 & 48,0 & 24,5 & 28.6 & 29,0 & 14,1 & 22,0 & 19.0 \\
\hline République tchèque & 59,2 & 57.8 & 55.4 & 35.7 & 35.4 & 34,0 & 23,6 & 22,4 & 21.4 \\
\hline Estonie & 74,8 & 67,3 & 61,0 & 36,6 & 39.2 & 39,3 & 38,2 & 28,0 & 21.6 \\
\hline Hongrie & 56,6 & 68.2 & 65,0 & 29.1 & 37.3 & 38,5 & 27,5 & 30,9 & 26.5 \\
\hline République de Slovaquie & 71.4 & 77.5 & 66.1 & 37.2 & 39.7 & 36.5 & 34,2 & 37.7 & 29.6 \\
\hline Irlande & 75,4 & 75.4 & 82,7 & 40,6 & 45.9 & 49,3 & 34,9 & 29.4 & 33,4 \\
\hline Luxembourg & 151,4 & 169.7 & 156.4 & 61,1 & 65,5 & 61.7 & 90,3 & 104,2 & 94,7 \\
\hline
\end{tabular}

Source : base ÉVA de l'OCDE. Composantes des exportations brutes : 1) valeur ajoutée domestique directe contenue dans les exportations brutes ; 2) valeur ajoutée domestique indirecte contenue dans les exportations brutes (provenant des intrants domestiques) ; 3) valeur ajoutée domestique réimportée contenue dans les exportations brutes ; 4) valeur ajoutée étrangère contenue dans les exportations brutes. X_GDP : exportations brutes en pourcentage du PIB (1-4); XVA_GDP : valeur ajoutée domestique contenue dans les exportations brutes en pourcentage du PIB (1-3). 


\section{Tableau 5 : Importations brutes et importations en valeur ajoutée (en pourcentage du PIB)}

\begin{tabular}{|c|c|c|c|c|c|c|c|c|c|}
\hline \multirow{2}{*}{ Pays } & \multicolumn{3}{|c|}{ J_GDP } & \multicolumn{3}{|c|}{ JVA_GDP } & \multicolumn{3}{|c|}{ DIFFÉRENCE } \\
\hline & 2005 & 2008 & 2009 & 2005 & 2008 & 2009 & 2005 & 2008 & 2009 \\
\hline Japon & 12,2 & 16.7 & 11.9 & 9.6 & 13.4 & 9.7 & 2.6 & 3,3 & 2,2 \\
\hline Brésil & 10,6 & 12,2 & 10,2 & 8,0 & 9,2 & 8.0 & 2,6 & 3.0 & 2,2 \\
\hline États-Unis & 14.4 & 16,2 & 12,8 & 10,6 & 12,0 & 9.6 & 3.9 & 4,2 & 3,2 \\
\hline Fédération de Russie & 20,2 & 20.7 & 19,3 & 15,9 & 15.6 & 15,4 & 4,3 & 5.1 & 4,0 \\
\hline Reste du monde & 43,2 & 45,5 & 43.8 & 38,9 & 40,0 & 39.5 & 4,3 & 5.5 & 4,3 \\
\hline Italie & 22,5 & 24,8 & 20.5 & 17,6 & 18.9 & 16.1 & 4,9 & 5,9 & 4,3 \\
\hline Inde & 20,6 & 27,1 & 23,3 & 16,6 & 21,1 & 18.8 & 4,0 & 5.9 & 4,5 \\
\hline Espagne & 26.1 & 26.4 & 21,1 & 19.5 & 19.9 & 16.4 & 6.6 & 6.4 & 4.7 \\
\hline Australie & 19.8 & 20.7 & 19,0 & 14,4 & 14,6 & 14,0 & 5.4 & 6.0 & 5,0 \\
\hline Turquie & 23,0 & 25.9 & 22,4 & 17,3 & 19.5 & 17,3 & 5.6 & 6.5 & 5,2 \\
\hline France & 24,9 & 26,9 & 23,3 & 18,3 & 20,2 & 18,0 & 6.6 & 6.7 & 5,3 \\
\hline Canada & 32,2 & 31,0 & 28.2 & 26,1 & 24,0 & 22.7 & 6.1 & 7,0 & 5,5 \\
\hline Indonésie & 29,1 & 28.4 & 21,1 & 20,8 & 19.7 & 15,3 & 8,3 & 8.7 & 5,8 \\
\hline Nouvelle-Zélande & 28.6 & 31,0 & 25.6 & 22,0 & 23,0 & 19.7 & 6.5 & 8.0 & 5,9 \\
\hline Chine & 31,0 & 27,1 & 22,1 & 22,1 & 18.9 & 16.1 & 9.0 & 8.1 & 6,0 \\
\hline Chili & 32,6 & 40,8 & 31,0 & 26.5 & 32,5 & 24.8 & 6.1 & 8.3 & 6.2 \\
\hline Mexique & 27,8 & 29.7 & 28.3 & 22,1 & 22,1 & 22,0 & 5,7 & 7,5 & 6,3 \\
\hline Grèce & 30,4 & 35.5 & 28,2 & 23,2 & 25.8 & 21.8 & 7,2 & 8.8 & 6.4 \\
\hline Royaume-Uni & 25,4 & 27.6 & 26,0 & 19,0 & 20,3 & 19.5 & 6.4 & 7,3 & 6,5 \\
\hline Finlande & 34,9 & 39.5 & 33,2 & 25,7 & 30.8 & 26.6 & 9.2 & 8.7 & 6.7 \\
\hline Norvège & 26,5 & 28,0 & 26.6 & 19.4 & 20,6 & 19.9 & 7,1 & 7.4 & 6.7 \\
\hline Israël & 40,4 & 39,0 & 30,3 & 31.2 & 29.8 & 23,5 & 9.2 & 9,2 & 6.8 \\
\hline Afrique du Sud & 25,2 & 35,2 & 25.7 & 18.2 & 24,4 & 18.7 & 7,0 & 10,8 & 7,0 \\
\hline Allemagne & 28,1 & 32,1 & 28,3 & 20,2 & 23,3 & 21,3 & 7,9 & 8.8 & 7,1 \\
\hline Portugal & 33.9 & 40,2 & 33.5 & 25.4 & 30.5 & 26,3 & 8.5 & 9.7 & 7,2 \\
\hline Pays-Bas & 35.1 & 39.4 & 35.9 & 26,8 & 30,3 & 28,3 & 8.2 & 9,1 & 7,6 \\
\hline Danemark & 35,2 & 41,2 & 35,1 & 27,0 & 31,0 & 27,2 & 8.2 & 10,2 & 7,9 \\
\hline Irlande & 63,9 & 66,3 & 67.8 & 54,4 & 56.8 & 59.7 & 9.5 & 9.6 & 8,1 \\
\hline Islande & 42,2 & 45,2 & 42,5 & 32,0 & 34,8 & 34,2 & 10,2 & 10.4 & 8,3 \\
\hline Autriche & 39.7 & 42,2 & 34,6 & 29,0 & 31,0 & 25.7 & 10.7 & 11,2 & 8,9 \\
\hline Suède & 35.6 & 40,9 & 36.6 & 26,3 & 30,0 & 27,7 & 9.3 & 10,9 & 9,0 \\
\hline Corée & 35,8 & 51.4 & 44,2 & 27,2 & 39.7 & 34,9 & 8.6 & 11.7 & 9,3 \\
\hline Pologne & 36,1 & 40,0 & 36,3 & 26,3 & 29,0 & 26,9 & 9.7 & 11,0 & 9,4 \\
\hline Belgique & 50,8 & 54.7 & 45.7 & 37,9 & 40,8 & 35,2 & 13,0 & 13,9 & 10,4 \\
\hline Suisse & 40,9 & 43.7 & 39.4 & 29,1 & 30,6 & 28.4 & 11.8 & 13,0 & 11,0 \\
\hline Slovénie & 51,4 & 55,8 & 45,3 & 36,9 & 39,6 & 33.8 & 14,5 & 16.1 & 11,5 \\
\hline République tchèque & 56,3 & 55,2 & 51,2 & 41,2 & 39,2 & 38,5 & 15,1 & 16,0 & 12.7 \\
\hline Estonie & 80.8 & 70,8 & 55,0 & 58.2 & 51,3 & 41,0 & 22,6 & 19.5 & 139 \\
\hline Hongrie & 58,2 & 67,2 & 60,0 & 41.6 & 48,4 & 44,6 & 16.6 & 18.8 & 15.4 \\
\hline Répriblique deSlovacque & 75,4 & 79,2 & 66.4 & 54,0 & 55.9 & 48,0 & 21,3 & 23,3 & 18,5 \\
\hline Luxembourg & 126,5 & 138,4 & 126,0 & 99.5 & 109.8 & 103,1 & 27,0 & 28.6 & 23,0 \\
\hline
\end{tabular}

Source : base ÉVA de l'OCDE. J_GDP : importations brutes (en pourcentage du PIB) ; JVA_GDP : valeur ajoutée étrangère contenue dāns les importations brutes (en pourcentage du PIB). 


\section{Indications bibliographiques}

Artus P., Gravet I., La Crise de l'Euro - Comprendre les causes, en sortir par de nouvelles institutions, Armand Colin, Paris, 2012

BCE, «The Eurosystem Household Finance and Consumption Survey », Statistic Paper Series, $n^{\circ} 2$, avril 2013, p. [n.c.]

Bertelsmann STIFTUNG, Wirtschaftliche Folgen eines Euro-Austritts der südeuropäischen Mitgliedsstaaten, Gütersloh, 2012 (Gutachten der Prognos AG)

DEUTSChE BundeSBANK, « Yields on Bunds under Safe Haven Effect », Monthly Report, octobre 2010, p. 30-31

FMI, Greece: Second Review Under the Stand-By Arrangement - Staff Report; Press Release on the Executive Board Discussion; and Statement by the Executive Director for Greece, Country Report No. 10/372, Washington DC, 2010

FMI, Euro Area Policies - 2012 Article IV Consultation, Washington DC, 2012

HenniCKe P., Welfens P.J.J., Energiewende nach Fukushima, Oekom Verlag, Munich, 2012

IWD, « Investitionsgüter führen zu Überschüssen », iw-dienst, vol. 39, n 15, 2013, p. 3

Kauffmann P., Uterwedde H., «Deutschland, Frankreich und die Euro-Krise », Aus Politik und Zeitgeschichte, vol. 43, 2010, p. 13-19

SVR, Jahresgutachten 2011/2012, Sachverständigenrat zur Begutachtung der gesamtwirtschaftlichen Entwicklung/Statistisches Bundesamt, Wiesbaden, 2011

WeLFENS P.J.J., Volkswirtschaftliche Auswirkungen der Euro-Staatsschuldenkrise und neue InstrumentE der Staatsfinanzierung in der EU [Avis rendu pour le Comité financier du Bundestag, séance du 9 mai 2012], [Bundestag], Berlin, 2012

Welfens P.J.J., Überwindung der Eurokrise und Stabilisierungsoptionen der Wirtschaftspolitik: Perspektiven für Nordrhein-Westfalen, Deutschland und Europa [Rapport rédigé pour le compte de la ministre pour les Affaires fédérales, l'Europe et les Médias du Land de Rhénanie du Nord-Westphalie] [en ligne], EIIW, Wuppertal, 2013a. Disponible sur : http://www.eiiw.eu/fileadmin/eiiw/Daten/Presse/2013/MIT_COVER_euroLandes regierungNRWwelfens2012.pdf [consulté le 21/01/2015]

Welfens P.J.J., Nachhaltige Überwindung der Euro-Krise - Marktdynamik und Politikoptionen [Étude réalisée pour la Fondation Hans Böckler], Lucius \& Lucius, Stuttgart, 2013 b. 


\section{OUVERTURE DE LA TABLE RONDE}

Rainer Klump : En tant que professeur d'économie à l'université Goethe de Francfort, je me réjouis d'animer cette table ronde de haut niveau, qui nous donnera l'occasion de revenir sur plusieurs thèmes abordés au cours de cette journée d'étude : les perspectives différentes de l'Allemagne et de la France en matière de stabilité monétaire, l'autonomie de la Banque centrale européenne $(\mathrm{BCE})$, ainsi que son indépendance et sa flexibilité.

Permettez-moi de faire une remarque en tant que représentant d'une université baptisée du nom de Goethe. Ce matin, Manfred Neumann a merveilleusement cité la lettre de la Reichsbank à Hitler, dans laquelle est mentionné le papier-monnaie. L'auteur y explique que la multiplication des billets ne peut pas entraîner un développement de l'économie réelle. Pour ceux qui connaissent la littérature allemande, cela renvoie à des éléments présentés de manière très intéressante par Goethe dans la deuxième partie de Faust. Faust II commence par la visite de Faust et Méphisto à un empereur dont le royaume est ruiné. Méphisto a l'idée géniale de proposer à l'empereur d'écrire des billets signés de son nom et de les distribuer au peuple. Il s'ensuit un bref boom économique... puis une grave crise survient, la révolution éclate et tout va de mal en pis! Ce thème est donc profondément ancré dans la littérature nationale allemande; à l'époque de Goethe, il s'appuie sur l'expérience française - aussi bien les expérimentations de John Law sous la Régence que l'inflation liée à la crise des assignats pendant la Révolution. On peut y trouver des imbrications franco-allemandes aux implications intéressantes en matière de politique monétaire.

\section{EXPOSÉS DES INTERVENANTS}

Rainer Klump : J'aimerais maintenant donner la parole à Luc Moulin. Le membre le plus expérimenté de cette table ronde a effectué une carrière d'ingénieur dans l'industrie métallurgique française. Il est donc en mesure d'apporter un point de vue très intéressant à cette discussion. De plus, il a été membre de l'Association pour l'union monétaire de l'Europe (AUME), un lobby qui a milité pour la mise en œuvre de l'Union monétaire. Son objectif atteint, cette association a été dissoute en 2002. Il reste intéressant d'entendre Luc Moulin nous redire son expérience du cheminement vers l'Union monétaire et du fonctionnement de cette union.

Luc Moulin : Dans un souci historique, il faut se placer dans le contexte d'une époque d'avant Maastricht. J'étais moi-même fabriquant de zirconium et de titane (en particulier le zirconium pour le combustible nucléaire). Parmi mes clients figurait un très bon ami, responsable de la partie combustible nucléaire de Siemens. Un jour, après une réunion à Erlangen, il m'a invité dans sa maison de famille en Haute-Franconie près de Bamberg, puis m'a amené devant le ri- 
deau de fer. Alors que je pensais être convaincu de savoir ce qu'était le rideau de fer, il m'a dit: "Tu vois Luc, le village qu'il y a juste en face? Tous mes cousins y habitent et je ne peux pas les voir... » Ce fut pour moi un choc! Peu après la chute du mur de Berlin, je me suis empressé de l'appeler et il m'a confié : « Eh bien maintenant, nous allons pouvoir faire l'Europe! »

À l'époque, en plus des incertitudes géopolitiques et des expériences monétaires cuisantes des années 1980 (c'est-à-dire les trois dévaluations réalisées en l'espace de 18 mois par les gouvernements sous Mitterrand), il était très difficile pour les industriels d'avoir une vision à long terme, ce qui est pourtant indispensable pour les investissements. Les dévaluations dites «compétitives 》 de divers pays avaient généralement, pour les industries de base, un impact supérieur au taux de marge sur les ventes. C'est dans ce contexte qu'un groupe de grands patrons européens a décidé de réagir en créant l'AUME, qui visait une action spécifique et coordonnée des entreprises pour acter la construction monétaire de l'Europe. L'association a dès lors contribué au travail en amont du traité de Maastricht. Après le traité de Maastricht, les bonnes intentions politiques se sont malheureusement enlisées...

Au début des années 1990, j'ai changé de secteur industriel pour travailler avec un sous-traitant spécialisé dans la fabrication d'éléments composant les turbines d'avions, fournisseur de Pratt \& Whitney, General Electric, RollsRoyce et Safran (ex-Snecma). Mon expérience personnelle témoigne de ces années d'incertitude jusqu'au sommet de Madrid (décembre 1995). Étant donné que le producteur doit s'engager dans des procédures de qualification longues et coûteuses, il se pose des questions monétaires : quel sera le coût en dollars des achats de métaux pour les cinq prochaines années? Quel sera le taux franc/dollar ensuite pour mes ventes (car je vends le plus souvent en dollars, mais mes coûts de production sont en francs) ? Quel sera le taux franc/sterling vis-à-vis de mon concurrent anglais ?... La gestion court-termiste est redoutable pour l'industriel! Par conséquent, il fallait concrétiser Maastricht. Les 600 sociétés membres de l'AUME ont réalisé un travail remarquable dans cette période pour aboutir à ce qu'on décide enfin, au sommet de Madrid, de la date butoir du $1^{\text {er }}$ janvier 1999 pour le démarrage de l'euro. Jacques Delors nous a remerciés de notre action par une lettre dans laquelle il a écrit : "Si vous n'aviez pas existé, il aurait fallu vous inventer! »

Dans le cadre de cette journée, je tiens à souligner l'intensité du dialogue entre les entrepreneurs allemands et français pour promouvoir à l'époque l'Union économique et monétaire. Les soutiens les plus actifs ont été, du côté allemand : Daimler, Deutsche Bank, Dresdner Bank, Robert Bosch, Siemens et Volkswagen, et, du côté français : Alcatel, la BNP, Cap Gemini, le CCF, Deloitte, LVMH, Paribas, Pathé, Renault et Total. Nous leur devons beaucoup! Soyons lucides face aux détracteurs actuels : pour les entrepreneurs, les acquis dus à l'euro sont énormes. La perte de l'euro serait une catastrophe, pour ne pas dire un désastre. 
Le temps a passé ; il a montré que l'Union économique et monétaire a été réalisée de manière imparfaite. En effet, les politiques de l'époque ont omis de tenir compte du mot « économique » pour se concentrer sur l'aspect monétaire. On vient de comprendre seulement maintenant le travail qu'il restait à fournir pour l'élaboration d'une union bancaire (qui reste toutefois éloignée d'une gouvernance économique davantage empreinte de fédéralisme).

Même si l'euro a apporté un progrès majeur, je voudrais rappeler quelques évidences présentes dans l'esprit des entrepreneurs, qu'ils soient allemands ou français :

- une entreprise ne peut pas être durablement en perte. Or l'État français budgète des pertes depuis plus de trente ans. Une entreprise peut emprunter pour investir, pas pour fonctionner! C'est pourtant ce que l'on constate actuellement ;

- il faut des conditions et des talents exceptionnels pour être compétitif en travaillant $1600 \mathrm{~h} /$ an quand le voisin travaille $1800 \mathrm{~h} / \mathrm{an}$, voire davantage ;

- il est encore plus difficile d'être compétitif quand les charges fiscales sont supérieures à celles de vos concurrents. Indépendamment du contexte français spécifique, la seule voie d'avenir pour tous est d'investir dans des innovations performantes. Si un trop grand nombre d'entreprises sont en perte de compétitivité, cela se traduit par le déficit de la balance commerciale, qui est ruineux à terme pour le pays. Dès lors qu'il n'y a plus d'échappatoire par la dévaluation, il faut réduire les dépenses publiques de fonctionnement pour pouvoir investir. Ceci ne pourra se faire probablement que dans un cadre plus fédéral sur le plan économique.

En conclusion, il n'y a aucune divergence entre entrepreneurs allemands et français sur ces choix économiques. Nous avons les mêmes convictions, mais une question demeure : quand nos dirigeants politiques auront-ils le courage de dire la vérité ?

Rainer Klump : Merci à Luc Moulin. Nous reviendrons certainement sur cette question. Je vais maintenant passer la parole à Christian Kastrop. Christian Kastrop, du ministère fédéral des Finances à Berlin, a étudié à l'université de Cologne. C'est l'une des villes dans lesquelles l'économie sociale de marché est née et a été discutée. Savoir si c'est encore le cas aujourd'hui est peut-être un autre thème qui serait digne d'intérêt. Christian Kastrop a exercé différentes fonctions au ministère fédéral des Finances et notamment participé de près aux étapes de développement du Pacte de stabilité et de croissance. Aujourd'hui, il est directeur adjoint de la division Questions économiques générales et directeur de la section Finances publiques, macroéconomie et recherche.

Christian Kastrop : Merci beaucoup, je suis très heureux d'être ici aujourd'hui. Les interventions précédentes montrent vraiment très bien combien 
l'Allemagne et la France ont encore du chemin à parcourir pour parvenir à une formulation commune de leurs intérêts. C'est donc une bonne chose que nous tentions, lors de manifestations comme celle-ci, de trouver un terrain d'entente sur une base plus large.

Personnellement, je ne fais pas partie des fidèles adeptes de concepts économiques donnés en Allemagne, mais plutôt de ceux qui recherchent des instruments permettant d'apporter des solutions à des problèmes concrets. Toutefois, je constate que parmi toutes les recettes visant à surmonter la crise financière et économique que vient d'énoncer le professeur Kauffmann, il n'y en a pas plus de $10 \%$ avec lesquelles je suis d'accord.

Cela est dû aussi bien aux concepts économiques proposés - que je considère en partie comme douteux, contre-productifs à long terme et politiquement prématurés - qu'à la philosophie sous-jacente selon laquelle c'est toujours le monde politique qui «tire les ficelles », c'est-à-dire qu'il est capable de diriger l'économie à sa guise. Dans ce domaine, les Allemands pensent différemment. Je ne crois ni à la compétence universelle des marchés pour résoudre les problèmes, ni à celle de la sphère politique. Les instruments de pilotage sont toujours insuffisants, souvent délicats stratégiquement; ils s'épuisent au fil du temps, ou bien les conditions de leur utilisation disparaissent tout simplement.

C'est pourquoi, du point de vue allemand, nous ne devons pas résoudre la crise « à tout prix », mais de manière à ce que tous les systèmes de pilotage envoient les incitations économiques adéquates aux acteurs privés et publics, afin d'avoir un effet préventif sur la prochaine crise. C'est un raisonnement typiquement allemand qui, chez de nombreux collègues français, ne déclenche que des hochements de tête dubitatifs face à de tels atermoiements.

Il reste donc beaucoup à faire. Nous devons simplement continuer à parler de ces conceptions économiques concrètes et des structures qui les justifient, nous devons échanger nos points de vue, et nous devons voir que nous progressons ensemble, parce que je suis intimement convaincu que le moteur franco-allemand doit faire avancer cette union et peut, dans certaines limites, la diriger. Il y a un point sur lequel je suis tout à fait d'accord avec Pascal Kauffmann : c'est le fait qu'il nous manque un concept économique d'ensemble raisonnable, ce à quoi l'on pourrait précisément s'attendre de la part des deux plus grands pays de l'UE.

À titre purement personnel, j'admets d'ailleurs que l'Allemagne porte à ce sujet une lourde responsabilité. Ces deux dernières années, nous avons agi de manière bien trop parcellaire, sans stratégie d'ensemble, en préférant dire ce que nous ne voulions pas plutôt que de proposer nos propres stratégies. D'autre part, jusqu'à présent nous avons toujours fini par trouver un compromis, qui me semble aller trop loin par endroits du point de vue économique. Mais c'est aussi un effet de cette politique des petits pas.

Il existe des différences dans la manière de comprendre et d'évaluer les risques des «mesures particulières » de politique monétaire, c'est-à-dire sur les opérations monétaires sur titres (outright monetary transactions, OMT), les 
opérations de refinancement à plus long terme (longer-term refinancing operations, LTROs), etc., sur la macropolitique, sur les eurobonds, sur le degré nécessaire de solidité des finances publiques et sa dynamique. Il existe également des points de vue différents sur l'organisation de l'UE après la crise, par exemple sur la conception du niveau fédéral nécessaire, de ses institutions, de ses règles fiscales, de son budget et de ses structures décisionnelles exécutives et législatives. Dans ce domaine, l'Allemagne et la France ont suivi des chemins totalement différents depuis le $\mathrm{XI}^{\mathrm{e}}$ siècle, dont l'héritage nous marque encore aujourd'hui malgré la mondialisation.

Désormais, la question est la suivante : comment peut-on assurer une direction commune sur cette base, que peut-on accomplir en commun malgré toutes nos divergences? Je suis fermement convaincu que nous avons besoin de l'euro et d'une perspective commune pour cette monnaie. Toutefois, les travaux de convergence nécessaires pour parvenir à une monnaie optimale prendront du temps.

Regardons la réalité en face : nous ne pourrons pas accomplir à court terme tout ce dont une union monétaire aurait idéalement besoin pour surmonter la crise, pour organiser ensuite un système préventif - ce qui inclut tout un éventail de domaines politiques - et aussi pour relever les défis mondiaux auxquels nous sommes confrontés sans mettre en danger l'idée européenne elle-même. Nous nous retrouvons ainsi dans une sorte d'entre-deux : aujourd'hui, il n'est pas réaliste d'envisager le Big Bang de l'intégration; ce sont les «peines de la plaine » qui nous attendent, comme disait Brecht ${ }^{2}$. L'utopie européenne, c'està-dire la création des États-Unis d'Europe, doit attendre. En économie, on appelle cela le «second best ", ou optimum de second rang. Mais même cette voie est exigeante, si l'on veut concilier des traditions différentes pour créer un système quelque peu cohérent, qui envoie au moins en principe les signaux économiques adéquats.

L'ancrage de ce débat est important. Mon prédécesseur vient de faire référence à la guerre froide, qui a été un élément d'identification important. Aujourd'hui, nous avons besoin d'un nouvel ancrage pour l'Europe. Toutefois, cela résulte moins de la résolution de nos problèmes internes que des défis mondiaux pour lesquels l'Europe doit s'armer. À ce sujet, je suis tout à fait convaincu qu'aucun pays ne peut y parvenir seul, même l'Allemagne. C'est un facteur crucial sur lequel j'insiste aussi dans toutes les discussions en Allemagne.

Comment combler un éventuel «delivery gap » entre ce qui est effectivement nécessaire ou souhaitable et ce qui est réalisable à moyen terme ? De

\footnotetext{
${ }^{2}$ Extrait du poème Wahrnehmung de Bertolt Brecht (1949). Die Mühen der Berge haben wir hinter uns, vor uns liegen die Mühen der Ebenen. Traduction française : Nous avons derrière nous les peines de la montagne, Les peines de la plaine sont devant nous. (Constatation, extrait de Manuel pour les habitants des villes Poèmes, L'Arche Éditeur, Paris, 2006), NdT.
} 
mon point de vue, cela est déjà extrêmement difficile. J'aimerais à ce sujet jeter rapidement un coup de projecteur sur la théorie du fédéralisme.

Quels sont les biens publics centraux au niveau européen ? La réponse à cette question en résoudrait bien d'autres, par exemple celle du budget fédéral centralisé que beaucoup réclament. Dans ce contexte, il est important d'aborder la question des effets de spillover, c'est-à-dire des répercussions des différentes politiques nationales sur les autres pays. La théorie du fédéralisme inclut également la question de la subsidiarité et des diverses préférences au niveau national, que l'on retrouve dans de nombreux domaines, pour l'organisation de l'Union monétaire, mais surtout dans le domaine de la politique économique et financière ainsi que dans les conceptions divergentes de l'État-providence.

Sans une définition commune, pas de fédération possible. On revient alors à la question: comment peut-on favoriser la "construction» de préférences communes? Encore une fois, je ne crois pas à un grand saut forcé vers l'intégration. Je crois que nous avons besoin d'un modèle de politique générale capable de fonctionner à moyen terme même avec des préférences différentes, tout en fournissant des résultats économiques réels.

C'est précisément parce que l'union politique et fiscale ne peut être tirée d'un chapeau - tout comme la stabilité des engagements politiques nationaux par un gouvernement central - que d'un point de vue allemand, il faut avant tout permettre à des marchés bien régulés d'assumer à nouveau leur rôle dans le cadre d'une union bancaire. Loin de moi l'idée de dire que les marchés ont toujours bien agi ces dernières années, mais pour que les marchés puissent travailler correctement, il faut que les signaux politiques soient clairs, compréhensibles et sans équivoque - ce qu'ils n'étaient certainement pas jusqu'à présent.

Par exemple, il s'agit de définir comment nous respectons le principe de no bail-out (non-renflouement). Dans les faits, nous avons déjà pris une certaine distance avec ce principe, alors que pour un bon fonctionnement économique des marchés, l'application du principe de non-renflouement serait certainement primordiale. Pour cela, il faudrait naturellement une procédure claire et bien réglementée de restructuration des pays en cessation de paiement. De même, les «solutions contractuelles » adoptées avec le pacte budgétaire sont très importantes. À chaque fois que l'on parvient à définir des préférences communes et à les consigner dans des accords bilatéraux ou multilatéraux, cela peut représenter une avancée importante, et même, par rapport à l'intervention d'aujourd'hui du professeur Neumann, une solution plus proche des électorats nationaux que si on l'imposait à l'échelle (abstraite) européenne.

Au niveau de l'UE, il faudrait certainement élaborer, sur la base des décisions prises jusqu'ici en réaction à la crise et pour la prévenir, une structure ouverte à l'intégration et génératrice de préférences. Celle-ci doit rester ouverte vers le haut et permettre un approfondissement sélectif. D'un autre côté - et dans ce domaine, le Royaume-Uni marque un point -, certains éléments qui re- 
lèvent aujourd'hui du niveau fédéral (par exemple la politique agricole !) devraient peut-être revenir au niveau national. Ainsi, pour une bonne fédération, nous ne devons pas seulement raisonner en bottom-up, du bas vers le haut, mais aussi en top-down, du haut vers le bas.

En attendant des institutions fédérales qui nous sont encore inaccessibles, une solution intermédiaire pourrait être la création d'institutions indépendantes, par exemple une autorité budgétaire disposant d'un mandat clair, à la place de la vision d'un ministère européen des Finances. Naturellement, cette solution présente un déficit de démocratie. D'un autre côté, nous en avons une bonne expérience, depuis assez longtemps d'ailleurs, et en ce moment même par notre politique monétaire avec une banque centrale indépendante. Elle est donc crédible, même si le désaccord règne en matière de politique budgétaire, ce qui peut avoir une fonction de stabilisation dont il ne faut toutefois pas abuser. L'Union bancaire également fera appel à des autorités indépendantes de supervision, de régulation, de recapitalisation et de liquidation.

Last but not least, je crois que nous autres économistes ne devons pas surestimer notre propre profession. Nous sommes ici réunis avec de nombreux historiens. Je pense qu'au-delà des défis mondiaux, on trouvera peut-être de nouveaux facteurs d'identification dans des éléments qui, traditionnellement, lorsqu'on considère l'histoire de fédérations réelles, ont toujours été mis très en avant : par exemple, la politique commune de défense et de sécurité. Cela représenterait un important budget central. Nous - et là, les économistes doivent peut-être rester modestes - ne créerons peut-être pas la nouvelle identité de l'Europe avec un nouveau pacte de stabilité, une union budgétaire, bancaire ou économique, mais plutôt dans des domaines très différents. Cela pourrait alors redonner des ailes à l'intégration économique.

Rainer Klump : Merci beaucoup. Jacques Mistral, qui va maintenant faire une courte intervention, est également économiste. Après une formation à l'École polytechnique ainsi qu'aux universités de Paris Dauphine et Paris I, il a mené une carrière bien plus typique de la France que de l'Allemagne : en effet, il a occupé des postes à responsabilité aussi bien dans l'administration - au sein du cabinet du Premier ministre et au ministère de l'Économie et des Finances - que dans le secteur privé, où il a été vice-président du groupe AXA. Jacques Mistral est aujourd'hui conseiller spécial à l'Ifri (Institut français des relations internationales).

Jacques Mistral : Le thème de cette table ronde est de débattre des convergences et divergences monétaires. Je vais essayer au cours de cet exposé de tenir compte des deux analyses précédentes.

Je comprends sans difficulté la ligne directrice défendue par l'Allemagne depuis le début de la crise. Selon une formule alors fréquemment utilisée à Berlin, le pays n'a pas voulu prêter «sa carte bleue ». L'Allemagne trouve par ailleurs la proposition des eurobonds - qui, selon moi, a de l'avenir - très intéressante. Tou- 
tefois, ces derniers ne sont pas d'actualité tant qu'un certain nombre de conditions préalables ne seront pas réunies. Dans ce contexte, je salue la politique allemande qui, dans cette épreuve pour le gouvernement et le peuple, a fait preuve d'une grande flexibilité en acceptant des initiatives et évolutions qui ne pouvaient à l'origine que susciter sa réticence, voire sa répulsion. Elle a demandé des garanties dans ce processus (quoi de plus naturel!) et a procédé à des réformes risquées, en permanence sous le contrôle démocratique (ce qu'il faut souligner, et dont la France pourrait s'inspirer).

Pourtant je souhaiterais formuler quelques critiques, constantes depuis 2009 :

- la première critique porte sur la façon dont le gouvernement a introduit, dans la perspective de ce qui allait devenir le sommet de Deauville, l'idée de l'implication du secteur privé (private sector involvement, PSI). C'est une idée absurde ! En effet, lorsque les emprunteurs ont besoin d'avoir un accès aux marchés financiers, leur clamer haut et fort qu'ils s'engagent sur un terrain miné car il y a de fortes probabilités qu'à l'avenir ils y laissent des plumes est la façon la plus sûre de conduire à la situation qui s'est produite, à savoir une année 2011 catastrophique, qui a failli aboutir à l'abîme ;

- en outre, parallèlement à toutes les qualités allemandes en matière budgétaire et industrielle, il existe une défaillance de la pensée financière. Cette défaillance s'est illustrée dans la façon dont a été abordée la crise grecque, considérée pendant des mois comme un problème de nature morale. Or quiconque possède une culture financière internationale et historique sait pertinemment qu'il s'agit là d'une façon absurde de poser le problème. Les désastres financiers sont monnaie courante ! À cet égard, j'oppose la culture financière du Trésor français qui gère le Club de Paris et qui a une longue expérience du sauvetage de pays en difficultés partout dans le monde (de l'Argentine à la Thaïlande) à l'esprit légaliste qui règne au ministère des Finances à Berlin et qui n'aide pas, souvent, à la recherche de solutions intelligentes ;

- la troisième critique a trait à la question des torts du créditeur. Lorsqu'une banque octroie des crédits hasardeux, la responsabilité d'un défaut de paiement tient bien entendu à l'emprunteur qui ne peut pas honorer sa dette. Mais je ne peux pas m'empêcher de penser que celui qui a prêté imprudemment porte aussi une part de responsabilité. Nous pouvons raisonnablement exposer à l'Allemagne le fait qu'à un moment ou à un autre, il faut que se traduise dans les attitudes la reconnaissance de ce que le fait d'avoir un énorme surplus et d'être créditeur des autres pays européens n'est pas simplement le fruit de la vertu mais le fait d'erreurs passées.

Cela étant dit, j'en termine avec les critiques et je souligne donc que grâce à l'Allemagne et sa flexibilité, l'Europe a franchi une étape formidable dans la 
réponse à cette crise, qui a été couronnée par le sommet de juin 2012 et l'accord des chefs d'État et de gouvernement européens sur la poursuite du projet d'Union monétaire. Ce sommet, déterminant, comprenait les quatre volets suivants : l'union politique, l'union bancaire, l'union budgétaire et l'union sur la compétitivité. Il a permis quelques semaines plus tard à Mario Draghi d'affirmer qu'eu égard à la volonté des chefs d'État et de gouvernement de poursuivre le projet d'Union monétaire, il allait mettre en œuvre tous les instruments dont il dispose à cette fin.

Je conclurais en regrettant que, malheureusement, nous traversions un climat franco-allemand défavorable en cette période du cinquantenaire du traité de l'Élysée. En raison des risques de récession générale en Europe, l'élément déterminant du débat dans les mois qui viennent sera le rythme du retour à l'équilibre. Avec tous les défauts des positions françaises, François Hollande a raison d'adopter une politique comparable à celle défendue par le FMI, à savoir qu'il faut être sûr de s'engager dans la direction d'un équilibre à moyen terme. Il faut prendre des engagements crédibles, vérifiables année après année. Néanmoins, le fait de vouloir précipiter ce retour à l'équilibre est une recette certaine pour aboutir à l'échec. Ce qui me rend confiant, c'est la flexibilité dont l'Allemagne a su faire preuve jusqu'à présent. Je ne peux pas croire qu'elle élève des obstacles absolus et permanents à la recherche d'une solution de ce genre. Je souhaite qu'elle trouve dans la France un partenaire crédible, qui lui permette précisément d'élaborer à deux, et pas simplement sur la base de sa force actuelle, un compromis, dont nous avons - comme si souvent par le passé - encore besoin.

Rainer Klump : Merci beaucoup, Jacques Mistral. Venons-en maintenant à Peter Schaefer, né dans ce qui s'appelait encore la RDA. Il a étudié les sciences économiques à Dresde avant d'entamer en quelque sorte une carrière européenne en tant qu'étudiant puis doctorant à l'université de Trente en Italie et à Paris IV. Dans sa thèse, qui sera prochainement terminée, il traite de la maîtrise des crises économiques en France, en Italie et en Allemagne. Il aborde également ce thème sous l'angle de sa perception par l'opinion publique, si je ne me trompe pas, c'est-à-dire du traitement de ces crises dans le discours public. Je pense qu'il s'agit d'un point de vue intéressant dans notre discussion.

Peter Schaefer : Comment surmonter les différences de politique monétaire entre l'Allemagne et la France ?

Avec Jochen Mayer, géographe et sociologue de l'Allemagne du XX $\mathrm{X}^{\mathrm{e}}$ siècle, j'ai organisé en 2011 à la Maison Heinrich Heine de Paris un colloque sur les évolutions différentes, en France et en Allemagne, des sciences économiques et les différents discours de politique économique qui y sont associés. Ont notamment pris part à cette manifestation intitulée «La compétition idéologique dans la discipline économique - Économistes et discours économiques en Allemagne 
et en France, 1980-2007 » les professeurs Frédéric Lebaron et Jan-Otmar Hesse. Pour ma part, je travaille sur une thèse dans laquelle je compare les interprétations allemandes et françaises des crises économiques de 1929 et 2008.

\section{Persistance de conceptions différentes de la politique monétaire}

Au moment de cette intervention, presque cinq ans se sont écoulés depuis que la banque d'investissement Lehman Brothers a fait faillite en 2008, et que les ÉtatsUnis et les États d'Europe occidentale ont pris des mesures d'une ampleur inégalée pour sauver le secteur financier. Considérant l'évolution de la situation, on peut affirmer que la crise économique et financière persistante est comparable à la crise économique mondiale de 1929, car, comme elle, elle va entraîner des modifications durables du capitalisme européen occidental qui se profilent déjà. Cette crise se caractérise par le conflit entre le capitalisme et la démocratie, ou plus précisément entre les élites économiques et politiques, un conflit marqué par la toute-puissance des premières et par la réduction des approches possibles pour les secondes au vu des imbrications économiques mondiales croissantes.

Face à cette évolution dramatique, les discours nationaux en Europe renvoient fréquemment à l'expérience historique de crises qui n'ont pourtant plus grand-chose à voir avec les problématiques actuelles. S'y ajoute le fait que ces discours rarement remis en question sont systématiquement avancés par des groupes d'intérêts spécifiques. Il s'ensuit une persistance de conceptions des politiques économiques et monétaires que l'on pourrait, avec un peu d'audace, comparer à la phase de la guerre de position pendant la Première Guerre mondiale. Dans ce conflit, l'industrie financière d'Europe occidentale profite des taux élevés sur les emprunts d'État de pays ployant sous les déficits, et les exportations allemandes profitent du cours de l'euro, trop faible par rapport à celui d'un deutsche mark indépendant, mais au prix de la hausse des soldes Target 2 de la Bundesbank. La guerre de position actuelle n'implique naturellement aucun affrontement militaire et ne fait pas de victimes, mais ses effets économiques sont comparables. Quelques grandes industries et banques profitent du conflit politique, les budgets publics souffrent et les risques toujours plus visibles sont principalement supportés par la classe moyenne.

\section{Relativiser l'expérience historique}

Aussi profitable et compréhensible qu'il soit pour les groupes d'intérêts en question d'exploiter les positions différentes de l'Allemagne et de la France en matière de politique monétaire, j'aimerais attirer l'attention sur le fait qu'une politique monétaire irresponsable peut rapidement avoir des conséquences indésirables : en voici quelques exemples historiques.

Après la Première Guerre mondiale, l'Allemagne s'est retrouvée prise au piège d'une dette qui exerçait une forte pression sur les gouvernements successifs de la République de Weimar et a fini par favoriser l'hyperinflation et l'ex- 
trémisme. Comme Michael Hudson le décrit très bien dans son ouvrage de 1972 intitulé Super Imperialism ${ }^{3}$, c'était principalement dû au fait que les États-Unis appliquaient un taux d'intérêt de $4 \%$ sur les dettes de guerre de la GrandeBretagne et de la France, ce qui a incité cette dernière à exiger des réparations très élevées et à insister pour les percevoir pendant la période agitée des années 1920, et ce malgré la montée de l'extrémisme ${ }^{4}$. Les conséquences de cette situation sont connues : l'hyperinflation de 1923-1924 a entrainé une paupérisation de la classe moyenne allemande et une crainte de l'inflation profondément ancrée en Allemagne ${ }^{5}$. Un État dépourvu de moyens d'action financiers était pratiquement incapable de maîtriser les poussées extrémistes à droite et à gauche. Lorsque la crise économique mondiale a éclaté, provoquant notamment le retrait des capitaux en majeure partie américains, l'État n'a guère pu ou voulu s'opposer à cette évolution. Le gouvernement social-démocrate (SPD) au pouvoir, qui ne voulait pas renoncer à l'allocation chômage introduite seulement en 1927, n'a pas pu se maintenir et a été démis par le président du Reich, Paul von Hindenburg. Lorsque plus tard, les national-socialistes ont mis en œuvre le programme d'investissements et d'emplois prévu par le SPD, les dégâts politiques étaient déjà irréparables.

La France a été moins exposée à la crise mondiale, principalement grâce à la faible intensité capitalistique de son économie, relativement autonome de par la prédominance de petites et moyennes entreprises. De plus, la France avait dévalué sa monnaie par rapport à la livre après 1926, et présentait déjà une inflation élevée 6 . Cela a non seulement réduit la valeur de la dette publique, mais a permis à la France d'échapper à la crise mondiale jusqu'à l'abandon de l'étalon-or par la Grande-Bretagne et les États-Unis. Elle était alors considérée comme un placement sûr et a vu affluer d'importantes quantités d'or. En 1929 et 1930, le taux de chômage avait atteint respectivement $13,3 \%$ et $22,7 \%$ en Allemagne, tandis qu'en France il ne dépassait pas $1 \%$ puis $2 \%$. Plus tard, en revanche, la France aurait elle aussi à affronter les conséquences de la crise mondiale.

Ces exemples montrent qu'il est judicieux de considérer les différends relatifs à la politique monétaire comme un élément important des conflits politiques des années 1930 et d'aujourd'hui. Ils montrent aussi que dans une crise économique d'ampleur mondiale, un pays peut rapidement basculer du statut de gagnant à celui de perdant. Il est au fond surprenant de constater que les questions macroéconomiques sont certes débattues en Allemagne, mais sans que l'on fasse suffisamment le lien avec la propre histoire du pays. Autrement, de nombreux citoyens allemands comprendraient peut-être mieux que, sans la possibili-

\footnotetext{
${ }^{3}$ Michael Hudson, Super Imperialism - The Origin and Fundamentals of U.S. World Dominance, nouvelle édition revue et corrigée de l'ouvrage de 1972, Pluto Press, Londres, 2003.

${ }^{4}$ Dès 1920, John Maynard Keynes était conscient des implications problématiques du traité de Versailles, comme il l'a écrit dans Les Conséquences économiques de la paix (Gallimard, Paris, 2002, Collection Tel).

${ }^{5}$ Cette crainte a été encore renforcée par la dévaluation de la monnaie après la Seconde Guerre mondiale.

${ }^{6}$ Cf. Paul Krugman, http://krugman.blogs.nytimes.com/2012/11/27/twenties-tales/ et http://krugman.blogs.ny times.com/2012/11/23/franc-thoughts-on-bond-vigilantes/ [consultés le 21/01/2015].
} 
té de dévaluer (une sortie de l'euro est un processus très complexe), de nombreux pays de la zone euro sont piégés par leur dette, dans une situation comparable à celle de l'Allemagne des années 1920. Mais la France aussi réagit de manière peu compréhensible. Naturellement, le pays peut se désengager de l'export, mais les visées autarciques auraient aujourd'hui d'autres conséquences que dans les années 1920, car la France est désormais nettement plus intégrée dans le réseau économique mondial. Il suffit de citer l'industrie automobile française, qui doit faire face à un net recul des ventes dans le sud de l'Europe.

Tandis que les Allemands ont une peur irraisonnée de l'inflation, les Français ne réagissent pas suffisamment. Certes, le président Hollande milite pour des augmentations de salaire (à mon avis utopiques) en Allemagne; en revanche, il semble que la sortie de l'euro, ou un retour commun au Système monétaire européen comme l'a conseillé le professeur Hankel, n'ait jusqu'à présent jamais fait l'objet de discussions sérieuses.

\section{Politique monétaire « neutre » et responsabilité des élites nationales et européennes}

$\mathrm{Au}$ lieu de rester prisonnières des vieux discours, les élites européennes devraient s'efforcer de mettre en place une politique monétaire qui réduise les déséquilibres économiques. Concrètement, cela signifie qu'il faut repenser la construction de la monnaie commune et élaborer une solution durable. Pour l'Allemagne, cela signifierait certes une réduction des excédents commerciaux, mais elle échapperait en contrepartie aux risques à long terme des plans de sauvetage permanents (MES, etc.). Les Allemands devraient aussi admettre que leur compétitivité supérieure à celle du reste de la zone euro n'est que partiellement liée à une meilleure structure économique, tandis qu'une autre part est due à la dévaluation interne contraire au traité obtenue par la baisse des salaires moyens depuis la mise en œuvre de la législation Hartz $I V^{7}$.

En parallèle, les élites nationales du sud de l'Europe devraient prendre conscience qu'elles ont vécu pendant des années dans l'illusion de la richesse et qu'il est temps de procéder à des coupes radicales et à des réformes d'avenir, car au vu de la hausse des faillites d'entreprises et des dépenses sociales, il semble vain d'imaginer que la crise puisse se régler toute seule. Il faut établir des structures d'entreprise et des mécanismes de sélection concurrentiels au niveau international, sinon l'on risque de retomber dans les visées autarciques et l'individualisme national qui remettraient en cause les avancées européennes de ces dernières décennies. Naturellement, il ne serait pas simple d'y parvenir réellement, parce que cela nécessiterait de modifier les structures et les réseaux, mais au moins, le projet Europe aurait à nouveau un avenir réaliste. Les élites européennes devraient expliquer aux citoyens comment relever les défis de la

\footnotetext{
${ }^{7}$ Cf. http://www.flassbeck-economics.de/der-europaische-traum-und-ein-schlimmes-erwachen/ [consulté le 21/01/2015].
} 
mondialisation, c'est-à-dire la montée en puissance des BRICS, au lieu de profiter de différentes manières du nouvel «empire fortuit » allemand, comme l'a fort bien exprimé Ulrich Beck ${ }^{8}$.

Une politique monétaire neutre mettrait fin à la mise en accusation et à la suprématie du modèle allemand en Europe et, espérons-le, améliorerait les conditions de l'économie réelle dans toute la zone euro. Le président de la Bundesbank, Jens Weidmann, a demandé début 2013 au Japon de renoncer à manipuler les taux de change, car cela n'entraînerait que des améliorations temporaires et pourrait provoquer, au niveau international, de véritables guerres monétaires ${ }^{9}$. Visiblement, il est plus simple d'accuser d'autres nations que de balayer devant sa porte. Cependant, on ne peut qu'être d'accord avec l'affirmation selon laquelle «observer les taux de change ou la politique monétaire détourne l'attention des défis structurels auxquels de nombreux pays devraient s'attaquer actuellement ».

L'Europe va devoir s'adapter aux nouvelles conditions de l'économie mondiale, avec un capital circulant dans le monde entier et des chaînes de création de valeur qui se déplacent sur toute la planète. Cette adaptation devra nécessairement donner lieu à de nouveaux compromis sociaux dans de nombreux pays. Je suis d'avis que les élites des différents États devraient endosser la responsabilité de ce processus, au lieu de simplement laisser les choses se dérouler. C'est ce qu'a fait l'Allemagne, dans une certaine mesure, avec l'adoption de la législation Hartz IV. Cependant, comme nous pouvons le constater maintenant, elle l'a fait aux dépens de ses partenaires européens. À une époque où une grande partie des pays émergents rêvent d'un avenir économique meilleur, l'Europe n'a pas besoin d'aventures postdémocratiques ou de nouveaux mouvements populistes.

Les Européens devraient peut-être aussi réfléchir à améliorer durablement les budgets publics, comme le prix Nobel Michael Spence ${ }^{10}$ le réclame. En effet, d'une manière ou d'une autre, il faut combler les failles déjà visibles.

\section{Traduction des propos de Paul J.J. WELFENS, Rainer KLUMP, Christian KASTROP et Peter SCHAEFER : Marie-Céline GEORG}

\footnotetext{
8 Cf. http://blogs.lse.ac.uk/europpblog/2013/03/25/five-minutes-with-ulrich-beck-germany-has-created-anaccidental-empire/ [consulté le 21/01/2015].

9 Cf. http://www.handelsblatt.com/politik/konjunktur/nachrichten/bundesbank-praesident-weidmann-warntvor-abwertungswettlauf/8089212.html [consulté le 21/01/2015].

10 Cf. http://www.project-syndicate.org/commentary/why-states-should-build-asset-portfolios-by-michaelspence [consulté le 21/01/2015].
} 



\section{- II -}

Cultures budgétAires en France et en AlLEmagne 



\section{Principes fondateurs et constantes de la politique budgétaire de la République fédérale d'Allemagne ${ }^{1}$}

\section{Heinz GROSSEKETTLER}

Le thème de cette journée d'étude consacrée aux cultures budgétaires en France et en Allemagne, ainsi que les sujets des deux contributions consacrées aux principes fondateurs et constantes de la politique budgétaire auraient pu être inspirés par un article intitulé Internationale Gemeinsamkeiten und nationale Eigenarten der Finanzpolitik (Convergences internationales et spécificités nationales de la politique financière), publié en $1948^{2}$. Son auteur, Fritz Neumark (1900-1991), spécialiste des finances, est très connu et très estimé en Allemagne ; il fut docteur honoris causa de l'université de la Sorbonne et Commandeur de l'Ordre des Palmes Académiques en France ${ }^{3}$. Neumark écrit (p. 120-121) : "Quels que soient les changements affectant au cours des siècles les données économiques et politiques d'une société, il semble que celles-ci comportent un certain nombre de "constantes" favorisant la formation d'une certaine tradition d'action publique. » II poursuit un peu plus loin : " [La] tendance de la politique financière d'un pays [n'est certes] pas absolument pérenne; elle [peut] elle-même être soumise à des changements. Cependant, ceux-ci s'opèrent en général très lentement, notamment du fait du pouvoir de la tradition [...] » (en italique dans l'original).

Mon analyse des principes fondateurs et des constantes de la politique budgétaire en République fédérale d'Allemagne (RFA) se limitera pour l'essentiel à la période allant de 1949 à la fin des années 1990. Ce découpage paraît opportun car, entre 1945 et l'adoption de la Loi fondamentale (Grundgesetz, GG) le 23 mai 1949, il n'existe ni République fédérale ni, par conséquent, de politique financière véritablement allemande, et car la période d'après $\mathbf{2 0 0 0}$ est couverte par d'autres contributions. Je commencerai par présenter le cadre juridique dans lequel s'inscrit la politique budgétaire allemande, à savoir la Constitution financière, et les évolutions récentes de ce cadre. J'aborderai dans un deuxième temps les constantes que l'on peut observer à moyen terme pendant des périodes précises de la politique budgétaire allemande. J'explorerai ensuite la question d'éventuelles constantes à long terme, au-delà des périodes définies.

\section{Bases juridiques et constitutionnelles de la politique budgétaire allemande}

\section{Le fédéralisme coopératif : cadre du droit budgétaire}

Le fait que l'Allemagne soit un État fédéral est crucial pour appréhender la politique budgétaire allemande. Un économiste s'attendrait à ce qu'un État de ce

\footnotetext{
${ }^{1}$ Cet article, rédigé en mars 2014, fait suite à une communication donnée dans le cadre de la journée d'étude Cultures budgétaires en France et en Allemagne, coorganisée par le CIRAC le 18 novembre 2013, NdE.

${ }^{2}$ Neumark (1948), cité ci-dessous d'après la réédition dans Neumark (1961), p. 96-121.

${ }^{3}$ Sur la biographie de Neumark et son importance, cf. Grossekettler (2013).
} 
type soit organisé suivant les principes de la théorie du fédéralisme fiscal et à ce que la mise à disposition de biens par des organisations publiques réponde à ce que l'on appelle le principe d'équivalence budgétaire. Cette orientation fondamentale suppose de satisfaire à deux principes ${ }^{4}$ :

- le groupe bénéficiant de l'utilisation d'un bien ou d'un ensemble de biens doit coïncider avec le groupe assumant les coûts de mise à disposition de ces biens (principe de l'équivalence fiscale);

- les contribuables participant au financement des biens et services doivent avoir, via les élections, un droit de contrôle sur ceux qui portent la responsabilité des décisions et de leur mise en œuvre, et ce de telle manière que ceux-ci aient à répondre au cours d'une seule et même élection du bénéfice comme des coûts afin que l'on ne se trouve pas dans une situation où, lors d'un scrutin 1 (par exemple les élections au Bundestag), les bénéfices seraient les seuls à jouer un rôle, tandis que lors d'un scrutin 2 (élections municipales par exemple) seuls les coûts interviendraient (principe de la responsabilité démocratique entière face aux coûts et bénéfices).

Une collectivité devrait donc assumer un ensemble donné de missions qu'elle financerait sur ses propres recettes (dont elle gérerait l'organisation), qu'elle mettrait en œuvre à l'aide de sa propre administration et dont elle devrait justifier les bénéfices comme les coûts devant les citoyens.

Or le fédéralisme allemand ne répond pas, même dans ses grandes orientations - contrairement à qui se fait en Suisse ou aux États-Unis -, au principe d'équivalence budgétaire. Cela tient pour l'essentiel à quatre facteurs majeurs.

D'abord et avant tout, le système de financement des entités publiques est conçu en Allemagne surtout comme un système fiscal commun et partagé : la Loi fondamentale définit les grandes lignes de la répartition des recettes fiscales entre État fédéral, Länder et communes, et seuls l'Etat fédéral et les municipalités se voient conférer des compétences autonomes limitées en matière fiscale. En revanche, tous les impôts dégageant des recettes significatives relèvent de la compétence de l'État fédéral et des Länder et sont définis par le Bundestag et le Bundesrat, puis répartis à travers un système de péréquation financière entre Bund et Länder ainsi que différents types d'aides financières ${ }^{5}$. Dans ce dispositif,

\footnotetext{
${ }^{4}$ La formulation qui suit est empruntée à Grossekettler (2005). Ces principes ont toutefois été posés par différents auteurs sous des formes diverses ; ils renvoient au fond aux principes de F.W. Raiffeisen - entraide, autonomie et responsabilité - déjà présentés dans les années 1840 comme les principes de l'organisation coopérative.

${ }^{5}$ Les impôts représentant des recettes importantes sont des impôts dits « communs » (Gemeinschaftssteuern), pour lesquels la compétence législative incombe à l'État fédéral sous la forme de lois soumises à l'approbation du Bundesrat, les recettes étant partagées entre les échelons administratifs et la compétence de gestion appartenant aux Länder. Pour les impôts dits «séparés », dont les recettes vont à un seul niveau administratif (Bund, Länder ou communes), la compétence législative incombe également à l'État fédéral, avec approbation nécessaire par le Bundesrat, tandis que la compétence de gestion relève généralement des Länder. On trouvera un aperçu sous forme de tableau dans les publications du ministère fédéral des Finances (Bundesministerium
} 
les communes sont considérées comme faisant partie des Länder et doivent se voir reverser une part des prélèvements régionaux - en fonction de la répartition des missions au sein de chaque Land - dans le cadre d'une péréquation financière municipale ${ }^{6}$. Dans un tel système, on perd complètement de vue le lien entre arbitrages en matière de dépenses et décisions relatives au financement. Pour ce qui est des Länder, il faut ajouter que, faute de compétence législative, ils ne peuvent pratiquement pas modifier l'impôt, dont ils ont pourtant la compétence de prélèvement ${ }^{7}$. Ainsi, un gouvernement régional social-démocrate qui souhaitait davantage d'intervention de l'État dans son champ de compétence ne disposait au mieux que d'une option : augmenter l'endettement ; cette possibilité disparaîtra elle aussi à l'avenir en raison du frein à l'endettement (« règle d'or »), sur lequel nous reviendrons en détail. C'est pourquoi le Conseil scientifique du ministère fédéral des Finances a demandé à plusieurs reprises, en vain à ce jour, l'introduction d'un droit d'augmenter les impôts sur le revenu pour les Länder.

Deuxièmement, les lois fédérales et les dépenses qu'elles impliquent ou autorisent ne sont en temps normal pas appliquées ou mises en œuvre par une administration fédérale spécifique mais - comme c'est le cas dans l'Union européenne (UE) - par les administrations régionales. Dans un scénario ordinaire, l'instance qui applique les lois participe aussi à leur élaboration et peut donc, par exemple, faire peser les frais généraux sur le Bund ou bien se permettre des mesures peu rentables - s'agissant par exemple de lever l'impôt - dont ce sont en fait les élus régionaux ou municipaux qui devraient avoir à répondre. Or c'est l'inverse qui se produit en Allemagne : les responsables politiques fédéraux se félicitent par exemple d'avoir obligé les communes à créer des places en crèche pour les moins de trois ans; or les dépenses qu'engendre cette décision doivent être, pour une part considérable, financées par les communes, au détriment des moyens dont celles-ci peuvent disposer librement à l'échelon local.

Troisièmement, certaines missions requièrent un minimum d'harmonisation nationale, mais leur centralisation au niveau fédéral n'est pas une obligation. Ainsi, en Allemagne, l'enseignement scolaire est une mission des Länder. Or le droit de libre circulation impose notamment que les parents d'enfants scolarisés puissent déménager sans difficulté particulière d'un Land à un autre. C'est pourquoi il existe une Conférence des ministres de l'Éducation qui définit des normes mini-

für Finanzen 2013a, p. 28-29) et de Heller (2010, p. 51-53). Une publication du ministère fédéral des Finances (Bundesministerium für Finanzen, 2012a) offre une représentation détaillée des relations financières entre Bund et Länder, avec des tableaux présentant les recettes fiscales et la péréquation financière.

${ }^{6}$ La répartition doit se faire de telle sorte que les communes aient au total (avec leurs propres recettes fiscales) les moyens de financer un certain nombre de missions municipales de manière autonome. La garantie d'autonomie de gestion est une notion importante, citée à l'art. 28 de la Loi fondamentale (Grundgesetz, GG), tout comme le principe dit de connexité, qui oblige les Länder à prendre en charge les coûts de missions transférées.

${ }^{7}$ L'imposition des casinos et celle des transactions immobilières font exception. Pour la première, ce sont les Länder qui détiennent la compétence législative et qui perçoivent les recettes; pour la seconde, les Länder prélèvent les recettes et ont le droit d'indexer le taux d'imposition sur l'assiette définie par l'État fédéral. Ces deux exceptions représentant seulement de faibles recettes, une synthèse sommaire peut en faire abstraction. 
males en termes de contenus et de modalités d'évaluation. D'autres domaines disposent également d'instances de coordination similaires, au sein desquelles l'État fédéral est parfois représenté ${ }^{8}$. La Conférence des ministres des Finances (FMK) est particulièrement importante en matière de processus décisionnel concernant la politique financière. Elle regroupe les ministres des Finances des 16 Länder. Ceux-ci se réunissent environ douze fois par an après les réunions de la commission des Finances du Bundesrat, l'objectif étant d'harmoniser entre eux la politique financière des Länder - en opposition à l'État fédéral le cas échéant. La présidence change chaque année : elle est confiée en alternance tantôt à un ministre des Finances d'un Land dirigé par le parti social-démocrate (SPD) (Land dit A), tantôt à celui d'un Land dirigé par l'union chrétienne-démocrate ou l'union chrétienne-sociale en Bavière (CDU/CSU) (Land « B »). Les Länder A et B se coordonnent également et choisissent pour chacun des deux groupes un ministre des Finances qui endosse le rôle de chef de file. Pour ne pas dépendre uniquement des données du ministère fédéral des Finances, la FMK a en outre créé une instance à son service, le «Centre de données des Länder » (ZDL). Un effet secondaire non négligeable de cette coordination horizontale des gouvernements régionaux à travers des conférences réside dans le fait que les parlements régionaux sont de facto dépossédés d'une partie de leur pouvoir : ils ne peuvent parfois plus qu'entériner les résultats des Conférences.

Quatrièmement, les lois fédérales doivent normalement recueillir une majorité non seulement au Bundestag, mais aussi au Bundesrat, la représentation des Länder. Il existe il est vrai des lois qui ne sont pas soumises à l'approbation du Bundesrat, par exemple celles relatives au budget fédéral, pour lesquelles le Bundestag peut de facto décider en dernier ressort ; mais si le Bundesrat souhaite peser sur une loi de ce type, il peut toujours menacer de faire obstacle à un autre texte qui, lui, est soumis à l'approbation de cette instance, et que le gouvernement fédéral tient à voir adopté. Il peut ainsi exercer tout de même une influence de façon indirecte.

Les quatre types de causes listés plus haut ont conduit à la configuration suivante : dans le fédéralisme à l'allemande, les responsables politiques sont dans l'obligation de coopérer au-delà des clivages partisans et corporatistes. C'est pourquoi, depuis les années 1970, on qualifie cette organisation de «fédéralisme coopératif», pour la différencier du fédéralisme concurrentiel pratiqué dans d'autres États. L'obligation de coopérer est renforcée par deux facteurs : d'une part, le Bund et les Länder sont normalement dirigés par des majorités politiques différentes; d'autre part, au cours d'une législature au Bundestag, les électeurs ont tendance à renforcer, lors des élections au Landtag, les partis qui n'ont pas la majorité au Bundestag. En Allemagne, le système du fédéralisme coopératif est loin de faire l'unanimité, bien qu'il soit en partie le reflet d'une tradition histo-

\footnotetext{
${ }^{8}$ En matière de politique des transports sur le long terme, l'État fédéral et les Länder coopèrent par exemple pour élaborer un plan des infrastructures de transports ; ces deux niveaux administratifs coopèrent également en matière de politique d'aménagement du territoire.
} 
rique. Ses partisans défendent la thèse, critiquée dans les milieux scientifiques depuis l'ouvrage d'Olson intitulé Logique de l'action collective (1978 pour la traduction française, Presses Universitaires de France/1965 pour l'original), selon laquelle la contrainte de mettre en œuvre de nombreux processus de négociation politique permettrait finalement une prise en compte adéquate de l'ensemble des intérêts. Le fédéralisme coopératif empêcherait en outre - à l'instar de toutes les formes de fédéralisme - une concentration dysfonctionnelle des pouvoirs. Il permettrait aussi en tant que tel de promouvoir un capital social sous la forme d'une disposition à coopérer dans une relation de confiance, en particulier sur des questions importantes pour l'intérêt général. Les détracteurs du fédéralisme coopératif y voient au contraire un système d' « irresponsabilité organisée »" : dans ce système, jugent-ils, chacun peut toujours se cacher derrière tous les autres, les petits exploitent les grands et les décisions nécessaires sont sans arrêt indûment différées car il faut convaincre, voire « acheter » un grand nombre d'acteurs disposant d'un droit de veto et prendre en compte des considérations externes qui ne devraient pas peser dans la décision. En tout cas, l'on ne saurait comprendre l'organisation du droit budgétaire du Bund, des Länder et des communes, que nous allons maintenant examiner, sans avoir à l'esprit ce fédéralisme à l'allemande. Ces deux facteurs influencent en pratique la politique budgétaire et font partie à ce titre de ses principes fondateurs et de ses constantes.

\section{Fondamentaux du droit budgétaire allemand}

Le droit budgétaire, composante du droit constitutionnel et administratif réglant la planification, l'adoption, la mise en œuvre et le contrôle des budgets publics ainsi que les prévisions financières et les calculs aidant à la décision qui y sont liés, avait à l'origine pour seule fonction d'obliger les responsables politiques à préparer des budgets. Il s'agissait d'adapter les dépenses souhaitées aux faibles ressources financières disponibles et d'engager le gouvernement comme l'administration à se tenir aux budgets prévus, avec des possibilités de suivi. Plus tard sont venues s'ajouter des fonctions supplémentaires, avec des objectifs en termes de conjoncture et de durabilité, ainsi que le contrôle de l'efficacité de l'action gouvernementale et administrative. On tenta d'abord de parvenir à gérer les recettes en répartissant les moyens financiers par types de dépenses (titres), administrations (chapitres) et secteurs (sections). Aujourd'hui, on désigne ce procédé de programmation et de contrôle axé sur les dépenses par le terme d' « ancien modèle de gestion ». On lui oppose un "nouveau modèle de gestion » axé sur l'allocation de ressources affectées à différentes missions et qu'il s'agit d'adapter progressivement au modèle de gestion des entreprises en calculant les financements, les charges et produits, et en établissant une comptabilité analytique.

\footnotetext{
${ }^{9}$ Allusion au titre de l'ouvrage d'Ulrich Beck, non traduit : Gegengifte - Die organisierte Unverantwortlichkeit, Suhrkamp, Francfort-sur-le-Main, 1988, NdT.
} 
La principale base constitutionnelle du droit budgétaire pour le Bund et les Länder réside dans les articles 104a à 115 GG de la Constitution financière ${ }^{10}$. Ces articles visent à régler la répartition des compétences entre État fédéral et Länder dans le domaine financier de sorte qu'ils s'acquittent non seulement de leurs missions de mise à disposition, mais soient aussi en mesure de répondre à leurs obligations pour assurer la stabilité financière en vertu du droit allemand et de la législation de l'UE et de l'Union économique et monétaire (UEM).

Les dispositions concrètes de la Constitution allemande en matière de droit budgétaire sont caractéristiques du fédéralisme coopératif: le principe général dit des « charges à supporter » énoncé à l'art. 104a GG indique d'abord que l'État fédéral et les Länder supportent chacun pour leur part les dépenses liées à leurs missions respectives. Les articles 105 à $108 \mathrm{GG}$ règlent ensuite la répartition des recettes fiscales, tandis que l'art. 109(1) GG pose le principe selon lequel l'État fédéral et les Länder sont autonomes et indépendants les uns des autres dans leur gestion budgétaire. Ce principe véritablement fédéraliste est toutefois relativisé par l'art. 109(4) nouvelle version, puisqu'il y est prévu un droit relatif aux principes (Grundsätzerecht), intégrant une dimension coopérative et dérivé de trois lois fédérales applicables au Bund et aux Länder ayant la primauté sur les constitutions et lois des Länder. Ce droit relatif aux principes regroupe :

- la loi sur les critères (Maßstäbegesetz) pour la péréquation financière entre Bund et Länder, qu'il n'y a pas lieu de développer ici et qui prend fin en 2019 ;

- la loi sur la stabilité et la croissance (Stabilitäts- und Wachstumsgesetz) de 1967, instaurant un pilotage global de l'économie et dont nous détaillerons les effets ;

- la loi relative aux principes budgétaires (Haushaltsgrundsätzegesetz, HGrG), importante dans le cadre traité ici et donc analysée en détail.

La loi HGrG vise à assurer la cohérence globale des règles budgétaires adoptées séparément par le Bund et les Länder. Par ailleurs, elle doit garantir que la législation municipale adoptée par les Länder et coordonnée entre eux de manière horizontale respecte elle aussi les principes budgétaires. Cette loi HGrG énonce deux moyens de remplir son objectif : sa première partie $(\S \S 1-48)$ formule les principes que l'État fédéral et les Länder doivent transposer dans leur législation respective ; ils l'ont été, principalement à travers le règlement budgétaire fédéral (Bundeshaushaltsordnung, $\mathrm{BHO}$ ) et les règlements budgétaires régionaux (Landeshaushaltsordnungen, LHO). La seconde partie ( $\S ~ 49-57)$ définit ensuite des règles directement valables à la fois pour le Bund et pour les Länder. Celles-ci concernent principalement trois volets : la compatibilité entre les programmations financières à moyen terme, la coopération s'agissant de ré-

\footnotetext{
${ }^{10}$ Intervient aussi, de manière provisoire, l'art. 143d GG, comportant des dispositions transitoires relatives à une réforme financière amorcée en 2009, avec échéance en 2019.
} 
pondre aux critères européens de stabilité ainsi que l'établissement de statistiques et l'échange de données.

Les règles énoncées par la $\mathrm{HGrG}$ ne peuvent être étudiées en détail dans le cadre de cet article. Cela ne devrait pas être un problème, dans la mesure où le droit budgétaire français comporte des dispositions semblables ${ }^{11}$. Il s'agit d'abord de principes de nature générale comme celui d'annualité (§ $4 \mathrm{HGrG}$ ), celui d'efficacité et de bonne gestion ( $§ 7 \mathrm{HGrG})$; viennent ensuite des principes spécifiques concernant l'élaboration, la mise en œuvre et le contrôle du budget ainsi, enfin, que d'autres principes spécifiques comme la gestion des ressources humaines ${ }^{12}$.

\section{Brève histoire du droit budgétaire allemand et de ses évolutions récentes}

Jusqu'à la fin des années 1960, la République fédérale était soumise aux dispositions du règlement budgétaire du Reich (Reichshaushaltsordnung) de 1922, bien que le fédéralisme de la République de Weimar fût en réalité bien plus unitaire que celui de la République fédérale et que les lacunes et possibilités de contourner ce règlement soient apparues dès l'époque de Weimar ${ }^{13}$. Ce règlement budgétaire était le prolongement du texte en vigueur en Prusse ${ }^{14}$. D'un point de vue strictement formel, le règlement budgétaire du Reich visait largement à éviter un endettement public "improductif» au sens défini par Lorenz von Stein, c'est-à-dire un endettement qui ne permettait pas d'augmenter les recettes ou de réduire les dépenses ${ }^{15}$. Le budget était divisé en deux volets : le budget « ordinaire », qui devait être couvert par les recettes ordinaires, donc essentiellement fiscales; le budget « extraordinaire », pour lequel un endettement ciblé était autorisé, s'il s'agissait de servir des fins "pérennes» (werbende Zwecke) c'est-à-dire de financer des investissements « rentables » ou "productifs ». Aucune considération en matière de stabilité ne figurait dans ce texte.

Cela changea avec l'introduction de la loi sur la stabilité et la croissance de 1967 puis la «grande réforme budgétaire » de 1969. Avec ces textes, les paramètres de ce que l'on appelait l'équilibre macroéconomique furent intégrés à la programmation budgétaire : stabilité du niveau des prix, taux d'emploi élevé, équilibre du commerce extérieur et croissance économique adéquate. L'État fédéral comme les Länder s'écartèrent en outre du principe de structuration en deux budgets et de l'endettement ciblé, pour tendre vers un solde du budget total visant à la stabilité. Furent également introduits une programmation finan-

\footnotetext{
${ }^{11}$ OCDE (2004), p. 189-190.

12 On trouvera un classement systématique des principes budgétaires sous forme de tableau chez Heller (2010), p. 230-231. Ces principes sont analysés de manière détaillée dans la partie B de l'ouvrage de Heller. Une reproduction de la loi HGrG y figure en annexe II.

${ }^{13}$ Sur l'histoire du droit budgétaire en Allemagne et pour une évaluation économique de ce droit, cf. Strube (2002).

${ }^{14}$ Cf. ibid., p. 70.

${ }^{15}$ Sur la doctrine de la dette publique selon Stein, cf. Grossekettler (1998a), p.102-107.
} 
cière à moyen terme pour la totalité des budgets et un conseil dédié, chargé de coordonner les programmations financières à moyen terme de l'État fédéral et des Länder. Le principe était qu'un déficit ne devait pas excéder la somme des investissements réalisés sur un budget, sauf si les objectifs énoncés plus haut étaient menacés. À l'époque, il fut parfois fait référence à cette règle sous le nom $d^{\prime}$ '《interdiction d'excès du financement par crédit $^{16} »$. Cette disposition jouait le rôle de frein à l'endettement. Mais ce frein à l'endettement «à l'ancienne » s'avéra par la suite insuffisant : en effet, la notion d'investissement n'était pas définie de manière assez précise et, s'agissant des nouvelles dettes, la contrainte était de rester en deçà non pas de la somme des investissements nets, mais des investissements bruts. Dans le cadre juridique de l'époque, un gouvernement souhaitant s'endetter - pour quelque raison que ce soit - pouvait par exemple déclarer des biens ayant une courte durée de vie comme étant des « investissements » et en financer régulièrement le remplacement par des dettes à long terme. Si l'on ajoute la tendance bien connue des gouvernements à s'endetter pendant les phases de ralentissement économique sans se désendetter en phase de conjoncture plus favorable, ce cadre juridique faisait courir le risque d'une progression par paliers de la dette publique ${ }^{17}$. Cette menace se trouve encore exacerbée en Allemagne dans la mesure où le système fédéral implique des élections fédérales et régionales presque en permanence, soit autant d'occasions de cadeaux électoraux.

$\mathrm{Au}$ cours des années qui suivirent la « grande réforme budgétaire », le droit budgétaire évolua, à travers plusieurs modifications de la HGrG et des procédures de programmation, et fut progressivement davantage tourné vers l'objectif à long terme d'un instrument politique de pilotage et de contrôle tel que celui dont dispose la direction de grandes entreprises privées. Citons les grandes mesures suivantes :

- la loi de développement du droit budgétaire (Haushaltsrechts-Fortentwicklungsgesetz) apporta à partir de 1998 plus de flexibilité dans la gestion budgétaire et renforça les incitations à une bonne gestion en :

○ élargissant la capacité de couverture réciproque et les possibilités de report de titres,

○ assouplissant le principe d'universalité budgétaire, l'objectif étant d'inciter ainsi à faire des économies et des recettes supplémentaires puisque les moyens supplémentaires dégagés restaient dans les caisses des services concernés,

\footnotetext{
${ }^{16} \mathrm{Cf}$. notamment ministère fédéral des Finances (Bundesministerium der Finanzen, 1989), p. 78.

${ }^{17}$ Le fait que la part de la dette dans le produit intérieur brut (PIB) augmente ou non dépend comme on le sait du déficit primaire et du ratio à long terme entre taux d'intérêt et taux de croissance. Si le taux d'intérêt applicable à la dette publique est supérieur au taux de croissance du PIB (ce qui est la situation normale sur le long terme), un déficit primaire entraîne une augmentation de la part de la dette dans le PIB. Sur l'évolution réelle du niveau d'endettement et de la part de la dette dans le PIB en Allemagne, voir la partie «Constantes à moyen et à long terme de la politique budgétaire allemande effective » dans cet article.
} 
- donnant plus d'importance à la comptabilité analytique et autres calculs de rentabilité,

- la loi de modernisation des principes budgétaires, entrée en vigueur en 2010, permit d'expérimenter différentes formes de comptabilité dans les collectivités territoriales. De nombreuses communes et quelques Länder sont par exemple passés à une comptabilité en partie double et se sont mis à faire des budgets par produits. Le Bund et les Länder ont mis en place des groupes de travail et des normes minimales, ce qui assure la comparabilité nécessaire de la comptabilité ;

- en 2011, lors de la programmation du budget 2012, l'État fédéral est passé du procédé ascendant (bottom-up) au procédé descendant (topdown). Cela signifie que ce ne sont pas les différents ministères qui formulent des demandes relatives à leur budget pour tenter ensuite de les imposer au ministère fédéral des Finances : c'est ce ministère qui définit les grandes lignes de cadrage d'un budget prévisionnel pour le compte du gouvernement. Ce budget prévisionnel définit de manière globale et contraignante les enveloppes budgétaires et financières pour l'ensemble des ministères. Le ministre des Finances est en position de force car il ne peut être mis en minorité que si le chancelier ou la chancelière vote également contre lui $(\S \S 28$ et $29 \mathrm{BHO}$, en lien avec le $\S 26$ $\mathrm{du}$ règlement du gouvernement fédéral - Geschäftsordnung der Bundesregierung, GOBReg). Sur la base du cadrage, les ministres peuvent ensuite programmer les détails pour chacun de leurs domaines. Ils doivent ensuite présenter ce projet de programmation au ministère des Finances. Puis le budget et la programmation financière sont une seconde fois définis par le gouvernement et transmis au Bundestag et au Bundesrat. Cette programmation descendante comporte deux grands avantages : d'une part, elle permet de mieux fixer les priorités politiques du gouvernement en termes de contenus et de calendrier; d'autre part, de mieux garantir le respect des règles définies par l'UE et l'UEM ainsi que du frein à l'endettement - qu'il convient maintenant de présenter grâce aux soldes budgétaires structurels (c'est-à-dire corrigés des variations conjoncturelles) préalablement définis.

L'introduction en 2009 du «nouveau » frein à l'endettement est sans doute le changement le plus important ayant affecté le droit budgétaire allemand depuis la "grande réforme budgétaire ». Il a fallu reformuler les articles 109, 109a et 115 GG et donc remplacer ainsi intégralement l' « ancien » frein à l'endettement - que l'on n'appelait toutefois pas encore ainsi - avec ses points faibles déjà évoqués ${ }^{18}$.

\footnotetext{
${ }^{18}$ Sur le sujet, cf. Kastrop/Meister-Scheufelen/Sudhoff (2010). On trouvera un rapport sur la première application du frein à l'endettement dans un article du ministère fédéral des Finances (Bundesministerium der Finanzen, 2012b).
} 
Dans les grands traits, l'article 109 GG (nouvelle version) prévoit que les budgets de l'Etat fédéral et des Länder doivent par principe être couverts sans recourir à des emprunts. Pour l'État fédéral, ce principe est considéré comme respecté dès lors que les recettes issues d'emprunts n'excèdent pas $0,35 \% \mathrm{du}$ produit intérieur brut (PIB) nominal ${ }^{19}$. Il doit parvenir à cet objectif d'ici 2016. Pour les Länder et les communes, aucun déficit structurel ne sera plus autorisé à partir de 2020. Le Bund et les Länder sont cependant autorisés à s'écarter de ce principe pour mener une politique atténuant les fluctuations d'activité et pour faire face à des catastrophes naturelles et d'autres urgences exceptionnelles. En cas de tels écarts, l'art. 115 GG (nouvelle version) prévoit cependant l'obligation de mettre en place un compte de contrôle sur lequel tous les déficits doivent être enregistrés. Ces déficits conjoncturels peuvent représenter jusqu'à 1,5\% du PIB. En cas de dépassement de ce taux-plafond, il convient de procéder à une diminution en fonction de la conjoncture. Les catastrophes naturelles et situations d'urgence peuvent amener à relever les plafonds de crédit si la majorité des membres du Bundestag ( Kanzlermehrheit») en a décidé ainsi et en accompagnant cette mesure d'un échéancier de remboursement approprié.

$\mathrm{Du}$ fait de ces règles, les plafonds d'endettement ne sont plus fixés en lien avec une notion d'investissement non définie - comme c'était le cas avec les versions précédentes des articles 109 et $115 \mathrm{GG}$ - mais en fonction de bases de calcul nettement plus concrètes. La tenue d'un compte de contrôle implique en outre que le dépassement des plafonds dans le passé ne peut plus être «oublié »; ces plafonds gardent désormais leur fonction de bornes. S'ajoute à cela que l'article 115 GG (nouvelle version) prend en compte l'endettement effectif ex post et non plus l'endettement prévu, sensiblement plus facile à manipuler.

L'article 109a GG instaure en outre un "Conseil de stabilité », qui reprend les fonctions de ce qui s'appelait jusque-là le «Conseil de programmation financière » (Finanzplanungsrat). Ses missions, décrites plus précisément dans la loi ad hoc (Stabilitätsratsgesetz), consistent à contrôler l'évolution des budgets de l'État fédéral et des Länder en s'appuyant sur des rapports de stabilité et un certain nombre d'indicateurs. Il s'agit au premier chef d'éviter les situations d'urgence budgétaire susceptibles d'avoir un impact sur la péréquation financière Bund/Länder. Dans le même temps, la publication de ces rapports par le Conseil de stabilité pourrait avoir également la conséquence suivante : informer l'opinion d'un risque de dérive assez tôt pour permettre un changement de cap, qui aurait de fortes chances de se produire.

L'efficacité du frein à l'endettement fait l'objet d'appréciations variables selon les sources ${ }^{20}$. Il me semble toutefois clairement exagéré de supposer que

\footnotetext{
${ }^{19}$ Pour le calcul des recettes issues de prêts, un certain nombre de transactions financières sont exclues suivant un procédé décrit à l'alinéa 3 de l'article 115 de la Loi fondamentale. Là encore, pour être bref, nous n'entrerons pas dans le détail, bien que des possibilités de contournement existent théoriquement.

${ }^{20}$ Citons comme exemple d'appréciation positive Meister-Scheufelen (2011); pour illustrer le scepticisme : Magin (2010). Cette règle a sans doute eu un impact positif sur la place de l'Allemagne sur les marchés financiers, puisque son adoption indiquait une volonté de consolidation et que le déficit a effectivement diminué
} 
ce dispositif est totalement inefficace. C'est sur cette note plutôt optimiste que se clôt cette partie sur l'évolution du droit budgétaire et que nous passons à la présentation d'évolutions caractéristiques pendant des périodes données de la politique budgétaire allemande.

\section{Constantes à moyen et à long terme de la politique budgétaire allemande effective}

\section{Remarques méthodologiques préliminaires}

Si l'on veut diviser la période allant de 1949 à 1999 en phases caractéristiques de politique budgétaire à moyen terme, il faudrait, afin d'opérer un découpage objectif à l'aide d'outils économétriques (avec un test de rupture structurelle adapté), que deux conditions soient réunies :

- un consensus - au moins parmi les économistes et, si possible, les responsables politiques - sur ce qui relève précisément de la «politique budgétaire » et sur les indicateurs permettant de décrire de manière satisfaisante la politique budgétaire menée dans un pays donné ;

- pour l'ensemble de ces indicateurs, l'existence, sur l'ensemble de la période, de valeurs mesurables ayant été obtenues à l'aide de définitions opérationnelles (conditions de mesure) valables et constantes.

Or aucune de ces deux conditions n'est malheureusement remplie. C'est pourquoi j'ai été contraint de procéder de manière subjective à la fois pour le choix des indicateurs permettant de décrire la politique budgétaire allemande et pour le découpage en périodes. J'ose toutefois espérer que le résultat comporte un certain degré d'objectivité qui apparaîtrait si l'on demandait à d'autres spécialistes de se prononcer.

Pour décrire la politique budgétaire, j'utilise les séries chronologiques des indicateurs suivants (pour l'ancienne RFA jusqu'à la réunification, pour l'Allemagne unie ensuite) :

- indicateurs de l'ampleur et du succès de la politique d'allocation des ressources menée par l'État, à savoir la part des dépenses publiques, celle des prélèvements fiscaux et le taux de croissance du produit intérieur brut. Chacun sait que la part des dépenses publiques et celle des prélèvements fiscaux ne donnent qu'un tableau imparfait du volume des ressources publiques allouées ${ }^{21}$. Ainsi, ces indicateurs ne rendent pas suffisamment compte d'arbitrages représentant un budget peu important

depuis. En revanche, les décisions prises par la grande coalition actuellement au pouvoir en matière de retraites sont préoccupantes car elles montrent que l'on peut remplacer une dette publique explicite par une dette implicite et de quelle manière; elles signalent qu'il est possible de ne pas respecter l'esprit du frein à l'endettement.

${ }^{21}$ Pour plus de détails sur ce point, cf. Schratzenstaller (2013). 
et influant sur l'affectation de ressources principalement à travers la mise en place d'un cadre juridique. En Allemagne, on peut citer l'exemple de la politique de protection de l'environnement, qui a pris de l'importance dans les années 1970, mais qui utilisait initialement avant tout le droit de la police. Si l'on avait au contraire opté pour un pilotage par des instruments fiscaux, le dispositif aurait eu une incidence sur la part des dépenses publiques et celle des prélèvements fiscaux, et l'enveloppe budgétaire aurait été modifiée. Mais à défaut de meilleures alternatives, il faut accepter ces erreurs de mesure (un argument que l'on peut également opposer aux autres grands indicateurs). S'agissant de la part des dépenses publiques dans le PIB, on a le choix entre la part des dépenses publiques au sens strict (la part de la consommation et des investissements des pouvoirs publics dans le PIB) et au sens large. Dans ce dernier cas, il s'agit d'une part artificielle, qui intègre, outre la part des dépenses publiques au sens strict, également les transferts sociaux et les subventions. L'ampleur de ces deux derniers facteurs, déterminée par l'État, ayant incontestablement des effets allocatifs, nous nous appuierons ci-dessous sur la part des dépenses publiques au sens large pour décrire la politique d'allocation des ressources. Si l'État ne peut agir directement sur le taux de croissance du PIB réel, il est toutefois en mesure d'éviter des pertes de croissance inutiles en créant des conditions favorables. De ce point de vue, un taux de croissance élevé en moyenne sur la période pourrait apparaître comme une preuve de réussite de la politique publique d'allocation des ressources, et une baisse de ce taux comme une preuve d'échec. Or l'expérience montre qu'en Allemagne tout comme dans la plupart des autres pays développés - la croissance du PIB réel est plutôt de type linéaire depuis 1950 (croissances moyennes constantes annuelles, en chiffres absolus), ce qui correspond à des taux de croissance moyens en baisse ${ }^{22}$. Il ne faut donc pas interpréter ces taux comme des indices d'échec de manière mécanique, mais uniquement lorsqu'il y a une explication plausible à cet échec ;

- des indicateurs relatifs à l'ampleur de la politique publique distributive, à savoir la part des prestations sociales dans le PIB (laquelle prend en compte l'ensemble des prestations sociales émanant des pouvoirs publics, y compris celles qui sont financées à travers l'impôt) et la part des prélèvements sociaux. Les données relatives à l'effet redistributif résultant de la progressivité de l'impôt sur le revenu ne sont pas prises en compte, pas plus que celles concernant la redistribution opérée par l'ensemble du système de transfert des recettes fiscales. En effet, il est difficile d'obtenir en la matière des données reconnues comme adaptées.

\footnotetext{
${ }^{22}$ Cf. l'Institut d'études de croissance (Institut für Wachstumsstudien, 2005). Des taux de croissance constants requéraient des accroissements exponentiels.
} 
- des indicateurs caractérisant l'ampleur et le succès de la politique de stabilité, autrement dit la part du déficit et celle de la dette dans le PIB, l'opinion de la population quant au risque lié à la dette publique ainsi que l'évolution des taux d'inflation et de chômage ;

- des déclarations politiques sur les priorités d'action au cours des différentes phases ainsi que les lectures qui en sont faites dans les publications spécialisées. Ces données sont de nature qualitative. On pourrait en principe également les quantifier à l'aide d'analyses quantitatives de contenu. Mais les conditions qui le permettraient ne sont en l'occurrence pas réunies. C'est la raison pour laquelle elles sont exploitées sous forme descriptive ${ }^{23}$.

Les indicateurs cités aux premier et troisième points vont de pair avec trois grands objectifs de la loi allemande sur la stabilité et la croissance : assurer la stabilité monétaire, éviter le chômage involontaire et assurer une croissance économique satisfaisante. L'objectif d'équilibre du commerce extérieur n'est pas pris en compte. La politique budgétaire peut théoriquement aussi jouer un rôle s'agissant d'atteindre cet objectif. C'est le cas lorsqu'un déficit budgétaire conduit - comme cela fut longtemps le cas aux États-Unis - à un déficit de la balance courante, ou quand les exportations sont subventionnées par les pouvoirs publics. Mais ce scénario ne s'applique pas pour l'Allemagne : il n'y a ni déficit de la balance courante induit par l'État, ni stratégie ou promotion publique d'exportation. Les excédents persistants de la balance courante sont davantage dus à la compétitivité allemande et au cours de l'euro, trop bas pour l'Allemagne, et/ou au fait qu'un placement à l'étranger est considéré plus rentable que dans le pays même. C'est pourquoi le solde courant n'est pas utilisé comme indicateur dans ce qui suit.

Pour la quasi-totalité des indicateurs cités (ainsi que d'autres, liés à l'évolution des investissements et des subventions, utilisés dans certains cas), on dispose de séries chronologiques, quoiqu'elles ne soient pas toujours continues. Pour le taux de croissance du PIB réel de 1950 à 1969, il n'y a pas encore de données établies officiellement sur la base des prix en euros 1995. C'est pourquoi je m'appuie pour cette période sur des données de l'Institut d'études de croissance ${ }^{24}$. Pour ce qui est de l'appréciation que les Allemands portent sur la dette publique, un facteur non négligeable à mon avis, on ne dispose curieusement pas de série chronologique continue ${ }^{25}$. Il faut donc s'appuyer sur des

\footnotetext{
${ }^{23}$ L'histoire de la guerre et la stratégie militaire ont beaucoup bénéficié de la présence, au sein des forces armées des pays développés, d'auteurs tenant un journal de guerre pendant les conflits : on y trouve en effet la vision (subjective) de la situation et les actions que décidait par conséquent un chef militaire suivant une méthode pour laquelle des normes internationales se sont développées. Il serait théoriquement envisageable de susciter la tenue analogue de journaux budgétaires et, passé un délai raisonnable, de permettre à des économistes d'y accéder pour exploiter ces données. Non seulement cette démarche serait intéressante pour les historiens de l'économie, mais il serait aussi plus facile de tester des hypothèses.

${ }^{24}$ Institut für Wachstumsstudien (2005).

${ }^{25}$ Neumark (1952, p. 655) estimait déjà lui aussi que l'opinion d'une population sur la dette publique a une importance politique, aussi et surtout pour l'efficacité de la politique fiscale en tant que telle. On peut au-
} 
études diverses menées à différents moments. Il a fallu attendre la crise financière pour que la Commission européenne prenne conscience de l'importance de ce facteur. Depuis 2010, l'Eurobaromètre donne des informations sur ce point ; depuis lors, les données recueillies montrent que la crainte d'une dette publique trop élevée est bien plus forte en Allemagne que dans d'autres pays (voir la sous-partie «Constantes à long terme» dans cet article). Cela tient sans doute au fait que le pays a, par deux fois, connu un endettement de l'État trop élevé, lequel avait entraîné l'inflation et une réforme monétaire à l'occasion desquelles une partie importante de la bourgeoisie avait subi d'importantes pertes patrimoniales et la perte d'une part non négligeable de ses réserves constituées au titre de la prévoyance vieillesse.

$\mathrm{Au}$ vu de l'évolution des indicateurs présentés et sur la base des déclarations de responsables politiques et des présentations faites dans la littérature ainsi que sur mes impressions d'observateur averti de la politique budgétaire en quelque sorte sur la base d'un test subjectif théorique de rupture -, j'ai acquis la conviction que l'on peut découper la politique budgétaire allemande de 1949 au tournant du millénaire en cinq périodes de moyen terme présentant des caractéristiques propres :

- la première phase de reconstruction, jusqu'au milieu des années 1950 ;

- la période souvent qualifiée d' «âge d'or de l'après-guerre », jusqu'au milieu des années 1960 ;

- la période des expériences keynésiennes jusqu'au début des années 1980 ;

- la période politique de consolidation dans les années 1980 ;

- la seconde période de reconstruction dans les années 1990, après la réunification.

L'on pourrait commenter des tableaux comportant les indicateurs cités, mais il me paraît utile de partir de descriptions qualitatives des différentes périodes et de présenter l'évolution des indicateurs sous forme narrative. Ce procédé permet de mieux saisir l'esprit de l'époque et de mettre en évidence les priorités d'action de la politique budgétaire au cours des différentes phases.

\section{Constantes à moyen terme de la politique budgétaire allemande}

$\mathrm{Au}$ cours de la première phase de reconstruction, la politique budgétaire visait surtout à résoudre le problème des réfugiés et du logement. Elle servait aussi,

\footnotetext{
jourd'hui décrire les canaux d'influence à l'aide des trois hypothèses suivantes : plus une population s'inquiète de l'étendue de la dette publique, (1) plus le niveau d'épargne est élevé (théorème de Ricardo/Barro), (2) plus le volume d'investissement est faible (effets d'éviction) et (3) plus la probabilité de réélection est faible pour les membres du gouvernement. Les deux premières hypothèses concernent l'efficacité d'un financement par le déficit; la troisième décrit une limite politico-psychologique à la poursuite de l'endettement. Il semble que cette limite ait gagné en importance depuis que les électeurs disposent, avec les critères européens de stabilité, d'un repère quant à ce qu'est une dette publique «trop élevée ", les responsables politiques allemands redoutant quant à eux de recevoir un avertissement de la part de Bruxelles.
} 
plus globalement, un objectif allocatif : augmenter la part des investissements et, ainsi, la croissance économique, laquelle atteignit au cours de cette période une valeur moyenne de $9,6 \%{ }^{26}$. Cette politique de soutien se traduisit par des investissements publics importants dans les infrastructures, des prêts accordés à des investisseurs privés par les collectivités locales et par le fonds de compensation (Lastenausgleichsfonds), ainsi que par des avantages fiscaux favorisant la constitution d'une épargne. La compensation évoquée devait servir un objectif distributif : dédommager les personnes - en particulier les réfugiés - qui avaient perdu une partie importante de leur patrimoine, en puisant dans les ressources de celles qui avaient été relativement épargnées. Mais comme les prélèvements au titre de la compensation se sont étalés sur trente ans, les revenus du patrimoine suffirent le plus souvent pour les couvrir ${ }^{27}$. Certaines parts d'indemnités étant versées avec du retard, un fonds se constitua, qui permit de couvrir des prêts en particulier pour la construction de logements et la création d'activités professionnelles indépendantes. L'État ouest-allemand devait alors financer l'intégration de millions de réfugiés qui arrivaient des anciens territoires de l'Est, de soldats qui revenaient de captivité et de réfugiés du territoire de la République démocratique allemande (RDA), ainsi que les dépenses liées à l'occupation par les Alliés d'un niveau qui représentait par exemple plus de $41 \%$ du budget fédéral en $1951^{28}$. La République fédérale parvint à réaliser des investissements et à verser des subventions au profit d'investissements considérables malgré ces charges et la faiblesse de la part des dépenses publiques et celle des prélèvements fiscaux dans le PIB (cf. infra); elle put également éviter de faire du deficit spending; en effet, la réforme monétaire et les lois qui l'accompagnaient lui avaient permis de se libérer de la masse des dettes internes publiques du pays, et les accords de Londres (1953) avaient effacé une partie des dettes extérieures allemandes ${ }^{29}$. À partir de 1951, les puissances d'occupation mirent en outre un terme au démontage des installations industrielles. Enfin, dans le sillage des tensions croissantes entre l'Est et l'Ouest, le plan Marshall entra en vigueur en 1948 ; si l'apport de ce dispositif n'était quantitativement pas très important pour l'Allemagne, il fit naître un climat économique favorable et, conjugué avec la politique commerciale américaine et la création de l'Organisation de coopération et de développement économiques (OCDE), il conduisit à

\footnotetext{
${ }^{26}$ Calcul de la valeur moyenne suivant les données de l'Institut d'études de croissance (Institut für Wachstumsstudien, 2005), p. 3. Cf. également Pötzsch (2006), p. 95. On trouve une synthèse des problèmes les plus urgents de cette époque aux Archives fédérales (Bundesarchiv, Kabinettsprotokolle, en particulier 1949 et 1950).

${ }^{27}$ Sur la péréquation des charges, cf. ministère fédéral des Finances (Bundesministerium der Finanzen, 1989), p. $150-151$.

${ }^{28}$ Cf. sur ce point ministère fédéral des Finances (Bundesministerium der Finanzen, 1989), p.155. Sur l'intégration des réfugiés, qui représentaient en 1950 environ $17 \%$ des habitants de l'ouest de l'Allemagne, cf. pour une vision à assez long terme Bauer/Braun/Kvasnicka (2013).

${ }^{29}$ Sur la gestion de la dette domestique et de la dette extérieure de l'Allemagne, cf. ministère fédéral des Finances (Bundesministerium der Finanzen, 1989), p. 152-154. Dans ce contexte, il faut également évoquer les cessions de biens de l'Allemagne au profit de pays étrangers, ainsi que les réparations versées en particulier à Israël. Ces aspects ne sont pas développés ici. Cf. sur ce point ibid., p. 147-150.
} 
une vague de libéralisation ${ }^{30}$. De 1953 à 1955, les Alliés renoncèrent de surcroît à une part des remboursements au titre des frais d'occupation. Sans pouvoir le démontrer, je présume que ce fut un geste accordé en échange du consentement de la RFA à créer la Bundeswehr. Toujours est-il que les moyens financiers ainsi économisés abondèrent un fonds spécial qui contribua par la suite à financer le réarmement. En référence à l'une des tours de la citadelle de Spandau, où était gardé avant 1914 le «trésor de guerre du Reich», ce fonds fut surnommé « Juliusturm »; il contribua à la stabilisation du développement du pays, bien qu'il n'y eût pas de politique visant spécifiquement la stabilisation ${ }^{31}$.

$\mathrm{Au}$ cours de cette première période, la part des dépenses publiques dans le PIB était de $31 \%$ à $32 \% 32$. Selon les données du ministère fédéral des Finances (2011), la proportion des prélèvements fiscaux restait, depuis les années 1950, proche de $22,6 \%$, sa valeur moyenne sur le long terme, et atteignit dans la période allant jusqu'en 1955 une valeur moyenne de $23,9 \%{ }^{33}$. Au cours de cette période, la politique de redistribution visant à réduire les écarts de revenu et de patrimoine n'était pas en soi un objectif politique prioritaire. La part des prestations sociales était néanmoins élevée, avec $28 \%$ à $29 \%$ du PIB, car il fallait régler d'importantes conséquences de la guerre ${ }^{34}$. Les prélèvements sociaux représentaient en moyenne $8,6 \%$ du PIB $^{35}$. Comme il a déjà été dit, il n'y avait pas de politique visant expressément la stabilisation. Le solde budgétaire (selon les critères de la comptabilité nationale) était positif et oscillait entre $0,6 \%$ et $11,1 \%$ du PIB, le taux d'endettement variait de $17,6 \%$ à $24,6 \% \mathrm{du} \mathrm{PIB}^{36}$. Le taux de chômage était élevé au début de la période (environ $11 \%$ en 1950), mais il diminua jusqu'en 1955 pour atteindre $5,6 \%{ }^{37}$. Le taux d'inflation connut des fluctuations importantes et tomba de $9,2 \%(1951)$ à $1,1 \%(1955)^{38}$. Quant à l'opinion de la population sur la dette publique, elle ne faisait pas l'objet de sondages à l'époque. Nous disposons uniquement d'une indication indirecte : à la question de savoir quelle opinion elles avaient de la constitution du fonds

\footnotetext{
${ }^{30}$ Cf. Pötzsch (2006), p. 53-55.

${ }^{31}$ Sous la pression des Alliés, il y eut en 1950 un «programme de création d'emplois ». C'était toutefois moins un véritable plan de relance qu'une panoplie de mesures présentée comme un tel programme dans le cadre d'un «stratagème ", tel que l'indique en 1950 l'introduction aux procès-verbaux de réunion ministérielle (Bundesarchiv, Kabinettsprotokolle, 1950).

${ }^{32}$ Je remercie l'Office fédéral des statistiques de m'avoir communiqué une vaste série de données sur la part des dépenses publiques dans le PIB, dont proviennent les chiffres ci-dessus. Il y est fait référence ci-dessous sous l'intitulé Statistisches Bundesamt (2014). Vaut à chaque fois la situation territoriale de l'époque.

${ }^{33}$ Le ministère fédéral des Finances a eu l'amabilité de me fournir des données sur la part des prélèvements fiscaux et sociaux dans le PIB de 1950 à 2013 (cité ci-dessous sous l'intitulé Bundesministerium der Finanzen, 2014).

${ }^{34}$ Cf. Ehrlicher (1981), p. 178-179.

${ }^{35}$ Données : ministère fédéral des Finances (Bundesministerium der Finanzen, 2014).

${ }^{36}$ La Bundesbank a eu l'amabilité de mettre à ma disposition de vastes séries de données sur le solde budgétaire et le taux d'endettement par rapport au PIB selon les critères de la comptabilité nationale (au sens de Maastricht à partir de 1991). Les chiffres ci-dessus en proviennent. Il y est fait référence ci-dessous sous l'intitulé Deutsche Bundesbank (2014a). Vaut à chaque fois la situation territoriale de l'époque.

${ }^{37}$ Cf. Statistisches Bundesamt (2013a).

${ }^{38}$ Cf. Statistisches Bundesamt (2013b).
} 
« Juliusturm » et des excédents associés, $46 \%$ des personnes interrogées répondirent en mars 1956 : «une bonne $»^{39}$. On peut en déduire que la création d'un déficit aurait sans doute été considérée «mauvaise», et ce avant même 1956 puisqu'il y avait eu des excédents budgétaires dans les années antérieures à cette date.

Globalement, cette phase se caractérise par les grandes lignes suivantes : priorité à une politique d'allocation des ressources orientée vers la croissance, avec un taux de dépenses publiques faible et un taux de prélèvements fiscaux légèrement supérieur à la moyenne. Une politique de redistribution non prioritaire, avec des prélèvements sociaux proportionnellement très faibles, mais un taux de prestations sociales gonflé durablement par les conséquences de la guerre. Malgré l'effet stabilisateur de la mise en place et (ultérieurement) de la suppression du fonds spécial, malgré également des taux d'inflation et de chômage initialement élevés puis en baisse rapide, aucune politique résolument tournée vers la stabilisation et, surtout, aucun financement par le déficit, mais un solde budgétaire positif et un niveau d'endettement très bas. Refus, chez une part importante de la population, du financement par le déficit budgétaire.

Dans ce que l'on a appelé l'âge d'or de l'après-guerre, de 1955 à 1965, les dépenses publiques représentaient $30 \%$ à $37 \%$ du $\mathrm{PIB}^{40}$ et les prélèvements fiscaux $23,4 \%$ en moyenne ${ }^{41}$. Les dépenses au titre de la défense du pays, venues remplacer les dépenses liées à l'occupation par les Alliés depuis l'adhésion de la RFA à l'Organisation du traité de l'Atlantique Nord (OTAN) et à l'Union de l'Europe occidentale (UEO) et, partant, la fin du régime d'occupation, étaient nettement inférieures à celles-ci. Mais, alors que la croissance avait été au cours de la période précédente la priorité absolue, l'objectif de redistribution passa au premier plan dans cette seconde période. Le taux de croissance moyen tomba à $5,9 \%{ }^{42}$. Le soutien à la croissance avait surtout augmenté les revenus et les patrimoines des entrepreneurs. Dorénavant, il s'agissait, selon les déclarations faites en ce sens par le gouvernement, de permettre aux salariés de se constituer un patrimoine ${ }^{43}$. Cela se traduisit notamment par la législation sur les primes d'épargne et le soutien fiscal à des prestations d'employeurs favorisant la constitution de patrimoine chez les salariés, mais aussi par l'augmentation des subventions aux secteurs en difficultés, y compris des subventions comme celles versées à l'agriculture ou au secteur minier, qui eurent pour effet la conservation des structures existantes. La part des prélèvements sociaux était en moyenne de $10,3 \%$ du PIB $^{44}$, celle des pres-

\footnotetext{
${ }^{39}$ Cf. Noelle/Neumann (1957), p. 213.

${ }^{40} \mathrm{Cf}$. Bundesministerium der Finanzen (2013b) et Statistisches Bundesamt (2014).

${ }^{41} \mathrm{Cf}$. Bundesministerium der Finanzen (2012c et 2014).

${ }^{42}$ Données : Institut d'études de croissance (Institut für Wachstumsstudien, 2005), p. 3. Cf. également Räth (2009), p. 204.

${ }^{43}$ Cf. Bundesministerium der Finanzen (1989), p. 71.

${ }^{44}$ Données : Bundesministerium der Finanzen (2012c et 2014).
} 
tations sociales, d'environ $21 \%{ }^{45}$. La plus importante mesure en termes de politique distributive fut la revalorisation des pensions de vieillesse avec la réforme des retraites de 1957, qui instaura l'indexation des pensions sur l'évolution des salaires. Cela permit de limiter l'appauvrissement de nombreux retraités lié à l'inflation et de faire profiter les bénéficiaires de pensions de vieillesse du boom économique. Au cours de cette phase, il n'y eut pas non plus de politique tournée délibérément vers la stabilisation. Jusqu'en 1965, le budget de l'État présentait un solde positif oscillant entre $-0,6 \%$ et $+4,7 \%$ du PIB ; le taux d'endettement public représentait entre $16,7 \%$ et $21,1 \%$ du $\mathrm{PIB}^{46}$. Le taux de chômage tomba à $0,7 \%{ }^{47}$; le taux d'inflation était en général entre $0 \%$ et $2 \%$, soit un corridor considéré aujourd'hui comme souhaitable ${ }^{48}$. Pour ce qui est de l'opinion de la population quant à la dette publique, on ne peut, faute d'autres sondages, que renvoyer à l'enquête de 1956 déjà citée, laquelle fait ressortir une aversion à l'égard du financement des dépenses par le déficit budgétaire.

L'on retiendra globalement les caractéristiques suivantes : priorité à la politique distributive, associée à une hausse de la part des prélèvements sociaux et des prestations sociales dans le PIB ; par ailleurs, des taux de croissance encore supérieurs à la moyenne, malgré une hausse de la part des dépenses publiques et une part de prélèvements fiscaux tout juste supérieure à la moyenne. Pas de politique visant expressément la stabilisation, un solde budgétaire positif et un niveau d'endettement très bas. Un taux de chômage extrêmement faible à la fin de la période, un taux d'inflation situé dans une fourchette aujourd'hui considérée comme souhaitable. Refus, chez une large part de la population, d'un financement des dépenses par le déficit.

Avec la période des expériences d'inspiration keynésienne, l'objectif de stabilisation passa au premier plan, au moins formellement (quoique ce ne fut pas vraiment une réussite). Les chercheurs avaient déjà très tôt formulé des demandes en ce sens, par exemple dans deux rapports d'expertise rédigés conjointement par les conseils scientifiques des ministères fédéraux des Finances et de l'Économie en 1955 et $1956^{49}$. Il s'agissait de recommandations en matière de politique conjoncturelle et d'inscription dans le droit d'instruments à cette fin. S'y ajouta en 1966 un rapport d'expertise de la Commission pour la réforme financière, favorable à une programmation financière à moyen terme $\mathrm{e}^{50}$. La mise en œuvre politique se trouva facilitée par deux facteurs : d'une part, la République fédérale connut en 1966-1967 une première récession, avec une hausse passagère du taux de chômage, de $0,7 \%$ à $2,1 \%$ (un chiffre qui semble au-

\footnotetext{
${ }^{45}$ Cf. ministère fédéral du Travail et des Affaires sociales (Bundesministerium für Arbeit und Soziales, 2007), p. 6.

${ }^{46}$ Cf. Deutsche Bundesbank (2014a).

${ }^{47}$ Cf. Statistisches Bundesamt (2013a).

${ }^{48}$ Cf. Statistisches Bundesamt (2013b).

${ }^{49}$ Cf. Wissenschaftliche Beiräte beim Bundesministerium der Finanzen und beim Bundesministerium für Wirtschaft (1955 et 1956).

${ }^{50}$ Kommission für die Finanzreform (1966).
} 
jourd'hui dérisoire $)^{51}$. Cette évolution ébranla la conviction, alors largement répandue chez les responsables politiques de l'époque, que le pays ne connaîtrait plus de fluctuations conjoncturelles. D'autre part, un nouveau gouvernement arriva aux commandes en 1966. Ce fut la première grande coalition du pays, et Karl Schiller devint ministre de l'Économie. Schiller avait largement contribué à l'adoption par le SPD du programme dit de Bad Godesberg, lequel entérinait la rupture avec l'économie planifiée et centralisée et le passage à l'économie de marché. En tant qu'universitaire - il était professeur d'économie à l'université de Hambourg -, Schiller aspirait à faire la synthèse entre l'ordolibéralisme souhaité par Eucken et la politique de relance prônée par Keynes. En sa qualité d'homme politique, il vit dans ses nouvelles fonctions l'opportunité de réconcilier l'aile gauche du SPD avec les principes de l'économie de marché. En effet, son souhait de mettre en place un pilotage global, auquel participeraient les syndicats et les employeurs dans ce qui était qualifié d'action concertée, rejoignait d'anciens projets du SPD visant à instituer un Conseil économique fédé$\mathrm{ral}^{52}$. Ces circonstances conduisirent finalement à l'adoption de la loi sur la stabilité et la croissance en 1967.

Cette loi était à orientation anticyclique et se heurta à ce titre aux critiques du Conseil d'experts pour l'évaluation de la situation économique qui craignait - à juste titre, comme les développements ultérieurs allaient le montrer - que la politique et l'administration ne soient dépassées. Ce Conseil était favorable à une stratégie de pérennisation, par-delà les cycles économiques ${ }^{53}$. La loi prévoyait, outre une série d'instruments d'information, de programmation et de coordination, douze dispositifs financiers d'intervention, qui devaient avoir pour moitié un effet d'expansion et pour l'autre moitié, de contraction sur les recettes ou les dépenses budgétaires ${ }^{54}$. Mais les interventions réelles pour la période 1970 à 1982 furent très majoritairement de nature expansive et consistèrent surtout à financer des dépenses publiques supplémentaires par le déficit budgétaire. À partir de 1974 , le déficit fut la norme ${ }^{55}$, y compris en l'absence de déséquilibre macroéconomique ou lorsque les causes d'un tel déséquilibre étaient à rechercher du côté de la demande. Ainsi, c'est une stratégie de la demande qui fut adoptée lorsque les syndicats, confiants dans les garanties d'emploi apportées par l'État, imposèrent en 1971 une hausse des salaires de $15 \%{ }^{56}$; c'est également cette stratégie qui fut retenue en réponse à la réduction des marges de manœuvre budgétaires qui résultèrent des deux chocs pétroliers des années 1970. La politique prétendument anticyclique eut en réalité surtout pour conséquence l'assouplisse-

\footnotetext{
${ }^{51} \mathrm{Cf}$. Conseil d'experts pour l'évaluation de la situation économique (Sachverständigenrat, 1968), p. 118.

${ }^{52}$ Cf. Grossekettler (2008), p. 227 avec d'autres références.

${ }^{53}$ Cf. Sachverständigenrat (1966), p. 143-145.

${ }^{54}$ Sur les effets prévus et pour une analyse critique, cf. Grossekettler (2008), p. 230-240.

${ }^{55}$ Cf. Deutsche Bundesbank (2014a) et Statistisches Bundesamt (2012).

${ }^{56}$ Cf. von Hagen/Strauch (2001), p. 12.
} 
ment des restrictions budgétaires ${ }^{57}$. Il se produisit ainsi une hausse de la part des dépenses publiques, des subventions, des prestations sociales et des prélèvements fiscaux dans le PIB, mais aussi du ratio des intérêts sur la dette publique et de la part des prélèvements sociaux, des taux de déficit et d'endettement. La part des dépenses publiques passa de $39 \%$ (1969) à 48,6\% du PIB (1975), avant de retomber à $47,5 \%(1982)^{58}$. La part des prélèvements fiscaux fut en moyenne de $23,7 \%^{59}$; celle des prélèvements sociaux grimpa de $10,3 \%$ à $14,0 \%{ }^{60}$ et la part des prestations sociales passa de $22,5 \%$ (1965) à $30 \%(1982)^{61}$. À partir de 1975, la part du déficit dans le PIB dépassa à plusieurs reprises le plafond européen (qui n'existait pas encore) de $3 \%$ et atteignit en 1975 une valeur record de $5,6 \%$ du $\mathrm{PIB}^{62}$. Le taux d'endettement grimpa de $19,1 \%$ à près de $39 \%$ du $\mathrm{PIB}^{63}$ et les subventions officiellement attestées doublèrent également, passant de 16 milliards d'euros (1970) à environ 32 milliards $(1982)^{64}$. La part des salaires dans le revenu national passa d'environ $60 \%$ (1970) à $73 \%$ (1982); la baisse du taux de bénéfice qui s'ensuivit entraîna un basculement d'investissements visant l'extension des capacités de production vers des investissements de rationalisation $^{65}$. Cela conduisit à une baisse des taux de croissance moyens, qui tombèrent à $2,8 \%{ }^{66}$, tandis que le taux de chômage passait de $0,7 \%(1970)$ à $8 \%(1982)^{67}$. Enfin, le taux d'inflation atteignit une valeur de $6,3 \%(1981)^{68}$. Ce repli du taux de croissance était pour partie lié à une tendance générale et dû pour une autre à des dysfonctionnements probablement exogènes comme les chocs pétroliers. Mais les mauvaises conditions politiques ont probablement aussi joué un rôle. Cette situation économique eut d'ailleurs des conséquences politiques : en 1971 et 1972, Alex Möller et Karl Schiller démissionnèrent successivement du poste de ministre des Finances ${ }^{69}$ en signe de protestation contre une hausse des dépenses publiques et de l'endettement qu'ils jugeaient irresponsable ; en 1982, le gouvernement de coalition réunissant le SPD et le parti libéral FDP de Helmut

\footnotetext{
${ }^{57}$ Pour des mesures précises, cf. Ehrlicher (1994), p. 226-230. Les augmentations des dépenses furent justifiées politiquement au nom de leur efficacité pour l'avenir. Mais Ehrlicher mentionne le fait que les dépenses d'investissement diminuaient tandis que les dépenses de personnel enregistraient une hausse disproportionnée.

${ }^{58}$ Cf. Bundesministerium der Finanzen (1989, p. 73-74) et Statistisches Bundesamt (2014).

${ }^{59}$ Données : Bundesministerium der Finanzen (2014).

${ }^{60}$ Données : ibid.

${ }^{61} \mathrm{Cf}$. Bundesministerium für Arbeit und Soziales (2007), p. 6.

${ }^{62}$ Cf. Deutsche Bundesbank (2014a).

${ }^{63}$ Cf. ibid. et Conseil d'experts pour l'évaluation de la situation économique (Sachverständigenrat, 2012), tableau « Verschuldung der öffentlichen Haushalte ».

${ }^{64}$ Cf. Bundesministerium der Finanzen (2013c), p. 22.

${ }^{65}$ Cf. Grossekettler (2008), p. 245 avec d'autres références.

${ }^{66}$ Données : Institut d'études de croissance (Institut für Wachstumsforschung, 2005, p. 3) et, à partir de 1971, Statistisches Bundesamt (2005).

${ }^{67}$ Cf. sur ce point ministère fédéral des Finances (Bundesministerium der Finanzen, 1989, p. 73) et Conseil d'experts pour l'évaluation de la situation économique (Sachverständigenrat, 2012), tableau «Eckdaten der Arbeitslosigkeit ».

${ }^{68} \mathrm{Cf}$. Statistisches Bundesamt (2013b).

${ }^{69}$ En 1971, Karl Schiller, alors ministre de l'Économie (depuis 1966), s'était vu confier également le portefeuille des Finances après la démission d'Alex Möller, NdE.
} 
Schmidt perdit sa majorité et fut remplacé par le gouvernement CDU/CSU-FDP de Helmut Kohl ${ }^{70}$. Les mauvais résultats économiques de cette période démontraient les failles de la théorie à l'appui d'une politique fiscale anticyclique et les problèmes liés à la mise en œuvre concrète d'une telle politique ${ }^{71}$. Ces expériences renforcèrent l'aversion de la population à l'encontre de la dette publique $^{72}$. Ces résultats encouragèrent en outre de nombreux responsables politiques à privilégier une politique de l'offre menée à plus long terme, comme celle que prônait le Conseil d'experts pour l'évaluation de la situation économique $^{73}$. Tout ceci eut sur la politique économique et financière allemande des répercussions qui perdurent encore aujourd'hui.

L'on retiendra schématiquement les caractéristiques suivantes pour cette période : priorité donnée à la politique de stabilisation sous la forme d'une politique anticyclique (qui se solda par un échec). Politique distributive non prioritaire, mais hausse considérable de la part des prestations sociales; hausse de la part des dépenses publiques, des prélèvements fiscaux et sociaux, des taux de déficit et d'endettement ainsi que des subventions ; taux de croissance en repli, associé à une hausse du taux de chômage et du taux d'inflation ("stagflation »). Du fait de ces évolutions, crainte exacerbée dans l'opinion de l'endettement public croissant.

Pendant la phase de politique de consolidation des années 1980, l'État fédéral en particulier tenta de réduire nettement la croissance de ses dépenses, de $9 \%$ en moyenne de 1970 à 1982 , tout comme le financement des dépenses par le déficit ${ }^{74}$. Cette politique correspondait à une promesse de Helmut Kohl répondant à la crainte largement répandue au sein de l'opinion publique que la dette publique ne continue de croître de manière incontrôlée ${ }^{75}$. Cette promesse fut d'une certaine façon tenue : à la fin des années 1980, le Conseil d'experts pour l'évaluation de la situation économique confirma au gouvernement qu'il avait bien progressé en matière de consolidation quantitative - entendue comme une baisse de la part des dépenses publiques dans le PIB et une diminution de la

\footnotetext{
${ }^{70}$ L'élément déclencheur fut la proposition de réforme économique («Lambsdorff-Papier ») du ministre de l'Économie libéral (FDP) Otto Graf Lambsdorff en septembre 1982 : son auteur demandait que cesse la politique d'endettement et se prononçait pour des réformes misant sur une politique de l'offre ; son rejet par Helmut Schmidt entraîna la démission de tous les ministres FDP en signe de protestation contre la poursuite de la politique en cours.

${ }_{71}^{71}$ Pour plus de détails sur ce point, Grossekettler (2008), p. 230-240.

${ }^{72}$ Selon Noelle-Neumann (1976, p. 228), en 1975, $88 \%$ des sondés s'inquiétaient de la dette publique. La même année, $25 \%$ des personnes interrogées considéraient ce thème comme la plus importante question politique qui soit (Noelle-Neumann/Piel). En 1980, $60 \%$ des sondés jugeaient particulièrement important de réduire la dette publique (ibid.), p. 336. En 1981, ils étaient $80 \%$ à considérer la dette publique comme trop élevée et seules $14 \%$ des personnes interrogées ne s'en inquiétaient pas ; la part des sondés jugeant irresponsable la dette publique augmenta entre janvier 1979 et juin 1982 : elle passa de $49 \%$ à $62 \%$, avec des résultats intermédiaires à $53 \%$ et $66 \%$ (ibid.), p. 382.

${ }^{73}$ Sur l'évolution de cette conception politique du Conseil, cf. Sievert (2003).

${ }^{74}$ Cf. Bundesministerium der Finanzen (1989), p. 74-75.

${ }^{75}$ Selon Noelle-Neumann/Piel (1983, p. 263), début 1983, $70 \%$ des sondés jugeaient bon que la CDU/CSU réduise la dette publique.
} 
dette structurelle ${ }^{76}$. La part des dépenses publiques passa en effet de $47,5 \%$ du BIP (1982) à 43,6\% $(1990)^{77}$; celle des prélèvements fiscaux était tombée à $22,4 \%$ en moyenne sur cette période ; la part des prélèvements sociaux était en hausse à $16,2 \%{ }^{78}$, tandis que celle des prestations sociales (mais non la valeur des prestations sociales) était en repli sensible à $26,8 \%{ }^{79}$. Le taux d'endettement restait cependant en hausse et atteignait $41,2 \%$ du PIB $^{80}$. Le Conseil déplorait l'absence de consolidation qualitative, l'absence d'une structure des dépenses favorable à la croissance et l'absence de baisse de la part des prélèvements dans le $\mathrm{PIB}^{81}$. En outre, la totalité des budgets de 1982 à 1988 se clôturait encore en déficit - mais avec une tendance à la baisse ; seul l'exercice 1989 arrivait à l'équilibre $(+0,1 \%)^{82}$. Ce résultat se faisait par surcroît au prix d'une chute - amorcée toutefois dès 1981 - des investissements publics nets, passés de l'équivalent de près de 14 milliards d'euros en 1980 à seulement 8 milliards en $1989^{83}$. Les subventions continuaient en revanche d'augmenter et atteignaient environ 45 milliards d'euros ${ }^{84}$. Le taux de croissance moyen était en léger repli (un phénomène normal à court terme dans les périodes de consolidation) à $2,7 \%{ }^{85}$. Le taux de chômage augmenta d'abord pour atteindre $9,3 \%$ en 1985 avant de retomber à $7,2 \%$ en $1990^{86}$; après une forte baisse, le taux d'inflation connut un léger rebond à $2,9 \%(1990)^{87}$. Les résultats n'étaient donc pas tous positifs ; mais cette forme de consolidation créait un point de départ plus favorable pour faire face aux coûts de la réunification imminente.

Pour cette période, l'on peut retenir schématiquement la caractérisation suivante : priorité à la politique de consolidation et amorce d'une stabilisation à long terme et d'une politique financière durable ${ }^{88}$. Baisse de la part des prélèvements fiscaux, des taux de déficit et d'endettement et diminution sensible de la part des dépenses publiques dans le PIB. Hausse de la part des prélèvements

\footnotetext{
${ }^{76}$ Cf. Sachverständigenrat (1989), paragraphe 169 sq.

${ }^{77}$ Cf. Bundesministerium der Finanzen (2013b) et Statistisches Bundesamt (2014).

${ }^{78}$ Données : Bundesministerium der Finanzen (2014).

${ }^{79}$ Cf. Bundesministerium für Arbeit und Soziales (2007), p. 6.

${ }^{80}$ Cf. Deutsche Bundesbank (2014a). Le Conseil d'experts pour l'évaluation de la situation économique (Sachverständigenrat, 2012, tableau « Verschuldung der öffentlichen Haushalte ») indique des chiffres qui diffèrent légèrement de ceux de la Bundesbank.

${ }^{81}$ Cf. Sachverständigenrat (1969), paragraphe 169 sq.

${ }^{82}$ Cf. Deutsche Bundesbank (2014a) et Statistisches Bundesamt (2012).

${ }^{83}$ Cf. Conseil d'experts pour l'évaluation de la situation économique (Sachverständigenrat 2012), tableau « Verwendung des Volkseinkommens ».

${ }^{84}$ Cf. Bundesministerium der Finanzen (2013c), p. 22.

${ }^{85}$ Cf. Statistisches Bundesamt (2013c). Chacun sait d'expérience que les mesures de consolidation entraînent à court terme une perte de croissance mais qu'elles sont néanmoins la condition d'un soutien du développement économique à long terme (Deutsche Bundesbank, 2014b, avec une synthèse d'études empiriques).

${ }^{86}$ Cf. Conseil d'experts pour l'évaluation de la situation économique (Sachverständigenrat, 2012), tableau « Eckdaten zur Arbeitslosigkeit ».

${ }^{87}$ Cf. Statistisches Bundesamt (2013b).

${ }^{88}$ Sur la notion de politique financière durable et les modalités de son évaluation, cf. Comité scientifique sous l'égide du ministère fédéral des Finances (Wissenschaftlicher Beirat beim Bundesministerium der Finanzen, 2001). Le ministère fédéral des Finances publie à intervalles de quelques années des analyses en ce sens, dites « rapports de viabilité » (Tragfähigkeitsberichte).
} 
sociaux et baisse de la part des prestations sociales (mais non des prestations elles-mêmes), augmentation des subventions. Baisse sensible des investissements publics, taux de croissance en léger repli et, en conclusion, baisse du taux de chômage et d'inflation. Crainte persistante d'un endettement public incontrôlé dans l'opinion publique.

La phase de reconstruction des années 1990 fut amorcée avant même la réunification officielle. Elle se basait sur une série d'accords et actes juridiques qui constituèrent l'assise juridique de l'unité étatique et de l'union économique, monétaire et sociale avec la RDA ${ }^{89}$ :

- 9 novembre 1989 : ouverture du Mur de Berlin;

- février 1990 : premières négociations Deux-plus-Quatre en vue de la réunification ;

- mars 1990 : premières élections libres en RDA ;

- mai 1990 : traité instituant l'Union monétaire, économique et sociale ;

- juillet 1990 : introduction du deutsche mark en RDA ;

- août 1990: accord électoral et traité d'unification; décision par la Chambre du Peuple (Volkskammer) d'adhérer à la Loi fondamentale ;

- septembre 1990: «traité portant règlement définitif concernant l'Allemagne », dit également « traité Deux-plus-Quatre » (c'est-à-dire de restauration de la souveraineté du pays);

- 3 octobre 1990 : réunification officielle ;

- 2 décembre 1990: premières élections législatives communes à l'ensemble du territoire allemand.

Dans les années suivantes, également mouvementées, la politique publique eut pour but l'adaptation du système centralisé de la RDA au fédéralisme de la République fédérale; la politique économique et financière avait, quant à elle, pour enjeu de transformer l'économie administrée d'ex-RDA en économie sociale de marché de la République fédérale. Le pays adopta trois ensembles de mesures à cet effet :

- ensemble I : la RDA avait supprimé les Länder qui existaient autrefois sur son territoire et réduit les municipalités autonomes à l'état de simples antennes de l'administration centrale. Le personnel administratif n'était en outre pas formé dans le cadre d'un État de droit et n'était $a$ fortiori pas familier du droit de la République fédérale dont le champ d'application s'étendait désormais, suite à la réunification, au territoire de la RDA. Il fallait donc créer des administrations régionales et municipales, et le personnel des administrations devait apprendre à appliquer le droit de la République fédérale. Des partenariats furent établis pour

\footnotetext{
${ }^{89}$ Sur les points suivants, cf. Grossekettler (1996 et 1998b). Ces deux articles décrivent dans le détail ce qui ne peut être ici que mentionné brièvement par manque de place. On trouvera les principaux documents juridiques réunis dans trois volumes de Stern/Bleibtreu (1990-1991), une chronique sous forme de tableau chez Heilemann/Gebhardt/von Loeffelholz (1996).
} 
faire face à cette mission : dans le cadre de ce qui fut appelé l'assistance administrative, les Länder et municipalités de l'Ouest se cherchèrent des partenaires à l'Est; ils mirent à leur disposition du matériel et des agents expérimentés qui endossèrent en quelque sorte le rôle de chefs d'équipe. Ainsi, la Bavière et le Bade-Wurtemberg apportèrent par exemple leur appui à la Saxe ${ }^{90}$. Comme l'Ouest envoyait surtout des cadres, certaines catégories de population, et en particulier certains intellectuels, virent dans cette démarche une sorte de colonisation. Mais en réalité c'était la seule façon de mettre rapidement sur pied une administration respectueuse de l'État de droit qui fût opérationnelle. En effet, ce n'est pas avec des personnalités issues de l'Église protestante et des écrivains apportant leur aide occasionnelle que l'on construit un État ;

- ensemble II : la banque d'État de la RDA fut dissoute. Une partie de ses missions fut transférée à la Bundesbank, tandis que les autres étaient reprises au fil de la privatisation par des banques d'affaires. Là encore, des partenariats utiles furent instaurés : les caisses d'épargne et banques coopératives ouest-allemandes cherchèrent des partenaires à l'Est et leur transmirent des compétences et du matériel. Les grandes banques firent de même avec leurs anciennes filiales ;

- ensemble III : il fallut privatiser le portefeuille d'entreprises subordonnées aux ministères de l'Industrie de la RDA. Ce processus fut confié à un établissement fiduciaire ad hoc (Treuhandanstalt). Cet établissement vendit ces entreprises non pas aux plus offrants mais aux acquéreurs qui présentaient le business plan le plus convaincant à long terme et étaient prêts à reprendre le plus de personnel possible. Le ministre fédéral de l'Économie de l'époque, Theo Waigel, avait à l'origine espéré que les activités de la Treuhand généreraient des bénéfices. Or il s'avéra que $30 \%$ des entreprises ne pouvaient être restructurées et l'établissement ferma en 1994, avec des pertes de 260 milliards de marks finalement épongées par l'État fédéral. Ces pertes avaient plusieurs causes importantes : en RDA, la productivité du travail représentait moins du tiers de celle enregistrée dans la République fédérale ; les entreprises de RDA avaient perdu une grande partie de leurs marchés dans l'ancien bloc de l'Est et elles ne pouvaient rivaliser avec les entreprises de l'Ouest en raison de la qualité produite et de coûts salariaux trop élevés. On peut néanmoins considérer le passage à l'économie sociale de marché de l'Allemagne de l'Ouest comme une réussite. Le chômage en Allemagne de l'Est passa toutefois à 10,2\% dès 1991, puis continua d'augmenter jusqu'en 2005 (20,6\%); il baissa ensuite du fait d'augmentations de la

\footnotetext{
${ }^{90}$ Le même procédé s'appliqua également aux universités puisque la RDA ne disposait pas de ressources humaines formées en droit ou en gestion dans le cadre d'une économie de marché. Les universités de l'Ouest du pays se mirent donc en quête de partenaires - Münster avec Leipzig par exemple - et assurèrent (via des cours d'été par exemple) la formation continue du personnel et des étudiants les plus avancés dans leur cursus.
} 
productivité et, après une flexibilisation du marché allemand de l'emploi menée jusqu'en 2012, il passa à $11,9 \%{ }^{91}$. Cela affecta bien sûr considérablement l'assurance chômage de l'ensemble du pays, d'autant que le taux de chômage augmentait sur l'ensemble du territoire : il atteignit $13 \%$ en 2005 , avant de retomber à $7,6 \%(2012)^{92}$. Cette évolution se fit également ressentir sur la part des prestations sociales dans le PIB : celle-ci atteignit $31,2 \%$ en 1999 pour l'ensemble du pays et est demeurée depuis lors à un niveau comparable ${ }^{93}$. Jusqu'en 2000, les subventions augmentèrent pour atteindre un volume de près de 60 milliards d'euros; depuis, elles sont toutefois en repli ${ }^{94}$. Les investissements nets publics, en augmentation, ont atteint un maximum de plus de 15 milliards d'euros (1992), avant de connaître une baisse considérable jusqu'à un niveau de 1,6 milliard d'euros (2002); depuis, ils sont même négatifs ${ }^{95}$. Le taux d'inflation a fait un bond à $5,1 \%$ (1992), avant d'enregistrer une baisse rapide; depuis le début du nouveau millénaire, il oscille généralement entre $1 \%$ et $2 \%{ }^{96}$.

En raison des charges liées à la réunification, la croissance de la productivité par heure de travail a chuté de 3,5\%, dernier taux enregistré dans l'ouest de l'Allemagne, à 2,5\% dans l'ensemble de l'Allemagne ${ }^{97}$. Ce facteur ainsi que la tendance des taux de croissance ont entraîné une baisse du taux de croissance à $1,4 \%$ de 1992 à 1999 , caractéristiques pour cette période ${ }^{98}$. Cela amène à poser la question des transferts globaux de l'Allemagne de l'Ouest vers les Länder d'ex-RDA et de leur financement. La réponse est la suivante : l'est de l'Allemagne a reçu une aide par le biais de divers fonds spéciaux, des dispositifs spécifiques dans la péréquation financière Bund/Länder dont le dernier en date fut le « Pacte de solidarité II » et, surtout, à travers des versements dans le domaine de l'assurance sociale. Un membre de la Commission européenne a estimé à $4,5 \%$ du PIB ouest-allemand les transferts de l'Ouest vers l'Est en cours ${ }^{99}$. Ils représenteraient à ce jour plus de deux mille milliards d'euros au tota ${ }^{100}$. La part des dépenses publiques est de ce fait passée de 43,6\% (1990) puis 46,2\% (1991) à 54,9\% (1995); elle s'est stabilisée jusqu'à la fin des années 1990 autour de $48 \%$ et $49 \%$ avant de retomber - après le remboursement des principales charges jusqu'en 2007 - à $43,5 \%$; après un rebond provisoire au cours de

\footnotetext{
${ }^{91}$ Cf. Sachverständigenrat (2012), tableau « Eckdaten der Arbeitslosigkeit ».

${ }^{92}$ Cf. ibid.

${ }^{93}$ Cf. Bundesministerium für Arbeit und Soziales (2007), p. 6.

${ }^{94} \mathrm{Cf}$. Bundesministerium für Finanzen (2013c), p. 22.

95 Cf. Conseil d'experts pour l'évaluation de la situation économique (Sachverständigenrat, 2012), tableau «Verwendung des Volkseinkommens ». Pour une relativisation de ces chiffres, cf. infra, p. 137.

${ }^{96} \mathrm{Cf}$. Statistisches Bundesamt (2013b).

${ }^{97}$ Cf. Bundesministerium für Wirtschaft (2013), p. 14.

${ }^{98}$ Calculé à partir des données de l’Office fédéral des statistiques (Statistisches Bundesamt, 2014).

${ }^{99}$ Cf. Jansen (2004), p. 4.

${ }^{100}$ Regnitz/Schirwitz/Scharfe (2009) obtiennent le chiffre de 1,6 billion d'euros pour l'année 2005 et une croissance de 100 milliards d'euros par an.
} 
la crise financière, elle s'est rétablie à $44,7 \%$ du PIB en $2012^{101}$. Les transferts ont été financés par des augmentations d'impôts (par exemple, introduction de la majoration dite de solidarité à l'impôt sur le revenu - Solidaritätszuschlag) et des prélèvements sociaux, mais également en grande partie par une hausse de l'endettement. La part moyenne des prélèvements fiscaux est restée pratiquement constante à $22,2 \%$, celle des prélèvements sociaux est montée à $18,4 \%{ }^{102}$. En 1995, la part du déficit au sens de Maastricht atteignait un taux maximal (faussé par la dissolution de la Treuhand) de 9,5\% (taux corrigé : 3,2\%); elle retomba avec des fluctuations jusqu'en 2008 à $0,1 \%$ et atteignit même une valeur négative (un solde budgétaire de $+0,1 \%$ ) après la sortie (provisoire ?) de la crise financière en $2012^{103}$. Le taux d'endettement selon les critères de Maastricht (parfois temporairement faussé en raison de l'intégration ou de l'externalisation de fonds) augmenta jusqu'en 1999 à $61,3 \%$; avec la lutte contre la crise financière, il connut une nouvelle hausse jusqu'en 2012 et atteignit $81,0 \%$ $\mathrm{du} \mathrm{PIB}^{104}$. La dette publique inquiéta la population dans la phase de reconstruction d'après la réunification alors que la répartition des dépenses qui y étaient associées avait une certaine justification ${ }^{105}$.

Globalement, on retiendra pour les années 1990 la caractérisation suivante : priorité à la gestion économique de la réunification et aux transferts de l'Ouest vers l'Est, un phénomène unique dans l'histoire. Jusqu'à la fin de la période, réalisation réussie des missions de fédéralisation, avec la mise en place de municipalités et de Länder opérationnels. Passage de l'économie administrée de la RDA à une économie de marché semblable à celle de la République fédérale. Hausse provisoire de la part des dépenses publiques, du déficit et des prélèvements sociaux ; hausse durable de la part des prestations sociales et de la dette ; parallèlement, part de prélèvements fiscaux pratiquement constante ; investissements publics nets élevés de 1991 à 1994, puis en repli important ; investissements nets négatifs à partir de 2003 ; nouvelle chute du taux de croissance moyen. La hausse des subventions se poursuit pour atteindre une

\footnotetext{
${ }^{101}$ Cf. Bundesministerium der Finanzen (2013b).

102 Données : Bundesministerium der Finanzen (2014).

${ }^{103}$ Cf. Deutsche Bundesbank (2014a). Sur la question de savoir si la crise financière est derrière nous ou non, cf. Fuest (2014).

${ }^{104}$ Cf. Deutsche Bundesbank (2014a). Sur l'évolution du taux d'endettement allemand depuis les années 1950 , on peut retenir les repères approximatifs suivants : valeur initiale au cours des deux premières périodes : $20 \%, 20 \%$ de plus à l'issue des expériences keynésiennes de la troisième période, $20 \%$ supplémentaires en raison de la réunification et, enfin, de nouveau $20 \%$ supplémentaires pour surmonter provisoirement la crise financière, soit au total environ $80 \%$.

${ }^{105}$ Selon Noelle-Neumann/Köcher (1997, p. 794), début 1993, 47 \% des sondés estimaient qu'il n'était plus possible d'attendre pour limiter la dette publique. En 1996, ils étaient $67 \%$ à juger important que la dette publique cesse d'augmenter (ibid., p. 796), $70 \%$ à juger important de la réduire (ibid., p. 868), et $53 \%$ à estimer que l'avenir de leurs enfants dépendait surtout de la réduction de la dette publique (ibid.), p. 18. Le rapport emnid constatait également pour 1996 que la dette publique avait été considérée pendant presque toute l'année comme un problème important (Schöppner, 1997, p. 19), même si les sondés n'avaient guère confiance dans les partis s'agissant de limiter la dette (ibid.), p. 23. En 2001 enfin, $70 \%$ de la population considérait la limitation de la dette publique comme un objectif important (Noelle-Neumann/Köcher, 2002), p. 700.
} 
valeur maximum en 2000. Rebond provisoire du taux d'inflation; principale hausse - en particulier dans les Länder de l'Est - : celle du taux de chômage qui demeure très élevé et ne se met ensuite à diminuer que très lentement. Clair mécontentement de la population face à l'évolution de la dette publique.

\section{Constantes à long terme}

Que montre maintenant ce panorama des périodes de la politique budgétaire allemande dans l'après-guerre en matière de constantes durables et influencées en quelque sorte culturellement, telles que les présentait Neumark dans son article de 1948 cité en introduction? Si l'on considère les missions prioritaires dans les périodes de moyen terme, on remarque premièrement que les responsables allemands de la politique financière durent surtout agir sous l'influence de facteurs extérieurs ; ils n'opéraient pas en acteurs libres ayant prise sur les événements : les mesures prises pendant la première période de reconstruction étaient de fait inévitables dans un pays détruit par la guerre, après le passage à l'économie de marché. Mais les faveurs accordées aux entreprises au cours de cette période entraînèrent une répartition des revenus et du patrimoine ressentie comme injuste par de vastes catégories de population. Cette situation généra une pression politique qui permit de rectifier le tir au cours de la deuxième période - l'âge d'or - car dans une démocratie, il fallait céder à cette pression. Le passage d'une politique prioritairement allocative et distributive dans les deux premières périodes à une politique de stabilisation dans la troisième était en soi un choix judicieux ; mais cette politique fut menée de manière anticyclique - en dépit des avertissements du Conseil d'experts pour l'évaluation de la situation économique - et le système administratif et politique se retrouva par là même dépassé ; or c'est là une erreur au sens de la Ordnungspolitik qui aurait pu être évitée. Rectifier le tir au cours de la quatrième période en faisant le choix de la consolidation était en revanche pratiquement inévitable car la population poussait en ce sens. La réunification au cours de la phase suivante est - d'un point de vue purement économique - semblable à un énorme choc exogène. Elle fut rapidement surmontée, ce qui est tout à fait remarquable, mais les réponses apportées n'étaient nullement le fruit d'un projet à long terme ; il s'agissait de réagir à court terme à une opportunité qui se présentait. Tout au long de cet enchaînement de périodes, les responsables politiques ont donc très largement dû réagir aux évènements exogènes survenus ou aux options unilatérales prises au cours de la période précédente. Ce schéma traverse les différentes périodes; on peut néanmoins à bon droit douter qu'il s'agisse d'une constante au sens d'une spécificité nationale. S'il faut admettre l'hypothèse contraire et que ce schéma ne tient pas uniquement aux circonstances propres à l'époque, alors il exprime sans doute la tendance à éviter les options unilatérales, à mener une politique budgétaire équilibrée à moyen ou long terme et à miser sur une coopération fondée sur la confiance, y compris au-delà des clivages politiques et corporatistes. Mais 
comme il est impossible de valider clairement cette hypothèse, cette observation ne peut pour l'instant être intégrée à la liste des constantes à long terme.

On peut en revanche considérer comme une première constante nationale à long terme en matière de politique budgétaire le positionnement de la population allemande dont les responsables politiques ne peuvent durablement s'affranchir: la peur d'une inflation causée par une dette publique galopante et, en conséquence, la tentative d'une politique de sérieux budgétaire. Comme il a été dit, cette peur a longtemps été de nature plutôt diffuse car il n'y avait pas de critère indiquant à quel moment la dette publique devenait trop importante. Après la signature du traité de Maastricht - donc après 1993 - un tel outil existait désormais pour l'opinion publique, avec les critères de déficit et d'endettement. C'est pourquoi les médias et la population sont depuis lors extrêmement réactifs quand le traité n'est pas respecté, en particulier lorsque c'est l'Allemagne qui enfreint les règles : un avertissement de la part de la Commission européenne est perçu comme la preuve d'une politique budgétaire peu sérieuse. En Allemagne, la population s'inquiète de la dette publique, et cela n'apparaît pas uniquement dans la période 1949-1999; il s'agit certainement d'une caractéristique constante dont l'action persistera sans doute à l'avenir, d'un facteur qui a des effets politiques. Dans une analyse des opinions en matière de politique européenne menée pour la Fondation Heinrich Böll, Göhring (2011, p. 9) écrit ainsi à juste titre : «Les questions de la dette publique et de l'inflation sont globalement perçues en Allemagne comme un problème central, d'une importance primordiale. » Les sondages Eurobaromètre réalisés sur ce thème confirment cette assertion ${ }^{106}$.

Le droit budgétaire constitue une deuxième quasi-constante. Comme cela a été montré, le droit est le reflet de la constitution fédérale de l'État; il évolue certes, mais lentement. Cela signifie d'une part que la politique budgétaire doit normalement respecter des principes juridiques fondamentaux, lesquels interdisent cependant d'élargir brutalement les marges de manœuvre budgétaires. Les modifications juridiques intervenues avec l'introduction de la loi sur la stabilité et la croissance font exception à cette règle. Elles ont ouvert une brèche qui eut pour conséquence l'évolution dévastatrice des années 1970, dont les effets négatifs se firent ressentir bien au-delà de la période définie. D'autre part, des élections ont lieu pratiquement chaque année en Allemagne : elles sont pour les gouvernements concernés l'occasion de faire des cadeaux électoraux et renforcent de ce fait une tendance à mener une politique conjoncturelle asymétrique. Cette

\footnotetext{
${ }^{106}$ Comme il a été dit, il fallut hélas attendre 2010 pour que commencent à apparaître des questions précises sur la dette publique. Dans l'Eurobaromètre 80 (automne 2013), il s'agit par exemple, dans les tableaux résumant les résultats publiés en annexe, des questions «QA4 : $\grave{A}$ votre avis, quels sont les deux problèmes les plus importants auxquels notre pays est actuellement confronté? » et «QA6 : Quels sont à votre avis les deux problèmes les plus importants auxquels l'UE est actuellement confrontée? 》 Il était possible de répondre à ces deux questions par: "la dette publique» ou «la réduction de la dette publique ». Autres questions: QC4.3 (approbation quant à la nécessité de réduire immédiatement le déficit public) et QC4.4 (approbation quant à la possibilité de différer la réduction du déficit public et la dette publique).
} 
configuration conduisit dans le passé à la tendance prédominante d'une progression par paliers de la dette publique. Il fallut attendre le nouveau frein à l'endettement pour pouvoir faire barrage - efficacement, espérons-le - à cette évolution. On peut voir dans l'introduction de ce dispositif une émanation de la constante évoquée ci-dessus d'une "peur chez la population d'une dette publique croissante » et de la pression exercée de ce fait sur les responsables politiques.

Abstraction faite de la période de consolidation des années 1980 et au vu des valeurs initiales et finales ainsi que de la tendance générale, on observe un troisième phénomène quasi constant : la hausse à très long terme de la part des dépenses publiques et des prestations sociales dans le PIB. Cette évolution correspond à la loi de Wagner et à une tendance que l'on observe partout dans le monde. La part des prélèvements fiscaux reste en revanche pratiquement stable, tandis que le taux d'endettement augmente. La hausse de ce dernier s'accompagne normalement d'une hausse de la charge de la dette en valeur et même d'une hausse de la part de la charge de la dette dans le PIB. Or, depuis la fin des années 1990, on n'observe pas cet effet car le niveau des taux d'intérêt a connu un important repli ${ }^{107}$. Cette évolution n'est toutefois pas sans risque : si les taux d'intérêt augmentaient d'un pour cent, la charge de la dette connaîtrait à long terme une hausse de 22 milliards par an compte tenu du niveau d'endettement actuel en Allemagne ${ }^{108}$. Une normalisation du niveau des intérêts, avec une hausse des taux d'environ $4 \%$ aurait donc, toutes choses égales par ailleurs, un impact considérable sur le budget public.

Une quatrième régularité apparaît non pas sur l'ensemble de la période observée, mais uniquement à partir des années 1980 : lorsque les responsables politiques se retrouvent dans une période de tension au cours de laquelle il leur faut prendre des mesures de consolidation, ils commencent par réduire non pas les dépenses publiques mais les investissements, un domaine dans lequel les réductions budgétaires sont moins vite ressenties par les électeurs. C'est un phénomène que l'on peut déjà observer pendant la période de consolidation antérieure à la réunification, et que l'on retrouve après 1992. Lorsque l'on étudie ces phénomènes, il faut prendre en compte différents facteurs : premièrement, les difficultés et controverses potentielles liées à la collecte de données statistiques relatives aux investissements publics nets; deuxièmement, l'impact dans les comparaisons internationales des écarts de taux d'inflation et d'effets temporaires - effets de rattrapage par exemple pour l'ex-RDA (si l'on compare le taux d'investissement total, effet tels que les bulles immobilières par exemple) ; troisièmement, l'existence dans les pays industrialisés comme l'Allemagne d'effets de saturation ${ }^{109}$. Dans une étude tenant compte de tous ces facteurs, l'institut ifo de Dresde aboutit néanmoins au constat global d'un déficit, en particulier dans le domaine des infrastructures de transports, lequel entrave la poursuite du dé-

\footnotetext{
${ }^{107}$ Cf. Deutsche Bundesbank (2013), p. 52.

${ }^{108}$ Cf. ibid., p.64.

${ }^{109}$ Pour des détails sur ce point, cf. ifo-Institut Dresden (2013).
} 
veloppement économique ${ }^{110}$. Un sondage vient à l'appui de cette observation : selon cette enquête, les entreprises allemandes continuent de trouver que les infrastructures sont bonnes, mais estiment qu'elles ne sont pas garanties à long terme $^{111}$. Contrairement à la hausse de la dette, l'insuffisance des investissements publics n'amène cependant pas la population à formuler des exigences ayant un impact politique : si la population se plaint des nids-de-poule sur certaines routes ou du manque de ponctualité des trains, elle n'établit pas de lien avec les investissements publics non réalisés au profit des dépenses publiques ou d'une redistribution opérée à la suite de promesses électorales. On peut donc affirmer - en forçant un peu le trait - qu'une dette trop élevée, des impôts trop élevés et des réductions trop importantes des dépenses publiques peuvent décider d'une élection en Allemagne, mais que cela ne vaut pas pour des investissements publics nets trop bas.

L'on observe ainsi au total quatre faits presque constants sur la durée, sans doute basés sur des singularités nationales :

- l'aversion de la population à l'égard de la dette publique et son corollaire : une préférence nette pour une politique de sérieux budgétaire ;

- l'organisation fédéraliste du droit budgétaire, sa quasi-constance mais aussi, si nécessaire, des corrections effectuées de manière à rendre possible un budget sain et vraisemblablement à le susciter ;

- l'évolution de la part des dépenses publiques et des prestations sociales conformément à la loi de Wagner ;

- un impact politique particulièrement faible d'investissements publics nets insuffisants.

Ces quatre régularités traversent les cinq périodes de politique budgétaire distinguées de 1949 à 1999 et resteront sans doute opératoires à l'avenir. Existe-t-il une cinquième singularité nationale qui serait une disposition particulièrement marquée à coopérer en confiance, au-delà des clivages partisans et corporatistes ? Il ne nous semble pas possible de trancher dès à présent cette question. Pour ce faire, il faudrait probablement analyser cet aspect en s'appuyant sur des comparaisons internationales.

Traduction de Marie GRAVEY

\section{Indications bibliographiques}

BaUer T.K., Braun S., KvaSniCKa M., « The Economic Integration of Forced Migrants: Evidence for Postwar Germany », The Economic Journal, vol. 123, 2013, p. 998-1024

BUNDESARCHIV (ed), Kabinettsprotokolle der Bundesregierung [en ligne]. Disponible sur http://www.bundes archiv.de/kabinettsprotokolle [consulté le 29/10/2014]

\footnotetext{
${ }^{110}$ Cf. ibid., p. 132.

${ }^{111}$ Cf. Institut der deutschen Wirtschaft (2014).
} 
BUNDESMINISTERIUM DER FINANZEN, 24. Subventionsbericht - Bericht der Bundesregierung über die Entwicklung der Finanzhilfen des Bundes und der Steuervergünstigungen für die Jahre 2011 bis 2014, Berlin, $2013 \mathrm{c}$

BUNDESMINISTERIUM DER FinANZEN, 40 Jahre Verantwortung für die Finanzen des Bundes - Das Bundesministerium der Finanzen - Geschichte, Aufgaben, Leistungen, Olzog, Munich, 1989

BUNDESMINISTERIUM DER FINANZEN, Bund/Länder-Finanzbeziehungen auf der Grundlage der Finanzverfassung, Berlin, 2012a

BUNDESMinisteriUM DER FINANZEN, Datensammlung zur Steuerpolitik 2012, Berlin, 2012c

BundeSMinisteriUm Der FinANZen, Entwicklung der Staatsquote [en ligne], Berlin, 2013b. Disponible sur : http://www.bundesfinanzministerium.de/Content/DE/Standardartikel/Themen/Oeffentliche_Finanzen/ Wirtschafts_und_Finanzdaten/Oeffentlicher_Gesamthaushalt/entwicklung-der-staatsquote.html [consulté le 12/09/2013]

BUNDESMinisteriUm DER FinANZEN, «Erstes Jahr mit Schuldenbremse erfolgreich abgeschlossen », Monatsbericht, $\mathrm{n}^{\circ} 10,2012$ b, p. 15-19

BUNDESMINISTERIUM DER FINANZEN, Steuern und Sozialabgaben im Verhältnis zum BIP, 2014 [communication personnelle]

BUNDESMinisterium DER FinANZEN, Steuern von A bis Z, Berlin, 2013a

BUNDESMINISTERIUM DER FINANZEN, «Struktur und Verteilung der Steuereinnahmen », Monatsbericht, $\mathrm{n}^{\circ} 6,2011$, p. $62-70$

BUNdeSMinisteriUm FÜr ARBEIT UND SozIAleS, Sozialbudget 2006 - Tabellenauszug, Berlin, 2007

BUNDESMINISTERIUM FÜR WIRTSCHAFT UND TECHNOLOGIE, « Datenreihe zum Wachstum der Arbeitsproduktivität », Wirtschaft.Wachstum.Wohlstand, 2013, p. 14

Deutsche BundeSBANK, « Die Entwicklung staatlicher Zinsausgaben in Deutschland », Monatsbericht, $\mathrm{n}^{\circ}$, 2013, p. 47-67

DEUTSCHE BUNDESBANK, Lange Reihen zum Haushaltssaldo und zum Schuldenstand im Verhältnis zum BIP [depuis 1991 au sens de Maastricht], 2014a [communication personnelle à l'auteur]

DEUTSCHE BUNDESBANK, «Zur kurzfristigen Wirkung fiskalpolitischer Maßnahmen auf das Wirtschaftswachstum », Monatsbericht, $\mathrm{n}^{\circ} 1,2014$ b, p. 49-50

EHRLICHER W., «Finanzpolitik seit 1945 », Vierteljahrsschrift für Sozial- und Wirtschaftsgeschichte, supplément $\mathrm{n}^{\circ} 114,1994$, p. 213-247

EHRLICHER W., «Finanzwirtschaft, öffentliche II: Die Finanzwirtschaft der Bundesrepublik Deutschland », in ALBERS W. et al. (eds), Handwörterbuch der Wirtschaftswissenschaft, vol. 3, Vandenhoeck \& Ruprecht, Stuttgart [et al.], 1981, p. 164-195

Fuest C., «Wie weit ist die Verschuldungskrise in der Europäischen Währungsunion überwunden? », Wirtschaftsdienst, vol. 94, numéro hors-série, 2014, p. 11-14

GöHRING R., Analyse europapolitischer Einstellungen - Auswertung von Meinungsumfragen zu europapolitischen Einstellungen der deutschen Bevölkerung sowie von Symphatisant_innen [sic.] von Bündnis90/Die Grünen [Analyse réalisée pour le compte de la Fondation Heinrich Böll], Heinrich-Böll-Stiftung, Berlin, 2011

GrosseKeTtLer H., « 40 Jahre Stabilitäts- und Wachstumsgesetz. Theoretische Analyse und statistische Evaluation einer verfassungsökonomischen Innovation », Jahrbuch für Wirtschaftsgeschichte, vol. 49, $\mathrm{n}^{\circ} 1$, 2008, p. 227-256

GROSSEKETTLER H., « Die ersten fünf Jahre - Ein Rückblick auf die gesamtdeutsche Finanzpolitik der Jahre 1990 bis $1995 »$, FinanzArchiv, vol. 53, n² 2, 1996, p.194-303

GrosSEKETTLER H., Fritz Neumark - Finanzwissenschaftler und Politikberater, Societäts-Verlag, Francfortsur-le-Main, 2013

GrosseKetTLeR H., "Lorenz von Stein und die moderne Staatswirtschaftslehre. Ein Führer durch die Steinsche „Finanzwirtschaft“ aus heutiger Sicht », in HAX H. (ed), Lorenz von Steins „,Lehrbuch der Finanz- 
wissenschaft “ - Vademecum zu einem Klassiker des Staatswissenschaft, Wirtschaft und Finanzen, Düsseldorf, 1998a, p. 73-110

GrosSEKeTtLeR H., "Verbände zwischen Markt und Staat aus finanzwissenschaftlicher Sicht», in SChmidT-Trenz H.-J., STOBER R. (eds), Jahrbuch Recht und Ökonomik des Dritten Sektors 2005/2006, Nomos, Baden-Baden, 2005, p. 13-35

GrosseKeTtLeR H., « Vereinigungs-Zwischenbilanz. Ein Rückblick auf die Wirtschafts- und Finanzpolitik zur Integration der neuen Bundesländer », in MEFFERT H., KRAWITZ N. (eds), Unternehmensrechnung und Besteuerung [Festschrift für Dietrich Börner zum 65. Geburtstag], Gabler, Wiesbaden, 1998b, p. 737-774

Hagen J. von, Strauch R. R., « German Public Finances: Recent Experiences and Future Challenges », ZEI working paper, $\mathrm{n}^{\circ} \mathrm{B} 13,2001$, p. 1-35

Heilemann U., GebHaRdT H., LOEFFElholz H.D. von, Wirtschaftspolitische Chronik der Bundesrepublik 1960 bis 1995, Lucius \& Lucius, Stuttgart, 1996 (Uni Taschenbücher für Wissenschaft ; 1778)

HELlER R.F., Haushaltsgrundsätze für Bund, Länder und Gemeinden - Handbuch zum Management der öffentlichen Finanzen, $2^{\mathrm{e}}$ édition revue et complétée, R. v. Decker, Heidelberg, 2010

IFO-INSTITUT DRESDEN, Endbericht zum Forschungsvorhaben „Öffentliche Infrastrukturinvestitionen: Entwicklung, Bestimmungsfaktoren und Wachstumswirkungen", Wirtschaftsforschung an der Universität München e.V., Dresde, 2013

INSTITUT DER DEUTSCHEN WIRTSCHAFT, « Immer noch ein Standortvorteil », iw-dienst, n8, 2014, p. 4-5

INSTITUT FÜR WACHSTUMSSTUdIEN, « Das Wachstum der deutschen Volkswirtschaft» [en ligne], IWSPapier, $\mathrm{n}^{\circ}$ 1, 2005, p. 1-7. Disponible sur : http://www.wachstumsstudien.de/Inhalt/Papiere/IWS-Papier1.pdf [consulté le 22/01/2015]

JANSEN H., «Transfers to Germany's Eastern Länder: a Necessary Price for Convergence or a Permanent Drag? », ECFIN Country Focus, vol. 1, n 16, 2004, p. [n.c.]

Kastrop C., Meister-Scheufelen G., Sudhoff M. (eds), Die neuen Schuldenregeln im GrundgesetzZur Fortentwicklung der bundesstaatlichen Finanzbeziehungen, Berliner Wissenschafts-Verlag, Berlin, 2010

Kommission Für DIE FINANZREFORM, Gutachten über die Finanzreform in der Bundesrepublik Deutschland, Kohlhammer, Stuttgart, 1966

Lienert J., M.-K. JUNG, « The Legal Framework for Budget Systems - An International Comparison », OECD Journal on Budgeting, Special Issue, vol. 4, n 3, 2004, p. 1-483

Magin C., «Die Wirkungslosigkeit der neuen Schuldenbremse: Warum die Staatsverschuldung weiterhin ungebremst steigen kann », Wirtschaftsdienst, vol. 90, n 4, 2010, p. 262-268

Meister-ScheufELEN G., «Die deutsche Schuldenbremse: Weg aus der permanenten Neuverschuldung », Wirtschaftsdienst, vol. 91, ${ }^{\circ}$ 9, 2011, p. 643-647

NeUMARK F., " Grundsätze und Arten der Haushaltsführung und Finanzbedarfsdeckung », in GERLOFF W., NeUMARK F. (eds), Handbuch der Finanzwissenschaft, $2^{\mathrm{e}}$ édition, vol. 1, Mohr Siebeck Verlag, Tübingen, 1952, p. 606-669

NEUMARK F., « Internationale Gemeinsamkeiten und nationale Eigenarten der Finanzpolitik » [1948], publié in NeUMARK F., Wirtschafts- und Finanzprobleme des Interventionsstaates, Mohr Siebeck Verlag, Tübingen, 1961, p. 96-121

Noelle E., NeumanN E.P. (eds), Jahrbuch der öffentlichen Meinung 1957, Verlag für Demoskopie, Allensbach, 1957

Noelle-Neumann E. (ed), Allensbacher Jahrbuch der Demoskopie 1974- 1976, vol. 6, Molden, Vienne/Munich/Zurich, 1976

Noelle-Neumann E. (ed), Allensbacher Jahrbuch der Demoskopie 1978-1983, vol. 8, K. G. Saur, Munich, 1983

Noelle-Neumann E., KöCher R. (eds), Allensbacher Jahrbuch der Demoskopie 1993 - 1997, vol. 10, K. G. Saur, Munich, 1997 
Noelle-Neumann E., KöCher R. (eds), Allensbacher Jahrbuch der Demoskopie 1998 - 2002, vol. 11, K. G. Saur, Munich, 2002

OLSON M., Die Logik des kollektiven Handelns - Kollektivgüter und die Theorie der Gruppen, $5^{\mathrm{e}}$ édition, Mohr Siebeck, Tübingen, 2004 [édition anglaise originale : The Logic of Collective Action: Public Goods and the Theory of Groups, 1965]

RAGNitz J., SCHIRwitz B., SCHARFE S., «Bestandsaufnahme der wirtschaftlichen Fortschritte im Osten Deutschlands 1989-2008 », ifo Dresden Studien, $\mathrm{n}^{\circ}$ 51, 2009, p. [n.c.]

RÄTH N., « Rezessionen in historischer Betrachtung », Wirtschaft und Statistik, n 3, 2009, p. 203-208

SACHVERSTÄNDIGENRAT ZUR BEGUTACHTUNG DER GESAMTWIRTSCHAFTLICHEN ENTWICKLUNG, Alternativen außenwirtschafticher Anpassung. Jahresgutachten 1968/69, W. Kohlhammer, Stuttgart, 1968

SACHVERSTÄNDIGENRAT ZUR BEgUTACHTUNG DER GESAMTWIRTSCHAFTLICHEN ENTWICKLUNG, $E x$ pansion und Stabilität. Jahresgutachten 1966/67, W. Kohlhammer, Stuttgart, 1966

SACHVERSTÄNDIGENRAT ZUR BEGUTACHTUNG DER GESAMTWIRTSCHAFTLICHEN ENTWICKLUNG, Stabile Architektur für Europa - Handlungsbedarf im Inland. Jahresgutachten 2012/13, Sachverständigenrat zur Begutachtung der gesamtwirtschaftlichen Entwicklung/Statistisches Bundesamt, Wiesbaden, 2012

SCHÖPPNER K.-P., emnid report - Meinungen, Trends, Fakten, Olzog, Munich, 1997

SChratzenstaller M., "Staatsquoten: Definitionen, Grenzen der Vergleichbarkeit und Aussagekraft », Wirtschaftsdienst, vol. 93, $\mathrm{n}^{\circ}$ 3, 2013, p. 204-206

Sievert O., "Vom Keynesianismus zur Angebotspolitik », in SACHVERSTÄNdigenRAT ZUR BEGUTACHTUNG DER GESAMTWIRTSCHAFTLICHEN ENTWICKLUNG (ed), Vierzig Jahre Sachverständigenrat 19632003, Sachverständigenrat zur Begutachtung der gesamtwirtschaftlichen Entwicklung/Statistisches Bundesamt, Wiesbaden, 2003, p. 34-46

STATISTISChes BUNDESAMT, Bruttoinlandsprodukt Deutschlands ab 1970 (in konstanten Preisen von 1995) [en ligne], 2005. Disponible sur : http://www.pdwb.de/w_biprei.htm [consulté le 22/01/2015]

STATISTISChEs BUNDESAMT, Destatis, lange Reihe „,Verbraucherpreisindizes für Deutschland“, Wiesbaden, $2013 b$

STATISTISChes BundeSAmt, Destatis, lange Reihe zum Arbeitsmarkt, Wiesbaden, 2013a

STATISTISCHES BUNDESAMT, Lange Reihe zur Staatsquote in der VGR gemäß jeweiligem Gebietsstand, 2014 [communication personnelle à l'auteur]

STATiSTiSches BundeSAMt, Statistisches Jahrbuch 2013 - Deutschland und Internationales [en ligne], Wiesbaden, 2013c. Disponible sur: https://www.destatis.de/DE/Publikationen/StatistischesJahrbuch/Statisti schesJahrbuch2013.pdf?_blob=publicationFile [consulté le 22/01/2015]

STATISTISChES BUNDESAMT, « Tab. 1.11: Einnahmen und Ausgaben sowie Finanzierungssaldo des Staates in \% des Bruttoinlandsprodukts », in STATISTISCHES BUNDESAMT, Volkswirtschaftliche Gesamtrechnungen - Inlandsproduktberechnung - Lange Reihen ab 1970 - Fachserie 18 Reihe 1.5, Wiesbaden, 2012, p. 41

STERN K., SCHMidT-BleibTREU B. (eds), Verträge und Rechtsakte zur Deutschen Einheit, 3 volumes, C.H. Beck, Munich, 1990-1991

STRuBE S., Die Geschichte des Haushaltsrechts vom Mittelalter bis zur Gegenwart - Eine ökonomische Analyse im Lichte der Budgetfunktionen, Duncker \& Humblot, Berlin, 2002 (Schriften zur wirtschaftlichen Analyse des Rechts ; 45)

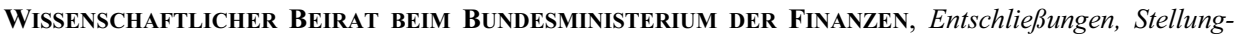
nahmen und Gutachten 1949-1973, Mohr, Tübingen, 1974

WisSEnSChAFTLICHER BEIRAT BEIM BUNDESMinisteriUm DER FinANZEN, Gutachten und Stellungnahmen 1999-2008, Richard Boorberg Verlag, Berlin, 2009

Wissenschaftlicher Beirat beim BUNDESMinisterium der FinANZEN, « Nachhaltigkeit in der Finanzpolitik - Konzepte für eine langfristige Orientierung öffentlicher Haushalte », [2001], in WISSEN- 
SCHAFTLICHER BEIRAT BEIM BUNDESMINISTERIUM DER FINANZEN, Gutachten und Stellungnahmen 19992008, Richard Boorberg Verlag, Berlin, 2009, p. 105-150

WISSENSCHAFTLICHE BEIRÄTE BEIM BUNDESMINISTERIUM DER FINANZEN UND BEIM BUNDESMINISTERIUM FÜR WIRTSCHAFT, « Gemeinsame Empfehlungen zu Folgerungen aus der gegenwärtigen Konjunkturlage ", [1955], in WISSENSCHAFTLICHER BEIRAT BEIM BUNDESMINISTERIUM DER FINANZEN, Entschließungen, Stellungnahmen und Gutachten 1949-1973, Mohr, Tübingen, 1974, p. 103-107

WISSENSCHAFTLICHE BEIRÄTE BEIM BUNDESMINISTERIUM DER FINANZEN UND BEIM BUNDESMINISTERIUM FÜR WIRTSCHAFT, « Gutachten über Instrumente der Konjunkturpolitik und ihre rechtliche Institutionalisierung », [1956], in WiSSENSCHAFTLICHER BEIRAT BEIM BUNDESMINISTERIUM DER FINANZEN, Entschließungen, Stellungnahmen und Gutachten 1949-1973, Mohr, Tübingen, 1974, p. 109-130. 


\section{Principes fondateurs et constantes en matière de politique budgétaire en France depuis $1945^{1}$}

\section{Frédéric TRISTRAM}

Le terme de culture politique a été utilisé dans le champ de l'histoire depuis une vingtaine d'années en France, à la suite des travaux pionniers de Serge Berstein. On pense notamment à un article sur l'historien et la culture politique qu'il a fait paraître dans la revue Vingtième Siècle en ${ }^{1992}{ }^{2}$. Ce terme a d'abord été utilisé dans l'histoire politique, puis a été étendu à d'autres domaines, et notamment à l'histoire économique à la suite d'une journée d'étude organisée en 2001 à Sciences Po sur les cultures économiques des hommes politiques à l'épreuve du pouvoir sous les IIle et IVe Républiques. II est aujourd'hui utilisé pour des politiques plus précises encore, le CIRAC ayant organisé une journée d'étude sur la politique monétaire et, aujourd'hui, sur la politique budgétaire dans un cadre qui plus est comparatif, ce qui montre l'efficience de la notion.

Je traiterai ici d'une politique budgétaire conçue au sens large, qui concerne la dépense, les recettes et qui inclut les modes de financement de l'État, en particulier sa politique fiscale. Ce dernier ne se réduit pas à l'État central mais comprend de plus en plus en tout cas sur le plan financier - les collectivités locales et les organismes de sécurité sociale. II ne s'agit pas ici - le terme de culture politique ou de culture économique ne s'y prête guère - de retracer l'histoire de la politique budgétaire entre 1945 et aujourd'hui, mais de prendre en compte plusieurs éléments de cette politique. Tout d'abord, les permanences qu'elle a pu présenter. Par-delà les circonstances et dans des contextes très différents, il s'agit de vérifier si apparaissent des réactions ou des comportements similaires chez les acteurs publics. Ensuite, il s'agit de voir en quoi ces comportements reflètent ou non des valeurs ou des préférences collectives, le terme de culture impliquant un véritable système de représentations partagées. II sera question d'en définir le contenu et les contours et de voir s'il est limité aux décideurs publics - qu'il s'agisse des membres de l'administration ou des hommes politiques qui prennent toujours la responsabilité des décisions budgétaires et fiscales - ou si au contraire il peut atteindre, sous des formes qui sont probablement plus diffuses, l'ensemble de la société. Par exemple, nous verrons que l'une des caractéristiques françaises réside dans un niveau élevé de dépenses publiques. II s'agira de vérifier si ce niveau élevé de dépenses publiques est révélateur de choix collectifs qui vont bien au-delà des spécialistes, par exemple un attachement à un niveau important de socialisation de la santé, ou plus largement, une vaste protection sociale. Enfin, cette culture budgétaire n'est pas figée. Elle évolue, se nourrit des transformations de l'État et, plus largement, de l'économie et de la société que la France a connues sur une période relativement longue, c'est-à-dire depuis 1945. J'essaierai ainsi dans un premier

\footnotetext{
${ }^{1}$ Texte issu d'une communication donnée dans le cadre de la journée d'étude Cultures budgétaires en France et en Allemagne, coorganisée par le CIRAC le 18 novembre 2013, NdE.

${ }^{2}$ Serge Berstein, «L'historien et la culture politique », Vingtième Siècle. Revue d'histoire, $\mathrm{n}^{\circ} 35$, juilletseptembre 1992, p. 67-77.
} 
temps d'inscrire cette notion dans une chronologie pour identifier la formation progressive d'une culture budgétaire française depuis 1945 et, dans un deuxième temps, d'en tirer un certain nombre de permanences et de constantes.

\section{La formation progressive d'une culture budgétaire française depuis 1945}

J'ai distingué, de façon chronologique, trois grandes périodes : la difficile mâ̂trise budgétaire d'un État reconstructeur, après la guerre et la crise des années 1930, entre 1945 et 1958, la période tout à fait singulière dans l'histoire française de l'équilibre budgétaire entre 1958 et 1973, qui correspond globalement à la politique gaullienne, dont elle est l'un des principes fondateurs, et le nouveau paradigme budgétaire depuis 1974 .

La difficile maîtrise budgétaire, 1945-1958

Selon moi, la période de difficile maîtrise budgétaire de 1945 à 1958 dans le cadre d'un État reconstructeur se caractérise par trois critères principaux. D'abord, la dépense publique est poussée vers le haut par le contexte de la Libération et par les impératifs de reconstruction. Les dépenses sont certes affectées à la protection sociale, avec la création de la sécurité sociale par les ordonnances des 4 et 19 octobre 1945, son extension progressive en dehors du champ des salariés et l'application de mesures de solidarité ou d'assistance allant audelà du cadre assurantiel, comme le minimum vieillesse qui, dans un cadre budgétaire pourtant très difficile en pleine guerre d'Algérie, est partiellement financé par la création d'impôts, et notamment par la vignette automobile en vertu de la loi du 30 juin 1956. Mais l'État va aussi et surtout prendre à sa charge une grande partie de la modernisation du pays après les destructions de la guerre. Les crédits passent directement par le budget de l'État ou par des comptes spéciaux du Trésor notamment, qui vont gérer l'aide Marshall via la Commission des investissements ou la direction du Trésor au sein du ministère des Finances. Il existe sur ce point une bibliographie abondante : je citerai notamment Michel Margairaz pour son travail pionnier sur l'État, les finances et l'économie ${ }^{3}$ ou encore, sous forme de témoignage, l'ouvrage paru en 1986 de François BlochLainé et Jean Bouvier sur la France restaurée ${ }^{4}$. Dans l'immédiat après-guerre, les fonds publics financent une part extrêmement importante des immobilisations en France, avec un pic en 1949, année lors de laquelle 64 \% des immobilisations sont financées directement ou indirectement par des fonds publics. Par la suite, si cette part va redescendre à des niveaux davantage compatibles avec l'économie de marché, elle se montera encore à $25 \%$ dans la seconde moitié des années 1950.

\footnotetext{
${ }^{3}$ Michel Margairaz, L'État, les finances et l'économie - Histoire d'une conversion, 1932-1952, 2 vol., Comité pour l'histoire économique et financière de la France, Paris, 1991 (Histoire économique et financière de la France : Série Études générales).

${ }^{4}$ François Bloch-Lainé, Jean Bouvier, La France restaurée - 1944-1954 - Dialogue sur les choix d'une modernisation, Fayard, Paris, 1986.
} 
Le deuxième critère qui caractérise cette période tient au fait que face aux exigences de la reconstruction, l'orthodoxie financière passe au second rang. On tolère par exemple une importante « impasse » (ce terme alors employé correspond au déficit public), qui atteint nettement plus de $5 \%$ du produit intérieur brut (PIB) dans les années de l'immédiat après-guerre. Surtout, le financement de cette impasse passe par des procédés hétérodoxes, et notamment par le mécanisme du circuit mis en place sous Vichy, qui est générateur d'inflation. L'épargne courte des Français, collectée notamment par les caisses d'épargne et la Caisse des dépôts, est ainsi distribuée au sommet de l'État pour financer des investissements. Cela est évidemment hétérodoxe, des immobilisations longues étant financées grâce à de l'épargne courte. Jean Bouvier disait dès lors, sur un ton quelque peu humoristique, qu'il avait financé des barrages hydroélectriques avec de l'épargne liquide. Cette hétérodoxie est revendiquée, y compris par des très hauts fonctionnaires de ce qui était alors la rue de Rivoli. Roger Goetze, directeur du Budget de 1949 à 1956, a pour sa part une position un peu plus orthodoxe que celle de François Bloch-Lainé. Dans toutes ses notes budgétaires sur l'état des finances publiques à destination des ministres, il met l'accent sur des dépenses qu'il juge disproportionnées.

La dernière caractéristique qui forge la culture budgétaire de cette période mais aussi des suivantes - et qui va quelque peu tempérer cette image de l'État reconstructeur est la conscience d'un problème institutionnel qui, indépendamment des contraintes financières très lourdes d'une France en reconstruction, rend compliquée la gestion des finances publiques. Ce problème est double: c'est d'abord l'absence de majorité stable dans le cadre d'un régime d'assemblée. Par exemple, en 1956-1957, le gouvernement de Front républicain, avec à sa tête Guy Mollet et comme ministre des Finances Paul Ramadier, est confronté à des problèmes de financement qui tiennent d'une part à son programme social avec le minimum vieillesse et, d'autre part, à la guerre d'Algérie. Ce gouvernement, qui souhaite augmenter les impôts pour financer ces dépenses nouvelles, est freiné dans ses projets par la composante radicale de la majorité, représentée au sein du gouvernement par Jean Filippi, secrétaire d'État au Budget, qui s'y oppose formellement et fait peser la menace d'une explosion de la coalition. De tels exemples politiques d'opposition à une augmentation des impôts et à un meilleur équilibre budgétaire sont légion sous la $\mathrm{IV}^{\mathrm{e}}$ République. Le second problème réside dans l'absence d'un texte qui encadre les procédures budgétaires. Il faut attendre le décret du 19 juin 1956 pour qu'apparaisse une première tentative d'encadrement des procédures budgétaires qui inspirera l'ordonnance organique de 1959. Cela a notamment pour conséquence une absence de maîtrise du temps budgétaire. Les lois de finances et les lois d'impôts sont adoptées à n'importe quel moment sous la $I^{\mathrm{e}}$ République. Le budget est voté avec retard. En 1953 par exemple, la loi de finances de l'année est votée le 7 février, le gouvernement ayant recours aux douzièmes provisionnels.

En conclusion vont se cristalliser à cette époque des éléments majeurs de la culture budgétaire dans le climat très symbolique de la Libération, ce qui leur 
donne une force supplémentaire. Celui du rôle d'un État reconstructeur, de l'arbitrage en faveur de l'expansion au détriment de la stabilité monétaire, mais aussi, et de plus en plus, celui de la réserve face à des institutions qui ne permettent pas une régulation optimale des budgets, qui existe certes dans la tradition gaulliste, mais qui en déborde à la veille de la $\mathrm{V}^{\mathrm{e}}$ République et qui touche notamment des socialistes.

\section{Le temps de l'équilibre budgétaire, 1958-1973}

La deuxième grande période qui va également forger des éléments de la culture budgétaire est celle située entre 1958 et 1973. Elle est tout à fait particulière puisqu'elle comprend une double rupture. La première rupture concerne la norme budgétaire : l'ordonnance organique du 2 janvier 1959 va encadrer strictement peut-être même trop strictement - les procédures budgétaires dans un calendrier ou dans des techniques censées assurer au mieux l'équilibre budgétaire. La deuxième rupture porte sur les pratiques politiques. On assiste à l'affirmation d'une doctrine gaullienne directement issue de l'expérience de 1958 et du plan de stabilisation Pinay-Rueff de décembre 1958, qui va inscrire l'ensemble de la nouvelle République dans la perspective d'un équilibre budgétaire. Cet exercice va être suivi jusqu'en 1973.

Au début des années 1960, les recettes courantes se situent à peu près à 36$37 \%$ du PIB. Elles font plus qu'équilibrer les dépenses totales de la sphère publique, qui oscillent autour de $35 \%$ du PIB. À la fin des années 1960, la puissance publique s'accroît pour se monter à $39 \%$ du PIB, le maximum étant atteint en 1968. Néanmoins, les recettes de l'État restent stables et le déficit, quand il existe, ne dépasse pas $0,5 \%$ de PIB.

Par conséquent, contrairement à ce qu'on pourrait penser, on a en France une référence de l'équilibre budgétaire qui s'ancre dans la culture gaulliste mais qui, curieusement, a beaucoup moins traversé les années que n'a pu le faire la politique gaullienne en matière par exemple de politique étrangère ou de défense. Cet héritage d'équilibre budgétaire n'est revendiqué aujourd'hui ni par la gauche ni par ceux qui pourraient passer pour les héritiers naturels du général de Gaulle. Cela aurait notamment pu être le cas en 2010 et 2011-2012, le gouvernement comportant alors dans ses rangs des élus gaullistes. C'est à la fois une surprise et sans doute une limite de cet héritage politique qui s'explique peut-être par le fait que cet équilibre budgétaire n'a été permis dans les années 1960 et jusqu'en 1973 que parce qu'il y avait de la croissance. Par conséquent, le contexte a beaucoup changé. Quand on regarde les budgets de l'époque, on n'assiste pas à une restriction budgétaire, mais bien au contraire à une augmentation des budgets très nette, à la fois en francs constants et en francs courants. Selon les chiffres de l'Organisation de coopération et de développement économiques (OCDE), les emplois totaux en part de PIB, c'est-à-dire l'ensemble des dépenses de l'ensemble des administrations publiques, passent d'un peu moins de $35 \%$ en 1960 à $39 \%$ en 1967. Ce mouvement est suivi d'une stabilisation, avec 38,5\% en 1973. 


\section{Un nouveau paradigme budgétaire depuis 1973}

La période depuis 1973 semble marquée par trois variations tout à fait singulières. D'abord, il s'agit d'une très forte poussée de la dépense et des recettes. On entend souvent dire que nous vivons une époque libérale où l'État aurait en quelque sorte réduit sa place. J'ai peine à le croire en regardant les chiffres des dépenses et des recettes qui me semblent ne pas être de mauvais indicateurs sur la place de l'État. Dans les années 1970, nous avons donc assisté à une poussée européenne de la place de l'État dans les économies occidentales, avec un moment de basculement dans les années 1973-1982/84, où l'on passe pour la dépense publique d'environ $38 \%$ à plus de $50 \%$ du PIB. Sur la même période, le taux de prélèvements obligatoires - qui ne représente certes pas l'ensemble des recettes publiques, mais qui en est un bon indicateur - a grimpé de $33,8 \%$ à $42,8 \%$. Depuis, si l'on excepte la situation tout à fait particulière depuis 2011, ce taux oscille entre $42 \%$ et $45 \%$, ce qui correspond au précédent pic atteint en 1999. En 2013, il se situe à $46 \%$. Par conséquent, les niveaux de dépenses et de recettes n'ont plus grand-chose à voir avec ceux des années 1960 .

Cette période se singularise également par une répartition différente des dépenses et des recettes, tout d'abord entre institutions. En 1978, l'État central représentait à peu près $18 \%$ de PIB en termes de prélèvements obligatoires, contre environ 14,5\% en 2008 avant la crise. On observe ainsi un déclin assez net du poids financier de l'État central en part de PIB. En revanche, cela est plus que compensé par la poussée des collectivités locales qui, en termes de prélèvements obligatoires, sont passées d'environ $3 \%$ à $6 \%$ entre 1978 et 1988 . Pour la première fois en 2006, les administrations de sécurité sociale ont pour leur part dépassé l'ensemble des autres partenaires, représentant ainsi plus de $50 \%$ des recettes et des dépenses publiques. La répartition nouvelle des dépenses et des recettes se fait également au sein même du budget de l'État. Pour reprendre une expression utilisée par Alexandre Siné dans son ouvrage sur l'ordre budgétaire, on est passé d'un État investisseur à un État «employeurdébiteur ", dont les principales fonctions en termes budgétaires consistent à payer de la masse salariale (les fonctionnaires) et à rembourser sa dette. On pourrait même employer un troisième terme : celui d'État « subventionneur ».

Le troisième changement fondamental réside dans l'apparition d'un niveau élevé de déficit et d'endettement. Le graphique 1 ci-après montre une croissance non régulière mais presque continue de la dette depuis le début des années 1980. En réalité, il y a eu le maintien d'une relative discipline budgétaire jusqu'en 1980-1981, avec un niveau d'endettement faible et un déficit public relativement maîtrisé. On peut distinguer cependant quatre moments. Dans un premier temps, la croissance lente mais régulière de la dette entre 1981 et 1990, suivie d'une très forte croissance de la dette sur fond de conjoncture très dégradée, qui passe ainsi de $35 \%$ à $60 \%$ du

\footnotetext{
${ }^{5}$ Alexandre Siné, L'ordre budgétaire - L'économie politique des dépenses de l'État, Économica, Paris, 2006 (Études politiques).
} 
PIB entre 1991 et 1997. Puis, on observe, malgré des conditions de croissance favorables, une absence de consolidation budgétaire et de désendettement entre 1997 et 2007, et enfin une explosion de la dette, phénomène que connaissent à peu près tous les pays européens depuis la crise de 2008.

J'aimerais apporter un commentaire sur ce schéma : ce qui pousse la dette, ce n'est pas l'alternance politique, c'est essentiellement la situation conjoncturelle. Les poussées de dette correspondent à des moments de conjoncture extrêmement déprimée. La période 1991-1996 notamment a été caractérisée par la pire crise conjoncturelle qu'ait connue la France de son histoire, si l'on excepte la crise actuelle.

\section{Graphique 1 : La dette publique (en \% du PIB)}

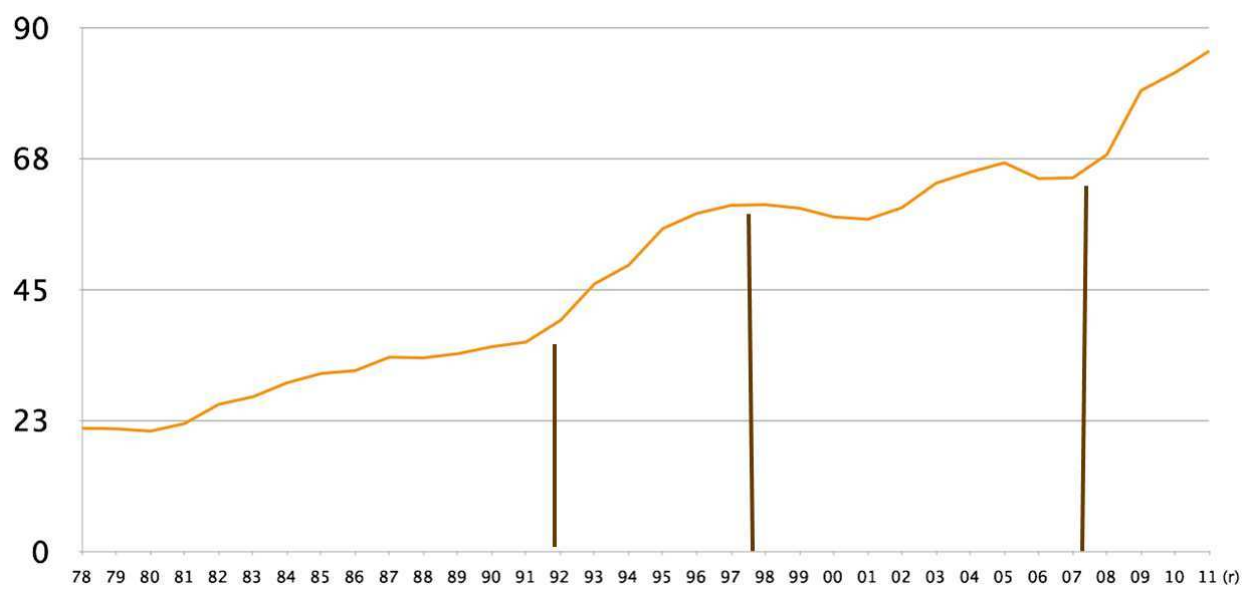

Source : présentation de l'auteur.

\section{Les permanences de l'histoire budgétaire}

Je distingue trois permanences de l'histoire budgétaire de ces 70 dernières années en France : le niveau structurellement élevé de la dépense publique, l'acceptation d'un niveau élevé de prélèvements obligatoires et surtout l'ambition inachevée d'un réglage conjoncturel par le budget.

\section{Un niveau structurellement élevé de la dépense publique}

Le niveau structurellement élevé de la dépense publique ne constitue pas un phénomène récent. L'écart existait déjà dans les années 1950 et 1960 entre la France et ses principaux pays partenaires. L'année 1965 est assez révélatrice du contexte budgétaire des années 1960. La France était à 38,4\% de dépenses publiques. C'était évidemment davantage que les États-Unis (28\%), et surtout, que le Japon $(18,6 \%)$, qui s'est toujours distingué par une place beaucoup plus limitée de l'État, à la fois en termes de dépenses et de recettes. Mais c'est également davantage que dans les autres grands pays européens, avec 34,3\% en Italie et $36,3 \%$ en 
République fédérale d'Allemagne. La situation s'inverse avec l'Allemagne dans les années 1970, notamment après 1973, parce que le pays mène alors des politiques plus keynésiennes que la France. À l'époque où Raymond Barre est Premier ministre et ministre des Finances, la France a une vision plus classique que celle des gouvernements socio-démocrates allemands sous Helmut Schmidt. Les courbes vont se croiser, l'écart le plus important étant atteint en 1977, avec 47,8 \% en Allemagne et 43,7\% en France. Au début des années 1980, les courbes se recroisent à nouveau et ne se réinverseront plus, de sorte que le différentiel France-Allemagne se monte à sept points dans les années 1990. Ce tropisme en faveur de la dépense s'explique essentiellement par une croissance importante de la dépense publique sociale en France, qui s'élevait à 15-17\% du PIB avant 1975 et qui se situe depuis les années 1990 au-dessus de $25 \%$. Ce niveau très élevé s'explique probablement par des choix collectifs et des choix culturels.

Deuxièmement, la France mène une politique budgétaire asymétrique depuis le début des années 1980, en ce sens qu'elle adopte une politique contracyclique en période de dépression et procyclique en période de croissance un peu plus soutenue, ce qui constitue un problème majeur. Le principal exemple en est la période 1997-2001 durant laquelle, face à des rentrées fiscales qui apparaissent exceptionnelles en période conjoncturelle favorable, le gouvernement de l'époque se retrouve complètement démuni face à cette manne providentielle qu'il finit par distribuer un peu à tout le monde et dans un relatif désordre. Inversement, dans le cadre de politiques de retour à l'équilibre budgétaire et visant au désendettement, la France a très nettement privilégié la hausse des prélèvements sur la baisse des dépenses. Cela a été le cas au cours des quatre périodes de consolidation budgétaire que la France a connues depuis 1945, c'est-à-dire celle de 1948 d'Henri Queuille notamment, celle de 1958 avec Antoine Pinay et le général de Gaulle, celle de 1995 lorsqu'il s'agissait de se qualifier à l'euro, et l'actuelle consolidation fiscale. Trois cas sur quatre se distinguent par une utilisation massive de l'impôt, au détriment d'une baisse des dépenses. La seule exception est la consolidation budgétaire de 1958, avec un plan beaucoup plus équilibré utilisant à peu près à parité la hausse des impôts et la baisse (ou la maîtrise) des dépenses publiques. Le symbole en est la fin de la retraite des anciens combattants, qui a beaucoup marqué les esprits. Après le plus fort de la crise conjoncturelle du début des années 1990, c'est-à-dire à partir de 1996-1997, la France mène une politique de consolidation à finalité européenne afin de respecter les critères de convergence définis par le traité de Maastricht. Cette consolidation est réalisée pour moitié par une baisse des dépenses et pour une autre moitié par une hausse des recettes, mais avec une chronologie extrêmement particulière: le gouvernement d'Alain Juppé commence par faire des hausses massives d'impôts - avec notamment l'augmentation de deux points de la taxe sur la valeur ajoutée (TVA) décidée à l'été 1995 - qui se traduisent par une augmentation d'un point et demi de PIB du taux de prélèvements obligatoires entre 1995 et 1997. La baisse des dépenses en points de PIB est pour sa part obtenue par l'effet automatique du retour 
de la croissance à partir de 1997, et non grâce aux mesures volontaristes de réduction des budgets. Si ce plan porte sur quatre points de PIB (deux points d'augmentation des recettes et deux points de diminution des dépenses), il s'agit en réalité d'une fausse symétrie parce que l'une est volontaire et l'autre, automatique. Ce type de démonstration est valable pour les autres plans de stabilisation que j'ai énoncés, celui de 1958 mis à part.

Troisièmement, il existe un difficile consensus sur les objectifs de restauration des grands équilibres. Il n'y a pas dans la société française - comme cela peut être le cas dans la société allemande - de sanction du corps électoral ou de pression de l'opinion publique en faveur de la réduction du déficit et de l'endettement. La consolidation budgétaire est un thème arrivé avec retard dans le débat politique français. La question de la dette apparaît au début des années 2000, d'abord dans des cercles de spécialistes. Une des grandes étapes de cette prise de conscience est marquée par la publication, en 2006, d'un rapport confié à Michel Pébereau sur la dette et l'endettement ${ }^{6}$. Ce rapport va pointer une «culture de la dépense » en matière de dépenses de fonctionnement de l'État, qui serait responsable du niveau élevé de la dette et du déficit en France, au détriment des dépenses d'investissements. On assiste aujourd'hui à une prise de conscience tardive, et surtout contrainte. En effet, après le point bas de la croissance en 2009, il a fallu attendre un an et demi pour entrer dans des logiques de consolidation budgétaire qui ne sont intervenues qu'à l'été 2011, et non au cours de l'année 2010, malgré des demandes assez insistantes de la Commission européenne et du Fonds monétaire international (FMI). Il a donc fallu attendre l'été 2011 pour qu'on entre dans une logique de réduction volontariste certes du déficit, mais pas de l'endettement, sous la pression de la crise des dettes souveraines et surtout sous la pression du risque de taux que pouvait connaître la dette française.

\section{L'acceptation d'un niveau élevé de prélèvements obligatoires}

Le deuxième élément de cette culture partagée sur 70 ans en France est l'acceptation d'un niveau élevé de prélèvements obligatoires. Avant les années 1950, il y avait en France une tradition ancienne du refus de l'impôt qui a conduit par exemple à une adoption tardive de l'impôt progressif sur le revenu (introduit plus tardivement que les impôts allemand ou britannique sur le revenu). Des travaux universitaires ont montré comment cette hostilité à l'égard de l'impôt progressif a été à l'origine des premières tentatives de syndicalisme des classes moyennes (je pense notamment à l'association de défense des classes moyennes de Maurice Colrat qu'a étudiée Gilles Le Béguec ${ }^{7}$ ).

\footnotetext{
${ }^{6}$ Michel Pébereau, Rompre avec la facilité de la dette publique - Pour des finances publiques au service de notre croissance économique et de notre cohésion sociale, La Documentation française, Paris, 2006 (Collection des rapports officiels).

${ }^{7}$ Cf. notamment Gilles Le Béguec, «Prélude à un syndicalisme bourgeois. L'association de défense des classes moyennes (1907-1939) », Vingtième Siècle. Revue d'histoire, n 37, janvier-mars 1993, p. 93-104.
} 
Or, depuis 1945, on est clairement entré dans une période d' "apaisement fiscal », pour reprendre l'expression utilisée par Nicolas Delalande dans son ouvrage sur l'acceptation de l'impôt ${ }^{8}$. Acceptation fiscale, alors même - et c'est un paradoxe - qu'on assistait à une poussée des prélèvements obligatoires. Les dernières oppositions fiscales, si on excepte ce qui se produit actuellement dans un contexte un peu différent, sont relativement anciennes. Il s'agit du poujadisme de 1953 à 1958 et du mouvement de la Confédération intersyndicale de défense et d'union nationale des travailleurs indépendants (CIDUNATI) de Gérard Nicoud au tournant des années 1960 et 1970. Ces mouvements n'ont pas eu d'incidence budgétaire, c'est-à-dire qu'ils n'ont pas empêché le recouvrement de l'impôt. Il y a eu des appels - notamment en 1955 par Poujade - à la grève de l'impôt. Toutefois, le mouvement n'a pas été suivi. En matière de contrôle fiscal, les interruptions sont beaucoup plus modestes qu'on ne le dit. Il y en a eu une (partielle) dans la deuxième partie de l'année 1955 et au début de l'année 1956. En revanche, ces mouvements de révolte fiscale ont différé des réformes fiscales qui étaient en cours. Je pense notamment à la TVA, qui est très liée à l'expérience poujadiste. Votée en avril 1954, la TVA a été dans un premier temps limitée au secteur de la production. Il faudra attendre 14 ans, en 1968, pour qu'elle soit étendue au secteur du commerce de détail. L'idée que l'on arrête la réforme à mi-chemin et qu'on la poursuivra dans un climat plus apaisé dans les années 1960 est un effet retardé du poujadisme, mais c'est bien le seul effet, du point de vue budgétaire, de ces mouvements de contestation de l'impôt.

Comment expliquer cette relative tolérance des Français vis-à-vis de la pression fiscale et des prélèvements obligatoires ? J'émettrai quelques hypothèses. Cela peut découler de l'attachement à un État assureur social et prestataire de services, dans la lignée de la Libération. On retrouve là cette référence assez constante dans notre histoire budgétaire. C'est aussi l'utilisation d'impôts relativement « indolores », mais qui passent sans doute mieux dans la population que d'autres types d'impôts, notamment la TVA dans les années 1960 et la contribution sociale généralisée (CSG) à partir des années 1990, qui a été introduite avec un taux extrêmement modeste de $1,1 \%$ et qui ensuite a vu son taux progressivement croître, jusqu'à devenir l'une des principales recettes de l'État au sens large puisqu'elle finance la protection sociale avec aujourd'hui à peu près 80 milliards d'euros de rendement. Cela nourrit une critique constante parce que ces impôts ont en commun de n'être pas progressifs, d'être des impôts qui sont - bien que la question soit discutée - de nature dégressive pour la TVA et proportionnelle (ou très légèrement progressive) pour la CSG. Dans des travaux menés dans les années 2000, la politiste nippo-américaine Junko Kato a montré que plus un État se développe, moins le prélèvement a tendance à être progressif?. En effet, ce n'est pas la même chose

\footnotetext{
${ }^{8}$ Nicolas Delalande, Les Batailles de l'impôt - Consentement et résistances de 1789 à nos jours, Seuil, Paris, 2011 (L’Univers historique).

${ }_{9}^{9}$ Junko Kato, Regressive Taxation and the Welfare State: Path Dependence and Policy Diffusion, Cambridge University Press, New York, 2003 (Cambridge Studies in Comparative Politics).
} 
d'avoir un impôt progressif à $25 \%$ ou à $45 \%$ de taux de prélèvements obligatoires. Il y a donc une classification des États qui, au fur et à mesure qu'ils s'étendent, tendent à adopter des impôts de plus en plus proportionnels pour financer notamment une protection sociale accrue. Ce n'est donc pas un phénomène ni choquant ni particulier que d'avoir recours à ce type de prélèvements pour financer une croissance des politiques publiques, et notamment des politiques sociales.

\section{L'ambition inachevée d'un réglage conjoncturel}

La dernière permanence de l'histoire budgétaire française réside selon moi dans l'ambition inachevée d'un réglage conjoncturel par le budget. La politique conjoncturelle apparaît dans les années 1960, dans un contexte d'équilibre global des finances publiques. Elle est d'autant mieux utilisée qu'elle s'inscrit dans un contexte d'équilibre à moyen terme du budget de l'État qui permet de dégager des marges de manœuvre pour infléchir l'évolution conjoncturelle quand cela s'avère nécessaire. Son apparition en France après 1966 est liée à l'expérience américaine Kennedy-Johnson. Il s'agit d'une transposition en France de la philosophie générale de ces politiques, et même parfois des instruments de ces politiques, notamment celui de la détaxation des investissements, beaucoup utilisé en France, qui correspond à une mesure prise aux États-Unis après 1962. Cette politique va se poursuivre en France dans les années 1970 et 1980, malgré l'échec des deux grands plans de relance de 1975 (mené au lendemain de la crise pétrolière dans le cadre de la politique de stop-and-go, et impliquant des masses budgétaires importantes) et de 1981-1982 (qui est plus symbolique parce que lié à la grande alternance politique). Malgré l'échec de ces politiques - et il s'agit peut-être là aussi d'une des constantes de la culture budgétaire française -, nous restons dans le cadre conceptuel de l'arbitrage entre l'équilibre budgétaire et la croissance, dont on a beaucoup de mal à sortir comme le montrent les débats récents. L'idée qu'un budget structurellement équilibré garantisse des conditions satisfaisantes de croissance structurelle n'est en effet pas ou peu envisagée en France. Nous sommes au contraire plutôt dans une logique court-termiste où l'on pratique de la relance continue. C'est un schéma mental qu'on a beaucoup de mal à quitter en France.

EN CONCLUSION, je dirai que trois éléments permettent de caractériser la culture budgétaire française : le rôle actif de l'État (notamment en matière sociale), une certaine tolérance au déficit qui est encore trop souvent perçu comme une condition de la croissance et qui se conjugue à la faiblesse d'une culture alternative - celle de l'expérience gaullienne de réduction du déficit dans les années 1960-1973 -, et une bonne tolérance à l'augmentation des impôts, même si quand on atteint $46 \%$ ou $47 \%$ de prélèvements obligatoires, cette tolérance tend évidemment à décliner. Néanmoins, on voit aujourd'hui que, sur ces trois constantes, la culture budgétaire française est entrée en crise, y compris dernièrement sur l'acceptation d'un niveau élevé de prélèvements obligatoires. 


\section{La politique budgétaire allemande après Maastricht : l'expression d'une culture budgétaire ?'}

\section{Michael THÖNE}

Avant de proposer, en ma qualité de spécialiste en économie financière, quelques remarques sur la politique budgétaire allemande depuis l'adoption du pacte de stabilité et de croissance de Maastricht, j'aimerais revenir sur le titre de cette journée d'étude et celui de ma contribution: quand on parle de "cultures budgétaires en France et en Allemagne", de quoi s'agit-il ? Le fait même que je prenne au mot cette thématique est peut-être caractéristique de la culture allemande. Dans cette contribution, j'aimerais en guise d'introduction poser une question générale : que sont les cultures budgétaires, par opposition à d'autres facteurs déterminant notre politique budgétaire ? Je commencerai par proposer quelques éclairages sur la politique budgétaire allemande et présenterai, en quelques données chiffrées, le budget de l'État fédéral depuis le début des années 2000. Je tenterai une comparaison avec les 27 autres États membres de l'Union européenne (UE) et, bien entendu, avec la France.

Ma contribution ne prétend pas être davantage qu'un " essai ", une tentative de brève réflexion personnelle sur ce qu'est une culture budgétaire et sur son influence potentielle sur la politique budgétaire.

\section{Des cultures budgétaires?}

\section{Est-il possible d'identifier les influences d'une culture budgétaire?}

L'on remarque de nombreuses différences d'un pays à un autre. Est-ce la culture ? Voici comment je vois les choses aujourd'hui, et c'est sûrement schématique : la culture ne se manifeste clairement que là où nous constatons des différences et tentons de nous les expliquer. Celles-ci correspondent aux spécificités qui, dans la perspective nationale, vont tellement de soi qu'on ne les perçoit peut-être pas comme des données culturelles. En matière de politique budgétaire et financière, nous constatons de nombreuses différences entre l'Allemagne et la France, et peut-être entre bon nombre d'autres pays au sein de l'Union européenne ou de l'Organisation de coopération et de développement économiques (OCDE) : parmi ces différences, combien sont vraiment de nature culturelle - quelle que soit la définition que l'on donne de la culture - et quelle part relève plutôt de facteurs fondamentaux et «objectifs »? En 2011, Alan Greenspan a par exemple avancé la thèse selon laquelle les déséquilibres

\footnotetext{
${ }^{1}$ Cet article, rédigé en décembre 2014, fait suite à une communication donnée dans le cadre de la journée
} d'étude Cultures budgétaires en France et en Allemagne, coorganisée par le CIRAC le 18 novembre 2013, NdE. 
macroéconomiques dans l'économie européenne s'expliqueraient au moins pour partie par des différences culturelles quasi inaltérables entre le nord et le sud de la zone euro.

L'approche d'un économiste consisterait à expliquer les différences en regardant d'abord ce qui diverge du point de vue macroéconomique. Autrement dit : dans la diversité que j'observe, quelle part tient aux disparités entre les économies nationales, entre leurs forces et leurs faiblesses respectives? Quel rôle jouent en outre les institutions économiques et politiques fondamentales, par exemple la constitution, ou encore l'organisation des rapports entre les partenaires sociaux?

La deuxième question est celle de la politique économique en cours : quelle part de ce que nous observons en matière de politique budgétaire s'explique par le fait que nous avons dans tel pays un gouvernement de gauche et dans tel autre un gouvernement de droite ? Ou par le fait que deux gouvernements d'une même sensibilité politique doivent néanmoins, dans deux pays distincts, offrir des prestations publiques différentes? Ce type de considération a bien sûr des répercussions claires sur les différences de politique budgétaire. Ces dissemblances sontelles déjà inscrites dans les cultures, autrement dit, les citoyens de pays distincts ont-ils des préférences différentes ? Ou bien cette culture commence-t-elle - et c'est ma thèse - là où, par-delà les facteurs fondamentaux, des routines, des traditions et des cultures politiques spécifiques marquent effectivement la politique budgétaire comme un facteur supplémentaire, un facteur à part entière ?

\section{La culture budgétaire de qui ?}

L'Allemagne est un État fédéral, et c'est un élément non négligeable. Je le souligne sciemment, même si maints observateurs issus d'États "véritablement » fédéraux, comme la Suisse ou les États Unis, pourraient tourner en dérision cette vision allemande au motif qu'ils ne voient pas en Allemagne la diversité effectivement à l'œuvre ni, surtout, la volonté de divergence caractéristique du fédéralisme. C'est un point de vue que l'on trouve également représenté en Allemagne, pays qualifié tantôt d' «État fédéral unitaire » tantôt d'« État unitaire déguisé ».

Abstraction faite de ces questions de fond - l'objet de nos travaux n'étant pas de débattre sur le fédéralisme - le fait est que les cultures budgétaires des Länder sont très présentes. Elles influencent la perception publique. Les différences résident au premier chef dans la perception que les acteurs ont d'euxmêmes. On doit par exemple à Klaus Wowereit, qui fut maire de Berlin pendant de nombreuses années, une expression devenue courante : en 2003, il déclarait que sa ville était "pauvre, mais sexy». À l'inverse, de riches Länder du Sud comme la Bavière et le Bade-Wurtemberg sont très privilégiés, y compris en termes de finances publiques. Dans ces régions, l'économie est très prospère ; les gouvernements présentent parfois un haut degré d'autosatisfac- 
tion. Et ils mènent souvent une politique budgétaire qui ressemble plutôt à celle de personnes riches : ils ne regardent pas toujours très précisément à la dépense. Les Länder de l'est du pays, plus pauvres, ou ceux du nord, pas particulièrement fortunés, doivent au contraire mener des politiques budgétaires bien plus restrictives.

Ces différences engendrent des conflits en matière de politique de redistribution, des tensions volontiers présentées par les acteurs comme des conflits culturels dont le motif implicite serait que les uns s'y prendraient mieux que les autres. Lorsque l'on observe tel ou tel acteur politique qui passe d'un Land à un autre et doit tout à coup faire de la politique dans des conditions différentes (passant d'un Land riche à un plus pauvre, ou inversement), on voit plus précisément ce qui, dans les différences, relève de la culture et ce qui relève d'un facteur fondamental.

Il convient de repréciser notre interrogation de départ : de qui sont-ce les cultures budgétaires? Dès lors que nous ne sommes plus chez nous, nous ne parlons plus de nous-mêmes et de notre pays que de manière collective. Lorsque je parle moi-même aujourd'hui, à Paris, de la (des) culture(s) budgétaire(s) en Allemagne, je dis parfois "nous». Qu'est-ce qui nous caractérise, "nous les Allemands » dans « notre » position en la matière ? Lorsque l'on est chez soi, ce n'est bien sûr pas du tout le cas. Nous avons, d'un parti à un autre et d'une institution à une autre, des représentations très différentes de ce que devrait être une culture budgétaire et des préférences qui lui sont associées. C'est un phénomène trivial, il en va ainsi pour toute personne. Mais il n'est parfois pas inutile de dire explicitement ce qui va de soi.

Les personnalités politiques sont parfois susceptibles d'avoir elles aussi une influence sur les choses qui auront lieu ou non. Mais, s'agissant de l'Allemagne, ce qu'il ne faudrait surtout pas sous-estimer, c'est réellement l'administration. Je pense que les Français voient bien ce que je veux dire. L'administration ministérielle allemande aime certes à se présenter au monde extérieur comme une entité qui ne fait que suivre les ordres du Parlement, mettre en œuvre le droit et la législation et qui ne prend elle-même aucune initiative. Il me semble cependant que l'administration sait perpétuer de façon utile les cultures et les traditions, et tente souvent ainsi de mener sa propre politique. Implicitement, le statut permanent des hauts fonctionnaires allemands sert également cet objectif : ils doivent parfois éviter le pire de ce que les électeurs et les responsables politiques, qui pensent à court terme, pourraient réaliser ensemble. Cette attribution élitiste des rôles est naturellement très contestable d'un point de vue constitutionnel.

Les citoyens ont-ils eux aussi une culture budgétaire ? Ou bien sont-ce simplement leurs préférences que nous, économistes du bien-être, nous plaisons à mettre au premier plan? Les citoyens du monde entier veulent-ils surtout des prestations de qualité, bon marché, et se différencient-ils peu pour le reste ?

Il existe à mon sens des différences bien réelles, y compris dans la façon dont les citoyens voient leur responsabilité collective : que pensent-ils fonda- 
mentalement de l'endettement public ? Quel niveau de dette publique considèrent-ils comme acceptable ? Ce type d'interrogation élémentaire quant aux valeurs apparaît aussi dans les enquêtes empiriques, par exemple le World Values Survey. L'on y voit très clairement où se trouvent les divergences entre les pays, que l'on peut attribuer plus ou moins aux cultures budgétaires.

\section{Lieux de la culture budgétaire}

En matière de culture budgétaire, il existe trois lieux, présentés sur le triangle ci-dessous. Lorsque nous parlons de cultures budgétaires, nous nous situons constamment à l'intérieur de ce triangle.

\section{Schéma 1 : Lieux de la culture budgétaire}
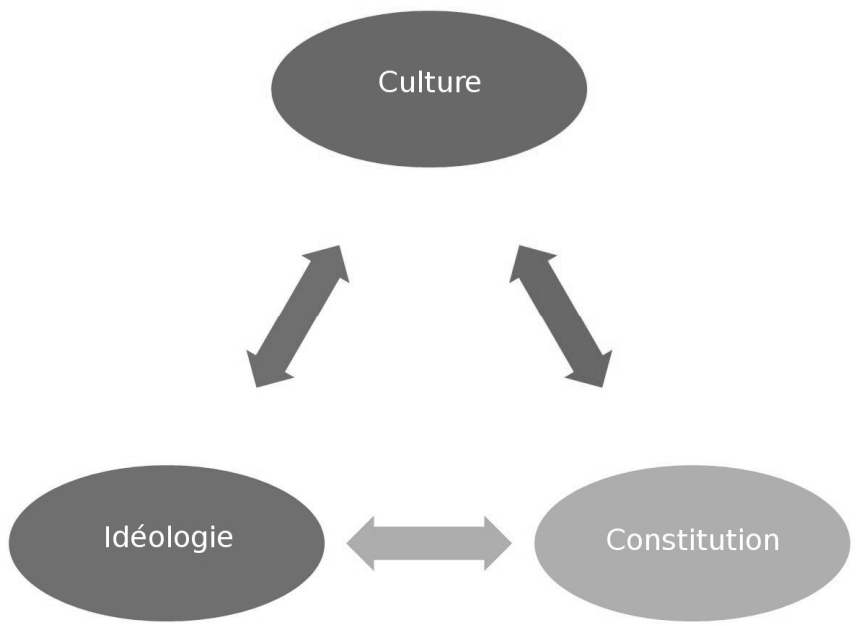

Source : présentation de l'auteur.

À l'un des angles se trouvent les idéologies - dans un sens non pas négatif, mais comprises comme des points de vue et des systèmes conceptuels différents. Il s'agit, pour simplifier, de la différence entre l'approche néoclassique («néolibérale ») et l'approche keynésienne. En Allemagne, on parlerait, en plus, d'approche ordolibérale.

À quel point les idéologies imprègnent-elles les cultures ? Dans quelle mesure la culture et l'idéologie marquent-elles les constitutions, au sens non seulement de constitutions écrites, mais également des institutions qui se sont développées sur leur base? Lorsque l'on parle par exemple de règles en matière d'endettement : celles qui ont été inscrites dans la constitution ne sortent pas de nulle part. Elles doivent en effet recueillir le soutien d'une majorité des deux tiers des parlementaires. Ce type de décision ne fait pas intervenir uniquement les idéologies, ni même uniquement les majorités politiques du pays concerné. Pour des décisions touchant à la constitution, les cultures budgétaires jouent 
elles aussi un rôle. En retour, la constitution influe sur la culture concernée. En effet, quel autre document pourrait marquer davantage une culture budgétaire ou une culture juridique que la constitution, produit d'une tradition? À aucun moment, l'on ne sort de cette dynamique triangulaire.

\section{Coup de projecteur sur la politique budgétaire allemande}

Je souhaite dans cette partie apporter quelques éclairages thématiques sur les finances publiques allemandes, sans pour autant retracer dans le détail la politique budgétaire de ces quinze dernières années, avec toutes ses fluctuations.

\section{Interaction avec le Pacte de stabilité et de croissance}

À l'échelon fédéral, la politique budgétaire allemande est fortement marquée par la référence au pacte de Maastricht ou à son rejet. Cela tient sans doute notamment au fait que le pacte de stabilité et de croissance de 1992 prétendait tenir compte très largement des attentes allemandes. En 1992, certains points clés de cet accord furent en effet formulés conformément à la vision allemande. Les limites fixées - endettement de l'État plafonné à $60 \%$ du produit intérieur brut (PIB), déficit autorisé de $3 \%$ du PIB par an - furent par exemple définies de telle manière que l'Allemagne ne les dépasserait jamais. C'est en tout cas ce que l'on pensait à l'époque, même si les choses ont changé, comme nous le verrons plus bas. La dette globale autorisée n'a en outre jamais joué un grand rôle d'un point de vue politique; aujourd'hui encore, à l'ère du pacte budgétaire, cet indicateur reste clairement secondaire.

La règle du déficit fut au contraire prise si au sérieux que la première entorse qui y fut faite, en 2002, doit être perçue comme un changement de culture budgétaire. Cinq ans après l'entrée en vigueur du Pacte de stabilité et de croissance, l'Allemagne et la France ont fait en sorte de le rendre inopérant. Les deux plus grands pays de l'UE, ses membres les plus connus, ont enfreint les règles encadrant le déficit d'une manière qui, en réalité, aurait dû entraîner des sanctions claires. Mais il ne s'est rien passé, ou presque : pour des raisons évidentes, la France et l'Allemagne ont reçu le soutien amical de la Grèce et de l'Italie, et les ministres de l'Économie et des Finances de l'UE se sont entendus pour que Bruxelles n'aille pas au-delà d'une simple mise en garde si les pays déficitaires rentraient dans les clous à compter de 2005 au plus tard.

Puis vint l'année 2008 - la grande césure, avec la faillite de Lehman Brothers. En 2009 et 2010 ensuite, la grande crise économique et financière, dont les pays ne se sont pas encore remis au plan budgétaire. Pour l'Allemagne, la crise eut l'effet d'un petit miracle. En 2009, l'État fédéral et les Länder adoptèrent le « frein à l'endettement » (la « règle d'or »), grâce au soutien d'une majorité qui permettait de modifier la Constitution. Les responsables politiques allemands ne se seraient jamais imposé cette règle d'eux-mêmes, en dépit de leur réputation de «rigueur» dans d'autres pays. À partir de l'année 2016, le 
déficit budgétaire structurel autorisé pour l'État fédéral est limité à seulement $0,35 \%$ du PIB ; quant aux Länder, ils doivent même présenter à partir de 2020 des budgets structurels équilibrés. Suivant l'exemple suisse, seuls sont autorisés des déficits conjoncturels qui doivent toutefois pouvoir être compensés en période de croissance économique grâce à des recettes publiques excédentaires.

Il avait déjà été question depuis longtemps de mettre en place des restrictions aussi sévères, mais ces discussions étaient plutôt le fait de cercles universitaires et du corps électoral, non des milieux politiques. Des économistes siégeant dans les instituts d'expertise et au gouvernement, exigeant depuis longtemps des finances publiques plus viables, avaient évoqué dans diverses tribunes des règles budgétaires plus contraignantes que celles alors en vigueur. $\mathrm{Ce}$ n'est toutefois pas parce que quelques experts tiennent de tels propos qu'ils vont être suivis d'effets. Mais la crise économique et financière produisit manifestement un choc culturel terrible dans les milieux politiques allemands. Ils se virent dans l'obligation de répondre aux déficits conjoncturels considérables en adoptant une mesure radicale de lutte contre les déficits durables et l'accroissement de la dette. Et c'est ce qui fut fait.

En 2011, on réforma le Pacte de stabilité et de croissance européen, notamment en mettant en place un encadrement plus strict de la dette publique (une sorte de «frein à l'endettement »), à l'instar de ce qui existait en Allemagne et en Suisse. Nous observons ici de nouveau une interaction très forte entre les politiques budgétaires menées aux niveaux national et européen, ou bien la tentative, du côté allemand, de faire intervenir le niveau européen au niveau national. Cet ancrage permanent dans l'Europe et avec elle est, à mon sens, une caractéristique importante de la culture budgétaire allemande.

\section{À l'intérieur : inertie face aux réformes}

Jetons maintenant un autre coup de projecteur, à l'intérieur du pays, sur l'inertie marquée face aux réformes. On parle volontiers de l'Allemagne comme étant une "locomotive», une "puissance économique de premier ordre ». Ces dénominations trouvent effectivement leur traduction politique dans les réformes du marché du travail, menées de 2003 à 2005 et qualifiées d' « Agenda 2010 »; ce furent des réformes profondes. Mais il est surtout question ici de politique budgétaire ; or, en Allemagne, nous sommes très réservés pour ce qui est de procéder à des réformes en la matière.

Il existe de nombreuses raisons institutionnelles à cela, sur lesquelles nous ne pouvons revenir ici de manière détaillée. Le fédéralisme politique joue en tout cas un rôle important : une élection a toujours lieu, à un moment ou à un autre, quelque part dans le pays. Les élections locales et régionales sont souvent considérées en Allemagne comme des baromètres de l'opinion en matière de politique nationale. Les élections au Landtag sont en effet presque autant d'occasions pour les citoyens de se prononcer sur le gouvernement fédéral. 
L'on observe certes des variations régionales, mais les influences de la politique fédérale sur les élections au Landtag sont absolument considérables et les décideurs politiques en tiennent compte. Dans ce contexte de campagne électorale permanente, le moment n'est jamais opportun pour mener des réformes certes nécessaires, mais impopulaires à court terme. Or c'est typiquement le lot des réformes en matière de politique budgétaire : les coûts politiques se ressentent au début, les bénéfices interviennent, au mieux, au bout de deux ou trois ans seulement. D'où la frilosité des responsables politiques en matière de réforme, puisque l'on ne dispose tout au plus que d'une année sans élection. Le risque de s'exposer à un vote sanction se trouve encore amplifié du fait que le fédéralisme allemand est très " coopératif », ce qui veut dire que les Länder ne se démarquent pas fortement comme entités politiques, et que les citoyens ne leur accordent pas tellement de valeur.

Le fédéralisme coopératif est en outre un facteur expliquant l'inertie face aux réformes. Nous tentons depuis de nombreuses années de mieux gérer cette étroite intrication en réformant le fédéralisme, à l'aide de correctifs de petite ou moyenne envergure. Mais ces modifications sont difficiles à réaliser : le fédéralisme donne lieu à un enchevêtrement serré de compétences, et pas uniquement entre les Länder. La réalisation des missions de l'État est aussi tellement imbriquée, depuis l'échelon fédéral jusqu'à celui des communes, en passant par l'échelon régional, que nous nous trouvons confrontés à d'importantes résistances dès lors qu'il s'agit de réformer. L'État fédéral ne peut par exemple procéder seul à aucune réforme en matière de politique sociale, quand bien même il y aurait de bonnes raisons de le faire. Dans presque tous les domaines, il faut intégrer les trois échelons administratifs, la Constitution interdisant de courtcircuiter l'échelon intermédiaire des Länder. De ce fait, les réformes ne sont souvent possibles en Allemagne que si le Bund apporte suffisamment de moyens financiers supplémentaires pour compenser la contribution des autres acteurs. Si la coopération ne fonctionne pas ou que les moyens financiers ne sont pas disponibles, les réformes nécessaires et même urgentes ne peuvent bien souvent pas se faire.

Mentionnons, pour conclure cette partie, la théorie dite « du nez » (NasenTheorie). Ce n'est pas une expression très répandue, mais il semble qu'elle ait cours au ministère fédéral des Finances depuis une vingtaine d'années déjà. Cette théorie explique la frilosité allemande en matière de réforme budgétaire par le fait que, ces dernières décennies, l'Allemagne ne s'est jamais "cassé le nez » assez sérieusement, autrement dit n'a jamais connu d'échec assez dur pour être incitée à modifier les choses en profondeur. On retrouve donc ici la même idée que dans la citation de Churchill : «Il ne faut jamais gaspiller une bonne crise (Never let a good crisis go to waste). »

Les nombreux pays de l'OCDE qui ont mené des réformes budgétaires fondamentales ne l'ont pas fait car ils se trouvaient désœuvrés. Les réformes profondes sont généralement une réaction à une grande crise macroéconomique, 
une crise bancaire ou un autre événement de ce type. L'Allemagne a souvent eu cette chance que l'impact des crises, auxquelles elle n'a pas échappé, ne fut pas aussi sévère que pour d'autres pays. Il a donc été possible d'atténuer les effets des crises avant que celles-ci ne conduisent à des réformes. Sans porter d'appréciation, on peut sans doute affirmer à juste titre que cette "atténuation » de l'action et de la réaction fait maintenant pleinement partie de notre culture politique. Cela explique peut-être pourquoi l'Allemagne est moins dynamique que d'autres pays dans certains domaines.

\section{Données budgétaires de l'Allemagne, de la France et de l'UE à 27}

Voici maintenant un bref aperçu de données budgétaires concernant la France et l'Allemagne, dans le contexte de l'UE, afin d'illustrer les effets des cultures budgétaires ou bien leur absence.

\section{Recettes publiques}

Le graphique 1 présente l'ensemble des recettes publiques pour l'Allemagne, la France et l'UE pour la période 2001-2012. La période mise en évidence correspond à la phase la plus aiguë de la crise financière. À en croire cet indicateur, il y aurait eu étonnamment peu de changement dans cette phase. Cette impression tient au fait que cet indicateur apparaît en pourcentage de PIB. Or, pendant la crise, le PIB a connu une chute massive dans les deux pays. Les recettes s'étant elles aussi effondrées, les taux de recettes publiques sont donc restés plutôt stables.

\section{Graphique 1 : Part des recettes publiques (en \% du PIB)}

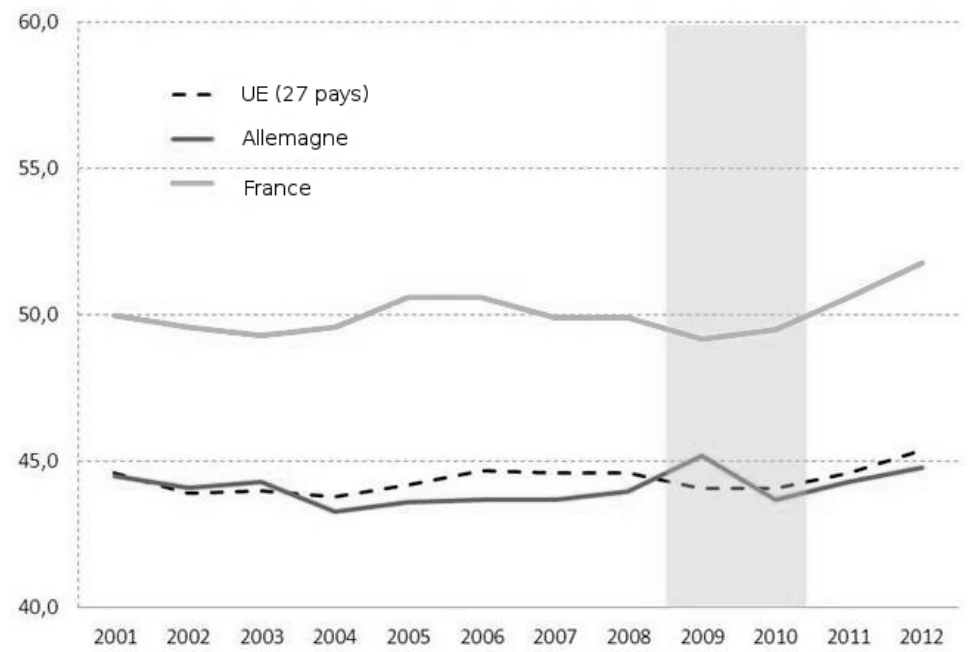

Source : Eurostat, présentation de l'auteur. 
Dépenses publiques

La situation est fort différente s'agissant des dépenses publiques (graphique 2) : dans tous les pays, comme dans l'ensemble de l'UE, la part des dépenses publiques dans le PIB a bien sûr explosé pendant la crise. Cette évolution était voulue et nécessaire. Les stabilisateurs automatiques déploient ici leurs effets.

Il est également intéressant d'observer l'évolution des graphiques avant et après la crise. Si le PIB joue également un rôle, on observe toutefois une certaine tendance à l'austérité en Allemagne, bien que la perception soit différente à l'intérieur du pays.

\section{Graphique 2 : Total des dépenses publiques (en \% du PIB)}

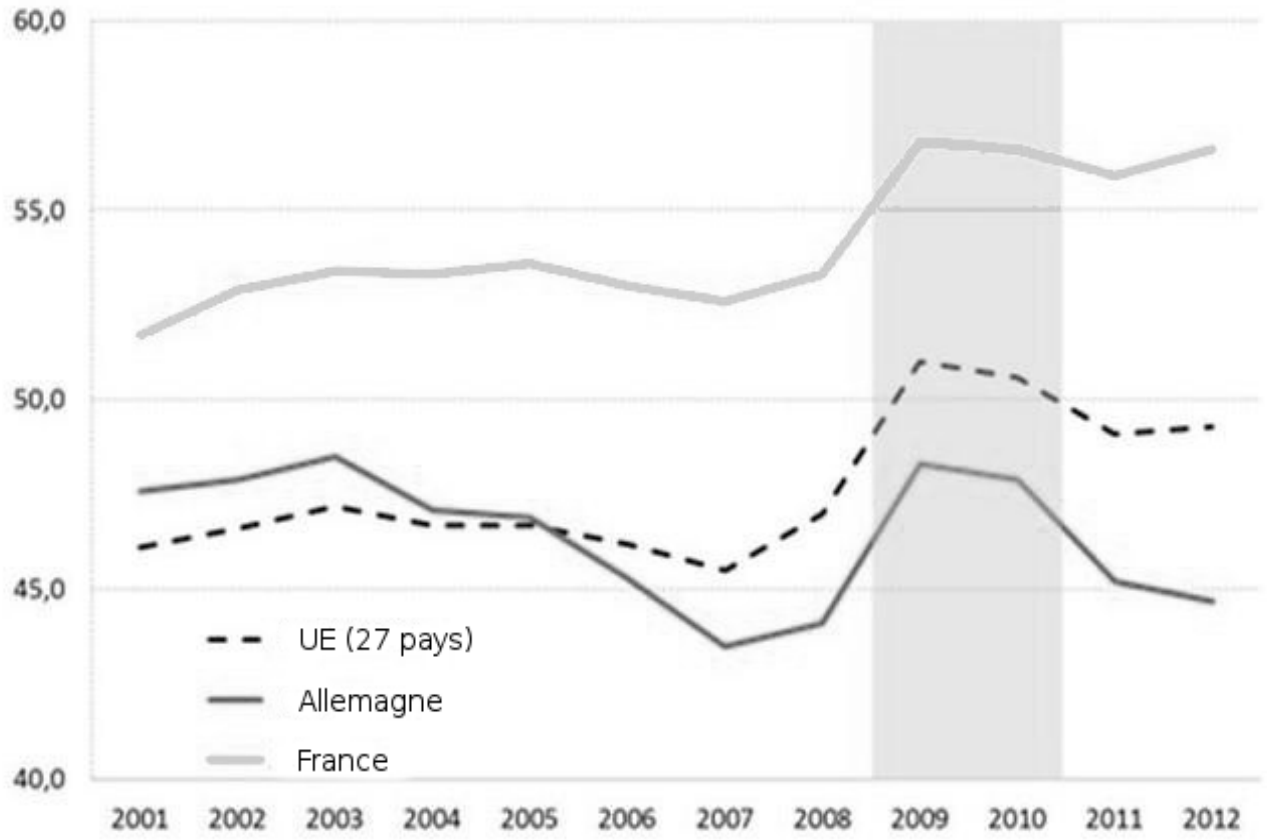

Source : Eurostat, présentation de l'auteur.

\section{Dépenses sociales}

Le graphique 3 met en évidence les dépenses sociales pour la même période. Si l'on observe là encore une hausse pendant la crise, on constate également un repli après en Allemagne. Cela ne veut pas dire que les programmes sociaux furent supprimés, mais plutôt que l'Allemagne a bien résisté à la crise, y compris dans le domaine social. 


\section{Graphique 3 : Dépenses sociales de l'ensemble des acteurs publics (en \% du PIB)}

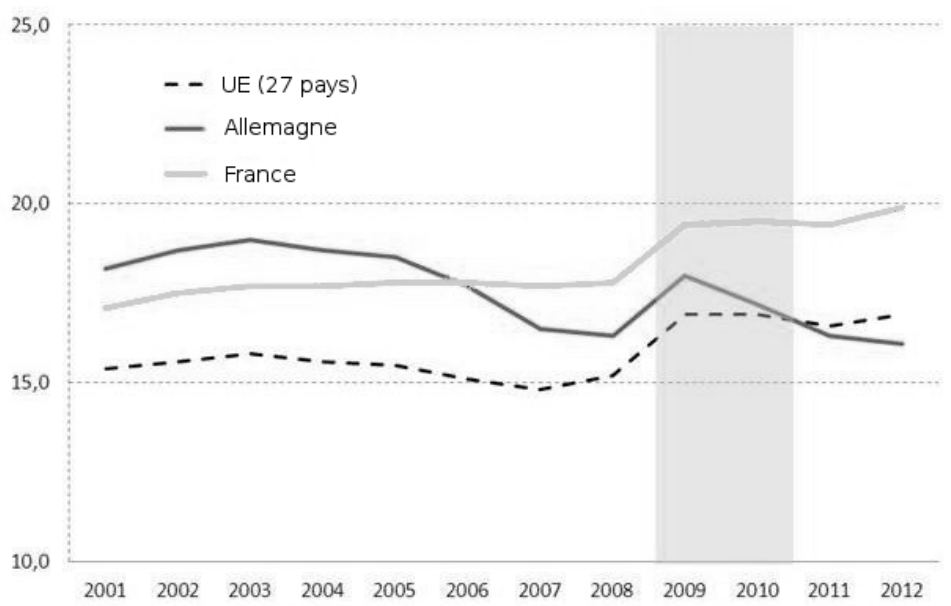

Source : Eurostat, présentation de l'auteur.

Investissements publics

Le niveau d'investissement public est très bas en Allemagne - et un vif débat a lieu actuellement sur la manière de combler le déficit d'investissement qui s'est constitué. Comment en est-on arrivé à un tel retard ? Lorsque l'on fait des économies, ou du moins lorsque l'on tente de maîtriser l'évolution du budget, cela se fait au détriment des dépenses qui, dans la concurrence avec la consommation publique en temps réel, ne sont pas défendues par des lobbys efficaces. À terme, ces choix se font donc au détriment des dépenses d'avenir.

\section{Graphique 4 : Total des investissements publics bruts (en \% du PIB)}

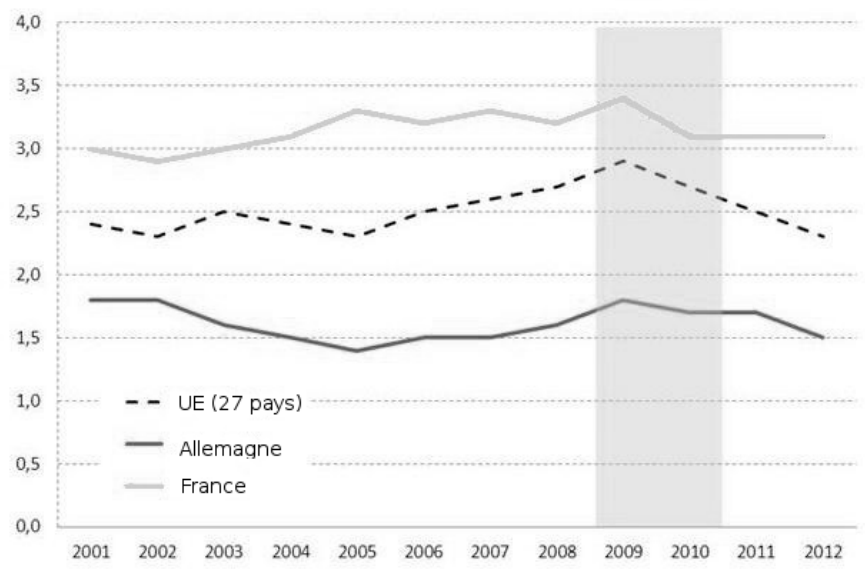

Source : Eurostat, présentation de l'auteur. 
En Allemagne, la part des investissements publics dans le PIB est très basse, depuis longtemps déjà. Cela crée un vrai problème de viabilité à long terme pour la politique budgétaire allemande. Je viens moi-même de Cologne. Dans cette ville, en 2013, deux des six ponts sur le Rhin n'étaient plus empruntables par les poids lourds, et ce, pour une longue durée, car ces infrastructures n'avaient pas été suffisamment entretenues depuis des décennies. Les investissements publics sont faibles, non pas que ces dépenses soient impopulaires, mais parce que lorsqu'il s'agit de réaliser des économies, on s'en prend aux dépenses les moins faciles à défendre, et il s'agit traditionnellement des investissements.

\section{Déficits}

Pour ce qui est des déficits, ce sont surtout les ordres de grandeur qui sont remarquables. Le graphique 5 représente la part de l'ensemble du déficit public dans le PIB (soldes budgétaires) pour l'Allemagne, la France et l'ensemble de l'UE. La ligne du zéro se trouve très haut sur le graphique car les déficits sont le plus souvent importants. Peu avant la crise, l'Allemagne était déjà parvenue une fois à l'équilibre sur l'ensemble de son budget public ; elle y revient en 2012. Ces moments traduisent un contexte macroéconomique très favorable, avec des recettes fiscales abondantes et des taux d'intérêts faibles. En 2012 (et en 2013), l'État fédéral et les Länder ont toujours des déficits, mais ils sont compensés par des excédents de la protection sociale.

\section{Graphique 5 : Solde du budget public (en \% du PIB)}

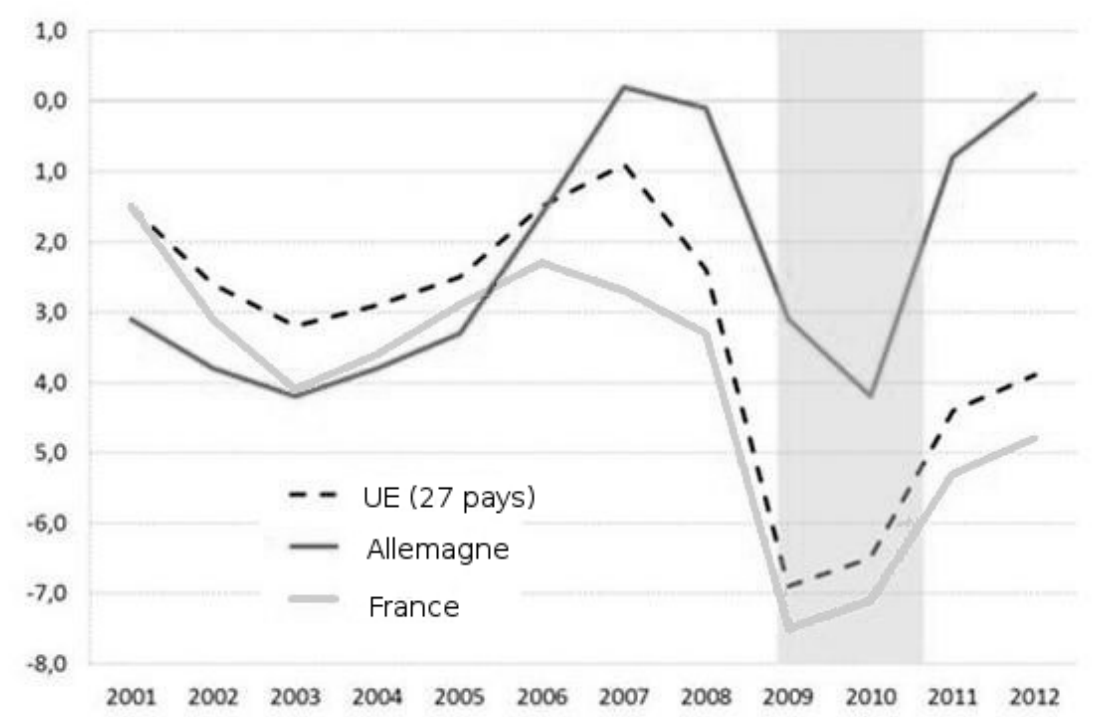

Source : Eurostat, présentation de l'auteur. 


\section{Dettes publiques}

Le graphique 6 présente le taux d'endettement public en pourcentage du PIB à partir de 1995. On voit en bas du graphique la limite de $60 \%$, inscrite dans le traité de Maastricht et confirmée récemment dans le cadre du pacte budgétaire. Il s'agit à la fois d'un objectif contraignant, convenu entre les États, et, pour l'instant, d'une simple réminiscence historique.

On note surtout la moyenne de l'UE à 27, de 1995 à 2007 : d'énormes mesures de consolidation ont été prises au cours de cette période, représentant 10 points de PIB en moyenne. Observons toutefois que l'Allemagne et la France ne participent pas à cette évolution. Par la suite, tous les pays de l'UE ont légèrement réduit leur dette publique peu avant la crise et, depuis, on note une hausse abrupte dans tous les pays, même si la courbe allemande est un peu plus plate que la moyenne.

\section{Graphique 6 : Ensemble de la dette publique (en \% du PIB)}

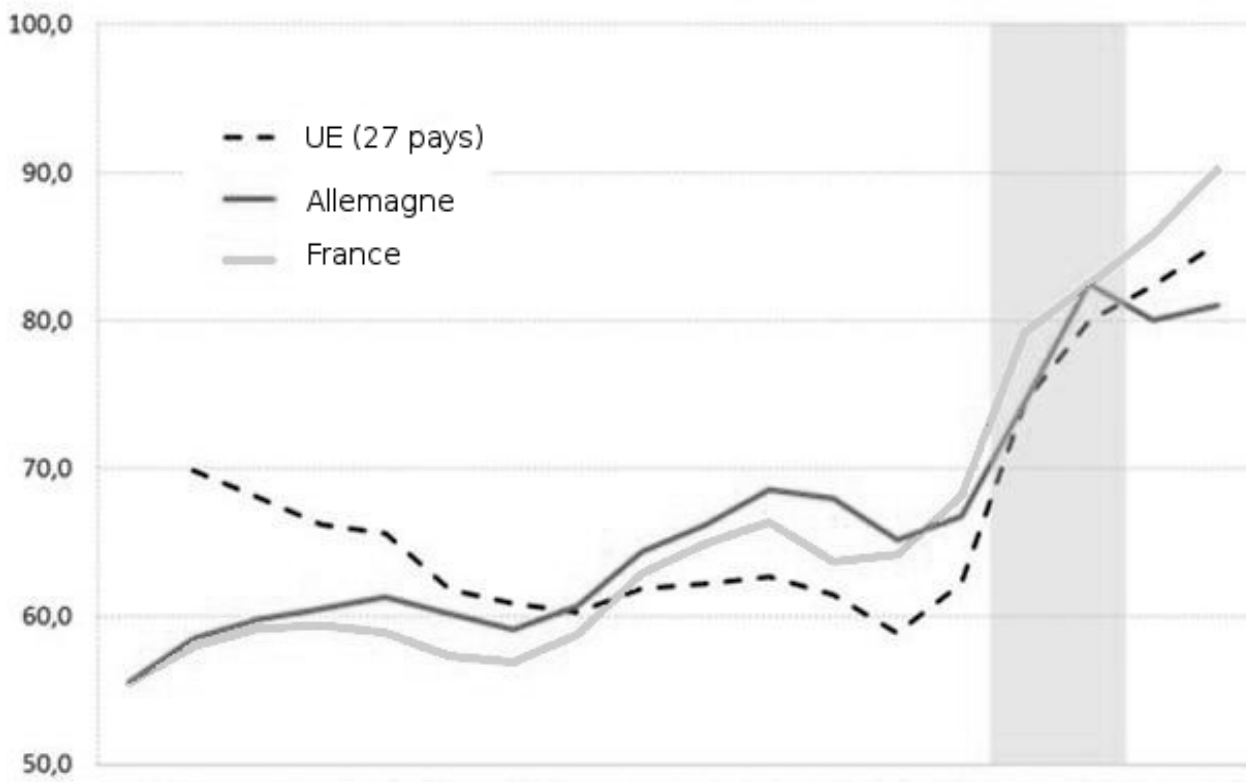

199519961997199819992000200120022003200420052006200720082009201020112012

Source : Eurostat, présentation de l'auteur.

Nous sommes très loin des $60 \%$ définis comme seuil repère dans le cadre de Maastricht. Ce seuil doit-il être respecté de manière dogmatique ? On pourrait sans doute en débattre longuement. Mais pour la viabilité des budgets publics, l'existence d'un plafond de cet ordre de grandeur joue tout de même un rôle important. 


\section{Institutions viables}

\section{Passerelles vers l'avenir}

Les cultures budgétaires peuvent nous aider à comprendre l'évolution historique - comprise comme les évolutions ayant eu lieu jusqu'à nos jours. Quelles conclusions convient-il d'en tirer pour l'avenir? Sur la route conduisant à l'État moderne du XXI siècle, les différences en matière de culture budgétaire fontelles partie de la solution ? Ou bien du problème ? Se trouvent-elles « au-dessus des choses » au sens où il faudrait accepter ces différences voire, dans certains cas, les cultiver? Nous faut-il au contraire laisser derrière nous certaines composantes de nos cultures budgétaires?

Ce sera certainement l'un des grands sujets de débats économiques, budgétaires et culturels à venir au sein de l'Union européenne.

\section{Pilotage budgétaire orienté résultats}

Lorsque l'on parle de cultures budgétaires, on a tôt fait d'oublier les institutions. Quelles sont les institutions viables à l'avenir? Quelles composantes des structures budgétaires publiques sont viables en l'état et lesquelles faudrait-il moderniser ? Le pilotage budgétaire orienté résultats peut jouer un rôle à cet égard. Ce principe signifie qu'en matière de gestion budgétaire, le succès d'une politique ne sera plus mesuré uniquement par le volume de financement injecté dans l'activité, mais aussi par les résultats mesurables. Ce sont les paramètres élémentaires à partir desquels il faudra à l'avenir conduire une politique budgétaire moderne si l'objectif est de pouvoir offrir de meilleures prestations publiques avec des moyens qui n'augmentent plus.

\section{Schéma 2 : Utilisation de la budgétisation axée sur la performance au niveau de l'État}

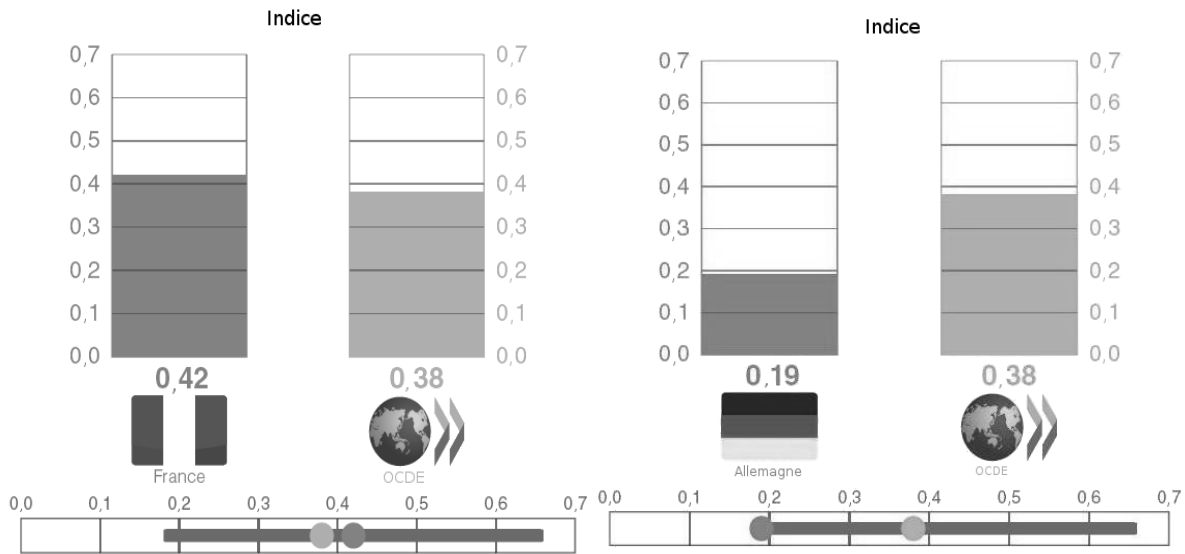

Sources : (C) OECD 2011 Survey on Performance Budgeting; OCDE, Panorama des administrations publiques 2013. 
C'est ce que l'on appelle la "budgétisation axée sur la performance». La France est déjà en bonne position en la matière, un peu au-dessus de la moyenne de l'OCDE (cf. schéma 2). L'Allemagne a en revanche encore beaucoup à faire, elle est de facto la lanterne rouge de l'OCDE. On peut peut-être interpréter ce retard comme un indicateur de la «théorie du nez»: en l'absence de phénomènes de crise sérieux, la nécessité de mener des réformes n'est pas perçue comme une urgence.

\section{Marchés publics}

La situation est tout à fait similaire dans le domaine des marchés publics. Ce secteur constitue en outre une interface très importante entre les pouvoirs publics, la population et l'économie du pays. Le mode de communication, de transparence et d'ouverture vis-à-vis de la concurrence internationale est un indicateur clé de la modernité des marchés publics. Là encore, la France fait mieux que l'Allemagne.

\section{Schéma 3 : Utilisation d'instruments innovants pour les marchés publics au niveau de l'État Fonctionnalités des systèmes d'acquisitions électroniques}

\begin{tabular}{|c|c|c|c|c|}
\hline & $\begin{array}{l}\text { Publication des } \\
\text { programmes d'achats }\end{array}$ & $\begin{array}{c}\text { Annonce des appels } \\
\text { d'offres }\end{array}$ & $\begin{array}{c}\text { Possibilité de } \\
\text { déposer des offres } \\
\text { par voie } \\
\text { électronique }\end{array}$ & $\begin{array}{l}\text { Possibilité d'envoyer } \\
\text { des factures par } \\
\text { voie électronique }\end{array}$ \\
\hline Allemagne & $\Omega$ & 0 & 0 & ) \\
\hline OCDE & $\begin{array}{r}20 \\
0 \quad 8 \\
0 \quad 10\end{array}$ & $\begin{array}{r}32 \\
7 \\
0\end{array}$ & $\begin{array}{r}16 \\
0 \quad 13 \\
0 \quad 6\end{array}$ & $\begin{array}{r}8 \\
0 \quad 10 \\
0 \quad 15\end{array}$ \\
\hline
\end{tabular}

électroniques nationaux et centraux

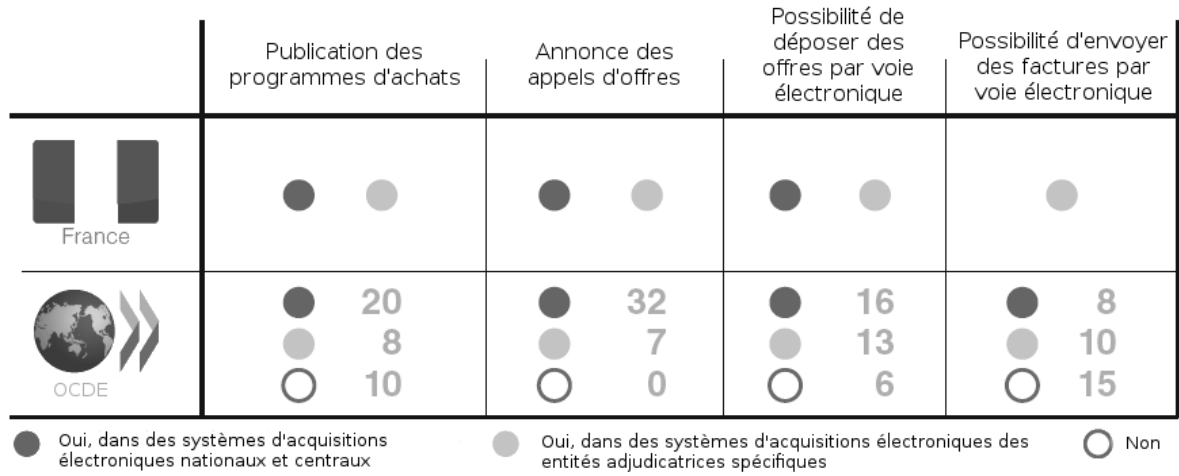




\section{Règles budgétaires}

Le schéma 4 présente le type de règles budgétaires et la manière dont elles sont mises en œuvre. Dans ce domaine, nous voyons également que les règles budgétaires européennes, sorte de « traité international »-il s'agit du pacte budgétaire - ont en Allemagne leur correspondance directe dans des règles de toute façon inscrites dans la Constitution. La France est quant à elle confrontée à un défi nettement plus grand puisque son cadre règlementaire en matière budgétaire est structuré différemment. Elle dispose de règles concernant les dépenses et les recettes, mais non de règle applicable au déficit ni à l'endettement. Il est donc très difficile de faire se rencontrer les systèmes des deux pays.

Schéma 4 : Règles budgétaires - types de règles et bases juridiques

\begin{tabular}{|c|c|c|c|}
\hline & \multicolumn{2}{|c|}{ Allemagne } & OCDE \\
\hline & $\begin{array}{l}\text { Existe-t-il ce } \\
\text { genre de règles? }\end{array}$ & $\begin{array}{l}\text { Si oui, sur quelle base } \\
\text { légale reposent-elles? }\end{array}$ & $\begin{array}{c}\text { Dans combien de pays ces règles } \\
\text { existent-elles? }\end{array}$ \\
\hline $\begin{array}{l}\text { Règle(s) sur les } \\
\text { dépenses }\end{array}$ & NON & & 21 \\
\hline $\begin{array}{l}\text { Règle(s) sur le } \\
\text { revenu }\end{array}$ & NON & & 5 \\
\hline $\begin{array}{l}\text { Règle(s) relative(s) } \\
\text { à l'équilibre } \\
\text { budgétaire }\end{array}$ & OUI & $\mathrm{TI}$ & 28 \\
\hline $\begin{array}{l}\text { Règle(s) relative(s) } \\
\text { à l'endettement }\end{array}$ & OUI & TI & 23 \\
\hline
\end{tabular}

Bases légales :

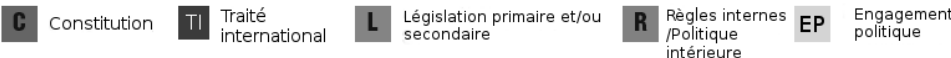

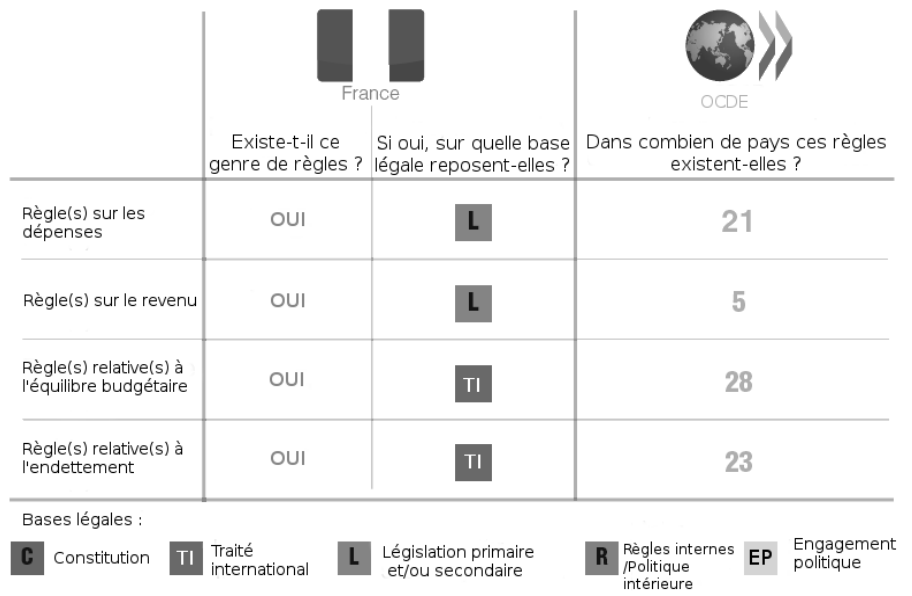

Source : (C) OCDE, Panorama des administrations publiques 2013. 


\section{Perspectives : des finances publiques viables?}

Puisque nous avons déjà tourné nos regards vers l'avenir, nous pouvons nous permettre de regarder maintenant à plus long terme. Voyons plus précisément la question du pacte budgétaire, sa mise en œuvre commune ou les controverses sur la question de savoir si nous pouvons (ou voulons) le mettre en œuvre et comment.

Quelle part de ce volet de la culture budgétaire pouvons-nous déléguer à Bruxelles ? Ou, pour formuler les choses autrement, quelle part voulons-nous déléguer à Bruxelles ?

Déléguer telle ou telle compétence à Bruxelles pourrait nous être tout à fait utile. Non pas parce que les choses y sont mieux faites, mais parce qu'il est parfois plus facile de laisser d'autres acteurs se charger des décisions douloureuses et désagréables. L'on concèderait à Bruxelles un certain pouvoir sur des questions fondamentales de politique budgétaire et l'on cesserait de s'y opposer fortement. On peut toutefois douter du fait qu'une centralisation croissante due à la faiblesse des politiques nationales serait, au-delà du répit accordé à la politique $\mathrm{au}$ jour le jour, également profitable à long terme au grand projet européen, $\mathrm{y}$ compris à la monnaie commune.

\section{Graphique 7 : Projections - part de la dette publique dans le PIB}

Allemagne

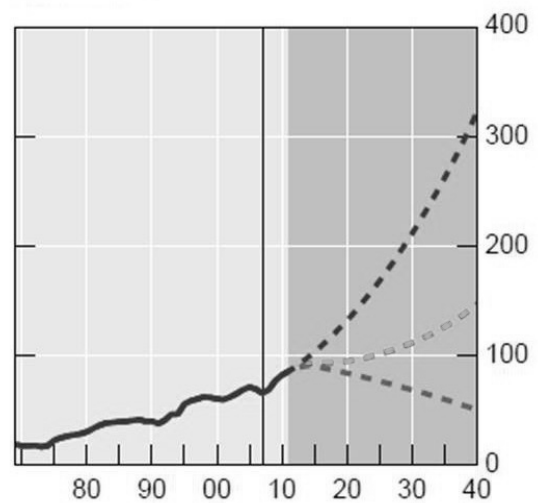

France

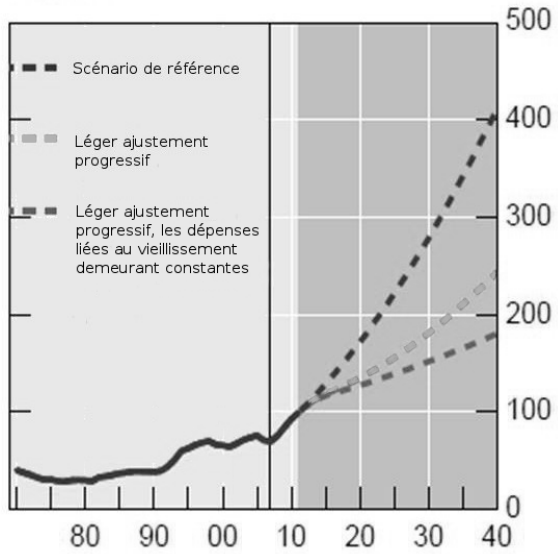

Source : (C) Cecchetti et al., 2010.

POUR CONClure, PROJETONS-NOUS À TRÈs LONG TERME : le graphique 7 propose une projection des dettes publiques en Allemagne et en France jusqu'en 2040, selon des calculs réalisés par la Banque des règlements internationaux. Ces calculs intègrent explicitement les évolutions démographiques. En effet, on trouve par ailleurs très souvent des indicateurs de durabilité budgétaire qui ne tiennent pas compte de ces évolutions et sont donc d'un optimisme irréa- 
liste. Si l'on prend en compte les répercussions négatives liées au vieillissement, on voit que l'Allemagne, avec sa structure démographique, est un pays qui, contrairement à la France, devra faire face à des difficultés importantes, susceptibles de grever fortement l'avenir. En effet, la part de la population active est de plus en plus réduite, et les enfants et adolescents sont de moins en moins nombreux pour compenser une société vieillissante qui, en outre, diminue.

Malgré des perspectives très différentes en Allemagne et en France, qu'il s'agisse de l'évolution démographique ou de leurs cultures budgétaires, les deux pays se trouveront, s'ils se contentent de poursuivre leurs politiques budgétaires actuelles, avec des niveaux de dette publique très problématiques. La question ne sera alors plus de savoir si le problème se situe à $60 \%$ ou $80 \%$ du PIB. L'on aboutira à des ordres de grandeur tout autres.

Je trouve notable que ce type de projections, dans le cadre de calculs de viabilité, procède exactement de la même manière que ce que nous avons observé comme le fondement des cultures budgétaires habituelles, c'est-à-dire selon la formule : « Continuons comme avant. »

À Cologne, il est un adage qui dit: "Les choses ont toujours fini par s'arranger. " Cet adage ne s'applique pas à la politique budgétaire, au contraire : si l'on ne change rien, seul l'impact négatif se développe. Si l'on ne modifie pas les cultures budgétaires, la situation poursuivra son évolution, et se dégradera. À l'inverse, une nouvelle culture budgétaire de la prévision et de la durabilité budgétaire pourrait permettre de mieux défendre les «valeurs anciennes » d'un État fort et performant.

\section{Traduction de Marie GRAVEY}

\section{Indications bibliographiques}

Cecchetti S.G., Mohanty M. S., Zampolli F., « The Future of Public Debt: Prospects and Implications ", BIS Working Papers, n 300, mars 2010, p. 1-26

Greenspan A., «Europe's Crisis is All About the North-South Split»[en ligne], Financial Times, 06/10/2011, p. [n.c.]. Disponible sur : http://www.ft.com/intl/cms/s/0/678b163a-ef68-11e0-bc88-00144feab4 9a.html\#axzz3RLEsCM8T [consulté le 10/02/2015]

OCDE, Panorama des administrations publiques 2013, Paris, 2013. 



\section{La politique budgétaire depuis le Pacte de stabilité et de croissance : point de vue français ${ }^{1}$}

\section{Jérôme CREEL}

Cette contribution est consacrée à une analyse des pratiques françaises de politique budgétaire depuis la création de l'euro, après l'établissement des critères de convergence dits de Maastricht, puis du Pacte de stabilité et de croissance (PSC). L'exemple français sera mobilisé pour discuter des marges de manœuvre et des contraintes qu'impose le dispositif institutionnel européen, y compris le traité budgétaire.

Dans le présent chapitre, je reviendrai sur la logique sous-jacente aux critères de Maastricht puis au PSC ; j'insisterai sur le glissement théorique intervenu entre ces différents dispositifs de surveillance des politiques budgétaires. Ensuite, je rappellerai les différents concepts de déficit public; je discuterai des contraintes qu'impose le nouveau traité budgétaire et dégagerai les sources des déficits français au cours du temps. J'évoquerai ainsi l'état des finances publiques françaises et poserai enfin la question de savoir si l'austérité en valait la peine.

Le tableau 1, issu des prévisions du département Analyse et prévision (DAP) de l'Observatoire français des conjonctures économiques (OFCE), rappelle l'évolution récente des finances publiques françaises. Après le contrecoup de la crise financière internationale démarrée à l'été 2007, on observe en 2010 un solde public négatif considérable de $7,1 \%$. Il était prévu d'atteindre $-4,1 \%$ du PIB à la fin de l'année 2013 et seulement -3,5\% fin 2014. Compte tenu de la persistance de la crise économique, la Commission européenne a octroyé aux autorités françaises en mai 2013 un sursis de deux années supplémentaires pour revenir sous la barre des $3 \%$ du produit intérieur brut (PIB) et respecter le Pacte de stabilité et de croissance (PSC). Selon la prévision du tableau 1, la France atteindrait effectivement un déficit de $3 \%$ du PIB en 2015.

L'une des raisons à l'origine de ces déficits réside dans le niveau élevé des dépenses en proportion du PIB. En 2013, 57 \% du PIB était consacré aux dépenses publiques alors que le taux de prélèvements obligatoires n'était que (!) de $46 \%$. Malgré cet écart important, il convient de noter que le taux de croissance de la dépense publique depuis 2010 est situé en deçà du taux d'inflation. Le taux de croissance des dépenses publiques progressant moins vite que le taux d'inflation en France, les volumes de dépenses publiques régressent désormais. Le tableau 1 fait également apparaître un ratio de dette publique par rapport au PIB situé à 93,3 \% à la fin de l'année 2013, ce qui place la France bien loin des $60 \%$ fixés par le traité de Maastricht.

\footnotetext{
${ }^{1}$ Article rédigé en juin 2014, NdE.
} 
Tableau 1 : Principaux agrégats des finances publiques (en \% du PIB)

\begin{tabular}{|l|c|c|c|c|c|}
\cline { 2 - 6 } \multicolumn{1}{l|}{} & $\mathbf{2 0 1 0}$ & $\mathbf{2 0 1 1}$ & $\mathbf{2 0 1 2}$ & $\mathbf{2 0 1 3}^{*}$ ) & $\mathbf{2 0 1 4}^{*}$ ) \\
\hline Solde public & $-7,1$ & $-5,3$ & $-4,8$ & $-4,1$ & $-3,5$ \\
\hline Dépenses publiques (DP) & 56,6 & 55,9 & 56,6 & 57,0 & 56,5 \\
\hline $\begin{array}{l}\text { Taux de croissance des DP } \\
\text { (en \%, en euros constants) }\end{array}$ & 1,4 & 0,8 & 1,4 & 0,8 & 0,4 \\
\hline Taux de prélèvements obligatoires & 42,5 & 43,7 & 45,0 & 46,0 & 46,1 \\
\hline Dette publique au sens de Maastricht & 82,4 & 85,8 & 90,2 & 93,3 & 94,9 \\
\hline
\end{tabular}

Sources : Institut national de la statistique et des études économiques (Insee), ministère de l'Économie et des Finances, calculs OFCE, prévision France, DAP, octobre 2013. *) Prévision OFCE, octobre 2013.

\section{La logique des critères de Maastricht et du Pacte de stabilité et de croissance}

De Maastricht à Amsterdam, 178 km qui font une différence

Les critères de Maastricht (ou critères d'entrée dans la zone euro) sont au nombre de cinq : l'inflation, le taux d'intérêt, le taux de change, le déficit et la dette publique. Si les trois premiers critères (d'inflation, de taux d'intérêt et de taux de change) étaient des critères relatifs permettant d'évaluer le degré de convergence vers les «meilleurs élèves » de la future zone euro, les critères de déficit et de dette publics étaient quant à eux exprimés sous forme absolue. Le déficit public devait être inférieur à $3 \%$ du PIB et la dette publique inférieure à $60 \%$ du PIB ou converger régulièrement vers ce ratio.

Ce ratio de $60 \%$ fixé dans le traité de Maastricht constitue en réalité la moyenne des dettes publiques européennes à la fin des années 1980 et au début des années 1990. Malheureusement, personne n'a jamais été capable de démontrer qu'il s'agissait là d'un niveau optimal de dette publique, celui qu'il faudrait atteindre pour maximiser le bien-être des citoyens européens. En tout état de cause, la notion de dette optimale est très largement contingente aux circonstances économiques : le niveau de dette publique optimale n'est pas invariant au cours du temps. En imposant ce critère de dette, les autorités européennes (gouvernements, Commission européenne) ont pris le risque d'assurer la cohérence des critères de Maastricht avec un taux de croissance nominale de $5 \%$ par an (qu'il était possible d'obtenir à l'époque) : en effet, avec une telle croissance, la dette publique est stable à $60 \%$ du PIB si le déficit public est égal à $3 \%$ du PIB. On remarquera en passant que la stabilité de la dette publique est confondue avec son optimalité. Était-il pour autant sous-optimal de voir la dette pu- 
blique diverger de son niveau stable à $60 \%$ du PIB après que la crise financière et économique s'est déclenchée, à un moment où les entreprises et les ménages devaient se désendetter? Le danger de limiter arbitrairement la dette publique à $60 \%$ du PIB vient aussi du fait qu'un taux de croissance nominale à $5 \%$ est très éloigné du rythme de croissance actuel. Avec un taux d'inflation ciblé à $2 \%$ par la Banque centrale européenne (BCE), cette croissance nominale revient à un taux de croissance en volume de $3 \%$. La France, qui devrait avoir atteint $+0,1 \%$ de croissance en 2013, a ainsi 3 points d'écart avec le fonctionnement sous-jacent des critères de finances publiques de Maastricht.

Les critères de Maastricht sont un moyen de faire converger les économies européennes préalablement à leur entrée dans la zone euro. Ils évitent ainsi que l'adoption de la monnaie unique ne constitue un choc macroéconomique pour l'économie en question. C'est la raison pour laquelle il fallait voir converger l'inflation et les taux d'intérêt pour ne pas se retrouver du jour au lendemain confrontés à des différentiels d'inflation très importants, qui auraient produit des chocs de balance commerciale, ou de taux d'intérêt, qui auraient produit des chocs financiers dans une zone intégrée financièrement (les contraintes à la mobilité européenne des capitaux ont été levées en 1990). Parallèlement, la convergence préalable sur les déficits et les dettes était supposée favoriser la convergence car elle permettait de peser sur des déterminants de l'inflation (par la demande) ou des taux d'intérêt (par effet d'éviction).

La logique budgétaire ayant prévalu à l'adoption d'un PSC, en marge du traité d'Amsterdam en 1997, est assez différente : il ne s'agit pas de soutenir des efforts de convergence préalable, qui n'ont qu'un temps par nature, mais bel et bien d'imposer une règle budgétaire de bonne conduite permanente qui consiste - pour tous les États membres de la zone euro et de l'Union européenne (UE) au sens large - à avoir des déficits constamment inférieurs à $3 \%$ du PIB, sauf circonstances exceptionnelles. Avec l'indépendance accordée à la BCE et le PSC, l'UE a adopté toute la panoplie des outils censés renforcer sa crédibilité.

Proposition allemande initialement, le PSC aura pour effet de réduire drastiquement la pratique budgétaire aux stabilisateurs automatiques, dans la mesure où il ne limite pas simplement le déficit public à moins de $3 \%$ du PIB, mais vise également à l'équilibre budgétaire à moyen terme. Si à cet horizon, le PIB a atteint son niveau potentiel non inflationniste - l'output gap (l'écart de production) est refermé -, les dépenses publiques structurelles doivent être financées par des recettes courantes. Le recours aux stabilisateurs automatiques n'est plus requis et le déficit public devient nul. En revanche, si l'économie s'écarte de son potentiel, il lui sera possible d'utiliser des stabilisateurs automatiques jusqu'à atteindre un déficit d'au plus $3 \%$ du PIB. En vertu de cette règle de bonne conduite, la politique budgétaire revient à laisser jouer exclusivement les stabilisateurs automatiques. Dès lors, les politiques structurelles (ou discrétionnaires) vont disparaître de l'agenda politique. Cela ne sera pas sans créer une sorte de séisme lorsque, confrontés à une puissante crise financière internatio- 
nale, les États n'auront d'autre choix que d'intervenir massivement au-delà des stabilisateurs automatiques. Il faudra remettre à l'agenda politique la pratique de politique budgétaire discrétionnaire, qui n'était pas prévue dans le PSC. Cela aide à expliquer pourquoi les plans de relance seront mis en œuvre aussi tardivement, après le démarrage de la récession en 2009.

Une spécificité de la première mouture du Pacte de stabilité réside dans le fait qu'il n'existait qu'une seule circonstance exceptionnelle. Un pays qui dépassait la limite de déficit à $3 \%$ du PIB pouvait le justifier uniquement par l'occurrence d'une récession économique, c'est-à-dire d'une chute du PIB. Or, à l'époque, les récessions économiques étaient rares. Les Allemands et les Français n'ont pas pu utiliser cette circonstance en 2002, quand ils ont subi des déficits publics excessifs. Ainsi, le Pacte de stabilité et de croissance dans sa première mouture était-il voué à n'être pas respecté : bien qu'ils n'aient pas de circonstances à faire valoir pour justifier du dépassement de leur déficit public, certains gouvernements ont consciemment enfreint les règles de bonne conduite qu'ils avaient instaurées en 1997.

Le nouveau Pacte de stabilité et de croissance de 2005

En 2005, une nouvelle mouture du PSC a été adoptée, laquelle rappelait que la discipline budgétaire imposait un déficit total inférieur à $3 \%$ du PIB. Toutefois, les promoteurs de cette nouvelle version voulaient que le PSC soit (mieux) appliqué ; en un mot, ils souhaitaient que les infractions aux règles du pacte deviennent beaucoup plus rares. Pour cela, il fallait s'assurer que les circonstances exceptionnelles le fussent moins désormais, il fallait pouvoir expliquer et justifier par une foule d'évènements économiques un déficit public temporairement supérieur à $3 \%$ du PIB.

Pour améliorer la mise en œuvre du PSC, on assiste ainsi à l'extension des circonstances exceptionnelles, qui comprennent désormais :

- le ralentissement économique ;

- la mise en œuvre de réformes structurelles (par exemple sur les retraites) ;

- l'innovation et les politiques en faveur de la R\&D ;

- la politique en faveur de l'accroissement des taux d'emploi ;

- les politiques d'investissement public.

Il est très important de constater que le ralentissement économique, c'est-à-dire le fait qu'une économie ait moins de croissance qu'attendu, peut suffire à expliquer et à excuser un déficit excessif. Avec moins de croissance qu'espéré, il y a en effet moins de recettes fiscales et plus de dépenses sociales : la limite autorisée des $3 \%$ du PIB peut être dépassée. Autre circonstance exceptionnelle, la mise en œuvre de réformes structurelles (sur le marché du travail, par exemple) qui, à court terme, est très coûteuse pour l'État en termes structurels (il y a des 
populations qui doivent continuer d'être indemnisées car elles ont préalablement cotisé), avant de ne plus l'être grâce aux bénéfices qu'elle permet d'engendrer. Par ailleurs, les politiques innovantes en termes de recherche et développement peuvent désormais expliquer les dépassements du déficit public au-delà de $3 \%$. Les politiques en faveur du taux d'emploi des femmes, des seniors et/ou des taux d'emploi globaux ou encore les politiques d'investissement d'une nation peuvent également donner lieu à un dépassement. Elles portent toutes en germe des bénéfices à long terme pour une croissance durable. Assez paradoxalement cependant, ces circonstances exceptionnelles ne permettent de dépasser les déficits publics autorisés que temporairement. Les pays disposent d'une à trois années pour rétablir l'équilibre de leurs finances publiques au sens $\mathrm{du}$ PSC. Or certaines de ces politiques structurelles ne produiront leurs effets favorables potentiels qu'à long terme. Le nouveau dispositif fait certes gagner quelques années à ceux, parmi les gouvernements, qui font le pari de renforcer R\&D et taux d'emploi mais, en même temps, il les oblige à arbitrer à moyen terme entre ces politiques et d'autres sources de dépenses. L'élargissement des circonstances exceptionnelles n'est pas synonyme d'abandon de la discipline budgétaire.

La nouvelle mouture de 2005 voit aussi l'introduction dans le PSC de l'objectif de dette publique. En effet, entre 1997 et 2005, les institutions européennes ont totalement oublié le critère de dette publique qui avait disparu des textes législatifs européens. Présent parmi les critères de finances publiques du traité de Maastricht, il n'était pas mentionné dans la première mouture du PSC. Ainsi, une fois qu'un pays était entré dans la zone euro, il n'était pas susceptible de subir les foudres de ses partenaires en raison d'une dette publique jugée trop élevée.

Le critère de dette publique est donc réintroduit en 2005, avec une nouvelle spécificité. Il y a un avantage accordé aux pays qui ont des dettes publiques plutôt faibles, au détriment de ceux qui ont des dettes publiques plutôt fortes : les premiers disposeront de circonstances atténuantes en cas de déficit excessif, pas les seconds.

\section{Bilan du Pacte de stabilité et de croissance}

À peu près tous les pays membres de l'Union européenne, à un moment ou à un autre, ont dépassé les limites du PSC. Cela pose la question de la pertinence d'un pacte qui n'est pas respecté par tous et, qui plus est, est fréquemment enfreint. Un principe de sanctions financières était prévu en cas de dépassement du déficit public au-delà de $3 \%$ du PIB. Or ces sanctions financières n'ont jamais été appliquées en dépit des nombreux dépassements constatés. Avec la réforme de 2005 et l'extension des circonstances exceptionnelles, un gouvernement trouvera toujours une bonne excuse (une politique structurelle en cours) pour justifier un dépassement de la limite autorisée. Dès lors, la validité du pro- 
cessus budgétaire européen peut être mise en doute et sa crédibilité très amoindrie. Enfin, il a fallu mettre entre parenthèses le Pacte de stabilité et de croissance au démarrage de la crise financière internationale. Bien qu'en contradiction avec la discipline budgétaire, les politiques discrétionnaires expansionnistes ont été jugées nécessaires pour résoudre la chute de croissance que la crise avait produite. La mise entre parenthèses du Pacte a aussi découlé de la constatation que les déficits publics n'étaient pas responsables des déséquilibres des balances des comptes courants à l'origine de la seconde phase de la crise, la fameuse crise des dettes souveraines, largement alimentée par les pertes de compétitivité des entreprises privées des pays périphériques de la zone euro.

En dépit de son application toute relative, le Pacte de stabilité et de croissance a cependant très certainement limité les marges de manœuvre des autorités budgétaires en Europe au plan national ; il agit comme une épée de Damoclès au-dessus des ministères des Finances qui ne peuvent concevoir de préparer des lois de finances aux antipodes de leurs engagements européens. Certes, les déficits publics peuvent-ils dépasser la limite autorisée, mais si celle-ci n'existait pas, il y a fort à parier que les dépassements auraient été plus conséquents. Cette internalisation des limites au déficit public est visible dans la réaction de l'ensemble des pays de la zone euro après la crise financière internationale, quand on la compare à celle des États-Unis ou du Royaume-Uni : ces deux pays ont mis en œuvre des politiques budgétaires beaucoup plus expansionnistes qu'en zone euro. Depuis lors, la zone euro traverse une situation conjoncturelle certainement plus préoccupante que celle des États-Unis ou du Royaume-Uni.

\section{Le traité budgétaire (TSCG)}

En réponse aux défauts du Pacte de stabilité et de croissance, la réforme du traité budgétaire de juin 2011 donne lieu à la création de deux nouvelles règles :

- une règle de limitation du déficit structurel à $0,5 \%$ du PIB ;

- la suppression progressive, pour les États membres de l'UE, de l'écart de leur dette publique par rapport aux $60 \%$ prévus dans le traité de Maastricht. Ces derniers doivent ainsi revenir à une dette publique à hauteur de $60 \%$ de leur PIB à un horizon de 20 ans.

La nouvelle règle portant sur le déficit structurel présente le problème de dépendre de l'output gap et, derrière l'output gap, de la notion de PIB potentiel, variable inobservable. Or les économistes ne disposent pas d'une méthode unique pour calculer le PIB potentiel et leurs estimations peuvent incorporer un certain nombre d'erreurs. L'autre problème que pose cette cible de déficit structurel réside dans l'éventuelle instrumentalisation du PIB potentiel. Prenons un exemple à titre d'illustration. Mettons que la France ait un déficit public de $4,1 \%$. Si le gouvernement français prétend que cette situation résulte de pro- 
blèmes conjoncturels - et strictement conjoncturels -, on peut lui accorder des circonstances exceptionnelles. Dès lors, le gouvernement français peut très bien prétendre que le PIB de la France est 8 points au-dessous de son potentiel. Les 8 points d'output gap négatif aboutissent à un déficit conjoncturel de 4 points et à un déficit structurel de 0,1 point. Ainsi, avec cette norme de déficit structurel, il existe un très fort risque de voir différents États membres utiliser la notion d'output gap comme cela les arrange. Par conséquent, ce n'est pas une très bonne réponse à la recherche d'une pratique optimale de politique budgétaire.

Le déficit structurel présente un autre défaut: tel qu'il est spécifié par la Commission européenne, il inclut les charges d'intérêt. Or les charges d'intérêt résultent du paiement des dettes passées et des déficits passés accumulés par d'autres gouvernements dans d'autres circonstances. Inclure les intérêts issus indifféremment des déficits conjoncturels et structurels passés dans le déficit structurel courant, et limiter en même temps ce dernier, c'est un peu la double peine : les charges d'intérêt ont grevé les capacités de relance du passé, alors même que peut-être les déficits étaient justifiés par une conjoncture défavorable, et elles limitent les capacités de relance courantes. Un État ayant une dette élevée, donc des charges d'intérêt importantes, va en effet devoir consentir plus d'efforts d'austérité qu'un pays ayant une dette plus faible, comme nous allons le discuter dans la section suivante.

\section{Les différents concepts de déficit}

Les formules qui suivent permettent de mieux distinguer entre les différents concepts de déficit public et vont servir à tenter de clarifier les enjeux du retour à l'équilibre budgétaire selon que l'on se place dans le cadre du PSC ou dans celui du traité budgétaire.

$\begin{array}{llll} & b & \text { dette publique } \\ b=b_{-1}-d & d p & : & \text { déficit primaire } \\ d=d p-c i & c i & \text { charges d'intérêt } \\ d=d c+d s & d c \quad: & \text { déficit conjoncturel } \\ d c=0,5 O G & d s \quad: & \text { déficit structurel } \\ d s=d s p-c i & \text { OG } & \text { écart du PIB effectif à son potentiel, ou output gap }\end{array}$

La première équation ci-dessus est celle de l'accumulation de la dette. La dette actuelle en proportion du PIB moins la dette passée est égale au déficit (exprimé par convention avec un signe négatif). Le déficit se partage entre les charges d'intérêt et le déficit primaire. Il peut aussi se partager entre déficit conjoncturel et déficit structurel. Le déficit conjoncturel (aussi dénommé stabilisateurs automatiques) dépend de l'output gap. Si le PIB effectif est supérieur au PIB potentiel, il y aura un excédent conjoncturel. Si le PIB effectif est en deçà de son potentiel, le pays accumulera des déficits conjoncturels. Il est possible également 
de séparer dans le déficit structurel ce qui provient des charges d'intérêt de ce qui provient du déficit structurel primaire. Ce dernier concept est la caractérisation la plus discrétionnaire de la politique budgétaire d'un État puisque les charges d'intérêt dépendent de la dette passée et des taux d'intérêt sur la dette qui dépendent de la politique monétaire. Ainsi, les responsabilités sur les charges d'intérêt se partagent entre plusieurs acteurs, ce qui n'est pas le cas de celles sur les déficits structurels primaires.

Les tableaux 2 à 4 ci-après visent à illustrer les difficultés d'application du traité budgétaire ou TSCG (pour «traité sur la stabilité, la coordination et la gouvernance »). Dans le tableau 2, on calcule le déficit public maximal qu'une économie pourrait atteindre si elle respectait scrupuleusement le critère d'un déficit structurel maximal à $0,5 \%$ du PIB en fonction de l'état de sa conjoncture, en considérant un spectre d'output gap allant du très positif ( $+4 \%)$ au très négatif $(-4 \%)$. On observe que la règle portant sur les déficits structurels est plus contraignante que la règle de Maastricht. En effet, il serait possible d'atteindre au maximum un déficit public de $2,5 \%$ du PIB (contre $3 \%$ du PIB selon les critères de Maastricht).

Le tableau 3 renvoie vers les déficits structurels maximaux compatibles avec la règle des $3 \%$ selon que l'output gap est très positif ou très négatif. On observe qu'avec un output gap très fort à $4 \%$, il serait possible d'avoir un déficit structurel de 5 points. Cela n'est évidemment pas tout à fait conciliable avec une pratique optimale de la politique budgétaire : une limite portant sur le déficit public total peut inciter à une dérive des finances publiques en phase haute de conjoncture, en contradiction avec le principe de régulation macroéconomique cher à Musgrave. En phase basse de conjoncture, à l'inverse, la règle des $3 \%$ du PIB donne des marges de manœuvre adaptées aux circonstances : avec un output gap de $-4 \%$, le respect de la règle des $3 \%$ permettait à tous les États membres de l'UE d'avoir un déficit structurel d'au plus $1 \%$ du PIB, au lieu du $0,5 \%$ du PIB du TSCG. Les capacités de réaction européenne à un choc macroéconomique ont donc été divisées par deux en passant d'une règle à $3 \%$ sur l'ensemble du déficit à la règle de déficit structurel à $0,5 \%$ du PIB.

Le tableau 4 illustre les contraintes qu'imposera le traité budgétaire sur la politique budgétaire discrétionnaire (ici représentée par le déficit structurel primaire) selon le niveau de dette publique. La première ligne fait évoluer la dette publique de $40 \%$ à $120 \%$ du PIB. Il convient toutefois de noter que la dette publique de certains États membres de la zone euro est supérieure à $120 \%$ du PIB. Sous l'hypothèse que les économies ont un output gap nul, respecter un déficit structurel de $0,5 \%$ du PIB, avec des charges d'intérêt qui sont d'autant plus élevées que le pays est très endetté, oblige in fine à faire des politiques de contraction budgétaire qui peuvent être particulièrement fortes (de -1,5\% d'impulsion budgétaire si la dette est faible à $-5,5 \%$ si la dette est élevée). C'est bien cette logique qui s'est imposée aux États tels la Grèce, le Portugal ou l'Espagne, soumis à des contractions budgétaires d'autant plus fortes que leurs 
dettes publiques et leurs charges d'intérêt s'étaient alourdies. Les résultats d'une telle épreuve sont bien connus : ces pays ont plongé dans la récession.

Tableau 2 : Déficit public maximal autorisé par le TSCG (les déficits sont exprimés en $\%$ du PIB)

\begin{tabular}{|c|c|c|c|c|c|}
\cline { 2 - 6 } \multicolumn{1}{c|}{} & $\mathbf{( 1 )}$ & $\mathbf{( 2 )}$ & $\mathbf{( 3 )}$ & $\mathbf{( 4 )}$ & $\mathbf{( 5 )}$ \\
\hline $\mathbf{O G}(\%)$ & $\mathbf{4}$ & $\mathbf{2}$ & $\mathbf{0}$ & $\mathbf{- 2}$ & $\mathbf{- 4}$ \\
\hline $\mathbf{d c}$ & 2 & 1 & 0 & -1 & -2 \\
\hline $\mathbf{d s}$ & $-0,5$ & $-0,5$ & $-0,5$ & $-0,5$ & $-0,5$ \\
\hline $\mathbf{d}_{\max }$ & 1,5 & 0,5 & 0,5 & $-1,5$ & $-2,5$ \\
\hline
\end{tabular}

Source : exemples de l'auteur.

Tableau 3 : Déficit structurel maximal compatible avec la règle de $3 \%$ du PIB (les déficits sont exprimés en \% du PIB)

\begin{tabular}{|c|c|c|c|c|c|}
\cline { 2 - 6 } \multicolumn{1}{c|}{} & $\mathbf{( 1 )}$ & $\mathbf{( 2 )}$ & $\mathbf{( 3 )}$ & $\mathbf{( 4 )}$ & $\mathbf{( 5 )}$ \\
\hline OG (\%) & 4 & 2 & 0 & -2 & -4 \\
\hline $\mathbf{d}$ & -3 & -3 & -3 & -3 & -3 \\
\hline $\mathbf{d c}$ & 2 & 1 & 0 & -1 & -2 \\
\hline ds & -5 & -4 & -3 & -2 & -1 \\
\hline
\end{tabular}

Source: exemples de l'auteur.

Tableau 4 : Efforts budgétaires requis pour satisfaire au TSCG, pour un taux d'intérêt sur la dette de $5 \%$ l'an et un output gap nul (les déficits et la dette sont exprimés en $\%$ du PIB)

\begin{tabular}{|c|c|c|c|c|c|}
\cline { 2 - 6 } \multicolumn{1}{c|}{} & $\mathbf{( 1 )}$ & $\mathbf{( 2 )}$ & $\mathbf{( 3 )}$ & $\mathbf{( 4 )}$ & $\mathbf{( 5 )}$ \\
\hline b & 40 & 60 & 80 & 100 & 120 \\
\hline dc & 0 & 0 & 0 & 0 & 0 \\
\hline ds & $-0,5$ & $-0,5$ & $-0,5$ & $-0,5$ & $-0,5$ \\
\hline ci & 2 & 3 & 4 & 5 & 6 \\
\hline dsp & 1,5 & 2,5 & 3,5 & 4,5 & 5,5 \\
\hline
\end{tabular}

Source : exemples de l'auteur. 
Ces quelques exemples illustrent combien le traité budgétaire est ou va être très contraignant pour les États membres de l'Union européenne. Nous y reviendrons plus loin.

\section{L'état des finances publiques françaises}

À la fin de l'année 2013, l'Allemagne était un des rares États au monde à toujours bénéficier d'une notation triple A délivrée par Standard \& Poor's - ils étaient alors au nombre de 13 - et l'un des très rares, avec le Royaume-Uni seulement, à disposer d'un montant de PIB très important. Les autres États notés triple A étaient tous des petits, voire des très petits pays. Aussi, la France, qui a perdu son triple A en janvier 2012, est-elle loin d'être une exception. Elle ne l'est pas plus si l'on s'intéresse à l'évolution de ses déficits publics depuis le début des années 1980 (graphique 1).

\section{Graphique 1 : Déficits publics - Moyennes décennales}

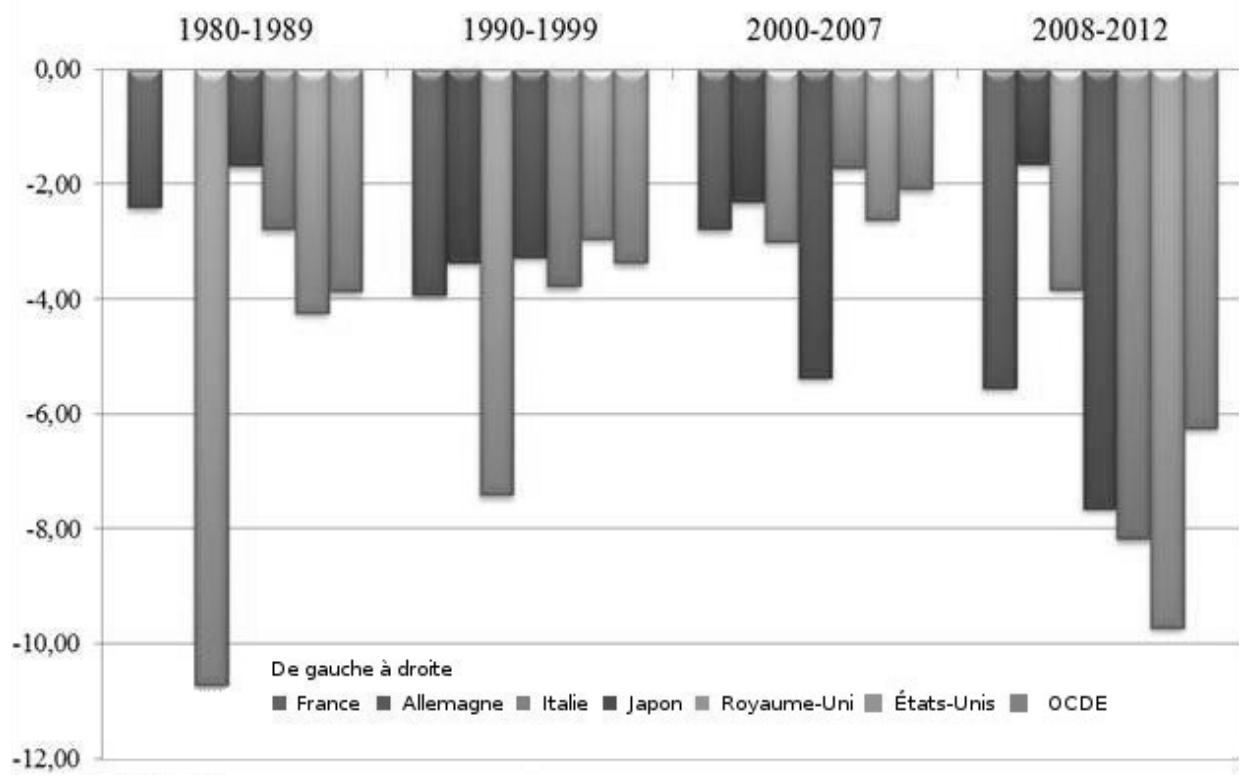

Source : Organisation de coopération et de développement économiques (OCDE).

Si l'on compare sur le graphique la première colonne négative (qui représente le déficit public français) à la dernière colonne (c'est-à-dire le déficit dans les pays membres de l'OCDE), la décennie 1990-1999 mise à part, la France se situe peu ou prou dans la moyenne des pays de l'OCDE, en dépit du fait qu'elle est très décriée sur le plan de ses finances publiques. 
Tableau 5 : Niveaux de dette publique brute en proportion du PIB

\begin{tabular}{|l|r|r|r|}
\cline { 2 - 4 } \multicolumn{1}{c|}{} & $\mathbf{2 0 0 7}$ & $\mathbf{2 0 1 2}$ & $\mathbf{2 0 1 7}$ \\
\hline Allemagne & 65,355 & 83,038 & $\mathbf{7 3 , 7 0 1}$ \\
\hline Italie & 103,082 & 126,332 & 120,635 \\
\hline France & 64,215 & 89,968 & 86,457 \\
\hline États-Unis & 67,161 & 107,18 & 114,015 \\
\hline Royaume-Uni & 43,713 & 88,684 & 93,722 \\
\hline Japon & 183,012 & 236,564 & 250,328 \\
\hline G7 & 83,47 & 125,146 & 127,566 \\
\hline Zone euro & 66,432 & 93,624 & 89,509 \\
\hline
\end{tabular}

Source : Fonds monétaire international, Perspectives de l'économie mondiale, 2013.

Le tableau 5 montre que la France avait une dette publique de $90 \%$ de son PIB en 2012, ce qui la place dans la moyenne de la zone euro (voire un peu mieux que la zone euro). Or le principe selon lequel l'état des finances publiques françaises est extrêmement dégradé ne peut pas se concevoir sans une comparaison avec la zone euro, compte tenu des relations commerciales, économiques et financières entre ses États membres.

\section{Composition des dépenses publiques françaises}

Le niveau des dépenses publiques françaises en proportion du PIB est plus élevé en France qu'en Allemagne. Depuis les années 1970, la France connaît une très nette augmentation de ses dépenses publiques par rapport au PIB, qui la place au-dessus de la moyenne de la zone euro. Néanmoins, ces dépenses publiques servent à financer des prestations sociales. Ainsi, la France n'a pas autant recours que d'autres pays à des prestations privées.

La France a un système social généreux. Par conséquent, le pays peut laisser jouer les stabilisateurs automatiques en cas de crise. Ainsi, au début de la crise internationale, l'OCDE a vanté les mérites du système fiscalo-social français qui avait permis à l'économie française de moins subir la crise que le reste du monde. Malheureusement depuis lors, la générosité du système français a plutôt tendance à diminuer, sous l'effet notamment de l'alourdissement de l'impôt sur le revenu suite au gel du barème et de la hausse de la taxe sur la valeur ajoutée (TVA), deux mesures qui vont réduire la progressivité du système et conduire à baisser l'efficacité des stabilisateurs automatiques.

\section{Composition des recettes fiscales françaises}

L'évolution des recettes fiscales montre également une augmentation. Toutefois, sur le plan de la répartition des recettes fiscales en France en 2011, la part du lion 
est constituée des cotisations sociales employé-employeur, l'impôt sur le revenu ne représentant que la portion congrue de nos recettes fiscales. Or c'est le seul impôt progressif en France, toutes les autres fiscalités étant plutôt de caractère régressif. La fiscalité indirecte est régressive puisque les mêmes taux d'imposition sont imposés à des populations qui n'ont absolument pas les mêmes niveaux de revenus. Les cotisations sociales ont également un caractère régressif, dans la mesure où les durées de cotisation sont homogènes entre employés, mais pas les espérances de vie : celle des cadres supérieurs est plus élevée que celle des ouvriers, aussi les cadres supérieurs profiteront-ils plus longtemps que les ouvriers des prestations que leurs cotisations ont financées. Par ailleurs, le système fiscal français, extrêmement complexe, ne comprend pas moins de 200 niches fiscales, ce qui rend difficile sa compréhension ou sa réforme.

\section{Les déficits publics français}

L'évolution des finances publiques de la France depuis 1987 montre que le pays a certes eu constamment des déficits publics, mais que la part la plus régulière et la plus importante du déficit public français correspond au financement de sa dette (graphique 2).

\section{Graphique 2 : Contributions au déficit public (en \%)}

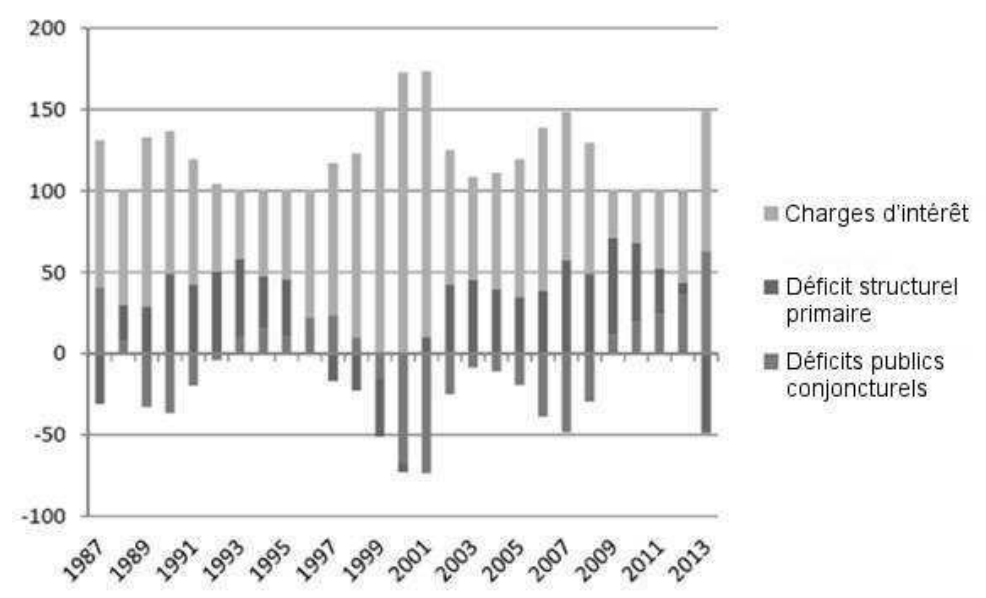

Sources : OCDE, calculs de l'auteur.

Les charges d'intérêt apparaissent en gris clair. La stabilité des charges d'intérêt malgré une dette publique en hausse s'explique par la baisse des taux d'intérêt sur la dette publique française. La composante en gris moyen, qui représente les soldes publics conjoncturels, est tantôt déficitaire, tantôt excédentaire, tout simplement en vertu du jeu des stabilisateurs automatiques. Outre les charges d'intérêt et la composante cyclique des déficits publics, les impulsions budgé- 
taires en gris foncé ont généralement été positives : il y avait alors des expansions budgétaires discrétionnaires. Depuis les années 2007-2008, des impulsions budgétaires importantes ont été mises en œuvre, mais il convient de constater qu'elles ont finalement régressé assez vite. En 2013, la contraction budgétaire discrétionnaire des finances publiques françaises est assez frappante. Si le déficit public reste élevé, le gouvernement français a mis en place une très forte austérité budgétaire pour tenter d'y remédier.

La France mène une politique d'austérité budgétaire qui pose donc la question de son effet sur la croissance économique. Personne ne peut ignorer qu'un grand débat a eu lieu à propos de l'effet multiplicateur de la politique budgétaire. Or il existe aujourd'hui un consensus parmi les économistes sur le fait qu'une contraction budgétaire qui intervient alors que l'output gap est très négatif aura des effets multiplicateurs très élevés, c'est-à-dire qu'elle sera très coûteuse en termes de croissance. Dans une telle situation conjoncturelle, il est préférable de faire de la relance budgétaire, comme en témoignent les travaux du Fonds monétaire international (FMI) et de l'OFCE dès 2011 (cf. Creel et al., 2011). Il est sans doute utile de rappeler que les évaluations de l'effet multiplicateur menées avant la crise financière internationale concluaient très majoritairement en faveur d'un effet positif. Il a été d'autant plus fort en France que la France subissait un output gap négatif. Dès lors, le pays a subi de plein fouet la politique budgétaire restrictive menée depuis 2010 .

\section{La dette publique française}

Concernant la dette publique, un premier élément d'analyse consiste à comparer la dette publique française avec les actifs détenus par l'État (les routes, les droits et licences, les droits sur l'eau...). Selon les données de comptabilité nationale, les montants d'actifs publics sont tels que l'État français dispose d'une richesse nette positive. Il reste qu'elle a baissé depuis 1978, puisqu'elle était alors proche de $80 \%$ du PIB et qu'elle se situait à $20 \%$ du PIB en 2012. La valorisation de certains actifs étant difficile à réaliser en l'absence d'un marché d'échange, il est préférable d'avoir une vision en termes d'actifs financiers. Or la dette publique nette de la France, c'est-à-dire la dette brute réduite des seuls actifs financiers, s'est très fortement accrue depuis le début des années 1980 et a connu une nouvelle augmentation sous l'effet de la crise financière internationale que nous subissons, avec des niveaux de dette publique certes considérablement élevés, mais qui correspondent à peu près à la moyenne de la zone euro.

Il est certainement intéressant de chercher les raisons de la baisse des taux d'intérêt malgré une dette en hausse. Au-delà de la recherche par les épargnants de valeurs refuges pendant les crises - la dette publique française en aurait fait partie -, l'action de l'Agence France Trésor, créée en 1999, a permis de gérer au mieux les intérêts des citoyens français en assurant à la dette publique française 
des rendements les plus bas possibles. Pour y parvenir, l'Agence France Trésor émet chaque jour des titres de dette française à des maturités extrêmement variées afin de profiter au mieux de la liquidité sur les différents segments du marché des dettes souveraines. Cela a permis à la France d'augmenter la maturité de sa dette pour en faire l'une des dettes publiques à la maturité la plus élevée au monde. La dette parmi les pays avancés dont la maturité est la plus élevée est celle du Royaume-Uni, celle de la France étant située légèrement au-dessus de celle de l'Allemagne. Dès lors, on prête à plus longue échéance en France qu'en Allemagne et considérablement à plus longue échéance qu'aux États-Unis puisqu'ils émettent en moyenne à 4,4 années, contre 7 années pour la France. Par conséquent, les risques d'insoutenabilité de la dette publique française sont atténués par la baisse des taux d'intérêt.

\section{Coûts et bénéfices du traité budgétaire}

On observe ainsi que les finances publiques françaises se sont détériorées sous l'effet d'une mauvaise conjoncture. Une reprise de la croissance - qui ne se décrète pas, cependant - permettrait d'atténuer les problèmes de déficit public, en même temps que la gestion de la dette publique française permet d'être assez serein sur l'évolution des taux d'intérêt futurs et, partant, sur la soutenabilité des finances publiques de la France.

C'est dans ce contexte de crise économique et financière que le dispositif budgétaire européen s'est renforcé, au travers notamment de l'adoption du TSCG. Deux études récentes (Creel/Hubert/Saraceno, 2012 ; 2013) peuvent être mobilisées afin d'évaluer les coûts pour différents pays - dont la France - du passage à une nouvelle règle : celle de la limite à $0,5 \%$ du PIB portant sur le déficit structurel ou la règle de convergence sur 20 ans de la dette à la limite supérieure de $60 \%$ du PIB. La première étude porte sur une évaluation empirique des liens entre output gap et taux d'inflation, avec prise en compte des effets du déficit structurel et de la dette publique. Après avoir fait cette évaluation empirique sur données passées, les auteurs établissent un scénario contrefactuel en simulant ce qu'aurait imposé à l'économie française le passage à la règle budgétaire du traité budgétaire, le maintien du traité de Maastricht tel qu'il était ou l'adoption d'une règle d'or des finances publiques (proche de celle un temps adoptée au Royaume-Uni).

Dans la deuxième étude, plus théorique, les auteurs utilisent un modèle néokeynésien de type $\operatorname{DSGE}^{2}$ (modèles dynamiques et stochastiques d'équilibre général) de petite dimension, avec des anticipations rationnelles et rétrospectives. Les impulsions budgétaires interviennent dans l'équation de demande agrégée, contrairement à la littérature (les auteurs montrent que ce comportement est microéconomiquement fondé). Les auteurs introduisent des primes de

\footnotetext{
${ }^{2}$ De l'anglais Dynamic Stochastic General Equilibrium, NdE.
} 
risque sur la dette et rendent le calibrage cohérent avec le modèle sous-jacent du traité de Maastricht, c'est-à-dire un taux de croissance nominale de $5 \%$.

Les résultats de la première étude sont que d'une part, la nouvelle règle de limitation des dettes publiques permettrait finalement, par une contraction budgétaire extrêmement forte à court terme, d'avoir un output gap plus élevé à long terme, c'est-à-dire qu'en suivant cette pratique budgétaire, on ferait un sacrifice à court terme au bénéfice du long terme, mais avec le risque que l'output gap n'augmente trop vite et provoque des tensions inflationnistes. Évidemment, si on en restait au schéma de Maastricht, on aurait un retour de l'output gap à un niveau positif plus lent qu'avec les règles en vigueur mais on aurait aussi moins d'inflation, ce qui serait également le cas si on était dans le système que permettrait une règle d'or des finances publiques. Les conclusions sont donc qu'à long terme, les pertes seraient moindres en France si on adoptait une règle d'or des finances publiques, la deuxième meilleure option étant la poursuite de la règle inscrite dans le traité de Maastricht. La situation la plus défavorable serait d'adopter la limite du déficit structurel à $0,5 \%$ du PIB, car elle provoquerait une telle récession à court terme qu'elle en deviendrait extrêmement coûteuse pour l'économie française.

La seconde étude parvenait à des résultats qualitativement similaires. En partant d'une situation (bien réelle) d'output gap fortement négatif, l'adoption du TSCG provoquerait des déséquilibres importants. En effet, la baisse de l'inflation s'écarterait très sensiblement de la cible à $2 \%$ de la $\mathrm{BCE}$, ce qui serait très coûteux pour l'économie française. Par conséquent, l'économie française se porterait mieux avec une règle d'or à la britannique de ses finances publiques et beaucoup moins bien si elle adoptait strictement le traité budgétaire.

Pour CONClure, la France a CONNU Des déFICITS et des dettes très élevés. La France a eu recours, à bon escient, aux stabilisateurs automatiques. Malheureusement, la générosité de son système étant aujourd'hui mise à mal, elle aura moins de facilité à l'avenir pour faire face à une autre crise financière et économique. La France a su gérer sa dette raisonnablement. Elle a connu une très forte politique d'austérité qui explique certainement les très mauvaises performances économiques françaises. Elle a donc subi les contraintes du dispositif budgétaire européen et, en termes de culture budgétaire, il me semble qu'il n'y a pas de grande spécificité française. In fine, malgré des contrariétés passagères, que l'on peut qualifier de marginales dans les relations budgétaires entre la France et la Commission européenne, les autorités françaises semblent appliquer plutôt scrupuleusement les recommandations et autres injonctions de la Commission européenne. C'est certainement regrettable dans la mesure où l'orientation récente de la politique budgétaire qu'a recommandée la Commission européenne est allée à contre-courant du consensus économique sur la valeur, élevée, des multiplicateurs budgétaires de dépenses en phase basse de conjoncture. Rappelons que si la Commission européenne a accepté des délais dans 
le retour à l'équilibre des finances publiques de plusieurs pays, dont la France, elle est une des rares institutions internationales au monde à ne s'être pas excusée pour avoir préconisé de mauvaises politiques budgétaires au très mauvais moment, au contraire du FMI qui a exprimé ses regrets pour avoir imposé une austérité beaucoup trop importante à des pays comme la Grèce ou le Portugal.

La France peine à sortir d'une situation de croissance molle et il semble que les politiques budgétaires sont responsables, au moins pour partie, de cette situation. La politique budgétaire aujourd'hui est un enjeu majeur. Il est nécessaire d'en revenir au traité préalable et de s'écarter des conditions drastiques qu'impose le traité budgétaire. Dans le Pacte de stabilité et de croissance, il est précisé qu'un État qui a un déficit public excessif, c'est-à-dire un déficit public au-delà de $3 \%$ du PIB, doit réaliser chaque année un effort structurel d'au moins un demi-point de PIB de son économie. Pendant presque trois ans, la France a fait trois fois plus d'efforts structurels que ce qui était requis par le PSC. Difficile, dans une conjoncture aussi morose, de s'expliquer pourquoi la France continue de pratiquer une politique aussi austère alors que les traités lui laissent des marges de manœuvre. La crédibilité d'une institution doit porter sur la capacité de celle-ci à atteindre ses objectifs. En France, comme en Europe, les finances publiques sont devenues un objectif de politique économique, alors qu'elles auraient dû être un moyen d'atteindre d'autres objectifs, tels que la croissance, l'inflation, l'emploi, l'innovation ou la transition écologique. Combien de décennies perdues avant de voir les finances publiques redevenir un outil de politique économique?

\section{Indications bibliographiques}

Creel J., Heyer E., Plane M., «Petit précis de politique budgétaire par tous les temps - Les multiplicateurs budgétaires au cours du cycle », Revue de l'OFCE, n²116, janvier 2011, p. [61]-88

Creel J., Hubert P., Saraceno F., « An Assessment of Stability and Growth Pact Reform Proposals in a Small-Scale Macro Framework », Journal of Economic Dynamics and Control, vol. 37, n 8, août 2013, p. $1567-1580$

Creel J., Hubert P., SARAceno F., « The European Fiscal Compact - A Counterfactual Assessment », Journal of Economic Integration, vol. 27, n 4, décembre 2012, p. [537]-563. 


\section{Table ronde : Les contraintes budgétaires liées au Pacte de stabilité et de croissance et au pacte budgétaire européen (TSCG), dont le MES, sont-elles praticables et adaptées pour sortir l'économie européenne de la crise ? ${ }^{1}$}

\section{OUVERTURE DE LA TABLE RONDE}

Rainer Klump : D'une part, cette table ronde va être animée par deux grands praticiens, des personnes qui ont une longue expérience concrète de la politique financière ainsi que de la coopération franco-allemande dans ce domaine. Nous en arriverons peut-être à constater que de nombreux débats qui semblent très disputés au niveau universitaire s'avèrent nettement moins controversés sur le plan de la coopération pratique. D'autre part, je pense que pour répondre à la question «Les contraintes budgétaires liées au Pacte de stabilité et de croissance (PSC) et au pacte budgétaire européen (inclus dans le traité sur la stabilité, la coordination et la gouvernance, TSCG), dont le Mécanisme européen de stabilité (MES), sont-elles praticables et adaptées pour sortir l'économie européenne de la crise ? »- un titre très allemand, c'est-à-dire long et compliqué -, il faut probablement aussi se tourner vers l'avenir après avoir discuté en profondeur des traditions respectives de la France et de l'Allemagne en matière de politique budgétaire. Nous devrions d'ailleurs jeter un coup d'œil sur le futur dès maintenant, du point de vue des praticiens de la politique financière qui ont l'habitude de coopérer afin de faire avancer le projet européen et d'élaborer des perspectives d'avenir.

J'aimerais maintenant vous présenter les participants à cette table ronde : Sandrine Gaudin est sous-directrice des Affaires européennes à la Direction générale du Trésor, au ministère français de l'Économie et des Finances. Juriste de formation, Sandrine Gaudin a étudié à l'ÉNA avant d'entamer une carrière au Trésor public, au sein du ministère des Finances. Au poste qu'elle occupe actuellement, elle se consacre particulièrement à la coordination des politiques financières allemande et française, dans la perspective des questions européennes. Christian Kastrop est directeur adjoint de la division Questions économiques générales et directeur de la section Finances publiques, macroéconomie et recherche au ministère fédéral des Finances à Berlin. Christian Kastrop a déjà apporté une contribution précieuse à notre débat sur la politique monétaire. Il provient - c'est intéressant de le mentionner du point de vue de la tradition universitaire - de l'école de sciences économiques de Cologne. En Allemagne, l'université de Co-

${ }^{1}$ Cette table ronde s'est tenue dans le cadre de la journée d'étude Cultures budgétaires en France et en Allemagne, coorganisée par le CIRAC le 18 novembre 2013, NdE. 
logne a longtemps été un haut lieu de l'ordolibéralisme et de l'économie sociale de marché. Christian Kastrop y a étudié avant de partir à Harvard, puis a très rapidement trouvé un poste au ministère fédéral des Finances, qui se trouvait encore à Bonn à l'époque. Je me réjouis d'entendre l'opinion de Christian Kastrop pour commencer, puis la réponse de Sandrine Gaudin.

\section{EXPOSÉS DES INTERVENANTS}

Christian Kastrop : Merci beaucoup pour cette amicale présentation. Je suis heureux d'être de retour ici, parce que je suis convaincu que la politique financière, budgétaire et économique est un domaine dont on ne discute pas assez souvent. Nous avons certainement bien des différences, mais nous avons aussi des points communs. Ce ne sont pas seulement les résultats, mais aussi la manière dont l'Allemagne et la France s'entendent en matière de politique financière, budgétaire et économique, qui détermineront l'avenir plus ou moins positif de l'Europe. À ce titre, pas une minute de la discussion que nous menons ici n'est gaspillée.

Dans sa contribution, Michael Thöne a très clairement évoqué les différentes cultures allemandes. Au ministère fédéral des Finances également, il existe différentes sensibilités et cultures. La division Questions économiques générales, dont je fais partie, suit parfois des conceptions différentes de celles, plutôt opérationnelles, de la division Europe. De même, les positions de la division Europe et de la division Questions économiques générales ne correspondent pas automatiquement à l'opinion du ministre ou de ses secrétaires d'État. Nous sommes dans une discussion permanente pour trouver la meilleure voie, et c'est très bien ainsi. C'est pourquoi $\mathrm{j}$ 'aimerais préciser que les propos qui vont suivre reflètent exclusivement mon opinion personnelle.

\section{La crise de la dette dans la zone euro - les leçons}

\section{Le cadre institutionnel de l'UE avant la crise}

Le cadre réglementaire que nous sommes en train d'élaborer dans l'Union européenne (UE) pour la politique financière et économique est largement différent de l'existant, et en grande partie nouveau. Pour de nombreux aspects de cette entreprise, nous n'avons pas vraiment d'exemple historique à suivre, et nous avançons tous ensemble en terrain inconnu.

Dans ce contexte, nous devons commencer par nous demander quel a été le point de départ de la crise. Tout d'abord, on peut mentionner les faiblesses des règles de politique financière et économique. Se pose aussi la question de l'énorme erreur commise par la France et l'Allemagne en 2003. Je pourrais parler longtemps de cette histoire, des raisons pour lesquelles l'Allemagne et la France se sont retrouvées ensemble en difficulté par rapport à ces règles, et de 
ce qu'elles en ont fait. De nombreuses légendes circulent à ce sujet, pas toutes véridiques. Mais il est certain qu'à l'époque, la règle fiscale du Pacte de stabilité et de croissance a été affaiblie par les décisions politiques. Depuis lors, on avait cessé de croire que les règles pouvaient aboutir à des sanctions, ce qui a certainement contribué au fait qu'en fin de compte, les marchés ont supposé que non seulement les «pécheurs » ne seraient pas punis, mais qu'au contraire la communauté viendrait toujours à leur secours. Aux doutes sur la cohérence en matière de respect des règles s'est ajouté le manque de crédibilité de la clause de non-renflouement (no bail-out). Un élément capital pour que les marchés contribuent à la stabilité a ainsi été sapé.

D'un autre côté, la réforme engagée à l'époque a aussi eu des résultats positifs, ce qui a d'une certaine manière permis la création du pacte budgétaire, qui complète en les améliorant nettement les règles budgétaires de l'UE. Mais à mon avis, pour déterminer si l'UE peut ou non envoyer les bons signaux en temps de crise, il faut surtout savoir si le principe de non-renflouement sera maintenu ou non. C'est la pierre angulaire de tout ce débat, et dans toutes les réformes que nous avons connues et sur lesquelles je vais brièvement revenir, je pense qu'il est clair que c'est le point qui reste à éclaircir. À ce sujet, tout le monde cherche à éviter d'envoyer un message clair, car on craint les risques encourus. Cependant, c'est parfois précisément le manque de clarté qui représente le plus gros risque, et qui pourrait porter en soi les germes de la prochaine crise.

Dans l'ancien système réglementaire, nous avons ignoré deux éléments. J'ai participé à la première proposition allemande de ce qui s'appelait alors le Pacte de stabilité et qui, après les discussions avec les collègues français, a ensuite été rebaptisé Pacte de stabilité et de croissance. Dans ce cadre, on voit bien que l'Allemagne et la France sont parfaitement capables de coopérer lorsqu'elles le veulent bien. Cependant, à l'époque, nous sommes passés à côté de l'importance des déséquilibres macroéconomiques. Nous avons supposé que si nous fixions correctement les données de politique financière - c'est-à-dire le taux d'endettement et le déficit - dans le Pacte de stabilité et de croissance, les acteurs d'une politique économique responsable sous le régime d'une monnaie européenne unique devraient réagir et feraient pression pour des mesures de politique structurelle - maintien de la compétitivité de l'économie -, c'est-àdire pour une politique d'adaptation active, dans une situation où la politique des changes n'est plus applicable au sein de l'Union monétaire.

Le deuxième aspect que nous avons sous-estimé réside dans les interactions entre le secteur bancaire et les finances publiques, c'est-à-dire entre la dette privée et la dynamique qui peut toucher aussi la dette publique si les dettes privées sont incontrôlables, par exemple dans le secteur bancaire, comme cela a été le cas en Islande, en Irlande et en Espagne. Les finances publiques irlandaises, islandaises ou espagnoles étaient pourtant saines. Mais les bilans des banques ont été gravement déséquilibrés par l'éclatement de la bulle immobilière. Le problème est alors passé des mains des banques à celles des États souverains 
lorsque ceux-ci ont couvert les dettes des banques - rétrospectivement, une opération pas assez réfléchie. On s'efforce aujourd'hui de trouver une solution en formant une union budgétaire et bancaire.

Mais il y a aussi des choses que l'on ne pouvait pas savoir. Je me souviens encore parfaitement qu'à la fin des années 1990, le ministre allemand des Finances de l'époque, Theo Waigel, m'avait demandé s'il pouvait se passer quelque chose de grave si la Grèce, dont les chiffres n'étaient pas au mieux, faisait défaut. Je lui ai répondu : «Oh, Monsieur le Ministre, ils sont si petits, ne vous faites donc pas de souci. » Voilà ce que j'ai dit, et d'autres en ont certainement fait de même. Pourtant, je me suis trompé et j'ai ainsi contribué à un grave problème. D'un autre côté, toute la dynamique des marchés financiers, des nouveaux produits structurés, de ce que nous considérons aujourd'hui comme une infection et une contamination, n'existait même pas encore à l'époque, et n'était peut-être même pas prévisible. Beaucoup de gens, a posteriori, prétendent volontiers qu'ils savaient tout. J'aurais tendance à en douter et, à la fin des années 1990, le sujet n'était certainement pas aussi médiatisé.

\section{Nécessité de mener des réformes}

On peut en déduire que des réformes sont nécessaires, en particulier des réformes de consolidation et des réformes structurelles au plan national, ainsi que des cadres plus efficaces et de meilleures règles pour une économie stable et des finances solides.

Dans les débats publics, on oppose toujours systématiquement la consolidation à la croissance. Quoi qu'on pense du rôle de la macroéconomie par rapport à la politique structurelle, la croissance potentielle est le facteur décisif en fin de compte. C'est quelque chose qu'il faut aborder de manière particulièrement engagée, en particulier dans des pays tels que l'Allemagne. À ce sujet, il existe une différence fondamentale par rapport à la France : en Allemagne, le taux de natalité est seulement de 1,4 tandis que la France a un taux supérieur à 2. Ainsi, dans l'immédiat, on n'a pas besoin de se soucier autant de la croissance potentielle que les Allemands lorsqu'on est français. À court terme, l'Allemagne et la France auront des devoirs quelque peu différents, mais à long terme, elles auront plutôt les mêmes tâches. Il s'agit d'un point très important qui relève aussi de la solidité des finances publiques. La dette publique implicite n'est pas une question importante qu'en Allemagne. Si l'on suppose une synchronisation des systèmes de sécurité sociale à long terme, l'Allemagne rencontrera toutefois bien plus de problèmes que la France pour s'assurer des finances solides dans la durée. S'y ajoutent peut-être d'autres décisions prises en Allemagne, par exemple la transition énergétique. Si la France continue à miser largement sur l'énergie nucléaire, au premier abord plus économique - je m'interroge cependant sur la véracité de cette hypothèse après la catastrophe de Fukushima -, cela offrira un avantage compétitif important à l'industrie 
française, que l'Allemagne devra compenser à d'autres niveaux par une politique de croissance astucieuse.

L'Allemagne n'est pas opposée par principe aux instruments macroéconomiques. Nous ne pensons pas non plus que la consolidation soit la voie à suivre dans tous les cas, mais nous sommes d'avis qu'il s'agit d'une stratégie importante à long terme pour la pérennité financière et qu'elle ne devrait pas être totalement occultée par le fonctionnement d'instruments macroéconomiques qui ne peuvent que nous donner un peu de temps, mais ne créent aucun potentiel de croissance. Voilà en tout cas notre opinion de principe. Je ne sais pas ce que Sandrine Gaudin va dire tout à l'heure, mais je ne pense pas que nous soyons en désaccord sur ce point. C'est plus une question de temps et de manière.

\section{An V de la crise : où en sommes-nous ?}

En 2013, les dettes ont atteint un niveau record. Toutefois, dans plusieurs pays, les déficits structurels se sont fortement améliorés. On pourrait par exemple en déduire que les instruments adoptés jusqu'à présent ont eu des effets positifs. Il s'agit non seulement de la politique budgétaire et économique, mais aussi d'une procédure de réaction à la crise rapidement développée et de plans d'action pour les États faisant l'objet de programmes d'aide, ainsi que de la politique de la Banque centrale européenne (BCE). La question essentielle est de savoir quel élément domine dans cette évolution positive. À ce sujet, les avis divergent. Certains diraient que les réformes structurelles et les mesures budgétaires se font déjà sentir, et ont permis un regain de confiance. D'autres pensent que cela est dû à l'annonce claire de Mario Draghi qui a promis de «tout faire », avec l'hypothèse que la règle de non-renflouement a été affaiblie et, de facto, n'est pas appliquée.

Ces dernières années, la compétitivité des pays en crise tels que l'Irlande, la Grèce, l'Espagne, l'Italie et le Portugal s'est nettement améliorée par rapport à l'Allemagne. De plus, les soldes des balances commerciales se sont quelque peu stabilisés à moyen terme. En ce qui concerne les écarts de rendement, c'est-àdire les spreads, il est évident que là aussi, la politique de la BCE joue un rôle prééminent. Je suis convaincu que pendant de nombreuses années, les spreads et les notes des grandes agences n'ont pas suffisamment reflété les différentes politiques budgétaires et économiques des pays. Au moment où la crise a été transférée dans le domaine de souveraineté des États et s'est intégrée dans la crise des finances publiques, les agences de notation ont alors naturellement réagi de manière excessive, tout comme elles avaient brillé par leur absence de réaction auparavant. Je pense également que la forte augmentation des spreads en 2011 et en 2012 reflète non seulement le problème de performance des pays en crise, mais également le manque de confiance des marchés financiers dans la capacité des institutions européennes telles qu'elles existent aujourd'hui à résoudre ce problème. 
C'est également la raison pour laquelle nous avons besoin d'une solution de toute urgence. Même si les marchés aiment à comparer l'Europe au Japon, à l'Angleterre ou aux États-Unis, nous ne jouons pas dans la même division. Il faut dire qu'en Europe, nous procédons en quelque sorte à une expérimentation sur nous-mêmes. Nous rêvons d'une union monétaire qui nous mettra sur la voie de l'union budgétaire et politique. Pourtant, même elle n'est pas pour tout de suite. Tous les acteurs, comme les ministres des Finances - de la zone euro ou des 28 pays membres de l'UE - et la Banque centrale européenne, n'évoluent pas dans la même architecture institutionnelle que la Réserve fédérale américaine (Fed) ou la Banque d'Angleterre $(\mathrm{BoE})$ à Londres. Ainsi, cela exclut automatiquement certains modèles de solutions qui sont toujours recommandées par l'extérieur et par les marchés.

Prenons l'une de ces propositions: le célèbre eurobond. Certes, nous en avons déjà quelques-uns dans le cadre du MES, mais dans la situation actuelle, au moment où nous déciderions de mettre en place l'eurobond, c'est-à-dire de concrétiser la mise en commun de tous les risques, les marchés se réjouiraient bruyamment pendant environ 24 heures. Cependant, les pays qui possèdent encore un «triple A »-c'est-à-dire l'Allemagne, l'Autriche, les Pays-Bas, la Finlande et le Luxembourg - ne peuvent pas se permettre d'assurer l'ancrage de tous les autres. Les marchés s'en rendraient compte très rapidement, intégreraient les risques de cette situation, et l'ancre de la stabilité deviendrait subitement bien moins stable. C'est pourquoi il est très important que la France regagne très rapidement son triple $\mathrm{A}$.

\section{Bilan intermédiaire de "I'Union de la stabilité »}

Le bilan portant sur la solidité de l'Union européenne de la stabilité repose sur trois piliers. Le premier concerne la stabilité des politiques budgétaires. Il s'agit des modifications relatives au Pacte de stabilité et de croissance. Avec l'ancien pacte, l'Allemagne et la France avaient pu organiser assez facilement une majorité de blocage. Aujourd'hui, il existe cependant de meilleures procédures, plus simples et plus courtes. Le pacte budgétaire a été adopté, une autre étape positive. Il s'inspire du modèle allemand de frein à l'endettement, lui-même en grande partie inspiré du modèle suisse. Mais nous ne devons pas oublier que le pacte budgétaire repose aussi largement sur l'objectif à moyen terme de l'Union européenne (OMT) qui devient, à côté de l'objectif de $3 \%$ de déficit, l'ancrage de la nouvelle Union. À ce sujet, je suis d'un tout autre avis que Jérôme Creel. Je pense qu'en déplaçant le point d'ancrage vers le déficit structurel, on réalise un formidable progrès pour l'Union européenne, afin d'assurer durablement des finances solides, et en fin de compte, la croissance économique. De fait, je crois qu'il existe là aussi des effets de confiance qui se manifesteront dans les multiplicateurs, quelles que soient nos estimations. Nous ne devons pas suivre des modèles statiques, mais toujours penser de manière dynamique. De mon point 
de vue, on peut dès maintenant abolir le plafond des $3 \%$. En revanche, le critère du taux d'endettement de $60 \%$ reste important.

Pour une politique conjoncturelle anticyclique, nous avons surtout besoin de stabilisateurs automatiques puissants, à effet rapide et de grande qualité. Il faut aussi se demander de quelle politique conjoncturelle discrétionnaire nous avons également besoin. Dans ce domaine, une bonne voie a été tracée, et il serait très présomptueux d'affirmer qu'il s'agit encore une fois seulement de la voie allemande. C'est la voie sur laquelle on s'est déjà engagé par le Pacte de stabilité et de croissance, et même dès la réforme du Pacte en 2005.

Le deuxième pilier du bilan décrit la stabilité, la compétitivité et la capacité de croissance de l'économie. Dans ce contexte, la procédure relative aux déséquilibres macroéconomiques est une très bonne chose. Lorsque je faisais partie de l'Eurogroupe en tant que président du Comité de politique économique du Conseil ECOFIN, j'ai participé à de nombreuses discussions ministérielles en 2008 et en 2009. Le Comité de politique économique (CPE) a toujours milité énergiquement pour une réforme structurelle et pour une focalisation sur la politique de croissance, y compris en ce qui concerne les déséquilibres macroéconomiques persistants. Seules deux institutions nous ont écoutés, la Commission - qui a aussi fait des propositions intéressantes à l'époque - et la Banque centrale européenne. Jean-Claude Trichet a toujours été très amical et a toujours salué les efforts infatigables du CPE, mais les ministres, pour leur part, se sont plutôt concentrés sur l'examen du plafond ou de leur tasse de café. C'est seulement en 2010 que la situation a réellement changé.

Le troisième et dernier pilier du bilan réside dans la stabilité des marchés financiers. Dans ce domaine, la réglementation est un point essentiel. La réforme de la réglementation des marchés financiers prévoit de nouvelles règles de surveillance du marché financier européen (au niveau macroéconomique et microéconomique), une meilleure réglementation (notamment avec une augmentation des capitaux propres), et des règles de résolution des défaillances bancaires ainsi qu'un fonds de restructuration bancaire.

Cependant, il ne suffit pas de procédures pour assurer un budget stable, une économie stable et des finances stables. On en revient toujours à la question : comment le mécanisme de gestion de la crise est-il organisé ? Il s'agit d'un point décisif auquel nous devons encore réfléchir. Nous avons ce mécanisme de crise, mais nous avons également besoin d'un bon mécanisme pour la prévention ultérieure. Tout ce que nous avons déjà fait ou faisons encore pour mettre fin à la crise n'est peut-être pas particulièrement bon pour assurer une prévention optimale à l'avenir. J'ai mené de nombreuses discussions à l'occasion desquelles on m'a dit que les Allemands étaient trop compliqués, qu'ils devraient arrêter de toujours se projeter 10 ans en avant et qu'ils devraient plutôt commencer par aider à faire cesser la crise. Je réponds : oui, mais si on stoppe la crise en faisant quelque chose qui envoie à nouveau des signaux négatifs aux marchés, la prochaine crise surviendra encore plus vite et sera encore plus 
grave. Voilà la réalité. En fin de compte, les marchés doivent faire leur travail et avertir clairement les pays et leurs acteurs lorsque quelque chose va de travers. Pour cela, il faut des réglementations claires et des annonces politiques!

Je pense que les réformes que nous avons engagées jusqu'à présent sont très importantes. Mais il reste de grands défis à relever et des questions sans réponse. On ne peut donc pas exprimer de jugement définitif par rapport à la question de départ de cette table ronde. Nous devons voir ce qu'il en est avec une mise en ouvre crédible et systématique. Il faut cependant avouer que les premières expériences de la procédure relative aux déséquilibres macroéconomiques ne sont pas très encourageantes. Comme vient de le dire Jérôme Creel, l'Allemagne est mise au pilori par la Commission à cause des excédents de sa balance des transactions courantes. Certes, c'est justifié, car l'Allemagne n'a pas besoin de plus qu'elle a déjà. Mais je trouve que la Commission pourrait être plus calme. Je ne me fais aucun souci quant au résultat. En fin de compte, l'Allemagne finira certainement par devoir faire quelque chose, que j'approuverai certainement, car rien n'est pire que de se reposer sur ses lauriers. Et c'est un fait qu'en ce moment, nous sommes dans une situation qui nous offre d'importants effets d'aubaine, tant financiers qu'économiques. Nous devons vraiment faire quelque chose, et je m'attends à ce que la Commission intervienne. Le résultat sera que l'Allemagne ne pourra pas être accusée d'avoir commis une erreur en adoptant sa politique de renforcement de la compétitivité après le désastre des années 1990. Tout a été fait conformément aux règles du marché. Je pense qu'au bout du compte, on pourra dire qu'elle n'a pas vraiment eu tort, même au vu de la situation internationale en matière de concurrence. Je suis même convaincu que le rééquilibrage a déjà commencé en Allemagne. Les derniers chiffres de la croissance dans le pays montrent qu'actuellement, celleci est animée par les facteurs internes, et en aucun cas par les exportations. En fait, ce qui compte n'est pas seulement la compétitivité de l'Allemagne. Au final, ce qui importera, c'est de savoir comment les pays de la zone euro ou de l'Union européenne pourront s'imposer face aux marchés émergents.

Le nouveau mécanisme de contrôle est le nouveau bras correcteur du Pacte de stabilité. Même s'il s'est amélioré, je m'inquiète un peu de sa capacité à obtenir de meilleurs résultats. Il reste d'une grande complexité et offre donc encore de nombreux angles d'attaque stratégiques, certes moins simples à exploiter que dans l'ancien pacte, mais qui ouvrent toujours de nombreuses échappatoires. Ce mécanisme qui ne fonctionne pas encore de lui-même devrait encore être simplifié très bientôt.

\section{Options de réforme : questions conceptuelles}

Nous voilà face à la dernière question : est-ce que tout cela suffit? J'aurais tendance à répondre : "Non, cela ne suffit pas! » Nous devons regarder sérieusement s'il faudrait renforcer la centralisation au niveau de l'UE, par exemple 
sous la forme d'un ministère des Finances européen, d'un Fonds monétaire européen, ou d'une nouvelle compétence budgétaire (veto) de la Commission sur l'ensemble des pays de la zone euro. Cela compléterait le mécanisme de crise préventif. Cela améliorerait aussi certainement la coordination et supposerait une meilleure légitimité démocratique. Cependant, la logique interne dans le cadre de l'établissement d'une structure fédérale ainsi que la faisabilité politique de certaines de ces hypothèses me semblent très discutables.

Il existe également une approche décentralisée. Dans ce cas, il s'agirait d'un fédéralisme européen étendu, un peu sur le modèle allemand. Pour celui-ci, le principe de subsidiarité tiré de la théorie du fédéralisme est très important: avant toute décision publique et toute mesure politique, on vérifie à quel niveau elle doit être mise en œuvre. En cas de doute, elle est affectée au niveau le plus bas possible. Cette méthode a du charme, car elle tient mieux compte de l'hétérogénéité culturelle des États membres et de leurs préférences respectives. Toutefois, il faudrait vraiment qu'au préalable, l'Allemagne et la France travaillent particulièrement sur leurs conceptions différentes de l'État, afin de parvenir à des réflexions communes, et ce d'une manière convaincante pour les électorats, eux aussi différents, de chaque pays. J'ai toujours l'impression que l'Allemagne et la France pourraient certes consentir aux sacrifices nécessaires en matière de souveraineté nationale, mais malheureusement pas dans les mêmes domaines. Je pense que nous, les Allemands, nous n'aurions pas de gros problèmes à transférer à l'Europe nos missions de défense. Je pense aussi que la même chose serait encore impensable en France.

Sur le thème d'une stabilisation centralisée de la conjoncture, sur le budget de la zone euro, nous sommes actuellement en discussion, y compris avec nos collègues français. Mais là aussi, les opinions divergent largement. D'un autre côté, je pense que l'on verra s'il est possible de créer un noyau sur lequel s'appuyer à l'avenir.

Il est également important de conserver une forte intégration commerciale et une grande ouverture - des choses à ne pas oublier pour surmonter le choc au sein de l'Union monétaire. Dans plusieurs pays d'Europe, on voit de nouveau des partis ouvertement protectionnistes. Je trouve cela extrêmement malheureux. Il est important de garantir la flexibilité du marché du travail et de celui des produits, la flexibilité des prix et des salaires, ainsi que la mobilité. À ce sujet, le problème des langues est naturellement à prendre en compte. Les Allemands doivent plus apprendre le français, et les Français doivent plus apprendre l'allemand.

Des institutions indépendantes et plutôt technocratiques peuvent peut-être, malgré tous les déficits de démocratie, surmonter pendant un certain temps les préférences différentes et apaiser les conflits politiques. Le pacte budgétaire européen prévoit pour chaque pays un Conseil budgétaire (Fiscal Council). Les domaines dont nous avons impérativement besoin pour l'Union monétaire, mais où nous ne sommes peut-être pas encore en mesure de bâtir une institution fédérale européenne parce que nous ne pouvons pas nous mettre d'accord sur certaines 
préférences et décisions les concernant, pourraient exceptionnellement être transférés à des experts pour quelques années, jusqu'à ce que nous ayons progressé dans notre intégration. Ce n'est pas ma solution favorite, mais s'il y avait effectivement un problème de mise en œuvre dans l'Union monétaire, on pourrait au moins y réfléchir sans préjugés. Elle aurait peut-être aussi la capacité d'éviter les réactions populistes en cas de crise, dans les pays créditeurs et débiteurs. N'est-ce pas précisément pour cette raison que nous avons fait intervenir le Fonds monétaire international (FMI) ?

\section{Conclusions et perspectives}

Il est nécessaire d'établir des règles crédibles et efficaces pour compenser la faiblesse d'un mécanisme de marché qui doit néanmoins être renforcé. Concernant le rôle du MES : où en sont les attentes en matière de renflouement (bail$o u t)$ ? Comme je l'ai déjà dit, en ce moment, les spreads diminuent. C'est probablement dû non seulement aux efforts de redressement, mais aussi au MES et à l'annonce des opérations monétaires sur titres (outright monetary transactions, OMT) par la BCE. Je pense effectivement que les OMT sont une décision avisée, mais personne ne devrait les tenir pour la véritable solution au problème. La balle est désormais dans le camp des ministres de l'Économie et des Finances. Ils doivent faire le nécessaire au niveau national et au sein de la coordination européenne. Si nous ne comprenons pas cela, je prévois encore de gros problèmes. En ce qui concerne le mécanisme de gestion de la crise lui-même, à mon avis, on ne pourra pas échapper à la nécessité de clarifier encore et très nettement le principe de non-renflouement par des mesures adaptées.

Toutes les procédures de contrôle politique convenues dans le cadre de la surveillance des déséquilibres et de la politique budgétaire dépendent toujours, en fin de compte, de l'engagement politique dans chaque pays. La Commission n'a aucune possibilité d'intervention. C'est pourquoi je pense que les marchés doivent vraiment fonctionner au niveau actuel de fédération. Si les marchés signalent, par l'augmentation des spreads, que quelque chose ne tourne pas rond dans la politique financière et économique, un ministre européen des Finances réagira plus vite que si un groupe d'autres ministres déclare: "Nous avons constaté, dans le cadre de la surveillance macroéconomique, que quelque chose ne tournait pas rond. Nous allons rédiger un rapport, puis en discuter. » À partir du moment où le ministre des Finances ne peut mettre ses emprunts d'État sur le marché qu'en contrepartie de spreads accrus, les marchés doivent fonctionner. Je m'inquiète à l'idée que tout ce que nous avons convenu pour les pays bénéficiant de programmes d'aide puisse constituer une sorte de renflouement rampant. En effet, en fin de compte, le principe de non-renflouement reste tout aussi peu crédible qu'avant, les spreads baissent à nouveau et le cercle vicieux peut reprendre. La douleur est maîtrisée, mais la maladie n'est pas guérie. On aurait gelé la crise, tout en semant les graines de la prochaine. 
Rainer Klump : Merci beaucoup. Je pense que nous allons immédiatement passer la parole à Sandrine Gaudin.

Sandrine Gaudin : Merci beaucoup Christian. J'ai reconnu dans ton intervention tout le dynamisme qu'on te connaît à Berlin (et aussi lorsque tu étais président du Comité de politique économique) pour faire avancer de nombreuses idées.

Je suis assez d'accord avec ce que vient de dire Christian Kastrop, à deux ou trois choses près. Je vais peut-être revenir sur les éléments sur lesquels je suis d'accord. L'intérêt d'avoir ce débat dans cette enceinte est de montrer que la France et l'Allemagne n'ont effectivement pas les mêmes cultures économiques ni les mêmes cultures budgétaires. Cependant, lorsqu'il s'agit de l'euro et de la préservation de l'intégrité de la zone monétaire, de la lutte contre la crise et de la recherche de solutions durables pour l'avenir de l'Union économique et monétaire (UEM), les deux pays ont su démontrer qu'ils étaient d'accord sur l'essentiel.

\section{Nécessité de « banaliser » la mise en œuvre des règles et de discuter ouvertement des problèmes}

J'ai eu à la fois le plaisir et le malheur d'avoir été nommée dans mes fonctions au début de la crise, qui d'ailleurs n'est pas terminée aux yeux du Trésor français. De ce point de vue, Christian Kastrop a raison lorsqu'il dit qu'il ne faut pas s'endormir. Alors évidemment, le discours politique vise à être sans doute plus optimiste et heureusement, certaines choses avancent. On a résolu un certain nombre de problèmes dans la crise de la zone euro, mais le prochain chapitre de l'histoire de la zone euro n'est pas complètement fermé encore. Il faut notamment la stabiliser et arriver à une mise en œuvre de ces nouvelles règles qui soit parfaitement correcte et organisée. Il faut «banaliser » la mise en œuvre des nombreuses règles dont nous nous sommes dotés. De ce point de vue, l'approche allemande a prévalu. Les Allemands sont beaucoup plus attachés aux règles que les Français. Ces derniers voient dans la règle un cadre, mais dans lequel on peut manœuvrer. Les Allemands, sans vouloir faire de caricature, voient une règle qu'on doit appliquer. Le Pacte de stabilité et de croissance comportait de nombreuses règles. Les Français ne voulaient lire que les règles sur la mise en œuvre des circonstances exceptionnelles, qui permettaient la souplesse et la flexibilité, tandis que les Allemands n'ont souvent voulu voir que l'approche nominale pure et dure, comme la limite des $3 \%$ de déficit, et ce pendant des années, jusqu'à des épisodes très célèbres avant la crise où, ensemble, nous avons fait des choses qui allaient contre les règles.

Le nombre de règles actuellement en vigueur peut sembler effrayant. Or tout cela doit être relativisé. D'abord, ces règles étaient absolument indispen- 
sables. Il existait certes un encadrement de nos politiques budgétaires, mais sans procédure de surveillance et d'identification précoce des déséquilibres macroéconomiques. De ce point de vue, comme l'a dit Christian Kastrop, c'est une très grande avancée de pouvoir surveiller nos déséquilibres respectifs. On a vu dans les années 2000 des bulles immobilières se former en Espagne, en Irlande, lesquelles étaient pointées par les spécialistes. Nous-mêmes, dans les réunions techniques à notre niveau, disions aux Irlandais et aux Espagnols de faire attention. Nous leur précisions les conditions de l'éclatement à venir des bulles immobilières, qui a bel et bien eu lieu quelques années plus tard. Christian Kastrop a rappelé le cercle vicieux qui existe entre le souverain et le bancaire, c'est-àdire les conséquences de crises bancaires sur les finances publiques d'un État. Ces crises ont engendré des situations complexes dans les pays comme l'Espagne et l'Irlande, qui justifient la mise en place de ce que l'on appelle l'Union bancaire, c'est-à-dire une supervision renforcée des établissements bancaires, mais aussi la mise en œuvre de façon collective d'une régulation bancaire plus forte. On observe par conséquent de grandes avancées dans les règles, par l'ajout d'un pilier sur l'assurance des déséquilibres.

Sur ce point également, il faut banaliser le rapport qu'a publié la Commission en novembre $2013^{2}$, qui pointe du doigt les surplus commerciaux allemands, mais aussi d'autres déséquilibres. On a parlé de l'Allemagne, parce que c'était un peu nouveau. Je crois qu'il faut banaliser tout cela et surtout éviter de dire comme certains journalistes que l'Allemagne a été " punie ». Il faut arrêter les métaphores sur le bon élève et le mauvais élève. Il n'y a pas de bon élève et de mauvais élève, mais simplement 17, et bientôt 18 États membres ${ }^{3}$ embarqués dans une union monétaire, qui doivent ramer ensemble et dans le même sens pour gagner et retrouver de la croissance et pour éviter un scénario à la japonaise qui serait assez désastreux pour l'Europe. Pour marcher ensemble, on doit dire de manière très franche aux Allemands de faire attention à leurs surplus commerciaux. En effet, s'ils sont trop importants, cela crée des mouvements de capitaux internes à la zone euro préjudiciables à certains pays fragiles. On peut d'ailleurs imaginer des reconstitutions de nouvelles bulles dans la zone euro. Par ailleurs, il faut pouvoir dire aux Français de façon tout à fait banale de faire attention concernant le respect de la règle du déficit. Si les efforts et la politique budgétaire menés devraient permettre de réduire le déficit en France - et par extension le niveau de la dette -, le pays se situe tout de même à la limite. Et il faut pouvoir le dire dans une ambiance complètement dédramatisée. Il ne faut pas dire que l'Allemagne est menacée de sanctions comme j'ai pu le lire. Ce n'est pas parce qu'un rapport mentionne des déséquilibres excessifs qu'il sera suivi de sanctions. Il s'agit simplement de pointer du doigt le problème, puis

\footnotetext{
${ }^{2}$ Commission européenne, Alert Mecanism Report 2014 [en ligne], Commission européenne, Bruxelles, 2013. Disponible sur : http://ec.europa.eu/europe2020/pdf/2014/amr2014_en.pdf [consulté le 06/02/2015].

${ }^{3}$ La Lettonie est le $18^{\mathrm{e}}$ pays à avoir adopté l'euro au 1/01/2014. S'y ajoute la Lituanie depuis le 1/01/2015, NdE.
} 
d'en parler. Nous allons donc nous réunir à Bruxelles pour discuter des avis de la Commission. Je tiens par ailleurs à préciser que ce n'est pas la Commission qui émet un avis sur le projet de budget français. Ce n'est pas la Commission qui dit, qui prescrit ou qui punit s'il fallait punir. C'est la Commission qui produit une analyse mais qui est ensuite discutée par les États membres. Ce sont les États membres et la Banque centrale européenne qui en discutent et qui donnent leur avis. Les ministres des Finances de la zone euro vont par conséquent se réunir dans le cadre de l'Eurogroupe et donner leur avis sur tous les rapports qui ont été publiés en novembre 2013 par la Commission. Évidemment, en termes de médiatisation, il est plus percutant d'affirmer que c'est la Commission qui donne son avis. Or, en réalité, la pression vient des pairs. Elle ne vient pas que d'une institution technocratique, bureaucratique, sans légitimité démocratique qui s'appelle la Commission. Ce point peut toutefois être discuté, parce que la Commission est censée procéder du Parlement européen et a une certaine forme de légitimité démocratique.

Il faut donc banaliser la mise en œuvre des règles et le fait que l'Allemagne soit pointée du doigt. Banaliser ne veut pas dire minimiser, mais rendre normal le processus de surveillance multilatérale qui fut mis en place au moment du traité de Maastricht et que nous nous efforçons d'améliorer par ces nouveaux dispositifs qui sont, je l'admets, un peu technocratiques, mais très utiles pour cette surveillance multilatérale. Par ailleurs, surveiller n'est pas punir. Surveiller, cela veut dire détecter, identifier, discuter de nos contraintes réciproques. Ce n'est pas évident pour l'Allemagne de devoir se justifier sur ses surplus commerciaux. Leur réduction prendra du temps. Il y a tout un débat à avoir sur la question de l'investissement, de la consommation et du salaire minimum en Allemagne. Ces débats étaient d'ailleurs au cœur des discussions visant à aboutir à un accord de coalition pour le gouvernement allemand. De même en France, nous expliquons actuellement en quoi la réforme des retraites est - contrairement à ce que pense la Commission - pleinement suffisante pour répondre aux objectifs de soutenabilité des régimes de retraite, compte tenu notamment de notre situation démographique - dont Christian Kastrop parlait tout à l'heure -, qui est bien meilleure que celle de nombre d'États européens. Nous n'avons par conséquent pas besoin de dupliquer le modèle finlandais ou le modèle suédois en France pour affirmer que nous avons fait une bonne réforme des retraites. Ce copier-coller en Europe n'existe heureusement pas.

La mise en œuvre des règles existantes est la clé de tout. Nous avons sans doute été un peu laxistes dans le passé : nous n'avons pas bien vu le problème grec. Il s'agit sans doute d'une défaillance collective, à la fois de la Commission mais aussi du Conseil. Il me semble que six rapports consécutifs d'Eurostat ont été publiés sur la non-qualité des statistiques grecques présentées entre 1999 et 2006. Les ministres ont dû y consacrer cinq minutes, pas plus, dans leurs discussions au cours d'un ECOFIN. On se disait que ce n'était pas très grave, la 
Grèce étant un petit pays. Or depuis, on a vu l'ampleur de la catastrophe et le fait qu'un petit pays dans une zone monétaire peut affecter la viabilité de la zone monétaire. Si on n'avait pas su lui proposer un programme d'assistance en 2011-2012, la Grèce aurait pu faire exploser la zone euro.

\section{La crise est-elle derrière nous ?}

La question aujourd'hui est de savoir si tout cela nous permet de dire que la crise est dernière nous et que nous avons suffisamment avancé vers une union monétaire plus solide. Ce que nous expérimentons dans notre zone monétaire en Europe est assez unique. Il n'existe dans aucun schéma de zone monétaire d'organisation telle que celle mise en place au niveau européen. La Banque centrale européenne n'est pas la Réserve fédérale américaine, l'Union européenne ne correspond ni aux États-Unis, ni au Canada, ni à la Suisse. Nous travaillons au Trésor français sur le fédéralisme budgétaire et la manière dont les fédérations s'organisent, et notamment organisent la mise en place d'un budget pour la fédération (ce dont nous ne disposons pas au niveau de l'Union européenne).

Nous avons certes créé le Mécanisme européen de stabilité (MES) et le Fonds européen de stabilité financière (FESF), mais il s'agit d'outils de gestion de crise qui ne viennent pas consolider l'Union monétaire. Ils permettent seulement de prêter de l'argent à un pays qui en a besoin, au moment où c'est nécessaire. Sur ce point, je ne suis pas vraiment d'accord avec Christian Kastrop. La clause du no bail-out est effectivement incluse dans le traité, et elle doit y rester puisque c'est un principe important de l'UEM. J'ajouterais, bien que cela soit peut-être passé un peu inaperçu dans l'actualité, que le traité sur le fonctionnement de l'Union européenne a été révisé en 2011 pour permettre la création du MES, qui constitue une sorte de Fonds monétaire européen accordant des prêts aux États. Par conséquent, ce mécanisme d'assistance et d'aide existe et a même été autorisé par les États de l'Union européenne. Absolument utile et indispensable pour servir en dernier ressort, il devrait s'installer à l'avenir dans le paysage de la zone monétaire. Le concept du dernier ressort (ultima ratio) est beaucoup apprécié par l'Allemagne. Il s'agit d'abord pour l'État membre concerné de faire un certain nombre de réformes. Ce n'est vraiment qu'ultima ratio que l'aide lui sera apportée si nécessaire. De ce point de vue, ce dispositif est bon et fonctionne. On l'a vu avec la mise en œuvre des plans d'assistance irlandais et portugais, du programme bancaire espagnol et des deux programmes grecs. J'aimerais mentionner au passage que le 14 novembre 2013, les ministres de l'Eurogroupe ont salué la sortie de l'Irlande de son programme d'assistance. Il s'agit ici d'une success story qui doit être mise en avant. Les outils de crise ont été mis en place et fonctionnent. L'Irlande va retourner sur les marchés de manière normale, à des taux et avec un spread relativement apaisés. 


\section{Conforter le projet politique pour parachever l'UEM}

Au-delà de ces outils de gestion de crise, que faut-il pour parachever l'UEM ? Il existe plusieurs types de pistes. Les eurobonds ont été abordés il y a quelques années, avec l'idée que mutualiser l'émission de dette - et par conséquent la dette de la zone euro - pouvait être une des solutions. Les project bonds (obligations de projet) ont également été évoqués. On a parlé, à l'image de ce qui passe dans d'autres fédérations, de mettre en place une capacité budgétaire pour la zone euro, ce qui n'existe pas aujourd'hui. Aujourd'hui, le budget de l'Union européenne concerne ses 28 États membres. Toutefois, il n'a aucune fonction de stabilisation économique. Il n'a que des fonctions de redistribution ou de financement de politique sectorielle ou économique, dont l'impact économique est très limité, même si la politique de cohésion, la politique structurelle et la politique agricole commune peuvent avoir un impact économique. Quoi qu'il en soit, il n'a pas de fonction de stabilisation en cas de choc.

Nous travaillons beaucoup sur ces sujets, parce que nous pensons qu'il s'agit là d'une nouvelle page à écrire, de l'«Union économique et monétaire 2.0 ». De même que nous avons mis en place l'euro en trois étapes, nous pensons qu'il faut consolider l'UEM en établissant un nouveau projet. L'euro n'est pas un projet économique, mais un projet politique. J'ai récemment recensé les déclarations d'Angela Merkel, de Nicolas Sarkozy ou de François Hollande ces dernières années sur l'euro et la préservation de la zone euro : toutes leurs déclarations vont dans le même sens, ce qui est plutôt rassurant et très positif. Devant le Bundestag, Angela Merkel a clairement dit que l'euro était plus qu'une simple monnaie commune et que cette monnaie unique et irréversible représentait un projet politique. Cette déclaration est empreinte de symboles, le Bundestag allemand étant très proche de la gestion de la crise de la zone euro. Le fait d'avoir pu associer la légitimité démocratique à toutes les prises de décisions de l'Allemagne sur la crise constitue une particularité allemande que je salue.

Selon moi, il faut conforter ce projet politique. Sur ce point, je me permets d'évoquer notre publication Trésor-Eco, dont le numéro d'octobre $2013^{4}$ porte justement sur les raisons pour lesquelles une capacité budgétaire de la zone euro serait nécessaire. Ce papier vise à montrer ce que l'on peut faire de supplémentaire pour préserver l'intégrité de l'UEM, renforcer l'outil de coordination des politiques budgétaires, mais surtout créer un outil de stabilisation. On voit bien que la crise en Espagne a commencé par une bulle immobilière, une crise bancaire, un problème sur les finances publiques, un problème sur la croissance et un problème sur le chômage, avec $57 \%$ de chômage pour les jeunes de moins de 25 ans. C'est absolument inadmissible d'un point de vue économique, d'un point de vue politique et d'un point de vue tout simplement

\footnotetext{
${ }^{4}$ Nicolas Caudal, Nathalie Georges, Vincent Grossmann-Wirth, Jean Guillaume, Thomas Lellouch, Arthur
} Sode, « Un budget pour la zone euro », Trésor-Éco, n 120, octobre 2013, p. 1-12. 
démocratique. On a dès lors aidé l'Espagne par un plan d'assistance et d'assainissement bancaire, mais il n'existe aucun outil pour aider l'Espagne à faire face à des plans de formation accélérés pour les jeunes chômeurs et à tous les besoins liés à ce taux de chômage absolument phénoménal. Le budget de l'Union européenne pour l'emploi des jeunes s'élève à 6 milliards sur les sept prochaines années pour les 28 États membres, ce qui est vraiment très faible. Ne peut-on pas imaginer que, dans une logique de stabilisation de la zone euro, cette dernière puisse apporter son aide, prêter ou même donner de l'argent, dans la mesure où c'est un membre de la zone euro qui subit un choc, pour essayer d'avoir les bons outils contre le chômage des jeunes? Ce point me paraît essentiel.

\section{La question des transferts budgétaires}

J'aurais une dernière réaction par rapport à ce que disait Christian Kastrop concernant un sujet très controversé à l'échelle franco-allemande, dont nous commençons à peine à discuter : la notion de transfert budgétaire. Il n'existe pas pour l'instant d'analyse ni d'évaluation commune de la situation. Néanmoins, je ne désespère jamais de la relation franco-allemande. En effet, depuis 25 ans que je travaille dans le franco-allemand, j'observe que nous sommes toujours d'accord lorsqu'il le faut. Nous passons parfois par des moments un peu difficiles, mais nous y arrivons toujours à la fin. Par conséquent, je suis sûre que nous y arriverons.

\section{Renforcer la légitimité démocratique de l'Union européenne}

Quoi qu'il en soit, l'idée est de ne s'endormir ni sur la mise en œuvre des règles, ni sur la pédagogie. En effet, il me semble que nous avons tous manqué le défi de la communication. Nous sommes très mauvais pour expliquer les règles et quand nous les expliquons, nous disons qu'il s'agit des règles que Bruxelles nous a dit d'appliquer : c'est une catastrophe. Nous avons également manqué l'appropriation démocratique, mais nous pouvons imaginer que notre projet de capacité budgétaire de la zone euro puisse répondre à ce souci. Aujourd'hui, certains parlements nationaux sont très impliqués dans la mise en place des outils concernant la gestion de la crise. Ce n'est pas forcément le cas en France d'ailleurs et c'est sans doute dommage, mais c'est une question de culture. On peut néanmoins imaginer aussi que le Parlement européen puisse être davantage associé aux débats et à la prise de décision concrète et opérationnelle. Je crois que - même si, sur ce point, je sors de mon rôle et je suis obligée d'admettre que je vais vers d'autres terrains -, la question de l'appropriation politique de tous ces sujets est absolument capitale, notamment dans le cadre des élections pour le Parlement européen, dont la campagne ne sera simple dans aucun des pays de l'Union européenne. 


\section{CONCLUSION}

Rainer Klump : Merci beaucoup, Sandrine Gaudin. Voilà qui était, je crois, formulé de manière très incisive. Je constate qu'il règne une étonnante unanimité au niveau des administrations. C'est un bon signe. En 2013, nous avons célébré les 50 ans du traité de l'Élysée et les 55 ans du traité de Rome. On remarque que pendant ce temps, la coopération et la collaboration au quotidien ont beaucoup progressé et qu'à ce niveau, on est capable de surmonter ensemble les périodes problématiques. Sandrine Gaudin a encore rappelé que l'euro était initialement un projet politique. Du point de vue allemand, on serait certainement d'accord, tout en ajoutant qu'il ne faut néanmoins pas oublier les bases économiques. En effet, il serait fatal d'envisager un projet politique sans des bases économiques solides. C'est aussi de cela que nous nous préoccupons ensemble : poursuivre les ambitions politiques tout en établissant et en perfectionnant les bases économiques, afin que les deux s'accordent. Voilà le défi à relever. Vous avez tous les deux parlé de la réglementation, qui doit être sans cesse perfectionnée et affinée afin de pouvoir concrétiser cette vision politique jusque dans la vie quotidienne économique.

Traduction des propos de Rainer KLUMP et Christian KASTROP : Marie-Céline GEORG 



\section{- III -}

GOUVERNANCE ÉCONOMIQUE, FINANCIÈRE ET MONÉTAIRE DE L'UNION EUROPÉENNE 



\section{Table ronde : Quelle(s) politique(s) économique(s) pour la zone euro ?}

\section{OUVERTURE DE LA TABLE RONDE}

René Lasserre : Nous allons commencer par faire un tour d'horizon de la politique économique au sein de la zone euro pour en distinguer les grandes tendances. Je vais donner dans un premier temps la parole à Christian Kastrop. Après avoir longtemps travaillé pour le ministère fédéral des Finances, Christian Kastrop dirige désormais le département des Études de politique économique à l'Organisation de coopération et de développement économiques (OCDE). Autour de lui, Jean-Marc Daniel, professeur associé à ESCP Europe et économiste auprès de l'Institut de l'entreprise, reviendra sur la situation économique actuelle dans la zone euro et sur les enjeux de gouvernance. Puis Henrik Uterwedde, directeur adjoint de l'Institut franco-allemand de Ludwigsburg (DeutschFranzösisches Institut, DFI), nous exposera l'évolution de la position allemande vis-à-vis de la stabilité budgétaire et de la croissance tandis que Natacha Valla, directeur adjoint au Centre d'études prospectives et d'informations internationales (CEPII), abordera la position de la France dans le contexte européen.

\section{EXPOSÉS DES INTERVENANTS}

Christian Kastrop : Je me réjouis de pouvoir participer à nouveau à ce projet de recherche très réussi. Je pense que cette série de tables rondes est extrêmement utile et je crois que cela permet d'atteindre des diffuseurs d'idées afin, à moyen terme, de réconcilier quelque peu les perceptions et les perspectives respectives, parfois très divergentes, de la France et de l'Allemagne.

Je suis d'avis que, sans une très bonne coopération franco-allemande, l'Union européenne va connaître de grosses difficultés. Pour l'avenir de l'Union européenne, il me semble fondamental de parvenir à créer des passerelles sur le plan culturel, non seulement entre des concepts économiques, mais aussi entre des questions d'économie politique et de politique sociale. Les questions ne se limitent jamais aux concepts économiques, mais intègrent toujours une dimension de compréhension sociale. Même si l'Allemagne et la France ont des racines communes, elles ont développé des traditions très différentes. Il est très important de rappeler quelques aspects historiques pour comprendre pourquoi il est si difficile de se rapprocher aujourd'hui sur des questions fondamentales.

Lorsque ce projet de recherche a débuté, je travaillais encore au ministère fédéral des Finances à Berlin, où nous étions très engagés dans la promotion de projets franco-allemands, y compris au nom des ministres français et allemand

${ }^{1}$ Cette table ronde s'est tenue dans le cadre du colloque Gouvernance économique, financière et monétaire de l'Union européenne, coorganisé par le CIRAC les 6 et 7 octobre 2014, NdE. 
respectifs. Il est donc d'autant plus intéressant pour moi de me confronter à ces questions franco-allemandes sous un angle légèrement différent en tant que directeur des Études de politique économique à l'OCDE à Paris. Je me réjouis donc de pouvoir adopter aujourd'hui un point de vue global et de ne pas devoir représenter directement la position allemande, même si je suis prêt à le faire. Lorsqu'on observe la situation de l'extérieur, on constate en regardant l'Allemagne et la France des phénomènes très intéressants que j'aimerais vous présenter brièvement.

\section{Prévisions intermédiaires de l'OCDE, septembre 2014 : une croissance beaucoup plus faible dans la zone euro qu'aux États-Unis}

Comme la plupart des organisations internationales, l'OCDE établit des projections au printemps et à l'automne. Dans la projection de septembre 2014, on voit des relations très intéressantes en ce qui concerne les taux de croissance. La partie gauche du graphique ci-dessous montre qu'entre 2013 et 2015, les ÉtatsUnis vont connaître une très bonne croissance. La situation semble plutôt bonne au Japon également. Quant à la zone euro, ses performances ne sont pas très bonnes, même si elle devrait être un peu mieux placée que le Japon en 2015.

\section{Graphique 1 : Prévisions économiques intermédiaires de l'OCDE}
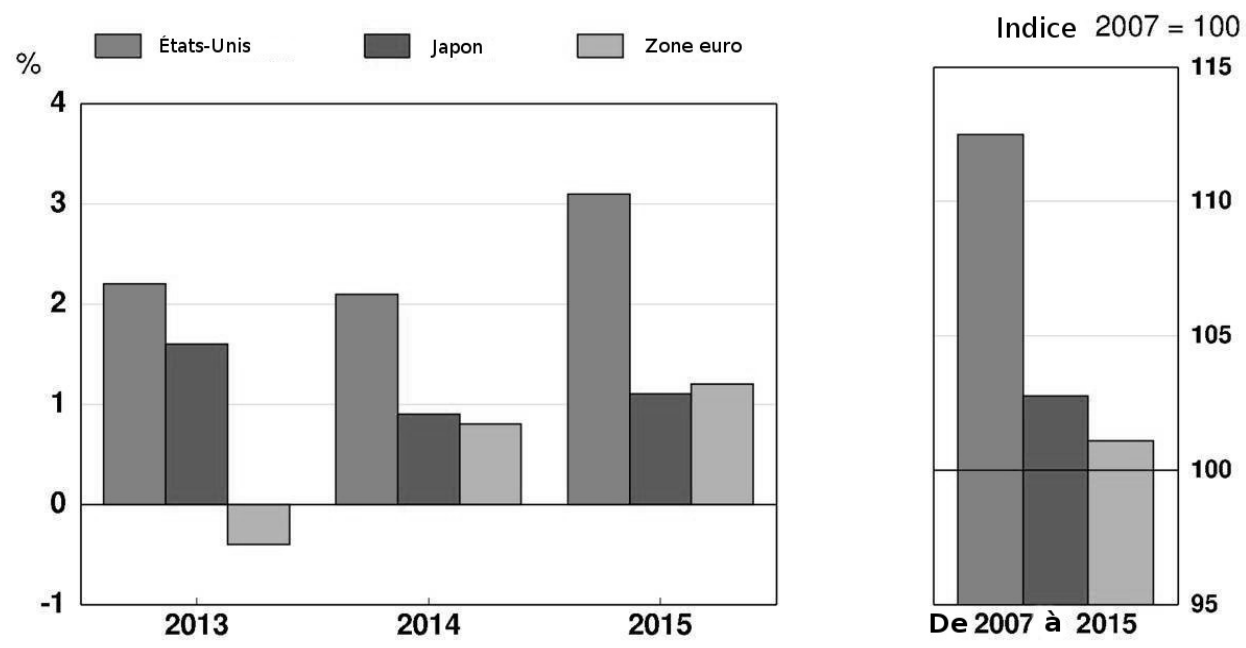

Source : base de données des Perspectives économiques de l'OCDE, n 95.

Sur la partie droite du graphique ci-dessus, on constate que, depuis 2007, les États-Unis ont connu dans l'ensemble une forte croissance malgré la crise. La situation du Japon est loin d'être aussi bonne, mais le grand perdant - même si on pourrait en dire beaucoup au sujet du Japon ou des États-Unis - est clairement l'Europe. Les raisons de cette faible performance sont assez claires, 
je n'entrerai pas dans les détails ici. Naturellement, ce chiffre constitue avant tout une moyenne entre des pays européens très différents. Pendant cette période, certains pays ont connu la récession ou ont même dû entrer en récession précisément pour résoudre leurs problèmes structurels. Le plus souvent, les réformes structurelles ont d'abord des effets négatifs sur la croissance actuelle de l'économie, et certainement aussi sur le marché du travail. Mais à long terme, elles devraient tout de même s'avérer d'une grande utilité ; naturellement, la mise en œuvre des programmes de réformes doit également tenir compte de la dimension sociale.

\section{L'austérité budgétaire depuis 2009 : \\ plus forte aux États-Unis que dans la zone euro}

Si l'on observe les chiffres consolidés depuis 2009, on constate dans l'ensemble que la zone euro n'est absolument pas un précurseur en matière de politique d'austérité. Le solde primaire 2009-2014 montre que la consolidation a été bien plus forte aux États-Unis qu'en moyenne dans la zone euro. Là aussi, il existe d'importantes différences, et naturellement la consolidation a dans la plupart des cas un effet négatif à court terme sur la croissance, qui est largement compensé par un potentiel de croissance supérieur à long terme. Naturellement encore, il faut appliquer la bonne stratégie d'ensemble, qui tient aussi compte de la position dans le cycle conjoncturel.

\section{Graphique 2 : Solde financier primaire sous-jacent des administrations publiques 2009-2014 (en \% du produit intérieur brut (PIB) potentiel, par an)}

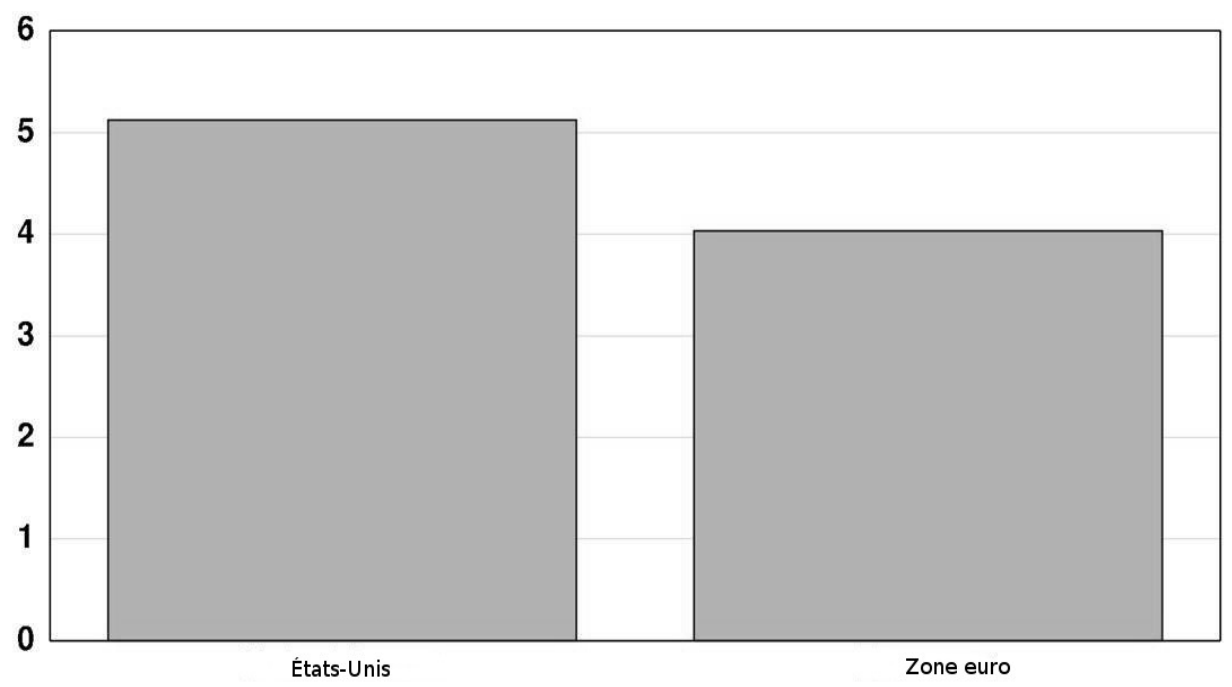

Source : base de données des Perspectives économiques de l'OCDE, $\mathrm{n}^{\circ} 95$. 


\section{L'assouplissement monétaire depuis 2009 : beaucoup plus marqué aux États-Unis que dans la zone euro}

Dans la gouvernance politique des nations industrielles vieillissantes et dépourvues de matières premières contre la stagnation, la baisse de la productivité et les risques de déflation, l'essentiel est toujours de déterminer un concept stratégique d'ensemble pour les « trois grands axes » : la politique budgétaire, la politique monétaire et la politique structurelle.

On remarque là de grandes différences dans l'orientation et la gouvernance politiques. Ainsi, les États-Unis ont fortement misé sur des allègements fiscaux, sur un rapide processus de désendettement (deleveraging) des ménages et des banques surendettés, ainsi que sur une politique d'assouplissement monétaire afin de redresser l'économie grâce à des mesures dites non conventionnelles, au-delà de la fameuse politique de baisse des taux jusqu'à un seuil plancher (zero lower bound).

Graphique 3 : Actifs des banques centrales en $\%$ du PIB (indice $2008=100$ )

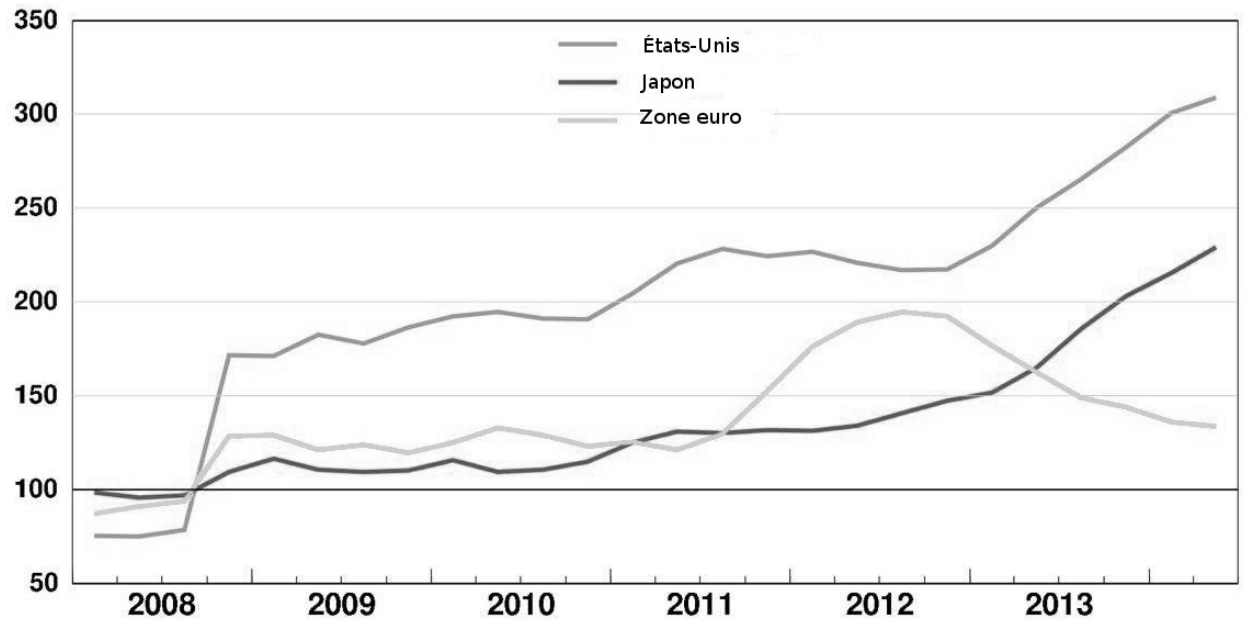

Source : Datastream.

La politique économique et financière de l'Europe, tout comme la Banque centrale européenne (BCE), est dans la même situation. En Europe, l'architecture institutionnelle de tous les principaux secteurs de gouvernance est très fragmentée, de sorte que les moyens d'action de la politique sont également différents. Il existe une forte incitation à l'aléa moral en matière de politique budgétaire et structurelle lorsque la politique monétaire fait «tout ce qu'il faudra » ( «whatever it takes »). À ce niveau, il n'existe pas de solution standard, même si l'OCDE a conseillé à la BCE d'intervenir plus énergiquement en adoptant 
elle aussi des mesures non conventionnelles, tout en sachant qu'en Europe, les moyens d'action ne sont pas les mêmes qu'aux États-Unis. Le graphique 3 montre les actifs des banques centrales. Mario Draghi a annoncé qu'il aimerait à nouveau atteindre le pic de 2012. Nous verrons en détail comment il met en œuvre cette stratégie par un paquet de mesures adéquates, la question n'étant pas anecdotique.

\section{La régulation freine davantage la croissance dans la zone euro qu'aux États-Unis}

Lorsqu'on observe les données de 2013 sur la réglementation du marché des produits et celle du marché du travail, on peut voir que les États-Unis sont plus engagés non seulement dans le domaine de la politique monétaire, mais aussi dans celui des réformes structurelles. En ce qui concerne la réglementation du marché des produits, l'Allemagne et la France ne sont pas si mal classées par rapport à la moyenne européenne, mais font nettement moins bien que les EtatsUnis, où la réglementation du marché du travail est nettement moins stricte que dans la zone euro. L'Allemagne et la France sont toutes les deux - l'Allemagne encore plus que la France - championnes en matière de réglementation.

\section{Graphique 4 : Réglementation sur les marchés des produits et du travail (2013)}

Réglementation sur le marché des produits

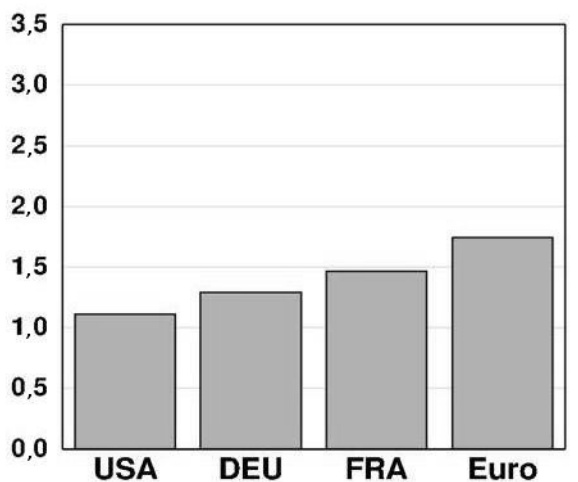

Réglementation sur le marché du travail

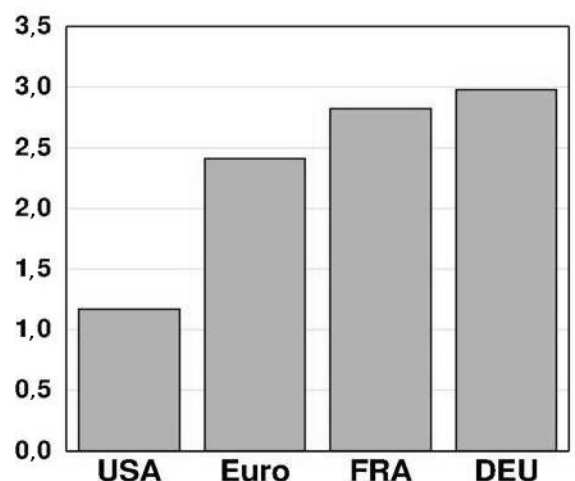

Source: Base de données OCDE des indicateurs de réglementation des marchés des produits et du travail. La réglementation sur le marché du travail correspond à la protection des salariés permanents contre les licenciements individuels et collectifs. Les données relatives à la réglementation américaine sur le marché des produits se rapportent à l'année 2008.

\section{Quels sont les grands enjeux?}

La question qui revient toujours est celle-ci : comment concilier des finances publiques durables et une réforme structurelle favorable à la croissance et à la concurrence, tout en "gagnant» du temps à travers la politique monétaire de 
la BCE ? À l'OCDE, nous nous penchons justement sur ces questions en ce moment, en particulier sur le séquençage et la composition des réformes. Nous avons pour cela un nouveau mandat du G20. On pourra donc peut-être surmonter un peu cette prétendue incompatibilité de principe entre la politique de l'offre et celle de la demande. Il ne s'agit même pas de mettre en œuvre une consolidation ou des réformes structurelles, mais de savoir comment on peut intégrer un programme de politique structurelle en cours dans une évolution macroéconomique. Cela pourrait aussi être une idée sur laquelle l'Allemagne et la France se rapprocheraient un peu. On ne va certainement pas vouloir appliquer impérativement une réforme structurelle aux effets fortement négatifs sur la demande précisément à une période de ralentissement économique. Cependant, les réformes structurelles sont bien plus variées que nous ne le pensons peut-être en général ; on peut donc sûrement trouver, dans le programme, des réformes structurelles favorables à la croissance qui auront dans l'immédiat moins d'effets néfastes sur la demande et sur la croissance.

La politique monétaire pourrait certainement jouer un rôle important. D'un autre côté, nous avons le problème dit de la gouvernance, à savoir que ni la politique financière, ni la politique économique ne sont dirigées en commun. Il y a certes des progrès dans la coordination, mais nous sommes encore loin d'une gouvernance commune, lorsqu'on compare la zone euro à des pays tels que la Grande-Bretagne et les États-Unis, où il existe également un État fédéral. Cela ouvre des possibilités totalement différentes pour mener une politique monétaire non conventionnelle de manière engagée.

Jean-Marc Daniel : Je vais à la fois réagir au panorama qui nous a été donné via trois remarques initiales et esquisser ma vision du problème.

\section{Remarques introductives sur la gouvernance européenne et la politique monétaire}

Ma première remarque concerne la question de la gouvernance en Europe. En premier lieu, on peut se demander quand on parle de l'Europe si on parle de la zone euro ou si l'on parle des 28 États membres de l'Union européenne (UE). On attribue par exemple à la zone euro et à l'euro des facteurs handicapants, notamment vis-à-vis du chômage. Toutefois, les cinq pays européens où le taux de chômage est le plus faible sont tous membres de la zone euro, c'est-à-dire Malte, le Luxembourg, l'Allemagne, l'Autriche, et les Pays-Bas. En revanche, les pays où le chômage augmente rapidement à l'heure actuelle sont la Croatie, la Suède et la France. Sur ces trois pays, deux se situent hors zone euro. Aux dernières élections en Suède, la population a sanctionné la situation économique du pays. Hors zone euro, des politiques économiques d'austérité ont été menées. La Hongrie notamment a adopté une politique de même nature que celle menée 
par la Grèce, avec des résultats plutôt positifs, mais dans le cadre de laquelle la contraction de l'activité économique a été assez forte.

Deuxième remarque à titre de prolégomènes : je pense que la focalisation sur la politique monétaire est aussi liée au fait que personne ne comprend la politique monétaire. Quand vous dites à un citoyen que l'on va changer la donne sur le plan fiscal, même s'il ne comprend pas au départ, il comprend assez vite quand il voit arriver sa feuille d'impôt. Quand vous lui dites que Mario Draghi a lancé un programme d'opérations de refinancement ciblées à long terme (targeted longer-term refinancing operations, TLTROs), il n'en mesure pas forcément l'impact; il fait confiance. Le banquier central peut alors passer d'une image extrêmement positive à la menace d'être traîné en prison. Alan Greenspan a connu ce destin. Il était l'homme qui parlait à l'oreille des marchés, l'homme qui comprenait tout, puis il est devenu responsable du séisme financier que nous avons connu à partir de 2007-2008.

Ma troisième remarque constitue un élément de réflexion sur un plan plus politique : est-ce que les solutions politiques envisagées, et notamment la marche vers une Europe fédérale plus affirmée, sont réalistes ou sont-elles des leurres pour essayer de se masquer la réalité des problèmes?

\section{Trois problèmes d'interprétation de la situation économique actuelle}

Le premier problème réside dans l'absence de croissance. Selon moi, la situation dans les blocs homologues que sont le Japon et les États-Unis est moins favorable qu'on pourrait le penser. Les États-Unis continuent à vivre à crédit avec un déficit de balance des paiements courants qui demeure important, même s'il s'est réduit pour deux raisons : la remontée du taux d'épargne des ménages et l'effort structurel réalisé par le pays en réaction à une situation très dégradée dans les années 2008-2009. Quant au Japon, son gouvernement a été obligé de démissionner à la suite d'une crise d'inflation qu'on n'avait pas connue dans le pays depuis très longtemps. L'inflation en 2014 sera de $3 \%$ au Japon. En Europe, il existe actuellement un problème de croissance. Une des raisons pour lesquelles nous sommes dans une situation délicate, c'est que nous interprétons systématiquement le problème de croissance comme étant un problème d'emploi. Or je pense que la priorité d'une politique économique, c'est la croissance et non l'emploi. Sinon, les anciennes démocraties populaires des années 1960 qui avaient le plein emploi seraient une sorte de modèle et d'accomplissement ultime.

Le deuxième problème, c'est l'analyse que nous faisons de notre situation monétaire en termes de déflation. Le discours selon lequel nous serions menacés de déflation n'est à mon avis pas pertinent, d'abord parce que la masse monétaire continue à augmenter (+1\% en zone euro en 2013). En France en particulier, la Banque de France vient de publier les dernières statistiques montrant que le crédit aux entreprises - qui est la dimension positive de la 
création monétaire - a recommencé à augmenter à partir de mai 2014. Par conséquent, nous ne nous situons pas dans une dynamique de déflation. Certes, le prix de l'énergie baisse, mais cela représente un facteur positif. La dernière fois que nous avons eu un taux d'inflation aussi faible en équivalent de la zone euro en 1998, cela correspondait à un contre-choc pétrolier qui a nourri la période de croissance des années 1998-2000. Donc parler de déflation, c'est passer à côté du problème, de même que parler de chômage, c'est passer à côté du problème.

Le troisième élément qui à mon avis est un élément d'erreur dans l'analyse, c'est l'interprétation que l'on doit faire de notre excédent extérieur et du taux de change de l'euro. La France a une position qui me paraît surprenante, qui consiste à accuser la Chine de réaliser des excédents extérieurs trop importants et à réclamer systématiquement l'appréciation du yuan, en déclarant par ailleurs que l'euro est trop fort et qu'il faut le dévaluer. Or la zone euro a davantage d'excédents extérieurs que la Chine. Il faudrait donc selon cette logique qu'il y ait une appréciation de l'euro.

\section{II faut respecter le TSCG...}

Derrière ces trois erreurs, nous passons à côté de deux types de réformes à faire selon moi. La première réforme est de respecter le traité sur la stabilité, la coordination et la gouvernance (TSCG) de l'Union européenne. Les autorités européennes et les peuples européens se sont dotés d'un traité qui améliore la situation de l'interprétation que l'on doit faire d'une politique économique partagée. Ce qu'il y a d'intéressant en France, c'est que les journalistes comme les hommes politiques évoquent souvent Maastricht, qui correspond à l'entrée dans l'Union économique et monétaire (UEM). Or nous sommes passés désormais au Pacte de stabilité et de croissance (PSC). Les $3 \%$ mythiques qui persécutent nos dirigeants et ne les empêchent manifestement pas de dormir mais qui les empêchent d'être crédibles -, sont d'une certaine manière obsolètes. L'enjeu aujourd'hui est le TSCG. Il n'interdit pas le déficit budgétaire en tant que tel, mais il interdit le déficit structurel, c'est-à-dire qu'il appelle à une remise en ordre de nos finances publiques sur la base non pas du déficit, mais de la qualité de la dépense réalisée. Derrière cette notion de déficit structurel, la vraie question n'est pas de savoir si nous dépensons trop ou pas assez, mais plutôt de savoir si la population estime que les dépenses ainsi réalisées rendent les services auxquels elle s'attend et si elle est prête à payer la quantité d'impôts qui permettent de financer les dépenses publiques. Si oui, il n'y a pas de déficit structurel. Selon moi, le véritable enjeu dans les finances publiques est de respecter le TSCG et de réduire le déficit structurel. Si ces engagements sur le déficit structurel ne sont pas mesurables au milliard près, ils sont mesurables dans une logique intellectuelle d'appréciation de ce que sont les finances publiques. 


\section{... et mener des réformes structurelles}

La deuxième chose à faire, ce sont les réformes structurelles. La zone euro est assez bien positionnée sur le marché des produits et moins bien positionnée sur le marché du travail. En Europe, la politique de la concurrence menée à la fois par les autorités de Bruxelles et les autorités locales de concurrence est efficace. Cela ne se fait pas sans mal. Bien qu'on ait l'impression, à entendre certains commentaires, qu'on a confié l'UE à des fous, la concurrence a fait des progrès considérables en Europe depuis la politique de Roy Jenkins et de Jacques Delors à l'issue de l'Acte unique. Néanmoins, l'UE est en retard dans la gestion de la population active. Cela ne concerne pas le chômage en tant que tel, mais la capacité à générer de la concurrence sur le marché du travail et de l'efficacité dans la politique de formation. En effet, la politique de formation, pour être efficace, doit également être raisonnée et gérée en concurrence. La prochaine réforme structurelle consistera donc à introduire de la concurrence dans le processus non seulement de formation des salaires, mais également de formation du niveau de compétences de la population active. Pour être clair et simple, je suis professeur à ESCP Europe. Les étudiants se précipitent davantage vers notre antenne de Londres que vers celle de Berlin certes parce qu'on y parle l'anglais, mais aussi parce qu'ils ont la vision qu'à Londres, il y a un esprit concurrentiel car le mécanisme des MOOC (massive online open courses) s'y est généralisé. Je pense que la qualité de l'information et de la formation sera améliorée par l'utilisation des nouvelles technologies.

\section{L'importance des NTIC}

Sur le plan de la croissance, la véritable force des États-Unis est de jouer à fond le jeu des nouvelles technologies de l'information et de la communication (NTIC), y compris en gérant les enjeux qui ont trait à la concurrence. Je ne souhaite pas revenir à Franklin Delano Roosevelt, mais à Theodore Roosevelt qui s'était attaqué à l'industrie pétrolière. J'attends en effet du prochain président des États-Unis une vraie politique de concurrence face à Google et Facebook. J'attends aussi qu'en Europe, on prenne conscience que l'enjeu, c'est la productivité, que la productivité, c'est le progrès technique et que le progrès technique passe en ce moment par les NTIC.

\section{Les trois Charles}

Dans un livre récent ${ }^{2}$, je conclus en disant que l'Europe a toujours fonctionné sur des Charles. Charlemagne associe les Français et les Allemands. Puis, au $\mathrm{XVI}^{\mathrm{e}}$ siècle, Charles Quint, le deuxième Charles, s'est allié à l'Angleterre de Henry VIII lorsque les Allemands et les Français étaient dans une phase d'op-

\footnotetext{
${ }^{2}$ Jean-Marc Daniel, L'État de connivence - En finir avec les rentes, Odile Jacob, Paris, 2014.
} 
position. Ce qu'il y a d'intéressant, c'est qu'au moment de la guerre de Sept Ans, dont on commémorait en 2013 les 250 ans, Frédéric II de Prusse avait dit : "En associant l'Allemagne et l'Angleterre, $j$ 'associe l'Allemagne à une flotte et une banque », c'est-à-dire que l'Angleterre était la flotte et la banque. Et puis il $\mathrm{y}$ a eu l'alliance entre Louis XIV et le troisième Charles, Charles II d'Angleterre. Selon moi, l'Europe a tout intérêt à sortir des trois Charles et à retrouver un moyen d'insérer l'Angleterre dans le dispositif. Au moment de la crise du Système monétaire européen (SME) en 1992, nous avons commis l'erreur de ne pas régler le cas anglais de façon digne. Les Anglais sont sortis du SME de façon ignominieuse. Je parle sous le contrôle de Jacques Mistral qui y était et avec qui j'ai déjà eu l'occasion d'en parler.

\section{La difficulté d'aller vers une union fédérale}

Enfin, sur le plan politique, je ne pense pas que nous puissions aller vers une union fédérale. Lorsque Jean Monnet soumet son texte à Robert Schuman en 1950 , il comprend le terme «abandon de souveraineté». Robert Schuman répond à Jean Monnet que cela va trop loin et qu'il faut préparer les esprits. Puis, lorsque Charles de Gaulle apprend cette anecdote, il ajoute que la France va avoir l'arme atomique. Par conséquent, un abandon de souveraineté entre la France et l'Allemagne poserait le problème de savoir qui va gérer le siège de membre permanent au Conseil de sécurité de l'Organisation des Nations Unies (ONU) ou qui va décider de l'usage de l'arme atomique.

Autour d'un noyau composé de la France, de l'Allemagne et du RoyaumeUni, l'Europe doit développer un modèle intellectuel né selon moi au sommet de Bonn en 1978. Dans un article publié par l'OCDE à l'occasion de ses 50 $\mathrm{ans}^{3}$, il y avait l'idée que le vrai changement dans l'OCDE était intervenu avec le rapport McCracken ${ }^{4}$ et le sommet de Bonn de 1978. Le rapport McCracken posait la question de savoir si l'inflation pouvait être un moyen de lutter contre le chômage (arbitrage de Phillips). Données statistiques à l'appui, il arrivait à la conclusion qu'avec le chômage, on réduirait l'inflation mais qu'avec l'inflation, on ne réduirait certainement pas le chômage. Or l'administration Carter décida tout de même en 1978 d'utiliser l'inflation et de faire du quantitative easing, c'est-à-dire d'injecter de la monnaie par des déficits budgétaires dans l'espoir de créer éventuellement de la croissance. Le chancelier allemand Helmut Schmidt refusa alors de s'engager dans cette logique, arguant que l'inflation n'était pas un outil, mais un problème. Cela avait frappé les esprits. En 1978, il n'était alors pas courant qu'un chancelier allemand exprime son désaccord face

\footnotetext{
${ }^{3}$ OCDE, « Les 50 ans de l'OCDE - Changements de paradigme dans la conduite de la politique économique », Perspectives économiques de l'OCDE [en ligne], $\mathrm{n}^{\circ}$ 89, mai 2011, p. 339-364. Disponible sur : http://www.oecd-ilibrary.org/docserver/download/1211012e.pdf [consulté le 19/02/2015].

${ }^{4}$ Paul W. McCracken, Towards Full Employment and Price Stability - A Report to the OECD by a Group of Independent Experts, OCDE, Paris, 1977.
} 
aux Américains. Mais Helmut Schmidt a tenu ferme. Je regrette néanmoins qu'à l'époque, seul Raymond Barre en France ait voulu le soutenir (et pas Valéry Giscard d'Estaing). C'est selon moi à ce moment précis qu'est née l'idée que l'inflation était un problème et non une solution et que la politique monétaire ne détenait pas l'avenir. Ce qui détient l'avenir, c'est un modèle intellectuel qui favorise le progrès technique, le profit et la concurrence en vertu du théorème d'Helmut Schmidt: "Les profits d'aujourd'hui sont les investissements de demain et les emplois d'après-demain. » L'Europe peut en être le porteur, car c'est ce qui unit pour l'instant la démarche britannique et allemande. Intellectuellement, cela pourrait être la démarche française. Le problème de la France est que nous n'avons pas de dirigeants qui soient assez courageux pour assumer ce qu'ils pensent en eux-mêmes; parce que je suis persuadé qu'ils sont persuadés que les profits d'aujourd'hui sont les investissements de demain et les emplois d'après-demain, mais qu'ils n'osent pas le dire à leur population. L'issue n'est donc pas de mettre en commun la bombe atomique mais le théorème d'Helmut Schmidt.

Henrik Uterwedde : Des trois Charles à Wolfgang Schäuble et Michel Sapin ou à Angela Merkel et François Hollande, mon propos sera d'analyser l'évolution possible de la position allemande vis-à-vis des grands thèmes de la stabilité budgétaire d'une part et de la croissance d'autre part. Pour commencer avec un constat, je pense que la plupart des analyses de Jean-Marc Daniel plairaient aux responsables allemands.

\section{Position allemande vis-à-vis de la stabilité budgétaire}

En 2010, c'est-à-dire au début de la crise de la zone euro, la position allemande initiale tournait autour du problème de la stabilité, avec une certaine rigidité d'abord. Au moment de la signature du traité de Maastricht, l'Allemagne avait été prête à abandonner le mark, qui était sa force de frappe (tandis que la France n'avait pas abandonné le nucléaire). Ce renoncement était conditionné pour l'Allemagne à un contrat moral accepté bon gré mal gré par les autres partenaires. L'approche adoptée comprenait des règles chiffrées, des sanctions, une $\mathrm{BCE}$ indépendante vouée à la stabilité et le principe de la responsabilité strictement nationale pour les dettes nationales (no bail-out). Sur le plan intellectuel, le drame de la crise pour les Allemands est qu'elle a montré les failles de l'architecture de Maastricht, dans lesquelles de nombreux pays se sont engouffrés. En 2010, la grande peur des Allemands était que l'on assiste au détricotage du traité de Maastricht, pour passer à une nouvelle lecture de l'UEM que les Allemands n'auraient jamais acceptée au départ. Toutefois, en l'espace de quatre ans, l'Allemagne et la France - souvent en première ligne - ont été les artisans des compromis nécessaires, comme par exemple l'aide à la Grèce, le Mécanisme européen de stabilité (MES), les fonds de solidarité et l'Union ban- 
caire. Dans ce contexte, l'Allemagne a pu faire prévaloir certains principes à travers l'octroi d'aides strictement conditionnées, le renforcement des règles du PSC et le TSCG. Par conséquent, l'Allemagne reste stricte sur son refus de mutualiser la dette via des eurobonds, mais elle admet implicitement une certaine souplesse dans l'application du PSC et dans le délai accordé à la consolidation budgétaire.

\section{La consolidation doit aller de pair avec la croissance}

Certes, au début de la crise de la zone euro, l'Allemagne a joué le rôle de gardien de la stabilité budgétaire, mais il ne faut pas croire que les Allemands ne voulaient pas de la croissance. Il y a une ouverture dans le discours sur la croissance après l'élection de François Hollande avec le Pacte européen pour la croissance et l'emploi de juin 2012. Dès lors, le débat se déplace vers les questions suivantes : quelle croissance et comment y arriver? Si avec l'entrée du parti social-démocrate (SPD) dans le gouvernement allemand à l'issue des élections au Bundestag de 2013, on pouvait s'attendre à une évolution plus accentuée de la position allemande dans le contrat de grande coalition, ce document s'inscrit en réalité dans la continuité de la politique jusqu'alors menée par Angela Merkel. ${ }^{5}$ Mais il y a ouverture car ce contrat comprend le rappel d'une stratégie équilibrée qui doit combiner d'une part des réformes structurelles pour une compétitivité améliorée et, d'autre part, une consolidation budgétaire stricte et soutenable avec des investissements d'avenir pour la croissance et l'emploi. Par conséquent, la consolidation doit aller de pair avec la croissance, laquelle s'inscrit dans une politique de l'offre. Sur le refus de mutualisation de la dette, le SPD a souscrit à la phrase suivante: "Toute forme de communautarisation des dettes publiques nationales porterait atteinte à la nécessaire orientation des politiques nationales dans chacun des États membres. »" Cela traduit la crainte que toute souplesse "excessive » minimise l'urgence, pour les responsables politiques nationaux, de faire les réformes nécessaires. La grande coalition précise dans la phrase suivante que «la responsabilité strictement nationale pour la politique budgétaire et une responsabilité supranationale commune pour les dettes sont incompatibles ». Sans acceptation du droit d'ingérence et d'une intégration plus forte des politiques fiscales, il ne peut y avoir de véritable mutualisation de la dette.

\section{Vers une relance des investissements au niveau national...}

Au niveau national, la grande coalition a mis en œuvre un programme social, avec des mesures liées aux retraites et avec l'introduction d'un Smic dans un contexte de demande de hausses de salaires plus marquées de la part des

\footnotetext{
${ }^{5}$ Cf. Henrik Uterwedde, « Grands défis, Grande coalition, grande politique ? », Regards sur l'économie allemande. Bulletin économique du CIRAC, $\mathrm{n}^{\circ}$ 111, hiver 2013, p. 21-32.
} 
syndicats. En outre, un programme d'investissement est lancé, composé de huit actions prioritaires. Il fait suite à un débat, qui s'est intensifié en 2013, sur le manque d'investissements publics (Investitionslücke) dans les infrastructures. Le président de l'Institut allemand pour la recherche économique de Berlin (DIW), Marcel Fratzscher, a estimé à 80 milliards d'euros annuels le manque d'investissements dans les infrastructures. Or ce que prévoit le gouvernement actuel dans son contrat de coalition est minime en comparaison. Dans ce contexte, des voix s'élèvent pour critiquer le fait que des sommes importantes aient été consacrées aux mesures sociales (en puisant dans les réserves de la sécurité sociale et dans les marges budgétaires), peut-être au détriment du financement des mesures d'investissement. Le débat transpartisan sur la nécessité de stimuler les investissements s'amplifie jusque dans les milieux économiques. Le ministre SPD de l'Économie, Sigmar Gabriel, vient de créer une commission d'experts pour renforcer les investissements publics. Marcel Fratzscher, qui est une étoile montante en tant qu'économiste, avec un positionnement très intéressant qui essaye d'échapper au clivage gauche-droite en Allemagne, prône quant à lui une politique davantage orientée vers la croissance, le défi pour les années à venir étant de générer une croissance durable et saine en Allemagne et dans la zone euro. Comme l'a dit Christian Kastrop, je pense aussi que ce genre de débat, quand il prend de l'ampleur, peut finir par infléchir la politique. L'idée prônée par Marcel Fratzscher est que pour combler ce manque d'investissements, il faut redéployer les dépenses publiques vers davantage d'investissements en créant des conditions économiques, fiscales et juridiques qui favorisent la mobilisation de capitaux privés. Un nouveau débat s'instaure ainsi dans le contexte de la publication du livre de Marcel Fratzscher, Die Deutschland-Illusion ${ }^{6}$, qui a eu un écho dans la presse française. Dans cet ouvrage, l'auteur met en garde sur la situation économique de l'Allemagne, qui n'est pas préparée aux défis de l'avenir. En effet, depuis Gerhard Schröder, l'audace réformatrice en Allemagne est toute relative.

\section{... et au niveau européen}

Au niveau européen, on observe par ailleurs une nouvelle sensibilité à la question de la croissance et de l'emploi. Angela Merkel a souscrit au Pacte européen pour la croissance et l'emploi et elle est ouverte au programme d'investissements de Jean-Claude Juncker. En septembre 2014, Wolfgang Schäuble et Michel Sapin ont transmis une lettre au président du Conseil comprenant des propositions communes pour lancer le programme d'investissements, avec l'idée notamment de donner un nouveau mandat à la Banque européenne d'investissement (BEI) pour qu'elle puisse gérer des fonds abondés par les États membres. Cela dit, l'approche de la croissance est une approche par l'offre : pas

\footnotetext{
${ }^{6}$ Marcel Fratzscher, Die Deutschland-Illusion - Warum wir unsere Wirtschaft überschätzen und Europa brauchen, Hanser Verlag, Munich, 2014. Ce livre paraîtra également en langue française.
} 
de programme de relance keynésien avec du deficit spending. L'idée que l'Allemagne devrait jouer le rôle de locomotive économique en Europe est réfutée. On revient sur le triptyque finances publiques consolidées, réformes structurelles et politique de compétitivité des entreprises, qui passe par une révision de la fiscalité des entreprises et du mode de financement de la sécurité sociale ainsi que par la voie de l'innovation. Le quatrième point consiste à mobiliser des capitaux privés en Europe pour les investissements. On observe certes une ouverture outre-Rhin sur la nécessité de mettre l'accent sur l'investissement, à la fois au niveau national et européen, mais en restant fidèle à la doctrine ordolibérale allemande.

\section{Une convergence franco-allemande réelle...}

Objectivement, nous avons la chance d'avoir une certaine convergence de fait entre l'approche politique allemande et française. La France admet la nécessité d'une politique de l'offre et se lance dans une politique de consolidation et de réformes, tandis que l'Allemagne admet davantage que la demande intérieure et le cycle économique peuvent jouer un rôle et montre un nouvel intérêt envers la croissance passant par l'investissement. Lorsqu'au cours de la table ronde « Les contraintes budgétaires liées au Pacte de stabilité et de croissance (PSC) et au pacte budgétaire européen (TSCG), dont le MES, sont-elles praticables et adaptées pour sortir l'économie européenne de la crise? 》 du 18 novembre $2013^{7}$, Christian Kastrop, qui travaillait encore au ministère fédéral des Finances et Sandrine Gaudin, du ministère français de l'Économie et des Finances, ont montré la façon dont les deux ministères géraient les points y compris litigieux, on voyait bien que le travail de convergence était à l'œuvre. Je vous renvoie également à une interview croisée de Michel Sapin et Wolfgang Schäuble ${ }^{8}$, parue dans Les Échos et dans le quotidien économique allemand Handelsblatt, qui montre une large convergence de vues entre les ministres des Finances français et allemand.

\section{... mais fragile}

Toutefois, cette convergence est fragile car elle résulte davantage des dynamiques économiques, sociales et politiques nationales que d'un véritable débat ou d'une négociation européenne. Le tournant de la politique de l'offre, des réformes et de la consolidation en France n'est pas dû à une demande de Bruxelles ou d'Angela Merkel, mais plutôt à l'état de l'économie française qui exige ces changements. De même, l'orientation vers l'investissement et la crois-

\footnotetext{
${ }^{7}$ Voir dans cet ouvrage, p. 187-203.

8 «Paris-Berlin : deux visions alternatives de l'Europe économique et financière »[en ligne], Les Échos, 20/07/2014. Disponible sur : http://www.lesechos.fr/20/07/2014/lesechos.fr/0203653948987_paris-berlin---deuxvisions-alternatives-de-l-europe-economique-et-financiere.htm\#GowO8YWa8XgAA0KT.99] [consulté le 25/11/2014].
} 
sance en Allemagne ne résulte pas de l'appel de Christine Lagarde, il y a quelques années, à augmenter les salaires et la demande intérieure. L'infléchissement de la politique allemande s'explique par des raisons économiques, sociales et politiques nationales. Les syndicats qui, après une phase de modération salariale, souhaitaient un réajustement des salaires, ont été soutenus dans leur demande par le parti chrétien-démocrate (CDU) et, plus récemment, par la banque centrale allemande. De même, le manque d'investissement est un problème spécifiquement allemand.

On assiste ainsi à un rapprochement réel des positions des gouvernements, dans une souplesse qui n'est souvent pas comprise par les opinions publiques et par une bonne partie de la classe politique française et allemande. Le parti Alternative für Deutschland (AfD) et de nombreux eurosceptiques allemands appellent le gouvernement allemand à plus de rigueur et de fermeté vis-à-vis de la France. Les commentaires désobligeants dans la presse allemande et chez les seconds couteaux de la CDU contre Pierre Moscovici témoignent de la pression exercée en ce sens. Le même genre de pression publique existe en France. Les forces de convergence sont ainsi soumises à des pressions publiques qui vont vers la divergence et qui rendent fragile cette convergence politique.

\section{La nécessité de mener un débat citoyen sur l'Europe}

Néanmoins, les débats s'ouvrent et se différencient en France et en Allemagne, ce qui me semble une bonne chose. L'approche allemande et l'approche française perdurent peut-être dans les discours dominants mais en France, de nombreux économistes vont désormais dans un sens plutôt allemand, favorisant des politiques de l'offre, avec à titre d'exemple le livre de Philippe Aghion, Gilbert Cette et Élie Cohen sur la sortie de modèle ${ }^{9}$. En Allemagne, on observe la même tentative d'ouverture ${ }^{10}$, dont le livre précité de Marcel Fratzscher est un bon exemple, tout comme la constitution du Groupe de Glienicke réunissant une dizaine d'économistes, politologues et juristes. Il existe un nouveau réseau d'intellectuels, soutenu par des responsables politiques, qui pousse vers de nouveaux compromis en Europe. Marcel Fratzscher demande qu'on en finisse avec les compromis bilatéraux a minima (comme le compromis budgétaire passé à Deauville entre Nicolas Sarkozy et Angela Merkel en 2010) et que l'on ait une vision européenne. Il faut sortir de la défensive qui consiste à ne pas assumer la politique européenne par crainte des opinions publiques et passer à l'offensive en menant une campagne d'explication et un débat public - controversés peutêtre - sur la nécessité de l'Europe et sur les illusions de tous ceux qui pensent qu'ils seraient mieux tout seuls. Sur ce point, les choses évoluent dans les deux pays. Je m'en réjouis car le rejet de l'Europe par de nombreux citoyens, tant en

\footnotetext{
${ }^{9}$ Philippe Aghion, Gilbert Cette, Élie Cohen, Changer de modèle, Odile Jacob, Paris, 2014.

${ }^{10}$ Cf. Henrik Uterwedde, «Zone euro : nouveaux débats allemands », Allemagne d'aujourd'hui, ${ }^{\circ} 210$, octobre-décembre 2014, p. 5-11.
} 
France qu'en Allemagne, tient notamment au fait que les choix européens n'ont pas été exposés et assumés et qu'ils n'ont pas donné lieu à un débat pour emporter l'adhésion des citoyens. Le débat est devant nous pour qu'à l'avenir, nous parvenions à une politique plus approfondie en Europe.

Natacha Valla : Par rapport aux propos qui ont été tenus, il me semble que nous sommes sur un ton de débat qui associe des visions allemandes et françaises qui ne sont pas les plus radicales et qui ne sont pas forcément même très représentatives de la vision majoritaire dans les deux pays. Mais ce n'est peutêtre pas plus mal car il faut essayer de converger en allant chacun l'un vers l'autre. Je suis ravie d'entendre que le livre de Marcel Fratzscher a été commenté avec beaucoup de détails. Nous avons fait notre doctorat ensemble dans un institut qui s'occupait des questions européennes. Ce n'est donc peut-être pas un hasard que nous soyons tous impliqués dans le projet européen et que nous souhaitions continuer à le faire vivre parce que nous avons grandi dans cette culture européenne.

Je vais structurer mes quelques remarques en fonction de ce qui a été dit, en commençant par ce que la France n'a pas fait ou n'a pas assumé en termes de position, de décisions politiques et de responsabilités. Ensuite, j'essaierai de montrer comment on peut comprendre - ou en tout cas expliquer - la position quelque peu ambiguë de la France vis-à-vis des réformes structurelles, de la rigueur budgétaire et de l'association qu'on doit pouvoir en faire aujourd'hui dans le contexte européen. Je dirai quelques mots sur le rôle de la BCE vu de France puis j'évoquerai l'investissement, qui me semble une opportunité institutionnelle absolument historique aujourd'hui parce que nous avons besoin d'un symbole, d'un pas supplémentaire pour dépasser la dynamique de crise et consolider ce qui a été construit pendant la crise (la création du MES et la modification de la perception des rôles institutionnels de la Commission et de la $\mathrm{BCE}$ ). Nous devons maintenant pouvoir laisser cela derrière nous et créer de nouvelles institutions, ou encore créer des modes opératoires qui permettent de partir sur un nouveau modèle après-crise. Sur ce point, il est vraiment important que les visions franco-allemandes convergent de façon structurante.

\section{Ce que la France n'a pas fait}

Dans la dynamique européenne d'aujourd'hui, il est dommageable que la France n'ait pas avancé, malgré la crise financière de 2008, puis la crise des dettes souveraines à partir de 2010, sur la nécessité d'une Europe politique derrière la monnaie. C'est devenu un poncif. On s'est rendu compte avec la crise qu'il manquait une Europe budgétaire et une Europe politique. Jean-Marc Daniel et Henrik Uterwedde ont rappelé que l'Allemagne a renoncé au mark et à la Bundesbank, alors que la France n'a pas renoncé à son armée, à sa flotte et à son arme nucléaire. Je pense qu'il s'agit là d'éléments essentiels à l'Europe, 
qui vont bien au-delà de la dynamique économique. Le débat public à avoir, qui est encore complètement tabou et inhibé aujourd'hui en France, est donc celui de la question politique derrière la monnaie unique.

La deuxième chose que la France n'a pas très bien fait est d'assumer une position dans la gouvernance collective, c'est-à-dire de prendre en compte dans la définition de ses propres politiques économiques des externalités que ces politiques économiques nationales pouvaient avoir sur la dynamique européenne. Ces externalités aujourd'hui au niveau budgétaire sont complètement imbriquées dans la surveillance macroéconomique, le TSCG... Mais on ne prend pas du tout en compte les implications des faibles réformes structurelles sur la pérennité du projet européen. La France en tant que grand pays en termes de PIB de la zone euro ne met-elle pas en péril, par une externalité massive, le scénario souhaitable d'une continuation du projet de monnaie unique vers quelque chose de vraiment pérenne? Si nous sommes tous apaisés par rapport à ce que nous avons pu vivre il y a deux ans, certains aspects fondamentaux n'ont pas encore été pris en compte. Selon moi, les externalités des atermoiements de politique économique nationale devraient presque faire l'objet de recommandations de la part du Fonds monétaire international (FMI) quand il regarde la France en article IV, de la part de l'OCDE quand elle fait une étude pays et de la part de la Commission européenne. La Commission européenne le fait structurellement car elle doit regarder de nombreuses variables.

Le troisième point sur la position française est la question du rapport au respect des règles. La France et l'Allemagne ont une approche absolument différente de la règle et de l'interprétation du texte. C'est quelque chose de tellement profond culturellement qu'on a besoin de beaucoup de bonne volonté pour générer une dynamique symbiotique et non divergente au niveau macroéconomique et institutionnel. Cela a été déjà beaucoup dit, mais il est utile de le répéter aujourd'hui parce qu'un certain nombre de solutions qui ont été développées pendant la crise sont basées sur d'autres règles chiffrées. Or nous n'avons pas encore réussi à converger sur une façon pragmatique de gérer les chiffres que nous avons mis dans les contrats que nous avons tous signés. Par conséquent, la France doit réaliser qu'elle a signé ces contrats et que, même si elle considère que l'Allemagne est un peu trop dogmatique sur son interprétation des textes, il faut accepter cela comme une différence culturelle et faire en sorte qu'une règle soit quand même malgré tout une règle respectée.

\section{La position ambiguë de la France vis-à-vis des réformes structurelles et de la rigueur budgétaire}

J'aimerais revenir sur le lien entre la consolidation budgétaire et les réformes structurelles. Il me semble que, sur ce sujet, la France cultive assez maladroitement l'art de l'ambiguïté. En septembre 2014, Benoît Cœuré, membre du directoire de la BCE, et Jörg Asmussen, ancien membre du directoire de la BCE et 
actuellement secrétaire d'État au Travail au sein du gouvernement allemand, ont décidé de publier une tribune très courageuse ${ }^{11}$. Le propos était de dire que les pays qui avaient de la marge de manœuvre pour faire moins de consolidation budgétaire et aider du côté budgétaire devaient le faire. Dans la foulée, la France annonçait qu'elle n'allait pas atteindre les fameux $3 \%$ à l'horizon escompté, que le déficit global et essentiellement le déficit structurel dépasseraient très largement les efforts qui avaient été promis et sur lesquels la France était revenue graduellement année après année. Je pense qu'il est très maladroit d'essayer d'interpréter des propos qui n'ont certainement pas été prononcés à l'intention de la France, et ce alors que la BCE a vraiment très largement essayé d'aller au plus loin dans les limites du compromis possible avec les différentes lectures de ses prérogatives.

La France est dans une situation un peu compliquée puisqu'elle a pris beaucoup de retard sur les réformes structurelles. L'Allemagne par le passé a bien compris que si elle voulait faire des réformes structurelles, il fallait qu'elle soit beaucoup plus souple sur les réformes budgétaires. Toutefois, je pense qu'il n'est pas très responsable d'aller chercher l'exemple de la remise en question du Pacte de stabilité et de croissance en 2003 pour justifier le fait qu'on ait besoin de réformes structurelles aujourd'hui en France, lesquelles sont très intimement liées - et peut-être beaucoup plus que dans d'autres cas passés pour des pays membres de la zone euro - à la dynamique des finances publiques, et notamment à la dépense publique. Quand on doit mener des réformes structurelles, sur les marchés du travail certes, mais aussi sur le volume et la structure des dépenses publiques, cela contraint de façon très douloureuse la consolidation budgétaire. Ne faut-il pas à un certain point accepter cette contrainte, y compris par respect pour les autres pays de la zone euro qui ont fait de la consolidation budgétaire de façon très marquée, sans doute deux fois plus intense que ce que la France a pu faire jusqu'à présent? Il faudrait aussi avoir une certaine responsabilité par rapport à cela et accepter peut-être, si effectivement il y a beaucoup de difficultés sur la consolidation budgétaire à très court terme, une conditionnalité de l'assouplissement de cette consolidation à des réformes structurelles qui passe par la mise en place de processus juridiques parlementaires. La Constitution en France permet de rendre le processus parlementaire plus rapide - ou en tout cas plus affirmé - que les procédures standards. Sur ce point, j'ai été un peu dure et dans ce sens peut-être pas des plus représentatives de la majorité, mais je pense que c'est important de comprendre qu'en France, on pense aussi comme cela et que ce n'est pas forcément trahir son pays que de dire qu'il faut qu'on arrive à converger vers quelque chose de plus coopératif en matière de gestion de la chose publique.

\footnotetext{
${ }^{11}$ Benoît Cœuré, Jörg Asmussen, « Ce que la zone euro doit faire pour retrouver la croissance » [en ligne], Les Echos, 19/09/2014. Disponible sur : http://www.lesechos.fr/idees-debats/editos-analyses/0203787167425-ceque-la-zone-euro-doit-faire-pour-retrouver-la-croissance-1044363.php [consulté le 20/11/2014].
} 


\section{Le rôle de la BCE vu de France}

Les pouvoirs publics en France se sont longtemps défiés de la banque centrale. Il faut se rappeler qu'au moment de la création de la Caisse des dépôts et consignations (CDC) en 1816, il était utile d'avoir une institution capable de canaliser l'épargne de la population après les dépenses de guerre réalisées. Louis XVIII se méfiait de la Banque de France comme de la peste et avait envie d'avoir une autre institution capable de générer des flux financiers, en dehors de la mainmise de la banque centrale sur la création monétaire. La relation de la France à sa banque publique d'investissement est par conséquent beaucoup moins saine que celle de l'Allemagne avec la sienne. La France a bien accepté de voir partir les prérogatives monétaires à Francfort mais elle reste aujourd'hui encore très floue et ambiguë par rapport à ce qu'elle attend de la Caisse.

Depuis le texte initial du traité de Rome, on a assisté à une évolution absolument colossale de l'acceptation par l'Allemagne de l'interprétation de l'article 123 du traité sur le fonctionnement de l'Union européenne (TFUE), qui prohibe le financement monétaire par les banques centrales. À titre d'anecdote, j'ai travaillé au début des années 2000 à la BCE. Une partie de ma mission consistait à vérifier les comptes des banques centrales nationales. J'avais sur mon bureau l'article du traité - qui était à l'époque l'article 101 - et la législation secondaire, c'est-à-dire une série de directives européennes qui déclinaient l'article 101 et qui définissaient des seuils d'achat par les banques centrales nationales sur les marchés secondaires de titres de dette souveraine. Quand une banque centrale, comme la banque centrale de Grèce - car c'est elle qui était toujours en infraction par rapport au traité -, achetait plus de $0,01 \%$ en valeur de PIB sur les marchés secondaires de dette publique émise par son souverain ce qui est dérisoire par rapport aux montants dont il est question aujourd'hui -, une lettre était envoyée au gouverneur de la banque de Grèce qui devait s'en expliquer au conseil des gouverneurs. Si les choses n'étaient pas corrigées et que le pays n'était pas en règle avec le droit européen, cela pouvait aller jusqu'en cour de justice. Il y avait une approche extrêmement rule-based de l'essence et de l'esprit du traité. L'Allemagne a fait un effort considérable car nous sommes actuellement vraiment éloignés de cette lecture initiale du texte, à laquelle nous n'allons certainement jamais revenir.

Par conséquent, en fonction de cet effort, il faut être très prudent par rapport à ce qu'on peut demander à la BCE aujourd'hui. La France l'est d'une certaine manière par les non-dits, mais ce serait très malvenu que le pays appelle la $\mathrm{BCE}$ à faire du quantitative easing, au sens de l'achat de titres publics sur les marchés souverains. Ce point du débat me semble en suspens en France. Il est très actif en Allemagne, avec le débat autour de Karlsruhe et de la légitimité des opérations monétaires sur titres (outright monetary transactions, OMT). Ce débat est très proche du cœur des Allemands. Les Français en revanche sont très éloignés de la politique monétaire car ils n'ont jamais eu à vivre cela de façon 
aussi prégnante que les Allemands au cours de leur histoire. Il faudrait peut-être que la France prenne ce sujet un peu plus au sérieux et l'intègre plus profondément dans le débat public.

D'autant qu'une nouvelle question fait débat : celle du rôle, dans l'environnement des institutions économiques européennes, de preneur de risque ultime. Je pense que le débat sur le prêteur en dernier ressort est presque obsolète et que, dans un contexte macroéconomique où l'on discute volontiers de stagnation séculaire et de risque sur la croissance à long terme, la vraie question est de définir qui sera le preneur de risque en dernier ressort. Il y a matière à débattre pour réfléchir au rôle de la BCE sur ce sujet. Pour ma part, j'aurais une position assez conservatrice sur les achats souverains, et libérée sur la prise de risque de la banque centrale. Mais je me méfie aussi beaucoup de la proposition concernant les garanties publiques destinées à permettre à la $\mathrm{BCE}$ de jouer un jeu plus proactif. L'idée ici est de demander aux États membres de la zone euro de devenir des garants de titres, dettes ou investissements afin de permettre à cette dette ou à cet investissement d'être éligible comme collatéral aux opérations de refinancement de la banque centrale. Je pense que la garantie publique est un objet assez pernicieux, notamment dans le contexte actuel de dettes excessives où on a à gérer un recul de l'endettement général. Ce ne sera pas facile si en plus on fait peser sur les États des garanties publiques dont les valeurs atteignent plusieurs points de PIB. On ne sait pas comment cela va finir. Pour relier cela à la dynamique franco-allemande et aux positions relatives, je pense qu'il est assez délicat aujourd'hui d'accumuler du "off-balance sheet » pour permettre de faire semblant de bien se comporter sur le «on-balance sheet ». Cela dit, il existe des façons plus inventives d'impliquer la $\mathrm{BCE}$ sur la prise de risque et je serais plus allante sur ces autres moyens.

\section{L'investissement}

En ce qui concerne l'investissement, il existe de nombreuses pierres d'achoppement et de nombreux malentendus dans le dialogue franco-allemand sur la désirabilité de flux de transfert, c'est-à-dire de flux financiers liés à des revenus budgétaires transfrontaliers. La France a été assez maladroite en poussant longtemps et à plusieurs reprises l'idée d'un budget de la zone euro qui servirait à financer des politiques contracycliques, en particulier une assurance chômage. L'idée en elle-même n'est peut-être pas mauvaise, mais dans une dynamique où on ne veut pas avoir en Allemagne la possibilité du moindre doute sur le risque de hasard moral par rapport à ces transferts, ce n'est pas le moment d'insister sur cette proposition. En revanche, si on arrivait à s'accorder sur des questions qui préservent les flux financiers des cycles politiques nationaux et qui permettent en même temps d'avoir un projet commun et de soutenir concrètement la croissance potentielle européenne, si possible sur un modèle basé sur les technologies de l'innovation, alors il faudrait s'engouffrer dans cette fenêtre de tir. Il me semble que la question de l'investissement public européen en est une. 
Le graphique 5 montre une comparaison entre la zone euro et les États-Unis en matière d'investissement privé, en points de pourcentage du PIB. Aux ÉtatsUnis, on observe que l'investissement privé fluctue beaucoup plus en pourcentage du PIB. C'est sans doute parce que dans l'économie américaine, les cycles ont été jusqu'à maintenant beaucoup plus amples que dans la zone euro. Ce qui est encore plus intéressant sur ce graphique, c'est que depuis la crise de 2008, l'investissement privé a baissé d'à peu près deux points de PIB dans la zone euro. Il ne semble pas que le redressement soit pour demain. Aux États-Unis en revanche, le pays est déjà reparti sur une dynamique beaucoup plus vive.

\section{Graphique 5 : Zone euro - investissement privé en-deçà de $20 \%$ du PIB}

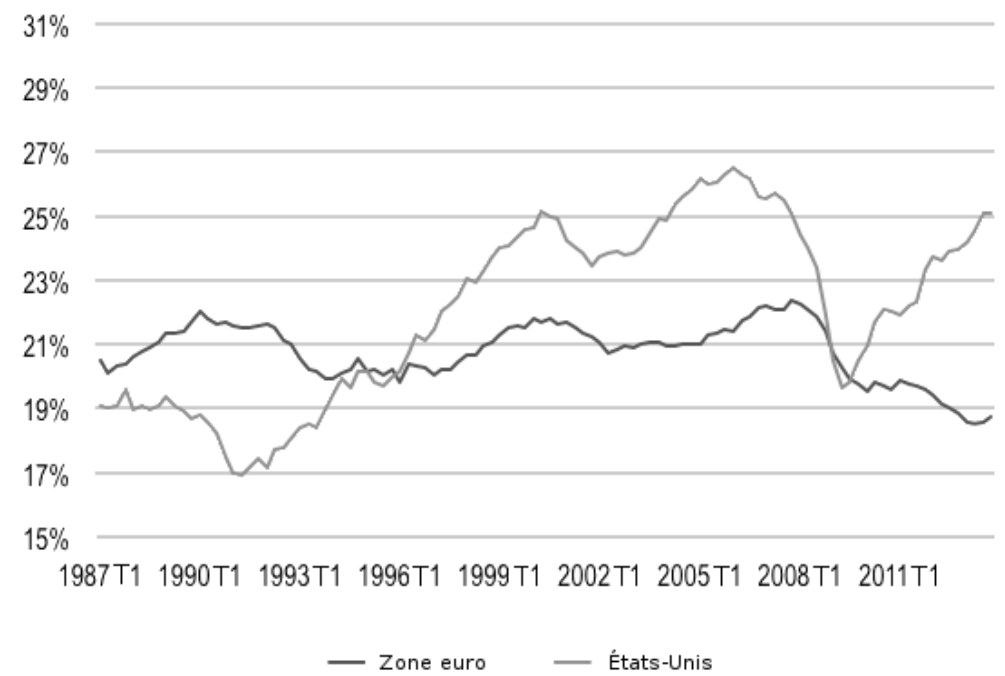

Source : Eurostat ; Giannone et al., 2012 $2^{12}$. Dernières données : $4^{\mathrm{e}}$ trimestre 2013.

Le graphique 6 compare les États-Unis et la zone euro en matière d'investissement public exprimé en points de PIB, avec une profondeur historique plus longue. Depuis les années 1980, on se rend compte de deux choses essentiellement. La première est qu'il y a un écart persistant d'un point et demi à deux points de PIB entre les investissements publics réalisés aux États-Unis et dans la zone euro, et cela en toutes circonstances macroéconomiques. La deuxième est que l'investissement public a été réduit par paliers. Ces paliers ont exactement coïncidé avec les phases de consolidation budgétaire associées à la stabilisation ou à la réduction des dettes. Ainsi, jusqu'à maintenant, les consolidations ont été faites au détriment de l'investissement public. Nous avons regardé au CEPII si nous pouvions trouver une complémentarité entre l'investissement public et

\footnotetext{
${ }^{12}$ Domenico Giannone, Jérôme Henry, Magdalena Lalik, Michele Modugno, « An Area-Wide Real-Time Database for the Euro Area », Review of Economics and Statistics, vol. 94, n 4, 2012, p. 1000-1013.
} 
l'investissement privé, pour voir si le fait que l'État investisse permettait au secteur privé d'investir plus ou de façon plus efficace. Il se trouve que non seulement c'est le cas (les deux sont complémentaires), mais aussi que plus le stock initial de capital public est important, plus l'investissement privé est efficace. Il y a donc un argument en matière de politique publique au niveau européen pour renforcer l'investissement public afin de stimuler l'investissement privé et, partant, de redresser la croissance potentielle.

\section{Graphique 6 : Zone euro - investissement public divisé par deux en 35 ans}

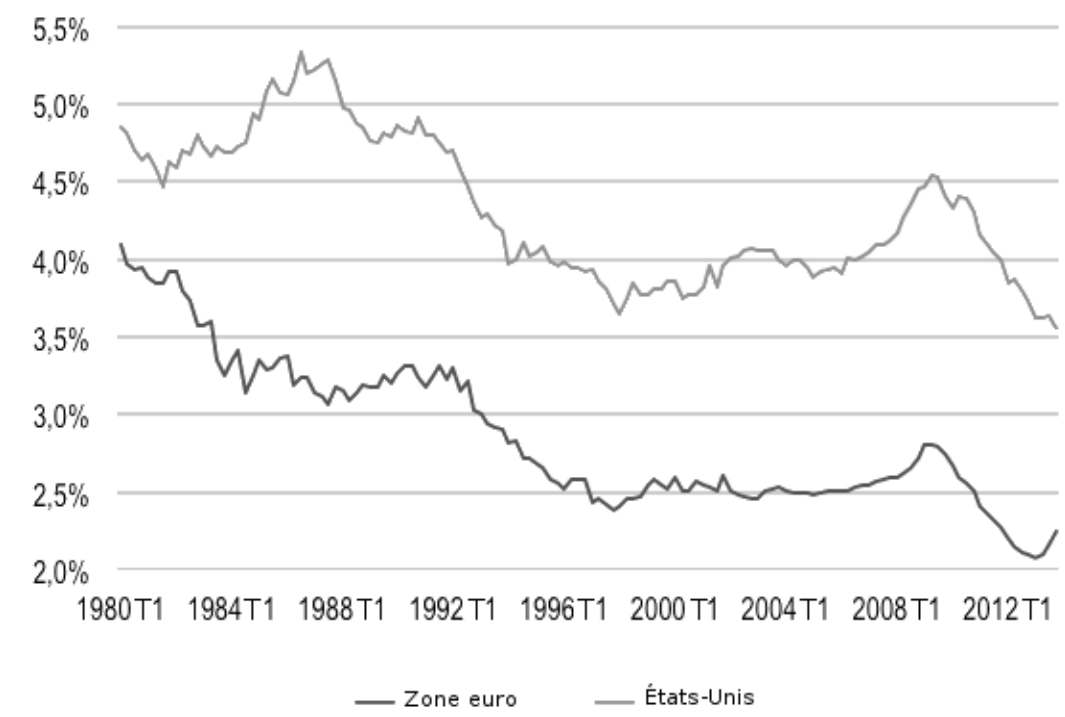

Sources : Fred ; Eurostat; Paredes et al., 2009 ${ }^{13}$; Giannone et al., 2012. Dernières données : $4^{\mathrm{e}}$ trimestre 2013.

En matière de mise en commun des ressources publiques d'investissement, nous avons beaucoup discuté des propositions institutionnelles avec Marcel Fratzscher, mais aussi avec les Polonais qui ont eux aussi une proposition assez explicite. C'est un terrain un peu miné car on parle de grosses sommes d'argent. Le bilan des banques d'investissement nationales, comme la Kreditanstalt für Wiederaufbau (KfW) en Allemagne, la CDC en France ou la Cassa Depositi e Prestiti en Italie, est d'environ $20 \%$ du PIB. Ces banques canalisent des quantités massives d'épargne nationale. Étant donné qu'il est politiquement très délicat de commencer à mettre en commun ou à coordonner ces masses, il est nécessaire d'avoir une impulsion politique au plus haut niveau européen pour que les projets puissent aboutir.

\footnotetext{
${ }^{13}$ Joan Paredes, Diego J. Pedregal, Javier J. Pérez, « A Quarterly Fiscal Database for the Euro Area Based on Intra-Annual Fiscal Information », ECB Working Paper, n 1132 , décembre 2009, p. [1]-47.
} 
Parmi les propositions, la mienne était de réformer la BEI et de mettre en réseau les banques d'investissement nationales sur le même modèle que ce qu'on a fait au début de l'Union monétaire, avec la mise en réseau des banques centrales nationales, qui a abouti au Système européen des banques centrales (SEBC). Au regard des 15 dernières années, cela peut sembler un miracle de constater ce qu'on a pu atteindre en matière de compromis au niveau national, notamment au niveau de l'Allemagne. Nous avons réussi à faire rendre complètement les armes à tous les pays fondateurs de ce système sur le choix du taux d'intérêt et le choix de la conduite de la politique monétaire. C'était une véritable déflagration mais elle a été possible. Aujourd'hui, si nous parvenions à sortir des dynamiques budgétaires nationales ne serait-ce qu'une infime partie des décisions d'investissement public, nous aurions réglé une grande partie du problème car nous aurions une capacité d'investissement affranchie de tout ce qui constitue des pierres d'achoppement au niveau de la progression fédérale de l'Europe.

La proposition de réformer la $\mathrm{BEI}$, de mettre en réseau les acteurs nationaux et de régler soigneusement la gouvernance de l'investissement appelle à une réforme en profondeur des statuts de la BEI pour plusieurs raisons. Il faudrait que dans le capital de la nouvelle BEI, il y ait les pays membres mais aussi des acteurs neutres du secteur privé qui ne soient pas forcément des hedge funds américains, mais par exemple des grandes compagnies d'assurance européennes, des grandes banques européennes et des institutions qui ont l'expérience de la gouvernance en matière de prise de décision d'investissement sur les destinations de ces investissements. Sur ce point, la réflexion peut être complètement novatrice et libre. Nous ne sommes plus obligés d'aller financer des infrastructures, des ronds-points et de doubler des autoroutes sur certains tronçons. Vous avez sans doute tous les mêmes exemples que moi en termes d'investissements publics absurdes réalisés. Cela permettrait de mener une vraie réflexion sur la notion même d'infrastructures du XXI siècle, qui sont des infrastructures tout autant technologiques que matérielles. On retrouve également derrière cela la politique énergétique. Une gouvernance qui réduirait l'influence des gouvernements sur cette politique offrirait aussi l'avantage d'avoir une vision à beaucoup plus long terme, beaucoup moins confinée derrière des frontières géographiques artificielles, de sortir de la discussion myope cyclique liée au cadre budgétaire européen et des incertitudes d'après-crise et de définir un cap à long terme. Ce message sur l'investissement que je voulais faire passer est pour moi le plus porteur d'espoir. Cela permettrait aussi à la $\mathrm{BCE}$ de rester dans son rôle, c'est-àdire de ne pas avoir besoin de réaliser des missions pour lesquelles elle n'a pas été conçue, notamment le financement des États.

Traduction des propos de Christian KASTROP : Marie-Céline GEORG 



\section{Table ronde : Quel rôle doit jouer la BCE aux yeux des responsables politiques français et allemands et quelle est sa politique actuelle ? ${ }^{1}$}

\section{OUVERTURE DE LA TABLE RONDE}

Christian de Boissieu : Nous allons parler de politique monétaire, de la Banque centrale européenne (BCE) et de sujets qui y sont liés. Il se trouve que compte tenu des thèmes abordés, nous allons donner la parole dans un premier temps aux deux intervenants français, puis aux deux intervenants allemands.

\section{EXPOSÉS DES INTERVENANTS}

Christian de Boissieu : Nous allons commencer avec Jaques Mistral qui a été professeur d'université, qui est membre du Cercle des économistes et qui, après avoir eu des fonctions éminentes à Washington, reste attaché à la Brookings Institution. Jacques Mistral a publié en 2014 un livre qui a fait pas mal de bruit sur le système ou le non-système monétaire international ${ }^{2}$. Jacques Mistral, merci d'être avec nous.

Jacques Mistral : C'est toujours un plaisir de participer aux rencontres conjointes du CIRAC et du DFI et je remercie une nouvelle fois René Lasserre et Henrik Uterwedde pour leur invitation. On a remarqué lors de la première table ronde que les deux intervenants français n'étaient pas très représentatifs des débats français et je crains de ne pas l'être non plus. Je vais tenter d'aborder rapidement sept points comme introduction à la table ronde.

\section{La politique de la BCE}

Depuis le début de la crise des dettes souveraine, la BCE a eu un comportement exemplaire. Sans la BCE, je ne sais pas où en seraient l'Europe et le système monétaire international. Je tire ce jugement du fait que la $\mathrm{BCE}$ a agi très exactement dans le cadre du mandat qui était le sien, en faisant preuve à la fois d'une très grande rigueur dans la définition de ses politiques et d'une très grande audace dans leur mise en œuvre dans une conjoncture qui était absolument terrible. Je me souviens encore de Jean-Claude Trichet expliquant comment la communauté des banquiers centraux étaient passée au bord de l'abîme et comment il souhaitait ne plus jamais avoir à traiter des sujets semblables.

\footnotetext{
${ }^{1}$ Cette table ronde s'est tenue dans le cadre du colloque Gouvernance économique, financière et monétaire de l'Union européenne, coorganisé par le CIRAC les 6 et 7 octobre 2014, NdE.

2 Jacques Mistral, Guerre et Paix entre les monnaies, Fayard, Paris, 2014.
} 
Évidemment, beaucoup ne trouvent pas satisfaction dans cette attitude exemplaire. Cette politique de la $\mathrm{BCE}$ a suscité des réactions, souvent désenchantées en France, mais qui sont quasiment automatiques, c'est-à-dire l'idée que la banque centrale n'en fait pas assez, qu'elle dispose d'outils qu'elle devrait utiliser plus largement pour aider à la croissance, l'emploi, le pouvoir d'achat... Cette idée est très profondément répandue en France. Or la BCE est souvent critiquée à tort à mes yeux, en France comme en Allemagne. Par plus tard que cette semaine, Arnaud Montebourg a critiqué la BCE selon la ligne que je viens d'évoquer, tandis qu'en Allemagne, $\mathrm{j}$ 'ai noté que quatre éminentes personnalités avaient exprimé des réserves à l'encontre du programme d'achat d'actifs sur lequel le président de la BCE Mario Draghi a donné des détails la semaine dernière.

Quand je dis que la BCE a agi avec beaucoup de prudence et d'audace, j'ai en tête nombre de situations, de choix et de décisions, mais je voudrais juste mentionner un exemple. Au moment de la crise sur les marchés financiers, les marchés demandaient instamment à la BCE d'utiliser un «gros bazooka » et la BCE s'y est refusée jusqu'au fameux mois de juillet 2012, au moment où Mario Draghi a pu prononcer sa phrase célèbre : "Nous ferons tout ce qu'il faut et croyez-moi, ce sera suffisant. » Ce qui est intéressant, c'est que contrairement à ce qu'ont compris les marchés qui voyaient enfin leur demande satisfaite, ce ne sont pas les marchés qui ont décidé du comportement de la BCE, mais les chefs d'État et de gouvernement qui, au conseil de juin précédent, avaient lancé les quatre unions (économique, budgétaire, politique et bancaire) et confirmé de ce fait la volonté unanime des responsables politiques de renforcer l'Union monétaire plutôt que de la laisser aller à vau-l'eau. À partir du moment où la décision politique des chefs d'État et de gouvernement avait été prise, il devenait de la responsabilité de la $\mathrm{BCE}$ de donner aux responsables politiques les moyens d'atteindre leurs objectifs. C'est un exemple formidable à la fois de rigueur, d'attente de la décision politique et, une fois que celle-ci est donnée, de réponse à la hauteur des enjeux.

\section{La France et la politique monétaire}

Depuis toujours, il subsiste en France une séduction de la dévaluation, c'est-àdire l'idée qu'on s'en sortirait mieux seul, sans les contraintes de l'environnement international. C'est une attitude extrêmement curieuse parce que - à la lumière de mes thèses sur les dévaluations du franc puis de mes responsabilités dans des cabinets ministériels - s'il y a une chose qui est certaine, c'est que la dévaluation n'est pas le meilleur moyen d'améliorer le pouvoir d'achat des salariés. La dévaluation est le constat d'un échec de la politique économique qui a été menée et le constat de grands désordres financiers. Elle se traduit toujours par un vrai plan de rigueur et une perte de pouvoir d'achat pour les ménages. Il $\mathrm{y}$ a dans ce pays, et j'en suis triste, une espèce de résistance aux enseignements de l'expérience qui est très troublante. 
Il faut néanmoins ajouter à cela le fait que les Français restent très attachés à l'euro ; et d'ailleurs ils ne sont pas les seuls. Les sondages démontrent que tous les peuples y sont très attachés, de la Grèce à l'Allemagne, en passant par l'Espagne ou la France. Cela a des conséquences politiques puisque Marine Le Pen, par exemple, avait un temps fondé ses propositions économiques sur la sortie de l'euro et avait adopté une attitude assez combative sur ce sujet. Elle s'est rendu compte que ce n'était pas très populaire pour son électorat, et ce pour une raison très simple : l'électorat est vieillissant et attend donc le paiement des retraites. Les retraités ou futurs retraités préfèrent de toute évidence avoir des retraites en euros plutôt qu'en francs dévalués.

Par ailleurs, les Français ne sont pas aidés dans leur éducation économique et financière par la bienveillance des marchés. Les marchés financiers financent aujourd'hui notre dette à des taux d'intérêt ridiculement bas au regard de critères historiques. Par conséquent, l'incitation à agir pour corriger cette trajectoire est extrêmement faible. Par le passé, les dévaluations se sont évidemment produites à contrecœur, mais au moment où le matelas des devises et les réserves de change s'épuisaient. Or aujourd'hui, on ne parle même pas des réserves de change; la dette se renouvelle de manière constante à des taux sans précédent historique. Donc il faut bien dire qu'il y a non seulement une part de responsabilité française, mais également une part de responsabilité des marchés dans l'espèce de négligence française dans cette gestion. Toutefois, les marchés financiers ont plusieurs raisons d'adopter cette attitude à l'égard de la dette française. L'une d'entre elles est qu'ils préfèrent ne pas voir la France tomber dans une récession grave. Ils ne sont pas les seuls d'ailleurs, le Fonds monétaire international (FMI) non plus ne le souhaite pas et je suspecte que le gouvernement allemand lui-même n'a pas un grand intérêt à voir la France tomber dans une récession grave. Car si en plus de la Grèce, l'Espagne, l'Italie, le Portugal et l'Irlande, la France avait eu un produit national brut (PNB) en chute de 2-3\%, on imagine où en serait la conjoncture européenne et allemande. La bienveillance envers la France s'explique aussi par le fait que maintenir un niveau d'activité suffisant, ne pas pousser les économies qui peuvent éviter de tomber en récession et leur permettre de maintenir un niveau d'activité ou d'entrevoir des perspectives de reprise facilite la gestion de la dette et donc la sécurité des créanciers.

\section{Le problème du chômage et de la déflation}

Je voudrais me mettre en faux par rapport à ce qu'a dit Jean-Marc Daniel lors de la première table ronde, déclarant que ni le chômage, ni la déflation n'étaient le vrai problème et que c'étaient de faux débats. Je pense qu'il a un point de vue un peu biaisé sur cette question. Selon moi, ce sont de vrais sujets, pour des raisons totalement indépendantes l'une de l'autre. Sur le sujet du chômage, et plus généralement sur les conditions sociales, j'ai participé à un livre de la Broo- 
kings Institution ${ }^{3}$ sur la crise européenne - avec Friedrich Heinemann notamment -, dans lequel j'ai écrit, outre un chapitre assez sévère sur la France, un chapitre sur l'évolution des systèmes sociaux en Europe. Le constat est que dans l'Union européenne (UE), dont les États membres avaient traditionnellement en commun des régimes démocratiques d'économie de marché ou d'économie mixte et un niveau de protection sociale très élevé au résultat assez homogène, ce troisième pilier se délabre actuellement dans le sud de la zone, nous acheminant vers une économie duale. Je pense que cette évolution vers des économies duales n'est pas tenable et que si l'euro a bien survécu à la crise financière, il pourrait être mis en péril maintenant par la montée de tensions sociales dont on a vu quelques indices au moment des élections européennes de mai 2014.

Concernant la déflation, les derniers indices sur une année sont de $0,3 \%$ pour l'inflation agrégée et de $0,75 \%$ pour l'inflation structurelle. Ce sont des niveaux très bas et nous ne sommes pas loin d'imaginer un scénario dans lequel les prix deviendraient dangereusement stables ou négatifs. Par déflation, on entend le déclin d'une mesure agrégée des prix et non la baisse de certains prix relatifs. Certes, la déflation n'est pas une menace imminente et la BCE se veut rassurante en annonçant $1,25 \%$ pour fin 2015 . Néanmoins, le fait que la conjoncture ralentisse, notamment en Allemagne au deuxième et au troisième trimestre 2014, reste un vrai sujet de préoccupation pour la BCE.

\section{L'Union monétaire}

L'Union économique et monétaire (UEM) est certes monétaire, mais pas économique. Sur le plan monétaire, j'aimerais caractériser ce qui distingue cette union de ce qu'est une économie monétaire comme celle des États-Unis, du Royaume-Uni ou du Japon. Cette union monétaire a en effet un caractère très hybride. D'un côté, elle est beaucoup plus que n'importe quel système monétaire européen, puisqu'il ne s'agit pas d'un accord entre États pour gérer en commun leur monnaie. Elle comprend une monnaie, une banque centrale et un système de paiement à la taille du continent. Par conséquent, cela donne l'impression d'une union monétaire. Mais la réalité est qu'en l'absence d'un pouvoir politique et de circuits privés de financement qui assurent l'homogénéité intime de toute la zone, la fragmentation entre gouvernements nationaux, espaces nationaux et financements nationaux reste très présente. En situation de crise, l'homogénéité s'évapore et il ne reste qu'une sorte de régime de change fixe entre des monnaies qui devraient ne pas être liées par des changes fixes. Cette seconde caractéristique est très préoccupante car cela fait référence aux années 1920 et aux tentatives - en particulier en Grande-Bretagne - de revenir à une parité avec l'or par la baisse des prix, comme on cherche à le faire dans les pays du sud de l'Europe. Mal engager cette politique monétaire de fixité des

\footnotetext{
${ }^{3}$ Kemal Derviş, Jacques Mistral (eds), Europe's Crisis, Europe's Future, Brookings Institution Press, Washington, 2014.
} 
changes par rapport à une référence extérieure par l'intermédiaire de la baisse des prix encourage la déflation. C'est une raison pour laquelle, au-delà des indices conjoncturels, $\mathrm{j}$ 'invite nos partenaires allemands à être sensibles à cette question dans le débat autour de l'inflation et de la déflation.

Entre parenthèses d'ailleurs, j'indique qu'à mes yeux, l'utilisation de l'hyperinflation dans les références culturelles économiques allemandes est hypertrophiée et cela sans raison, parce que l'hyperinflation allemande des années 1920 est le résultat de fautes de gestion caractérisées de l'institut d'émission allemand. Dès lors, il n'y a aucune espèce de raison d'imaginer que de pareilles erreurs puissent être reproduites. Quand on fait un lien entre l'hyperinflation et les drames de l'entredeux-guerres et le nazisme, c'est évidemment un raccourci sans objet puisque l'Allemagne est sortie de l'hyperinflation très vite dans les années 1920. C'est en réalité le chômage de masse, la Grande Dépression et l'incapacité des politiques à répondre au problème du chômage qui ont provoqué les élections de 1933.

\section{L'importance du rôle du Royaume-Uni en Europe}

À propos du Royaume-Uni et de la zone euro, Jean-Marc Daniel a mentionné à juste titre que la Grande-Bretagne apporte une contribution très utile à la culture européenne, à la vision du monde et au sort démocratique de l'UE. Depuis l'éjection ignominieuse de la livre en 1992, il se trouve que le peuple britannique n'a apparemment pas une grande volonté de se joindre à l'aventure de la monnaie unique. Il a toujours considéré qu'il avait plus à gagner d'un système de libre échange bien organisé plutôt que de s'intégrer dans un espace plus homogène comme un marché commun et ce que cela implique, c'est-à-dire une monnaie qui soit à la taille de ce marché. Par conséquent, depuis le début de la crise des dettes, les Britanniques ont souvent montré qu'ils ne souhaitaient pas participer à la recherche des solutions puisqu'ils ne font pas partie de la zone euro. Je vois assez mal comment on pourrait les intégrer à cette recherche, même si c'est une perte pour le continent de ne pas avoir la Grande-Bretagne, son expertise financière et ses qualités politiques avec nous.

Pour l'avenir de l'Europe et de sa gouvernance, il me semble plus raisonnable d'envisager l'intégration renforcée de la zone euro, vers laquelle nous nous dirigeons depuis le lancement des quatre unions (l'union fiscale, l'union bancaire, l'union autour de la compétitivité et à terme l'union politique) et à laquelle ni le Royaume-Uni, ni la Suède n'ont envie de se joindre. Il faut donc définir une relation qui soit adaptée aux préférences des Britanniques et à la dynamique du continent. Au surplus, ce sera quelque chose d'utile puisque comme l'économiste et homme politique turc Kemal Derviș le dit souvent, si on trouve une solution avec les Britanniques pour avoir cette nouvelle conjonction, cela sera également propice pour accueillir un pays comme la Turquie dont on voit bien qu'il est dans une situation pour l'instant un peu instable quant à sa perspective d'adhésion. 


\section{La politique monétaire ne peut pas tout}

Comme l'a répété Mario Draghi et comme l'avait dit Jean-Claude Trichet aux chefs d'État et de gouvernement en 2011, je pense que la politique monétaire ne peut pas tout, bien que $\mathrm{j}$ 'en aie fait l'éloge au début de cette intervention. La $\mathrm{BCE}$ fait ce qu'elle peut : elle a répondu aux défis des trois dernières années à travers les opérations de refinancement à long terme (longer-term refinancing operations, LTROs) ou encore via l'annonce d'un possible programme d'opérations monétaires sur titres (outright monetary transactions, OMT). J'interprète le lancement des nouvelles initiatives de 2014 comme un indice d'inquiétude sur ce que nous réserve le proche avenir en termes de croissance et d'évolution des prix. C'est une habitude que j'ai prise aux États-Unis. En effet, lorsqu'ils ne comprennent pas bien ce que fait la banque centrale, les experts à Washington cherchent en général ce que la banque sait et que le public ignore encore. La BCE ne s'est pas lancée dans son programme d'achat de titres adossés à des actifs (asset-backed securities, ABS) par simplicité d'esprit. Je me demande si elle sait des choses que nous ignorons et cela ne me rassure pas énormément, parce que je comprends le cri d'alerte de Mario Draghi. Une fois encore, ce n'est pas avec ce programme que la BCE va relancer la croissance et l'espoir en Europe, c'est évident. Elle peut seulement aider.

J'en viens donc au point de vue qui a été développé avec talent par Natacha Valla lors de la première table ronde : l'Europe a besoin d'investissements. Il n'est pas impossible que l'investissement reparte spontanément après une période de stagnation depuis plusieurs années, due à l'immense incertitude dans laquelle baignaient les entreprises. En couverture de The Economist et en première page du Financial Times, on annonçait la fin imminente de l'euro. Dans un tel contexte, il est difficile pour un chef d'entreprise qui investit sur 5, 10 ou 15 ans de se lancer dans de grands programmes. Par conséquent, l'insuffisance de l'investissement privé européen ne me surprend pas. Désormais, ces incertitudes sont en grande partie levées mais je pense qu'il faut aller au-delà à travers une initiative qui redonne une vision et qui incite à l'investissement en Europe.

\section{Le sens d'une aventure commune en Europe}

En conclusion, sur la question de savoir si nous convergeons ou non en Europe, je vais citer une anecdote. J'ai enseigné récemment aux États-Unis à la Kennedy School, où j'avais une classe d'une trentaine d'étudiants dont la moitié américaine et l'autre moitié européenne. J'ai été absolument fasciné, bien qu'étant un Européen assez convaincu et actif depuis pas mal d'années, de voir que les 15 Européens (c'est-à-dire des Allemands, des Autrichiens, des Grecs, des Espagnols, des Italiens et des Français) formaient un ensemble plus uni que les Américains qui étaient du Texas, du Minnesota, de Nouvelle-Angleterre et de Californie. Les Américains eux-mêmes m'ont dit qu'ils trouvaient cela très étonnant 
car ils s'attendaient à ce que, entre l'Allemand et le Grec, il y ait du tirage. Et effectivement, il y avait un certain tirage, mais il y avait le sens d'une aventure commune, d'un engagement commun et d'une histoire qui était la leur. Pour conclure, il suffit que nos grands responsables politiques développent une vision qui soit à l'image de ce que les jeunes attendent.

Christian de Boissieu : Merci Jacques Mistral. Nous allons continuer avec l'exposé de Philippe Moutot. Avant d'occuper son poste actuel, Philippe Moutot a eu des responsabilités à la Banque de France, puis il a travaillé à l'Institut monétaire européen (IME), présidé par Alexandre Lamfalussy entre 1994 et 1997, puis par Wim Duisenberg qui a fait la transition entre l'IME et la BCE. La BCE a vu le jour le $1^{\text {er }}$ juin 1998 pour préparer l'arrivée de l'euro le $1^{\text {er }}$ janvier 1999. Philippe Moutot a été directeur général adjoint à la BCE à la Direction générale de l'économie. Aujourd'hui, il est conseiller principal au sein de cette direction. Je lui laisse donc la parole.

Philippe Moutot : Je suis très heureux aujourd'hui de me retrouver à Paris. Cela fait pratiquement vingt ans que j'ai quitté mon emploi à la Banque de France pour exercer à l'IME, et j'ai l'impression quelquefois que le temps s'est arrêté dans le sens où j'ai souvent tendance à penser à mon expérience à Paris comme une chose qui s'est passée hier. C'est pour cela qu'il est tout à fait intéressant pour moi aujourd'hui d'essayer de parler des opinions des responsables politiques français et allemands sur le rôle de la $\mathrm{BCE}$, que j'ai observées pendant un certain nombre d'années.

Mon premier propos sera de dire que le rôle de la BCE aux yeux des hommes politiques - allemands comme français - reflète souvent le poids des expériences historiques nationales. Dès lors, il peut être temps pour nous d'essayer de reconsidérer ces expériences historiques et de comprendre comment les utiliser de manière positive. Le deuxième point est que je crois que le rôle de la $\mathrm{BCE}$ ces dernières années a été dicté par son mandat et par la nécessité d'assurer la pérennité de la zone euro. Selon moi, on ne peut pas différencier ces deux types d'actions : l'une soutient l'autre. L'ensemble des citoyens comme l'ensemble des juges qui peuvent avoir à discuter ou à évaluer la zone euro doivent bien avoir cela à l'esprit. Autrement, le raisonnement ne peut qu'être un raisonnement absurde. Et cela m'amènera au besoin d'un compromis qui réponde aux défis actuels de la part de nos hommes politiques. Bien sûr, cela commence par une discussion à un niveau plus analytique, de façon à ce que les gens soient convaincus qu'ils analysent bien le même problème, mais il est essentiel d'aboutir à un compromis sur ce genre de questions.

\section{Le poids de l'histoire}

Les perceptions nationales française et allemande s'opposent en matière de centralisation et de fédéralisme. La France est plutôt positive vis-à-vis de la centra- 
lisation et le contraire est vrai en Allemagne. Toute une liste d'expériences pourrait expliquer la vision positive qu'ont les Français de la centralisation, qui a permis de répondre à différents problèmes au cours du temps. La guerre de Cent Ans n'en est qu'un exemple : la manière dont on la raconte en France est positive pour la royauté et la centralisation. En contrepoint, la Révolution française est pour une large part le résultat des déficits budgétaires créés par les Parlements régionaux à l'époque. $\mathrm{Au} \mathrm{XIX}$ siècle, lors de la révolution industrielle, la centralisation a facilité et accéléré la colonisation d'une large partie du monde par des pays comme la France et la Grande-Bretagne. On peut donc comprendre dans cette situation que la centralisation ait été vue de manière positive. Les choses sont très différentes pour l'Allemagne qui est un État-nation tardif, qui probablement avait envie d'imiter la "Grande Nation »- comme on appelle souvent la France en Allemagne - mais qui n'y est pas parvenu aussi rapidement que souhaité et qui finalement, à travers l'expérience du début du siècle et son contraste avec celle qui l'a précédée comme avec celle de la Bundesrepu$b l i k$, ne peut qu'avoir une vue positive du fédéralisme.

Une seconde opposition existe sur la perception du lien entre risque d'inflation et risque d'instabilité financière. Pour les Français, le lien n'est pas direct parce que l'expérience de l'instabilité financière est historiquement associée au nom de John Law. Elle n'est pas liée du tout à l'hyperinflation des assignats, qui est notre première expérience en France de l'hyperinflation - et la seule. Du côté allemand, les évènements sont plus proches de nous. L'année 1924 est souvent mentionnée en Allemagne. Or elle cache une autre date, celle de 1931, lorsque le chancelier Heinrich Brüning n'a pas souhaité soutenir le système bancaire allemand par peur d'un retour de l'inflation, ce qui a favorisé ensuite l'arrivée au pouvoir d'Adolf Hitler en 1933.

Même des événements simultanés et communs peuvent être vus de manière différenciée. Par exemple, lorsque la France et l'Allemagne se sont vu poser la question de la participation à une union monétaire avec le traité de Maastricht, cela a été perçu en France plutôt comme une grande aventure internationale et comme un échange de bons procédés, la réunification de l'Allemagne contre un partage de la monnaie. Ces mêmes termes en Allemagne ont résonné de manière beaucoup plus directe pour la population allemande. Par ailleurs, la décision en France d'avoir une banque centrale indépendante - ce qui nous permettait de respecter le traité de Maastricht et de parvenir à l'Union monétaire - a été prise pendant la campagne électorale qui précédait l'élection d'Édouard Balladur, par le premier ministre Pierre Bérégovoy. Adopter cette position pendant qu'il y avait des tensions sur le franc était une manière de limiter les tensions de taux de change qui recommençaient à s'exprimer sans avoir à faire les frais d'un grand débat public. C'est passé relativement facilement. En Allemagne, les choses sont beaucoup plus complexes et ont donné lieu à procès auprès de la Cour de Karlsruhe, dont a résulté une décision que je qualifierais d'ambiguë puisqu'elle consiste à dire qu'il y a problème mais que 
ce n'est pas à la Cour d'en juger. Tous ces points me font penser que la discussion doit vraiment être approfondie - au niveau national et au niveau européen - pour mener à des progrès.

\section{Le rôle effectif de la BCE}

Pendant que ces expériences étaient tentées au niveau politique, à la BCE, nous avons essayé d'être le plus rationnel possible et de remplir notre mandat. La crise qui s'est propagée était une crise au début financière et fiscale, pas monétaire. Pourtant, nous avions dès le début de l'Union mis en œuvre une stratégie de politique monétaire qui prenait en compte la dimension financière en alliant l'analyse économique et l'analyse monétaire. Cela nous a amenés à une gestion très souple de la liquidité. Mais nous avons aussi souvent été critiqués sur ce choix de stratégie monétaire - en ce qui me concerne, je pense que c'est un choix qui a été extrêmement utile car nous avons pu, à travers l'analyse monétaire, avoir la confirmation de craintes que nous pouvions avoir dans d'autres domaines mais qui n'apparaissaient pas naturellement dans nos prévisions économiques. J'ai notamment le souvenir de discussions très difficiles avec le FMI et l'Organisation de coopération et de développement économiques (OCDE), lorsque nous devions expliquer qu'imiter la Réserve fédérale américaine (Fed) qui soutenait la demande américaine grâce à l'immobilier n'était pas une très bonne idée. Personnellement, j'ai dû le faire dans le cadre de l'OCDE, et je peux vous dire que nous étions bien seuls à défendre notre point de vue. Tout cela pour dire que parfois, suivre simplement le point de vue du FMI ou de l'OCDE n'est pas la meilleure solution. On peut peut-être nous critiquer pour n'avoir pas agi assez fortement en 2005, mais on ne peut pas nous reprocher de n'avoir pas compris la situation et l'impact de la politique américaine sur l'immobilier.

$\mathrm{Au}$ début de la crise, nous avons donc descendu les taux à toute vitesse, et autant que possible, bien que certains eussent aimé que nous l'ayons fait plus tôt ou différemment. Ceci a été complété par des mesures non standard. Au début de l'année 2010, nous pensions ainsi que nous avions réussi à contrôler la situation et étions sur le point de sortir de la crise. Toutefois, à la fin de l'année, les primes de certaines obligations souveraines ont augmenté très rapidement. Certes, il y avait une détérioration des soldes budgétaires. Mais pour nous, cela n'expliquait pas tout puisque nous avions des déficits et un niveau de dette qui, bien que croissants, n'étaient pas plus élevés que dans d'autres pays. L'autre élément qui demandait à être expliqué était celui de la taille du secteur financier. Il est de fait que lorsqu'un pays a un secteur financier très important, celui-ci peut avoir un impact sur le solde budgétaire. Mais là encore, nous n'étions pas si mal placés au niveau de la zone euro dans son ensemble. Certes pas aussi bien que les États-Unis, mais mieux que la Suisse ou la Grande-Bretagne. Au niveau national en revanche, les situations de certains pays comme l'Irlande ou Chypre pouvaient mieux s'expliquer. 
Globalement, la crise financière a révélé que divers éléments, insignifiants au niveau de la zone euro, peuvent se conjuguer au niveau national pour former un risque systémique puissant qui finit par menacer toute la zone euro. Elle a montré non seulement qu'un secteur bancaire prenant des risques excessifs crée des risques pour les autres pays et les autres secteurs bancaires, mais aussi que la stabilité financière et les risques budgétaires interagissent fortement. L'exemple de la Grèce, bien qu'atypique, a révélé cette combinaison de tensions aux marchés et au public. Une union bancaire était nécessaire.

Cette nécessité, que n'ignoraient pas les négociateurs du traité de Maastricht, avait été remise à plus tard. Ainsi, jusqu'en 2008, la soutenabilité budgétaire et stabilité financière n'avaient pas de grand soutien institutionnel. Toutefois, c'est parce que nous n'étions pas dans une situation d'ignorance que nous avons réussi ensuite, avec l'ensemble des États, à créer très rapidement un ensemble d'administrations susceptibles d'aider à gérer le système de manière plus efficace. Par conséquent, les discussions sur le Mécanisme de résolution unique (MRU), l'Union bancaire et le Mécanisme de surveillance unique (MSU) ont été menées très rapidement. Ce travail, qui s'ajoutait à la politique monétaire, a constitué l'ensemble de notre effort sur la période 2010-2014. Par ailleurs, les OMT ont été un élément très intéressant au niveau économique. Bien que discuté, voire critiqué, ce plan d'action a eu soudain l'air de convaincre les marchés et a mené à une diminution du pessimisme, sans même que nous ayons à l'utiliser. À partir de cette annonce, la situation s'est peu à peu améliorée.

\section{Le besoin d'un compromis qui réponde aux défis actuels}

Depuis 2007, les perspectives de croissance en Europe ne sont plus les mêmes et c'est le premier défi. Dès lors, même l'Allemagne a des perspectives de croissance bien inférieures à ce qu'elles étaient en 2007. Le problème de gouvernance associé à ce constat doit être résolu. Il nous faut nous-mêmes trouver et explorer des solutions pour devenir plus productifs et générer suffisamment d'investissements en Europe. Dans certains pays, il peut s'agir de faire des progrès sur le marché du travail, tandis que dans d'autres pays, cela peut passer par des investissements plus structurels. Pour essayer de bien comprendre ce qu'il est utile de faire, il est nécessaire de tenir compte de la multiplicité des nations souveraines à l'intérieur de la zone euro.

Dans un article que j'ai publié en $2014^{4}$, j'ai essayé d'analyser cette situation avec un modèle parfaitement néo-classique à deux agents, c'est-à-dire deux pays. Ils ont un marché où ils échangent les biens, mais pas de banques. À chacun des pays sont infligées des contraintes de trésorerie dans les deux variantes : contraintes de transactions au comptant (variante 1) ou liquidités positives à la

\footnotetext{
${ }^{4}$ Philippe Moutot, «Macroeconomics and systemic risk: must central bankers go back to their future?», Banks and Bank Systems, vol. 9, n 3, 2014, p. 103-119.
} 
fin de chaque exercice comptable (variante 2). Ce sont deux manières complémentaires de concevoir le rôle de la monnaie.

Dans la variante 1, lorsque la distribution de la richesse est trop inégale, les agents ont intérêt à sortir de l'union monétaire, ce qui veut dire que pour qu'une union soit pérenne et stable, elle doit être en mesure de punir et d'empêcher l'agent A ou l'agent B de sortir de la zone. Il ne peut y avoir de politique monétaire rationnelle sans un système d'amendes imposées à celui qui veut sortir de la zone. Or la mise en œuvre d'une amende est justement ce que la France et l'Allemagne se sont accordées à éviter en 2004. En somme, un transfert de souveraineté fiscale est nécessaire dans une union monétaire.

La deuxième variante explique quant à elle le fonctionnement des marchés financiers, lorsque des bons souverains sont discutés et valorisés par quelques grandes banques qui emploient des traders intéressés directement, pour leurs salaires, aux résultats des spéculations ou des transactions qu'ils entreprennent. Quand l'impatience de ces «market makers » augmente, les prix des bons souverains peuvent très fortement varier et les transactions disparaître des marchés alors même qu'ils restent ouverts. Cela n'est pas parce que le marché continue d'exister que celui-ci continue de jouer sa fonction. Les primes de risque peuvent atteindre des niveaux excessifs au vu des fondamentaux.

C'est cela à mon avis qui permet d'expliquer l'intérêt des OMT. Une fois que la banque centrale a bien fait apparaitre qu'elle se sentait responsable, le marché peut fonctionner de nouveau. Certes, il peut mal fonctionner si les autorités se mettent à acheter des bons souverains uniquement pour soutenir l'action d'un gouvernement quelconque, mais cela peut aller aussi très bien si la banque centrale s'est d'ores et déjà engagée à n'intervenir que lorsqu'il y a une situation excessive au regard des fondamentaux en termes du fonctionnement des marchés et que les programmes économiques nécessaires pour redresser la situation sont présents et suivis à l'intérieur de ce type de pays.

Pour conclure, la BCE ne peut pas tout faire. Elle ne peut pas assurer la pérennité de l'euro sans la collaboration des États. Dès lors, les États doivent trouver des compromis durables, c'est-à-dire qui créent une confiance financière entre eux. Cette confiance financière est essentielle pour que les réformes structurelles qui doivent être faites puissent être menées à bien.

J'aimerais terminer cette contribution en image. J'aurais pu utiliser La Fontaine, en particulier son lièvre, mais j'aurais pu aussi utiliser Münchhausen j'ai pensé que c'était un peu délicat dans une ambiance franco-allemande donc j'ai choisi d'utiliser Mary Poppins, qui a la chance d'être proche de la Cité, tout simplement parce que dans ce film, le père travaille pour la Banque d'Angleterre - mais on ne vous le dit pas lorsque vous regardez le film. Ce qu'il fallait éviter à mon avis, c'est l'ambiance tendue qui se dégage de ce film lorsqu'on revoit la banque centrale réagir à la situation qui était celle des années 1930. Ce qu'il faut obtenir, c'est ce qu'on voit à la fin du film, c'est-à-dire les gens regar- 
dant ensemble vers le futur. C'est une manière peut-être un peu innocente de présenter les choses, mais je crois qu'elle a une certaine vérité.

Christian de Boissieu : Ce n'est plus Mary Poppins, c'est presque la famille Trapp, pour les anciens qui ont vu ce film. Merci Philippe Moutot. Nous passons maintenant à l'exposé de Friedrich Heinemann, qui travaille au Centre d'études économiques européennes (ZEW) de Mannheim.

Friedrich Heinemann : Ma contribution à cette table ronde consiste à présenter le rôle que la Banque centrale européenne (BCE) devrait jouer aux yeux des responsables politiques allemands. Je remplacerai ici «les responsables politiques » par «les électeurs », c'est-à-dire les habitants de l'Allemagne. La base empirique que j'utilise pour estimer cette perception de l'opinion publique provient de diverses sources : premièrement du débat dans les médias allemands, deuxièmement de divers exposés et débats dans lesquels j'essaie d'expliquer toute la crise de l'euro au grand public depuis quelques années, et troisièmement de sondages d'opinion publiés. D'après mon expérience des débats, je peux dire qu'en Allemagne, il a parfois été très difficile d'expliquer la politique de sauvetage de l'euro et de la faire accepter.

C'est dans ce contexte que j'aimerais vous rapporter ici comment la BCE et ses mesures sont actuellement perçues. Je souligne que je n'expose pas là ma position personnelle, mais que j'aimerais essentiellement dépeindre l'opinion publique telle que je l'ai comprise.

\section{Quelles sont les inquiétudes de l'opinion publique allemande? Quel rôle la BCE devrait-elle jouer à ses yeux ?}

Ma première observation - qui semblera peut-être surprenante - est que ce n'est plus l'inflation qui est au centre des préoccupations des Allemands. En Allemagne aussi, les gens ont fini par comprendre que l'inflation n'était pas le problème dominant de l'Europe actuellement. Il y a d'autres soucis qui les préoccupent lorsqu'ils observent la politique monétaire ou l'évolution de la situation européenne.

Ce qui les inquiète le plus, c'est que la $\mathrm{BCE}$ est bien trop impliquée dans la politique de crise. J'ai trouvé très intéressant ce que Jacques Mistral a dit sur le fait qu'en France, on a l'impression que la BCE n'en fait pas assez. C'est le contraire en Allemagne. La perception dominante est que la BCE en fait beaucoup trop et outrepasse ses compétences. Dans ce contexte, la décision de la BCE en septembre sur la baisse des taux d'intérêt a fait l'objet de commentaires très négatifs dans les médias - elle a été qualifiée de décision activiste, relevant presque même de la panique. Il y a une inquiétude à l'idée que la BCE endosse un rôle qui ne lui a jamais été confié par les traités. Beaucoup se sentent confortés dans cette opinion par la décision de la Cour constitutionnelle fédérale sur les OMT, dont Philippe Moutot a déjà parlé. 
La deuxième préoccupation porte sur les conséquences de cette politique : on craint qu'elle entraîne des risques de pertes pour les épargnants allemands et qu'elle participe à la construction secrète et opaque d'une sorte d'union de transfert à la charge des épargnants. À ce sujet, une expression est devenue très populaire en Allemagne : celle de "répression financière ». Lors d'une répression financière, les taux sont manipulés à la baisse par des mesures publiques afin de déposséder de fait les épargnants. Je le répète, ce n'est pas mon opinion. Je ne fais que citer la perception des gens telle qu'elle m'apparaît.

La troisième inquiétude, que Philippe Moutot a déjà abordée, réside dans le fait que la BCE ne peut pas tout faire. C'est certainement l'opinion qui domine en Allemagne. Beaucoup de gens disent que la BCE peut certes apporter son aide, mais qu'elle ne peut pas résoudre tous les problèmes - manque de compétitivité, problèmes liés aux sites de production, excès de régulation. Elle ne peut ni éliminer la bureaucratie, ni améliorer le système de formation, ni rendre un pays plus innovant, ni abolir une taxation ou une réglementation excessive, ni discipliner les syndicats militants. Par ses mesures, la BCE peut tout au plus gagner du temps afin que la politique de réformes bénéficie d'un peu plus de calme, car les réformes - comme on le sait bien en Allemagne - se font dans la durée. Dans ce contexte, le troisième grand souci réside dans la crainte que le temps gagné par la $\mathrm{BCE}$ ne soit pas mis à profit pour mener les réformes nécessaires. L'inquiétude s'est désormais déplacée et ne porte plus sur les pays qui ont subi la première phase de la crise. Aujourd'hui, les Allemands reconnaissent qu'en Grèce, en Espagne, au Portugal et en Irlande, les gens ont beaucoup travaillé et accepté des changements profonds. Les médias en parlent. Maintenant, les préoccupations visent plutôt la France et l'Italie. Les questions qui me sont sans cesse posées expriment l'impression que la BCE a beau gagner du temps, celui-ci n'est pas suffisamment mis à profit dans tous les pays, et en particulier dans ces deux-là.

\section{La BCE outrepasserait son mandat et serait trop active}

Cette inquiétude ne s'observe pas seulement chez des eurosceptiques ou des populistes quelconques. On sait que Hans-Werner Sinn, qui dirige l'institut ifo de Munich, a fait connaître à l'opinion publique le problème des soldes TARGET élevés. Je peux vous assurer que dans les conférences, une personne sur deux sait ce que sont les soldes TARGET de la BCE. Le public français n'est peutêtre pas aussi bien renseigné. Mais des détails de ce genre sont bien connus de l'opinion publique allemande, ce qui indique un niveau élevé d'information ainsi qu'un bon niveau des débats.

Naturellement, l'arrêt du 14 janvier 2014 de la Cour constitutionnelle fédérale sur le mécanisme des OMT de la BCE a aussi un impact notable. On peut dire sans ambages que la Cour constitutionnelle fédérale a pratiquement balayé toute l'argumentation de la BCE. Le premier argument de la Cour constitutionnelle fédérale est le suivant : "Ce n'est pas à la BCE de prendre des décisions 
sur la composition de la zone euro. » La Cour de Karlsruhe ne croit pas que la $\mathrm{BCE}$ soit chargée de préserver la composition de la zone euro, comme elle le fait aujourd'hui, et juge qu'elle outrepasse son mandat.

De même, la BCE a soumis les OMT à la condition d'un accord sur le Mécanisme européen de stabilité (MES). Pour Karlsruhe, cela montre clairement que la BCE s'occupe ici de politique économique. Il ne s'agit plus seulement de politique monétaire. Dans les OMT, Karlsruhe critique en outre l'achat sélectif d'emprunts d'État, c'est-à-dire le fait que contrairement à une mesure générale de quantitative easing (assouplissement quantitatif) où l'on achète des emprunts d'État de manière très large, l'on n'achèterait dans ce cas des emprunts d'État qu'à quelques pays donnés. Pour Karlsruhe, il ne s'agit donc pas d'un programme classique de politique monétaire. La BCE a opposé quelques arguments pour se défendre : dans le cadre des OMT, elle n'achèterait pas directement les emprunts, mais passerait par le marché libre. Mais la Cour constitutionnelle a rejeté cette excuse pour de bonnes raisons, car l'achat indirect a les mêmes effets que l'achat direct. Lorsqu'un investisseur sait que la BCE est prête à jouer le rôle d'acheteur final, il est naturellement plus enclin à acheter temporairement ces emprunts.

Je cite l'arrêt de la Cour constitutionnelle fédérale : "Pour ces motifs, la décision de procéder aux OMT ne devrait pas être couverte par le mandat de la $B C E$. Il ne s'agit plus d'une mesure de politique monétaire, mais en grande partie d'une mesure de politique économique. Les OMT ne devraient donc pas pouvoir se justifier comme mesure de soutien de la politique économique. Dans ces conditions, il existe des doutes sérieux quant à leur validité. » L'article 123 du traité sur le fonctionnement de l'Union européenne (TFUE), qui interdit le financement monétaire des budgets, contient également une interdiction de contournement. Le principe des OMT devrait aussi s'y heurter.

Les juges suprêmes d'Allemagne ont ainsi déclaré que, selon eux, le programme OMT n'était pas conforme au droit européen. Ils ont ensuite fait part de leurs réserves à la Cour de justice de l'Union européenne (CJUE). Ceux qui pensent que la CJUE pourrait simplement écarter le point de vue de Karlsruhe risquent de se tromper. En effet, la Cour constitutionnelle fédérale a indiqué qu'elle se réservait le droit de juger en dernier recours et qu'elle vérifierait par exemple si la CJUE limitait quantitativement le programme OMT. Dans le pire des cas, la Cour de Karlsruhe pourrait interdire à la Banque fédérale allemande de continuer à participer au programme OMT. Les OMT seraient alors mortes. Si les marchés n'ont pas réagi plus nerveusement à la décision de Karlsruhe, c'est simplement parce qu'ils attendent que la BCE procède, en remplacement, à un quantitative easing général.

J'ai présenté en détail ce jugement sur les OMT parce qu'il montre bien que le refus du rôle activiste de la $\mathrm{BCE}$ en Allemagne a cessé depuis longtemps d'être seulement un thème populiste, mais qu'il a également un solide ancrage constitutionnel et donc qu'il s'agit d'une opinion partagée par de larges pans de la bourgeoisie modérée. 


\section{La peur des pertes pour les épargnants}

Comment les Allemands justifient-ils précisément leur peur des pertes de patrimoine ? À ce sujet, j'ai déjà cité l'expression de « répression financière ». On s'inquiète donc à l'idée que la politique monétaire intervienne artificiellement au bénéfice du fisc, et bloque les intérêts sous le taux de l'inflation. Nous avons reçu, en Allemagne, les premiers signalements de taux négatifs sur le marché, du moins pour les entreprises. Quant à la République fédérale d'Allemagne, elle peut pratiquement se refinancer à $0 \%$ à court terme. Un problème est que les épargnants allemands, ces dernières années, ont appris qu'ils devaient faire quelque chose pour leur prévoyance retraite. Cependant, avec les taux au plus bas, c'est devenu extrêmement difficile. Les assurances-vie sont loin de rapporter les taux auxquels les clients s'attendaient à la signature des contrats. Tout le système de retraite par capitalisation mis en place ne rapporte plus les taux attendus. Le problème fait l'objet d'âpres discussions en Allemagne, et en particulier dans les milieux bourgeois. Même pour les électeurs fidèles à Angela Merkel, tout cela va trop loin, et c'est l'Europe et la BCE que l'on accuse.

À ce sujet, je pense personnellement que la perception de l'opinion publique allemande repose assez largement sur une analyse erronée. Ce terme de "répression financière » ne s'applique tout simplement pas à l'Allemagne. La notion de répression financière est née dans le contexte des pays en voie de développement et désigne un régime dans lequel les taux d'intérêt sont régulés à la baisse par des moyens législatifs. De cette manière, le secteur public gagne un accès privilégié au marché des capitaux. Cette définition ne correspond pas à la République fédérale d'Allemagne et aux conditions favorables de refinancement dont elle bénéficie actuellement. Celles-ci ne résultent pas d'artifices de régulation, mais font partie d'un équilibre de marché qui est caractérisé par une forte aversion au risque de la part de larges groupes d'investisseurs, et par une importante offre globale de capitaux d'épargne.

Dans ce contexte, selon moi, l'opinion publique allemande surestime largement l'influence de la $\mathrm{BCE}$ sur les taux à long terme. Après tout, ces taux à long terme suivent une pente décroissante continue depuis 15 ans déjà. Une cause en est notamment l'excès d'épargne mondiale (saving glut) dont Ben Bernanke parle régulièrement. Pourtant, dans le débat allemand, cet aspect est largement ignoré. À la place, on accuse unilatéralement Mario Draghi d'être responsable des faibles taux d'intérêt qui exposent aujourd'hui de nombreux épargnants à des pertes.

Ces faibles taux obligent les épargnants allemands qui mettent de côté pour leur retraite à épargner encore plus afin d'atteindre leurs objectifs. Il n'est même pas certain que la faiblesse des taux ait obligatoirement un effet positif sur la consommation. Si un ménage a un objectif d'épargne retraite donné, et si les rendements tombent aussi bas, ce ménage doit augmenter ses efforts d'épargne afin de pouvoir réaliser son objectif. Le fisc allemand également, qui supporte 
d'importantes charges de retraites, doit entre-temps aller bien plus loin dans la consolidation afin de pouvoir assurer ces dépenses à l'avenir.

\section{Le temps gagné par la BCE ne serait pas mis à profit}

Le fait que la BCE soit engagée de manière trop active dans des systèmes de transferts secrets entraîne toute une série de problèmes pour le dialogue francoallemand. Mais il y a encore d'autres difficultés. Naturellement, il y a le contraste bien connu qui revient actuellement sur le devant de la scène : les Allemands sont plutôt dans une politique de l'offre, tandis que les Français privilégient la demande. Du point de vue allemand, cette orientation nette de notre voisin vers la demande, souvent ressentie comme unilatérale, est de plus en plus considérée comme une fuite vis-à-vis de la réalité. On pense que l'économie française a besoin de réformes structurelles urgentes, que ce soit en rapport avec le marché du travail, le système fiscal, l'État social ou la régulation du marché des biens. Si l'on prétend que la France n'a essentiellement qu'un problème de demande, l'opinion publique allemande n'y croit pas.

De même, la suggestion de mettre en place de nouvelles capacités budgétaires pour la zone euro se heurte plutôt à un refus. Le point de vue dominant est que ces nouveaux mécanismes de dépenses constituent plutôt une réaction d'évitement aux nouvelles limites de dettes. À peine a-t-on interdit aux pays d'accéder au marché de la dette au niveau national que démarre une recherche créative de nouvelles possibilités de contourner cette interdiction, en déplaçant l'endettement au niveau de l'Union européenne. Ce comportement n'engendre pas l'enthousiasme, mais est également considéré comme une fuite vis-à-vis de la réalité.

Un autre problème pour le consensus franco-allemand est que l'utilité de cet argent peu cher de la BCE fait l'objet de jugements très divergents. Du point de vue allemand, la politique des taux nuls est plutôt associée à un coût, celui des rendements perdus sur les placements sûrs tels que les avoirs issus de l'épargne ou les emprunts fédéraux. De plus, l'Allemagne n'est guère intéressée par une dévaluation de l'euro. Les entreprises allemandes n'ont pas vraiment besoin d'une dévaluation pour assurer leur compétitivité. Au contraire, l'Allemagne se retrouve au banc des accusés en raison des forts excédents de sa balance des transactions courantes. Un euro faible pourrait encore renforcer ce problème. Quant aux consommateurs allemands, dont on connaît la propension à prendre leurs vacances à l'étranger, ils ne sont pas enthousiasmés par l'affaiblissement de l'euro à l'extérieur.

Sur le thème de la solidarité européenne aussi, les perceptions sont largement divergentes. De l'extérieur, on reproche souvent à l'Allemagne de ne pas avoir fait preuve d'assez de solidarité vis-à-vis de l'Europe du Sud ces dernières années. La perception allemande est totalement différente : pour l'opinion, le pays est allé très loin dans l'acceptation des garanties sous la direction d'Angela 
Merkel, et même bien trop loin au goût de nombreux électeurs. Je pense qu'il serait faux de compter sur l'Allemagne pour être en mesure d'assumer sans plus de difficultés des garanties encore plus importantes si la crise s'aggravait. Le problème serait immense, tant constitutionnellement que politiquement.

Pour terminer, encore une réflexion sur ce qui pourrait redonner de la crédibilité à la $\mathrm{BCE}$ en Allemagne dans sa ligne actuelle. Je pense qu'il est très important pour la réputation de la $\mathrm{BCE}$ en Allemagne qu'elle se repose à nouveau simplement sur des arguments de politique monétaire, et non de politique européenne. Je déconseillerais vivement à la BCE d'utiliser encore des arguments tels que la nécessité de défendre la composition actuelle de la zone euro pour justifier ses mesures de politique monétaire. Elle devrait se montrer bien plus réservée et ne pas prétendre qu'elle est capable de résoudre les grands problèmes de la politique économique européenne. Elle devrait également rappeler les limites de ses propres actions et insister sur le fait que ses mesures ne sont destinées qu'à gagner du temps, mais ne permettent pas de résoudre des problèmes plus profonds. Puis, dans les prochaines années, la responsabilité devrait absolument repasser des mains de la politique monétaire à celles des capitales de l'UE. Les principaux problèmes de l'Europe ne se résolvent pas à Francfort, mais en partie à Bruxelles et, selon l'opinion allemande, surtout à Rome, à Paris, à Berlin et dans les autres capitales.

Christian de Boissieu : Merci beaucoup Friedrich Heinemann. Je trouve que le débat qui s'est amorcé autour de la table est tout à fait passionnant. Mais ce n'est pas fini, car Gerald Braunberger va nous donner son point de vue. Comme Friedrich Heinemann, Gerald Braunberger connaît bien la France. Il est rédacteur en charge de la rubrique Marchés financiers au quotidien allemand Frankfurter Allgemeine Zeitung (FAZ).

Gerald Braunberger : Le titre de mon bref exposé est : «Aide-toi, le ciel t'aidera. » Cela ne vient pas du président de la Bundesbank, mais je l'ai trouvé par hasard sur Internet. C'était le nom d'une société politique libérale française qui, dans les années 1820-1830, s'opposait à quelques tendances autoritaires dans la politique française de l'époque. Mais honnêtement, jusqu'à la semaine dernière, je l'ignorais complètement.

Tandis que Friedrich Heinemann a parfaitement décrit le sentiment ressenti en Allemagne, j'aimerais pour ma part évoquer les relations entre la politique monétaire et les banques et marchés financiers, parce que je suis journaliste financier.

\section{Deux points de vue différents?}

Le prix Nobel américain Paul Samuelson a dit: "The Lord gave us two eyes to watch both demand and supply. » En français, cela signifie que Dieu a donné deux yeux aux économistes pour qu'ils regardent l'offre et la demande. Comme 
le disait Friedrich Heinemann et comme cela a été évoqué lors de la première table ronde, la France aurait peut-être tendance à regarder davantage la demande et l'Allemagne, l'offre. Mais comme le disait Henrik Uterwedde précédemment, il faut se méfier des généralisations. Il y a des économistes de tous bords dans tous les pays. Néanmoins, les traditions culturelles exercent aussi une influence sur la pensée économique. Je ne pense pas que cela soit trop grave et suis de l'avis qu'il faut en discuter et que nous pouvons traverser le fossé franco-allemand en regardant à la fois l'offre et la demande.

\section{Le poids des banques}

Des années 1980 jusqu'à la crise de 2008, les banques ne jouaient aucun rôle dans les analyses macroéconomiques. Même la BCE utilisait des modèles économiques dépourvus de banques. C'était une époque où on pensait que ce n'était pas très intéressant car cela fonctionnait très bien. Depuis 2007-2008, l'image est complètement différente. En Europe continentale, les banques sont devenues très importantes pour le financement des entreprises, contrairement aux États-Unis où beaucoup d'entreprises se financent par la vente des titres, donc sur le marché des capitaux.

En Allemagne, depuis la crise de 2007-2008, les banques n'ont pas réduit leurs financements aux entreprises. Les grandes banques ont réduit leurs crédits aux entreprises, mais les petites et les moyennes banques les ont augmentés. En revanche, en France et en Italie, les banques sont beaucoup plus hésitantes. Or l'économie allemande se porte mieux que l'économie française ou italienne. Je ne dis pas que le financement des entreprises par les banques explique cela, mais il peut constituer un facteur.

\section{La formule magique : « macrofinance »}

Des économistes européens et américains essaient dorénavant d'intégrer le secteur financier dans les modèles macroéconomiques. Cela avait déjà été fait avant 1980 pour être ensuite presque oublié. Cette recherche contemporaine est très riche. Par exemple, le professeur français Hélène Rey à Londres a récemment reçu un prix très important de la part de l'association allemande des économistes. Je vais utiliser dans cet exposé un outil de recherche élaboré par l'Allemand Markus Brunnermeier et par l'Ukrainien Yuliy Sannikov, qui travaillent tous les deux à l'université de Princeton aux États-Unis.

\section{Deux cercles vicieux}

Supposons qu'il y ait une crise économique avec une récession et une déflation. $\mathrm{Si}$ on place le secteur bancaire au centre de l'analyse, deux cercles vicieux apparaissent. Les actifs d'une banque comprennent surtout des crédits aux entreprises et aux particuliers, ainsi que des titres - en particulier des obligations 
d'État. Le passif du bilan est le financement de ces actifs, composé à $90 \%$ de dettes, par exemple les dépôts des clients (particuliers et entreprises). Les fonds propres, qui sont normalement très minces, sont les réserves.

En cas de crise, les actifs et le passif de la banque seront touchés par un cercle vicieux. En situation de récession en effet, la banque va beaucoup perdre sur les crédits aux entreprises, car de nombreuses entreprises vont se retrouver en faillite. Si la récession est forte et que règne la crainte de voir la dette publique devenir insoutenable, les rendements des obligations d'État vont augmenter, ce qui signifie que les cours des obligations vont baisser - ce qui touche aussi la banque. Dès lors, dans une situation de crise profonde, les actifs de la banque vont se réduire. Du côté du passif, la valeur réelle des dettes augmentera dans un contexte de déflation profonde. Les banques perdront beaucoup d'argent. Or elles n'ont pas beaucoup de réserves parce que les fonds propres sont réduits.

\section{La BCE ne fait pas cavalier seul}

Il existe un lien étroit entre la stabilité des prix, la stabilité du secteur financier et la stabilité de la dette publique. Par conséquent, il existe un lien étroit entre la politique monétaire (européenne), la régulation du secteur financier (mieuropéenne avec l'Union bancaire, et mi-nationale) et la politique budgétaire (nationale). Dans une telle configuration, la banque centrale a un rôle à jouer. La plupart des économistes, qu'ils soient français, allemands, américains ou autres sont de l'avis que dans une telle crise profonde, la banque centrale doit injecter de l'argent dans le système bancaire. La banque centrale devient alors un assureur contre des risques macroéconomiques graves.

Toutefois, il convient de garder à l'esprit le risque moral (moral hazard) lié à une assurance. En effet, si l'assureur est omnipotent et si les banques savent que la BCE les sauvera, les banques risquent de ne pas augmenter leurs fonds propres. Elles risquent également d'accorder facilement des crédits. De même, si l'État sait qu'il sera sauvé financièrement par la BCE, il ne s'occupera pas de la solidité de la dette publique. La politique monétaire peut perdre son indépendance et se retrouver sous le contrôle soit de la politique budgétaire des Etats (fiscal dominance), soit des banques (financial dominance).

Si la BCE hésite à augmenter ses taux d'intérêt en période conjoncturelle favorable face à un État qui avance le volume de ses dettes publiques ou à une banque qui prétexte une réserve faible de fonds propres, alors elle perd son indépendance. La domination financière constitue un sujet actuellement discuté en Allemagne. Le fait que la BCE veuille racheter des crédits des banques partout dans la zone euro crée un certain esprit de dépendance envers ces banques. La politique monétaire a besoin d'une stabilité du secteur financier et d'une stabilité de la dette publique. C'est la raison pour laquelle il est impératif de regarder également la dette publique. Or le Wall Street Journal écrivait ce matin (le 6 
octobre 2014) que, d'après des sources proches du dossier, la Commission européenne envisageait de rejeter le budget 2015 du gouvernement français.

\section{Illusions sur la politique monétaire}

La politique monétaire est un assureur dans le sens où elle peut stabiliser une économie au moins pendant quelques années. Toutefois, on attend trop de la politique monétaire parce qu'on veut qu'elle crée de la croissance. Actuellement, on évoque un risque de déflation. Une déflation sérieuse serait certainement un grave danger, mais le cercle vicieux précédemment décrit s'est très rarement produit dans l'histoire. Philippe Moutot a évoqué la situation de l'année 1931, qui ne concernait pas seulement l'Allemagne. La zone euro ne se trouve pas en déflation et la BCE ne prévoit pas de déflation. On observe certes une baisse des prix, mais il ne s'agit pas nécessairement d'une déflation. Il convient également de noter que si les prix baissent, cela augmente le pouvoir d'achat des ménages et la compétitivité des entreprises. En outre, une dévaluation interne telle que celle qui a eu lieu en Espagne, où l'on a réduit les coûts de production, n'est pas une déflation.

Par ailleurs, j'aimerais attirer l'attention sur le fait que la politique monétaire n'est pas très efficace dans un régime sans taux d'intérêt (zero lower bound), et dans un monde où le secteur bancaire est fragile, comme c'est le cas dans quelques pays de la zone euro. À un niveau de taux d'intérêt proche de zéro, une banque centrale peut augmenter le bilan des banques. C'est une mesure utile pour pallier un manque de liquidités de la part des banques commerciales. Or les banques sont liquides. Nombre d'entre elles ne sont pas rentables, mais ce n'est pas la même chose. De plus, le rachat de titres (quantitative easing) ne sert absolument à rien en théorie pure. Cela a été décrit par un économiste américain, Neil Wallace, au début des années $1980^{5}$. On pourrait avancer que la neutralité de Wallace reposait sur un monde artificiel, et que notre monde est plus compliqué et différent. Néanmoins, une étude réalisée sur le quantitative easing montre que le rachat de titres par la Fed à hauteur de 1,5 billion de dollars s'était soldé par une baisse du taux de rendement des obligations à 10 ans de 55 points de base. C'est peu. Le rachat des obligations d'État ne sert pas à grand-chose. Le rachat de titres privés aura peut-être un effet, mais il sera transitoire, incertain et difficile à calculer.

\section{Enfin, le taux de change !}

Depuis 25 ans que je participe à des discussions franco-allemandes, le taux de change a toujours constitué un point de divergence. L'idée en France est d'utiliser le taux de change comme arme économique, sous prétexte que les autres le

\footnotetext{
${ }^{5}$ Neil Wallace, «A Modigliani-Miller Theorem for Open-Market Operations », American Economic Review, vol. $71, n^{\circ} 3$, juin 1981 , p. 267-274.
} 
font. Un Allemand préfère pour sa part un taux de change flexible, qui évolue de temps en temps. Actuellement, le taux de change est probablement l'instrument le plus puissant de la $\mathrm{BCE}$, mais cela ne signifie pas grand-chose étant donné que les autres instruments de la BCE n'ont presque aucun effet. Tout de même, les effets de la politique monétaire sur le taux de change sont incertains à long terme. Il ne faut pas uniquement regarder le dollar. De nombreux pays ont lié leur monnaie à l'euro - en Europe du Nord, en Europe de l'Est et en Afrique. En 2009-2011, l'euro a été dévalué face aux monnaies émergentes. Cela n'a pas eu d'effet sur les exportations. Donc oui, c'est un instrument, mais je pense qu'il ne faut pas trop nourrir d'espoir en lui.

\section{Le secteur bancaire}

Il y a trop de banques en Europe et nombre d'entre elles ne sont guère rentables. La qualité de leurs actifs est souvent fragile, en particulier en ce qui concerne les banques des pays du sud de la zone euro. De plus, les banques européennes ont une insuffisance de fonds propres. À mon avis, c'est l'un des problèmes les plus graves qui touche la zone euro car elle a besoin d'un secteur financier sain. Mais assainir les banques n'est pas la tâche de la politique monétaire. Un marché $\mathrm{ABS}$ transparent serait bénéfique à l'UEM, comme le montrent les résultats des travaux de Markus Brunnermeier et de Yuliy Sannikov. Mais il n'y a aucune raison que la $\mathrm{BCE}$ achète des titres $\mathrm{ABS}$. Il faudrait chercher des acquéreurs dans le secteur privé (assureurs, fonds de pensions, hedge funds...).

Pour conclure, il ne faut pas surestimer la puissance de la politique monétaire. En outre, la stabilité du secteur financier et une politique budgétaire crédible au niveau national renforcent la cohérence et la puissance de la politique monétaire. En ce qui concerne la demande en Europe, la politique monétaire ne peut pas faire plus, la politique budgétaire n'a pas beaucoup de marge de manœuvre et surtout, je ne crois pas que la politique budgétaire soit une arme puissante. En Allemagne, il y a une discussion pour déterminer s'il faut augmenter ou non les salaires. La Bundesbank a contribué à ce débat. Toutefois, dans la plupart des pays de la zone euro, je ne crois pas que des augmentations de salaire significatives soient probables. Il faut vraiment espérer qu'un secteur financier sain apporte de l'aide à la politique monétaire telle qu'elle existe, parce que ce n'est pas la BCE, mais ce sont les banques commerciales qui donnent des crédits aux entreprises. Et si les banques commerciales ne fonctionnent pas correctement, il faut les consolider.

\section{CONCLUSION}

Christian de Boissieu : Merci à Gerald Braunberger et aux trois autres intervenants de cette session. Si on distingue des convergences entre eux sur des points que je trouve intéressants, certaines divergences apparaissent, lesquelles ne doivent pas être sous-estimées. 


\section{Les conséquences d'une baisse des prix}

Par exemple, il y a un débat sur les risques ou non de déflation. J'ai senti une petite divergence sur ce point entre Jacques Mistral et Gerald Braunberger. Sans vouloir trancher sur ce point, je voudrais dire un mot à ce sujet. Quand on aborde les problèmes d'inflation zéro, voire de baisse des prix dans certaines économies ou dans certains secteurs, cela ne concerne pas que le pouvoir d'achat et la compétitivité des entreprises. Il y a la question de l'impact de la baisse des prix sur la consommation des ménages. Est-ce que cela retarde ou non la consommation des ménages ? La baisse des prix est-elle favorable ou non à l'investissement des entreprises? Ce sont des questions qui me paraissent importantes dans le débat sur les conséquences macroéconomiques d'une possible baisse des prix. Quand vous avez $0,3 \%$ d'inflation en moyenne au mois de septembre dans la zone euro (en glissement sur 12 mois), cela veut dire que certains pays et certains secteurs sont en déflation. Même dans les secteurs qui ne sont pas en déflation, certaines entreprises sont en déflation. Je voudrais élargir un peu le débat sur les conséquences d'une baisse des prix dans certains secteurs avec, par-delà la compétitivité et le pouvoir d'achat que vous avez tous évoqués, l'impact sur la répartition dans le temps de la consommation. Est-ce que cela décourage la consommation et l'investissement? N'y a-t-il pas un danger ici?

\section{Les stress tests bancaires}

Vous avez été plusieurs à parler des banques. Je pense que c'est un sujet très important. Nous sommes à quelques jours de la publication de l'asset quality review (AQR) et des stress tests par la BCE. Personne ne sait ce qui va sortir de ces études. J'espère que personne ne le sait parce que si quelqu'un le savait, cela signifierait qu'il y a des risques de délits d'initiés sur les marchés. Je suis membre du Collège de l'Autorité des marchés financiers (AMF). En 2014, une question nous paraissait importante à nous, régulateurs de marchés financiers : celle d'éviter les fuites sur ces études parce que toute fuite sur les résultats des stress tests et de l'AQR pourrait entraîner des mouvements de marché. Je pense que le problème est le même du côté du régulateur allemand, du régulateur néerlandais et de tous les régulateurs. Peut-être que cet état des lieux des systèmes bancaires en Europe va nous réserver des surprises, en positif comme en négatif. Je ne sais pas ce qu'il va se passer du côté des banques françaises, mais un certain nombre d'experts pensent qu'il pourrait y avoir des nouvelles négatives sur certaines banques dans les Länder allemands par exemple.

\section{Le rôle de la $B C E$}

À propos des banques, je me tourne vers les deux intervenants allemands qui disent qu'il faudrait que la BCE applique strictement son mandat. Le pro- 
blème est que son mandat a changé avec l'accord sur l'Union bancaire de novembre 2013. La BCE est désormais en charge de la supervision des 120 banques les plus significatives de la zone euro, en liaison avec les autorités nationales compétentes. Ce n'est pas la BCE qui a décidé de superviser les banques. Il y a eu un accord européen, que les Allemands regrettent peut-être aujourd'hui, mais qu'ils ont quand même signé. Cet accord crée l'Union bancaire et donne ce pouvoir à la BCE. Par conséquent, quand vous nous dites qu'il faudrait que la BCE revienne sur un concept plus étroit de son mandat, le problème est qu'on vient de l'élargir avec l'Union bancaire. Derrière ce débat, il y a la question de savoir si la BCE pourrait être confrontée à un trade-off dans les années qui viennent entre sa mission de politique monétaire et sa mission prudentielle. Les économistes doivent déjà commencer à réfléchir à ce problème qui ne peut pas être écarté. Supposons que, dans quelques années, on soit en danger d'inflation dans la zone euro et que la BCE relève ses taux directeurs au titre de sa mission de politique monétaire. Si elle relève ses taux d'intérêt, il est possible que cela fragilise certaines banques de la zone euro qui se financent sur les marchés. Pour qu'elle puisse traiter ce genre de situation, deux organes différents ont été créés à l'intérieur de la BCE : le conseil des gouverneurs qui existait déjà et l'autorité de supervision. Toutefois, il existe des passerelles entre ces deux organes, qui ont des membres communs. À côté de Danièle Nouy qui dirige l'autorité de supervision à Francfort à la $\mathrm{BCE}$, le vice-président de la BCE, Vítor Constâncio, siège de droit. On aurait pu établir une muraille de Chine entre les deux institutions pour éviter tout conflit d'intérêts. On aurait certes évité les conflits d'intérêts mais pas les conflits d'objectifs.

Je suis totalement d'accord avec ce qu'a dit Friedrich Heinemann sur le fait que la BCE achète du temps. En tant que Français, cela ne me pose pas de problème de reconnaître que je pense que la France utilise mal ce temps. Ce n'est pas parce qu'on est Français qu'on est obligé de dire que tout va bien en France et ce n'est pas parce qu'on est Allemand qu'on est obligé de dire que tout va bien en Allemagne. À un moment donné, il faut arriver à dépasser sa propre nationalité, comme vous l'avez tous fait d'ailleurs. Néanmoins, j'émettrais une réserve en ce qui concerne les propos de Friedrich Heinemann sur la Cour constitutionnelle fédérale. La distinction entre les interventions directes de la $\mathrm{BCE}$ en faveur des États - c'est-à-dire le bail-out - et les interventions indirectes est au cœur de l'interprétation du traité de Maastricht. J'entends ce que dit Friedrich Heinemann sur les critiques adressées par la Cour de Karlsruhe sur la politique de la BCE. En même temps, la Cour de Karlsruhe a validé les plans de sauvetage en faveur de la Grèce et des autres pays de la zone euro. Si elle ne les avait pas validés, nous n'en serions pas là aujourd'hui. J'ai donc le sentiment que contrairement à certaines attentes, la Cour constitutionnelle allemande a été finalement plutôt bienveillante à l'égard d'une certaine pratique de la zone euro dont on pouvait discuter a priori. 


\section{Conséquences des règles de Bâle III}

Par rapport à l'exposé de Gerald Braunberger, nous sommes d'accord sur l'article d'Irving Fisher de 1933 sur la déflation par la dette ${ }^{6}$. Mais quand vous dites que le montant de capital est faible, tenez-vous compte de l'arrivée de Bâle III, qui consiste à augmenter les ratios de fonds propres des banques et à créer des ratios de liquidité ? Ce dernier point me pose d'ailleurs problème parce que ces ratios de liquidité vont modifier le business model des banques, qui vont dès lors faire moins de transformation d'échéance. Cela pose un autre problème que celui qui est évoqué dans le modèle de Markus Brunnermeier et de Yuliy Sannikov, parce que je pense que nous entrons en Europe dans une phase de désintermédiation des financements comme conséquence de Bâle III. Les banques vont moins prêter aux PME. Dès lors, la question est de savoir comment gérer un système financier dans lequel les structures de financement vont se modifier, en partie sous l'effet des conséquences des nouvelles réglementations prudentielles. Personne autour de la table n'a évoqué Bâle III. Or la $\mathrm{BCE}$ va être concernée par Bâle III à travers sa fonction de supervision des banques, qui consistera aussi à vérifier que les banques respectent les règles prudentielles. Par conséquent, j'aimerais qu'on introduise cette dimension réglementaire dans le débat, qui à mon avis va avoir des conséquences qui n'ont, au départ mais pas à l'arrivée, pas grand-chose à voir avec la politique monétaire de la BCE.

\section{L'opinion publique allemande}

L'exercice de Friedrich Heinemann n'était pas facile. Il a expliqué l'attitude de l'opinion publique allemande à l'égard de la BCE et à certains moments, il a donné - à juste titre - son avis. Les moments où il a donné son avis m'ont aussi donné envie de lui demander son avis justement lorsqu'il faisait état de l'opinion publique allemande sur un certain nombre de sujets posés par la BCE. Personnellement, je trouve que l'opinion publique allemande est réaliste sur beaucoup de sujets. La BCE achète du temps, nous sommes tous d'accord sur ce point.

\section{Le système de sanctions en cas de non-respect du traité}

Philippe Moutot a évoqué le système d'amende. Je pense que la France a eu tort de refuser une proposition d'Angela Merkel à l'automne 2010 sur le système de sanctions dans le Pacte de stabilité et de croissance (PSC). Notre système de sanctions pour les pays qui ne respectent pas les règles budgétaires et les ratios n'est absolument pas crédible. Un pays dans ce cas devrait constituer un dépôt

\footnotetext{
${ }^{6}$ Irving Fisher, «The Debt-Deflation Theory of Great Depressions », Econometrica, vol. 1, n 4, 1933, p. 337-357.
} 
non rémunéré auprès de la $\mathrm{BCE}$ - ce qui équivaut aux amendes. Or ce système n'a jamais été appliqué, ni à l'égard de la France, ni à l'égard de l'Allemagne, ni à l'égard du Portugal ou de l'Italie lorsque ces pays n'ont pas respecté les critères du PSC. Certes, nous avons amélioré la gouvernance budgétaire de la zone euro à la lumière de la crise, mais nous n'avons pas changé le système de sanctions. Or Angela Merkel avait proposé en 2010 un système de sanctions qui me paraît beaucoup plus crédible, qui consistait à dire qu'un pays qui ne respectait pas les règles budgétaires une fois qu'il était dans la zone euro pouvait se voir privé de droit de vote dans les instances communautaires (sanction politique) et/ou d'accès aux fonds structurels (sanction financière). À l'époque, Nicolas Sarkozy avait refusé cette proposition allemande et le débat s'était arrêté là. Par conséquent, nous vivons aujourd'hui avec un système de sanctions qui à mon avis n'a aucune effectivité et donc pas de crédibilité.

Sur le dernier point qu'évoque Gerald Braunberger, c'est-à-dire le possible refus du budget français par Bruxelles - vraisemblablement pour de bonnes raisons de mon point de vue d'économiste -, le fait est qu'il ne se passera rien. La France révisera un peu sa copie et donnera le sentiment de faire quelques concessions. Toutefois, nous n'avons pas de système de sanctions crédible, c'est-àdire que si la France met plus de temps que promis à revenir vers les $3 \%$ et si, à la limite, le pays reste pendant plusieurs années à $4 \%$, va-t-on lui appliquer le système de dépôt auprès de la $\mathrm{BCE}$ ? Quand un pays ne respecte pas les règles du jeu, même si on a donné des pouvoirs nouveaux à Bruxelles sur la coordination des politiques budgétaires, même si on a créé le semestre européen, que se passe-t-il concrètement ? Je n'ai pas compris ce qu'il allait se passer en dehors de la renégociation, mais tout cela a une efficacité en question.

Traduction des propos de Friedrich HEINEMANN : Marie-Céline GEORG 



\section{Table ronde : Regards communautaires sur les enjeux de la politique économique et financière européenne ${ }^{1}$}

\section{OUVERTURE DE LA TABLE RONDE}

Guntram B. Wolff : On m'a demandé d'animer une session sur les enjeux de la politique économique et financière de l'Union européenne non seulement du point de vue de la France et de l'Allemagne, mais aussi de celui d'autres pays membres. Je suis donc très heureux de pouvoir avoir ce débat aujourd'hui avec trois experts éminents venant de trois pays très importants pour l'avenir de l'euro et de l'Union européenne. Nous commencerons avec Miguel Moltó Calvo, professeur à l'université San Pablo de Madrid, qui nous donnera tout d'abord, je pense, son propre point de vue - celui d'un excellent universitaire -, mais aussi un aperçu de l'opinion espagnole sur notre situation actuelle en ce qui concerne les politiques économique et financière européennes.

Permettez-moi peut-être de dire, pour commencer, que nous nous trouvons dans une situation plutôt étrange, puisqu'aujourd'hui, les économistes sont plus identifiés par leur pays d'origine qu'autre chose. Je me souviens d'une époque, avant cette crise, où l'on faisait essentiellement la différence entre bons et mauvais économistes. Désormais, on distingue les économistes allemands, français, espagnols, italiens, irlandais, etc. Je trouve cela assez triste, et je pense que nous devrions plutôt essayer d'avoir un débat en tant qu'économistes et universitaires, mais bien entendu, chacun d'entre nous connaît probablement le mieux son propre pays, et nous en apprendrons certainement beaucoup en écoutant également ce que la population du pays peut penser.

Après la présentation du professeur Calvo, nous écouterons l'intervention du professeur Piga, de l'université de Rome. Je connais les recherches de Gustavo Piga depuis le début des années 2000, alors qu'il était l'un des économistes qui parlaient des grandes difficultés du secteur public et de la manière dont certains chiffres du déficit et de la dette étaient manipulés pour répondre à des critères budgétaires dans plusieurs pays. C'est le professeur Murphy qui succédera à Gustavo Piga : il est professeur au Trinity College de Dublin et je le connais également par son ouvrage The Fall of the Celtic Tiger ${ }^{2}$, coécrit avec Donal Donovan. Il s'agit d'un livre très intéressant qui m'a aidé à écrire un article sur l'évaluation de ce que la Troïka a effectivement accompli. En effet, il y a plus d'un an, nous avons publié une évaluation du travail de la Troïka dans trois des pays qui ont reçu un soutien financier, à savoir la Grèce, l'Irlande et le Portu-

\footnotetext{
${ }^{1}$ Cette table ronde s'est tenue dans le cadre du colloque Gouvernance économique, financière et monétaire de l'Union européenne, coorganisé par le CIRAC les 6 et 7 octobre 2014, NdE.

${ }^{2}$ Donal Donovan, Antoin E. Murphy, The Fall of the Celtic Tiger - Ireland and the Euro Debt Crisis, Oxford University Press, Oxford, 2013.
} 
$\mathrm{gal}^{3}$. Ce livre nous a vraiment aidés à mieux comprendre ce qui s'est réellement passé en Irlande.

Il est certain qu'aujourd'hui à Bruxelles, l'un des sujets brûlants est celui de l'égalité de traitement des pays quant au respect des règles budgétaires. Lors des auditions au Parlement européen, Pierre Moscovici a été assailli de questions par les pays de petite et moyenne taille qui ont entamé des programmes d'ajustement macroéconomique, lui demandant en somme s'il traiterait la France de la même manière que la Commission précédente et la Commission actuelle traitaient les petits pays. Dans les années à venir, on constatera des tensions sur l'égalité de traitement des pays quant au respect des règles, mais aussi sur la possibilité d'appliquer les règles de la même manière aux grands et aux petits pays. Je préviens toujours les gens, en particulier ceux qui viennent des pays baltes, qu'ils ne doivent pas s'imaginer que les ajustements opérés en Lituanie, en Lettonie, et dans une certaine mesure en Estonie, peuvent être copiés à l'identique en France et en Italie, parce que de petites économies ouvertes ne peuvent pas s'adapter de la même manière que des économies plus grandes et moins ouvertes. À mon sens, il y a un réel danger à penser que nous pouvons appliquer les mêmes règles partout de la même manière. Bien sûr, il en va de la crédibilité des règles, mais l'économie a besoin d'un traitement quelque peu différencié. Nous avons besoin de déterminer quelle est la part de politique et quelle est la part d'économie.

Le second problème est celui des (tristement) célèbres réformes structurelles. J'ai hâte d'entendre Antoin Murphy s'exprimer à leur sujet, car le discours officiel sur le programme irlandais reposait sur trois piliers : le financier, le budgétaire et le structurel. J'ai toujours pensé que le volet structurel était surdimensionné dans les documents officiels de la Troïka. En fin de compte, tout était question de budget et de finances. De nombreux pays qui ont fait l'objet d'un programme d'aide, en particulier la Grèce et le Portugal, ont entrepris des réformes structurelles très importantes. Aujourd'hui, il y a un large débat sur le type de réformes structurelles dont les grands pays ont effectivement besoin, et sur ce que l'Italie et la France devraient faire. Mais puisque je suis le participant allemand de cette table ronde, laissez-moi aussi vous dire ce que l'Allemagne devrait faire. Je dis depuis assez longtemps qu'elle aussi a besoin de réformes structurelles substantielles pour faciliter le processus d'adaptation au sein de l'Union monétaire. Je trouve toujours que le débat sur cette question à Berlin est extrêmement défensif, au lieu d'accepter le fait que les réformes structurelles nécessaires en Allemagne aideraient le pays ainsi que le reste de la zone euro. Elles n'aideront peut-être pas certaines couches de la société allemande, mais elles créeront certainement de nouvelles possibilités d'emplois et d'investissements, et généreront plus de croissance en général pour l'Allemagne. En ce qui

\footnotetext{
${ }^{3}$ Jean Pisani-Ferry, André Sapir, Guntram B. Wolff, EU-IMF Assistance to Euro Area Countries: an Early Assessment [en ligne], Bruegel, Bruxelles, 2013 (Bruegel Blueprint Series; 19). Disponible sur: http://www.bruegel.org/publications/publication-detail/publication/779-eu-imf-assistance-to-euro-area-coun tries-an-early-assessment/ [consulté le 19/01/2015].
} 
concerne la Banque centrale européenne (BCE), il existe une grande variété d'opinions philosophiques sur ce que la BCE fait et sur ce qu'elle devrait faire, ainsi que sur la manière dont elle devrait agir.

Enfin, mon dernier élément d'introduction concerne le moyen à long terme. Dans les cinq à dix prochaines années, dans quelle direction l'Union monétaire devrait-elle aller, selon nous ? Là aussi, les philosophies divergent largement. Certains diront que sur la base de l'existant, nous n'avons qu'à continuer à apporter des ajustements marginaux ici et là, et que l'on insiste trop sur la légitimité ainsi que sur les processus visant à déterminer comment établir la légitimité au niveau européen. D'autres pensent que pour la prochaine étape, nous avons besoin d'une révision nettement plus substantielle des traités afin d'établir des mécanismes de légitimité très différents, des mécanismes de recettes très différents et des ressources propres au niveau de la zone euro. Là encore, une partie des divergences de vues provient de la diversité des approches culturelles et historiques du système juridique, politique et judiciaire.

Ce n'étaient que quelques considérations de ma part en guise d'introduction, mais je suis sûr que vous aurez tous bien d'autres points à soulever et que nous trouverons de nombreux autres sujets de discussion au fil de cette table ronde. Je passe maintenant la parole au professeur Calvo.

\section{EXPOSÉS DES INTERVENANTS}

Miguel Moltó Calvo ${ }^{4}$ : Bonjour à tous. Tout d'abord, je voudrais remercier René Lasserre pour son invitation à participer à cette table ronde. Le CIRAC m'a demandé d'aborder les difficultés de ces dernières années en Espagne dans le contexte de la crise de l'euro et de traiter les sujets des deux premières tables rondes sous l'angle espagnol.

Mon exposé sera structuré en cinq points. Le premier point concerne les déséquilibres macroéconomiques en Espagne pendant la phase d'expansion (19992007). En effet, afin de mieux comprendre les effets sur l'Espagne de la triple crise qui a affecté la zone euro (la crise financière, puis la crise de l'économie réelle et, à partir de 2009 , la crise de la dette souveraine), il convient de mentionner les déséquilibres macroéconomiques cumulés en Espagne durant la période qui a précédé la crise. Le deuxième point a trait à la stratégie appliquée par le gouvernement espagnol pour faire face à la crise et les principaux effets de cette crise sur l'économie et le domaine social. Le troisième point fait référence au programme de stabilité 2014-2017 présenté par l'Espagne à Bruxelles. J'évoquerai les points forts et les points faibles de l'Espagne à l'aune de ce programme, qui d'ailleurs vient d'être modifié le 26 septembre lors de la présentation par le gouvernement espagnol du budget pour 2015. Le quatrième point signale certains comportements de la BCE qui ont affecté plus directement

\footnotetext{
${ }^{4}$ Texte revu et complété par l'auteur le 23/12/2014, NdE.
} 
l'Espagne. Enfin, le dernier point essaie d'esquisser une réponse au titre de cette table ronde. À mon avis, pour faire face aux enjeux communautaires, l'Union économique et monétaire (UEM) devrait avancer vers le fédéralisme.

\section{Les déséquilibres macroéconomiques en Espagne durant la phase d'expansion (1999-2007)}

Concernant les déséquilibres macroéconomiques en Espagne, j'aimerais rappeler que le pays a dû réaliser un effort macroéconomique considérable pour répondre aux critères de Maastricht afin d'appartenir à la zone euro dès 1999. Cependant, certains déséquilibres existaient entre 1999 et 2007. Le taux d'inflation, qui s'élevait à $3 \%$, était ainsi supérieur à la moyenne de la zone euro $(2,1 \%)$, avec pour conséquence l'appréciation du taux de change effectif en termes réels. En outre, la hausse du coût salarial unitaire était plus forte en Espagne $(2,7 \%)$ que dans la moyenne de la zone euro $(1,5 \%)$, tandis que l'augmentation de la productivité par heure travaillée était plus faible (1,9\%) que dans la zone euro $(3,2 \%)$. La balance commerciale présentait un déficit de $5,8 \%$, contre un excédent de $1,1 \%$ dans la zone euro. Toutefois, le financement de ces déficits ne posait pas de problème car les taux d'intérêt sur les marchés financiers étaient bas, les liquidités étaient abondantes et les primes de risque n'existaient pas. L'Espagne a obtenu plus de financements qu'elle n'en avait besoin. L'épargne nationale était inférieure à l'investissement national, d'où un besoin de financements externes. Les institutions financières espagnoles obtenaient des fonds par cette voie, mais également par l'émission de titres hypothécaires sur les marchés internationaux.

Les sociétés financières en ont profité pour investir, augmentant leur capacité de production et achetant des immeubles et des actifs à l'étranger, ce qui a mené à l'internationalisation des entreprises espagnoles. De leur côté, les ménages en ont profité pour réaliser des achats immobiliers (en Espagne, $83 \%$ des ménages sont propriétaires d'un logement, en France, $58 \%$, et en Allemagne, 44\%). Cela s'est traduit par une augmentation de l'endettement du secteur non financier (entreprises et ménages) et des institutions financières du secteur privé.

Le secteur immobilier a joué un rôle clé dans la phase d'expansion 19992007. Le secteur de la construction représentait $12 \%$ du produit intérieur brut (PIB) et l'investissement dans le secteur de la construction résidentielle se situait à environ $7 \%$ du PIB durant cette période. Les raisons de ce boom immobilier ont été la disponibilité de crédits à un taux d'intérêt bas, la disponibilité d'une main-d'œuvre abondante non qualifiée et la hausse des prix du secteur résidentiel. Dès lors, le principal élément déclencheur de la récession en Espagne à partir du troisième trimestre 2008 a été l'explosion de la bulle immobilière.

Pour résumer, les facteurs de vulnérabilité avant la crise résidaient dans le différentiel entre le taux d'inflation et l'accroissement de la productivité - dont 
a résulté une perte de compétitivité -, dans la bulle immobilière et dans le niveau de l'endettement privé, du déficit extérieur et de la dette extérieure.

En revanche, l'Espagne disposait de quelques points forts avant la crise. Son taux de croissance du PIB était de 3,7\% durant cette période (contre 2,2 \% en moyenne dans la zone euro), la situation budgétaire était satisfaisante en 2007, avec un déficit public situé à $3 \%$ du PIB et une dette publique à $36 \%$ du PIB. La situation sur le marché du travail était également favorable, avec un taux de chômage de $8 \%$ en 2007.

\section{La crise en Espagne, la stratégie du gouvernement et les effets de la crise}

La crise en Espagne comprend un volet interne et un volet externe. Concernant le volet interne, les prévisions relatives au PIB deviennent dès lors plus réalistes, les conditions d'accès au crédit plus difficiles, l'endettement privé est excessif tandis que les prix du secteur de la construction résidentielle et la demande nationale se réduisent. Sur le plan externe, la crise financière internationale augmente le coût du financement. Il faut procéder à la réduction de l'endettement des institutions financières et du crédit pour le secteur privé.

Pour faire face à la crise, le gouvernement va adopter une politique budgétaire expansive pendant la période 2008-2009, avec une augmentation des lignes de crédit officielles. Toutefois, à partir de 2010, la politique d'austérité va s'imposer à la zone euro et la stratégie du gouvernement espagnol sera axée sur trois piliers :

- la consolidation budgétaire via l'augmentation des impôts, un budget restrictif en 2010, un plan d'austérité 2011-2013 et l'accord cadre du gouvernement, des régions et des communes pour essayer de réduire le déficit public ;

- l'adoption de réformes structurelles. On peut citer la loi sur l'économie soutenable, la réforme du marché des biens et des services (libéralisation) et du marché du travail (application d'une dévaluation interne afin d'améliorer la compétitivité espagnole, le but étant que le secteur extérieur puisse compenser la faible demande interne) ainsi que la réforme des retraites comprenant le report de l'âge de départ à la retraite de 65 à 67 ans et un changement dans le mode de calcul du montant des pensions, basé non plus sur les 15, mais sur les 25 dernières années de cotisation ;

- le renforcement du système financier. Il y avait une grande concentration des risques dans le secteur immobilier, notamment au niveau des caisses d'épargne qui représentaient $50 \%$ du secteur financier en Espagne. Avec la crise, toutes les caisses d'épargne - sauf deux petites vont disparaître. La crise a mis en évidence un problème : le superviseur, la Banque d'Espagne, n'a pas réagi lorsque les problèmes ont commencé. Si les autorités ont agi sur les problèmes de liquidité, le sec- 
teur financier espagnol était également touché par des problèmes de solvabilité, qui n'ont pas été traités dans un premier temps. L'approche suivie par les autorités espagnoles aura tout d'abord été d'injecter massivement des liquidités et de ne pas évaluer les actifs immobiliers au prix du marché (non-mark-to-market). Ensuite, et pour améliorer l'efficience des caisses d'épargne, l'approche suivie sera de faciliter leur intégration. Mais la fusion de plusieurs caisses insolvables générera une caisse plus insolvable encore (c'est le cas de Bankia, qui par la suite aura besoin d'environ 23 milliards d'euros pour être sauvée). En juin 2009, le Fonds pour la restructuration bancaire (FROB) sera créé pour financer la fusion des caisses d'épargne.

Il a fallu attendre 2011 pour que l'Espagne réagisse à la situation concernant la solvabilité par la mise en place de lois venant renforcer le secteur financier. En février 2011, le besoin de capital du secteur financier espagnol était estimé à 15 milliards d'euros. En février 2012, 50 milliards d'euros supplémentaires étaient nécessaires, ce à quoi sont venus s'ajouter 30 milliards d'euros en mai 2012. En juin 2012, l'Espagne a effectué une demande de financement pour les banques espagnoles auprès de l'Eurogroupe qui a consenti à mettre à disposition des autorités espagnoles un montant de 100 milliards d'euros à 15 ans, à un taux d'intérêt de 1,5\%. En contrepartie, l'Espagne devait accepter les 32 conditions figurant dans un Memorandum of Understanding (MoU), parmi lesquelles figurait un audit sur le système financier espagnol effectué par des experts externes. En septembre 2012, le cabinet d'audit indépendant mandaté par le gouvernement espagnol Oliver Wyman a estimé qu'il y avait un besoin supplémentaire de 59 milliards d'euros. Au total, le besoin de capital depuis 2011 était de 158 milliards d'euros. Il est à souligner que, finalement, le gouvernement espagnol n'a demandé qu'environ 42 milliards d'euros sur les 100 mis à sa disposition par l'Eurogroupe.

La crise en Espagne a généré deux récessions en 2009 et 2012. Le taux de chômage actuel est de $24 \%$ (53\% pour les jeunes). Le seuil de pauvreté a augmenté pour atteindre $27 \%$. Néanmoins, il convient de rappeler que le taux de pauvreté dans l'Union européenne (UE) est de $20 \%$, ce qui correspond à 100 millions de personnes. En matière d'inégalité, le coefficient de Gini a été en 2012, avec 0,35, le plus élevé de l'UE à 27 (en France, il était de 0,30; en Allemagne de 0,28 ). Une dévaluation interne a été menée sur les salaires. Il fallait en effet augmenter la compétitivité et stimuler la demande externe afin de compenser la baisse de la demande nationale. En outre, la balance courante espagnole a été déficitaire jusqu'en 2013. À l'heure actuelle, la dette publique se monte à près de $100 \%$ du PIB. Hors institutions financières, la dette privée s'élève pour sa part à environ $200 \%$ du PIB, tandis que la dette externe nette correspond à $100 \%$ du PIB. Enfin, dans le cadre de la crise, il a fallu capitaliser les institutions financières. Toutefois, l'économie espagnole connaît une reprise depuis le deuxième semestre 2013. Selon les dernières données disponibles, 
pour le deuxième semestre 2014, la croissance du PIB sera de 0,6 \% en Espagne (contre -0,2 en Allemagne, 0 en France et -0,2 en Italie).

Tableau $1:$ Indicateurs en Espagne (2007-2013)

\begin{tabular}{|l|c|c|c|c|c|}
\cline { 2 - 6 } \multicolumn{1}{c|}{} & $\mathbf{2 0 0 7}$ & $\mathbf{2 0 0 9}$ & $\mathbf{2 0 1 0}$ & $\mathbf{2 0 1 2}$ & $\mathbf{2 0 1 3}$ \\
\hline Taux de croissance du PIB & 3,5 & $-3,8$ & $-0,2$ & $-1,6$ & $-1,2$ \\
\hline Taux de chômage & 8 & 18 & 20 & 25 & 26 \\
\hline Déficit public (en \% du PIB) & 2,2 & -11 & -11 & -10 & -7 \\
\hline Dette publique (en \% du PIB) & 36 & 54 & 62 & 86 & 94 \\
\hline $\begin{array}{l}\text { Dette des sociétés non financières } \\
\text { (en \% du PIB) }\end{array}$ & 114 & 124 & 124 & 107 & 98 \\
\hline Dette des ménages (en \% du PIB) & 83 & 86 & 86 & 81 & 77 \\
\hline $\begin{array}{l}\text { Position d'investissement international } \\
\text { (en \% du PIB) }\end{array}$ & -78 & -94 & -89 & -93 & -98 \\
\hline Balance courante (en \% du PIB) & -10 & -5 & $-4,6$ & $-1,2$ & 0,8 \\
\hline
\end{tabular}

Sources : Institut national de statistiques espagnol et Banque d'Espagne.

L'Institut national de statistiques a estimé que pendant la période 2008-2013, le PIB a reculé de 7,3\%. Entre 2007 et 2009 notamment, le PIB s'est réduit de presque 7 points, ce qui est considérable. Entre 2007 et 2013, le taux de chômage a très fortement augmenté, passant de $8 \%$ à $26 \%$. Tandis que le solde public était excédentaire en 2007, il a par la suite été fortement déficitaire (-7\% en 2013). La dette nette des sociétés non financières a reculé, se situant dorénavant à près de $100 \%$ du PIB, après un pic à $124 \%$ en 2009 et 2010 . La dette des ménages s'est quant à elle légèrement réduite, passant de $83 \%$ à $77 \%$ du PIB sur l'ensemble de la période. La dette externe nette avoisine pour sa part les $100 \%$ du PIB en 2013 (contre $78 \%$ en 2007). Enfin, la balance des comptes courants, après avoir été fortement négative, est devenue positive en 2013 , avec $0,8 \%$ du PIB.

Tableau 2 : La dette totale en Espagne, en France et en Allemagne en 2013 (en \% du PIB)

\begin{tabular}{|l|c|c|c|}
\cline { 2 - 4 } \multicolumn{1}{c|}{} & Espagne & France & Allemagne \\
\hline Dette publique & 94 & 94 & 78 \\
\hline $\begin{array}{l}\text { Dette des sociétés } \\
\text { non financières }\end{array}$ & 99 & 68 & 43 \\
\hline Dettes des institutions financières & 109 & 165 & 95 \\
\hline Dette des ménages & 84 & 68 & 58 \\
\hline TOTAL & $\mathbf{3 8 6}$ & $\mathbf{3 9 5}$ & $\mathbf{2 7 4}$ \\
\hline
\end{tabular}

Source : Global Financial Stability Report, Fonds monétaire international (FMI), avril 2014. 
Comme le montre le tableau 2, la dette totale en Espagne (qui comprend la dette publique, la dette des sociétés non financières, la dette des institutions financières ainsi que la dette des ménages) représente désormais $386 \%$ du PIB espagnol. En France, elle se monte à $395 \%$ du PIB, contre $274 \%$ en Allemagne.

\section{Le programme de stabilité espagnol (2014-2017)}

Sur la période 2014-2017, les points forts de l'Espagne sont les suivants : les prévisions de croissance du PIB sont bonnes, le déficit public va se réduire et la capacité financière sera positive. En outre, une réforme fiscale prévoyant une réduction d'impôts devrait s'appliquer à compter de 2015. En effet, le gouvernement espagnol considère que la croissance du PIB permettra de financer la réduction d'impôts, estimée à 9 milliards d'euros.

Toutefois, le pays possède encore quelques points faibles. Le taux de chômage continue à être très élevé. Bien qu'elle tende à se réduire, la dette reste très importante. Par ailleurs, la pression fiscale est assez réduite en Espagne.

\section{Tableau 3 : Prévisions économiques 2014-2017 - quelques indicateurs clés (variation annuelle en \%)}

\begin{tabular}{|l|c|c|c|c|}
\cline { 2 - 5 } \multicolumn{1}{c|}{} & $\mathbf{2 0 1 4}$ & $\mathbf{2 0 1 5}$ & $\mathbf{2 0 1 6}$ & $\mathbf{2 0 1 7}$ \\
\hline Taux de croissance du PIB & 1,2 & 1,8 & 2,3 & 3,0 \\
\hline Taux de chômage & 24,9 & 23,3 & 21,7 & 19,8 \\
\hline Indice des prix à la consommation & 0,3 & 0,9 & 1,3 & 1,6 \\
\hline Coût salarial unitaire & $-0,4$ & 0,0 & 0,4 & 1,0 \\
\hline Productivité/personne occupée & 0,6 & 0,6 & 0,7 & 0,7 \\
\hline Déficit public (en \% du PIB) & $-5,5$ & $-4,2$ & $-2,8$ & $-1,1$ \\
\hline Dette publique (en \% du PIB) & 99,5 & 101,7 & 101,5 & 98,5 \\
\hline Solde primaire (en \% du PIB) & $-2,0$ & $-0,6$ & 0,9 & 2,7 \\
\hline Pression fiscale (en \% du PIB) & 33,9 & 34,2 & 34,5 & 34,7 \\
\hline $\begin{array}{l}\text { Balance des biens et des services } \\
\text { (contribution au PIB) }\end{array}$ & 0,6 & 0,5 & 0,4 & 0,3 \\
\hline Capacité financière (en \% du PIB) & 2,0 & 2,4 & 2,5 & 2,5 \\
\hline
\end{tabular}

Source : ministère espagnol de l'Économie et de la Compétitivité.

Le tableau 3 ci-dessus montre notamment qu'à l'horizon 2017, le taux de chômage reste élevé et le déficit public se réduit. La Commission européenne a établi que l'Espagne parviendrait à respecter la limite des $3 \%$ de déficit d'ici à 2016, ce qui est corroboré par les estimations espagnoles. La dette publique devrait commencer à reculer en 2016. Cette même année, le solde primaire deviendra positif. Néanmoins, la pression fiscale continue à être de $9 \%$ inférieure 
à la moyenne communautaire. La balance des biens et des services sera quant à elle positive, de même que la capacité financière. Le cadre macroéconomique que vient de présenter l'Espagne améliore même certains indicateurs : le PIB devrait ainsi augmenter de $1,3 \%$ en 2014 et de $2 \%$ en 2015.

\section{Le rôle de la BCE et l'Espagne}

Concernant le rôle qu'a joué la BCE en Espagne, la faiblesse des taux d'intérêt plusieurs années avant la crise a favorisé les pays du centre de l'Europe tels que l'Allemagne. Durant cette période, l'Espagne aurait eu davantage besoin d'une politique monétaire plus restrictive. Or la BCE tient bien entendu compte de l'ensemble des pays de la zone euro.

En août 2011, la prime de risque en Espagne et en Italie était d'environ 400 points. La BCE a acheté de la dette publique de ces deux pays sur les marchés secondaires. En décembre 2011 et février 2012, un billion d'euros ont été injectés dans les banques de la zone euro. Les banques espagnoles se sont retrouvées dans une logique de carry trade: elles se sont financées auprès de la $\mathrm{BCE}$ au taux de $1 \%$ et ont préféré acheter de la dette publique espagnole à $4-5 \%$ plutôt que d'augmenter le crédit au secteur privé. En juillet 2012, la prime de risque était au-dessus de 600 points en Espagne et d'environ 500 points en Italie. Or, étonnamment, la BCE n'a alors pas acheté de dette publique de ces deux pays. Le 18 septembre 2014, la demande de fonds à la BCE (concernant la première tranche des TLTROs, targeted longer-term refinancing operations adoptés en juin 2014 pour un montant total de 400 milliards d'euros) par les banques espagnoles a été d'environ 15 milliards d'euros (sur un montant total demandé par les banques de la zone euro de 82 milliards d'euros). On s'attendait à une demande plus élevée de la part des banques espagnoles, mais il semblerait qu'elles soient en attente des tests sur les qualifications d'actifs que la BCE achève ce mois-ci et des stress tests de l'autorité bancaire européenne.

Dernier point : la fragmentation des marchés monétaires se poursuit en Espagne, c'est-à-dire qu'une entreprise espagnole doit encore se financer à un taux plus élevé qu'en France ou en Allemagne.

\section{Pour faire face aux enjeux communautaires : avancer vers le fédéralisme dans l'UEM}

La nouvelle gouvernance économique de la zone euro depuis 2010 avait trois objectifs : le renforcement du pilier économique, l'adoption de mécanismes d'aide financière et l'amélioration de la supervision et de la régulation bancaire. La nouvelle gouvernance est un grand pas en avant, mais elle est à mon avis insuffisante. En effet, même si la discipline budgétaire a été renforcée, la politique budgétaire relève des États membres et, partant, l'asymétrie entre politique monétaire et politique budgétaire se maintient. 
L'Union bancaire est presque achevée, bien que la prise de décision pour la résolution des banques soit complexe. Le mécanisme de soutien budgétaire (fiscal backstop) est insuffisant et le fonds européen de garantie des dépôts n'est pas adopté. De plus, l'union budgétaire n'avance pas. Les budgets des États membres sont envoyés à Bruxelles dans le cadre du semestre européen, mais la décision finale continue de relever des États membres. Il est prévu que les déséquilibres macroéconomiques soient sanctionnés, mais l'expérience montre qu'aucune sanction n'a été appliquée dans les pays dépassant les $3 \%$ de déficit et les $60 \%$ de dette publique par rapport au PIB figurant dans le traité de Maastricht, puis dans le traité de Lisbonne.

Ma conclusion est la suivante : la seule façon d'avancer vers une union économique et monétaire stable et permanente est l'adoption d'un système fédéraliste.

\section{Quelles seraient les mesures à court terme de ce système?}

Il s'agirait d'avancer dans la convergence économique et d'instaurer une flexibilité dans l'approche de l'austérité. La politique monétaire et la politique budgétaire devraient être plus expansives. La politique monétaire ne peut pas tout faire, comme l'a dit le président de la BCE Mario Draghi. Il faudrait également instaurer une flexibilité dans l'application du Pacte de stabilité et de croissance (PSC), avec par exemple le retrait des investissements de l'État dans le calcul du déficit public ou la prolongation des délais pour revenir à $3 \%$ de déficit public. Il faudrait également davantage de fonds européens pour augmenter l'investissement et contribuer à la croissance et à l'emploi.

Concernant la proposition du nouveau président de la Commission européenne, Jean-Claude Juncker, à savoir l'investissement de 315 milliards d'euros en trois ans, on peut se demander d'où proviendront les fonds étant donné que le cadre financier pluriannuel 2014-2020 est déjà adopté et que plusieurs pays ne semblent pas vouloir le modifier. En outre et pour le moment, les États membres n'ont pas décidé s'ils allaient contribuer au Plan Juncker 5 .

Mesures à moyen et à long terme (5-10 ans)

Il faudrait avancer à mon avis vers une structure fédérale. Sur le plan économique, il s'agirait :

- de créer un ministère des Finances à l'échelon communautaire avec les compétences suivantes :

\footnotetext{
${ }^{5}$ La Communication de la Commission COM(2014) 903 du 26 novembre 2014 précise que le montant initial de fonds disponibles sera de 21 milliards d'euros, dont 16 milliards proviendront de fonds déjà existants issus du budget communautaire et 5 milliards de la Banque européenne d'investissement (BEI). L'effet multiplicateur global du fonds sera de 15. Autrement dit, 1 euro mobilisé par le fonds pourra générer en moyenne 15 euros d'investissement dans l'économie réelle.
} 
- la supervision macroéconomique et budgétaire des États membres,

- l'harmonisation fiscale (le Conseil d'analyse économique a émis une note en juillet $2014^{6}$, à la rédaction de laquelle Guntram Wolff a participé),

- d'établir un budget de la zone euro d'une taille suffisante ;

- d'instaurer un Trésor européen (avec un mandat pour l'émission d'eurobonds) une fois que les indicateurs de déficit public et de dette publique se seront améliorés ;

- de créer un instrument pour faire face à des chocs asymétriques ;

- d'élargir le mandat de la BCE (prêteur en dernier ressort et contribuant à la croissance et à l'emploi) ;

- de modifier les traités, comme le disait Guntram Wolff précédemment, et de rédiger un traité sur l'euro (c'est la proposition du Groupe de Glienicke ${ }^{7}$ );

- ou d'aller vers une coopération renforcée (c'est la proposition d'António Vitorino et d'Yves Bertoncini ${ }^{8}$ ).

Pour établir un système économique tel que présenté ci-dessus, il faudrait avancer sur le plan politique et institutionnel avec un pouvoir législatif sur le modèle du bicaméralisme entre le Parlement européen et le Conseil - le « Parlement de l'euro » proposé par le groupe de Glienicke -, un pouvoir exécutif - qui serait la Commission élue par le Parlement européen, un pouvoir judiciaire - la Cour de justice avec toutes ses compétences -, la réduction au minimum des traités intergouvernementaux et le Conseil européen pour établir des lignes directrices. Merci à tous.

Guntram B. Wolff : Merci pour votre exposé et pour avoir mentionné le groupe de Glienicke dont je fais partie. Je laisse maintenant la parole à Gustavo Piga.

Gustavo Piga : S'il est difficile d'expliquer mon opinion, imaginez celles de 60 millions de personnes. Puisque je participe activement au débat en Italie, pour irrationnel et/ou peu conventionnel que puisse être mon avis, j'espère qu'il permettra de jeter un peu de lumière sur l'état du débat européen dans un pays qui fait preuve d'une irritation croissante vis-à-vis des politiques européennes, jusque dans les mots de son Premier ministre.

\footnotetext{
${ }^{6}$ Agnès Bénassy-Quéré, Alain Trannoy, Guntram Wolff, « Renforcer l'harmonisation fiscale en Europe », Les notes du conseil d'analyse économique, $\mathrm{n}^{\circ} 14$, juillet 2014, p. 1-12.

${ }^{7}$ Groupe de Glienicke, Vers une Union de l'euro, Tribune de Notre Europe - Institut Jacques Delors [en ligne], 23 octobre 2013. Disponible sur: http://www.notre-europe.eu/media/tribune11allemandsneijdoct2013.pdf?pdf=ok [consulté le 15/12/2014].

${ }^{8}$ Yves Bertoncini, António Vitorino, «Réformer la 'gouvernance' européenne - Pour une fédération d'États nations plus légitime et plus efficace », Notre Europe. Études et Rapports, n ${ }^{\circ}$ 105, septembre 2014, p.71-72.
} 


\section{Un moment dramatique de l'Histoire}

Pour moi, nous vivons un moment dramatique. Le graphique 1 provient du discours de Mario Draghi à Jackson Hole en 2014. Il montre, du côté gauche, l'évolution incroyable de l'emploi par secteur. Il s'agit d'une moyenne, donc on peut imaginer que les pays les moins bien lotis sont tombés encore plus bas. Il existe une inégalité croissante entre les pays de la zone euro, qui menace la stabilité sociale en Europe. C'est pourquoi il est crucial d'étudier les chiffres de l'emploi avant même de s'occuper de la production et des revenus. Les problèmes d'emploi sont importants pour comprendre l'Europe et son avenir.

Du côté droit de l'illustration, on voit un autre type d'inégalité, qui se remarque à l'intérieur des pays : l'évolution cumulée de l'emploi dépend du niveau d'études, ceux qui ont le moins étudié (souvent les plus pauvres) étant les plus touchés par cette crise. C'est encore un avertissement pour la stabilité future de la zone euro, y compris en termes politiques.

\section{Graphique 1 : Évolution de l'emploi dans la zone euro par secteur et par niveau d'études}
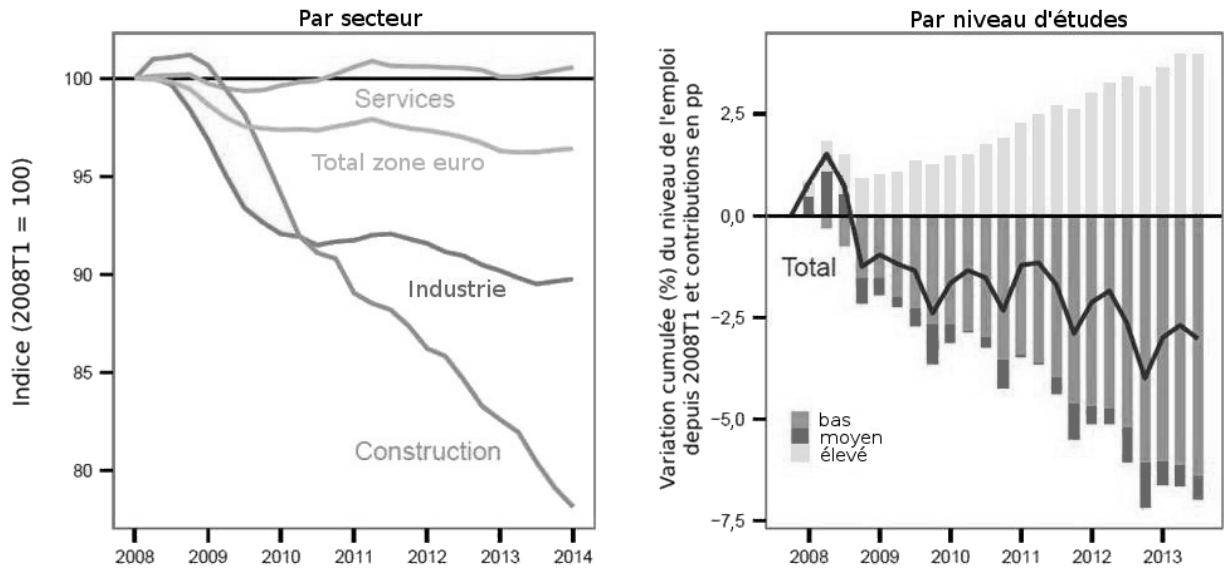

Source : Mario Draghi, discours à Jackson Hole, 22 août 2014.

\section{La nécessité d'une demande intérieure accrue}

Tous les six mois, la Banque centrale européenne (BCE) publie des entretiens menés avec un échantillon représentatif de PME européennes. Depuis cinq ans, elle demande à ces entreprises quel est leur plus gros problème. Réponse : ce n'est ni la concurrence, ni l'accès aux financements, le coût de la production, la réglementation ou la bureaucratie ; c'est la difficulté à trouver des clients - et, de toute évidence, des clients au sein de la zone euro. L'Italie, par exemple, n'a pas tant un problème de demande extérieure - c'est-à-dire extérieure à l'Union 
européenne - qu'un énorme problème de demande intérieure, c'est-à-dire au niveau national et, dans une moindre mesure, au sein de l'Union européenne.

\section{Graphique 2 : Le problème le plus pressant des PME européennes (pourcentage des répondants)}

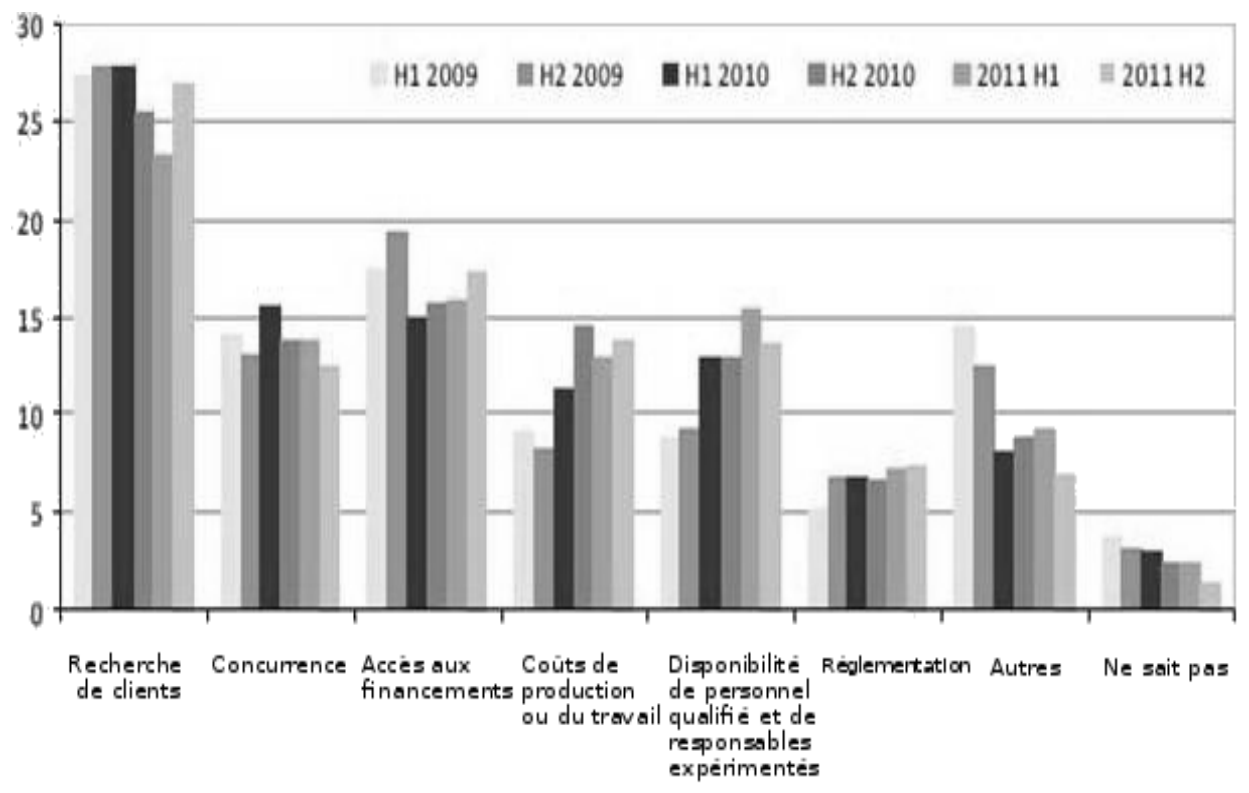

Source : BCE. Base : toutes les PME.

\section{Des politiques cycliques}

Il devrait être clair que nous parlons de la structure économique d'un continent qui est en crise parce que ses échecs sont survenus pendant des périodes d'expansion. En Italie au moins - mais je pense que cela peut s'appliquer à d'autres pays également -, nous n'avons pas procédé à des réformes pendant les périodes d'expansion, mais nous avons préféré des politiques budgétaires procycliques. Alors que l'austérité est la meilleure chose à faire en période conjoncturelle favorable et alors qu'entre 1998 et 2007, le produit intérieur brut (PIB) de l'Italie et celui de l'Allemagne ont progressé au même rythme (respectivement de $1,5 \%$ et $1,7 \%$ par an), l'Italie n'a pas corrigé son propre budget comme on aurait pu l'espérer, mais a attendu les temps difficiles. Ses dépenses primaires (hors dépenses en capital et paiements d'intérêts) ont augmenté de 2,1\%, tandis qu'elles sont restées stables en Allemagne.

Aujourd'hui, en pleine récession causée par la demande, nous parlons de remédier à ces échecs et nous reproduisons exactement les mêmes erreurs, en adoptant des politiques budgétaires procycliques telles que l'austérité pendant 
une récession. De toute évidence, beaucoup de ceux qui utilisent les statistiques sur l'Italie en s'intéressant au rapport des dépenses publiques sur le PIB observent que ce chiffre ne bouge pas. Cela est dû à l'effondrement concomitant des dépenses publiques et du PIB. En Italie, entre 2010 et 2014, les dépenses primaires (hors retraites) ont montré une baisse nominale de $3 \%$. Cela signifie qu'en termes réels (avec l'inflation), l'impact est encore plus fort. En ce qui concerne l'investissement public, la première victime de l'austérité constitue l'élément le plus pertinent à considérer : les dépenses en capital ont diminué de $12 \%$ (en valeur nominale). Par ailleurs, dès que la réforme des retraites adoptée par le gouvernement Monti est entrée en vigueur pendant la récession, de nombreuses grandes entreprises ont annulé leurs plans d'embauche de jeunes. Depuis 2012, le chômage des jeunes a explosé en Italie, passant de $20 \%$ à $44 \%$.

En d'autres termes, l'austérité est une bonne chose et les réformes sont réalisables quand tout va bien, mais elles n'ont aucun sens lorsque la situation est dégradée. Deux erreurs d'affilée ne font pas une réussite.

Concernant la situation actuelle, la logique des fables ne s'applique que jusqu'à un certain point. Dans la célèbre fable de La Fontaine, la fourmi dit à la cigale d'aller «danser » à l'arrivée de l'hiver, ce qui la condamne à mort. La fable aurait pu s'appliquer jusqu'au déclenchement de la crise, mais c'est impossible maintenant, puisque nous avons affaire à des pays qui ont décidé de réaliser un ambitieux projet ensemble. Allons-nous danser ou - une solution que La Fontaine n'avait visiblement pas envisagée - allons-nous aider la cigale à survivre pendant l'hiver en nous assurant que l'été prochain, pas cet hiver, elle travaillera assidûment?

J'irai encore plus loin dans cette métaphore de la cigale et de la fourmi. Je pense que l'on devrait en fait rejeter l'accent moral de cette fable, qui divise le monde en deux catégories, les bons et les mauvais pays. La diversité est une aide, non une entrave à la réussite de l'Europe. Ces dix dernières années, nous avons vécu au son de deux mots, «austérité » et «stabilité »; je me souviens d'une époque où nous rêvions de «liberté » et de « diversité ». Pour citer Jean Monnet: "La grande révolution européenne de notre époque, la révolution qui vise à remplacer les rivalités nationales par une union de peuples dans la liberté et la diversité, la révolution qui veut permettre un nouvel épanouissement de notre civilisation, et une nouvelle renaissance, cette révolution a commencé avec la Communauté européenne du charbon et de l'acier.»

En tout cas, en ce qui concerne l'économie, je regrette que le débat ne s'attache pas aux deux problèmes cruciaux suivants :

- peut-on trouver un moyen de résoudre le véritable problème qui explique pourquoi nous nous retrouvons aujourd'hui dans une situation de crise, c'est-à-dire peut-on discuter de la manière d'appliquer l'austérité en période d'expansion, puisque c'est cela qui semble être le vrai 
problème ? L'Espagne et l'Italie ont appliqué des politiques procycliques pendant ces années et, à l'époque, personne n'est venu nous dire de faire ce qu'il fallait comme c'est le cas aujourd'hui. On nous disait au contraire de faire les mauvaises choses ;

- nous sommes tous d'accord: les réformes mettent du temps à faire effet. Si des pays engagent réellement des réformes qui n'ont pas d'impact aujourd'hui, mais qui en auront un demain, nous devrions les récompenser immédiatement pour ce qu'ils ont fait. J'ai un sentiment très mitigé vis-à-vis de la réforme des retraites de Mario Monti. D’un côté, elle a été très néfaste à l'emploi des jeunes, mais d'un autre côté, comme l'a dit le vice-président de la BCE Vítor Constâncio, "c'est précisément dans le domaine des réformes visant à contenir le poids à long terme des populations vieillissantes sur les dépenses publiques [c'est-à-dire les retraites et la santé] que les pays sous pression ont déjà apporté des ajustements. L'Italie et le Portugal [il oublie de citer aussi la Grèce], par exemple, prévoient des augmentations négligeables des dépenses liées à l'âge. » Alors pourquoi l'Italie n'aurait-elle pas plus de marge de manœuvre aujourd'hui en raison des réformes qu'elle a mises en place? Même si leur impact ne se fera sentir que dans le futur, c'est maintenant que nous avons besoin d'argent et de flexibilité. Et par «nous », j'entends « nous les Européens » : l'Europe n'est rien sans l'Italie, la France ou l'Espagne, tout comme elle ne serait rien sans l'Allemagne.

\section{Le pacte budgétaire}

On pourrait demander au président américain Barack Obama ou au Premier ministre japonais Shinzo Abe s'ils savent ce qu'est le pacte budgétaire et ce que sont les déficits structurels. Dans toute l'histoire de l'humanité, personne n'avait jamais eu l'idée de mener une politique budgétaire de cette manière. Certes, les inventions peuvent avoir du bon. Mais pas toujours. Alphonse X de Castille, dit le Sage (1221-1284), a déclaré devant la théorie astronomique de Ptolémée : «Si le Seigneur tout-puissant m'avait consulté avant de se lancer ainsi dans la Création, je lui aurais recommandé quelque chose de plus simple. » Je crois qu'il est temps de procéder à notre révolution copernicienne dans la manière de gérer la politique budgétaire.

Le graphique 3 montre comment le chômage évolue depuis le début de la crise. Si la courbe revient vers zéro, cela signifie que le chômage est redescendu à son niveau d'avant la crise. Au début de la crise, puisqu'elle est née aux ÉtatsUnis, c'est là-bas que le chômage a augmenté le plus rapidement. Puis quelque chose a dû fonctionner aux États-Unis, puisque le chômage est en train de revenir à son niveau antérieur. Il est intéressant de noter qu'en Europe, l'accroissement du chômage est plus faible au départ, puisque la crise était externe. 
En 2010-2011, il semble que la situation va s'améliorer, puis soudainement, en 2011, au début du printemps, le chômage repart à la hausse.

\section{Graphique 3 : Évolution du taux de chômage depuis 2008 - zone euro et États-Unis}

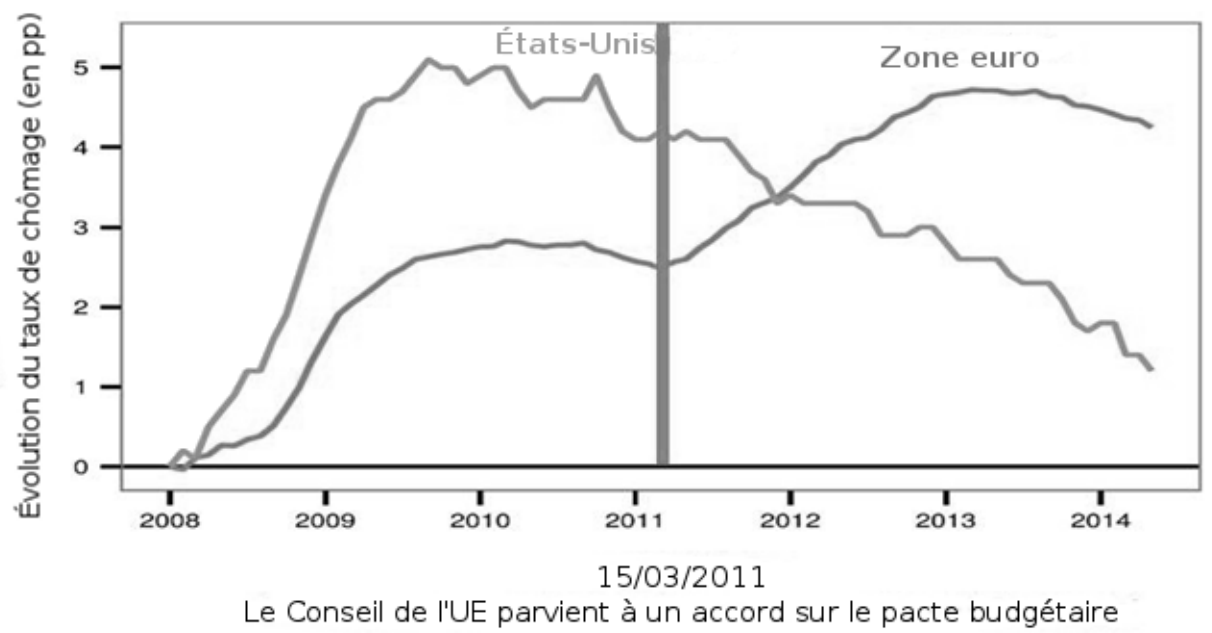

Source : BCE, discours de Mario Draghi à Jackson Hole.

En mars $2011^{9}$, l'Europe a signé l'ancêtre du pacte budgétaire, le " Six-Pack », qui en contient les principaux aspects. À l'exception du Royaume-Uni et de la République tchèque, ce pacte va devenir le principal instrument d'orientation de la politique budgétaire pour tous les pays de l'Union européenne, gravé dans le marbre de l'accord intergouvernemental. À partir de là, les familles et les entrepreneurs savent qu'ils vont vivre sur un continent où, à n'importe quel stade du cycle, les gouvernements devront réduire leurs déficits et leur endettement à un rythme croissant. Personne ne viendra à leur aide. Il n'y aura plus ni diminution de la fiscalité, ni hausse des dépenses lorsque la situation se dégrade, comme on l'a toujours appris en première année d'économie. Imaginez un entrepreneur qui sait que dans les dix prochaines années, si les choses tournent mal, le gouvernement va augmenter les impôts et réduire les investissements publics. Voudriez-vous investir dans un tel pays ? Voilà qui pourrait expliquer pourquoi, en termes de demande, l'Italie s'effondre, tout comme l'investissement privé en Europe.

L'Irlande a approuvé le pacte budgétaire après référendum le 31 mai 2012. La loi qui importait le pacte budgétaire en Italie a été adoptée le 24 décembre 2012 derrière les portes closes du Parlement, lors d'un débat très secret. Cela n'a certainement pas amélioré la perception de l'Europe.

\footnotetext{
${ }^{9}$ Conseil de l'Union européenne, Le Conseil parvient à un accord sur des mesures visant à renforcer la gouvernance économique [communiqué de presse] [en ligne], Bruxelles, 15/03/2011. Disponible sur: http://data.consilium.europa.eu/doc/document/ST-7691-2011-INIT/fr/pdf [consulté le 19/02/2015]
} 
J'aimerais vous montrer à quel point nous ne comprenons pas l'effet du pacte budgétaire. Aujourd'hui, tout le monde parle de la France et de l'Italie, qui auraient laissé tomber le pacte budgétaire et finalement abandonné l'austérité. Ce n'est pas totalement vrai. Le ministre français de l'Économie et des Finances, Michel Sapin, a déclaré : «Nous assumons le sérieux budgétaire, nous rejetons l'austérité. » Qu'est-ce que cela signifie, pour les dix prochaines années, pour un entrepreneur qui a besoin d'investir en France ? Pas grand-chose. Cela le remplit d'incertitude. Et il faut faire attention au choix des mots : en faisant moins de coupes dans les dépenses, Michel Sapin ne rejette pas l'austérité ; il fait moins d'austérité.

Le Premier ministre italien, Matteo Renzi, a déclaré cette année que le déficit se maintiendrait à $3 \%$ du PIB en 2015. En même temps, il a déclaré qu'il serait progressivement ramené à zéro d'ici 2018, c'est-à-dire, pour vous, l'austérité. Bien que toute la presse débatte sur le fait que Matteo Renzi va à l'encontre du pacte budgétaire, c'est faux: les données montrent clairement qu'il ne rejette pas l'austérité. Le même gouvernement italien ajoute dans son plan de stabilité que s'il n'est pas capable de réduire les dépenses, «il augmentera la taxe sur la valeur ajoutée (TVA) de $1 \%$ chaque année entre 2016 et 2018, avec une perte de production de 0,7\% à la fin de la période à cause de la contraction de 1,3\% de la consommation et des investissements ». Visiblement, les attentes - qui constituent l'essentiel pour l'investissement, car il s'agit d'une décision à long terme - s'effondrent. Il en va de même pour les ménages.

On pourrait me dire que j'ai tort, parce que les déficits de l'Europe tiennent compte du cycle. La Commission européenne a réalisé une modélisation permettant de calculer la production potentielle et le taux naturel de chômage. Les déficits structurels, tels qu'ils sont calculés par la Commission européenne, constituent un problème. Si l'on pense que la production potentielle va baisser à cause des mesures d'austérité, d'après le modèle, l'écart de production sera bien plus faible. Si l'écart de production est plus faible, la composante dite cyclique est moins importante, donc le déficit structurel augmente et nous sommes obligés de renforcer l'austérité, ce qui diminue encore la production potentielle. Dans toute l'Italie, nous avons appliqué différents modèles qui ont donné des résultats complètement différents, tant en termes de production potentielle (plus élevée) que de taux naturel de chômage (plus bas). Ainsi, le déficit structurel qui serait calculé serait complètement différent. Il s'agit d'un énorme problème technique qui est délégué à la Commission européenne, et sur lequel devrait plutôt porter le débat politique.

Avons-nous une autre solution? Je pense, et le Fonds monétaire international (FMI) semble désormais d'accord avec moi, que l'Italie devrait fortement augmenter l'investissement public pour stimuler la demande interne, en le finançant par la seule réforme que le pays n'a pas encore engagée et dont il a besoin, à savoir un examen radical des dépenses. Il devrait s'autofinancer à un déficit de $3 \%$ du PIB, sans s'éloigner de ces $3 \%$. La dette, en pourcentage du 
PIB, finirait par diminuer, ce qu'elle n'a jamais fait pendant toutes ces années d'austérité inutile, bien au contraire : elle a atteint son plus haut niveau depuis 1928, rien que pour souligner l'échec total de l'austérité.

Au lieu de cela, le gouvernement italien réduit l'investissement public et ne réexamine pas ses dépenses.

J'ai entendu aujourd'hui qu'en Allemagne, un débat sur la nécessité d'augmenter l'investissement public était en train de s'amplifier. Je comprends aussi qu'en revanche, il n'y a aucun débat sur la nécessité de réduire les impôts. Ces deux outils stimuleraient la demande en Allemagne, ce qui favoriserait les exportations des pays très touchés par la récession. Je préférerais une baisse des taxes à une augmentation de l'investissement public en Allemagne. À mon avis, la hausse de l'investissement public créerait plus de problèmes pour le pays, car l'une des choses que nous devons corriger est son déséquilibre du commerce extérieur, avec un énorme excédent de la balance des transactions courantes. L'investissement public accroît la productivité et la compétitivité, et pourrait aggraver les déséquilibres du commerce extérieur dans la zone euro. Privilégier l'investissement public à la baisse des taxes implique une perspective nationale, plutôt qu'internationale et eurocentrée. Baisser les taxes pour les travailleurs allemands serait une meilleure chose pour l'Italie, car un plus grand nombre d'Allemands viendraient en vacances au lac de Côme, ce qui permettrait de réduire l'écart entre les balances des transactions courantes.

Christopher A. Sims, lauréat du prix Nobel d'Économie 2011, a déclaré en 2014 lors d'une réunion des prix Nobel à Lindau que, pour sortir de la crise, l'Europe avait besoin "d'une politique budgétaire expansionniste maintenant, sans engagement de réduire les dépenses futures ou d'augmenter les taxes futures afin de préserver la stabilité actuelle des prix ». Il est évident que selon lui, la politique monétaire à elle seule ne peut pas tout faire.

Malheureusement, le professeur Sims pense que c'est impossible, étant donné le climat politique actuel : "Malheureusement, les gens sont totalement convaincus que ce n'est pas notre politique budgétaire actuelle et qu'il faudrait un changement radical du mode de pensée des décideurs ainsi que de leurs discours destinés à convaincre les gens que c'est ce qui va vraiment se produire. Le système politique doit prendre des engagements sur la durée et s'y tenir, ce qui est très difficile pour les responsables politiques. "

Ce discours a une autre implication plus subtile : la politique monétaire de la zone euro ne fonctionne pas. Les banques italiennes disposent de liquidités abondantes, mais personne ne demande de crédits. Les gens ne veulent ni investir, ni embaucher. Certaines banques refusent alors ces liquidités, car il serait coûteux de les conserver à la $\mathrm{BCE}$ à Francfort. Pourquoi ? Mario Draghi a toujours affirmé que la BCE allait apporter son aide et proposer une politique monétaire plus expansionniste, mais que dans le même temps, les pays devaient respecter le pacte budgétaire en augmentant les taxes et en réduisant leurs dépenses : "Par conséquent, l'orientation d'ensemble de la politique gagnerait 
à ce que la politique budgétaire puisse jouer un rôle plus important aux côtés de la politique monétaire, et je pense qu'il existe suffisamment de latitude pour ce faire, tout en tenant compte de la spécificité de nos conditions initiales et de nos contraintes juridiques. Au nombre de ces conditions initiales, il faut citer entre autres les niveaux de dépenses publiques et d'imposition de la zone euro qui, rapportés au PIB, sont déjà parmi les plus élevés au monde. Nous évoluons en outre dans le cadre d'un ensemble de règles budgétaires - le Pacte de stabilité et de croissance - qui constitue un point d'ancrage pour la confiance et qu'il serait contre-productif d'enfreindre. " (Mario Draghi, discours à Jackson Hole, 22 août $2014^{10}$ ). Si je suis entrepreneur, je ne vais pas investir en pareil contexte, même si les taux sont nuls : si la politique monétaire promet d'une main et menace de l'autre, cela ne peut guère inciter le secteur privé à l'optimisme.

\section{Les unions budgétaires}

Les unions budgétaires sont une question de culture. Des gens se réunissent au sein d'un système fédéral lorsqu'ils ont l'impression de partager la même culture. Les États-Unis d'Amérique sont nés sous la forme d'une union monétaire regroupant des États très différents. Il leur a fallu longtemps avant de se faire confiance : plus de 100 ans.

Les États-Unis sont devenus un système fédéral centralisé dans les années 1930, 150 ans après le début de l'union monétaire. Entre-temps, il leur a fallu une guerre civile, l'invention du train qui a permis aux cultures de se rencontrer, et la Première Guerre mondiale pour comprendre qu'ils avaient besoin de devenir un leader mondial sur la planète. Tous les États ont décidé d'abandonner volontairement leur pouvoir à Washington quand et parce que Franklin Delano Roosevelt est venu en aide aux États qui étaient le plus en difficulté pendant la récession. Cet acte de solidarité a à lui seul créé parmi les États un niveau de confiance basé sur la croyance qu'ils recevraient de l'aide en cas de nécessité. À cette époque, tout le monde comprit qu'il était temps de mettre les différences de côté et de s'unir.

C'est une histoire importante pour l'Europe. Nous n'aurons pas d'union budgétaire avant que les cultures changent et qu'un acte de solidarité déclenche ce changement.

\section{La (seule) solution}

Voilà pourquoi je pense que nous avons besoin de gagner du temps et d'éteindre la récession maintenant, quel que soit l'endroit où se trouve l'incendie, avant qu'il n'embrase encore la forêt. Nous n'avons pas le temps d'attendre encore deux ans. Marine Le Pen est proche. Il y a énormément de gens en Italie, dont

${ }^{10} \mathrm{Cf}$. http://www.ecb.europa.eu/press/key/date/2014/html/sp140822.fr.html [consulté le 19/02/2015], NdE. 
une part croissante de jeunes, qui sont contre l'euro. Nous devons prendre cela au sérieux. Nous devons cesser d'être des architectes et d'imaginer de nouvelles structures légales. À la place, nous devons être des pompiers et supprimer le pacte budgétaire. Nous devons prêter de la solidarité : c'est le meilleur prêt que l'on puisse faire, car il est toujours remboursé. Par ailleurs, nous devons imaginer des symboles, bloquer et repousser les problèmes bancaires, et privilégier la résolution du problème des jeunes une fois pour toutes, grâce à un service civil, par exemple. Enfin, nous avons besoin d'unification. Cela ressemble peut-être beaucoup à Franklin Delano Roosevelt, mais nous n'avons jamais connu une telle crise depuis 80 ans. Nous vivons des temps extraordinaires qui nécessitent des solutions extraordinaires, sinon nous regretterons toujours, en tant qu'Européens, d'avoir perdu quelque chose d'aussi précieux pour une bête erreur de politique économique.

Guntram B. Wolff : Merci beaucoup. Je vous propose de passer maintenant à la dernière présentation de cette table ronde, qui a pour thème la chute du Tigre celtique : l'Irlande et la crise de la dette dans la zone euro.

Antoin E. Murphy : Merci Guntram, et encore merci de m'avoir invité à m'exprimer lors de ce colloque.

Dans cette contribution, j'aborderai les problématiques suivantes, dont certaines sont traitées dans The Fall of the Celtic Tiger: Ireland and the Euro Debt Crisis (Oxford University Press, 2013), dont je suis le co-auteur avec Donal Donovan :

- les quatre crises interdépendantes qui ont provoqué l'implosion de l'économie irlandaise ;

- l'émergence d'un système de Ponzi dans la création de la bulle immobilière irlandaise ;

- le rôle de la Banque centrale européenne (BCE) ;

- l'avenir de l'économie irlandaise.

\section{Les quatre crises interdépendantes}

La chute du Tigre celtique entre 2008 et 2010 est l'histoire de quatre crises interdépendantes. Elle a été provoquée par l'effondrement du marché de 1'immobilier, lequel a précipité une crise bancaire et une crise budgétaire. Combinées, ces deux dernières sont à l'origine du quatrième élément, la crise financière. La crise du marché de l'immobilier résulte de l'éclatement d'une bulle spéculative classique. Cette bulle impliquait des surévaluations massives, liées à la conviction générale que l'immobilier représentait le moyen le plus rapide et le plus facile de devenir riche et qu'au pire, si les prix baissaient, le marché «atterrirait en douceur ». Le marché de l'immobilier a été financé à l'aide d'un excès de prêts, consentis par un système bancaire qui a confondu les profits avec la maximisation des ventes de prêts, qui a abandonné la diversification des porte- 
feuilles pour se concentrer sur les prêts au secteur de l'immobilier et de la construction, et qui a négligé la gestion de la trésorerie ainsi que le principe de conservation d'un équilibre raisonnable entre sa base de dépôts et le montant des prêts. Dans la zone euro récemment créée, les banques irlandaises avaient accès à des financements internationaux de gros apparemment illimités, à des taux d'intérêt très bas. En même temps, elles ne subissaient pratiquement aucune réglementation financière nationale efficace, un environnement en partie né d'une idéologie économique qui portait aux nues l'efficacité des marchés et les attentes rationnelles des agents économiques. Ce type d'idéologie a mené à l'opinion que les marchés pouvaient s'autoréguler, ou qu'au pire, ils n'avaient besoin que d'une réglementation allégée. L'éclatement de la bulle immobilière a entraîné la crise bancaire. En s'appuyant trop sur l'emprunt externe, les banques se sont retrouvées exposées à des problèmes de liquidité croissants à partir de mi-2007, problèmes qui ont atteint une ampleur critique en septembre 2008 après la faillite de Lehman Brothers. Cela a entraîné une quasi-ruée sur les banques irlandaises à la fin de ce mois de septembre, et le gouvernement a dû garantir les dettes du système bancaire. Peu après, toutefois, des questions de solvabilité ont commencé à se poser, et il a fallu une aide majeure des finances publiques tout au long des années 2009 et 2010 pour maintenir les banques à flot.

En parallèle, la crise budgétaire s'est développée. Les recettes publiques étaient devenues exagérément dépendantes des taxes liées aux transactions immobilières : droit de timbre, impôts sur les plus-values, taxe sur la valeur ajoutée (TVA), et impôts directs prélevés sur le secteur immobilier. Lorsque le marché de l'immobilier a commencé à s'effondrer, l'État a subi une chute vertigineuse des recettes provenant de ces sources. Au même moment, alors que la croissance économique devenait fortement négative, la hausse du chômage a fait peser des contraintes supplémentaires sur les dépenses de protection sociale. Ainsi, avant même que les coûts budgétaires liés à la nécessité de recapitaliser les banques aient été pris en compte, le budget est passé très rapidement d'un excédent à un grave déficit. Ensemble, les crises immobilière, bancaire et budgétaire ont provoqué la crise financière fin 2010. Cette crise impliquait plusieurs éléments. Premièrement, l'incapacité croissante des banques à se procurer des liquidités sur le marché interbancaire les a rendues dépendantes des financements de la BCE, directement et indirectement, via la Banque centrale d'Irlande. En novembre 2010, ces prêts de la BCE et de la Banque centrale d'Irlande avaient atteint un total de 140 milliards d'euros, l'équivalent de $88 \%$ du PIB irlandais de l'époque. Deuxièmement, à partir de début 2010, l'énorme coût budgétaire de la recapitalisation des banques s'est révélé plus clairement. Troisièmement, malgré divers trains de mesures d'urgence, le déficit budgétaire sous-jacent - abstraction faite des coûts liés aux banques - a continué à dépasser $10 \%$ du PIB. Tous ces éléments se sont traduits par une forte dégradation de la note de la dette souveraine irlandaise, et le spread des rendements obligataires irlandais a explosé. 
Début novembre 2010, tous les partenaires extérieurs de l'Irlande ainsi que le G7, étant parvenus à la conclusion qu'une intervention était nécessaire pour sauver la situation, ont insisté pour que l'Irlande demande un renflouement à l'Union européenne (UE) et au Fonds monétaire international (FMI).

\section{Le système de Ponzi et la bulle immobilière irlandaise}

Après une chute notable en 2001, les prix de l'immobilier sont repartis à la hausse à partir de 2002, annonçant la seconde phase du boom du marché immobilier, lequel s'est rapidement métamorphosé en bulle. Le caractère de cette seconde phase était assez distinct de celui de la première phase, qui a duré jusqu'en 2000. Les prêts hypothécaires utilisés pour financer le marché de l'immobilier résidentiel ont recommencé à augmenter rapidement, aidés par les nouvelles incitations fiscales, les faibles taux d'intérêt et les innovations financières sous la forme de crédits hypothécaires à taux variable, de prêts hypothécaires à $100 \%$ (et parfois plus) de la valeur des biens ou d'extensions de la durée des prêts à trente ans et plus. Cependant, ce boom des prix de l'immobilier résidentiel, quoiqu'important, n'a pas été le principal rouage de l'engrenage spéculatif qui a caractérisé le boom du marché de l'immobilier à partir de 2002. En effet, si celui-ci était resté cantonné au marché de l'immobilier résidentiel, il aurait probablement créé une bulle relativement limitée sur ce marché, sans les conséquences que la plus grande bulle immobilière a produites, à savoir l'effondrement du système bancaire. Quelque chose d'infiniment plus sinistre que les hypothèques résidentielles avait commencé à faire son effet sur le marché immobilier, sous la forme du prêt direct de sommes toujours plus importantes aux promoteurs immobiliers. Ces prêts, comme on le verra, s'élevaient à plusieurs fois les montants prêtés dans l'immobilier résidentiel. Plus insidieusement, ils contenaient les véritables germes de la destruction du système bancaire.

Hyman Minsky, éminent chercheur keynésien, a analysé la manière dont les bulles émergeaient, en se concentrant sur leur financement. Il a suggéré trois phases de financement : le financement prudent, le financement spéculatif et le système de Ponzi. Dans la phase de financement prudent, une banque s'attache à s'assurer que les intérêts et le capital des prêts sont remboursés. Le financement spéculatif intervient lorsque la banque se concentre sur le remboursement des intérêts des prêts, mais néglige le remboursement du capital. Dans un système de Ponzi, la banque prête seulement sur la base de l'appréciation des prix de l'immobilier, et ne s'intéresse pratiquement pas (voire pas du tout) au remboursement des intérêts et du capital. Le système bancaire irlandais est rapidement passé du financement prudent au financement spéculatif, et encore plus rapidement au système de Ponzi entre 2002 et 2008.

En 2003, la banque Anglo Irish Bank, spécialisée dans les prêts aux constructeurs/promoteurs, s'était emparée d'une portion notable de ce marché 
apparemment rentable et en croissance rapide. La croissance de la part de marché et de la valorisation d'Anglo sur le marché constituait une menace pour les banques irlandaises plus prudentes, Allied Irish Bank (AIB) et Bank of Ireland. Plutôt que d'étudier la nature du portefeuille de prêts d'Anglo au secteur de l'immobilier et de la construction, et le profil très risqué de ces prêts, il semble qu'AIB et Bank of Ireland soient devenues obsédées par le taux de croissance d'Anglo. L'encours de prêts d'Anglo a successivement augmenté de $34 \%$ (2004), $41 \%$ (2005), $45 \%$ (2006), $34 \%$ (2007) et $10 \%$ (2008). De tels taux auraient dû alerter non seulement l'autorité de régulation financière et la banque centrale irlandaises, ainsi que la Banque centrale européenne, mais aussi AIB et Bank of Ireland. Au lieu de s'interroger sur la qualité des prêts qu'une telle croissance impliquait, AIB comme Bank of Ireland se sont engouffrées dans le secteur bien plus risqué des prêts immobiliers, sous la rubrique «immobilier et construction ». Ainsi, pendant qu'AIB accroissait son encours d'hypothèques de $139 \%$ entre 2003 et 2008, son encours de prêts pour l'immobilier et la construction progressait de $332 \%$. Bank of Ireland, malgré sa réputation largement répandue de plus grande prudence, a étendu son volume de prêts à l'immobilier et à la construction de $439 \%$, c'est-à-dire encore plus rapidement qu'AIB, même si elle partait de plus bas. Le contraste est flagrant avec l'augmentation des prêts hypothécaires de $95 \%$ pendant la même période. Encouragée par son succès apparemment durable, Anglo a pour sa part augmenté ses prêts à l'immobilier et à la construction de $303 \%$ pendant cette période.

C'est cet afflux de prêts pour l'immobilier et la construction aux promoteurs qui constitue la principale source de développement de la bulle immobilière entre 2003 et 2008. Le total des prêts hypothécaires consentis par AIB et Bank of Ireland a augmenté de 48 milliards d'euros, tandis que le total des prêts pour l'immobilier et la construction consentis par AIB, Bank of Ireland et Anglo a augmenté de 122 milliards d'euros.

\section{Le rôle de la Banque centrale européenne}

Il y a beaucoup de questions qui attendent une réponse concernant le rôle de la Banque centrale européenne pendant la crise financière irlandaise, en particulier en septembre 2008, lorsque le gouvernement irlandais a pris la décision de garantir le passif des banques irlandaises, et en novembre 2010, lorsque le gouvernement irlandais a été forcé d'accepter le plan de renflouement de la Troïka. On se souviendra que lorsque ces événements se sont produits, la BCE était une banque centrale toute neuve, et à cause de l'accent mis sur l'objectif de maîtriser l'inflation en négligeant le problème de la stabilité financière, il n'y avait en fait aucun cadre réglementaire détaillant les moyens de faire face aux problèmes d'un système financier en difficulté tels qu'ils se sont posés.

En 2008, après la faillite de Lehman Brothers, lorsque la fuite de capitaux a provoqué d'énormes problèmes de liquidité pour le système bancaire irlandais, 
le gouvernement s'est senti obligé d'apporter une garantie globale au passif de ce système bancaire (pour les déposants comme pour les détenteurs de titres). En arrière-plan, il semblerait que la BCE ait décrété qu'il s'agissait d'un problème irlandais, nécessitant une solution irlandaise ; en même temps, il était entendu que la solution irlandaise aurait besoin de l'approbation de la BCE. C'est pourquoi la décision irlandaise d'opter pour la garantie bancaire, qui protégeait les créanciers seniors, était une décision que la $\mathrm{BCE}$ était prête à accepter. De la part de la BCE, il s'agissait d'une approche relevant de la formule «qui ne dit mot consent». D'un autre côté, si le gouvernement irlandais avait envisagé de prendre une décision autorisant le système bancaire à faire défaut, la $\mathrm{BCE}$ aurait immédiatement rejeté une telle proposition.

Guntram B. Wolff : Excusez-moi, mais est-ce que vous parlez de 2008 ou de 2010 ?

Antoin E. Murphy : Je pense que c'était implicite en 2008, mais encore plus vrai en 2010.

Guntram B. Wolff : Avez-vous connaissance de documents à ce sujet?

Antoin E. Murphy : Eh bien, il existe des documents qui n'ont pas été publiés. [Récemment, en novembre 2014, la BCE a publié la correspondance de JeanClaude Trichet avec le ministre irlandais des Finances de l'époque, feu Brian Lenihan, avant le renflouement par la Troïka en novembre 2010. Ces lettres montrent clairement l'énorme pression exercée par Jean-Claude Trichet sur le ministre des Finances pour qu'il accepte les conditions du renflouement ${ }^{11}$.]

En 2010, la BCE s'est elle-même retrouvée dans une position plutôt étrange. À ce stade, environ un quart des sommes qu'elle avait prêtées - environ 140 milliards d'euros - étaient allées au système bancaire irlandais. Devant la nécessité d'injecter de telles quantités de liquidités, Jean-Claude Trichet devait faire des cauchemars à l'idée que le système financier irlandais puisse s'effondrer totalement. Il semble que le gouvernement irlandais ait répugné à passer à l'action. C'est pourquoi la BCE a orchestré une campagne médiatique d'envergure pour informer les journalistes et les diffuseurs qu'il y allait y avoir un gros scoop financier à Dublin. Ainsi, d'une manière très bizarre, la presse était aux portes du parlement irlandais avant même que le gouvernement du pays ait pris une décision. Au même moment, la BCE, soutenue par Timothy Geithner, alors secrétaire au Trésor américain, a certifié que l'accord éventuel de renflouement par la Troïka garantirait que les créanciers seniors seraient protégés. La BCE comme les États-Unis craignaient un second krach du style de Lehman, qui aurait pu se produire si les créanciers seniors avaient été lésés par l'Irlande. On pense que les retombées d'une telle action auraient eu un énorme pouvoir de

\footnotetext{
${ }^{11}$ Actualisation de l'auteur le 2/12/2014, NdE.
} 
contagion, qui aurait pu faire imploser non seulement le système financier européen, mais tout le système financier mondial.

La chorégraphie des événements imposée par la BCE en novembre 2010 en Irlande mérite une analyse attentive. Même si l'on peut prétendre que la fragilité du système financier irlandais et son éventuel effet domino sur la zone euro imposaient à l'Irlande une action corrective, on peut aussi avancer que la BCE n'aurait pas dû brandir le type de puissance politique qu'elle a utilisé à l'époque. Lorsqu'une banque centrale dicte ce qu'il doit faire à un gouvernement national, cela soulève d'importants problèmes de souveraineté nationale. Lorsque la BCE établira un cadre réglementaire sur la manière de gérer de telles crises à l'avenir, espérons qu'il inclura des méthodes plus diplomatiques pour ce genre de situation.

Une autre question qu'il faut se poser est la suivante : pourquoi une petite nation telle que l'Irlande aurait-elle été obligée de transférer son passif bancaire dans le bilan de sa dette souveraine afin de protéger non seulement ses propres banques, mais aussi celles de la zone euro ? C'était une chose que le système bancaire irlandais ait consenti des prêts très risqués, et on admet en Irlande que nous devons assumer une part considérable du coût de ces prêts, mais les banques françaises et allemandes, qui prêtaient fort imprudemment à leurs homologues irlandaises, n'ont supporté aucun coût. Ironiquement, au moment où les contribuables irlandais étaient contraints de payer pour l'imprudence excessive des banques irlandaises et internationales, la chancelière allemande Angela Merkel et le président français Nicolas Sarkozy expliquaient lors du sommet de Deauville, mi-octobre 2010, que cette «souverainisation » forcée du passif bancaire ne serait plus permise à l'avenir !

\section{L'avenir}

En novembre 2010, lorsque la Trö̈ka a dû intervenir pour gérer l'économie irlandaise, rares sont ceux qui auraient dit qu'en l'espace de trois ans, celle-ci serait capable de se passer des «soins intensifs » de la Troïka. C'est un résultat assez remarquable, et il convient d'applaudir la BCE, les institutions de l'UE et le FMI pour leur considérable expérience et les généreux financements qu'ils ont fournis afin de parvenir à cette issue très favorable.

Aujourd'hui, en Irlande, il y a des raisons d'espérer. Premièrement, en tant que membre de la zone euro, l'Irlande voit son avenir étroitement lié à celui de la monnaie commune. La zone euro est devenue plus stable depuis le discours «whatever it takes» (quoi qu'il en coûte) de Mario Draghi. C'est incroyable de penser qu'au moment où les tabloïds britanniques prédisaient la chute de l'euro avec des titres comme «L'euro n'a plus que sept jours à vivre » ou « La zone euro va s'effondrer la semaine prochaine », ces trois mots du président de la BCE ont suffi à faire cesser la spéculation des fonds d'investissement contre les pays de la zone euro. Par ces mots, la zone euro est arrivée à maturité, et depuis 
lors, les taux d'intérêt de l'euro ont nettement diminué, ce qui reflète la nouvelle confiance internationale dans cette devise.

Deuxièmement, l'Irlande est très liée aux économies des États-Unis et du Royaume-Uni ; ces deux pays étant actuellement en phase de croissance, cela va indubitablement profiter aux exportations irlandaises.

Troisièmement, le secteur multinational, qui a été le moteur de la première phase du Tigre celtique et qui est resté solide pendant la seconde phase, semble réémerger en cette troisième phase qui vient de débuter. Les entreprises de médias sociaux telles que Twitter, Facebook et Google ont ajouté une dimension supplémentaire aux investissements multinationaux américains en Irlande.

Quatrièmement, l'assiette des coûts pour le secteur national de l'industrie et des services s'est nettement améliorée, car les gens ont dû accepter des coupes très substantielles dans leurs salaires pendant les années d'austérité.

Cinquièmement, la perception de l'Irlande au plan international est devenue plus positive. Le fait que l'Irlande n'ait pas fait défaut (l'un des avantages de ne pas avoir restructuré la dette envers les créanciers seniors) a ajouté à la solvabilité croissante du pays sur les marchés financiers internationaux.

Pour conclure, la crise financière irlandaise a entraîné une austérité considérable, en termes de rigueur budgétaire et de réduction des salaires. Mais au lieu de s'attacher à l'insistance des populistes sur l'austérité, il serait peut-être plus approprié de remplacer le mot austérité par une autre expression : le bon sens. Le bon sens a remplacé la folie collective à l'origine de la bulle immobilière et de son éclatement. Au lieu de brûler les banques, de se perdre en grèves interminables ou de faire sans cesse étalage du ressentiment de l'opinion publique, le pays a admis qu'il était nécessaire de procéder à des améliorations considérables, en particulier en matière budgétaire. Ce bon sens, allié à l'aide financière internationale considérable de la Troïka, a aidé l'Irlande à sortir du programme de renflouement de 2010. Cela montre que dans le monde moderne où l'argent, la banque, la finance et le commerce sont interconnectés à l'échelon international, aucun pays n'est une île.

Traduction des propos de Guntram B. WOLFF, Gustavo PIGA et Antoin E. MURPHY : Marie-Céline GEORG 


\section{LES AUTEURS}

\section{Christian DEBOISSIEU}

Professeur d'économie à l'université Paris 1 Panthéon-Sorbonne, professeur au Collège d'Europe à Bruges, membre du Cercle des économistes et du Collège de l'Autorité des marchés financiers, Paris

\section{Gerald BRAUNBERGER}

Rédacteur en charge de la rubrique Marchés financiers, Frankfurter Allgemeine Zeitung

\section{Jérôme CREEL}

Directeur du département des Études de l'Observatoire français des conjonctures économiques (OFCE), Paris et professeur associé à ESCP Europe

\section{Jean-Marc DANIEL}

Économiste, professeur associé à ESCP Europe

\section{Sandrine GAUDIN}

Sous-directrice des Affaires européennes, direction générale du Trésor (jusqu'en 2014) Actuellement chef du service des Affaires bilatérales et de l'Internationalisation des entreprises, direction générale du Trésor, ministère des Finances et des Comptes publics - ministère de l'Économie, de l'Industrie et du Numérique, Paris

\section{Heinz GROSSEKETTLER}

Professeur émérite à l'Institut für Finanzwissenschaft, université de Münster et ancien président du Conseil scientifique du ministère fédéral des Finances, Berlin

\section{Solène HAZOUARD}

Ingénieur d'études au Centre d'information et de recherche sur l'Allemagne contemporaine (CIRAC), Cergy-Pontoise

\section{Friedrich HEINEMANN}

Chef du département Fiscalité des entreprises et finances publiques, Centre d'études économiques européennes (ZEW), Mannheim

\section{Christian KASTROP}

Directeur adjoint de la division Questions économiques générales, directeur de la section Finances publiques, macroéconomie et recherche, ministère fédéral des Finances, Berlin (jusqu'en 2014) Actuellement directeur du département des Études de politique économique, Organisation de coopération et de développement économiques (OCDE), Paris

\section{Pascal KAUfFMANN}

Professeur de sciences économiques, chercheur au Centre de recherche et de documentation européennes et internationales (CRDEI), université Montesquieu-Bordeaux IV 


\section{Rainer KLUMP}

Vice-président de l'université Goethe de Francfort-sur-le-Main

et directeur du département Développement économique et intégration (jusqu'en 2014)

Actuellement président de l'université du Luxembourg

\section{Hans-Helmut KoTz}

Directeur de programme au SAFE Policy Center (Sustainable Architecture for Finance in Europe), université Goethe de Francfort-sur-le-Main, Resident Fellow/directeur de programme au Center for European Studies, université de Harvard (Cambridge, États-Unis)

et ancien membre du directoire de la Deutsche Bundesbank, Francfort-sur-le-Main

\section{René LASSERRE}

Directeur du Centre d'information et de recherche sur l'Allemagne contemporaine (CIRAC) et professeur à l'université de Cergy-Pontoise

\section{Michel MARGAIRAZ}

Professeur d'histoire économique contemporaine, université Paris I Panthéon-Sorbonne, directeur de l'IDHES (Institutions et dynamiques historiques de l'économie et de la société)CNRS (UMR 8533) et responsable de la Mission historique de la Banque de France, Paris

\section{Jacques MISTRAL}

Économiste, conseiller spécial à l'Institut français des relations internationales (Ifri), membre du Cercle des économistes, Paris et Senior Fellow, Brookings Institution, Washington DC

\section{Miguel Moltó Calvo}

Professeur, Institut universitaire d'études européennes, université San Pablo de Madrid

\section{LUC MOULIN}

Ancien PDG de Howmet au sein du groupe Pechiney et membre de l'ancienne Association pour l'union monétaire de l'Europe (AUME), Paris

\section{Philippe Moutot}

Conseiller principal au sein du directorat général Économie de la Banque centrale européenne (BCE), Francfort-sur-le-Main

\section{Antoin E. MURPHY}

Professeur émérite d'économie, Trinity College, Dublin

\section{Manfred J.M. NEUMANN}

Professeur émérite, Institut de politique économique internationale, université de Bonn

\section{Gustavo PIGA}

Professeur d'économie politique, université de Rome

\section{Peter SCHAEFER}

Doctorant, université de Trente/Paris IV Sorbonne 


\section{Michael THÖNE}

Directeur exécutif de l'Institut de recherche de sciences financières de l'université de Cologne (FiFo)

\section{Frédéric TRISTRAM}

Maître de conférences, chercheur à l'IDHES (Institutions et dynamiques historiques de l'économie et de la société)-CNRS (UMR 8533), université Paris 1 Panthéon-Sorbonne

\section{Henrik UTERWEDDE}

Directeur adjoint de l'Institut franco-allemand de Ludwigsburg (DFI) (jusqu'en 2014)

Actuellement chercheur associé au DFI

\section{Natacha VALLA}

Directeur adjoint du Centre d'études prospectives et d'informations internationales (CEPII), Paris et responsable du programme Macroéconomie et finance internationales

\section{Paul J.J. WELFENS}

Président de l'Institut européen des relations économiques internationales (EIIW), chaire de macroéconomie et professeur Jean Monnet, université de Wuppertal, chaire Alfred Grosser 2007-2008, Sciences Po, Paris, Research Fellow, Institut de recherche sur l'avenir du travail (IZA), Bonn et Non-Resident Senior Fellow, AICGS (American Institute for Contemporary German Studies), université Johns Hopkins, Washington DC

\section{Guntram B. WoLFF}

Directeur de l'Institut Bruegel, Bruxelles 


Composition : CIRAC http://www.cirac.u-cergy.fr CIRAC, c/o Université de Cergy-Pontoise 33, boulevard du Port - 95011 CERGY-PONTOISE CEDEX Impression : Dupli-print 2, rue Descartes - Z.I. Sezac - 95330 DOMONT

$\mathrm{N}^{\circ}$ d'impression : 2015031705

Dépôt légal : mars 2015

Imprimé en France 
Depuis plusieurs décennies au sein de I'Union européenne, les controverses franco-allemandes en matière de coopération économique sont légion : politique commerciale, politique agricole commune dans les années 1960, politique industrielle, politique monétaire dans les années 1970 et 1980... Mais depuis le déclenchement de la crise de la zone euro en 2010, les gouvernements français et allemands ont su, malgré de fortes divergences, trouver les compromis nécessaires pour stabiliser et renforcer I'Union économique et monétaire (UEM).

L'objectif de cet ouvrage est triple : il s'agit d'identifier les constantes des cultures monétaires et budgétaires françaises et allemandes depuis 70 ans, d'analyser l'interaction entre ces cultures nationales dans la mise en œuvre de l'UEM et de se demander si l'existence de visions et de choix différents dans les deux pays freine ou peut, au contraire, favoriser l'émergence d'une gouvernance économique, financière et monétaire efficace dont l'Europe a besoin.

Couverture : @ BCE.
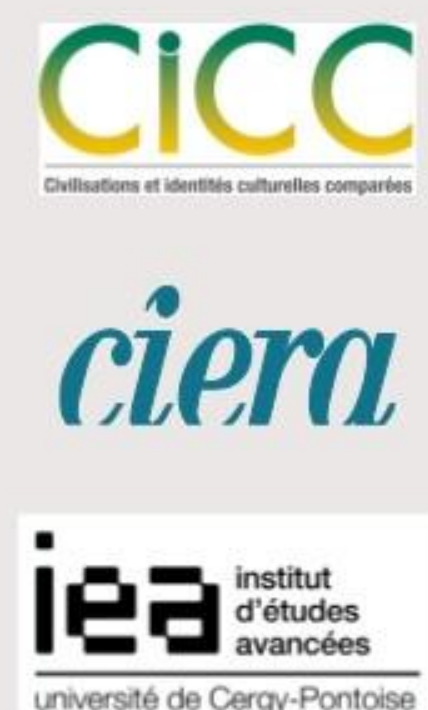

université de Cergy-Pontoise

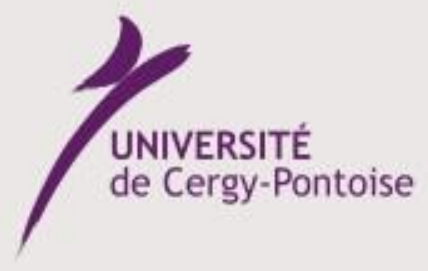

UNIVERSITÉ de Cergy-Pontoise

$25 €$

ISBN : 978-2-905518-48-4

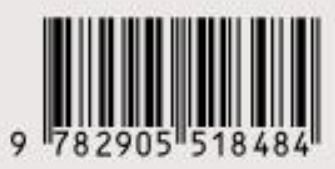

\section{CIRAC}

Université de Cergy-Pontoise - 33, boulevard du Port - 95011 Cergy-Pontoise cedex 